\title{
An investigation of basin-scale controls on upper ocean export and remineralization
}

\author{
by \\ Erin E. Black \\ M.S. Geosciences, Dartmouth College, 2006 \\ B.A. Earth Sciences, Skidmore College, 2002 \\ Submitted in partial fulfillment of the requirements for the degree of \\ Doctor of Philosophy \\ at the \\ MASSACHUSETTS INSTITUTE OF TECHNOLOGY \\ and the \\ WOODS HOLE OCEANOGRAPHIC INSTITUTION
}

February 2018

(C) 2018 Erin Elizabeth Black. All rights reserved.

The author hereby grants to MIT and WHOI permission to reproduce and to distribute publicly paper and electronic copies of this thesis document in whole or in part in any medium now known or hereafter created.

Author

Joint Program in Oceanography and Applied Ocean Science and Engineering Massachusetts Institute of Technology and Woods Hole Oceanographic Institution November 30, 2017

Certified by

Dr. Ken O. Buesseler Thesis Supervisor Woods Hole Oceanographic Institution

Accepted by

Dr. Shuhei Ono Chair, Joint Committee for Chemical Oceanography Massachusetts Institute of Technology and Woods Hole Oceanographic Institution 


\title{
An investigation of basin-scale controls on upper ocean export and remineralization
}

\author{
by
}

Erin E. Black

Submitted to the Massachusetts Institute of Technology and the Woods Hole Oceanographic Institution on November 30, 2017, in partial fulfillment of the requirements for the degree of Doctor of Philosophy in Chemical Oceanography

\begin{abstract}
The biological carbon pump (BCP) helps to moderate atmospheric carbon dioxide levels by bringing carbon to the deep ocean, where it can be sequestered on timescales of centuries to millennia. Climate change is predicted to decrease the efficiency of the global BCP, however, the magnitude and timescale of this shift is largely uncertain and will likely impact some areas of the global ocean more significantly than others. Therefore, it is imperative that we (1) accurately quantify surface export and remineralization of particulate organic carbon (POC) via the BCP over large regions of the global ocean, (2) examine the factors controlling these POC fluxes and their variability, which includes the cycling of biologically-relevant trace metals, and (3) establish if and how the BCP is changing over time.

This thesis focuses on addressing various aspects of these objectives using the ${ }^{234} \mathrm{Th}-{ }^{238} \mathrm{U}$ method across basin-scale GEOTRACES transects. First, the export and remineralization of POC were examined across large gradients in productivity, upwelling, community structure, and dissolved oxygen in the southeastern tropical Pacific Ocean. Although low oxygen zones are traditionally thought to have decreased POC flux attenuation relative to other regions of the global ocean and the low oxygen Pacific locations followed this pattern, regions that were functionally anoxic had enhanced attenuation in the upper $400 \mathrm{~m}$. Second, trace metal export and remineralization were quantified across the Pacific transect. Because many trace metals are necessary for the metabolic functions of marine organisms and can co-limit marine productivity, the controls on the cycling of trace metals in the upper ocean were examined. Lastly, POC export was determined across two transects in the Western Arctic Ocean, where light and nutrient availability drive the biological pump. Upper ocean export estimates in the central basin did not reflect a substantial change in the biological pump compared to studies from the last three decades, however, an extensive maximum in ${ }^{234} \mathrm{Th}$ relative to ${ }^{238} \mathrm{U}$ deeper in the water column indicated that rapid vertical transport had occurred, which could suggest a more efficient biological pump in the Arctic Ocean.
\end{abstract}

Thesis Supervisor: Dr. Ken O. Buesseler

Title: Senior Scientist, Marine Chemistry and Geochemistry Department, Woods Hole Oceanographic Institution 


\section{Acknowledgements}

My advisor, Ken Buesseler, has graciously provided me with numerous opportunities to work on every aspect of oceanographic work, as well as encouraged me to try out different fields of study during my time at WHOI. I am so grateful that I was given the option to participate in several field campaigns (to amazing locations like Tahiti and the North Pole) and that Ken actively involved me in many of his own projects. Over the last five years, I was truly able to observe and/or experience all areas of being a researcher and this has prepared me for a future in science. It has been an honor to learn from someone who is as knowledgeable about so many topics, who has been part of (and continues to be a part of) some of the largest oceanographic field programs to date, and who has achieved the level of success that Ken has.

My committee members, Matt Charette, Phoebe Lam, and Mick Follows, have also helped me immeasurably throughout my time at WHOI. I was fortunate to work closely with Phoebe and Matt on the Arctic GEOTRACES project, sailing with Phoebe to the North Pole and developing a new measurement method with Matt. I would try not to bug them too often, but when I did they always invested in that time and I left the meeting or call with the exact piece of information or advice that I needed. Each of them had a unique combination of honesty, pragmatism, and compassion that I greatly respected and that has made me a better person and scientist.

I would not have been able to complete my research without the help of the members of Café Thorium and the Charette Lab. Steven Pike instructed me in laboratory and ship analyses for all of the methods used in this thesis and a few other $\mathrm{Cs}$ and $\mathrm{Pu}$ techniques that are not. Spike is a wealth of information and I wouldn't be pipetting properly without him. Spike, along with Paul Henderson, have shown me the importance of teamwork, hard work, and making it work, all of which are vital in the lab and at sea. Other past and present members of the labs that have helped with various aspects of my research are Jennifer Kenyon, Crystal Breier, Jess Drysdale, Stephanie Owens, Meg Estapa, and Collen Durkin.

I want to thank Bill Jenkins, Scott Doney, Ben Van Mooy, Bill Martin, Jeff Seewald, Scott Wankel, Dave Glover, and Mak Saito for keeping an open door to all students and taking the time to discuss anything from classwork to careers. I particularly want to thank our former Education Coordinator and current Department Chair, Bernhard Peucker-Ehrenbrink, who has been a constant supportive presence in my academic life over the past five years, both in and out of the classroom. To Meg Tivey, Phoebe Lam, Amanda Spivak, Amy Aprill, Rachel Stanley, Colleen Hansel, Liz Kujawinksi and Julie Huber, I want to thank you for being role models of success, providing a kaleidoscope of a what a female scientist is, and giving your time when it was needed (even when you had little time to spare).

I would not have made it through the last 5 years without the love and understanding of my family and close friends. The extent of the thanks needed cannot fit here, but in short, Mom and Dad, your support and generosity have been unfailing through the past three decades. You brought me up to believe I could do anything I set my mind to and were always there to make sure I continued believing it. Candace, you are my rock and my support. Sean, you are my motivator and devil's advocate. Ryan, you are my scientific compass. Lauren, Megan, and Gabi, you show me every day how to be a better version of myself and a stronger scientist. You have been here every step of this thesis to cheer, suggest, prod, critique, or give whatever was needed and so are a big reason it's finally completed. Virginie, Net, Maja, Niya, and Jay, thank you for all of your support and I can't wait to see the great things you achieve in the coming years.

I also need to thank everyone in the Academic Programs Office at WHOI, including Meg Tivey, Jim Yoder, Julia Westwater, Lea Fraser, Valerie Caron, and Christine Charette. Special thanks go out to Donna Mortimer and Sheila Clifford, without whom nothing would get done. 
Over the past five years I have received funding from a variety of sources, without whom this research would not have been possible. I was funded under the NASA Earth and Space Science Fellowship Program grant (NNX13AP31H) for three years. I was also funded for work on the U.S. Pacific and Arctic GEOTRACES campaigns under two National Science Foundation grants (OCE1232669 and OCE- 1458305). The MIT Henry G. Houghton Fund provided support for the purchase of computers and textbooks and the MIT Scurlock Fund allowed for my travel to Bermuda for cruise training. WHOI Academic Programs supplemented the aforementioned funding and also provided additional support for travel to conferences. 


\section{Table of Contents}

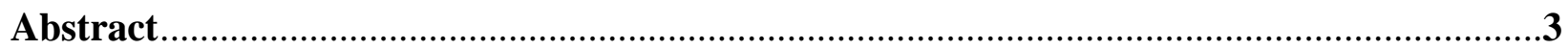

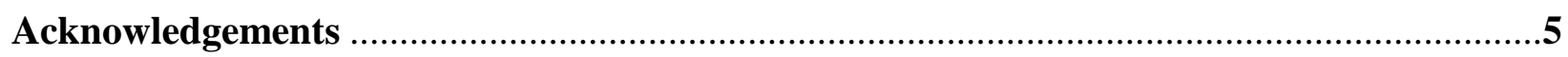

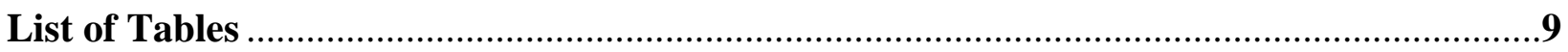

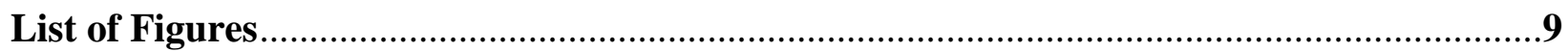

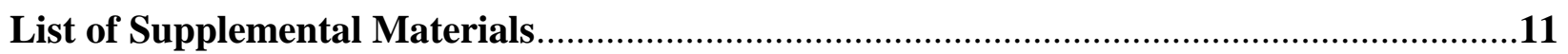

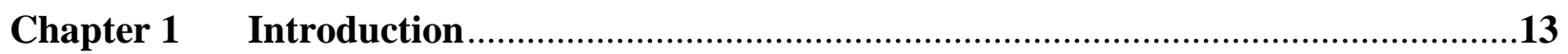

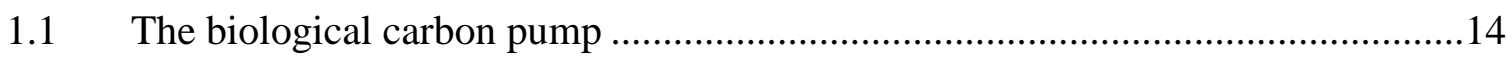

$1.2 \quad{ }^{234} \mathrm{Th}$ as a tracer of export and remineralization ................................................16

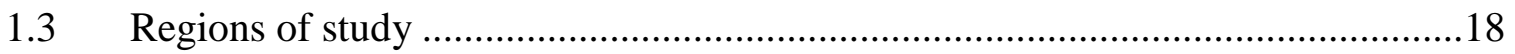

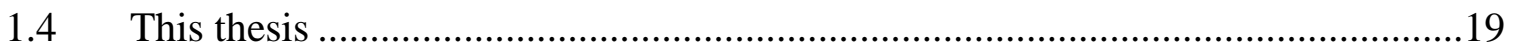

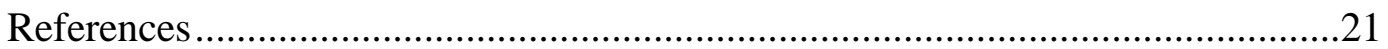

Chapter $2 \quad{ }^{234} \mathrm{Th}$ as a tracer of particulate export and remineralization in the Southeastern Tropical Pacific .........................................................................

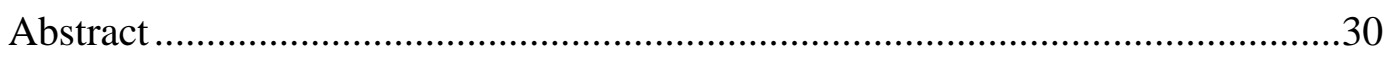

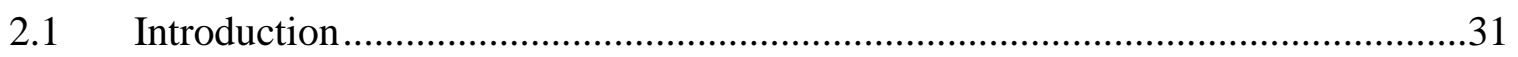

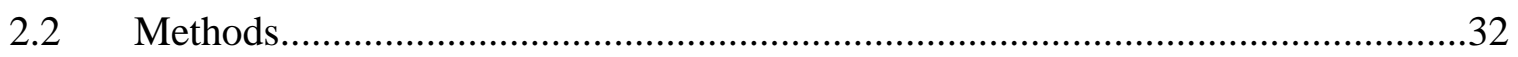

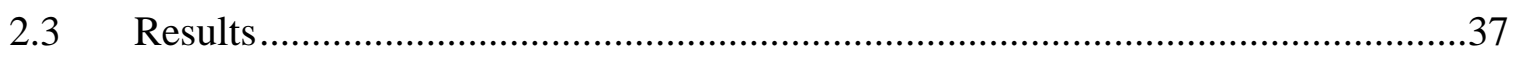

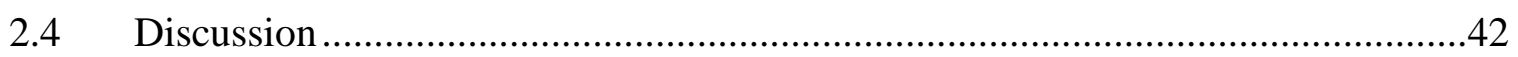

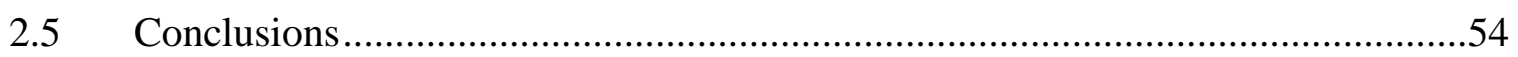

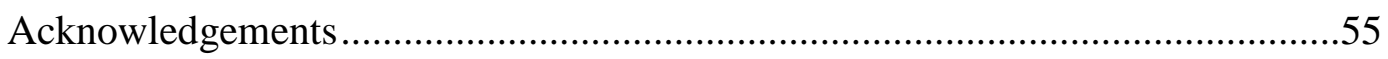

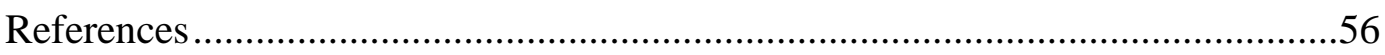

Chapter $3 \quad A^{234} \mathrm{Th}$-based method for deriving particulate export and upper ocean residence times of trace metals ......................................................................90

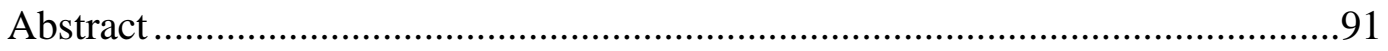

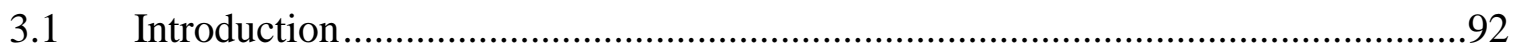

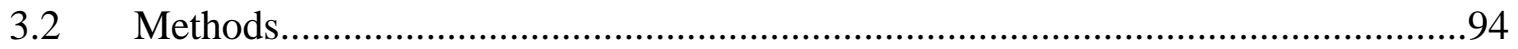

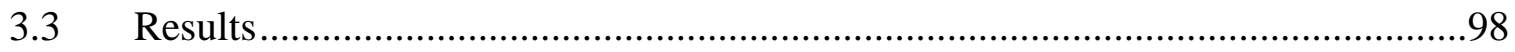

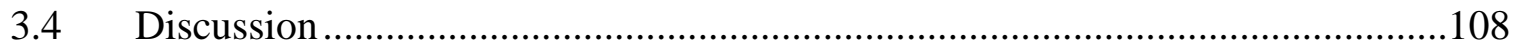

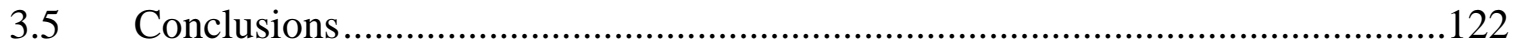

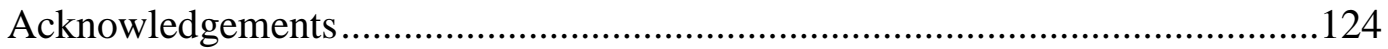

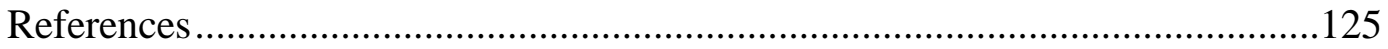

Chapter 4 Particulate export and remineralization in the Western Arctic Ocean:

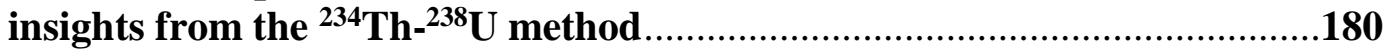

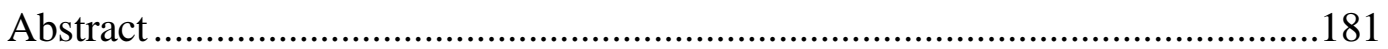




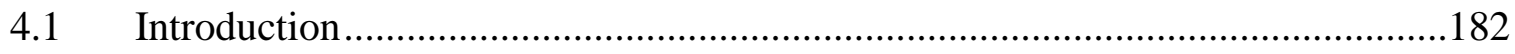

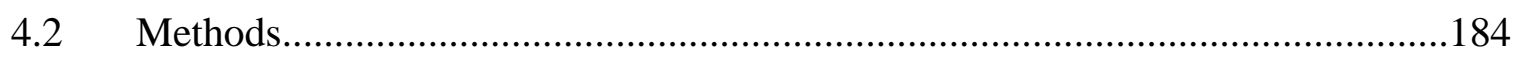

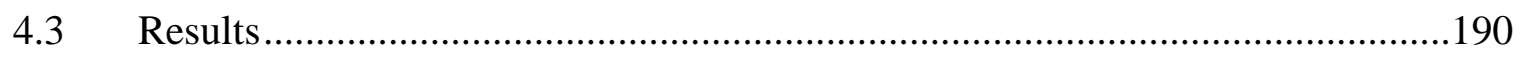

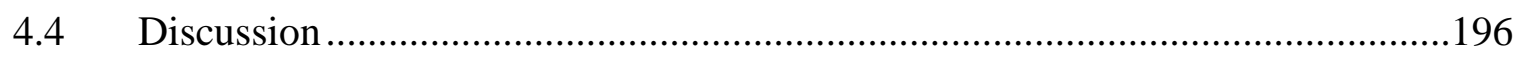

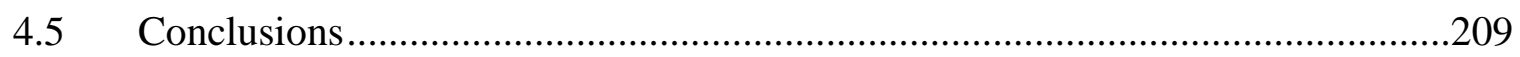

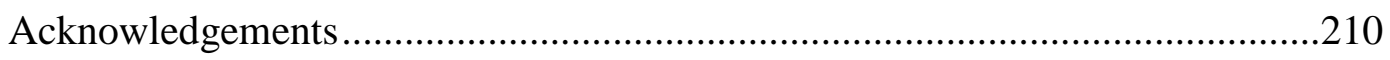

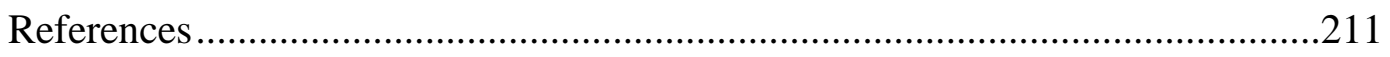

Chapter 5 Concluding Remarks and future directions ..........................................245

5.1 Export and remineralization of POC across the Peruvian OMZ ........................246

5.2 Trace metal cycling across the Peruvian OMZ ...............................................248

5.3 Export and Remineralization of POC in a changing Arctic.............................250

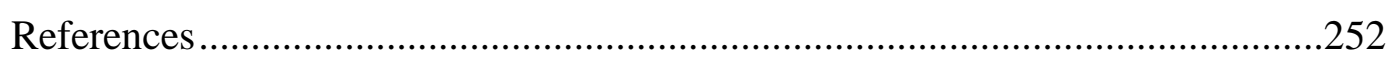

Appendix Method development for the measurement of ${ }^{228} \mathrm{Th}$ on $>51 \mu \mathrm{m}$ particulate

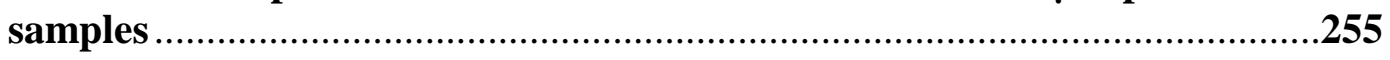

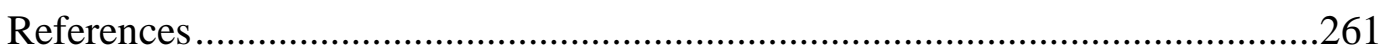




\section{List of Tables}

\section{Chapter 2}

Table 1: Summary of ${ }^{234} \mathrm{Th}$ results by station including ${ }^{234} \mathrm{Th}$-derived parameters .....................62

Table 2: Summary of zonal characteristics for the Shelf, Offshore, and Gyre zones..................64

Table $3:{ }^{234}$ Th-derived carbon and nitrogen export and remineralization results, net primary production, and Ez-ratios by station

\section{Chapter 3}

Table 1: Large and small particle E: ${ }^{234}$ Th ratios

Table 2: Fluxes, flux ratios, and \%lithogenic fraction........................................................135

Table 3: Upper ocean residence times

\section{Chapter 4}

Table 1: Station Information

Table 2: ${ }^{234} \mathrm{Th}, \mathrm{POC}$, and PON fluxes.................................................................................218

Table 3: ${ }^{234}$ Th removal rates and residence times for the shelf-slope region ..........................220

Table 4: Summary of ${ }^{234}$ Th and POC flux statistics

\section{List of Figures}

\section{Chapter 1}

Figure 1: The solubility pump and the biological carbon pump

Figure 2: The major constituents and processes composing the biological carbon pump in the surface ocean

Figure 3: Completed U.S. GEOTRACES campaigns with available ${ }^{234}$ Th-derived POC export

estimates

\section{Chapter 2}

Figure 1: Station locations with oxygen saturation at 400 meters

Figure 2: ${ }^{234} \mathrm{Th}$ results and relevant CTD sensor parameters with oxygen saturation $\%$.................68

Figure 3: ${ }^{234} \mathrm{Th}$ and ${ }^{238} \mathrm{U}$ activity profiles in $\mathrm{dpm} \mathrm{L}^{-1}$ for the upper 500 meters of the water column.

Figure 4: Carbon: ${ }^{234} \mathrm{Th}$ on large particles $(>51 \mu \mathrm{m})$ in $\mu \mathrm{mol} \mathrm{dpm}{ }^{-1}$ for the upper 400 meters .....70

Figure 5: ${ }^{234} \mathrm{Th}$ export at Ez and remineralization through the $200 \mathrm{~m}$ below Ez.........................71

Figure 6: Regional ${ }^{234}$ Th-derived carbon fluxes ..................................................................72

Figure 7: ${ }^{234}$ Th-derived Ez-ratios (export at Ez/NPP) and $\mathrm{T}_{100}$ (flux at $100 \mathrm{~m}$ below Ez/export at

Ez) values for POC

\section{Chapter 3}

Figure 1: Station locations with prior 0 to $400 \mathrm{~m}$ study sites for trap- and ${ }^{234} \mathrm{Th}$-based flux estimates

Figure 2: Element concentration and $\mathrm{E}:{ }^{.34} \mathrm{Th}$ 
Figure 3: Element ratios and fluxes for characteristic stations in the Shelf (4), Offshore (9), and

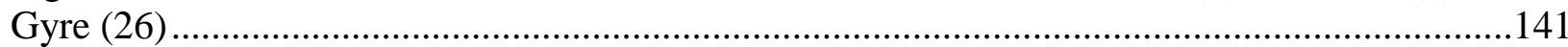

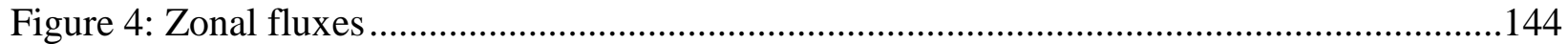

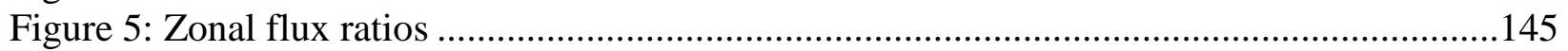

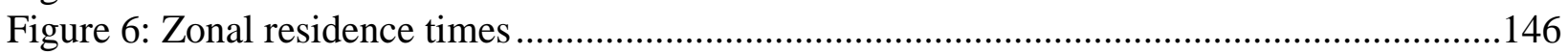

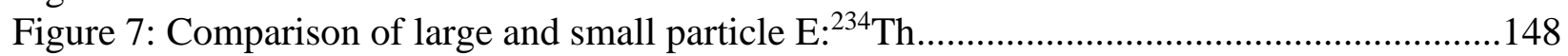

Figure 8: Particulate cycling of Cd, Co, Mn, and Fe in the Southeastern Tropical Pacific .........149

\section{Chapter 4}

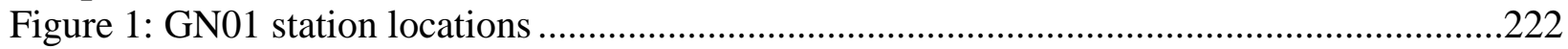

Figure 2: Typical hydrographic and biogeochemical features of the Western Arctic Ocean ......223

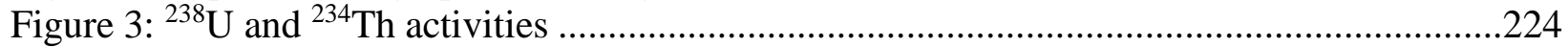

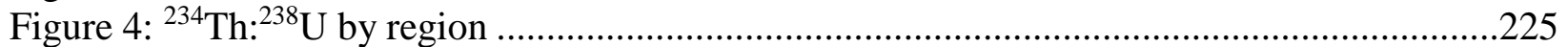

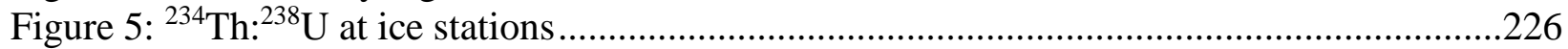

Figure 6: Full water column ${ }^{238} \mathrm{U}$ activity minus ${ }^{234} \mathrm{Th}$ activity $\left(\mathrm{dpm} \mathrm{L} \mathrm{L}^{-1}\right)$............................222

Figure 7: Oxygen saturation, the fraction of dissolved ${ }^{234} \mathrm{Th}$, large particle ${ }^{234} \mathrm{Th}$ activity, and

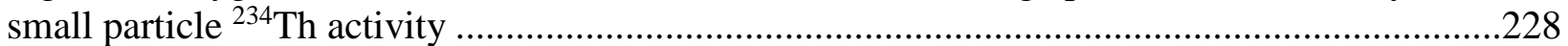

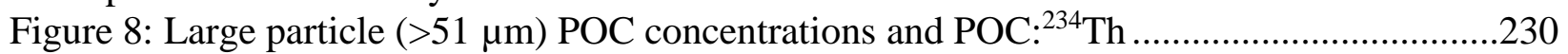

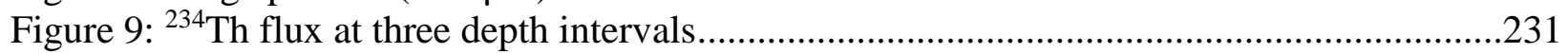

Figure 10: Three potential causes of the mid-deep water ${ }^{234} \mathrm{Th}$ activity excesses at NB stations

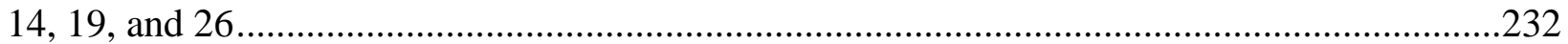

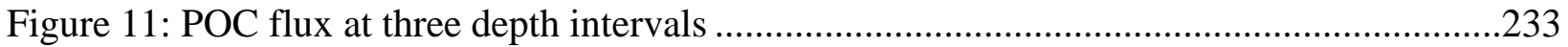

\section{Chapter 5}

Figure 1: ${ }^{234}$ Th-derived POC export fluxes from the three completed U.S. GEOTRACES campaigns 


\section{List of Supplemental Materials}

\section{Chapter 2}

Supplemental Table 1: Depth of relevant physical parameters for the shallow ${ }^{234} \mathrm{Th}$ cast and the

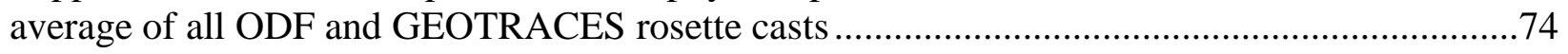

Supplemental Table 2: Results for $100 \mathrm{~m}$ calculations.......................................................76

Supplemental Table 3: Parameters used to derive the ${ }^{234}$ Th flux component due to upwelling in

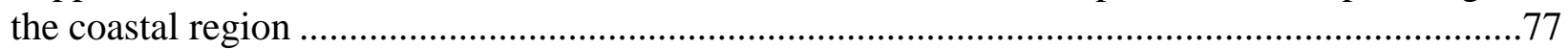

Supplemental Figure 1: Example of inter-cast variability in oxygen ....................................78

Supplemental Figure 2: Examples of intra-cast variability in oxygen for casts where ${ }^{234} \mathrm{Th}$ was collected

Supplemental Figure 3: Nitrogen: ${ }^{234} \mathrm{Th}$ on large particles $(>51 \mu \mathrm{m})$ in $\mu \mathrm{mol} \mathrm{dpm}{ }^{-1}$ for the upper

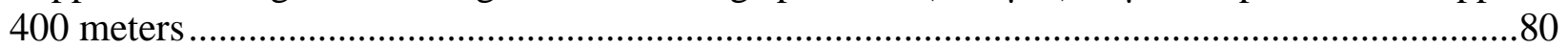

Supplemental Figure 4: Average surface current velocities for October 2013 ..........................81

Supplemental Figure 5: ${ }^{238} \mathrm{U}$-minus ${ }^{234} \mathrm{Th}$ activity (colors) with density anomaly $\left(\rho_{\theta}\right)$ and

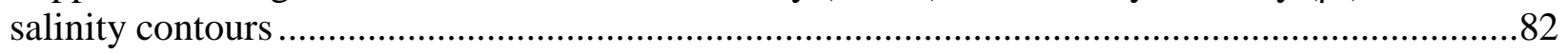

Supplemental Figure 6: Potential density profiles for coastal stations (upper $140 \mathrm{~m}$ ) ...............83

Supplemental Figure 7: Flux of ${ }^{234}$ Th due to upwelling calculated at the base of the euphotic

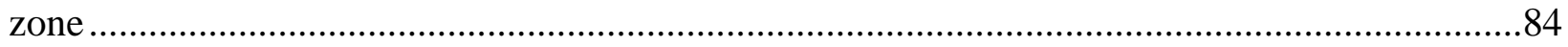

Supplemental Figure $8:{ }^{234} \mathrm{Th}$ results plotted relative to the upper $30 \%$ oxygen saturation boundary

\section{Chapter 3}

Supplemental Table 1: Zonal boundaries and ${ }^{234}$ Th flux results ............................................153

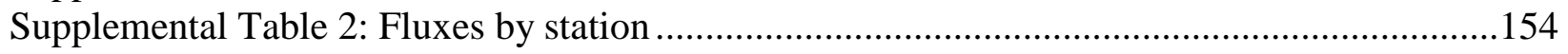

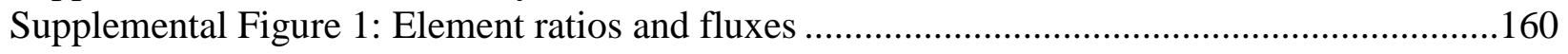

Supplemental Figure 2: Element fluxes at $100 \mathrm{~m}$ and $200 \mathrm{~m}$ by station ................................178

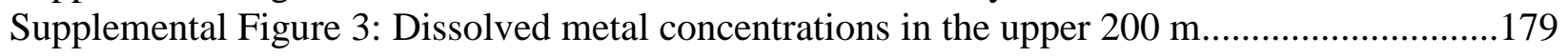

\section{Chapter 4}

Supplemental Figure 1: CPM for total ${ }^{234}$ Th samples versus chemical recovery......................234

Supplemental Figure 2: Chemical recoveries by location for all total ${ }^{234} \mathrm{Th}$ samples ................235

Supplemental Figure 3: Fluorescence data with salinity contours for the NB and SB transects .236

Supplemental Figure 4: Average monthly Chl-a concentrations at station locations .................237

Supplemental Figure 5: Average monthly Chl-a concentrations for the Western Arctic ............238

Supplemental Figure 6: Physical and geochemical data from three NB stations with observed

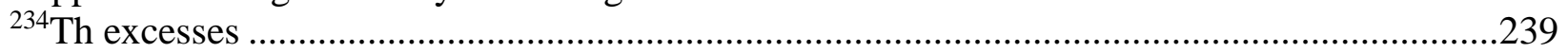

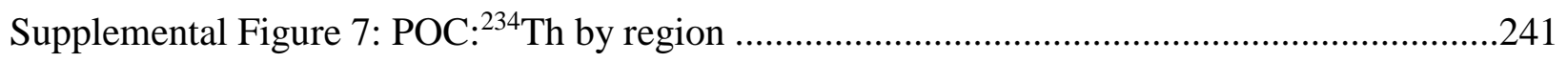

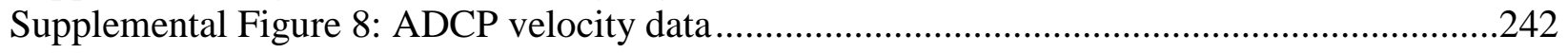

\section{Appendix}

Table 1: Summary of RaDeCC detector efficiency results...................................................262

Figure 1: Preliminary ${ }^{228}$ Th activities along the northbound Arctic transect............................263

Figure 2: Preliminary ${ }^{228} \mathrm{Th}$ activities along the southbound Arctic transect ..................................264

Figure 3: Small volume chamber construction schematics..............................................265

Figure 4: Small volume chamber interior construction schematics .....................................266 
Figure 5: Photographs of small volume chambers

Figure 6: Example calibration for large particle ${ }^{228} \mathrm{Th}$ on RaDeCCs with small volume chambers versus traditional alpha spectrometry .268

Figure 7: RaDeCC results for wet versus dry measurements ... 
Chapter 1:

Introduction 


\subsection{The biological carbon pump}

The distribution of carbon in the global ocean is controlled by two mechanisms: the solubility pump and the biological pump (Fig. 1). The biological carbon pump (BCP) refers to processes by which carbon dioxide $\left(\mathrm{CO}_{2}\right)$ is first fixed by organisms into organic carbon in the shallow ocean and subsequently transported to depth. Most of the fixed carbon that is incorporated into organic matter via the $\mathrm{BCP}$ is remineralized, converted to inorganic forms, and recycled within the water column, never making it to deep ocean sediments (Martin et al., 1987). The BCP encompasses a suite of physical, chemical, and biological processes (Fig. 2), while the solubility pump is governed by thermodynamics and physical mixing (Ito and Follows, 2003; Toggweiler et al., 2003). Sinking particles can transport particulate organic carbon (POC) below the depth of winter mixing to the deep ocean in a matter of days to weeks (McDonnell and Buesseler, 2010), much faster than the solubility pump, which works on the centennial to millennial timescales of ocean circulation (Khatiwala et al., 2012; Matsumoto, 2007). It has been predicted that without the BCP, atmospheric levels of $\mathrm{CO}_{2}$ could increase up to 200 parts per million (Sarmiento and Toggweiler, 1984). Therefore, understanding how the BCP functions as a mechanism for facilitating the long term storage of carbon is central to elucidating the oceanic controls on climate change.

The ability to accurately quantify the amount of carbon transported via the BCP is vital to our

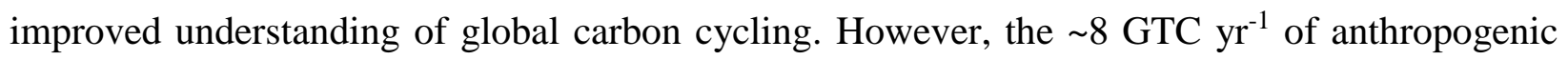
$\mathrm{CO}_{2}$ added to the atmosphere from fossil fuel burning (Ciais et al., 2013) is as large as the 5 GTC

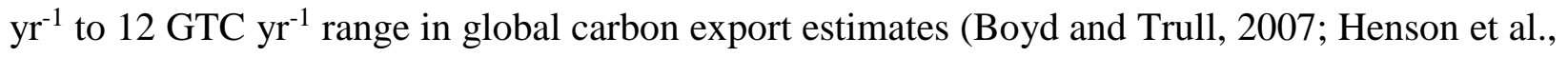
2011; Siegel et al., 2014). These estimates cannot be improved without a greater knowledge of the spatial and temporal variability of upper ocean POC fluxes and their incorporation into global climate models. The large range in global carbon estimates stems from the individual uncertainties associated with each of the processes that compose the BCP (Fig. 2), such as vertical mixing and zooplankton migration, as well as the necessary, but simplified boundary conditions used in these models. For instance, global carbon export is frequently estimated at $100 \mathrm{~m}$ when the euphotic zone (the upper sunlit portion of the ocean) can vary by more than $100 \mathrm{~m}$ worldwide (Buesseler and Boyd, 2009; Henson et al., 2011; Siegel et al., 2014). Model sensitivity analysis has shown that a small change $(24 \mathrm{~m})$ in the remineralization depth of POC can increase of atmospheric $\mathrm{CO}_{2}$ 
by 10 to 27 parts per million (Kwon et al., 2009), which illustrates the importance of high resolution estimates of export and remineralization in the upper $400 \mathrm{~m}$ of the water column.

A few key parameters and metrics are used to quantify and characterize the BCP. First, net primary production (NPP) represents the gross production by primary producers (GPP) in the surface ocean minus the respiration by these organisms (NPP $=$ GPP - respiration). Export is the quantity of organic matter leaving the surface ocean, usually given at a fixed depth (i.e. $100 \mathrm{~m}$ ), or at the euphotic zone base (Ez). In theory, Ez marks the depth in the water column where net autotrophic production reaches zero and below this depth POC would only be remineralized or produced chemosynthetically. Export divided by the NPP is referred to as the 'strength' of the BCP or how much of the surface production is being utilized by euphotic communities and how much makes it to depth. Below Ez, remineralization or the breakdown of organic matter is larger than any non-chemosynthetic production and the rate of these processes control how much organic matter makes its to depth. In this region of the water column, we refer to the 'efficiency' of the $\mathrm{BCP}$ as the amount of carbon left at a given depth (i.e. that is not remineralized or attenuated) relative to how much was exported at Ez.

Many factors impact the strength and efficiency of the BCP, which makes it difficult to determine exactly how each process acts (and interacts) to contribute to the downward transport of POC (Fig. 2). The surface community structure has been shown to be important to the magnitude of export (e.g. Boyd and Trull, 2007; Siegel et al., 2014). In areas like the Peruvian upwelling zone, the diversity of food-webs (Maas et al., 2014; Rii et al., 2016; Seibel et al., 2016; Stevens and Ulloa, 2008) is linked to the supply of nutrients from the deep ocean and water column chemistry (Pennington et al., 2006). Therefore, factors like oxygen concentrations and water mass transport can influence the BCP at a regional scale (Paulmier et al., 2006). The availability of micronutrients, such as iron $(\mathrm{Fe})$, which are essential for cellular processes like photosynthesis, can also impact regional productivity and community structure (Sunda, 2012). Mineral ballast, protection, or association have often been suggested as an addition control on export and remineralization, but the relationship between export and the amount of carbonate or silicate in sinking particulate matter is not a simple one (Boyd and Trull, 2007; De La Rocha and Passow, 2007; Francois et al., 2002; Hedges et al., 2001). The extent of vertical mixing and zooplankton 
migration also play a role in the amount of material exported (Buesseler and Boyd, 2009; Marra et al., 1995; Steinberg and Landry, 2017).

Models have indicated that global climate change decreases the ocean's ability to take up carbon by decreasing the strength of the biological and solubility pumps (Matsumoto et al., 2010) and thus, it is important to establish how the strength and efficiency of the BCP is being altered regionally and globally. Therefore, it is imperative that we (1) accurately quantify the magnitude of surface export and remineralization of POC via the BCP over large regions of the global ocean, (2) examine the factors controlling these magnitudes and their variability, which includes the cycling of biologically-relevant trace metals, and (3) establish if and how the BCP is changing over time. Each of these objectives has been addressed to some extent in Chapters 2 through 4 of this thesis using the ${ }^{234} \mathrm{Th}^{2}{ }^{238} \mathrm{U}$ disequilibrium method as the primary tool.

\section{$1.2{ }^{234} \mathrm{Th}$ as a tracer of export and remineralization}

Partition coefficients for thorium in seawater (ratio of $\mathrm{Bq} \mathrm{kg}^{-1}$ suspended to $\mathrm{Bq} \mathrm{L}^{-1}$ dissolved) are generally on the order of $10^{6}$, making it one of the most particle reactive of all the elements (Geibert and Usbeck, 2004; Santschi et al., 2006). Due to this high affinity for particles, naturally occurring thorium isotopes have become one of the most extensively utilized and reliable means of tracking particle dynamics and export in the ocean (Cochran and Masque, 2003; Rutgers van der Loeff et al., 2006). ${ }^{234}$ Th has a relatively long history of use as a flux proxy (Bhat et al., 1969; Buesseler et al., 1995; Cai et al., 2008; Coale and Bruland, 1987; Rutgers van der Loeff et al., 2011). The majority of ${ }^{234}$ Th-based flux studies have focused on tracking carbon export out of the upper $200 \mathrm{~m}$ to $400 \mathrm{~m}$ (Le Moigne et al., 2013), although nitrogen (N) and silicon (Si) are two other elements of interest to which this method has been applied (e.g. Buesseler et al., 2001a). The

${ }^{234} \mathrm{Th}$ method provides an alternative to traditional sediment trap export studies (e.g. surfacetethered traps) that can have sample preservation, swimmer, and hydrodynamic issues (Buesseler et al., 2007).

${ }^{234} \mathrm{Th}\left(\mathrm{t}_{1 / 2} \sim 24.1\right.$ days $)$ forms via the decay of ${ }^{238} \mathrm{U}$, which is highly soluble in seawater, ubiquitous in the ocean, long-lived ( $\mathrm{t}_{1 / 2} \sim 4.47$ billion years), and conservative in the ocean with respect to salinity. If ${ }^{234} \mathrm{Th}$ attached to sinking particles is transported out of the surface ocean at a faster rate than it can be replenished by the decay of ${ }^{238} \mathrm{U}$, a disequilibrium is created between the parent and daughter isotopes. This resulting deficit of ${ }^{234} \mathrm{Th}$ in the surface can be integrated to 
determine a net particulate flux of ${ }^{234} \mathrm{Th}$ at a given depth $z$. If the relative abundance of a given element and ${ }^{234} \mathrm{Th}$ (element: ${ }^{234} \mathrm{Th}$ ) are measured on sinking particles at depth $z$, a flux of that element can be calculated by multiplying the particulate ${ }^{234} \mathrm{Th}$ flux by this ratio (Buesseler et al., 2006). The same principle can be applied to ${ }^{234} \mathrm{Th}$ excesses observed below the sunlit upper ocean to determine remineralization rates (Maiti et al., 2010).

In Chapters 2 through 4 , the ${ }^{234} \mathrm{Th}^{-238} \mathrm{U}$ disequilibrium method was employed to quantify and assess the export and remineralization of POC. While ${ }^{234} \mathrm{Th}$-based estimates of POC flux are numerous, many regions of the world's ocean have yet to be visited (Le Moigne et al., 2013) and many earlier studies have had limited depth resolution due to the large volumes needed for cartridge- and gamma-based methods (Buesseler et al., 1995). The reduction of the sample volume required to $4 \mathrm{~L}$ (Buesseler et al., 2001b) and the switch to shipboard beta counting have made higher resolution sampling more manageable for large field studies, such as the two U.S. GEOTRACES campaigns discussed in this thesis. These basin-scale transects are vital to constrain the variability in the global BCP and specifically, to quantify remineralization and transfer efficiencies. Remineralization features can be difficult to capture if they occur over a narrow section of the water column (Maiti et al., 2010). Only a few prior studies have attempted to use ${ }^{234} \mathrm{Th}$ to assess remineralization (Buesseler et al., 2008; Maiti et al., 2010; Savoye et al., 2004), but accurate estimates of NPP, export, and remineralization are all needed to determine the amount of an element that has been incorporated into particulate matter in the surface ocean that makes it to depth over a given period of time.

Since the first published effort that utilized ${ }^{234} \mathrm{Th}$ a flux proxy for particulate aluminum ( $\left.\mathrm{Al}\right)$, $\mathrm{Fe}$, lead $(\mathrm{Pb}$ ), and barium (Ba) (Weinstein and Moran, 2005), the number of studies applying variations of the ${ }^{234} \mathrm{Th}^{-238} \mathrm{U}$ method to estimate fluxes for elements besides $\mathrm{C}, \mathrm{N}$, and $\mathrm{Si}$ has gradually increased. In the last decade the technique has most often been used to derive metal fluxes (see Chapter 3), although a few studies have branched into novel areas, such as polycyclic aromatic hydrocarbons (Adhikari et al., 2016) and black carbon (Fang et al., 2016). While the assumptions involved in utilizing the ${ }^{234} \mathrm{Th}^{238} \mathrm{U}$ method to quantify the export of POC have been well documented (Buesseler et al., 2006; Rutgers van der Loeff et al., 2006; Savoye et al., 2006), the application of the method to other elements brings new challenges that have yet to be fully explored. However, many trace metals are critical to metabolic processes (Sunda, 2012; Sunda and 
Huntsman, 1997) and determining the degree to which surface ecosystems retain and are restricted by these micronutrients is vital to our understanding of the BCP. In other words, the distribution and flux of these elements can both aid in controlling and are controlled by the processes composing the BCP, which is why large-scale efforts to quantify the factors contributing to the export and remineralization of key trace metals are so important (see Chapter 3).

\subsection{Regions of study}

Global export and climate models depend on our understanding of how various regions of the world's ocean take up, transform, and transport carbon. Greater investigation of how the BCP functions in diverse ocean systems, such as shallow coastal and deep ocean regions, low and high latitudes, and central gyres and high nutrient-low chlorophyll regions (HNLCs), and how each of these systems collectively contribute to the global export and sequestration of carbon is needed. This thesis focuses on two regions already being altered by climate change that could experience shifts in the overall efficiency of their respective regional BCPs. Depending on the scale of these changes, the BCP in these locations may play a more important role in the global sequestration of carbon over the next few decades.

The first study region is the Peruvian oxygen minimum zone (OMZ), where sampling took place during the austral fall and winter of 2013 (Fig. 3). OMZs are regions of low oxygen in the upper water column which are permanently maintained by a combination of physical and biological factors, such as upwelling, stratification, poor ventilation, and high coastal productivity. OMZs have been growing over the last few decades, with intensifying, functionally anoxic cores and broadening edges, both horizontally and vertically (Stramma et al., 2010, 2008). These zones are predicted to continue growing with the increased effects of climate change. In short, warming temperatures decrease the solubility of dissolved gases, including oxygen, and increased stratification means decreased exchange to replenish the depleted low oxygen regions (Matear and Hirst, 2003; Stramma et al., 2008). A few limited studies have looked at export through the world's largest OMZs (e.g. Devol and Hartnett, 2001; Van Mooy et al., 2002), but not many with the resolution needed to project how the growth of OMZs could impact the regional BCPs, as suggested by the study of small changes in remineralization length scales (Kwon et al., 2009).

The second region of interest is the Western Arctic Ocean, which was sampled along two North-South transects starting in August 2015 (Fig. 3). The broad coastal shelves in the Arctic can 
boast NPP rates that rival any other region in the world's ocean during short periods in spring and summer (Arrigo and van Dijken, 2015; Arrigo and Van Dijken, 2011) and the regional BCP could change drastically as sea ice cover and sea ice thickness continue to decrease (Arrigo and van Dijken, 2015). Prior studies of the central Arctic basin have indicated that it is a highly oligotrophic region, with an export efficiency similar to that of the Pacific gyres (Cai et al., 2010; Moran et al., 1997), but we have a poor understanding of the connection between shelf-slope processes and the central Arctic BCP and there is no consensus on what the net effect of climate change will be on carbon sequestration in the Arctic Ocean as a whole (Walsh et al., 2004; Wassmann et al., 2004; Wassmann and Reigstad, 2011). The limited satellite-derived NPP estimates that are available from coastal regions have been increasing in the Arctic Ocean (Arrigo and van Dijken, 2015) and massive under ice blooms have been reported (Arrigo et al., 2012). Therefore, in-situ measurements of export are vital to creating a historical record of changes in the Arctic BCP and understanding what controls, or could impact, the BCP of both the present and future Arctic Ocean.

\subsection{This thesis}

Three independent chapters have been included in this thesis that strive to constrain the magnitude of basin-scale surface export and remineralization in the two areas of study detailed above and attempt to elucidate the role of various controls on the regional BCPs. With the unprecedented sampling resolution of the two GEOTRACES datasets that have been utilized here, each chapter is able to push the boundaries of how the ${ }^{234} \mathrm{Th}^{238} \mathrm{U}$ method can be applied in the upper $400 \mathrm{~m}$ of the world's ocean to understand what impacts export and remineralization at a regional scale. A final chapter summarizes the thesis, discusses any outstanding questions, and puts forth suggestions for future research directions in this field.

Chapters 2 and 3 focus on the southeastern tropical Pacific transect. The first data chapter examines the relationship between satellite-derived surface NPP, ${ }^{234} \mathrm{Th}$-derived export at Ez, and the amount of remineralization occurring at $100 \mathrm{~m}$ and $200 \mathrm{~m}$ below Ez. These relationships are examined in three types of low oxygen regions: the coastal oxygen deficient zone (ODZ; $\left.\mathrm{nM} \mathrm{O}_{2}\right)$; the offshore ODZ; and the low nutrient $\left(<5 \mu \mathrm{mol} \mathrm{kg}{ }^{-1}\right)$, low oxygen $(1-100 \mu \mathrm{M} \mathrm{O}$,), tropical Pacific gyre. The largest remineralization feature ever observed with the ${ }^{234} \mathrm{Th}^{2}{ }^{238} \mathrm{U}$ method (>7500 $\mathrm{km}$ ) is characterized in Chapter 2 and has provided unique insight into the differences between the strength and efficiency of the BCP in these three types of low oxygen regions. 
Chapter 3 is one of the first basin-scale attempts to apply the ${ }^{234} \mathrm{Th}^{-238} \mathrm{U}$ method to trace metal cycling in the upper ocean, specifically for $\mathrm{Fe}$, manganese (Mn), cadmium (Cd), and cobalt (Co). Although all of these trace metals are biologically relevant, the combination of factors controlling the export observed at $100 \mathrm{~m}$ and $200 \mathrm{~m}$ across the Pacific transect was unique to each element. The patterns in export and remineralization of nutrients along this transect, such as $\mathrm{N}$ and phosphorus $(\mathrm{P})$, closely followed those of POC, but the distribution and fluxes of elements like Fe and $\mathrm{Mn}$ appeared to be impacted by additional factors, such as source inputs (e.g. lateral advection and dust), redox conditions (e.g. oxygen levels), and scavenging within the water column. Because trace metals are necessary for many cellular functions and their abundance or scarcity can influence the $\mathrm{BCP}$, it is important to quantify the surface export of these elements and understand the drivers of trace metal cycling in the upper ocean. Efforts to include metals in global biogeochemical models have illustrated our lack of understanding of the controls on trace metal distributions in the global ocean (Tagliabue et al., 2016; van Hulten et al., 2017) and the surface export estimates contained within Chapter 3 are a first step to improving these models.

Lastly, Chapter 4 focuses on results from the U.S. Arctic GEOTRACES campaign. The export and remineralization of POC and PON are quantified across the shallow shelves and the upper 200 $\mathrm{m}$ of the central Arctic basin. Since ${ }^{234} \mathrm{Th}$ has been measured in the central Arctic on a few occasions over the last three decades, this chapter on the Western Arctic BCP is also able to draw conclusions about whether a significant change in POC export has occurred over this time period. In addition, few historical studies have sampled deeper than $200 \mathrm{~m}$ in the central Arctic because this region is thought to be oligotrophic and thus, ${ }^{234} \mathrm{Th}$ would be at equilibrium with ${ }^{238} \mathrm{U}$ here without significant particle production. This study sampled the full water column and thus, was able to examine this assumption of equilibrium and pose hypotheses for how carbon cycling might be different in the Arctic Ocean than in other larger, non-ice covered ocean basins. Specifically, the possibilities of sinking algae from massive under-ice bloom events (vertical transport) or shelfderived inputs of carbon to the deep basin (lateral transport) were assessed. 


\section{References}

Adhikari, P.L., Maiti, K., Bosu, S., Jones, P.R., 2016. ${ }^{234}$ Th as a tracer of vertical transport of polycyclic aromatic hydrocarbons in the northern Gulf of Mexico. Mar. Pollut. Bull. 107, 179-187. doi:10.1016/j.marpolbul.2016.04.002

Arrigo, K.R., Perovich, D.K., Pickart, R.S., Brown, Z.W., van Dijken, G.L., Lowry, K.E., Mills, M.M., Palmer, M.A., Balch, W.M., Bahr, F., Bates, N.R., Benitez-Nelson, C., Bowler, B., Brownlee, E., Ehn, J.K., Frey, K.E., Garley, R., Laney, S.R., Lubelczyk, L., Mathis, J., Matsuoka, A., Mitchell, B.G., Moore, G.W.K., Ortega-Retuerta, E., Pal, S., Polashenski, C.M., Reynolds, R.A., Schieber, B., Sosik, H.M., Stephens, M., Swift, J.H., 2012. Massive Phytoplankton Blooms Under Arctic Sea Ice. Science 336, 1408-1408. doi:10.1126/science.1215065

Arrigo, K.R., van Dijken, G.L., 2015. Continued increases in Arctic Ocean primary production. Prog. Oceanogr. 136, 60-70. doi:10.1016/j.pocean.2015.05.002

Arrigo, K.R., Van Dijken, G.L., 2011. Secular trends in Arctic Ocean net primary production. J. Geophys. Res. Ocean. 116, 1-15. doi:10.1029/2011JC007151

Bhat, S.G., Krishnaswamy, S., Lal, D., Rama, Moore, W.S., 1969. ${ }^{234} \mathrm{Th} /{ }^{238} \mathrm{U}$ ratios in the ocean. Earth Planet. Sci. Lett. 5, 483-491.

Boyd, P.W., Trull, T.W., 2007. Understanding the export of biogenic particles in oceanic waters: Is there consensus? Prog. Oceanogr. 72, 276-312. doi:10.1016/j.pocean.2006.10.007

Buesseler, K.O., Andrews, J.A., Hartman, M.C., Belastock, R., Chai, F., 1995. Regional estimates of the export flux of particulate organic carbon derived from thorium-234 during the JGOFS EqPac program. Deep. Res. II 42, 777-804.

Buesseler, K.O., Antia, A.N., Chen, M., Fowler, S.W., Gardner, W.D., Gustafsson, O., Harada, K., Michaels, A.F., Rutgers van der Loeff, M., Sarin, M., Steinberg, D.K., Trull, T., 2007. An assessment of the use of sediment traps for estimating upper ocean particle fluxes. J. Mar. Res. $65,345-416$.

Buesseler, K.O., Ball, L., Andrews, J., Cochran, J.K., Hirschberg, D.J., Bacon, M.P., Fleer, A., Brzezinski, M., 2001a. Upper ocean export of particulate organic carbon and biogenic silica in the Southern Ocean along 170 W. Deep. Res. II 48, 4275-4297.

Buesseler, K.O., Benitez-Nelson, C., Rutgers van der Loeff, M., Andrews, J., Ball, L., Crossin, G., Charette, M.A., 2001b. An intercomparison of small- and large-volume techniques for thorium234 in seawater. Mar. Chem. 74, 15-28.

Buesseler, K.O., Benitez-Nelson, C.R., Moran, S.B., Burd, A., Charette, M., Cochran, J.K., Coppola, L., Fisher, N.S., Fowler, S.W., Gardner, W.D., Guo, L.D., Gustafsson, O., Lamborg, C., Masque, P., Miquel, J.C., Passow, U., Santschi, P.H., Savoye, N., Stewart, G., Trull, T., 2006. An assessment of particulate organic carbon to thorium-234 ratios in the ocean and their impact on the application of ${ }^{234} \mathrm{Th}$ as a POC flux proxy. Mar. Chem. 100, 213-233. doi:10.1016/j.marchem.2005.10.013

Buesseler, K.O., Boyd, P.W., 2009. Shedding light on processes that control particle export and flux attenuation in the twilight zone of the open ocean. Limnol. Oceanogr. 54, 1210-1232.

Buesseler, K.O., Trull, T.W., Steinberg, D.K., Silver, M.W., Siegel, D.A., Saitoh, S.-I., Lamborg, C.H., Lam, P.J., Karl, D.M., Jiao, N.Z., Honda, M.C., Elskens, M., Dehairs, F., Brown, S.L., Boyd, P.W., Bishop, J.K.B., Bidigare, R.R., 2008. VERTIGO (VERtical Transport In the Global Ocean): A study of particle sources and flux attenuation in the North Pacific. Deep. Res. II 55, 1522-1539. doi:10.1016/j.dsr2.2008.04.024 
Cai, P., Chen, W., Dai, M., Wan, Z., Wang, D., Li, Q., Tang, T., Lv, D., 2008. A high-resolution study of particle export in the southern South China Sea based on ${ }^{234} \mathrm{Th}:{ }^{238} \mathrm{U}$ disequilibrium. J. Geophys. Res. 113, 1-15. doi:10.1029/2007JC004268

Cai, P., Rutgers Van der Loeff, M., Stimac, I., Nothig, E.M., Lepore, K., Moran, S.B., 2010. Low export flux of particulate organic carbon in the central Arctic Ocean as revealed by ${ }^{234} \mathrm{Th}:{ }^{238} \mathrm{U}$ disequilibrium. J. Geophys. Res. Ocean. 115, 1-21. doi:10.1029/2009JC005595

Chisholm, S.W., 2000. Stirring times in the Southern Ocean. Nature 407, 685-687. doi: $10.1038 / 35037696$

Ciais, P., Sabine, C., Bala, G., Bopp, L., Brovkin, V., Canadell, J., Chhabra, A., DeFries, R., Galloway, J., Heimann, M., Jones, C., Le Quéré, C., Myneni, R.B., Piao, S., Thornton, P., 2013. Carbon and Other Biogeochemical Cycles, in: Stocker, T.F., Qin, D., Plattner, G.-K., Tignor, M., Allen, S.K., Boschung, J., Nauels, A., Xia, Y., Bex, V., Midgley, P.M. (Eds.), Climate Change 2013: The Physical Science Basis. Contribution of Working Group I to the Fifth Assessment Report of the Intergovernmental Panel on Climate Change. Cambridge University Press, Cambridge, United Kingdom and New York, NY, USA, pp. 465-570.

Coale, K.H., Bruland, K.W., 1987. Oceanic stratified euphotic zone as elucidated by ${ }^{234} \mathrm{Th}:{ }^{238} \mathrm{U}$ disequilibria. Limnol. Oceanogr. 32, 189-200.

Cochran, J.K., Masque, P., 2003. Short-lived U/Th series radionuclides in the ocean: Tracers for scavenging rates, export fluxes and particle dynamics, in: Bourdon, et al. (Ed.), Uranium Series Geochemistry, Reviews in Mineralogy and Geochemistry. pp. 461-492.

De La Rocha, C., Passow, U., 2007. Factors influencing the sinking of POC and the efficiency of the biological carbon pump. Deep. Res. Part II 54, 639-658. doi:10.1016/j.dsr2.2007.01.004

Devol, A.H., Hartnett, H.E., 2001. Role of the oxygen-deficient zone in transfer of organic carbon to the deep ocean. Limnol. Oceanogr. 46, 1684-1690. doi:10.4319/lo.2001.46.7.1684

Fang, Z., Yang, W., Chen, M., Zheng, M., Hu, W., 2016. Abundance and sinking of particulate black carbon in the western Arctic and Subarctic Oceans. Sci. Rep. 6, 29959. doi:10.1038/srep29959

Feely, R.A., Sabine, C.L., Takahashi, T., Wanninkhof, R., 2001. Uptake and Storage of Carbon Dioxide in the Ocean: The Global C02 Survey. Oceanography 14, 18-32.

Francois, R., Honjo, S., Krishfield, R., Manganini, S., 2002. Factors controlling the flux of organic carbon to the bathypelagic zone of the ocean. Glob. Biogeochem. Cycles 16, 1087.

doi:10.1029/2001gb001722

Geibert, W., Usbeck, R., 2004. Adsorption of thorium and protactinium onto different particle types: Experimental findings. Geochim. Cosmochim. Acta 68, 1489-1501. doi:10.1016/j.gca.2003.10.011

Hedges, J.I., Baldock, J.A., Gelinas, Y., Lee, C., Peterson, M., Wakeham, S.G., 2001. Evidence for nonselective preservation of organic matter in sinking marine particles. Nature 409, 801-804.

Henson, S.A., Sanders, R., Madsen, E., Morris, P.J., Le Moigne, F., Quartly, G.D., 2011. A reduced estimate of the strength of the ocean's biological carbon pump. Geophys. Res. Lett. 38, 1-5. doi:10.1029/2011GL046735

Ito, T., Follows, M.J., 2003. Upper ocean control on the solubility pump of $\mathrm{CO}_{2}$. J. Mar. Res. 61, 465489. doi: $10.1357 / 002224003322384898$ 
Khatiwala, S., Primeau, F., Holzer, M., 2012. Ventilation of the deep ocean constrained with tracer observations and implications for radiocarbon estimates of ideal mean age. Earth Planet. Sci. Lett. 325-326, 116-125. doi:10.1016/j.eps1.2012.01.038

Kwon, E.Y., Primeau, F., Sarmiento, J.L., 2009. The impact of remineralization depth on the air-sea carbon balance. Nat. Geosci. 2, 630-635. doi:10.1038/ngeo612

Le Moigne, F.A.C., Henson, S.A., Sanders, R.J., Madsen, E., 2013. Global database of surface ocean particulate organic carbon export fluxes diagnosed from the ${ }^{234} \mathrm{Th}$ technique. Earth Syst. Sci. Data 5, 295-304. doi:10.5194/essd-5-295-2013

Maas, A.E., Frazar, S.L., Outram, D.M., Seibel, B.A., Wishner, K.F., 2014. Fine-scale vertical distribution of macroplankton and micronekton in the Eastern Tropical North Pacific in association with an oxygen minimum zone. J. Plankton Res. 36, 1557-1575. doi:10.1093/plankt/fbu077

Maiti, K., Nelson, C.R.B., Buesseler, K.O., 2010. Insights into particle formation and remineralization using the short-lived radionuclide, Thoruim-234. Geophys. Res. Lett. 37, 1-6. doi:10.1029/2010GL044063

Marra, J., Langdon, C., Knudson, C.A., 1995. Primary production, water column changes, and the demise of a Phaeocystis bloom at the Marine Light-Mixed Layers site (59 N, 21W) in the northeast Atlantic Ocean. J. Geophys. Res. 100, 6633-6643.

Martin, J.H., Knauer, G.A., Karl, D.M., Broenkow, W.W., 1987. VERTEX: carbon cycling in the northeast Pacific. Deep. Res. 34, 267-285.

Matear, R.J., Hirst, A.C., 2003. Long-term changes in dissolved oxygen concentrations in the ocean caused by protracted global warming. Global Biogeochem. Cycles 17, 1-32. doi:10.1029/2002GB001997

Matsumoto, K., 2007. Radiocarbon-based circulation age of the world oceans. J. Geophys. Res. 112, 1-7. doi:10.1029/2007JC004095

Matsumoto, K., Tokos, K.S., Chikamoto, M.O., Ridgwell, A., 2010. Characterizing post-industrial changes in the ocean carbon cycle in an Earth system model. Tellus, Ser. B Chem. Phys. Meteorol. 62, 296-313. doi:10.1111/j.1600-0889.2010.00461.x

McDonnell, A.M.P., Buesseler, K.O., 2010. Variability in the average sinking velocity of marine particles. Limnol. Oceanogr. 55, 2085-2096. doi:10.4319/lo.2010.55.5.2085

Moran, S.B., Ellis, K.M., Smith, J.N., 1997. ${ }^{234} \mathrm{Th} /{ }^{238} \mathrm{U}$ disequilibrium in the central Arctic Ocean: implications for particulate organic carbon export. Deep. Res. Part II 44, 1593-1606.

Paulmier, A., Ruiz-Pino, D., Garcon, V., Farias, L., 2006. Maintaining of the Eastern South Pacific Oxygen Minimum Zone (OMZ) off Chile. Geophys. Res. Lett. 33, 1-6. doi:10.1029/2006GL026801

Pennington, J.T., Mahoney, K.L., Kuwahara, V.S., Kolber, D.D., Calienes, R., Chavez, F.P., 2006. Primary production in the eastern tropical Pacific: A review. Prog. Oceanogr. 69, 285-317. doi:10.1016/j.pocean.2006.03.012

Rii, Y.M., Duhamel, S., Bidigare, R.R., Karl, D.M., Repeta, D.J., Church, M.J., 2016. Diversity and productivity of photosynthetic picoeukaryotes in biogeochemically distinct regions of the South East Pacific Ocean. Limnol. Oceanogr. 61, 806-824. doi:10.1002/lno.10255

Rutgers van der Loeff, M., Sarin, M.M., Baskaran, M., Benitez-Nelson, C., Buesseler, K.O., Charette, M., Dai, M., Gustafsson, Ö., Masque, P., Morris, P.J., Orlandini, K., Rodriguez y Baena, A., Savoye, N., Schmidt, S., Turnewitsch, R., Vöge, I., Waples, J.T., 2006. A review of present techniques 
and methodological advances in analyzing ${ }^{234} \mathrm{Th}$ in aquatic systems. Mar. Chem. 100, 190-212. doi:10.1016/j.marchem.2005.10.012

Rutgers van der Loeff, M.M., Cai, P.H., Stimac, I., Bracher, A., Middag, R., Klunder, M.B., van Heuven, S.M.A.C., 2011. 234Th in surface waters: Distribution of particle export flux across the Antarctic Circumpolar Current and in the Weddell Sea during the GEOTRACES expedition ZERO and DRAKE. Deep. Res. Part II Top. Stud. Oceanogr. 58, 2749-2766. doi:10.1016/j.dsr2.2011.02.004

Santschi, P.H., Murray, J.W., Baskaran, M., Benitez-Nelson, C.R., Guo, L.D., Hung, C.C., Lamborg, C., Moran, S.B., Passow, U., Roy-Barman, M., 2006. Thorium speciation in seawater. Mar. Chem. 100, 250-268. doi:10.1016/j.marchem.2005.10.024

Sarmiento, J.L., Toggweiler, J.R., 1984. A new model for the role of the ocean in determining atmospheric $\mathrm{PCO}_{2}$. Nature 308, 621-624.

Savoye, N., Benitez-Nelson, C., Burd, A.B., Cochran, J.K., Charette, M., Buesseler, K.O., Jackson, G.A., Roy-Barman, M., Schmidt, S., Elskens, M., 2006. ${ }^{234}$ Th sorption and export models in the water column: A review. Mar. Chem. 100, 234-249. doi:10.1016/j.marchem.2005.10.014

Savoye, N., Buesseler, K.O., Cardinal, D., Dehairs, F., 2004. 234Th deficit and excess in the Southern Ocean during spring 2001: Particle export and remineralization. Geophys. Res. Lett. 31, 1-4. doi:10.1029/2004GL019744

Seibel, B.A., Schneider, J.L., Kaartvedt, S., Wishner, K.F., Daly, K.L., 2016. Hypoxia tolerance and metabolic suppression in oxygen minimum zone Euphausiids: Implications for ocean deoxygenation and biogeochemical cycles. Integr. Comp. Biol. 56, 510-523. doi:10.1093/icb/icw091

Siegel, D.A., Buesseler, K.O., Doney, S.C., Sailley, S.F., Behrenfeld, M.J., Boyd, P.W., 2014. Global assessment of ocean carbon export by combining satellite observations and food-web models. Global Biogeochem. Cycles 28, 181-196. doi:10.1002/2013GB004743

Steinberg, D.K., Landry, M.R., 2017. Zooplankton and the Ocean Carbon Cycle. Ann. Rev. Mar. Sci. 132. doi:10.1146/annurev-marine-010814-015924

Stevens, H., Ulloa, O., 2008. Bacterial diversity in the oxygen minimum zone of the eastern tropical South Pacific. Environ. Microbiol. 10, 1244-1259. doi:10.1111/j.1462-2920.2007.01539.x

Stramma, L., Johnson, G.C., Sprintall, J., Mohrholz, V., 2008. Expanding oxygen-minimum zones in the tropical oceans. Science 320, 655-659.

Stramma, L., Schmidtko, S., Levin, L.A., Johnson, G.C., 2010. Ocean oxygen minima expansions and their biological impacts. Deep. Res. I 57, 587-595. doi:10.1016/j.dsr.2010.01.005

Sunda, W.G., 2012. Feedback interactions between trace metal nutrients and phytoplankton in the ocean. Front. Microbiol. 3, 1-22. doi:10.3389/fmicb.2012.00204

Sunda, W.G., Huntsman, S.A., 1997. Interrelated influence of iron, light and cell size on marine phytoplankton growth. Nature 2051, 389-392.

Tagliabue, A., Aumont, O., Death, R., Dunne, J.P., Dutkiewicz, S., Galbraith, E., Misumi, K., Moore, J.K., Ridgwell, A., Sherman, E., Stock, C., Vichi, M., Völker, C., Yool, A., 2016. How well do global ocean biogeochemistry models simulate dissolved iron distributions? Global Biogeochem. Cycles 30, 149-174. doi:10.1002/2015GB005289

Toggweiler, J.R., Murnane, R., Carson, S., Gnanadesikan, A., Sarmiento, J.L., 2003. Representation of the carbon cycle in box models and GCMs: 1. Solubility pump. Global Biogeochem. Cycles 17. doi:10.1029/2001GB001841 
United States Joint Global Ocean Flux Study, 2001. The biological pump.

van Hulten, M.M.P., Middag, R., Dutay, J.-C., de Baar, H.J.W., Roy-Barman, M., Gehlen, M., Tagliabue, A., Sterl, A., 2017. Manganese in the West Atlantic Ocean in context of the first global ocean circulation model of manganese. Biogeosciences 14, 1123-1152. doi:10.5194/bg-14-1123-2017

Van Mooy, B.A.S., Keil, R.G., Devol, A.H., 2002. Impact of suboxia on sinking particulate organic carbon: Enhanced carbon flux and preferential degradation of amino acids via denitrification. Geochim. Cosmochim. Acta 66, 457-465.

Walsh, J.J., Dieterle, D.A., Maslowski, W., Whitledge, T.E., 2004. Decadal shifts in biophysical forcing of Arctic marine food webs: Numerical consequences. J. Geophys. Res. 109, 1-30. doi:10.1029/2003JC001945

Wassmann, P., Bauerfeind, E., Fortier, M., Fukuchi, M., Hargrave, B., Moran, B., Noji, T., Nöthig, E.-M., Olli, K., Peinert, R., Sasaki, H., Shevchenko, V., 2004. Particulate Organic Carbon Flux to the Arctic Ocean Sea Floor, in: Stein, R., Macdonald, R.W. (Eds.), The Organic Carbon Cycle in the Arctic Ocean. Springer, pp. 101-138. doi:10.1007/978-3-642-18912-8_5

Wassmann, P., Reigstad, M., 2011. Future Arctic Ocean Seasonal Ice Zones and Implications for PelagicBenthic Coupling. Oceanography 24, 220-231. doi:10.5670/oceanog.2011.74

Weinstein, S.E., Moran, S.B., 2005. Vertical flux of particulate Al, Fe, Pb, and Ba from the upper ocean estimated from ${ }^{234} \mathrm{Th} /{ }^{238} \mathrm{U}$ disequilibria. Deep. Res. I 52, 1477-1488.

doi:10.1016/j.dsr.2005.03.008 


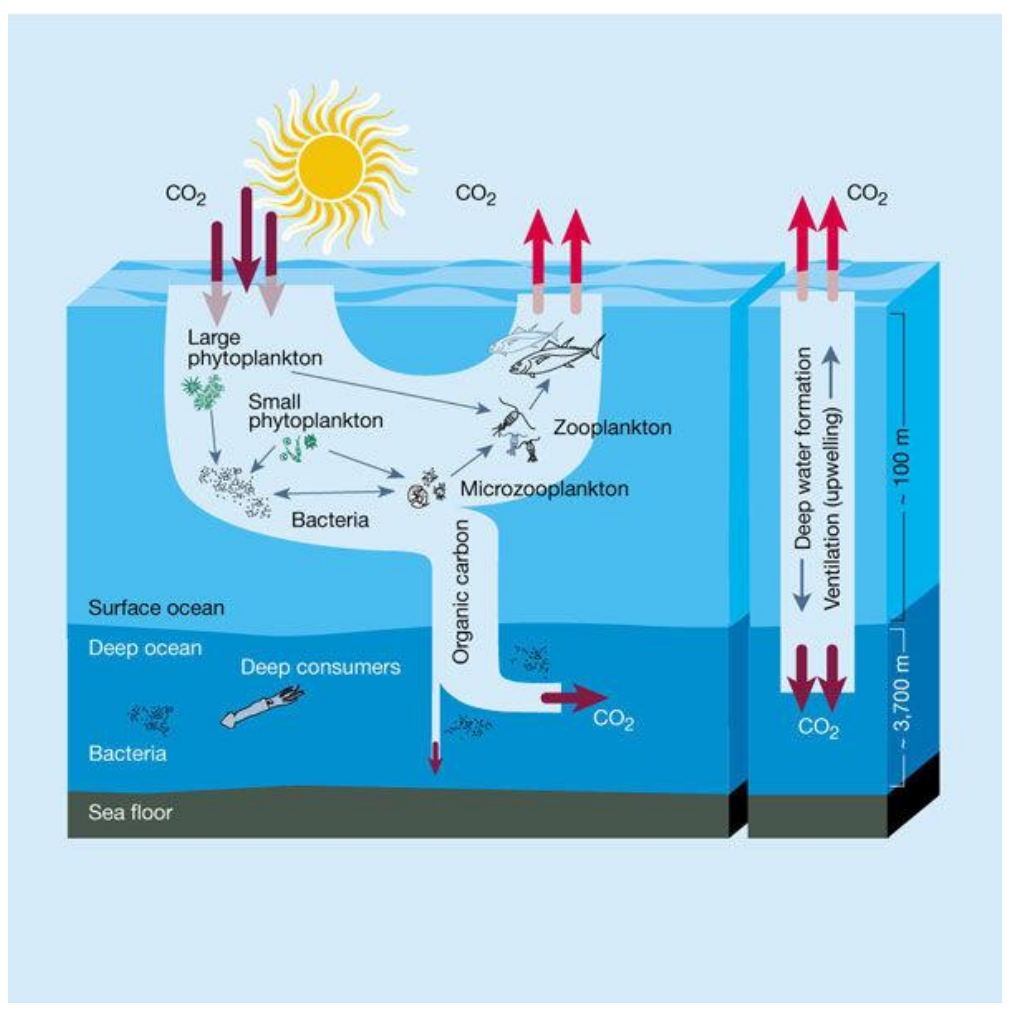

Figure 1: The solubility pump (right) and the biological carbon pump (left). (Chisholm, 2000). 


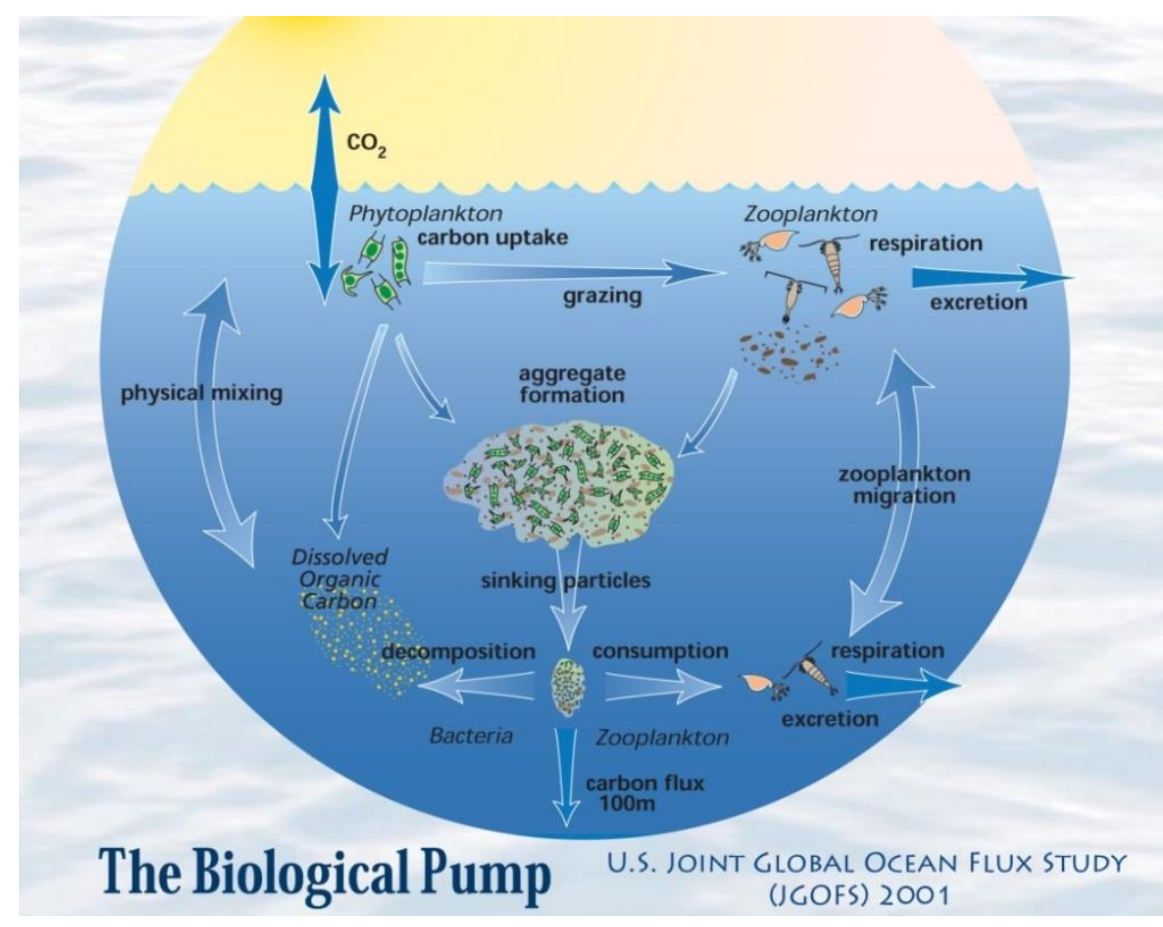

Figure 2: The major constituents and processes composing the biological carbon pump in the surface ocean (United States Joint Global Ocean Flux Study, 2001). 


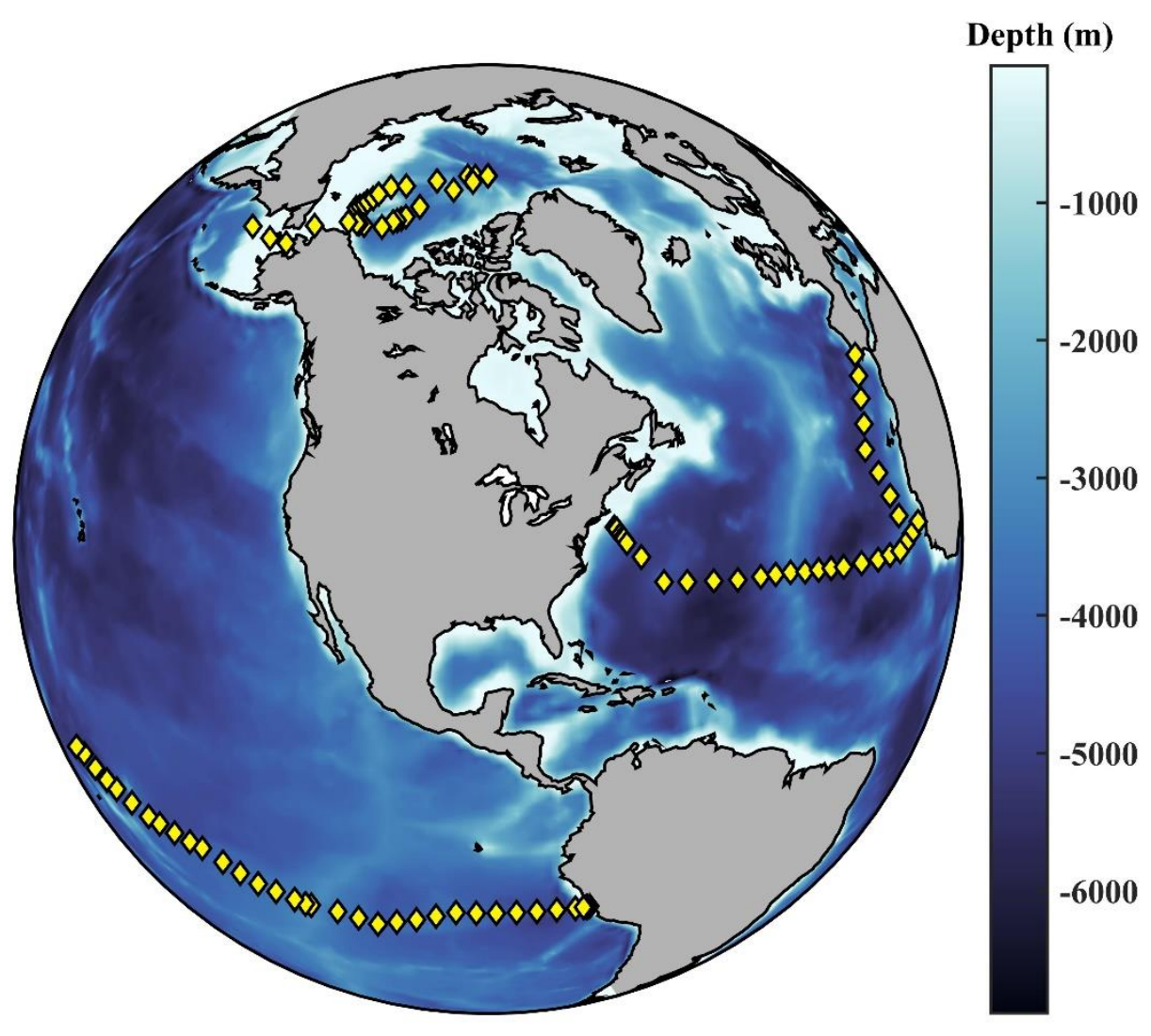

Figure 3: Completed U.S. GEOTRACES campaigns with available ${ }^{234}$ Th-derived POC export estimates. Chapters 2, 3, and 4 of this thesis focus on the southeastern tropical Pacific campaign (GP16) and the Western Arctic Ocean transects (GN01). References and comparisons are made to the first U.S. GEOTRACES cruises (GA03) that crossed the North Atlantic in 2010 and 2011. 


\section{Chapter 2:}

\section{${ }^{234} \mathrm{Th}$ as a tracer of particulate export and remineralization in the Southeastern Tropical Pacific}

This chapter was originally published in Marine Chemistry by Elsevier in 2017 and is reproduced here with their permission.

Black, E.E., Buesseler, K.O, Pike, S.M., and Lam, P.J., 2017, ${ }^{234}$ Th as a tracer of particulate export and remineralization in the Southeastern Tropical Pacific, Marine Chemistry: In press, doi: 10.1016/j.marchem.2017.06.009. 


\begin{abstract}
Oxygen minimum zones (OMZs) are thought to be regions of decreased carbon attenuation in the upper ocean with a biological pump that could deliver a greater percentage of exported carbon to the mesopelagic relative to surrounding waters. However, much is still unknown about carbon cycling through these zones and the areas of extreme oxygen minima $\left(\mathrm{nM} \mathrm{O}_{2}\right)$ or oxygen deficient zones (ODZs) within the OMZs. Paired sampling for ${ }^{234} \mathrm{Th}\left(\mathrm{t}^{1 / 2} \sim 24.1\right.$ days) and particulate organic carbon (POC) was performed along a zonal transect between $77^{\circ} \mathrm{W}$ and $152^{\circ} \mathrm{W}$ during the U.S. GEOTRACES Southeastern Tropical Pacific campaign in 2013 in order to constrain the magnitude of carbon export and remineralization through the Peruvian OMZ. POC export varied by an order of magnitude from the coast to $152^{\circ} \mathrm{W}$, reflecting a decrease in POC: ${ }^{234} \mathrm{Th}$ ratios $(>51 \mu \mathrm{m})$ with distance offshore and the influence of upwelling at the coast. Modeling indicated that ${ }^{234} \mathrm{Th}$ fluxes could be underestimated at coastal stations by up to 4-fold without adjustment for the impact of upwelling, which in turn would produce much lower carbon export estimates.

Low carbon Export:NPP ratios $(<0.15)$ at the base of the euphotic zone $(\mathrm{Ez})$ in the gyre support previous findings of inefficient surface export via the biological pump in the southeastern tropical Pacific. A broad remineralization feature beginning at Ez was observed across $>7500 \mathrm{~km}$ that resulted in, on average, $3 \%$ of the POC exported from the euphotic zone reaching $100 \mathrm{~m}$ below Ez. Although the highest percentages $(>10 \%)$ of total exported POC at $100 \mathrm{~m}$ below Ez were observed in the coastal ODZ region, the observed remineralization was also most pronounced these stations. While an average of $75 \%$ of the carbon export from the euphotic zone remained at Ez $+100 \mathrm{~m}$ in the gyre, a range of $10 \%$ to $50 \%$ was observed at ODZ stations, reflecting increased attenuation. Local subsurface minima in light transmission and maxima in fluorescence were observed in the regions of greatest remineralization at the upper ODZ boundary, suggesting that complex bacterial community dynamics play a role in increased attenuation through these zones. With ODZs and OMZs predicted to grow worldwide with climate change, these areas require further large-scale and seasonal studies to assess the permanency of these attenuation features and the impact of high Gyre and lower ODZ transfer of POC on the overall efficiency of carbon export in the Pacific.
\end{abstract}




\subsection{Introduction}

Oxygen minimum zones (OMZs) are permanent subsurface $(50 \mathrm{~m}$ to $1000 \mathrm{~m})$ features in the global ocean where dissolved oxygen can drop to extreme minima due a combination of weak ocean ventilation and the respiration of sinking organic matter (Karstensen et al., 2008). Oxygen deficient zones (ODZs) are defined as the regions within OMZs where oxygen levels reach near or below detection using traditional methods $\left(\mathrm{O}_{2}<1 \mu \mathrm{M}\right.$, see Section 2.2.6). These ODZs are characterized by coastal upwelling, which brings nutrient rich water to the surface and creates extensive, high productivity regions (Barber et al., 2001; Fiedler et al., 1991). Although these coupled high productivity-low oxygen regions currently compose only $8 \%$ of the world's ocean area (Paulmier and Ruiz-Pino, 2009), ODZs are also one of the only regions of the ocean where nitrogen loss occurs (Codispoti et al., 2001; Lam et al., 2009) and their overlying waters boast some of the highest, non-coastal annual rates of net primary production (NPP) worldwide (Siegel et al., 2014). The Peruvian upwelling system, in particular, has tremendously productive shelf waters, supporting one of the world's largest natural fisheries (Montecino and Lange, 2009). Local marine ecosystems could be substantially impacted as changes in Earth's climate result in further expansion of the world's OMZs and ODZs (Stramma et al., 2010, 2008).

In order to predict and model the impact of global climate change on oceanic carbon and nutrient budgets, the controls on regional export via the biological pump need to be better understood. The biological pump composes the processes that transform and transport organic matter beginning with the fixation of inorganic carbon dioxide in the shallow ocean (photosynthesis) and ending with the transport to depth or remineralization of particulate organic carbon (POC) to dissolved forms (Matear and Hirst, 2003). It has been predicted that without this means of sequestration and transport of carbon to the deep ocean, the earth could see an increase in atmospheric carbon dioxide of 200 ppm (Sarmiento and Toggweiler, 1984). Sequestered POC need not reach the sediments to be removed from ocean-atmospheric interactions for millennia (Kwon et al., 2009) and so quantifying the export and remineralization of POC and nutrients in the upper 100s of meters is vital. Shallow, low oxygen zones have the potential to produce enhanced export at depth relative to more oxygenated regions of the ocean as a result of observed decreases in the attenuation of organics through OMZs (Roullier et al., 2014; Van Mooy et al., 2002). 
The ${ }^{234} \mathrm{Th}-{ }^{238} \mathrm{U}$ disequilibrium method is one of the most established means of studying export and particle dynamics in the upper ocean over time scales of days to weeks (Bacon et al., 1996; Benitez-Nelson et al., 2001; Bhat et al., 1969; Buesseler et al., 1992; Coale and Bruland, 1987). ${ }^{234} \mathrm{Th}\left(\mathrm{t} 1 / 2=24.1\right.$ days) is highly particle reactive, while its long-lived ${ }^{238} \mathrm{U}$ parent $(\mathrm{t} 1 / 2=4.47$ billion years) behaves conservatively with respect to salinity in the ocean (Not et al., 2012; Owens et al., 2011). As a result of its long half-life and ubiquity in the ocean, ${ }^{238} \mathrm{U}$ is generally at secular equilibrium with its ${ }^{234} \mathrm{Th}$ daughter (activity ratio $=1$ ) throughout much of the water column. In the surface ocean, a deficit between ${ }^{234} \mathrm{Th}$ and its parent is created when particles transporting ${ }^{234} \mathrm{Th}$ sink faster than new ${ }^{234} \mathrm{Th}$ is created via decay. This disequilibrium is used to calculate a particulate ${ }^{234} \mathrm{Th}$ flux. If the element: ${ }^{234} \mathrm{Th}$ ratio on particles is known, the flux of the element can be found (Buesseler et al., 2006, 1992). As higher resolution profiles have become available, the ${ }^{234} \mathrm{Th}$ method has also been used to identify areas of intense remineralization just below the sunlit surface ocean (Buesseler et al., 2008; Maiti et al., 2010; Savoye et al., 2004).

Until the 2010s, the only prior ${ }^{234} \mathrm{Th}$ data from near the $12^{\circ} \mathrm{S}$ line came from the JGOFS equatorial Pacific program in the early 1990s (Bacon et al., 1996; Buesseler et al., 1995; Murray et al., 1996). Sampling methods for these studies varied from high volume, discrete depth pumping (Bacon et al., 1996) to drifting traps (Murray et al., 1996) to oscillating a pump over the upper 100 m to obtain an average surface value (Buesseler et al., 1995). Unlike previous studies, whose results were often limited spatially (a few locations with high sample numbers) or by a lack of depth resolution, this campaign allowed for an unprecedented look at this region's surface ocean with almost 340 total ${ }^{234} \mathrm{Th}$ samples in the upper $400 \mathrm{~m}$. The data presented here reflect distinct features along the transect that might have been missed with poorer sampling resolution.

\subsection{Methods}

Samples were collected aboard the R/V Thomas G. Thompson between October $29^{\text {th }}$ and December $18^{\text {th }}$, 2013. This cruise, the U.S. GEOTRACES Eastern Pacific Zonal Transect (GP16TN303) will be referred to as 'the transect' here. Sampling began off the coast of Lima, Peru and continued westward to Tahiti between $10^{\circ} \mathrm{S}$ and $20^{\circ} \mathrm{S}$. Sampling for total and particulate parameters was completed at a total of 22 stations (black squares and grey circles, Fig. 1). To increase spatial resolution, total samples were also taken at 13 additional stations in the upper 1000 $\mathrm{m}$ (white circles, Fig. 1). Although ${ }^{234} \mathrm{Th}$ samples were collected throughout the full water column, 
data available through BCO-DMO (http://www.bco-dmo.org/dataset/643213; see Database References), the analysis presented here will focus on the upper water column only (0 to $400 \mathrm{~m}$ ).

\subsubsection{Total ${ }^{234}$ Th collection and analysis}

Total ${ }^{234}$ Th samples were collected at 35 stations. At 'super' stations $(1,11,18,26$, and 36) and 'full' stations $(7,9,13,15,17,20,21,23,25,28,30,32), 8$ to 13 discrete depths were sampled through the upper $400 \mathrm{~m}$ (Fig. 1). At the remaining stations, 8 to 12 depths were sampled in the upper $400 \mathrm{~m}$, which for shallow shelf stations covered most of the water column. A standard, 12Niskin rosette was used to collect the total ${ }^{234} \mathrm{Th}$ samples from the upper $400 \mathrm{~m}$ and between 4 and 9 of the rosette depths were matched to those of in-situ pumping systems deployed on a subsequent cast to obtain particulate ${ }^{234} \mathrm{Th}$. At a third of the stations, surface samples for total ${ }^{234} \mathrm{Th}$ were collected from the ship's underway system ( $\sim 5 \mathrm{~m}$ below the surface) and on a few occasions 1 to 2 extra shallow depths were sampled using wire-attached Niskin bottles above in-situ pumps on later casts. Additional total samples were taken to improve the resolution of shallow features.

Total ${ }^{234} \mathrm{Th}$ analyses followed the small volume, $4 \mathrm{~L}$ method developed in Buesseler et al. (2001) and detailed in Owens et al. (2015). An exact $1 \mathrm{~mL}$ aliquot of ${ }^{230} \mathrm{Th}\left(46.34 \mathrm{dpm} \mathrm{g}^{-1}\right)$ was used as the yield monitor. Quartz microfiber filters (QMA) were used to collect the precipitate from the $4 \mathrm{~L}$ process and immediately dried. ${ }^{234} \mathrm{Th}$ measurements were made with RISO Laboratories anti-coincidence beta counters. Filters were counted twice for a minimum of $12 \mathrm{~h}$ at sea. As long as the calculated gross counts per minute from these 2 measurements were within $10 \%$, they were averaged for the at-sea ${ }^{234} \mathrm{Th}$ value. The filters were counted again $\sim 6$ months later to quantify the background radioactivity due to the beta decay of long lived natural radionuclides that are also precipitated. The mean value of the at-sea counts (decay-corrected to the time of collection) minus the background value for each filter is reported here as the ${ }^{234} \mathrm{Th}$ activity (dpm $\left.\mathrm{L}^{-1}\right)$.

In order to determine ${ }^{234} \mathrm{Th}$ activity deficits, ${ }^{238} \mathrm{U}$ activities were calculated using the salinity relationship described in Owens et al. (2011) and shown here:

$$
{ }^{238} U( \pm 0.047)=(0.0786 \pm 0.00446) * \text { Salinity }-(0.315 \pm 0.158) \quad(\text { Eq. } 1)
$$

where ${ }^{238} \mathrm{U}$ activity is in dpm $\mathrm{L}^{-1}$. The efficiency of the beta detectors was determined by minimizing the ${ }^{234} \mathrm{Th}$ deviation from ${ }^{238} \mathrm{U}$ for samples collected from regions of the water column 
where ${ }^{234} \mathrm{Th}$ and ${ }^{238} \mathrm{U}$ are expected to be at equilibrium. These included depths below $1000 \mathrm{~m}$ and above $500 \mathrm{~m}$ off the seafloor that were not near the coastal shelf or the hydrothermal vent. For these samples $(n=93)$ the mean derived ${ }^{238} \mathrm{U}$ activity and standard deviation (s.d.) were $2.407 \pm$ $0.004 \mathrm{dpm} \mathrm{L} \mathrm{L}^{-1}$, a value well within observed natural ranges (Owens et al., 2011). High activity ${ }^{238} \mathrm{U}$ standards were measured periodically to track any changes in detector operation.

The reported ${ }^{234} \mathrm{Th}$ activities were corrected for the chemical recovery efficiency of the ${ }^{234} \mathrm{Th}-$ Mn precipitate method. To determine the percent recovery of the added ${ }^{230} \mathrm{Th}$ tracer, the method detailed in Pike et al. (2005) was followed without the initial ion exchange column chemistry steps. Filters were leached in a nitric acid-hydrogen peroxide solution and 1 gram of a ${ }^{229} \mathrm{Th}$ yield monitor (68.87 $\left.\mathrm{dpm} \mathrm{g}^{-1}\right)$ was added. Samples were then sonicated for $20 \mathrm{~min}$, allowed to stand covered overnight, diluted, and prepared for analysis by ICP-MS. The mean chemical recovery for all samples was $90.8 \%$ with a median value of $91.6 \%$.

\subsubsection{Particulate ${ }^{234} \mathrm{Th}$, carbon, and nitrogen collection and analysis}

Particulate ${ }^{234}$ Th samples were taken using dual-filter head McLane in-situ pumping systems (Bishop et al., 2012). At super stations, 3 pump casts were performed with 5 to 9 depths in the upper $400 \mathrm{~m}$ (black squares, Fig. 1). Pumps were deployed to capture 4 to 6 depths in the upper $400 \mathrm{~m}$ on 2 casts at full stations and on 1 cast at shelf stations 2 to 5 and at station 34 (grey circles, Fig. 1). The filter heads each contained a $51 \mu \mathrm{m}$ pore size pre-filter followed by either a Supor filter or a pre-combusted and acid-leached QMA filter with a nominal pore size of $1 \mu \mathrm{m}$ (Lam et al., 2015b). The material on the $51 \mu \mathrm{m}$ pre-filter from the Supor filter head was rinsed onto silver filters using $0.1 \mu \mathrm{m}$ filtered seawater and dried. The $142 \mathrm{~mm}$ QMA filter was oven dried and subsampled with a $25 \mathrm{~mm}$ punch. Both the silver filter and the $25 \mathrm{~mm}$ QMA subsample were counted once at sea and $\sim 6$ months later. The average sample volume through the $51 \mu \mathrm{m}$ pre-filter was $404 \mathrm{~L}$ and for the area of the QMA subsample was $41 \mathrm{~L}$. The $>51 \mu \mathrm{m}$ sample will be referred to as the large size fraction (LSF) and the $1 \mu \mathrm{m}$ to $51 \mu \mathrm{m}$ as the small size fraction (SSF).

Particulate organic carbon (POC) and organic nitrogen (PON) were measured after all ${ }^{234} \mathrm{Th}$ analyses were completed. The silver and $25 \mathrm{~mm}$ QMA were acid-fumed with concentrated $\mathrm{HCl}$ and then POC and PON were measured by combustion. Measurements were made on 2, $12 \mathrm{~mm}$ diameter punches of the QMA filters with a Flash EA1112 Carbon/Nitrogen Analyzer and using a Dynamic Flash Combustion technique. Half of the silver filters were used for POC analysis on 
either a Carlo Erba 1108 or a CE Instruments NC2500 elemental analyzer interfaced to a ThermoFinnigan Delta Plus XP isotope ratio mass spectrometer. Measurements were blank corrected using dipped blanks housed near the filter heads on 1 pump from each cast. The s.d. of the blank filters was used to estimate the error in the POC and PON measurements. The SSF dip blank s.d. for the full water column was $21.8 \mu$ molC/filter, with a maximum sample value (0 $\mathrm{m}$ to $500 \mathrm{~m}$ ) of $9.4 \mu \mathrm{molC}$. The SSF dip blank s.d. for nitrogen was $6.1 \mu \mathrm{molN} /$ filter. The LSF s.d. for carbon was $0.50 \mu \mathrm{molC} /$ filter and the maximum surface sample value was $3.3 \mu \mathrm{molC}$. The LSF s.d. for nitrogen was $0.13 \mu$ molN/filter.

\subsubsection{Net primary production}

NPP was estimated at each station using MODIS.r2014 satellite products. NPP data from a $0.017^{\circ}$ by $0.017^{\circ}$ region $\left(\sim 335 \mathrm{~km}^{2}\right)$ containing each station were derived with a carbon-based productivity model (CbPM, Westberry et al., 2008) by Oregon State University Ocean Productivity (http://www.science.oregonstate.edu/ocean.productivity/). An average NPP for each station was determined for the previous 16 days prior to the sampling date using a weighted average of 8-day NPP products. This time period is similar to the integrated residence time of ${ }^{234} \mathrm{Th}$ in the upper water column, which has been estimated at days to a couple weeks (Coale and Bruland, 1987; Turnewitsch et al., 2008) and provides a better time scale match for the ${ }^{234} \mathrm{Th}$-derived export estimates than $12 \mathrm{~h}$ to $24 \mathrm{~h}$ incubations (Henson et al., 2011). The response time scale of ${ }^{234} \mathrm{Th}$, equivalent to $1 /(\lambda+\mathrm{k})$, accounts for both the mean life of the radioisotope $\left(1 / \lambda\right.$ or 24.1 days $\left./ \log _{\mathrm{e}} 2\right)$ and scavenging $(\mathrm{k})$ by particles (Turnewitsch et al., 2008). NPP was also determined for the 30 days prior to sampling and with the exception of Shelf stations 2 to 5, the 30-day NPP values did not differ from 16-day averages by $>5 \%$ or $2 \mathrm{mmolC} \mathrm{m}^{-2} \mathrm{~d}^{-1}$, on average.

\subsubsection{Particulate export flux of ${ }^{234} \mathrm{Th}$}

${ }^{234} \mathrm{Th}$ activity in the surface ocean can be described as a balance between production from ${ }^{238} \mathrm{U}$, decay, sinking flux, and transport in the following equation:

$$
\frac{\partial^{234} T h}{\partial t}={ }^{238} U \lambda_{T h}-{ }^{234} T h \lambda_{T h}-P+V
$$

where ${ }^{234} \mathrm{Th}$ is the activity of total ${ }^{234} \mathrm{Th}$ in $\mathrm{dpm} \mathrm{L} \mathrm{L}^{-1},{ }^{238} \mathrm{U}$ is the salinity-derived activity of uranium, $\lambda_{T h}$ is the decay constant for ${ }^{234} \mathrm{Th}\left(0.0288 \mathrm{day}^{-1}\right), P$ is the net removal of ${ }^{234} \mathrm{Th}$ on sinking particles (dpm $\left.\mathrm{L}^{-1} \mathrm{~d}^{-1}\right)$, and $V$ is the sum of the advective and vertical diffusive fluxes (dpm $\left.\mathrm{L}^{-1} \mathrm{~d}^{-1}\right)$. If a 
system is at steady state, the change in activity with time is assumed to be equal to zero. In many open ocean locations, horizontal and vertical transport processes can be assumed to be negligible ( $<10 \%$ of total flux contribution) over the timescales relevant to the sinking flux of ${ }^{234} \mathrm{Th}$ and the uncertainties of the flux estimates (Cai et al., 2010, 2008; Savoye et al., 2004). If the steady state assumption is valid and the advective and diffusive fluxes are ignored, particulate ${ }^{234}$ Th export can be determined using the following equation:

$$
P_{T h @ z}=\lambda_{T h} \int_{0}^{z}\left({ }^{238} U-{ }^{234} T h\right) d z \quad \text { (Eq.3). }
$$

The particulate flux of ${ }^{234} \mathrm{Th}$ or $P\left(\mathrm{dpm} \mathrm{m} \mathrm{m}^{-2} \mathrm{~d}^{-1}\right)$ is integrated to depth $\mathrm{z}$, the base of the euphotic zone $(\mathrm{Ez})$ or $100 \mathrm{~m}$. It can be difficult to determine whether steady state and one-dimensional (1D) assumptions hold true without repeated sampling campaigns to the same region or without the use of other tracers. These assumptions are discussed in more detail in Sections 2.3.5 and 2.4.1.

\subsubsection{Euphotic zone}

The euphotic zone, generally defined from the ocean's surface to the point in the water column where $0.1 \%$ to $1 \%$ of the flux of incident photosynthetically available radiation (PAR) is reached, is a desirable depth range of integration for surface POC export (Boyd et al., 2008; Siegel et al., 2014). In theory, Ez marks the point at which NPP goes to zero (net production = gross production - phytoplankton respiration). Above Ez, POC is created and remineralized, while below this boundary POC is only remineralized or produced by chemosynthetic pathways of much smaller magnitudes. Because PAR data were not available, the 'particle production zone' method was adopted as a euphotic zone proxy (Owens et al., 2015). Ez was determined by locating the depth where fluorescence reached $10 \%$ of its maximum (Ohnemus et al., 2016). The average particle production zone depths for all stations are referred to here as the average Ez depth. Although the variable-depth Ez is informative for looking at surface export efficiency, $100 \mathrm{~m}$ flux estimates for ${ }^{234} \mathrm{Th}$ and POC were also determined for comparison with prior studies.

\subsubsection{Oxygen data usage and saturation calculations}

Oxygen levels can directly influence community structure and zooplankton migration, indicate the extent of remineralization occurring, and will undoubtedly influence carbon and nutrient cycling (Bianchi et al., 2013; Goericke et al., 2000; Maas et al., 2014; Seibel et al., 2016). A separate Sea-Bird CTD oxygen sensor (SBE 43, limit $~ 3 \mu \mathrm{M})$ was deployed with the 
GEOTRACES (GTC) and Ocean Data Facility (ODF) rosettes. Only the ODF data from the shallow ${ }^{234}$ Th cast was used because of the observed variation in the raw oxygen sensor data from one CTD to the next and from one cast to the next (Supp. Fig. 1). In addition, only the upcast data was used due to the large difference in the response of the oxygen sensor between the up- and downcasts (Supp. Fig. 2). Upcast data also matched well with the Winkler bottle measurements (limit $\sim 1 \mu \mathrm{M}$, Supp. Fig. 2).

Oxygen is expressed as a percent, referred to as oxygen saturation, to facilitate station to station comparison across the $>7500 \mathrm{~km}$ transect (Ohnemus et al., 2016). Oxygen saturation values were generated using the 1-m binned ODF SBE 43 oxygen data and the Gibbs-SeaWater Oceanographic Toolbox (McDougall and Barker, 2011). The depths below the mixed layer where oxygen saturation drops rapidly to the local oxygen minimum, will be referred to as the upper oxycline. In the most offshore stations, the upper oxycline ended in a minimum of 30\% saturation, so a $30 \%$ oxygen saturation boundary was used when comparing parameters between all stations.

Similar to what was performed in Ohnemus et al. (2016) using the GTC data, the upper boundary of the oxygen deficient zone was defined as the first appearance of significant curvature in the ODF saturation data starting at the water column oxygen minimum and moving towards the surface. The gradient criterion was a $0.05 \%$ change in saturation between 3 successive 1-m data bins. In broad terms, oxygen deficient suggests levels below the limit of traditional Winkler titrations $(\sim 1 \mu \mathrm{M})$, but aerobic processes could still occur and nanomolar concentrations are typical in these low oxygen cores (Tiano et al., 2014). Because of the SBE and Winkler method limits, as well as the low resolution of the Winkler measurements through the base of the oxycline, we do not state an exact oxygen saturation value that marks the boundary between low oxygen and oxygen deficiency. We used the curvature criterion to define the depth of this boundary, where the oxygen sensor data definitively indicated that oxygen saturation began to change rapidly.

\subsection{Results}

The following section contains a brief summary of the results for total ${ }^{234} \mathrm{Th}$, particulate ${ }^{234} \mathrm{Th}$, NPP, and ${ }^{234}$ Th flux. The results for derived parameters that required more complex assessments and calculations, including the consideration of upwelling impacts on ${ }^{234} \mathrm{Th}$ export, the estimation of ${ }^{234} \mathrm{Th}$ remineralization, and the determination of POC fluxes, are contained in the Discussion.

\subsubsection{Zonal delineation}


The transect was divided into 3 biogeochemical zones by Ohnemus et al. (2016), including the Shelf ODZ (stations 1 to $5,77^{\circ} \mathrm{W}$ to $79.5^{\circ} \mathrm{W}$ ), the Offshore ODZ (6 to $13,79.5^{\circ} \mathrm{W}$ to $100^{\circ} \mathrm{W}$ ), and the Gyre ( 14 to $36,100^{\circ} \mathrm{W}$ to $152^{\circ} \mathrm{W}$ ). Patterns in ${ }^{234} \mathrm{Th}$ deficits, ${ }^{234} \mathrm{Th}$ excesses, POC: ${ }^{234} \mathrm{Th}$ ratios, and NPP generally followed these zones and so the same station delineations are used here, with one exception (Fig. 1, Table 1). Stations 14 and 15 have been included in the Offshore ODZ instead of in the Gyre, making the zonal boundary $105^{\circ} \mathrm{W}$. As will be discussed below, these stations did not reach oxygen deficiency, but were more similar to the Offshore ODZ stations in other biological characteristics. The zones will be referred to here as the 'Shelf', 'Offshore' and 'Gyre'.

In summary, the Shelf was dominated by microplankton (diatoms and dinoflagellates, Ohnemus et al., 2016) with an average mixed layer depth (MLD) of $21 \mathrm{~m}$ and an Ez of $43 \mathrm{~m}$ (Table 2, Supp. Table 1). The averages for the Offshore showed a $>2$-fold deepening of all features with a MLD of $59 \mathrm{~m}$ and Ez of $123 \mathrm{~m}$. The Offshore boundary was moved to station 15 because pigment analysis showed a dominance of nanoplankton in the top $100 \mathrm{~m}$ until station 15 (Ohnemus et al., 2016) and a large contrast in SSF ${ }^{234}$ Th activities was also apparent between stations 15 and 17 (Fig. 2D). Below $100 \mathrm{~m}$, picoplankton dominated in the Offshore. In the Gyre, the MLD average was similar to that of the Offshore at $60 \mathrm{~m}$ and the average Ez was the deepest of the 3 zones at $169 \mathrm{~m}$. The Gyre was dominated by picoplankton.

Oxygen saturation varied from a low of $0.2 \%$ within the $\mathrm{ODZ}$ at $89^{\circ} \mathrm{W}$ to $>100 \%$ in the surface mixed layer (Fig. 2, Supp. Table 1). Saturation values were lowest in the Shelf where the average upper boundary of the ODZ was $54 \pm 12 \mathrm{~m}$ (s.d.). The depth of minimum oxygen saturation in the Shelf was $90 \pm 45 \mathrm{~m}$ with an average oxygen saturation value at this depth of $0.4 \pm 0.1 \%$. In the Offshore, stations to the east of $100^{\circ} \mathrm{W}$ (station 13) contained zones of oxygen deficiency and those to the west contained only oxygen minima. For those stations with an ODZ boundary, the average depth was $152 \pm 56 \mathrm{~m}$. The average depth of the oxygen minimum in the Offshore was $200 \pm 85 \mathrm{~m}$ and the average saturation at this depth was $1.5 \pm 2 \%$. The deepest oxygen minima were found in the Gyre with an average value of $14 \pm 8 \%$ and a depth of $337 \pm 25 \mathrm{~m}$.

\subsubsection{Total and particulate ${ }^{234} \mathrm{Th}$}

Total ${ }^{234}$ Th activities ranged from $0.52 \mathrm{dpm} \mathrm{L}^{-1}$ to $2.95 \mathrm{dpm} \mathrm{L}^{-1}$ for all depths sampled (Fig. 3). All stations that were occupied showed pronounced surface deficits of ${ }^{234} \mathrm{Th}$ relative to ${ }^{238} \mathrm{U}$. 
Shelf stations had the greatest surface deficits of ${ }^{234} \mathrm{Th}$ (up to $2 \mathrm{dpm} \mathrm{L} \mathrm{L}^{-1}$ ) with ${ }^{234} \mathrm{Th}$ activities dropping as low as $0.5 \mathrm{dpm} \mathrm{L}^{-1}$ (Fig. 3). Although the MLD and Ez deepened with distance offshore (Supp. Table 1), ${ }^{234} \mathrm{Th}$ surface activities were remarkably consistent from $80^{\circ} \mathrm{W}$. In a typical open ocean setting, ${ }^{234} \mathrm{Th}$ and ${ }^{238} \mathrm{U}$ profiles (Fig. 3) can resemble those found at Station 33 (no measureable excess ${ }^{234} \mathrm{Th}$ in the subsurface) or those at Station 9 (excess ${ }^{234} \mathrm{Th}$ in the subsurface). The Shelf profiles generally did not show an excess of ${ }^{234} \mathrm{Th}$ and reached equilibrium $10 \mathrm{~m}$ to $30 \mathrm{~m}$ below Ez, but above $100 \mathrm{~m}$. The influence of upwelling on these profiles is discussed in Section 2.4.1. Subsurface excesses were observed at most other stations. The transition from deficit to excess was reached at or near Ez from the Shelf until $110^{\circ} \mathrm{W}$. After $110^{\circ} \mathrm{W}$ and starting at station 18, Ez dropped below this transition point into the zone of excess ${ }^{234} \mathrm{Th}$. The coincidence of Ez and the deficit-excess transition occurred again between $142^{\circ} \mathrm{W}$ and $152^{\circ} \mathrm{W}$.

The SSF composed the majority of particulate ${ }^{234} \mathrm{Th}$ measured along the transect. The SSF ranged from $20 \%$ to $50 \%$ of the total ${ }^{234} \mathrm{Th}$ in the top $100 \mathrm{~m}$ and $10 \%$ to $40 \%$ between $100 \mathrm{~m}$ and $200 \mathrm{~m}$ (Fig. 2D). Almost all values above $40 \%$ for the SSF were confined to the top $50 \mathrm{~m}$ between $120^{\circ} \mathrm{W}$ and $130^{\circ} \mathrm{W}$. Less than $10 \%$ of ${ }^{234} \mathrm{Th}$ total was observed to be in the LSF, except from $91.5^{\circ} \mathrm{W}$ to $96.5^{\circ} \mathrm{W}$, where values reached $10 \%$ to $20 \%$ of the total ${ }^{234} \mathrm{Th}$ (Fig. 2E). A distinct high in the percentage of both particulate size fractions was also observable along the oxycline at the top of the ODZ in the Shelf and the Offshore regions, although it was most pronounced in the SSF. This feature also aligned with a decrease in light transmission (Fig. 2B).

\subsubsection{Particulate carbon: ${ }^{234} \mathrm{Th}$ and nitrogen: ${ }^{234} \mathrm{Th}$}

For this study, the analysis is limited to element: ${ }^{234} \mathrm{Th}$ ratios between the MLD and $400 \mathrm{~m}$, the region of the water column that is relevant for ${ }^{234} \mathrm{Th}$-derived export at $\mathrm{Ez}$ and subsurface remineralization calculations. In general, LSF POC: ${ }^{234} \mathrm{Th}$ ratios decreased with depth and with distance offshore (Fig. 4). The highest euphotic zone ratios (non-mixed layer) were found in the Shelf, reaching $>15 \mu \mathrm{mol} \mathrm{dpm}{ }^{-1}$, and in the Offshore and Gyre these ratios were $<4 \mu \mathrm{mol} \mathrm{dpm}^{-1}$. Below $100 \mathrm{~m}$, very little change in ratios with depth was observed. The mean subsurface POC: ${ }^{234} \mathrm{Th}$ was calculated for each zone by averaging all samples below Ez and above $400 \mathrm{~m}$ (Table 2). The Shelf average was highest at $1.4 \pm 0.3 \mu \mathrm{mol} \mathrm{dpm}^{-1}$ (s.d.) and dropped to $0.9 \pm 0.2$ $\mu \mathrm{mol} \mathrm{dpm}{ }^{-1}$ and $0.7 \pm 0.1 \mu \mathrm{mol} \mathrm{dpm}{ }^{-1}$ in the Offshore and Gyre, respectively. 
LSF particulate organic nitrogen (PON) to ${ }^{234} \mathrm{Th}$ showed a decrease across the transect of similar magnitude to that observed for the LSF POC: ${ }^{234}$ Th (Supp. Fig. 3). Ratios decreased with depth and the maximum value measured below the MLD in the top $400 \mathrm{~m}$ was $\sim 3 \mu \mathrm{mol} \mathrm{dpm}{ }^{-1}$. The subsurface (Ez to $400 \mathrm{~m}$ ) Shelf average was highest at $0.2 \pm 0.05 \mu \mathrm{mol} \mathrm{dpm}^{-1}$. The averages for the Offshore and Gyre were about half at $0.10 \pm 0.04$ and $0.08 \pm 0.03$, respectively.

LSF element: ${ }^{234} \mathrm{Th}$ ratios are generally used when calculating export because this fraction has been operationally defined as representative of 'sinking' particles (e.g. Bishop et al., 1977). Differences in the LSF and SSF element: ${ }^{234} \mathrm{Th}$ ratios were assessed as a check on how similar sinking ratios were to suspended ones. Like the LSF, the $\mathrm{SSF}^{234} \mathrm{Th}$ ratios for both POC and PON generally decrease with depth and east to west across the transect (Table 2). In each zone, the subsurface (Ez to $400 \mathrm{~m}$ ) average of SSF POC: ${ }^{234} \mathrm{Th}$ ratios was within one standard deviation of the LSF ratio average. While the overlap of LSF and SSF POC: ${ }^{234} \mathrm{Th}$ within the estimated uncertainty ensures that flux estimates using the LSF ratios are representative of all potentially sinking material, this overlap is atypical. For example, in the Gulf of Mexico pump samples taken at $120 \mathrm{~m}$ showed an increase from $2.8 \mu \mathrm{mol} \mathrm{dpm}^{-1}$ to $34.4 \mu \mathrm{mol} \mathrm{dpm}^{-1}$ to $124.7 \mu \mathrm{mol} \mathrm{dpm}^{-1}$ when going from the $10-50 \mu \mathrm{m}$ to the $50-150 \mu \mathrm{m}$ and to the $>150 \mu \mathrm{m}$ size fraction, respectively (Hung et al., 2010).

\subsubsection{Net primary production}

NPP estimates for the 16 days prior to sampling ranged from $34 \mathrm{mmolC} \mathrm{m}^{-2} \mathrm{~d}^{-1}$ to $61 \mathrm{mmolC}$ $\mathrm{m}^{-2} \mathrm{~d}^{-1}$ in the Offshore and Gyre, while the Shelf NPP values ranged from $63 \mathrm{mmolC} \mathrm{m} \mathrm{m}^{-2} \mathrm{~d}^{-1}$ to $188 \mathrm{mmolC} \mathrm{m}^{-2} \mathrm{~d}^{-1}$ (Table 3). The highest NPP observed was at station 2 in the Shelf and the lowest was station 36, in the Gyre. NPP in the Shelf varied substantially in the month prior to sampling, dropping as low as $8 \mathrm{mmolC} \mathrm{m} \mathrm{d}^{-2}$ at station 3 in late October and rising to >3000 mmolC $\mathrm{m}^{-2} \mathrm{~d}^{-1}$ at station 2 just 8 days after. The 16-day NPP for the non-Shelf stations was very consistent at $48 \pm 6 \mathrm{mmolC} \mathrm{m}^{-2} \mathrm{~d}^{-1}$ (s.d.).

Averages for the 8 days prior to sampling and annual estimates (1997 to 2008) from the SeaWIFS-based GBC14 global carbon export model (Siegel et al., 2014) have been included here for comparison (Table 3). The 8-day and annual averages for NPP were within $1 \mathrm{mmolC} \mathrm{m} \mathrm{m}^{-2} \mathrm{~d}^{-1}$ to $4 \mathrm{mmolC} \mathrm{m}^{-2} \mathrm{~d}^{-1}$ except for a few stations in the Shelf. Annual and 8-day export estimates derived with the GBC14 model were also very similar. The largest differences between the 8-day 
SeaWIFS and the 16-day MODIS NPP values were seen in the Shelf and the Gyre. Despite these differences, the same general trend was observed in both datasets: higher NPP in the Shelf and the region around $140^{\circ} \mathrm{W}$ (Table 3). Considering all stations, the SeaWIFS 8-day NPP values were only 13\% higher (and the annual only 9\% higher) than the MODIS NPP estimates, on average (Table 2).

\subsubsection{Particulate flux of ${ }^{234} \mathrm{Th}$}

Steady state, 1-D estimates of ${ }^{234} \mathrm{Th}$ fluxes using $100 \mathrm{~m}$ and Ez as integration depths produced similar results for most stations (Tables 1 and 2, Supp. Table 2). The $100 \mathrm{~m}$ estimate was not consistently higher or lower than the Ez-based estimate, but in the Offshore and Gyre results from both methods were within uncertainties, except for at stations 14 and 26. Including estimates derived at both depths, ${ }^{234}$ Th fluxes ranged between $900 \mathrm{dpm} \mathrm{m}^{-2} \mathrm{~d}^{-1}$ and $2700 \mathrm{dpm} \mathrm{m} \mathrm{d}^{-1}$. The average ${ }^{234}$ Th flux at Ez for the Shelf, Offshore, and the Gyre zones was $1400 \mathrm{dpm} \mathrm{m} \mathrm{m}^{-1}, 1900$ $\mathrm{dpm} \mathrm{m} \mathrm{m}^{-2} \mathrm{~d}^{-1}$, and $1600 \mathrm{dpm} \mathrm{m}^{-2} \mathrm{~d}^{-1}$, respectively (Table 2). This consistency in ${ }^{234} \mathrm{Th}$ flux with distance offshore was unsurprising considering the observed similarities in surface deficit magnitude and depth (Fig. 2F). The average ${ }^{234}$ Th flux (un-adjusted for upwelling) for the transect was $1600 \mathrm{dpm} \mathrm{m}^{-2} \mathrm{~d}^{-1}$ for both methods.

The steady state assumption for the ${ }^{234}$ Th flux calculations was evaluated using the MODISderived NPP for each station. Between September and December, the region between $80^{\circ} \mathrm{W}$ and $152^{\circ} \mathrm{W}$ showed very little change in surface NPP. Excluding stations 2, 3, and 4, the average percent change in NPP across the transect between the 16-days prior to sampling and the previous 16 days was $1 \pm 13 \%$. While variations in NPP cannot be directly related to changes in ${ }^{234} \mathrm{Th}$ activities over the same time period, the negligible change in NPP suggested that the Offshore and Gyre regions were not experiencing a significant bloom that could have impacted ${ }^{234} \mathrm{Th}$ activities and that a steady state model was sufficient. The Shelf stations 2,3 , and 4 ( $77^{\circ} \mathrm{W}$ to $78^{\circ} \mathrm{W}$ ), on the other hand, showed a larger change in NPP between these 16 day periods, with increases of $\sim 760 \%, 410 \%$, and $130 \%$. Coastal zones are notoriously difficult for quantifying spatial and temporal changes in NPP and export, especially with satellite-derived data (Saba et al., 2011). The observed swings in progressive 8-day NPP estimates for these 3 stations are evidence of the challenges inherent in estimating NPP for coastal regions. For example, between late October and the end of December the average NPP at station 2 was $130 \mathrm{mmolC} \mathrm{m}^{-2} \mathrm{~d}^{-1}$ with a s.d. of almost 90 
mmolC $\mathrm{m}^{-2} \mathrm{~d}^{-1}$. Due to the lack of in-situ NPP and temporal ${ }^{234} \mathrm{Th}$ data along this $1^{\circ}$ of longitude, we do not attempt to constrain the potential temporal variability in ${ }^{234}$ Th distributions, and assume steady state.

\subsection{Discussion}

\subsubsection{Influence of upwelling and horizontal advection on ${ }^{234} \mathrm{Th}$ export estimates}

Prior ${ }^{234} \mathrm{Th}$, Beryllium-7, and helium studies off Peru indicated that upwelling could substantially impact the ${ }^{234} \mathrm{Th}$ activity balance at coastal stations (Haskell et al., 2015, 2013; Steinfeldt et al., 2015). If physical factors are taken into account, particulate ${ }^{234} \mathrm{Th}$ flux is calculated as follows:

$$
P_{T h @ z}=\int_{0}^{z}\left(\lambda_{T h}\left({ }^{238} U-{ }^{234} T h\right) \pm w \frac{\partial^{234} T h}{\partial z} \pm u \frac{\partial^{234} T h}{\partial x} \pm v \frac{\partial^{234} T h}{\partial y}\right) d z \text { (Eq. 4) }
$$

where $w$ is the upwelling velocity, $u$ is the zonal velocity, and $v$ is the meridional velocity. The gradients ( $\partial^{234}$ Th terms) are vertical (with depth), west to east, and south to north, respectively.

The impact of east-west horizontal advection on ${ }^{234} \mathrm{Th}$ fluxes during the month prior to sampling was determined to be negligible based on calculated ${ }^{234} \mathrm{Th}$ surface gradients and average zonal $(u)$ and meridional $(v)$ geostrophic current velocities (Supp. Fig. 4). The east-west gradient in average ${ }^{234} \mathrm{Th}$ activities for a $30 \mathrm{~m}$ surface layer across the transect was considered (Brink et al., 1983). No significant horizontal gradient was observed between $89^{\circ} \mathrm{W}$ and $152^{\circ} \mathrm{W}$, but a gradient of $3.5 \times 10^{-7} \mathrm{dpm} \mathrm{L} \mathrm{m}^{-1}$ was observed between $89^{\circ} \mathrm{W}$ and the Peruvian coast. Monthly satellitederived surface currents for October, were examined along $12^{\circ} \mathrm{S}\left(0.5^{\circ}\right.$ by $0.5^{\circ}$ scale $)$. Although the absolute zonal and meridional velocities reached maxima of $0.09 \mathrm{~m} \mathrm{~s}^{-1}$ and $0.19 \mathrm{~m} \mathrm{~s}^{-1}$, respectively, the cumulative (taking into account direction) average velocities observed across this area were $<0.008 \mathrm{~m} \mathrm{~s}^{-1}$. This suggested that a consistent pattern in surface currents did not exist in the region and would not affect ${ }^{234}$ Th gradients. However, if the east-west coastal gradient of 3.5 $\times 10^{-7} \mathrm{dpm} \mathrm{L} \mathrm{L}^{-1} \mathrm{~m}^{-1}$ was applied to a maximum current velocity of $\sim 0.2 \mathrm{~m} \mathrm{~s}^{-1}$, the result would be a meager $180 \mathrm{dpm} \mathrm{m} \mathrm{m}^{-2} \mathrm{~d}^{-1}$ change in ${ }^{234} \mathrm{Th}$ flux calculated at Ez. In this extreme case, the zonal horizontal advection term in Eq. 4 would only total $11 \%$ of the average ${ }^{234} \mathrm{Th}$ flux from Ez for stations between $89^{\circ} \mathrm{W}$ and the coast (Table 1). Therefore, the horizontal advection terms in Eq. 4 were disregarded. 
Previous ${ }^{234}$ Th modeling of the equatorial Pacific suggests that the influence of upwelling in the region between $95^{\circ} \mathrm{W}$ to $140^{\circ} \mathrm{W}\left(10^{\circ} \mathrm{S}\right.$ to $\left.15^{\circ} \mathrm{S}\right)$ should be negligible $\left(w<0.5 \mathrm{~m} \mathrm{~d}^{-1}\right.$; Buesseler et al., 1995), but prior ${ }^{7} \mathrm{Be}$-, temperature-, and helium-derived upwelling rates for coastal waters between $78^{\circ} \mathrm{W}$ to $85^{\circ} \mathrm{W}\left(10^{\circ} \mathrm{S}\right.$ to $15^{\circ} \mathrm{S}$ ) can vary from $0 \mathrm{~m} \mathrm{~d}^{-1}$ to $3 \mathrm{~m} \mathrm{~d}^{-1}$ (Haskell et al., 2015; Steinfeldt et al., 2015). Total ${ }^{234}$ Th distributions and curved isohalines and isopycnals in the upper $100 \mathrm{~m}$ of the Shelf indicated some degree of upwelling (Supp. Figs. 5 and 6). The following simplification of Eq. 4 can be used to determine the particulate ${ }^{234} \mathrm{Th}$ flux adjusted for the impact of upwelling, assuming that any upwelled waters reaching the surface contained ${ }^{234} \mathrm{Th}$ at equilibrium with ${ }^{238} \mathrm{U}$ :

$$
P_{T h @ z}=\int_{0}^{z}\left(\lambda_{T h}\left({ }^{238} U-{ }^{234} T h\right)+w \frac{\partial^{234} T h}{\partial z}\right) d z
$$

Based on equilibrium activities observed from $100 \mathrm{~m}$ to $200 \mathrm{~m}$ in the Shelf zone (Figs. 2F and 3), this assumption is valid if the upwelled waters originate locally. Stations in the Offshore could have been supplied with upwelled waters containing an excess of ${ }^{234} \mathrm{Th}$, which would lead to slightly conservative estimates of upwelling-adjusted fluxes. However, it will be shown that the impact of upwelling on Offshore stations was substantially less than in the Shelf, with only two stations showing any distinguishable gradient of ${ }^{234} \mathrm{Th}$ with depth in surface waters.

Both a vertical gradient $\left(\partial^{234} \mathrm{Th} / \partial z\right)$ and upwelling rate $(w)$ are needed to determine the upwelling component of ${ }^{234} \mathrm{Th}$ flux in Eq. 5. The gradient was determined at each station using 3 to 8 sampling depths beginning at the base of the mixed layer. The number of sample points used depended on the sampling resolution at that station and the depth extent of the gradient. The termination of the gradient often coincided with a change in the curvature (slope) of the ${ }^{234} \mathrm{Th}$ profiles (Fig. 3, Supp. Table 3) and was often at or just below the depth of equilibrium between ${ }^{234} \mathrm{Th}$ and ${ }^{238} \mathrm{U}$. The depth where the ${ }^{234}$ Th gradient terminated also occurred very close to the depth where large surface gradients in potential density stabilized near $1026.2 \mathrm{~kg} \mathrm{~m}^{-3}$ (density difference between $1 \mathrm{~m}$ sampling bins dropped below 0.025 for at least $40 \mathrm{~m}$, Supp. Fig. 6). Gradients were calculated for all stations, so as to provide a range for the typical gradients that could exist in areas without upwelling. Much larger gradients in ${ }^{234} \mathrm{Th}$ with depth were observed at stations 1 to 7 , with

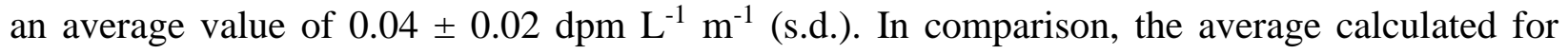
stations 8 to 36 was $0.009 \pm 0.002 \mathrm{dpm} \mathrm{L}^{-1} \mathrm{~m}^{-1}$. Stations 8 and 9 had ${ }^{234} \mathrm{Th}$ gradients that were 
indistinguishable $( \pm 0.002)$ from those found in the far western part of the transect. Therefore, Eq. 5 was used to calculate upwelling-adjusted fluxes for stations 1 through 7 and ${ }^{234} \mathrm{Th}$ fluxes for the remaining stations west of $85^{\circ} \mathrm{W}$ (8 to 36 ) were calculated using Eq. 3 only.

Using data collected during this campaign and paired ${ }^{7} \mathrm{Be}$-temperature modeling, Kadko (2017) determined upwelling rates for six stations located between $79^{\circ} \mathrm{W}$ and $104^{\circ} \mathrm{W}$ (Supp. Table 3). Kadko (2017) and other prior studies (Haskell et al., 2015; Kadko and Johns, 2011) determine upwelling rates using the dilution of the ${ }^{7} \mathrm{Be}$ inventory in the surface ocean by upwelling of ${ }^{7} \mathrm{Be}$-free waters from below. ${ }^{7} \mathrm{Be}$-derived upwelling rates are well suited for use with ${ }^{234} \mathrm{Th}$ because of the comparable half-life ( $\sim 53$ days) and ${ }^{7} \mathrm{Be}$-derived rates will reflect trends observed over a greater portion of the euphotic zone than tracers like helium (Haskell et al., 2015). A 1-D model was used by Kadko (2017) to determine the ${ }^{7} \mathrm{Be}$-derived upwelling rate at the base of the mixed layer for cases of invariant upwelling with depth (singular, constant value) and depthdependent upwelling (linear decrease of upwelling rate with depth).

To determine upwelling-adjusted ${ }^{234} \mathrm{Th}$ fluxes for stations 1 to $7\left(77^{\circ} \mathrm{W}\right.$ and $\left.84^{\circ} \mathrm{W}\right)$, stationspecific ${ }^{234} \mathrm{Th}$ gradients $\left(\partial^{234} \mathrm{Th} / \partial z\right.$, Supp. Table 3$)$ were paired with the appropriate upwelling rates (w) from Kadko (2017) using Eq. 5 for the depths over which a gradient existed (Supp. Table 3, Fig. 3). For the depth region above and below this gradient zone, which included the mixed layer, the $w$ and $\partial^{234} T h / \partial z$ terms were set to zero. While ${ }^{234} \mathrm{Th}$ gradients are available for all stations between $77^{\circ} \mathrm{W}$ and $84^{\circ} \mathrm{W}$, upwelling rates (constant model, $\mathrm{CM} ; 0 \mathrm{~m} \mathrm{~s}^{-1}$ horizontal advection, OHA) were only determined by Kadko (2017) for stations 1 at $79.2^{\circ} \mathrm{W}\left(3.0 \mathrm{~m} \mathrm{~d}^{-1}\right)$ and 7 at $84^{\circ} \mathrm{W}$ $\left(1.1 \mathrm{~m} \mathrm{~d}^{-1}\right)$. These $w$ were used at stations 1 and 7; at coastal stations 2 through 5 , the $w$ from station 1 was applied; and at station 6, located approximately halfway between station 1 and 7 , the average $w$ from these surrounding stations was used (Supp. Table 3). The constant model results were utilized because the upwelling structure was unknown and the $w$ values converged near Ez, where Kadko (2017) suggested that model choice was relatively unimportant.

To assess the uncertainties in the $w$ values, at any chosen depth the flux component due to upwelling was determined for both the constant and depth-dependent models, for horizontal advection of $0 \mathrm{~m} \mathrm{~s}^{-1}$ to $2 \mathrm{~m} \mathrm{~s}^{-1}$, and for the range of $w$ shown in Supp. Table 3. Although it was determined that horizontal advection would not impact ${ }^{234} \mathrm{Th}$ surface fluxes significantly, a $0.1 \mathrm{~m}$ $\mathrm{s}^{-1}$ horizontal advection term is consistent with historical results and ${ }^{7} \mathrm{Be}$, which with a half-life 
more than double that of ${ }^{234} \mathrm{Th}$, has the potential to be affected by physical factors over longer time scales. The range in the upwelling component of ${ }^{234} \mathrm{Th}$ flux, as calculated using all of these cases (vertical lines, Supp. Fig. 7), was used as the uncertainty on the CMOHA results (open circles, Supp. Fig. 7) which are summarized in Supp. Table 3.

Upwelling adjustments raised fluxes at the stations between the Peruvian coast and $84^{\circ} \mathrm{W}$ by an average of $2500 \mathrm{dpm} \mathrm{m}^{-2} \mathrm{~d}^{-1}$ or $\sim 175 \%$ (Supp. Table 3). Figure 5 shows the original ${ }^{234} \mathrm{Th}$ flux estimates (black bars) with the added upwelling component (white bars), the sum of which is equivalent to the total ${ }^{234} \mathrm{Th}$ flux at Ez. Values in the $3000 \mathrm{dpm} \mathrm{m}^{-2} \mathrm{~d}^{-1}$ to $6000 \mathrm{dpm} \mathrm{m} \mathrm{d}^{-1}$ range have also been seen in the Equatorial Pacific, the Southern Ocean, and the Arabian Sea (Buesseler et al., 2001a, 1998, 1995). Similar ${ }^{234}$ Th flux increases (1 to 5 times) due to upwelling were also observed near Hawaii ( $w=1$ to $3 \mathrm{~m} \mathrm{~d}^{-1}$; Maiti et al., 2008). Even with conservative uncertainty estimates on the upwelling component of ${ }^{234} \mathrm{Th}$ flux at Ez, which approached $50 \%$ at many of the coastal stations (Fig. 5, Supp. Fig. 7), the impact of upwelling on ${ }^{234}$ Th surface activities and fluxes was significant and should be accounted for when performing similar studies in this region.

\subsubsection{Upper ocean remineralization of ${ }^{234} \mathrm{Th}$ and particle cycling}

The processes composing the biological pump result in a net transport of particulate ${ }^{234} \mathrm{Th}$ from surface waters and a ${ }^{234}$ Th deficit above Ez. Below Ez, any process that attenuates flux or transfers sinking particles containing ${ }^{234} \mathrm{Th}$ to suspended particles, results in an excess in total ${ }^{234} \mathrm{Th}$. Without high resolution ${ }^{234} \mathrm{Th}$ profiles, however, the signature of these particle remineralization processes are often missed (Maiti et al., 2010). Remineralization may be occurring but not observed if the majority of it occurs over a narrow depth zone within the water column that is not sampled, or if the rates are too slow over a large depth zone within the water column for the signal to be captured within the time scale of the ${ }^{234} \mathrm{Th}$ method.

The extensive sampling along the transect captured a defined remineralization feature in the first $200 \mathrm{~m}$ below Ez at 27 of the 35 stations where total ${ }^{234}$ Th was measured (Fig. 3, Table 1). With changing Ez, remineralization is best compared by looking at the first $100 \mathrm{~m}$ below Ez, where flux attenuation is largest (Buesseler and Boyd, 2009). The decrease in ${ }^{234} \mathrm{Th}$ flux was calculated between the Ez and $100 \mathrm{~m}$ below it $\left(\mathrm{R}_{100}\right)$ and from $100 \mathrm{~m}$ below Ez to $200 \mathrm{~m}$ below Ez $\left(\mathrm{R}_{200}\right)$. After upwelling-adjustments were made, 9 of 10 stations in the Offshore region had identifiable remineralization features, while these features were only seen in the Gyre at 15 of 20 stations. 
Some of the Shelf stations had no discernable remineralization features before or after upwellingadjustments (1 and 2) and in the cases of stations 3, 4, and 5, ${ }^{234}$ Th flux was still increasing through the Ez (Supp. Table 3). Remineralization was more difficult to assess here due to the shallow depth, condensed vertical profiles, and offset of Ez from deeper ${ }^{234} \mathrm{Th}^{238} \mathrm{U}$ equilibrium depths.

The average $\mathrm{R}_{100}$ across the entire transect was $400 \pm 200 \mathrm{dpm} \mathrm{m}^{-2} \mathrm{~d}^{-1}$ (s.d.). $\mathrm{R}_{100}$ was highest in the Offshore with an average decrease in flux of $600 \mathrm{dpm} \mathrm{m}^{-2} \mathrm{~d}^{-1}$. In the Gyre, the $\mathrm{R}_{100}$ dropped to an average of $400 \mathrm{dpm} \mathrm{m}^{-2} \mathrm{~d}^{-1}$. If $\mathrm{R}_{200}$ is considered, the mean values for the Offshore and Gyre were indistinguishable and averaged between $400 \mathrm{dpm} \mathrm{m} \mathrm{d}^{-2}$ and $500 \mathrm{dpm} \mathrm{m} \mathrm{m}^{-2}$. An increase in relative uncertainties between $R_{100}$ and $R_{200}$ values is apparent in Table 1. This increase in uncertainties is inherent in flux with depth calculations (Supp. Fig. 8E) and is not due to any change in the uncertainty on the total ${ }^{234} \mathrm{Th}$ measurements themselves (Coale and Bruland, 1987). For those stations which had remineralization features, ${ }^{234} \mathrm{Th}$ flux decreased on average by $30 \%$ between Ez and $100 \mathrm{~m}$ below it, and 60\% between Ez and $200 \mathrm{~m}$ below it (sum of $\mathrm{R}_{100}$ and $\mathrm{R}_{200}$ ).

In general, remineralization features were broad (10s to 100s of m, Fig. 2F). Remineralization features in the Offshore in particular appeared to penetrate to almost $400 \mathrm{~m}$ at some stations (Figs. $2 \mathrm{~F}$ and 3). Peaks this broad are not unheard of as ${ }^{234} \mathrm{Th}$ excesses attributed to remineralization observed in the Southern Ocean have reached $400 \mathrm{~m}$ to $500 \mathrm{~m}$ (Savoye et al., 2004), but the consistency in the magnitude of $\mathrm{R}_{100}$ and $\mathrm{R}_{200}$ across these zones is atypical for a basin-scale transect, especially in a gyre (Owens et al., 2015). Although ${ }^{234}$ Th-derived remineralization has been quantified by a few other studies in the Weddell Sea (Usbeck et al., 2002), Northwest Pacific (Maiti et al., 2010) and the Southern Ocean (Savoye et al., 2004), flux attention has been assessed most often relative to carbon and nutrient losses, which are covered in more detail in Sections 2.4.4 and 2.4.5.

Further insight was gained by comparing ${ }^{234} \mathrm{Th}$ flux at a given depth with the distribution of ${ }^{234} \mathrm{Th}$ between the three measured phases (total, SSF, LSF) and expressing all parameters relative to the depth where oxygen saturation first reached $30 \%$ in the surface ocean (Supp. Fig. 8). Typical open ocean profiles here (Supp. Figs. 8D and 8E), have low total ${ }^{234} \mathrm{Th}$ and elevated particulate ${ }^{234} \mathrm{Th}$ in the surface ocean due to scavenging. Particles originating in the surface ocean, where photoautotrophic primary productivity is high, scavenge ${ }^{234} \mathrm{Th}$ and transport it deeper into the water column. As particles sink and are remineralized, a decrease in particulate ${ }^{234} \mathrm{Th}$ and an 
increase in dissolved ${ }^{234} \mathrm{Th}$ is expected. A local excess of ${ }^{234} \mathrm{Th}$ over its ${ }^{238} \mathrm{U}$ parent is created as many sinking particles become suspended or dissolved. These excess ${ }^{234} \mathrm{Th}$ features represent a decrease in flux with depth because less ${ }^{234} \mathrm{Th}$ will be reaching farther into the water column. This pattern was observed in most of the Gyre stations (last panel, Supp. Figs. 8D and 8E). The location of the subsurface ${ }^{234} \mathrm{Th}$ excess, when present, coincided with a drop in particulate ${ }^{234} \mathrm{Th}$.

The particulate activity profiles for both size fractions in the Shelf and Offshore did not follow those in the Gyre (Supp. Figs. 8A to 8C) as most stations showed a distinct peak in both size fractions that began below the $30 \%$ oxygen saturation point (at or just below the upper boundary of the ODZ, Figs. 2D and 2E). These particulate peaks sometimes coincided with remineralization features (decreases in flux, Supp. Fig. 8A) but often the peak in ${ }^{234} \mathrm{Th}$ flux and subsequent drop in flux occurred well above the local subsurface particle maxima (Supp. Fig. 8C). These particulate peaks were especially pronounced in the SSF and were located progressively deeper in the water column from stations 7 to $13\left(84^{\circ} \mathrm{W}\right.$ to $\left.99^{\circ} \mathrm{W}\right)$. These peaks also coincided with a decrease in light transmission and an increase in fluorescence (Fig. 2). Prior studies in this region could have missed the subsurface local maxima in particulate ${ }^{234} \mathrm{Th}$ with poorer resolution.

Oxygen deficient zones have been shown to be unique hotspots of microbial processes and particulate features. The coincidence of nitrate minima, nitrite maxima, intermediate nephloid layers, and enhanced microbial activity in the low oxygen zones of Peru and the Arabian Sea are well established (Morrison et al., 1999; Naqvi et al., 1993), although the impact of these boundaries on the particle cycling within the ODZs is not. Studies of the southeastern Pacific and the Arabian Sea have observed similar features in light transmission or backscatter in the core of the ODZ (Garfield et al., 1983; Kullenberg, 1982; Naqvi et al., 1993; Spinrad et al., 1989; Whitmire et al., 2009). A few of these studies have linked these trends to bacterial abundance and denitrification (Morrison et al., 1999; Naqvi et al., 1993). A bacterial origin is the favored explanation for these observed particle layers over inorganic origins because the features only exist at the top of the ODZ and not throughout the entire zone. In addition, Stevens and Ulloa (2008) determined that the bacterial richness, or the number of different species in the OMZ, was higher in the upper ODZ region than the richness determined for the oxic surface and deeper oxycline (across the lower boundary of the ODZ). The prokaryotic nature of the upper ODZ in the Shelf and Offshore regions of this transect have been discussed in more detail in Ohnemus et al. (2016), but in summary, their 
analysis suggested the presence of both denitrifying prokaryotes and autotrophic communities near the upper ODZ boundary.

The co-occurrence of subsurface, local minima in light transmission with maxima in particulate ${ }^{234} \mathrm{Th}$ and fluorescence along the transect suggested that these bacterial communities and their metabolic products have a strong impact on particle cycling and particle reactive metals, such as thorium, in the upper Peruvian ODZ (Fig 2, Supp. Fig. 8). The net signal in ${ }^{234}$ Th through the top of the ODZ was remineralization, as shown by an excess in total ${ }^{234} \mathrm{Th}$ and a decrease in flux (Fig. 2E, Supp. Fig. 8). Therefore, if the system was 1-D, particles from the surface ocean must have been stopped, slowed down with respect to sinking, and/or degraded in this zone, transferring ${ }^{234} \mathrm{Th}$ to a dissolved or non-sinking pool. However, the higher particulate activities suggest that ${ }^{234} \mathrm{Th}$ may also be scavenged onto new particles being produced below the upper boundary of the ODZ (likely by some combination of photoautotrophic and chemoautotrophic processes).

Bacteria have been shown to be important agents for trace element scavenging (Cowen and Bruland, 1985). The particulate iron measured through the upper ODZ was composed mostly of $\mathrm{Fe}(\mathrm{III})$ in the form of high-surface-area Fe(III) oxyhydroxides (Heller et al., 2017). Without additional information, it is unclear whether denitrifying bacteria, which have been shown to form metal crusts when reducing nitrate (production of nitrite resulting in Fe-II oxidation), are creating these iron oxyhydroxides (Klueglein et al., 2014). However, the Fe(III) oxyhydroxides have the potential to scavenge ${ }^{234} \mathrm{Th}$, which would lead to a retention of ${ }^{234} \mathrm{Th}$ in the upper ODZ region of the Shelf and Offshore.

The data presented here suggest that the subsurface remineralization features captured by the ${ }^{234}$ Th-method could be largely driven by anaerobic denitrification processes up to station 9 or 10 $\left(\sim 90^{\circ} \mathrm{W}\right)$ and aerobic respiration west of station 10 . The observed subsurface excesses of total ${ }^{234} \mathrm{Th}$, which represent a switch between net production and net respiration in the water column, consistently began at the upper ODZ boundary until station 11, where the boundary dropped to almost 200 m (Figs. 2 and 3). Secondary, deep chlorophyll maxima were only present within the ODZ up to station 10 and for the remaining stations only a single, shallow chlorophyll maximum was observed. The large remineralization features observed up to station 10 appeared to be linked to the coexistence of both denitrifying and autotrophic communities (Ohnemus et al., 2016), which are tied to the presence of 'anaerobic' conditions and the depth of 'anaerobic' conditions (light 
levels), respectively. The ODZ was present at stations 11,12, and 13, but the depth of the upper ODZ boundary was much deeper than $150 \mathrm{~m}$. This suggested that a complex community structure is necessary to create the 'concentrated' remineralization feature directly below the upper ODZ boundary. In addition, the ${ }^{234} \mathrm{Th}$ activity profiles (Fig. 3) for stations 12 and 13 showed double remineralization peaks, with local maxima in excess ${ }^{234} \mathrm{Th}$ above and below the upper ODZ boundary. These double peak stations may represent a transition zone where both aerobic and anaerobic processes contributed to total remineralization.

\subsubsection{Regional trends in POC: ${ }^{234} \mathrm{Th}$ and PON: ${ }^{234} \mathrm{Th}$}

To determine macronutrient (e.g. nitrogen) or micronutrient trace metal (e.g. iron) export at a given depth the particulate concentration ratio of that element to ${ }^{234} \mathrm{Th}$ activity measured in the LSF ( $>51 \mathrm{um}$ ) at that depth is used to calculate the particulate flux with the following relationship:

$$
\text { Element Particulate Flux }=\frac{[\text { Element }]}{{ }^{234} \mathrm{Th}} * \text { Particulate Flux }{ }^{234} \mathrm{Th} \quad \text { (Eq. 6) }
$$

The major assumption in this empirical approach is that the element: ${ }^{234} \mathrm{Th}$ ratio measured at the depth of interest is representative of the sinking particles at this depth (Buesseler et al., 2006). Eq. 6 can be used to calculate an increase or decrease in flux that might result from production or remineralization processes. Samples above the MLD were excluded from POC: ${ }^{234} \mathrm{Th}$-depth relationships (Fig. 4). Mixed layer POC: ${ }^{234}$ Th in the same location can vary by orders of magnitude relative to those in the underlying euphotic zone (Buesseler et al., 2009; Passow et al., 2006).

Unlike the North Atlantic GEOTRACES dataset (Owens et al., 2015), the marked decline in POC: $:{ }^{234}$ Th ratios with longitude and the shift in Ez along the transect (Tables 1 and 2) warrant the use of regional estimates of particulate ratios. The distinct shift across the transect in both the Ez and subsurface LSF POC: $: 234$ Th averages can be seen in Fig. 4. Linear regressions are shown for the euphotic zones, where ratios decline sharply with depth. Vertical averages were used below Ez to $400 \mathrm{~m}$ because little variability in ratios was observed. These linear equations and subsurface vertical averages were used to determine the POC: ${ }^{234} \mathrm{Th}$ at any depth along the transect, not just those where particulate ${ }^{234} \mathrm{Th}$ was sampled. For example, to determine the POC flux at Ez for a given station, the (upwelling-adjusted) ${ }^{234} \mathrm{Th}$ flux was multiplied by the POC: ${ }^{234} \mathrm{Th}$ at the Ez depth

using the particulate ratio from one of the zones as described in Eq. 6. POC: ${ }^{234} \mathrm{Th}$ uncertainties for each depth and zone represent one standard deviation as shown in Fig. 4. 


\subsubsection{Upper ocean export and remineralization of POC}

With remarkable consistency in ${ }^{234} \mathrm{Th}$ export, calculated carbon fluxes primarily reflected the observed decrease in POC: ${ }^{234} \mathrm{Th}$ across the transect, modified by the increase in ${ }^{234} \mathrm{Th}$ flux in the coastal stations due to upwelling. The average Shelf POC export at Ez reached $42 \pm 27 \mathrm{mmolC} \mathrm{m}^{-}$ ${ }^{2} \mathrm{~d}^{-1}$ (s.d.) while the Offshore and Gyre POC export totaled only $3 \pm 2$ and $1.1 \pm 0.2 \mathrm{mmolC} \mathrm{m}^{-2} \mathrm{~d}^{-}$ ${ }^{1}$ (Table 2). Within a given zone, the range in export and s.d. reflected the variability in POC: ${ }^{234} \mathrm{Th}$ ratios (Table 3, Fig. 4). In the Offshore and Gyre, POC: ${ }^{234}$ Th ratios at the Ez depth were relatively consistent, so small variations in Ez did not lead to large changes in carbon flux. The Shelf stations, on the other hand, showed a linear decrease in POC: ${ }^{234} \mathrm{Th}$ from the shallowest Ez at $25 \mathrm{~m}$ to the deepest at $66 \mathrm{~m}$ and so stations with deeper Ez had lower POC flux. POC export differed greatly in the Shelf and the Gyre when using Ez or $100 \mathrm{~m}$ as the integration depth (Table 1 and Supp. Table 2). POC export in the Shelf was underestimated by up to a factor of 8 if $100 \mathrm{~m}$ was used instead of Ez. This supports the move to use the variable Ez as the export reference depth of choice for comparing export efficiencies (Buesseler and Boyd, 2009; Siegel et al., 2014).

Most of the prior carbon flux studies from this region were located in the Gyre (black, Fig. 6) and reported relatively similar export values to those calculated here (blue, Fig. 6). Austral spring POC fluxes from ${ }^{234} \mathrm{Th}$ in-situ pumping efforts at $12^{\circ} \mathrm{S}$ were determined to be between $2.5 \mathrm{mmolC}$ $\mathrm{m}^{-2} \mathrm{~d}^{-1}$ and $3 \mathrm{mmolC} \mathrm{m} \mathrm{d}^{-2}$ for $112.5^{\circ} \mathrm{W}$ and $2 \mathrm{mmolC} \mathrm{m} \mathrm{d}^{-2}$ and $2.5 \mathrm{mmolC} \mathrm{m}^{-2} \mathrm{~d}^{-1}$ for $142.5^{\circ}$ W (Buesseler et al., 1995). The paucity of ${ }^{234} \mathrm{Th}$-derived export values for this region and others worldwide has been and will continue to be improved by past and future GEOTRACES campaigns. However, more work is needed to not only fill in the gaps in the world's ocean where no data exists (Fig. 6), but to improve coverage throughout all seasons and to obtain more export values calculated at Ez.

Carbon remineralization was calculated in the same manner as carbon export: the decrease in ${ }^{234} \mathrm{Th}$ flux below Ez was multiplied by the POC: ${ }^{234} \mathrm{Th}$ ratio for that depth (Table 3). POC remineralization was examined in the $100 \mathrm{~m}$ below $\mathrm{Ez}$ and in the subsequent $100 \mathrm{~m}(\mathrm{Ez}+100$ to $\mathrm{Ez}+200)$. POC remineralization values had rather large uncertainties due to the lack of change in the POC: ${ }^{234} \mathrm{Th}$ ratios at these depths and increasing uncertainties on the ${ }^{234} \mathrm{Th}$ fluxes with depth. In the first $100 \mathrm{~m}$ below Ez, carbon flux decreased by a maximum of $66 \mathrm{mmolC} \mathrm{m}^{-2} \mathrm{~d}^{-1}$ (Shelf) and a minimum of $0.1 \mathrm{mmolC} \mathrm{m}^{-2} \mathrm{~d}^{-1}$ (Gyre), showing a much larger gradient in remineralization 
in the $100 \mathrm{~m}$ below Ez relative to ${ }^{234} \mathrm{Th}$. In the next $100 \mathrm{~m}$, from $\mathrm{Ez}+100$ to $\mathrm{Ez}+200$, the decrease in flux was relatively small outside the Shelf (maximum $10 \mathrm{mmolC} \mathrm{m}^{-2} \mathrm{~d}^{-1}$ decrease), ranging from only $0.1 \mathrm{mmolC} \mathrm{m} \mathrm{d}^{-2}$ to $0.8 \mathrm{mmolC} \mathrm{m} \mathrm{d}^{-1}$. The concept that POC attenuation, in general, is greatest at shallow depths has long been established in prior studies and enforces the concept of using Ez-based interpretation to compare studies (Buesseler and Boyd, 2009).

\subsubsection{Strength and export efficiency of the regional biological pump}

The fraction of surface production (NPP) that makes it out of the euphotic zone is often referred to as the strength of the biological pump, whereas the export efficiency of the biological pump refers to the fraction that is not remineralized in the twilight zone (Ez to $\sim 1000 \mathrm{~m}$, Buesseler and Boyd, 2009). Some studies have predicted that the strength and export efficiency of the biological pump will decrease as the global climate changes, leading to increased atmospheric levels of carbon dioxide (Manizza et al., 2010; Steinacher et al., 2010). We have assessed the biological carbon pump along the transect relative to two parameters, Ez-ratio and $\mathrm{T}_{100}$, that incorporate estimates of surface production, export, and remineralization. The Ez-ratio, defined as the POC flux at the Ez (export) divided by NPP, is an important parameter for characterizing the strength of the biological pump, or how much carbon makes it out of the sunlit upper ocean (Tables 2 and 3; Buesseler and Boyd, 2009). A large Ez-ratio for an area with low productivity would mean greater POC transport to depth than an area with moderate productivity, but a low Ez-ratio. Buesseler and Boyd (2009) also parameterized POC remineralization by relating the POC flux at $100 \mathrm{~m}$ below Ez to the POC flux at Ez. This parameter, $\mathrm{T}_{100}$, was chosen as the depth interval of maximum POC flux attenuation. These metrics were combined (Fig. 7) to illustrate the flow of carbon through the surface biological pump and to facilitate the comparison with other studies. If the Ez-ratio is multiplied by $\mathrm{T}_{100}$, the resulting value is the percentage of carbon reaching $100 \mathrm{~m}$ below Ez relative to what was produced in the euphotic zone (1\% and $10 \%$ contours, Fig. 7).

MODIS-derived 16-day NPP for the Offshore and Gyre were within $\sim 20 \mathrm{mmolC} \mathrm{m}^{-2} \mathrm{~d}^{-1}$ of the SeaWIFS-based 8-day and annual average NPP, while Shelf NPP values could differ by up to $\sim 74$ mmolC $\mathrm{m}^{-2} \mathrm{~d}^{-1}$ (Table 2 and 3). This large difference in the Shelf suggests that the coastal region is very dynamic and seasonal factors could greatly influence the productivity in this region. Prior studies of primary production in the southeastern tropical Pacific have shown similar results, where the Offshore and Gyre had lower, but consistent productivity throughout the year and the eutrophic 
coastal areas showed strong seasonality (Pennington et al., 2006). If the 16-day NPP values for the Offshore and Gyre (34-61 mmolC $\mathrm{m}^{-2} \mathrm{~d}^{-1}$ ) are considered relative to other locations worldwide (Fig. 7), they fall in between the low values observed at ALOHA (central North Pacific, 17 mmolC m $\mathrm{m}^{-2}$ ) and the $>100$ mmolC m $\mathrm{m}^{-2} \mathrm{~d}^{-1}$ NPP values observed in the Southern Ocean (SO) and Equatorial Pacific (EqPac). The coastal Shelf stations have comparable NPP values to locations under bloom conditions in the Southern Ocean and the North Atlantic (Natl).

Although NPP was higher in the Shelf relative to the Offshore and Gyre, POC export at Ez varied substantially from the Shelf to the Gyre, creating an order of magnitude difference in the estimated strength or Ez-ratio of the local biological carbon pump (Fig. 7). Stations in the Shelf had Ez-ratios (NPP/Export at Ez) varying from 0.5 to 0.1, while Ez-ratios in the Offshore and Gyre zones were more consistent and lower (Table 3). Average Ez-ratios in the Offshore and Gyre were $0.06 \pm 0.04$ (s.d.) and $0.02 \pm 0.005$, respectively. While up to $50 \%$ of carbon produced in the surface waters of the Shelf reached the base of the euphotic, the Offshore and Gyre saw $<15 \%$ exported at all stations (Table 3). Ez-ratios under 0.1 are relatively low compared to those found in productive regions like the North Atlantic and Northwestern Pacific, but are similar to those found at other low-export Pacific sites like Station Papa (Spring), ALOHA, and the Equatorial Pacific, where $<10 \%$ of POC is exported out of the euphotic zone (Fig. 7; Buesseler and Boyd, 2009). The GBC14 global export model, a food-web based model utilizing SeaWIFS satellite products, produces similar Ez-ratios in the Offshore and Gyre, with all estimated Ez-ratios under 0.2 for these regions and a consistent Ez-ratio for the Shelf stations of 0.2 (Siegel et al., 2014).

Carbon transfer ratios for the first $100 \mathrm{~m}$ below Ez $\left(\mathrm{T}_{100}\right)$ were larger, on average, in the Gyre at $0.8 \pm 0.1$ (s.d.), compared to the Offshore zone at $0.6 \pm 0.2$ and the Shelf zone at $0.4 \pm 0.4$ (Tables 2 and 3). This suggests lower remineralization of sinking POC in the Gyre relative to the Shelf and Offshore regions at depths just below Ez (Fig. 7). The $\mathrm{T}_{100}$ values for this transect cover the entire range of measured $\mathrm{T}_{100}$ reported for other locations worldwide in Buesseler and Boyd (2009). Most of this variability in $T_{100}$ was observed in the Offshore zone, as the Gyre stations had consistently high $\mathrm{T}_{100}$ values.

Based on these results from austral spring, the $10^{\circ} \mathrm{S}$ to $15^{\circ} \mathrm{S}$ region of the southeastern tropical Pacific has a strong coastal biological pump (higher Ez-ratio), but one that has high POC flux attenuation below the euphotic zone (low $\mathrm{T}_{100}$, Fig. 7). The biological pump of the Gyre, as 
expected from other Pacific studies, is relatively weak but has little POC flux attenuation below the euphotic zone (Buesseler and Boyd, 2009). The Offshore biological pump, surprisingly, was consistently poor in strength but had transfer efficiencies that ranged from 0.1 to 0.9 . On average, the amount of POC exported at $100 \mathrm{~m}$ below Ez was similar between the Offshore at $3 \pm 2 \%$ and the Gyre at $2 \pm 0.3 \%$ (Table 2), although it ranged from $0.4 \%$ (station 9) to $7 \%$ (station 14) in the Offshore. While there was a large spread in the data, almost all the stations from this study and prior studies fall within the $1 \%$ to $10 \%$ range for the amount of POC exported at $100 \mathrm{~m}$ below Ez. The percent of POC sequestered $100 \mathrm{~m}$ below Ez reaches a similar value at all stations by a combination of high export from the euphotic zone and rapid POC attenuation in the Shelf (shallowest Ez), progressively less export and less attenuation in the Offshore zone, and low export combined with less POC flux attenuation in the Gyre zone (deepest Ez).

While none of the stations from this study had POC export at $100 \mathrm{~m}$ below Ez close to the values seen at NABE (North Atlantic Bloom Experiment) or in the Southern Ocean, a 1\% to 13\% difference in export at this depth is not trivial if a large region of the ocean is impacted. The decrease in carbon attenuation below Ez in the Gyre zone is expected, as enhanced export through similar low oxygen regions has been shown (Roullier et al., 2014; Van Mooy et al., 2002). With expanding low oxygen regions worldwide, this trend of decreased attenuation through OMZs could lead to decreased POC export to depth over 8\% of the world ocean (Paulmier and Ruiz-Pino, 2009; Stramma et al., 2008). However, further study is needed to determine the cause of the relatively low transfer efficiency for some of the Shelf and Offshore stations and whether oxygen deficient zones indeed show greater attenuation of POC relative to zones of low oxygen. Station 9 (bottom left-most point in Fig. 7), in particular, had substantially more remineralization than any of the other stations in the Offshore region. Stations 6 to 10, the only Offshore locations with deep chlorophyll maxima at or below the upper ODZ boundary, had an average $\mathrm{T}_{100}$ of 0.4 . The remaining stations (11 to 15 ) in the Offshore zone, those that did not show signs of deep, light adapted autotrophs, had an average $\mathrm{T}_{100}$ of 0.8 . This suggests that the community structure through the upper oxycline and ODZ could play an important role in controlling the percent of POC that reaches $100 \mathrm{~m}$ below the euphotic. The extent of this role and the degree to which ODZs have the potential to increase carbon attenuation is vital to understanding the overall effect of expanding OMZs and ODZs on the global biological carbon pump. 


\subsection{Conclusions}

Here we have shown that the non-coastal southeastern tropical Pacific had relatively low carbon export out of the euphotic zone in the austral spring $\left(0.8-9 \mathrm{mmolC} \mathrm{m}^{-2} \mathrm{~d}^{-1}\right)$, but similar Ezratios (Export/NPP) to other open ocean locations in the Pacific, including the productive equatorial region (Fig. 7; Buesseler and Boyd, 2009). When estimating export in coastal regions similarly influenced by upwelling, surface fluxes of carbon or other elements could be underestimated by a factor of two to four if the influence of upwelling is not account for in ${ }^{234} \mathrm{Th}$ based estimates. Both particulate carbon and nitrogen export decreased with distance offshore, following shifts in plankton community dominance, increased Ez, and changes in POC: ${ }^{234} \mathrm{Th}$ and PON: ${ }^{234}$ Th ratios on sinking particles. The low transfer of carbon out of the euphotic zone along the transect produced some of the lowest Ez-ratios that have been quantified worldwide (excluding the Shelf). The $\mathrm{T}_{100}$ values observed here were only surpassed by regions under bloom conditions. This indicated that once POC left the euphotic zone, very little was attenuated in these low oxygen regions of the ocean. Currently covering $8 \%$ of the world's ocean area, these OMZs have the potential to enhance carbon transfer to depth, especially as OMZs are predicted to expand with changing global climate (Paulmier and Ruiz-Pino, 2009). However, greater attenuation at stations through the oxygen deficient region of the $\mathrm{OMZ}\left(77^{\circ} \mathrm{W}\right.$ to $\left.92^{\circ} \mathrm{W}\right)$ suggests that this expansion may have a more complex effect on the regional biological carbon pump than predicted.

The upper ODZ boundary is a unique setting where further research of trace metal and nutrient cycling is warranted. Simultaneous trace metal sampling with incubation experiments, measurements of bacterial counts, and genomic studies, would help in making definitive conclusions on the metabolic capacities of local bacteria (e.g. autotrophy, nitrate reduction via denitrification, anammox), how each population impacts ${ }^{234}$ Th scavenging and remineralization, and if the oxygen gradient accurately represents the relative transition from aerobic to anaerobicdominated metabolisms. As ODZs expand worldwide, their growth may drastically impact local planktonic communities, especially those who dwell just over or under the almost anoxic boundaries (Wishner et al., 2013). ${ }^{234}$ Th results suggests that these communities significantly affect subsurface particulate activities and multi-isotope analysis with longer-lived thorium isotopes $\left({ }^{228} \mathrm{Th},{ }^{232} \mathrm{Th},{ }^{230} \mathrm{Th}\right)$ could help shed light on the impact of the ODZ and its microbial communities on particle dynamics on longer time scales. 
Many trace metals, like iron, have been shown to be essential for ocean microbiota and much less is known about the quantities of these metals traveling or cycling through the world's ODZs compared to that which is known about elements like carbon and nitrogen. Whereas carbon concentrations tend to follow an exponential decrease from the surface, making export calculations relatively simple, trace metals are impacted by a host of processes that can create widely different distributions metal to metal and station to station (Lamborg et al., 2008). While trace metals

provide new challenges for utilizing the ${ }^{234} \mathrm{Th}$-method, radioisotope tracers from this and other GEOTRACES cruises will provide the tools necessary to determine basin-wide, carbon, nutrient, and trace metal export flux and remineralization rates, one of the key goals of the international GEOTRACES program.

\section{Acknowledgements}

This work was supported by the National Science Foundation (OCE-1232669) and E. Black was also funded by a NASA Earth and Space Science Graduate Fellowship (NNX13AP31H). The authors would like to thank the 3 anonymous reviewers for their thoughtful comments and the captain, crew, and scientists aboard the R/V Thomas G. Thompson. Particular thanks go to the chief scientists, James Moffett and Chris German, and to the members of the pump group including Matt Charette, Willard Moore, Dan Ohnemus, and Virginie Sanial. Laboratory assistance was provided by Gretchen Swarr, Paul Henderson, Lauren Kipp, and Crystal Breier. Additional thanks goes to Kelsey Bisson and Dave Siegel who provided model-derived carbon export estimates and Dave Kadko for his ${ }^{7} \mathrm{Be}$-derived upwelling rates. 


\section{References}

Bacon, M.P., Cochran, J.K., Hirschberg, D., Hammar, T.R., Fleer, A.P., 1996. Export flux of carbon at the equator during the EqPac time-series cruises estimated from ${ }^{234} \mathrm{Th}$ measurements. Deep. Res. II 43, 1133-1153.

Barber, R.T., Marra, J., Bidigare, R.C., Codispoti, L.A., Halpern, D., Johnson, Z., Latasa, M., Goericke, R., Smith, S.L., 2001. Primary productivity and its regulation in the Arabian Sea during 1995, Deep-Sea Research II.

Benitez-Nelson, C., Buesseler, K.O., Karl, D.M., Andrews, J., 2001. A time-series study of particulate matter export in the North Pacific Subtropical Gyre based on ${ }^{234} \mathrm{Th}:{ }^{.238} \mathrm{U}$ disequilibrium. Deep. Res. I 48, 2595-2611.

Bhat, S.G., Krishnaswamy, S., Lal, D., Rama, Moore, W.S., 1969. ${ }^{234} \mathrm{Th} /{ }^{238} \mathrm{U}$ ratios in the ocean. Earth Planet. Sci. Lett. 5, 483-491.

Bianchi, D., Galbraith, E.D., Carozza, D.A., Mislan, K.A.S., Stock, C.A., 2013. Intensification of openocean oxygen depletion by vertically migrating animals. Nat. Geosci. 6, 545-548. doi:10.1038/ngeo1837

Bishop, J.K., Edmond, J.M., Ketten, D.R., Bacon, M.P., Silker, W.B., 1977. The chemistry, biology, and vertical flux of particulate matter from the upper $400 \mathrm{~m}$ of the equatorial Atlantic Ocean. Deep. Res. 24, 511-548.

Bishop, J.K.B., Lam, P.J., Wood, T.J., 2012. Getting good particles: Accurate sampling of particles by large volume in-situ filtration. Limnol. Oceanogr. Methods 10, 681-710. doi:10.4319/lom.2012.10.681

Boyd, P.W., Gall, M.P., Silver, M.W., Coale, S.L., Bidigare, R.R., Bishop, J.L.K.B., 2008. Quantifying the surface-subsurface biogeochemical coupling during the VERTIGO ALOHA and K2 studies. Deep. Res. II 55, 1578-1593. doi:10.1016/j.dsr2.2008.04.010

Brink, K.H., Halpern, D., Huyer, A., Smith, R.L., 1983. The Physical Environment of the Peruvian Upwelling System. Prog. Oceanogr. 12, 285-305.

Buesseler, K.O., Andrews, J.A., Hartman, M.C., Belastock, R., Chai, F., 1995. Regional estimates of the export flux of particulate organic carbon derived from thorium-234 during the JGOFS EqPac program. Deep. Res. II 42, 777-804.

Buesseler, K.O., Bacon, M.P., Cochran, J.K., Livingston, H.D., 1992. Carbon and nitrogen export during the JGOFS North Atlantic Bloom Experiment estimated from ${ }^{234} \mathrm{Th}:{ }^{238} \mathrm{U}$ disequilibria. Deep. Res. $39,1115-1137$.

Buesseler, K.O., Ball, L., Andrews, J., Benitez-Nelson, C., Belastock, R., Chai, F., Chao, Y., 1998. Upper ocean export of particulate organic carbon in the Arabian Sea derived from thorium-234. Deep. Res. II 45, 2461-2487.

Buesseler, K.O., Ball, L., Andrews, J., Cochran, J.K., Hirschberg, D.J., Bacon, M.P., Fleer, A., Brzezinski, M., 2001a. Upper ocean export of particulate organic carbon and biogenic silica in the Southern Ocean along 170 W. Deep. Res. II 48, 4275-4297.

Buesseler, K.O., Benitez-Nelson, C., Rutgers van der Loeff, M., Andrews, J., Ball, L., Crossin, G., Charette, M.A., 2001b. An intercomparison of small- and large-volume techniques for thorium-234 in seawater. Mar. Chem. 74, 15-28.

Buesseler, K.O., Benitez-Nelson, C.R., Moran, S.B., Burd, A., Charette, M., Cochran, J.K., Coppola, L., Fisher, N.S., Fowler, S.W., Gardner, W.D., Guo, L.D., Gustafsson, O., Lamborg, C., Masque, P., 
Miquel, J.C., Passow, U., Santschi, P.H., Savoye, N., Stewart, G., Trull, T., 2006. An assessment of particulate organic carbon to thorium-234 ratios in the ocean and their impact on the application of ${ }^{234} \mathrm{Th}$ as a POC flux proxy. Mar. Chem. 100, 213-233. doi:10.1016/j.marchem.2005.10.013

Buesseler, K.O., Boyd, P.W., 2009. Shedding light on processes that control particle export and flux attenuation in the twilight zone of the open ocean. Limnol. Oceanogr. 54, 1210-1232.

Buesseler, K.O., Pike, S., Maiti, K., Lamborg, C.H., Siegel, D.A., Trull, T.W., 2009. Thorium-234 as a tracer of spatial, temporal and vertical variability in particle flux in the North Pacific. Deep. Res. I 56, 1143-1167. doi:10.1016/j.dsr.2009.04.001

Buesseler, K.O., Trull, T.W., Steinberg, D.K., Silver, M.W., Siegel, D.A., Saitoh, S.-I., Lamborg, C.H., Lam, P.J., Karl, D.M., Jiao, N.Z., Honda, M.C., Elskens, M., Dehairs, F., Brown, S.L., Boyd, P.W., Bishop, J.K.B., Bidigare, R.R., 2008. VERTIGO (VERtical Transport In the Global Ocean): A study of particle sources and flux attenuation in the North Pacific. Deep. Res. II 55, 1522-1539. doi:10.1016/j.dsr2.2008.04.024

Cai, P., Chen, W., Dai, M., Wan, Z., Wang, D., Li, Q., Tang, T., Lv, D., 2008. A high-resolution study of particle export in the southern South China Sea based on ${ }^{234} \mathrm{Th}:{ }^{238} \mathrm{U}$ disequilibrium. J. Geophys. Res. 113, 1-15. doi:10.1029/2007JC004268

Cai, P., Rutgers Van der Loeff, M., Stimac, I., Nothig, E.M., Lepore, K., Moran, S.B., 2010. Low export flux of particulate organic carbon in the central Arctic Ocean as revealed by ${ }^{234} \mathrm{Th}:{ }^{238} \mathrm{U}$ disequilibrium. J. Geophys. Res. Ocean. 115, 1-21. doi:10.1029/2009JC005595

Coale, K.H., Bruland, K.W., 1987. Oceanic stratified euphotic zone as elucidated by ${ }^{234} \mathrm{Th}:{ }^{238} \mathrm{U}$ disequilibria. Limnol. Oceanogr. 32, 189-200.

Codispoti, L.A., Brandes, J.A., Christensen, J.P., Devol, A.H., Naqvi, S.W.A., Paerl, H.W., Yoshinari, T., 2001. The oceanic fixed nitrogen and nitrous oxide budgets: Moving targets as we enter the anthropocene? Sci. Mar. 65, 85-105. doi:10.3989/scimar.2001.65s285

Cowen, J.P., Bruland, K.W., 1985. Metal deposits associated with bacteria: implications for Fe and Mn marine biogeochemistry. Deep Sea Res. 32, 253-272. doi:10.1016/0198-0149(85)90078-0

Fiedler, P.C., Philbrick, V., Chavez, F.P., 1991. Oceanic upwelling and productivity in the Eastern Tropical Pacific. Limnol. Oceanogr. 36, 1834-1850.

Garfield, P.C., Packard, T.T., Friederich, G.E., Codispoti, L.A., 1983. A subsurface particle maximum layer and enhanced microbial activity in the secondary nitrite maximum of the northeastern tropical Pacific Ocean. J. Mar. Res. 41, 747-768.

Goericke, R., Olson, R.J., Shalapyonok, A., 2000. A novel niche for Prochlorococcus sp. in low-light suboxic environments in the Arabian Sea and the Eastern Tropical North Pacific. Deep. Res. I 47, $1183-1205$.

Haskell, W.Z., Berelson, W.M., Hammond, D.E., Capone, D.G., 2013. Particle sinking dynamics and POC fluxes in the Eastern Tropical South Pacific based on ${ }^{234} \mathrm{Th}$ budgets and sediment trap deployments. Deep. Res. I 81, 1-13. doi:10.1016/j.dsr.2013.07.001

Haskell, W.Z., Kadko, D., Hammond, D.E., Knapp, A.N., Prokopenko, M.G., Berelson, W.M., Capone, D.G., 2015. Upwelling velocity and eddy diffusivity from 7Be measurements used to compare vertical nutrient flux to export POC flux in the Eastern Tropical South Pacific. Mar. Chem. 168, 140-150. doi:10.1016/j.marchem.2014.10.004

Heller, M.I., Lam, P.J., Moffett, J.W., Till, C.P., Lee, J.-M., Toner, B.M., Marcus, M.A., 2017. Accumulation of $\mathrm{Fe}$ oxides in the Peruvian oxygen deficient zone implies non-oxygen dependent $\mathrm{Fe}$ oxidation. Geochim. Cosmochim. Acta 211, 174-193. doi:10.1016/j.gca.2017.05.019 
Henson, S.A., Sanders, R., Madsen, E., Morris, P.J., Le Moigne, F., Quartly, G.D., 2011. A reduced estimate of the strength of the ocean's biological carbon pump. Geophys. Res. Lett. 38, 1-5. doi:10.1029/2011GL046735

Hung, C.C., Xu, C., Santschi, P.H., Zhang, S.J., Schwehr, K.A., Quigg, A., Guo, L., Gong, G.C., Pinckney, J.L., Long, R.A., Wei, C.L., 2010. Comparative evaluation of sediment trap and ${ }^{234}$ Thderived POC fluxes from the upper oligotrophic waters of the Gulf of Mexico and the subtropical northwestern Pacific Ocean. Mar. Chem. 121, 132-144. doi:10.1016/j.marchem.2010.03.011

Kadko, D., 2017. Upwelling and primary production during the U.S. GEOTRACES East Pacific Zonal Transect. Global Biogeochem. Cycles 31, 1-15. doi:10.1002/2016GB005554

Kadko, D., Johns, W., 2011. Inferring upwelling rates in the equatorial Atlantic using ${ }^{7}$ Be measurements in the upper ocean. Deep. Res. I 58, 647-657. doi:10.1016/j.dsr.2011.03.004

Karstensen, J., Stramma, L., Visbeck, M., 2008. Oxygen minimum zones in the eastern tropical Atlantic and Pacific oceans. Prog. Oceanogr. 77, 331-350. doi:10.1016/j.pocean.2007.05.009

Klueglein, N., Zeitvogel, F., Stierhof, Y.D., Floetenmeyer, M., Konhauser, K.O., Kappler, A., Obst, M., 2014. Potential role of nitrite for abiotic Fe(II) oxidation and cell encrustation during nitrate reduction by denitrifying bacteria. Appl. Environ. Microbiol. 80, 1051-1061. doi:10.1128/AEM.03277-13

Kullenberg, G., 1982. A comparison of distributions of suspended matter in the Peru and Northwest African upwelling areas, in: Richards, F.A. (Ed.), Coastal Upwelling. American Geophysical Union, pp. 282-290.

Kwon, E.Y., Primeau, F., Sarmiento, J.L., 2009. The impact of remineralization depth on the air-sea carbon balance. Nat. Geosci. 2, 630-635. doi:10.1038/ngeo612

Lam, P., Lavik, G., Jensen, M.M., van de Vossenberg, J., Schmid, M., Woebken, D., Dimitri, G., Amann, R., Jetten, M.S.M., Kuypers, M.M.M., 2009. Revising the nitrogen cycle in the Peruvian oxygen minimum zone. Proc. Natl. Acad. Sci. U. S. A. 106, 4752-4757. doi:10.1073/pnas.0812444106

Lam, P.J., Twining, B.S., Jeandel, C., Roychoudhury, A., Resing, J.A., Santschi, P.H., Anderson, R.F., 2015. Methods for analyzing the concentration and speciation of major and trace elements in marine particles. Prog. Oceanogr. 133. doi:10.1016/j.pocean.2015.01.005

Lamborg, C.H., Buesseler, K.O., Lam, P.J., 2008. Sinking fluxes of minor and trace elements in the North Pacific Ocean measured during the VERTIGO program. Deep. Res. Part II Top. Stud. Oceanogr. 55, 1564-1577. doi:10.1016/j.dsr2.2008.04.012

Le Moigne, F.A.C., Henson, S.A., Sanders, R.J., Madsen, E., 2013. Global database of surface ocean particulate organic carbon export fluxes diagnosed from the ${ }^{234} \mathrm{Th}$ technique. Earth Syst. Sci. Data 5, 295-304. doi:10.5194/essd-5-295-2013

Maas, A.E., Frazar, S.L., Outram, D.M., Seibel, B.A., Wishner, K.F., 2014. Fine-scale vertical distribution of macroplankton and micronekton in the Eastern Tropical North Pacific in association with an oxygen minimum zone. J. Plankton Res. 36, 1557-1575. doi:10.1093/plankt/fbu077

Maiti, K., Benitez-Nelson, C.R., Rii, Y., Bidigare, R., 2008. The influence of a mature cyclonic eddy on particle export in the lee of Hawaii. Deep. Res. II 55, 1445-1460. doi:10.1016/j.dsr2.2008.02.008

Maiti, K., Nelson, C.R.B., Buesseler, K.O., 2010. Insights into particle formation and remineralization using the short-lived radionuclide, Thoruim-234. Geophys. Res. Lett. 37, 1-6. doi:10.1029/2010GL044063

Manizza, M., Buitenhuis, E.T., Le Quere, C., 2010. Sensitivity of global ocean biogeochemical dynamics to ecosystem structure in a future climate. Geophys. Res. Lett. 37, 1-5. doi:10.1029/2010GL043360 
Matear, R.J., Hirst, A.C., 2003. Long-term changes in dissolved oxygen concentrations in the ocean caused by protracted global warming. Global Biogeochem. Cycles 17, 1-32. doi:10.1029/2002GB001997

McDougall, T.J., Barker, P.M., 2011. Getting started with TEOS-10 and the Gibbs Seawater (GSW) Oceanographic Toolbox (No. SCOR/IAPSO WG127).

Montecino, V., Lange, C.B., 2009. The Humboldt Current System: Ecosystem components and processes, fisheries, and sediment studies. Prog. Oceanogr. 83, 65-79. doi:10.1016/j.pocean.2009.07.041

Morrison, J.M., Codispoti, L.A., Smith, S.L., Wishner, K., Flagg, C., Gardner, W.D., Gaurin, S., Naqvi, S.W.A., Manghnani, V., Prosperie, L., Gundersen, J.S., 1999. The oxygen minimum zone in the Arabian Sea during 1995. Deep. Res. II 46, 1903-1931.

Murray, J.W., Young, J., Newton, J., Dunne, J., Chapin, T., Paul, B., Mccarthy, J.J., 1996. Export flux of particulate organic carbon from the central equatorial Pacific determined using a combined drifting trap- ${ }^{234}$ Th approach. Deep. Res. II 43, 1095-1132.

Naqvi, S.W.A., Kumar, M.D., Narvekar, P. V, De Sousa, S.N., George, M.D., D’Silva, C., 1993. An intermediate nepheloid layer associated with high microbial metabolic rates and denitrification. J. Geophys. Res. 98, 16469-16479.

Not, C., Brown, K.A., Ghaleb, B., Hillaire-Marcel, C., 2012. Conservative behavior of uranium vs. salinity in Arctic sea ice and brine. Mar. Chem. 130-131, 33-39. doi:10.1016/j.marchem.2011.12.005

Ohnemus, D.C., Rauschenberg, S., Cutter, G.A., Fitzsimmons, J.N., Sherrell, R.M., Twining, B.S., 2016. Elevated trace metal content of prokaryotic plankton communities associated with marine oxygen deficient zones. Limnol. Oceanogr. 62, 3-25. doi:10.1002/lno.10363

Owens, S.A., Buesseler, K.O., Sims, K.W.W., 2011. Re-evaluating the ${ }^{238} \mathrm{U}$-salinity relationship in seawater: Implications for the 238U-234Th disequilibrium method. Mar. Chem. 127, 31-39. doi:10.1016/j.marchem.2011.07.005

Owens, S.A., Pike, S., Buesseler, K.O., 2015. Thorium-234 as a tracer of particle dynamics and upper ocean export in the Atlantic Ocean. Deep. Res. II 116, 42-59. doi:10.1016/j.dsr2.2014.11.010

Passow, U., Dunne, J., Murray, J.W., Balistrieri, L., Alldredge, A.L., 2006. Organic carbon to ${ }^{234}$ Th ratios of marine organic matter. Mar. Chem. 100, 323-336. doi:10.1016/j.marchem.2005.10.020

Paulmier, A., Ruiz-Pino, D., 2009. Oxygen minimum zones (OMZs) in the modern ocean. Prog. Oceanogr. 80, 113-128. doi:10.1016/j.pocean.2008.08.001

Pennington, J.T., Mahoney, K.L., Kuwahara, V.S., Kolber, D.D., Calienes, R., Chavez, F.P., 2006. Primary production in the eastern tropical Pacific: A review. Prog. Oceanogr. 69, 285-317. doi:10.1016/j.pocean.2006.03.012

Pike, S.M., Buesseler, K.O., Andrews, J., Savoye, N., 2005. Quantification of ${ }^{234}$ Th recovery in small volume sea water samples by inductively coupled plasma mass spectrometry. J. Radioanal. Nucl. Chem. 263, 355-360.

Roullier, F., Berline, L., Guidi, L., Durrieu De Madron, X., Picheral, M., Sciandra, A., Pesant, S., Stemmann, L., 2014. Particle size distribution and estimated carbon flux across the Arabian Sea oxygen minimum zone. Biogeosciences 11, 4541-4557. doi:10.5194/bg-11-4541-2014

Saba, V.S., Friedrichs, M.A.M., Antoine, D., Armstrong, R.A., Asanuma, I., Behrenfeld, M.J., Ciotti, A.M., Dowell, M., Hoepffner, N., Hyde, K.J.W., Ishizaka, J., Kameda, T., Marra, J., Mlin, F., Morel, A., O’Reilly, J., Scardi, M., Smith, W.O., Smyth, T.J., Tang, S., Uitz, J., Waters, K., Westberry, T.K., 2011. An evaluation of ocean color model estimates of marine primary 
productivity in coastal and pelagic regions across the globe. Biogeosciences 8, 489-503. doi:10.5194/bg-8-489-2011

Sarmiento, J.L., Toggweiler, J.R., 1984. A new model for the role of the ocean in determining atmospheric $\mathrm{PCO}_{2}$. Nature 308, 621-624.

Savoye, N., Buesseler, K.O., Cardinal, D., Dehairs, F., 2004. 234Th deficit and excess in the Southern Ocean during spring 2001: Particle export and remineralization. Geophys. Res. Lett. 31, 1-4. doi:10.1029/2004GL019744

Seibel, B.A., Schneider, J.L., Kaartvedt, S., Wishner, K.F., Daly, K.L., 2016. Hypoxia tolerance and metabolic suppression in oxygen minimum zone Euphausiids: Implications for ocean deoxygenation and biogeochemical cycles. Integr. Comp. Biol. 56, 510-523. doi:10.1093/icb/icw091

Siegel, D.A., Buesseler, K.O., Doney, S.C., Sailley, S.F., Behrenfeld, M.J., Boyd, P.W., 2014. Global assessment of ocean carbon export by combining satellite observations and food-web models. Global Biogeochem. Cycles 28, 181-196. doi:10.1002/2013GB004743

Spinrad, R.W., Glover, H., Ward, B.B., Codispoti, L.A., Kullenberg, G., 1989. Suspended particle and bacterial maxima in Peruvian coastal waters during a cold water anomaly. Deep. Res. 36, 715-733.

Steinacher, M., Joos, F., Frolicher, T.L., Bopp, L., Cadule, P., Cocco, V., Doney, S.C., Gehlen, M., Lindsay, K., Moore, J.K., Schneider, B., Segschneider, J., 2010. Projected 21st century decrease in marine productivity: a multi-model analysis. Biogeosciences 7, 979-1005.

Steinfeldt, R., Sültenfuß, J., Dengler, M., Fischer, T., Rhein, M., 2015. Coastal upwelling off Peru and Mauritania inferred from helium isotope disequilibrium. Biogeosciences 12, 7519-7533. doi:10.5194/bg-12-7519-2015

Stevens, H., Ulloa, O., 2008. Bacterial diversity in the oxygen minimum zone of the eastern tropical South Pacific. Environ. Microbiol. 10, 1244-1259. doi:10.1111/j.1462-2920.2007.01539.x

Stramma, L., Johnson, G.C., Sprintall, J., Mohrholz, V., 2008. Expanding oxygen-minimum zones in the tropical oceans. Science 320, 655-659.

Stramma, L., Schmidtko, S., Levin, L.A., Johnson, G.C., 2010. Ocean oxygen minima expansions and their biological impacts. Deep. Res. I 57, 587-595. doi:10.1016/j.dsr.2010.01.005

Tiano, L., Garcia-Robledo, E., Dalsgaard, T., Devol, A.H., Ward, B.B., Ulloa, O., Canfield, D.E., Revsbech, N.P., 2014. Oxygen distribution and aerobic respiration in the north and south eastern tropical Pacific oxygen minimum zones. Deep. Res. I 94, 173-183. doi:10.1016/j.dsr.2014.10.001

Turnewitsch, R., Reyss, J.L., Nycander, J., Waniek, J.J., Lampitt, R.S., 2008. Internal tides and sediment dynamics in the deep sea - Evidence from radioactive ${ }^{234} \mathrm{Th} /{ }^{238} \mathrm{U}$ disequilibria. Deep. Res. I 55, $1727-$ 1747. doi:10.1016/j.dsr.2008.07.008

Usbeck, R., Rutgers Van der Loeff, M., Hoppema, M., Schlitzer, R., 2002. Shallow remineralization in the Weddell Gyre. Geochemistry Geophys. Geosystems 3, 1-18.

Van Mooy, B.A.S., Keil, R.G., Devol, A.H., 2002. Impact of suboxia on sinking particulate organic carbon: Enhanced carbon flux and preferential degradation of amino acids via denitrification. Geochim. Cosmochim. Acta 66, 457-465.

Westberry, T., Behrenfeld, M.J., Siegel, D.A., Boss, E., 2008. Carbon-based primary productivity modeling with vertically resolved photoacclimation. Global Biogeochem. Cycles 22, 1-18. doi:10.1029/2007GB003078 
Whitmire, A.L., Letelier, R.M., Villagrán, V., Ulloa, O., 2009. Autonomous observations of in vivo fluorescence and particle backscattering in an oceanic oxygen minimum zone. Opt. Express 17, 21992-22004. doi:10.1117/12.190060

Wishner, K.F., Outram, D.M., Seibel, B.A., Daly, K.L., Williams, R.L., 2013. Zooplankton in the eastern tropical north Pacific: Boundary effects of oxygen minimum zone expansion. Deep Sea Res. I 79, 122-140. doi:10.1016/j.dsr.2013.05.012

\section{Website and Database References}

Buesseler, K.O., Charette, M.A., \& Moore, W.S. (2017) Water-column total Th-234 and U-238 from R/V Thomas G. Thompson cruise TN303 in the Eastern Tropical Pacific in 2013 (U.S. GEOTRACES EPZT project). Biological and Chemical Oceanography Data Management Office (BCO-DMO). Version: 27 January 2017. http://www.bco-dmo.org/dataset/643213

Buesseler, K.O., Charette, M.A., \& Moore, W.S. (2016) Th-234 from in-situ pumps, including large size fraction (>51 um) and small size fraction (1-51 um), from R/V Thomas G. Thompson cruise TN303 in the Eastern Tropical Pacific in 2013 (U.S. GEOTRACES EPZT project). Biological and Chemical Oceanography Data Management Office (BCO-DMO). Version: 03 June 2016. http://www.bco-dmo.org/dataset/643316

NOAA, OceanWatch Central Pacific TDS THREDDS Data Server, http://pifscoceanwatch.irc.noaa.gov/thredds/catalog.html, Last Access: 10 Oct 2016.

NOAA, World Ocean Atlas 2009, https://www.nodc.noaa.gov/OC5/WOA09/pr_woa09.html, Last Access: 10 Oct 2016.

Ocean Productivity, Oregon State University, http://www.science.oregonstate.edu/ocean.productivity/, Last Access: 07 June 2017. 
Table 1: Summary of ${ }^{234}$ Th results by station including ${ }^{234}$ Th-derived parameters. Remineralization (R) values are shown for the decrease in ${ }^{234} \mathrm{Th}$ flux calculated between Ez (base of the euphotic zone) and $100 \mathrm{~m}$ below it $\left(\mathrm{R}_{100}\right)$ and from $100 \mathrm{~m}$ below the Ez to 200 $\mathrm{m}$ below Ez $\left(\mathrm{R}_{200}\right)$. $\mathrm{R}$ values were not reported if no remineralization $\left({ }^{234} \mathrm{Th}\right.$ excess) feature was observed (Fig. 3 ), fluxes increased below Ez, or uncertainties were $>3$ times the R values. Adjusted ${ }^{234} \mathrm{Th}$ fluxes incorporate the upwelling component (Eq. 5) for stations 1 to 7 (Supp. Table 3) and are the values to be used in determining other fluxes (e.g. particulate organic carbon). Stations are labeled as 'St' in all tables.

\begin{tabular}{|c|c|c|c|c|c|c|c|c|c|}
\hline Zone & St & $\begin{array}{c}\text { Lat } \\
\text { S }\end{array}$ & $\begin{array}{c}\text { Lon } \\
\text { W }\end{array}$ & $\begin{array}{l}\text { Average } \\
\text { Ez for } \\
\text { All Casts } \\
\quad(\mathbf{m})\end{array}$ & 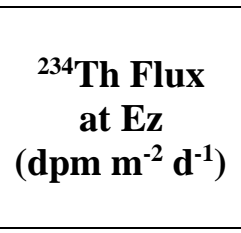 & $\begin{array}{c}\text { Upwelling- } \\
\text { Adjusted } \\
{ }^{234} \text { Th Flux } \\
\text { at Ez } \\
\left(\text { dpm m}^{-2} \mathbf{d}^{-1}\right) \\
\end{array}$ & $\begin{array}{c}\text { Upwelling- } \\
\text { Adjusted }{ }^{234} \text { Th } \\
\text { Flux at } \\
\text { Ez }+\mathbf{1 0 0} \\
\left(\mathbf{d p m ~ m}^{-2} \mathbf{d}^{-1}\right) \\
\end{array}$ & 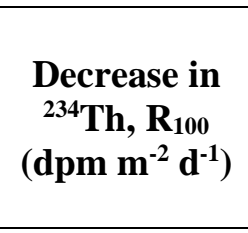 & 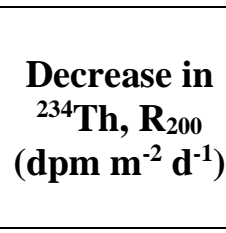 \\
\hline \multirow[t]{5}{*}{ Shelf } & 2 & 12.0 & 77.4 & 30 & $1400 \pm 30$ & $4000 \pm 600$ & & & \\
\hline & 3 & 12.0 & 77.7 & 25 & $1100 \pm 40$ & $5000 \pm 1000$ & $7000 \pm 1000$ & & \\
\hline & 4 & 12.0 & 77.8 & 43 & $1000 \pm 100$ & $4000 \pm 900$ & $6000 \pm 1000$ & & \\
\hline & 5 & 12.0 & 78.2 & 50 & $1700 \pm 70$ & $4000 \pm 700$ & $6000 \pm 800$ & & \\
\hline & 1 & 12.0 & 79.2 & 66 & $2000 \pm 90$ & $4000 \pm 800$ & $6000 \pm 900$ & & \\
\hline \multirow[t]{10}{*}{ Offshore } & 6 & 12.0 & 81.5 & 80 & $1900 \pm 100$ & $4000 \pm 700$ & $4000 \pm 700$ & & \\
\hline & 7 & 12.0 & 84.0 & 94 & $2000 \pm 100$ & $3000 \pm 400$ & $2000 \pm 400$ & $700 \pm 600$ & $500 \pm 800$ \\
\hline & 8 & 12.0 & 86.5 & 105 & $2300 \pm 200$ & $2300 \pm 100$ & $2000 \pm 200$ & $500 \pm 200$ & $300 \pm 400$ \\
\hline & 9 & 12.0 & 89.0 & 109 & $1400 \pm 100$ & $1400 \pm 100$ & $200 \pm 200$ & $1200 \pm 200$ & $200 \pm 400$ \\
\hline & 10 & 12.0 & 91.5 & 121 & $1600 \pm 300$ & $1600 \pm 200$ & $1000 \pm 300$ & $600 \pm 300$ & $400 \pm 400$ \\
\hline & 11 & 12.0 & 94.0 & 130 & $1900 \pm 200$ & $1900 \pm 100$ & $1600 \pm 200$ & $300 \pm 200$ & \\
\hline & 12 & 13.0 & 96.5 & 124 & $1400 \pm 200$ & $1400 \pm 200$ & $1100 \pm 200$ & $300 \pm 300$ & $500 \pm 400$ \\
\hline & 13 & 14.0 & 99.0 & 139 & $1200 \pm 200$ & $1200 \pm 100$ & $800 \pm 300$ & $400 \pm 300$ & $400 \pm 300$ \\
\hline & 14 & 15.0 & 101.5 & 164 & $2700 \pm 300$ & $2700 \pm 200$ & $2400 \pm 300$ & $300 \pm 300$ & \\
\hline & 15 & 16.0 & 104.0 & 168 & $2200 \pm 200$ & $2200 \pm 100$ & $1500 \pm 200$ & $700 \pm 300$ & $700 \pm 400$ \\
\hline
\end{tabular}




\begin{tabular}{|c|c|c|c|c|c|c|c|c|c|c|c|c|c|c|c|c|c|c|c|}
\hline \multirow{2}{*}{$\begin{array}{l}\text { Zone } \\
\text { Gyre }\end{array}$} & \multirow{2}{*}{$\begin{array}{l}\mathbf{S t} \\
\\
16\end{array}$} & \multirow{2}{*}{$\begin{array}{c}\text { Lat } \\
-15.4\end{array}$} & \multirow{2}{*}{$\begin{array}{c}\text { Long } \\
-106.5\end{array}$} & \multirow[t]{2}{*}{$\begin{array}{c}\begin{array}{c}\text { Average } \\
\text { Ez for All } \\
\text { Casts (m) }\end{array} \\
150\end{array}$} & \multicolumn{3}{|c|}{ 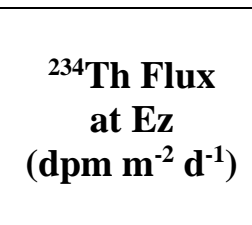 } & \multicolumn{3}{|c|}{$\begin{array}{c}\text { Upwelling- } \\
\text { Adjusted }{ }^{234} \text { Th } \\
\text { Flux } \\
\text { at Ez } \\
\left(\mathrm{dpm} \mathrm{m}^{-2} \mathbf{d}^{-1}\right) \\
\end{array}$} & \multicolumn{3}{|c|}{ 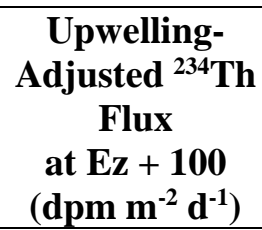 } & \multicolumn{3}{|c|}{$\begin{array}{c}\text { Decrease in } \\
{ }^{234} \mathbf{T h}, \mathbf{R}_{100} \\
\left(\mathbf{d p m} \mathbf{~ m}^{-2} \mathbf{d}^{-1}\right)\end{array}$} & \multicolumn{3}{|c|}{$\begin{array}{c}\text { Decrease in } \\
{ }^{234} \mathbf{T h}, \mathbf{R}_{200} \\
\left(\mathrm{dpm} \mathbf{m}^{-2} \mathbf{d}^{-1}\right)\end{array}$} \\
\hline & & & & & 2100 & \pm & 300 & 2100 & \pm & 300 & 1600 & \pm & 300 & 500 & \pm & 300 & 200 & \pm & 400 \\
\hline & 17 & -15.0 & -109.2 & 163 & 1300 & \pm & 200 & 1300 & \pm & 200 & 700 & \pm & 300 & 500 & \pm & 300 & 500 & \pm & 300 \\
\hline & 18 & -15.0 & -112.8 & 174 & 1600 & \pm & 200 & 1600 & \pm & 200 & 1000 & \pm & 400 & 600 & \pm & 300 & & & \\
\hline & 20 & -15.0 & -113.5 & 191 & 1600 & \pm & 300 & 1600 & \pm & 300 & 1300 & \pm & 400 & 300 & \pm & 300 & & & \\
\hline & 21 & -14.8 & -115.0 & 167 & 1300 & \pm & 200 & 1300 & \pm & 200 & 900 & \pm & 300 & 400 & \pm & 300 & & & \\
\hline & 22 & -14.4 & -117.5 & 179 & 2000 & \pm & 200 & 2000 & \pm & 200 & 1600 & \pm & 400 & 400 & \pm & 300 & 900 & \pm & 300 \\
\hline & 23 & -14.0 & -120.0 & 169 & 1700 & \pm & 300 & 1700 & \pm & 300 & 1400 & \pm & 400 & 300 & \pm & 300 & & & \\
\hline & 24 & -13.3 & -122.5 & 163 & 1300 & \pm & 300 & 1300 & \pm & 300 & 1000 & \pm & 400 & 300 & \pm & 300 & 500 & \pm & 300 \\
\hline & 25 & -12.5 & -125.0 & 163 & 1400 & \pm & 200 & 1400 & \pm & 200 & 1500 & \pm & 500 & & & & & & \\
\hline & 26 & -11.7 & -128.0 & 169 & 1000 & \pm & 200 & 1000 & \pm & 200 & 800 & \pm & 500 & 300 & \pm & 400 & & & \\
\hline & 27 & -11.7 & -130.0 & 184 & 1500 & \pm & 300 & 1500 & \pm & 300 & 1500 & \pm & 400 & & & & & & \\
\hline & 28 & -11.6 & -132.5 & 162 & 1500 & \pm & 200 & 1500 & \pm & 200 & 900 & \pm & 500 & 500 & \pm & 400 & & & \\
\hline & 29 & -11.6 & -135.0 & 175 & 1300 & \pm & 200 & 1300 & \pm & 200 & 1100 & \pm & 400 & 200 & \pm & 400 & & & \\
\hline & 30 & -11.6 & -137.0 & 168 & 1400 & \pm & 200 & 1400 & \pm & 200 & 1100 & \pm & 400 & 200 & \pm & 300 & 700 & & 300 \\
\hline & 31 & -11.3 & -140.0 & 146 & 1600 & \pm & 200 & 1600 & \pm & 200 & 1600 & \pm & 300 & & & & & & \\
\hline & 32 & -11.0 & -142.9 & 153 & 2000 & \pm & 200 & 2000 & \pm & 200 & 1600 & \pm & 300 & 400 & \pm & 300 & 400 & \pm & 400 \\
\hline & 33 & -10.9 & -145.0 & 186 & 1800 & \pm & 300 & 1800 & \pm & 300 & 1800 & \pm & 400 & & & & & & \\
\hline & 34 & -10.8 & -147.5 & 175 & 1600 & \pm & 200 & 1600 & \pm & 200 & 1400 & \pm & 400 & 200 & \pm & 300 & & & \\
\hline & 35 & -10.3 & -150.0 & 170 & 1900 & \pm & 200 & 1900 & \pm & 200 & 2100 & \pm & 400 & & & & & & \\
\hline & 36 & -10.5 & -152.0 & 179 & 1200 & \pm & 400 & 1200 & \pm & 400 & 800 & \pm & 600 & 400 & \pm & 400 & 300 & \pm & 600 \\
\hline
\end{tabular}


Table 2: Summary of zonal characteristics for the Shelf, Offshore, and Gyre zones. Included are ${ }^{234}$ Th estimates, carbon results, and biologically-relevant parameters. MODIS and SeaWIFS sources for NPP are discussed in Section 2.2.3. Ez-ratios are calculated using ${ }^{234} \mathrm{Th}$-derived POC export.

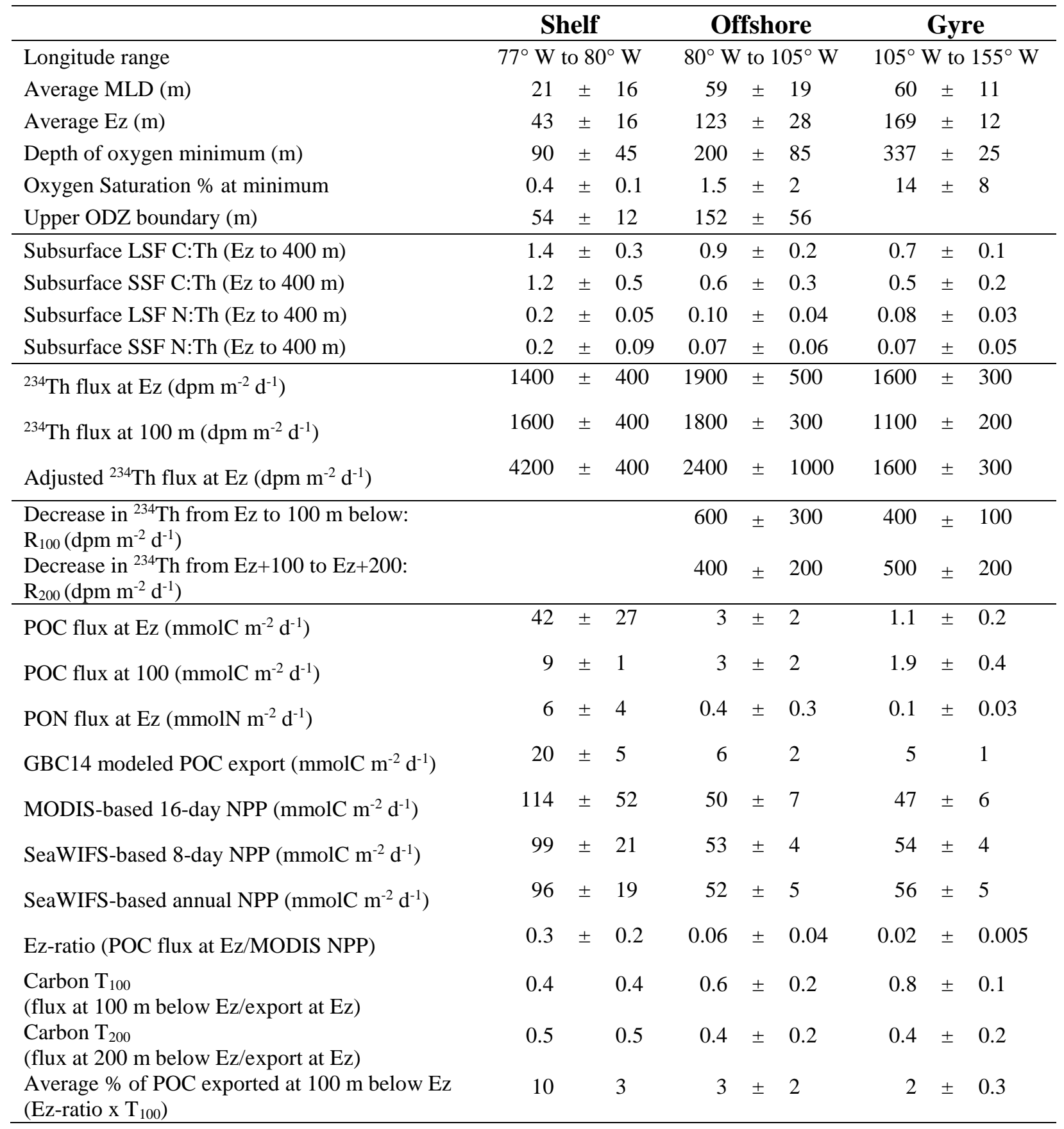


Table 3: ${ }^{234}$ Th-derived carbon and nitrogen export and remineralization results, net primary production, and Ez-ratios by station. MODIS and SeaWIFS sources for NPP are discussed in Section 2.2.3. All carbon fluxes, nitrogen fluxes, and NPP are reported in $\mathrm{mmol} \mathrm{m} \mathrm{m}^{-2} \mathrm{~d}^{-1}$. Ez-ratios were calculated using ${ }^{234} \mathrm{Th}$-derived POC export and represent the export of carbon at Ez divided by NPP. $\mathrm{T}_{100}$ values represent the POC flux at $100 \mathrm{~m}$ below the Ez divided by the POC flux at Ez. The GBC14 modeled export comes from the global model used in Siegel et al. (2014). The percent of POC exported at $100 \mathrm{~m}$ below Ez is equivalent to the Ez-ratio multiplied by the $\mathrm{T}_{100}$ value.

\begin{tabular}{|c|c|c|c|c|c|c|c|c|c|c|c|c|c|c|c|c|}
\hline \multirow{2}{*}{$\begin{array}{l}\text { St } \\
2\end{array}$} & \multicolumn{2}{|c|}{$\begin{array}{c}\text { Derived } \\
\text { C:Th at Ez } \\
\left(\mu \mathrm{mol} \mathbf{d p m}^{-1}\right)\end{array}$} & \multicolumn{2}{|c|}{$\begin{array}{c}\text { Derived } \mathrm{N}: \mathrm{Th} \\
\text { at } \mathbf{E z} \\
\left(\boldsymbol{\mu m o l} \mathbf{d p m}^{-1}\right)\end{array}$} & \multicolumn{2}{|c|}{$\begin{array}{c}\text { POC flux at } \\
\text { Ez }\end{array}$} & \multicolumn{2}{|c|}{$\begin{array}{c}\text { PON flux at } \\
\text { Ez }\end{array}$} & \multirow{2}{*}{$\begin{array}{c}\text { GBC14 } \\
\text { model } \\
\text { POC } \\
\text { flux at } \\
\text { Ez } \\
24\end{array}$} & \multirow{2}{*}{$\begin{array}{c}\text { 16-day } \\
\text { MODIS } \\
\text {-based } \\
\text { NPP } \\
188\end{array}$} & \multirow{2}{*}{$\begin{array}{c}\text { 8-day } \\
\text { SeaWIFS } \\
\text {-based } \\
\text { NPP } \\
112\end{array}$} & \multirow{2}{*}{$\begin{array}{c}\text { Annual } \\
\text { SeaWIFS } \\
\text {-based } \\
\text { NPP } \\
115\end{array}$} & \multirow{2}{*}{$\begin{array}{c}\begin{array}{c}\text { Ez- } \\
\text { ratio }\end{array} \\
0.3\end{array}$} & \multirow[t]{2}{*}{$\mathbf{T}_{100}$} & \multirow[t]{2}{*}{$\mathbf{T}_{200}$} & \multirow[t]{2}{*}{$\begin{array}{c}\% \text { POC } \\
\text { export at } \\
100 \mathrm{~m} \\
\text { below } \mathrm{Ez}\end{array}$} \\
\hline & 13 & \pm 4 & 2.2 & \pm 0.6 & 50 & \pm 20 & 9 & \pm 3 & & & & & & & & \\
\hline 3 & 15 & \pm 4 & 2.4 & \pm 0.6 & 80 & \pm 30 & 12 & \pm 4 & 24 & 147 & 112 & 115 & 0.5 & 0.1 & & 7 \\
\hline 4 & 9 & \pm 4 & 1.5 & \pm 0.6 & 40 & \pm 17 & 6 & \pm 3 & 24 & 93 & 113 & 115 & 0.4 & 0.2 & 0.2 & 9 \\
\hline 5 & 7 & \pm 4 & 1.1 & \pm 0.6 & 30 & \pm 20 & 4 & \pm 2 & 16 & 80 & 81 & 72 & 0.4 & 0.3 & 0.2 & 11 \\
\hline 1 & 2 & \pm 4 & 0.2 & \pm 0.1 & 8 & \pm 17 & 0.7 & \pm 0.2 & 14 & 63 & 77 & 63 & 0.1 & 1 & 1 & 13 \\
\hline 6 & 2.0 & \pm 0.6 & 0.3 & \pm 0.1 & 9 & \pm 3 & 1.2 & \pm 0.5 & 9 & 61 & 60 & 56 & 0.1 & 0.4 & 0.4 & 7 \\
\hline 7 & 2 & \pm 1 & 0.2 & \pm 0.1 & 5 & \pm 2 & 0.7 & \pm 0.3 & 8 & 51 & 57 & 54 & 0.1 & 0.4 & 0.3 & 4 \\
\hline 8 & 1 & \pm 1 & 0.2 & \pm 0.1 & 3 & \pm 1 & 0.4 & \pm 0.2 & 6 & 53 & 54 & 54 & 0.06 & 0.5 & 0.4 & 3 \\
\hline 9 & 1 & \pm 1 & 0.2 & \pm 0.1 & 2.0 & \pm 0.8 & 0.3 & \pm 0.1 & 6 & 53 & 54 & 53 & 0.04 & 0.1 & 0.0 & 0.4 \\
\hline 10 & 1.1 & \pm 0.6 & 0.1 & \pm 0.1 & 2.0 & \pm 0.9 & 0.2 & \pm 0.2 & 5 & 56 & 54 & 52 & 0.04 & 0.5 & 0.3 & 2 \\
\hline 11 & 0.9 & \pm 0.2 & 0.1 & \pm 0.1 & 2.0 & \pm 0.5 & 0.2 & \pm 0.2 & 5 & 49 & 52 & 51 & 0.04 & 0.9 & & 3 \\
\hline 12 & 1.0 & \pm 0.6 & 0.1 & \pm 0.1 & 1.4 & \pm 0.8 & 0.2 & \pm 0.1 & 5 & 46 & 50 & 52 & 0.03 & 0.7 & 0.4 & 2 \\
\hline 13 & 0.9 & \pm 0.2 & 0.1 & \pm 0.04 & 1.1 & \pm 0.3 & 0.1 & \pm 0.05 & 4 & 52 & 48 & 50 & 0.02 & 0.7 & 0.4 & 1 \\
\hline 14 & 0.9 & \pm 0.2 & 0.1 & \pm 0.04 & 3 & \pm 1 & 0.3 & $\pm \quad 0.1$ & 4 & 43 & 46 & 47 & 0.07 & 0.9 & 0.8 & 6 \\
\hline 15 & 0.9 & \pm 0.2 & 0.1 & \pm 0.04 & 2.1 & \pm 0.5 & 0.2 & $\pm \quad 0.1$ & 4 & 36 & 45 & 44 & 0.06 & 0.7 & 0.4 & 4 \\
\hline
\end{tabular}




\begin{tabular}{|c|c|c|c|c|c|c|c|c|c|c|c|c|c|c|c|c|}
\hline \multirow{2}{*}{$\begin{array}{l}\mathbf{S t} \\
\\
16\end{array}$} & \multicolumn{2}{|c|}{$\begin{array}{c}\text { Derived } \\
\text { C:Th at Ez } \\
\left(\mu \mathrm{mol} \mathrm{dpm}^{-1}\right)\end{array}$} & \multicolumn{2}{|c|}{$\begin{array}{c}\text { Derived } \mathrm{N}: \mathrm{Th} \\
\text { at } \mathbf{E z} \\
\left(\boldsymbol{\mu m o l} \mathbf{d p m}^{-1}\right)\end{array}$} & \multicolumn{2}{|c|}{$\begin{array}{c}\text { POC flux at } \\
\text { Ez }\end{array}$} & \multicolumn{2}{|c|}{ PON flux at Ez } & \multirow{2}{*}{$\begin{array}{c}\text { GBC14 } \\
\text { model } \\
\text { POC } \\
\text { flux at } \\
\text { Ez } \\
4\end{array}$} & \multirow{2}{*}{$\begin{array}{c}\text { 16-day } \\
\text { MODIS } \\
\text {-based } \\
\text { NPP } \\
44\end{array}$} & \multirow{2}{*}{$\begin{array}{c}\begin{array}{c}\text { 8-day } \\
\text { SeaWIFS } \\
\text {-based } \\
\text { NPP }\end{array} \\
48\end{array}$} & \multirow{2}{*}{$\begin{array}{c}\text { Annual } \\
\text { SeaWIFS } \\
\text {-based } \\
\text { NPP } \\
48\end{array}$} & \multirow{2}{*}{$\begin{array}{c}\begin{array}{c}\text { Ez- } \\
\text { ratio }\end{array} \\
0.02\end{array}$} & \multirow{2}{*}{$\begin{array}{l}\mathbf{T}_{100} \\
0.8\end{array}$} & \multirow{2}{*}{$\begin{array}{c}\mathbf{T}_{200} \\
0.7\end{array}$} & \multirow{2}{*}{$\begin{array}{c}\% \text { POC } \\
\text { export at } \\
\mathbf{1 0 0 ~} \mathbf{~ m} \\
\text { below Ez } \\
2\end{array}$} \\
\hline & 0.7 & \pm 0.1 & 0.08 & \pm 0.03 & 1.0 & \pm 0.2 & 0.2 & \pm 0.1 & & & & & & & & \\
\hline 17 & 0.7 & \pm 0.1 & 0.08 & \pm 0.03 & 1.0 & \pm 0.2 & 0.11 & \pm 0.03 & 4 & 42 & 50 & 49 & 0.02 & 0.6 & 0.2 & 1 \\
\hline 18 & 0.7 & \pm 0.1 & 0.08 & \pm 0.03 & 1.0 & \pm 0.2 & 0.13 & \pm 0.04 & 5 & 51 & 53 & 50 & 0.02 & 0.6 & & 1 \\
\hline 20 & 0.7 & \pm 0.1 & 0.08 & \pm 0.03 & 1.0 & \pm 0.2 & 0.13 & \pm 0.04 & 5 & 46 & 53 & 50 & 0.02 & 0.8 & & 2 \\
\hline 21 & 0.7 & \pm 0.1 & 0.08 & \pm 0.03 & 0.9 & \pm 0.2 & 0.10 & \pm 0.03 & 5 & 46 & 54 & 52 & 0.02 & 0.7 & & 1 \\
\hline 22 & 0.7 & \pm 0.1 & 0.08 & \pm 0.03 & 1.4 & \pm 0.2 & 0.17 & \pm 0.05 & 4 & 52 & 51 & 52 & 0.03 & 0.8 & 0.3 & 2 \\
\hline 23 & 0.7 & \pm 0.1 & 0.08 & \pm 0.03 & 1.2 & \pm 0.2 & 0.14 & \pm 0.05 & 5 & 58 & 52 & 52 & 0.02 & 0.8 & & 2 \\
\hline 24 & 0.7 & \pm 0.1 & 0.08 & \pm 0.03 & 0.9 & \pm 0.2 & 0.10 & \pm 0.04 & 5 & 46 & 56 & 53 & 0.02 & 0.8 & 0.4 & 2 \\
\hline 25 & 0.7 & \pm 0.1 & 0.08 & \pm 0.03 & 1.0 & \pm 0.2 & 0.12 & \pm 0.04 & 5 & 48 & 58 & 55 & 0.02 & & & \\
\hline 26 & 0.7 & \pm 0.1 & 0.08 & \pm 0.03 & 0.7 & \pm 0.2 & 0.09 & \pm 0.03 & 6 & 42 & 60 & 57 & 0.02 & 0.7 & & 1 \\
\hline 27 & 0.7 & \pm 0.1 & 0.08 & \pm 0.03 & 1.1 & \pm 0.2 & 0.12 & \pm 0.04 & 5 & 47 & 59 & 56 & 0.02 & & & \\
\hline 28 & 0.7 & \pm 0.1 & 0.08 & \pm 0.03 & 1.0 & \pm 0.2 & 0.12 & \pm 0.04 & 6 & 47 & 59 & 56 & 0.02 & 0.7 & & 1 \\
\hline 29 & 0.7 & \pm 0.1 & 0.08 & \pm 0.03 & 0.9 & \pm 0.2 & 0.10 & \pm 0.04 & 6 & 55 & 60 & 56 & 0.02 & 0.8 & & 1 \\
\hline 30 & 0.7 & \pm 0.1 & 0.08 & \pm 0.03 & 1.0 & \pm 0.2 & 0.11 & \pm 0.04 & 7 & 51 & 63 & 58 & 0.02 & 0.8 & 0.3 & 2 \\
\hline 31 & 0.7 & \pm 0.1 & 0.08 & \pm 0.03 & 1.1 & \pm 0.2 & 0.13 & \pm 0.04 & 8 & 46 & 67 & 62 & 0.02 & & & \\
\hline 32 & 0.7 & \pm 0.1 & 0.08 & \pm 0.03 & 1.4 & \pm 0.2 & 0.16 & \pm 0.05 & 6 & 54 & 58 & 57 & 0.03 & 0.8 & 0.6 & 2 \\
\hline 33 & 0.7 & \pm 0.1 & 0.08 & \pm 0.03 & 1.2 & \pm 0.2 & 0.14 & \pm 0.05 & 7 & 44 & 60 & 58 & 0.03 & & & \\
\hline 34 & 0.7 & \pm 0.1 & 0.08 & \pm 0.03 & 1.2 & \pm 0.2 & 0.13 & \pm 0.04 & 6 & 45 & 56 & 55 & 0.03 & 0.9 & & 2 \\
\hline 35 & 0.7 & \pm 0.1 & 0.08 & \pm 0.03 & 1.4 & \pm 0.2 & 0.16 & \pm 0.05 & 5 & 36 & 53 & 53 & 0.04 & & & \\
\hline 36 & 0.7 & \pm 0.1 & 0.08 & \pm 0.03 & 0.8 & \pm 0.2 & 0.10 & \pm 0.03 & 5 & 34 & 52 & 52 & 0.02 & 0.7 & 0.4 & 2 \\
\hline
\end{tabular}




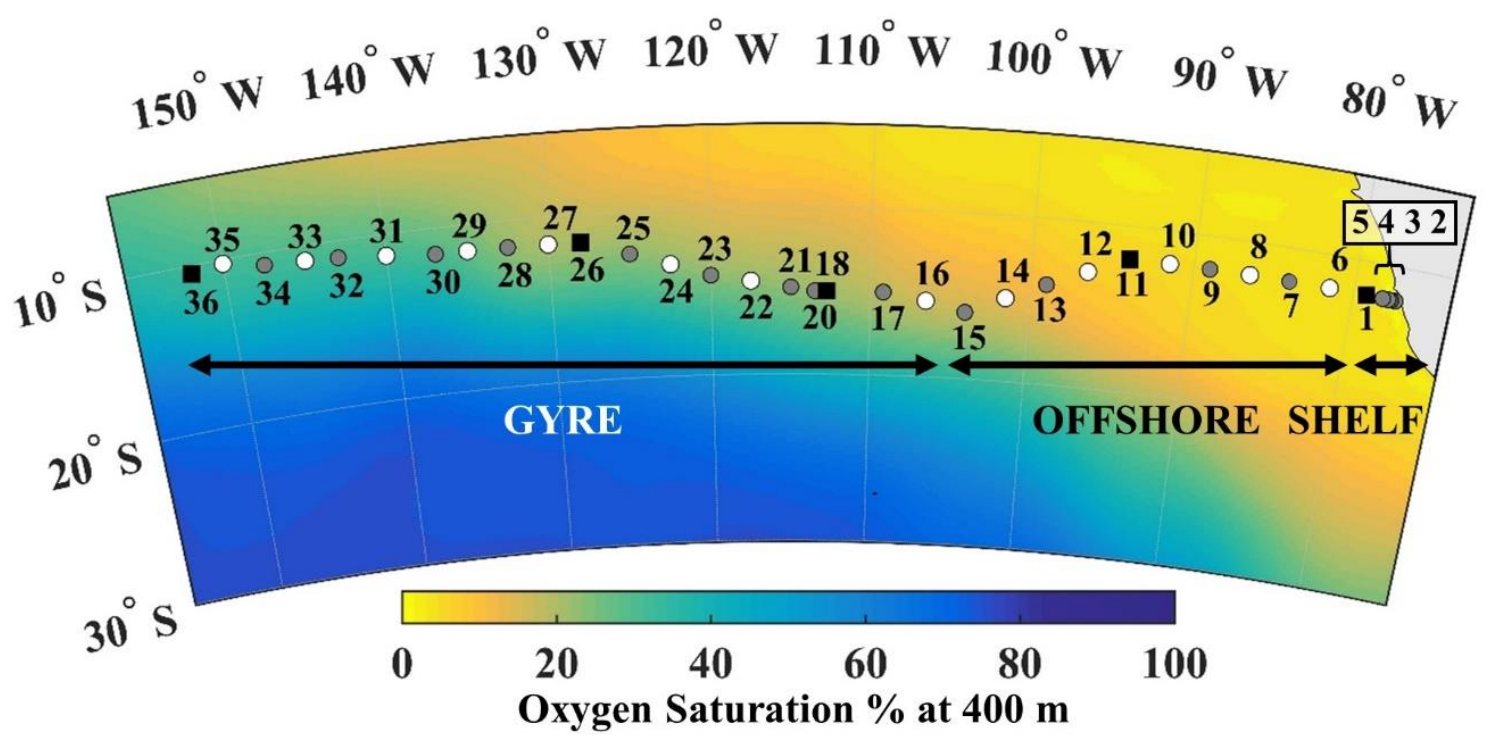

Figure 1: Station locations with oxygen saturation at $400 \mathrm{~m}$. Zonal designations are shown for stations 1-5 (Shelf), 6-15 (Offshore), and 16-36 (Gyre). Oxygen data are a historical compilation (NOAA World Ocean Atlas, 2009). Total and particulate ${ }^{234} \mathrm{Th}$ samples were taken at a minimum of 24 discrete depths throughout the full water column at super stations (black squares) and 8 to 16 depths at coastal and full stations (grey circles). Only total ${ }^{234} \mathrm{Th}$ was measured in the upper $1000 \mathrm{~m}$ at demi stations (12 depths; white circles). 

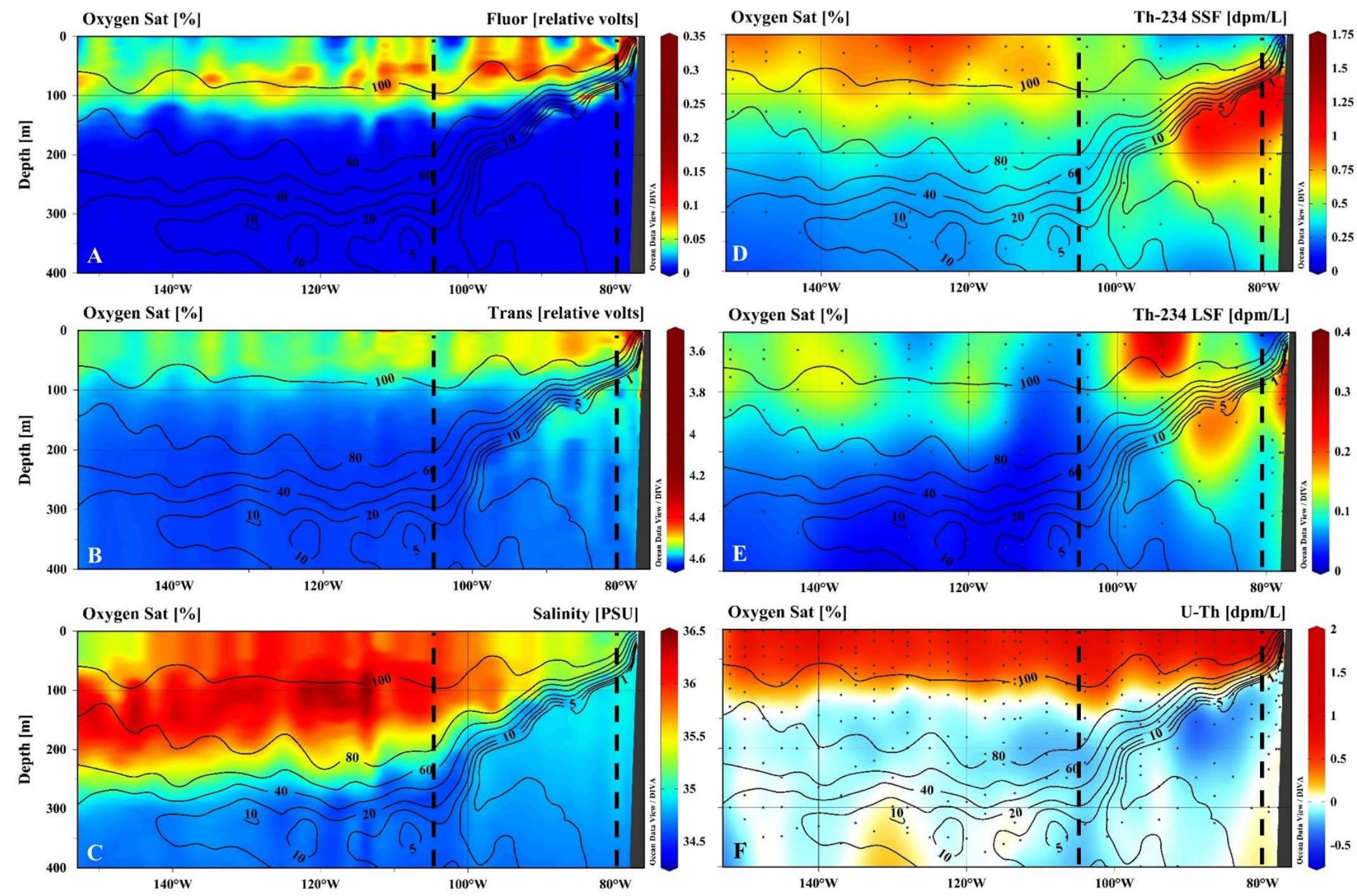

Figure 2: ${ }^{234}$ Th results and relevant CTD sensor parameters with oxygen saturation \%. Oxygen saturation (contours) is shown relative to Fluorescence (A), Light Transmission (B), Salinity (C), small particle ${ }^{234} \mathrm{Th}$ activities $\left(1-51 \mu \mathrm{m}\right.$, D), large particle ${ }^{234} \mathrm{Th}$ activities $(>51 \mu \mathrm{m}, \mathrm{E})$ and ${ }^{238} \mathrm{U}-{ }^{234} \mathrm{Th}$ activities $(\mathrm{F})$. Zones corresponding to Fig. 1 are delineated by dashed lines. Transmission, Salinity, and Fluorescence were from the ODF rosette. Oxygen saturation contours were determined using only data from the total ${ }^{234} \mathrm{Th}$ cast. 


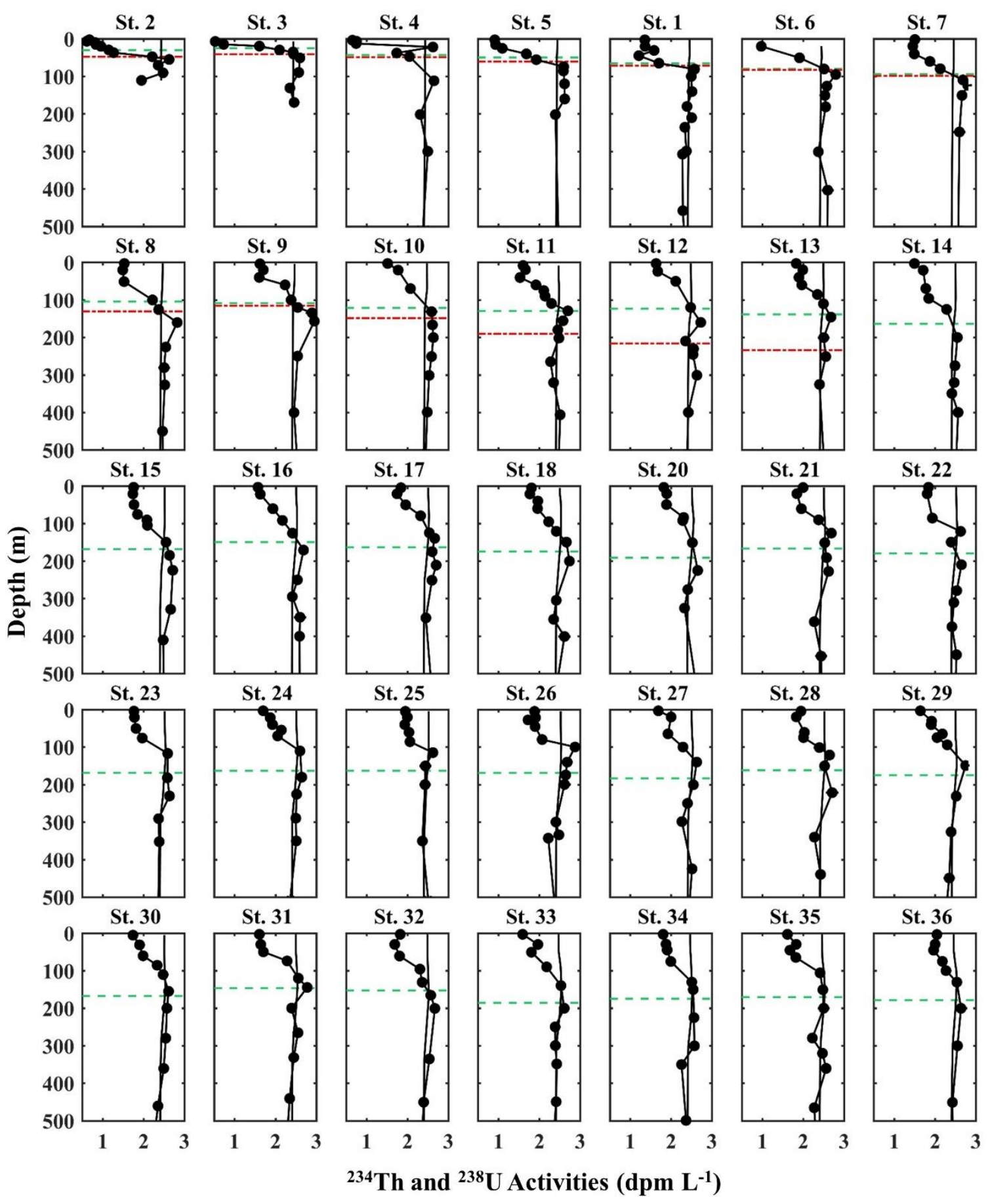

\begin{tabular}{|llll|}
\hline--- euphotic & ----- start of oxygen deficient zone $\quad-{ }^{238} \mathrm{U}$ & $-{ }^{234} \mathrm{Th}$ \\
\hline
\end{tabular}

Figure 3: ${ }^{234} \mathrm{Th}$ and ${ }^{238} \mathrm{U}$ activity profiles in $\mathrm{dpm}^{-1}$ for the upper $500 \mathrm{~m}$ of the water column. The average Ez for all casts is shown for each station (green) with the start of the oxygen deficient zone (red) for those stations with ODZs. All presented data was used in the export and remineralization flux calculations. 

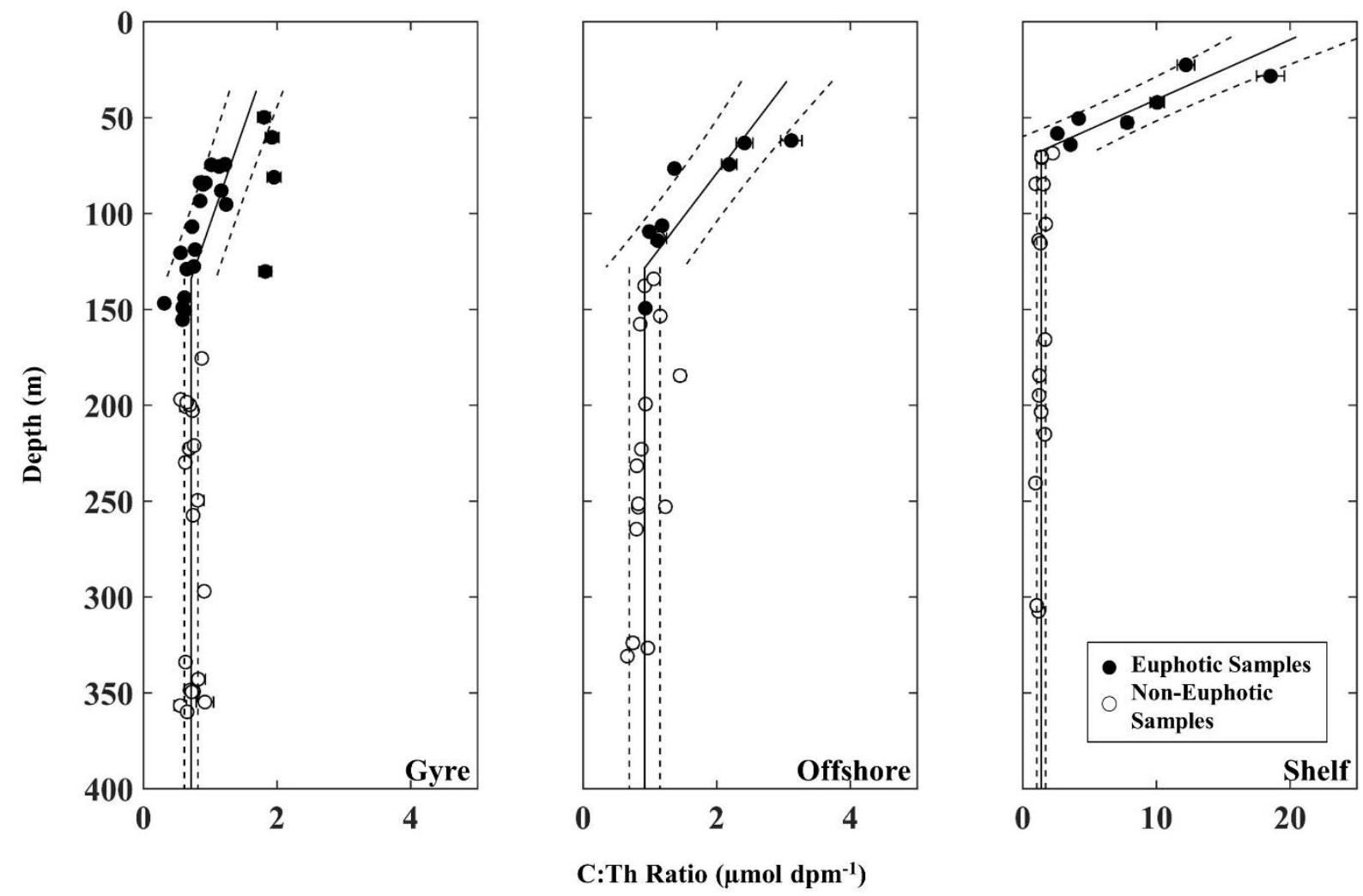

Figure 4: Carbon: ${ }^{234} \mathrm{Th}$ on large particles $(>51 \mu \mathrm{m})$ in $\mu \mathrm{mol} \mathrm{dpm}^{-1}$ for the upper $400 \mathrm{~m}$. Best fit linear regressions are shown for euphotic samples along with the average ratio for samples collected below the base of the euphotic to $400 \mathrm{~m}$ (solid lines). Both are shown with 1 standard deviation (dotted lines). Mixed layer samples are not shown and not included in ratio calculations. 


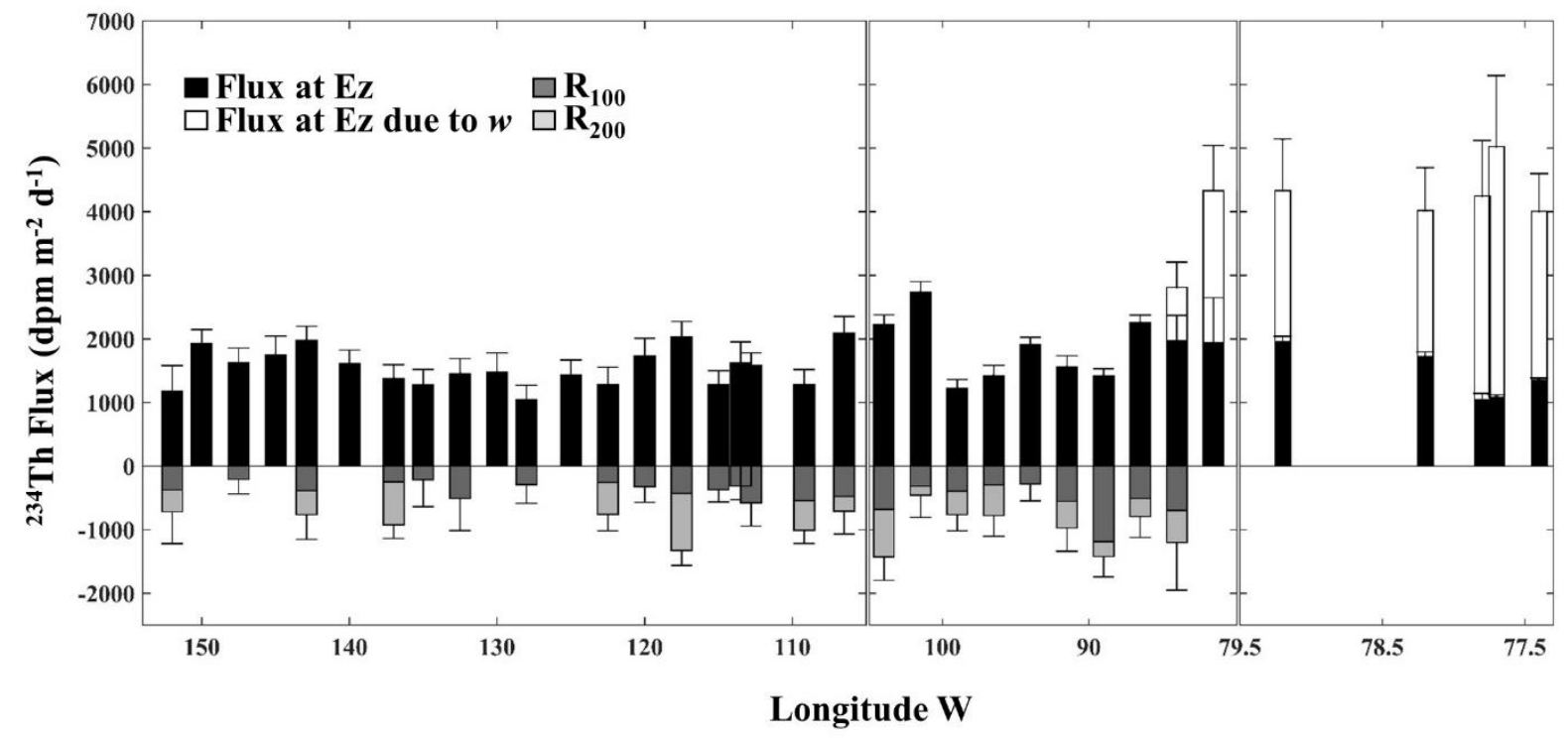

Figure 5: ${ }^{234} \mathrm{Th}$ Export at Ez and remineralization through the $200 \mathrm{~m}$ below Ez. Calculated ${ }^{234} \mathrm{Th}$ export is shown as the ${ }^{234} \mathrm{Th}$ flux without upwelling (black) and additional flux due to upwelling $w$ (white) at the base of the euphotic zone (Ez). The decrease in flux through the first $100 \mathrm{~m}$ below $\mathrm{Ez}\left(\mathrm{R}_{100}\right.$; dark grey) and subsequent $100 \mathrm{~m}$ to $200 \mathrm{~m}$ below $\mathrm{Ez}\left(\mathrm{R}_{200}\right.$; light grey) are also shown. Values of $R_{100}$ and $R_{200}$ are negative because ${ }^{234} \mathrm{Th}$ flux decreases below Ez at the stations indicated. See Table 1 for additional details on $\mathrm{R}$ values. 


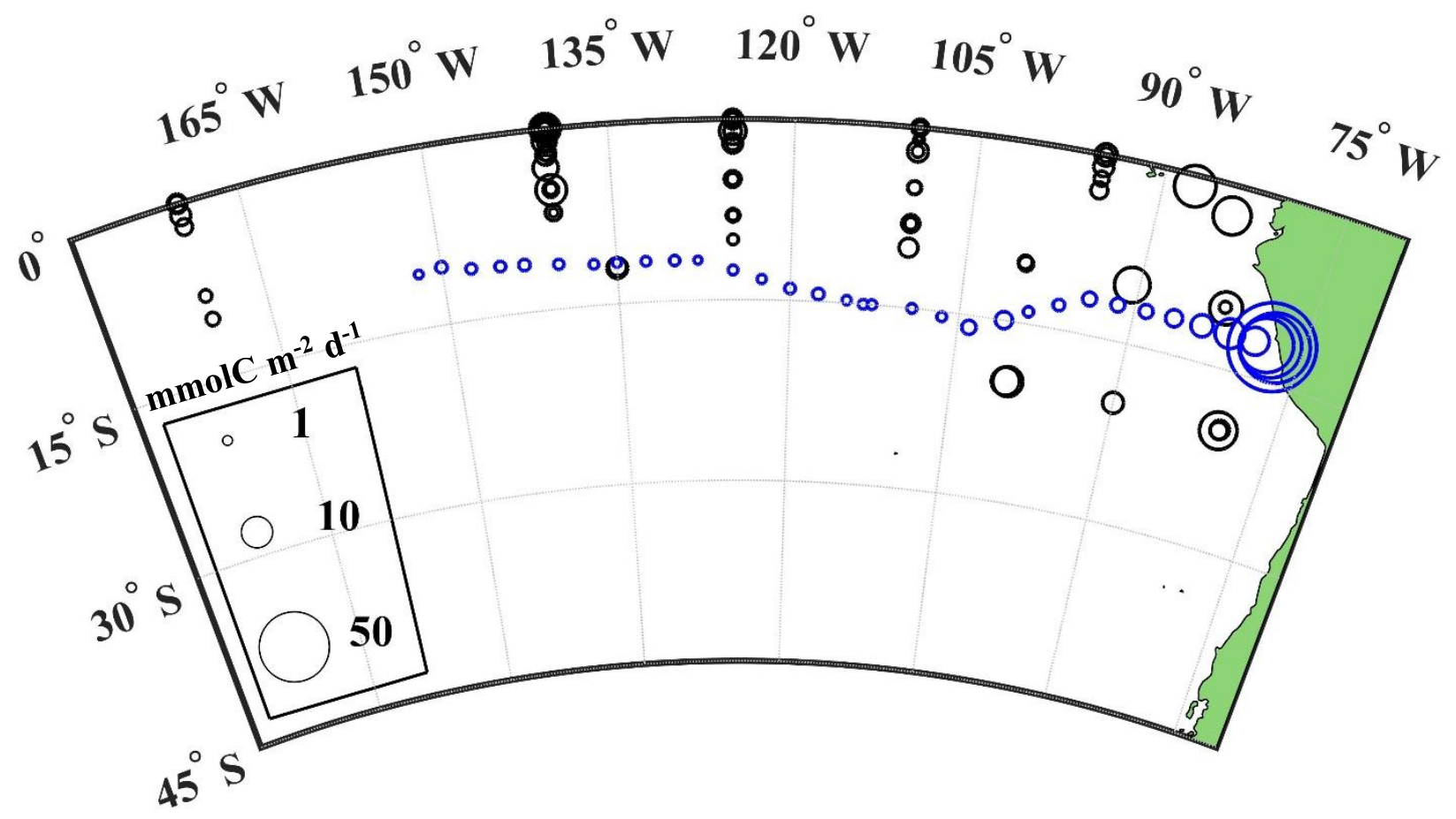

Figure 6: Regional ${ }^{234}$ Th-derived carbon fluxes. The magnitude of POC flux is indicated by circle area at each location. All prior carbon export values for this region are shown (black). Export was calculated at depths ranging from $100 \mathrm{~m}$ to $200 \mathrm{~m}$ and in different seasons. The equatorial data is from various studies compiled by Le Moigne et al. (2013) and the remaining locations were from Haskell et al. (2013). POC fluxes from this GEOTRACES campaign, calculated at Ez, are shown in blue (Table 3). 


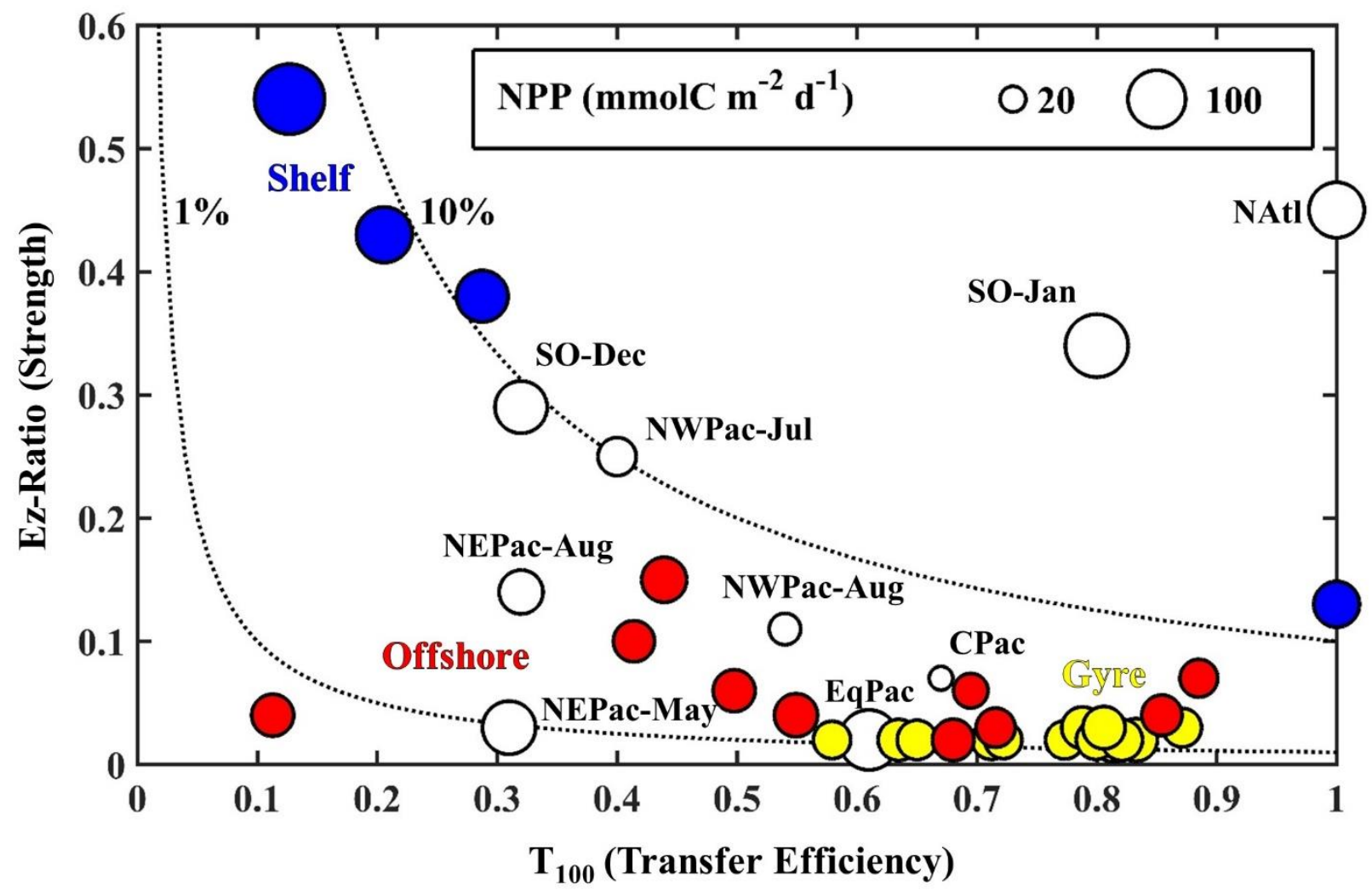

Figure 7: ${ }^{234}$ Th-derived Ez-ratios (export at Ez/NPP) and T100 (flux at $100 \mathrm{~m}$ below Ez/export at Ez) values for POC. Station specific values from this campaign are shown with results from Buesseler and Boyd (2009). The prior regions of study include the following: NAtl (NABE, North Atlantic Bloom Experiment; $47^{\circ} \mathrm{N}, 20^{\circ} \mathrm{W}$ ), NWPac (K2, Northwest Pacific; $47^{\circ} \mathrm{N}, 160^{\circ} \mathrm{E}$ ), CPac (ALOHA, Central North Pacific; $22^{\circ} \mathrm{N}, 158^{\circ} \mathrm{W}$ ), EqPac (Equatorial Pacific; $0^{\circ} \mathrm{N}, 140^{\circ} \mathrm{W}$ ), SO (KIWI, Southern Ocean; $61.5^{\circ} \mathrm{S}$ to $65.5^{\circ} \mathrm{S}, 170^{\circ} \mathrm{W}$ ), and NEPac (Station Papa, Northeast Pacific; $50^{\circ} \mathrm{N}, 145^{\circ} \mathrm{W}$ ). The area of the circles represents NPP in $\mathrm{mmolC} \mathrm{m}^{-2} \mathrm{~d}^{-1}$. The $1 \%$ and $10 \%$ contour lines show the relationships between the POC flux $100 \mathrm{~m}$ below Ez and NPP. 
Supplemental Table 1: Depth of relevant physical parameters for the shallow ${ }^{234}$ Th cast (Cast) and the average of all ODF and GEOTRACES rosette casts. Average mixed layer and Ez values (Ohnemus et al., 2016) were used for flux and upwelling calculations to allow comparison between this study and others. The shallow ${ }^{234} \mathrm{Th}$ cast depths are shown here for reference. As described in the text and shown in Supp. Figs. 1 and 2, oxygen data was highly variable cast to cast. Only the ${ }^{234}$ Th shallow cast (ODF rosette) values are shown here and used in this study, although values for the GEOTRACES rosette are described in Ohnemus et al. (2016).

\begin{tabular}{|c|c|c|c|c|c|c|c|c|}
\hline Zone & $\mathbf{S t}$ & $\begin{array}{c}\text { Average } \\
\text { MLD for all } \\
\text { casts (m) }\end{array}$ & $\begin{array}{c}\text { Cast } \\
\text { MLD } \\
(\mathbf{m})\end{array}$ & $\begin{array}{l}\text { Average Ez } \\
\text { for all casts } \\
\quad(\mathrm{m})\end{array}$ & $\begin{array}{c}\text { Cast Ez } \\
\operatorname{depth}(\mathrm{m})\end{array}$ & $\begin{array}{l}\text { Cast oxygen } \\
\text { minimum } \\
\text { depth (m) }\end{array}$ & $\begin{array}{c}\text { Cast oxygen } \\
\text { saturation \% at } \\
\text { minimum }\end{array}$ & $\begin{array}{c}\text { Cast upper } \\
\text { ODZ boundary } \\
\text { (m) }\end{array}$ \\
\hline \multirow[t]{5}{*}{ Shelf } & 2 & 8 & 10 & 30 & 28 & 69 & 0.4 & 48 \\
\hline & 3 & 8 & 12 & 25 & 22 & -- & 0.5 & 41 \\
\hline & 4 & 16 & 18 & 43 & 46 & 63 & 0.3 & 49 \\
\hline & 5 & 25 & 28 & 50 & 47 & 72 & 0.4 & 61 \\
\hline & 1 & 46 & 52 & 66 & 59 & 79 & 0.3 & 72 \\
\hline \multirow[t]{10}{*}{ Offshore } & 6 & 31 & 31 & 80 & 76 & 116 & 0.3 & 83 \\
\hline & 7 & 50 & 59 & 94 & 128 & 103 & 0.4 & 99 \\
\hline & 8 & 46 & 51 & 105 & 111 & 149 & 0.5 & 131 \\
\hline & 9 & 53 & 51 & 109 & 101 & 133 & 0.2 & 116 \\
\hline & 10 & 78 & 80 & 121 & 113 & 161 & 0.3 & 148 \\
\hline & 11 & 51 & 56 & 130 & 137 & 198 & 0.3 & 190 \\
\hline & 12 & 41 & 41 & 124 & 127 & 228 & 0.5 & 216 \\
\hline & 13 & 78 & 67 & 139 & 126 & 249 & 0.6 & 234 \\
\hline & 14 & 89 & 91 & 164 & 158 & 304 & 5 & -- \\
\hline & 15 & 74 & 74 & 168 & 162 & 362 & 7 & -- \\
\hline
\end{tabular}




\begin{tabular}{|c|c|c|c|c|c|c|c|c|}
\hline Zone & $\mathbf{S t}$ & $\begin{array}{c}\text { Average } \\
\text { MLD for all } \\
\text { casts (m) }\end{array}$ & $\begin{array}{l}\text { Cast } \\
\text { MLD } \\
\text { (m) }\end{array}$ & $\begin{array}{l}\text { Average } E z \\
\text { for all casts } \\
\quad(\mathrm{m})\end{array}$ & $\begin{array}{c}\text { Cast Ez } \\
\text { depth (m) }\end{array}$ & $\begin{array}{c}\text { Cast oxygen } \\
\text { minimum } \\
\text { depth }(m)\end{array}$ & $\begin{array}{c}\text { Cast oxygen } \\
\text { saturation \% at } \\
\text { minimum }\end{array}$ & $\begin{array}{c}\text { Cast upper } \\
\text { ODZ boundary } \\
(\mathbf{m})\end{array}$ \\
\hline \multirow[t]{20}{*}{ Gyre } & 16 & 56 & 56 & 150 & 140 & 350 & 5 & -- \\
\hline & 17 & 57 & 49 & 163 & 145 & 333 & 5 & -- \\
\hline & 18 & 36 & 31 & 174 & 165 & 358 & 5 & -- \\
\hline & 20 & 49 & 60 & 191 & 168 & 380 & 13 & -- \\
\hline & 21 & 59 & 63 & 167 & 142 & 357 & 4 & -- \\
\hline & 22 & 56 & 54 & 179 & 162 & 373 & 13 & -- \\
\hline & 23 & 45 & 51 & 169 & 164 & 375 & 11 & -- \\
\hline & 24 & 54 & 57 & 163 & 153 & 344 & 4 & -- \\
\hline & 25 & 76 & 76 & 163 & 156 & 353 & 12 & -- \\
\hline & 26 & 57 & 65 & 169 & 167 & 324 & 7 & -- \\
\hline & 27 & 60 & 58 & 184 & 171 & 302 & 10 & -- \\
\hline & 28 & 67 & 71 & 162 & 168 & 332 & 11 & -- \\
\hline & 29 & 66 & 67 & 175 & 154 & 325 & 16 & -- \\
\hline & 30 & 74 & 76 & 168 & 172 & 338 & 16 & -- \\
\hline & 31 & 65 & 61 & 146 & 126 & 332 & 9 & -- \\
\hline & 32 & 84 & 78 & 153 & 142 & 313 & 22 & -- \\
\hline & 33 & 65 & 64 & 186 & 176 & 325 & 26 & -- \\
\hline & 34 & 58 & 58 & 175 & 174 & 284 & 29 & -- \\
\hline & 35 & 67 & 70 & 170 & 169 & 306 & 27 & -- \\
\hline & 36 & 60 & 60 & 179 & 163 & 341 & 28 & -- \\
\hline
\end{tabular}


Supplemental Table 2: Results for $100 \mathrm{~m}$ calculations. For comparison with flux calculations at the base of the euphotic zone, fluxes for ${ }^{234} \mathrm{Th}$ (original and upwelling-adjusted) and particulate organic carbon are included.

\begin{tabular}{|c|c|c|c|c|c|c|c|c|c|c|}
\hline \multirow{2}{*}{$\begin{array}{r}\text { Zone } \\
\text { Shelf }\end{array}$} & \multirow{2}{*}{$\begin{array}{l}\text { St } \\
2\end{array}$} & \multicolumn{3}{|c|}{ 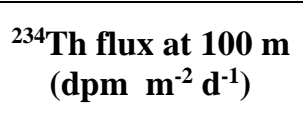 } & \multicolumn{3}{|c|}{ 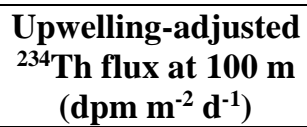 } & \multicolumn{3}{|c|}{ 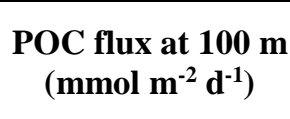 } \\
\hline & & 1800 & \pm & 100 & 7000 & \pm & 1000 & 10 & \pm & 3 \\
\hline & 3 & 900 & \pm & 100 & 7000 & \pm & 2000 & 10 & \pm & 3 \\
\hline & 4 & 1300 & \pm & 200 & 6000 & \pm & 1000 & 8 & \pm & 2 \\
\hline & 5 & 1800 & \pm & 100 & 6000 & \pm & 1000 & 8 & \pm & 2 \\
\hline & 1 & 2000 & \pm & 100 & 6000 & \pm & 1000 & 8 & \pm & 2 \\
\hline \multirow[t]{10}{*}{ Offshore } & 6 & 1800 & \pm & 200 & 4000 & \pm & 1000 & 7 & \pm & 3 \\
\hline & 7 & 1900 & \pm & 100 & 3000 & \pm & 1000 & 4 & \pm & 2 \\
\hline & 8 & 2200 & \pm & 200 & 2200 & \pm & 200 & 3 & \pm & 1 \\
\hline & 9 & 1400 & \pm & 100 & 1400 & \pm & 100 & 2 & \pm & 1 \\
\hline & 10 & 1700 & \pm & 200 & 1700 & \pm & 200 & 3 & \pm & 1 \\
\hline & 11 & 1900 & \pm & 100 & 1900 & \pm & 100 & 3 & \pm & 1 \\
\hline & 12 & 1400 & \pm & 200 & 1400 & \pm & 200 & 2 & \pm & 1 \\
\hline & 13 & 1300 & \pm & 200 & 1300 & \pm & 200 & 2 & \pm & 1 \\
\hline & 14 & 2200 & \pm & 200 & 2200 & \pm & 200 & 3 & \pm & 1 \\
\hline & 15 & 2000 & \pm & 100 & 2000 & \pm & 100 & 3 & \pm & 1 \\
\hline \multirow[t]{20}{*}{ Gyre } & 16 & 1900 & \pm & 200 & 1900 & \pm & 200 & 2 & \pm & 0.7 \\
\hline & 17 & 1400 & \pm & 100 & 1400 & \pm & 100 & 2 & \pm & 0.5 \\
\hline & 18 & 1600 & \pm & 100 & 1600 & \pm & 100 & 2 & \pm & 0.6 \\
\hline & 20 & 1500 & \pm & 200 & 1500 & \pm & 200 & 2 & \pm & 0.5 \\
\hline & 21 & 1400 & \pm & 200 & 1400 & \pm & 200 & 1 & \pm & 0.5 \\
\hline & 22 & 1900 & \pm & 200 & 1900 & \pm & 200 & 2 & \pm & 0.7 \\
\hline & 23 & 1800 & \pm & 200 & 1800 & \pm & 200 & 2 & \pm & 0.7 \\
\hline & 24 & 1400 & \pm & 200 & 1400 & \pm & 200 & 2 & \pm & 0.5 \\
\hline & 25 & 1500 & \pm & 100 & 1500 & \pm & 100 & 2 & \pm & 0.5 \\
\hline & 26 & 1400 & \pm & 200 & 1400 & \pm & 200 & 2 & \pm & 0.5 \\
\hline & 27 & 1500 & \pm & 200 & 1500 & \pm & 200 & 2 & \pm & 0.6 \\
\hline & 28 & 1500 & \pm & 200 & 1500 & \pm & 200 & 2 & \pm & 0.6 \\
\hline & 29 & 1500 & \pm & 200 & 1500 & \pm & 200 & 2 & \pm & 0.5 \\
\hline & 30 & 1400 & \pm & 200 & 1400 & \pm & 200 & 1 & \pm & 0.5 \\
\hline & 31 & 1700 & \pm & 200 & 1700 & \pm & 200 & 2 & \pm & 0.6 \\
\hline & 32 & 1800 & \pm & 200 & 1800 & \pm & 200 & 2 & \pm & 0.7 \\
\hline & 33 & 1600 & \pm & 200 & 1600 & \pm & 200 & 2 & \pm & 0.6 \\
\hline & 34 & 1600 & \pm & 100 & 1600 & \pm & 100 & 2 & \pm & 0.6 \\
\hline & 35 & 1800 & \pm & 200 & 1800 & \pm & 200 & 2 & \pm & 0.7 \\
\hline & 36 & 1100 & \pm & 100 & 1100 & \pm & 100 & 1 & \pm & 0.4 \\
\hline
\end{tabular}


Supplemental Table 3: Parameters used to derive the ${ }^{234}$ Th flux component due to upwelling in the coastal region. The depth ranges with gradients in ${ }^{234} \mathrm{Th}$ and potential density are shown here with the approximate depth of equilibrium (where the activity of total ${ }^{234} \mathrm{Th}$ first transitions from being lower than the activity of ${ }^{238} \mathrm{U}$ to higher than the local ${ }^{238} \mathrm{U}$ activity). The depth of equilibrium was approximated at each station using the slope of the ${ }^{234}$ Th gradient from the surrounding sampling points. The depth boundaries where the gradients existed in both ${ }^{234} \mathrm{Th}$ and potential density generally coincided, but some variability was observed due to the lower sampling resolution of ${ }^{234} \mathrm{Th}(\mathrm{n}=3$ to 8 , Fig. 3) compared to that of potential density ( $\mathrm{n}>50$, Supp. Fig. 6). To determine the upwelling component of ${ }^{234} \mathrm{Th}$ flux, ${ }^{234} \mathrm{Th}$ gradients from each station were multiplied by ${ }^{7} \mathrm{Be}$-derived upwelling rates that were calculated directly for stations 1 and 7 (constant upwelling model with $0 \mathrm{~m} \mathrm{~s}^{-1}$ horizontal advection; Kadko 2017), shown in bold and italics, or extrapolated from surrounding stations as indicated (Supp. Fig. 7). A range of models, $w$, and $u$ were tested to determine the uncertainty on the upwelling component of ${ }^{234}$ Th flux (Supp. Fig. 7).

\begin{tabular}{|c|c|c|c|c|c|c|c|c|c|c|c|c|c|c|}
\hline \multirow{2}{*}{$\begin{array}{l}\text { Zone } \\
\text { Shelf }\end{array}$} & \multirow{2}{*}{$\begin{array}{l}\text { St } \\
2\end{array}$} & \multicolumn{2}{|c|}{$\begin{array}{l}{ }^{234} \text { Th surface } \\
\text { gradient } \\
\text { depth range } \\
\text { (m) }\end{array}$} & \multirow{2}{*}{$\begin{array}{c}\begin{array}{c}\text { Estimated } \\
\text { equilibrium } \\
\text { depth (m) }\end{array} \\
57\end{array}$} & \multicolumn{2}{|c|}{$\begin{array}{l}\text { MLD to potential } \\
\text { density gradient } \\
\text { boundary }(m)\end{array}$} & \multirow{2}{*}{$\begin{array}{c}{ }^{{ }^{234}} \text { Th gradient } \\
\text { with depth } \\
\left(\mathbf{d p m} \mathbf{L}^{-1} \mathbf{~ m}^{-1}\right) \\
3.85 \mathrm{E}-02\end{array}$} & \multicolumn{3}{|c|}{$\begin{array}{l}\text { Range of } w \text { used } \\
\text { to assess model } \\
\text { variability } \\
\left(\mathrm{m} \mathrm{d}^{-1}\right)\end{array}$} & \multirow[t]{2}{*}{ 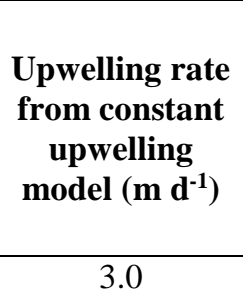 } & \multicolumn{3}{|c|}{$\begin{array}{c}\text { Upwelling } \\
\text { component of } \\
\text { 234 Th flux at Ez } \\
\text { using constant } \\
\text { model } \\
\left(\mathrm{dpm} \mathrm{m^{-2 }} \mathrm{d}^{-1}\right)\end{array}$} \\
\hline & & 6 & -55 & & 8 & -56 & & 2.4 & & 4.9 & & 3000 & \pm & 1000 \\
\hline & 3 & 7 & $-\quad 35$ & 35 & 8 & -54 & 7.14E-02 & 2.4 & & 4.9 & 3.0 & 4000 & \pm & 2000 \\
\hline & 4 & 13 & $\begin{array}{l}-\quad 47 \\
\end{array}$ & 57 & 16 & $\begin{array}{l}-\quad 49 \\
\end{array}$ & $3.79 \mathrm{E}-02$ & 2.4 & & 4.9 & 3.0 & 3000 & \pm & 1000 \\
\hline & 5 & 25 & $\begin{array}{l}-\quad 74 \\
\end{array}$ & 69 & 25 & -67 & $2.93 \mathrm{E}-02$ & 2.4 & & 4.9 & 3.0 & 2000 & \pm & 1000 \\
\hline & 1 & 45 & $\begin{array}{l}-\quad 81 \\
\end{array}$ & 80 & 46 & 79 & $3.74 \mathrm{E}-02$ & 2.4 & - & 4.9 & 3.0 & 2000 & \pm & 1000 \\
\hline \multirow[t]{2}{*}{ Offshore } & 6 & 20 & $\begin{array}{l}-80 \\
\end{array}$ & 75 & 31 & -95 & $2.55 \mathrm{E}-02$ & 1.4 & & 3.5 & 2.1 & 3000 & \pm & 700 \\
\hline & 7 & 40 & $-\quad 110$ & 96 & 50 & - 101 & $1.68 \mathrm{E}-02$ & 0.4 & - & 2.0 & 1.1 & 800 & \pm & 300 \\
\hline
\end{tabular}




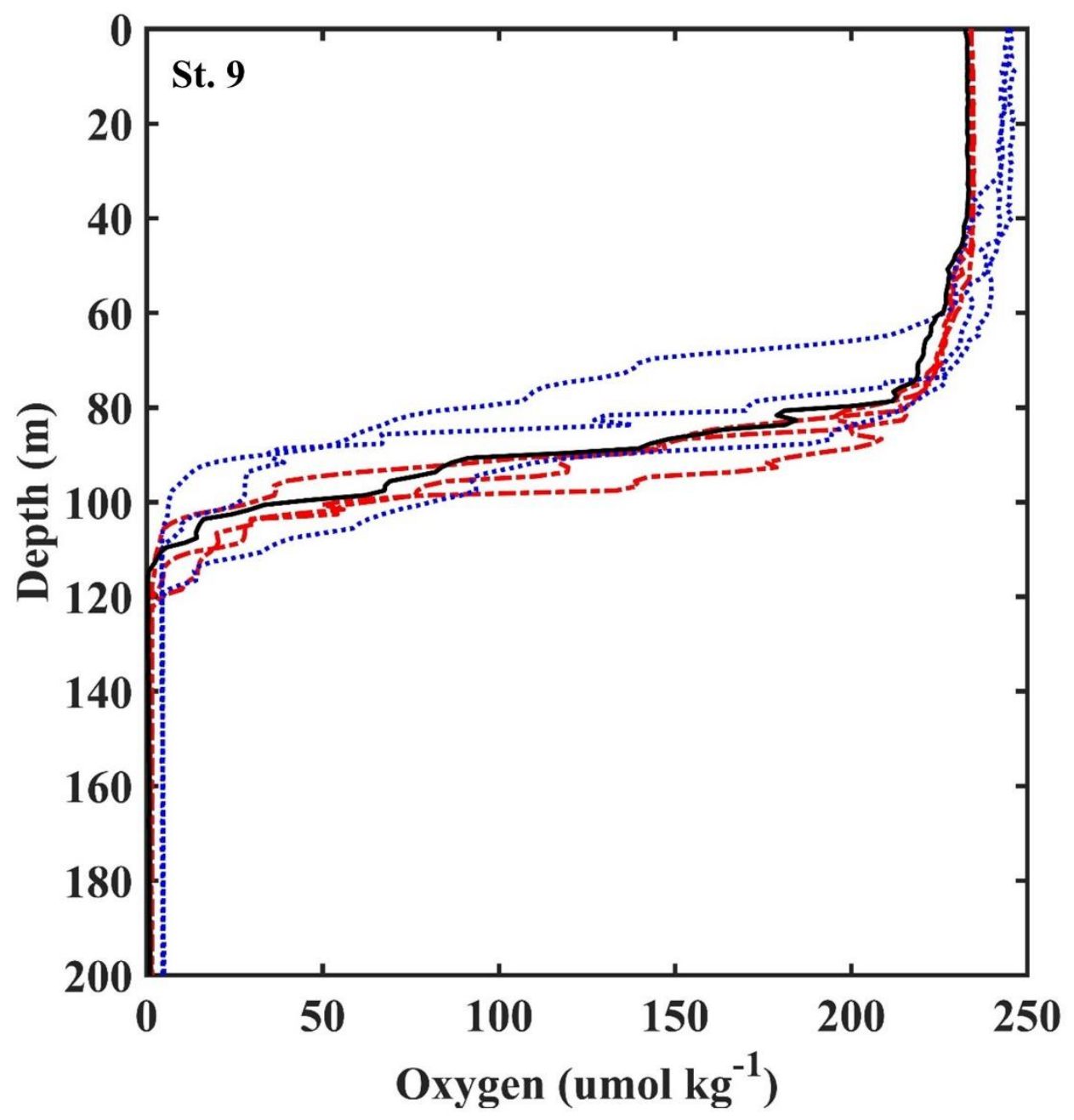

Supplemental Figure 1: Example of inter-cast variability in oxygen. Upcast data taken from station 9 is shown for casts performed with the GEOTRACES rosette CTD (red dashed) and the Ocean Data Facility rosette CTD (blue dashed). The data from the 3rd cast of the 7 shown here was the cast where ${ }^{234} \mathrm{Th}$ was sampled (solid black line). 

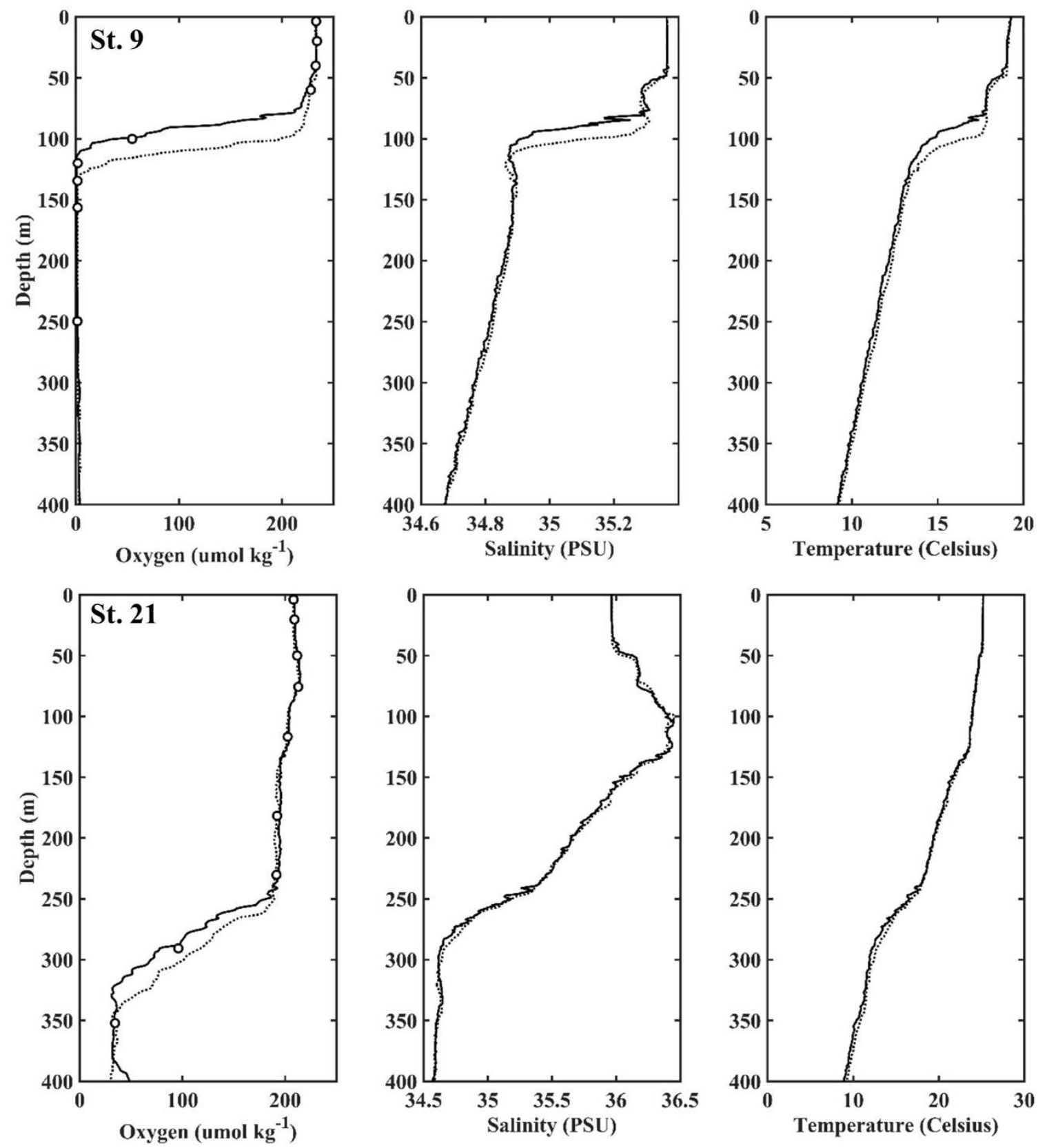

Supplemental Figure 2: Examples of intra-cast variability in oxygen for casts where ${ }^{234} \mathrm{Th}$ was collected. Downcast data (dashed line) and upcast data (solid line) are shown with bottlederived oxygen concentrations (circles). Bottle data match the upcast trace because niskins were tripped on the upcast. Substantial oxygen differences occurred between the up and down casts even when no difference was observed in salinity or temperature. This data suggests that only upcast traces should be used to estimate in-situ oxygen saturation. 


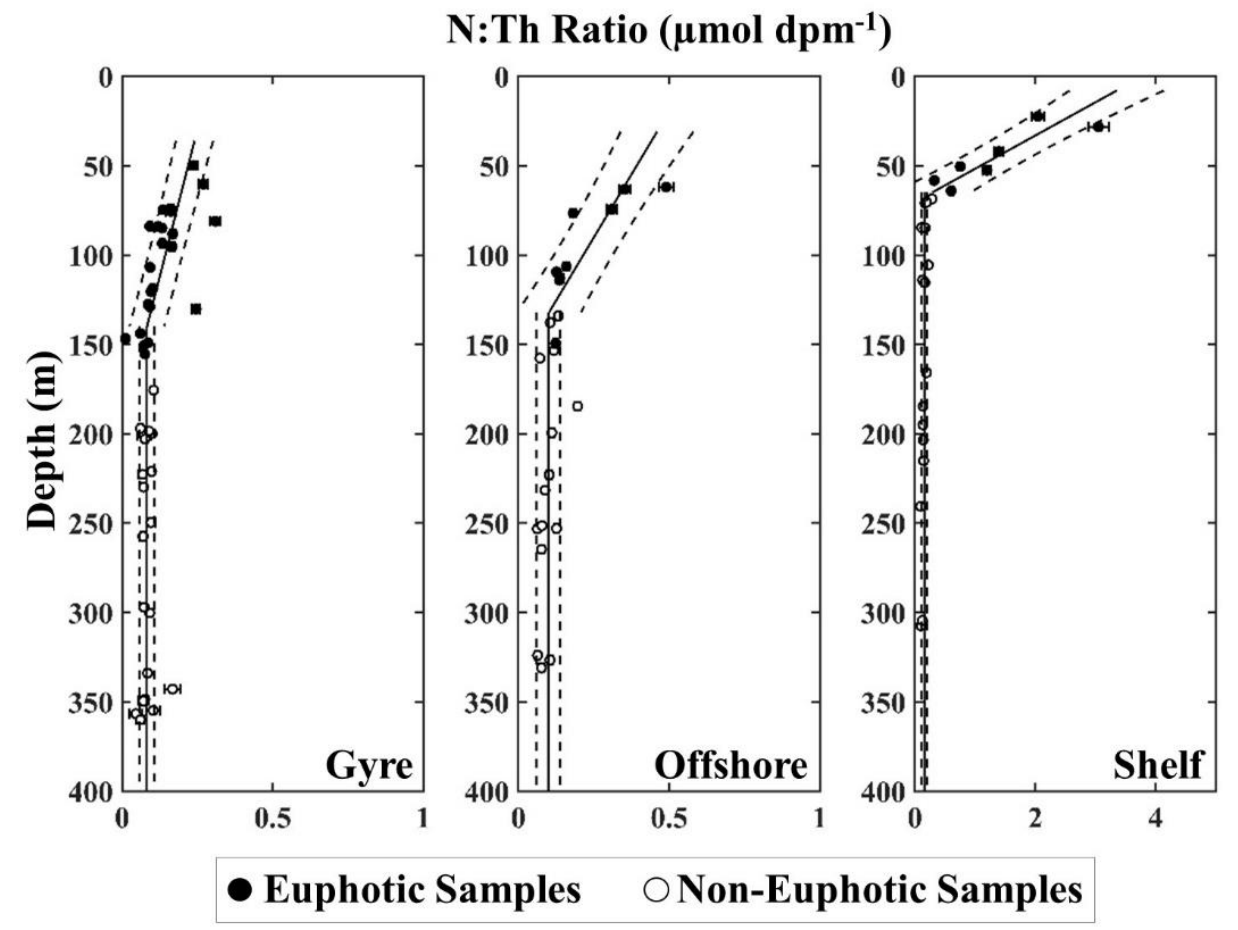

Supplemental Figure 3: Nitrogen: ${ }^{234} \mathrm{Th}$ on large particles $(>51 \mu \mathrm{m})$ in $\mu \mathrm{mol} \mathrm{dpm}^{-1}$ for the upper $400 \mathrm{~m}$. Best fit linear regressions are shown for euphotic samples along with the average ratio for samples collected below the base of the euphotic to $400 \mathrm{~m}$ (solid lines). Both are shown with 1 standard deviation (dotted lines). Mixed layer samples are not shown and not included in ratio calculations. 


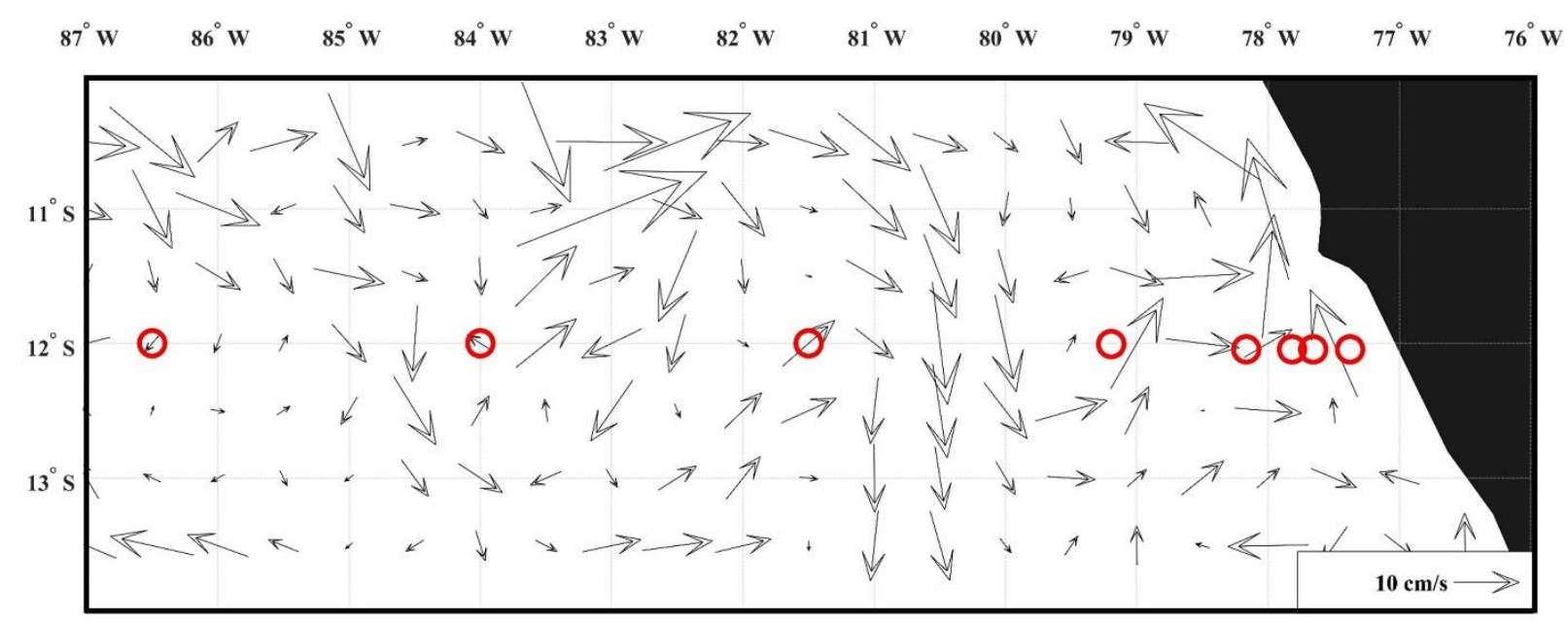

Supplemental Figure 4: Average surface current velocities for October 2013. Geostrophic current velocities were obtained from NOAA for the month prior to the sampling of coastal stations (October; http://pifsc-oceanwatch.irc.noaa.gov/thredds/catalog.html). Stations 1 to 8 are shown where red circles indicate location and approximate size of a $5 \mathrm{~cm} \mathrm{~s}^{-1}$ current (diameter of circle). 


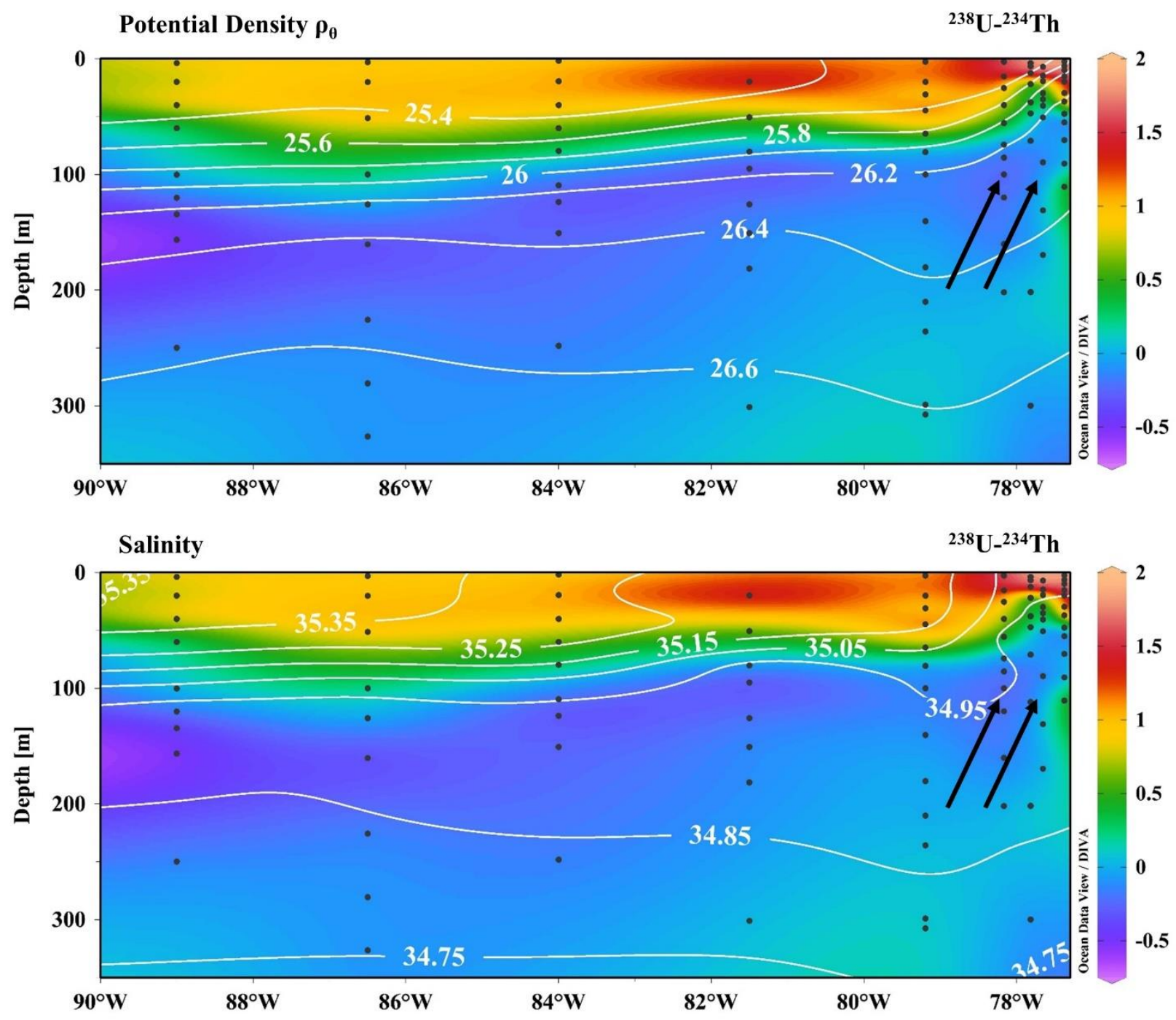

Supplemental Figure 5: ${ }^{238} \mathrm{U}$ minus ${ }^{234} \mathrm{Th}$ activity (colors) with density anomaly ( $\rho$ ) and salinity contours. Arrows show the proposed direction of upwelling, which would bring deeper waters close to equilibrium $\left({ }^{238} \mathrm{U}-{ }^{234} \mathrm{Th} \sim 0\right)$ to the shelf. The most impacted locations appear to be from $77^{\circ} \mathrm{W}$ to $78.5^{\circ} \mathrm{W}$. 


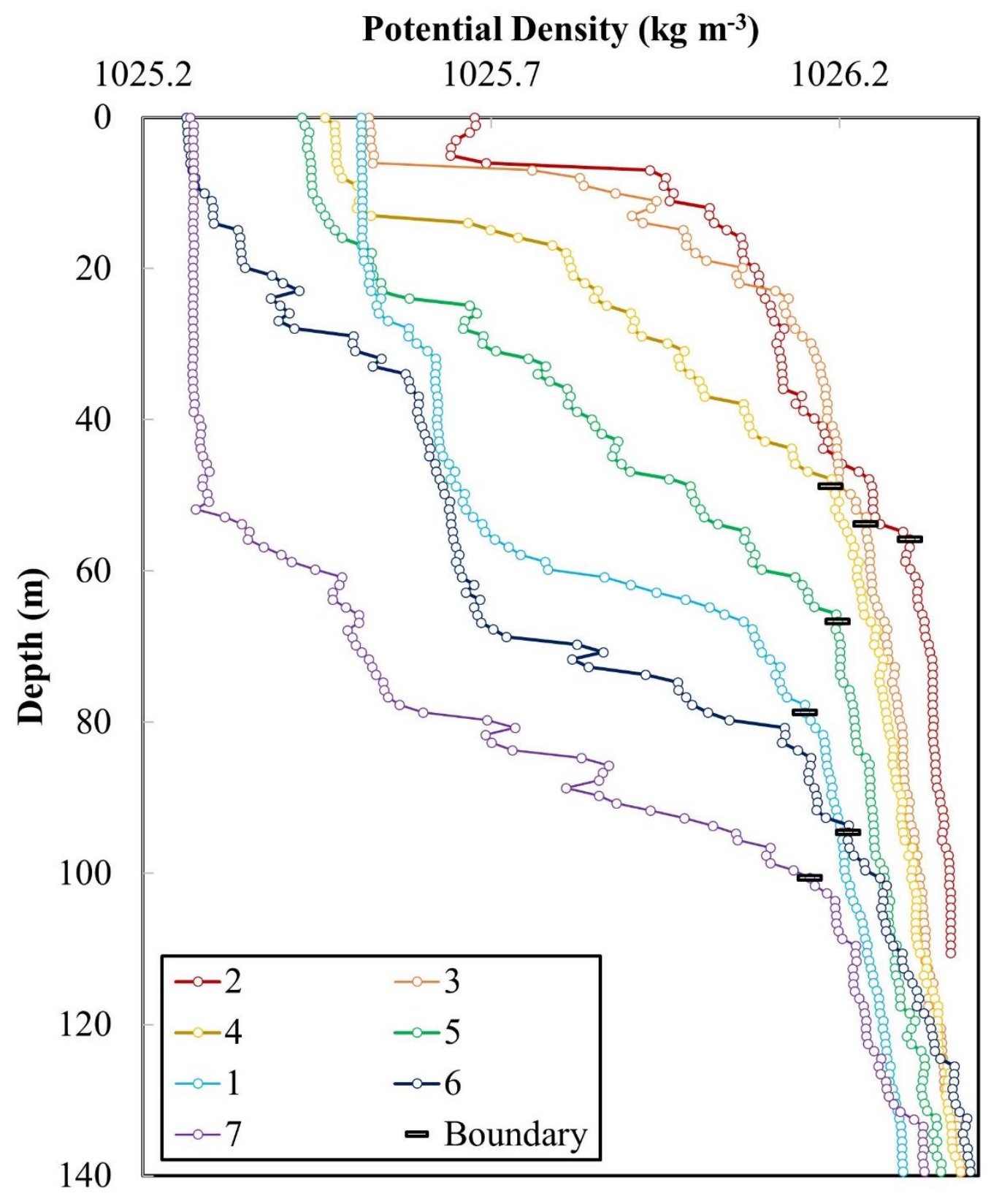

Supplemental Figure 6: Potential density profiles for coastal stations (upper $140 \mathrm{~m}$ ). Potential density profiles are shown here for Stations 1 through $7\left(77^{\circ} \mathrm{W}\right.$ to $\left.84^{\circ} \mathrm{W}\right)$ with a black marker indicating the depth at which successive $1 \mathrm{~m}$ data bins showed a difference $<0.025 \mathrm{~kg} \mathrm{~m}^{-3}$. This profile stability (difference $<0.025$ ) lasted for at least $40 \mathrm{~m}$ below the marker. Due to low variability throughout the entire density profile at station 9 , a more stringent difference limit between $1 \mathrm{~m}$ bins (0.01) was applied at this station. The marker depth is referred to in the text as the 'Gradient Boundary' (Supp. Table 3) and the depth often coincided with the gradient termination depth for total ${ }^{234} \mathrm{Th}$. Stations 1 to 7 are shown here because relatively large surface gradients in ${ }^{234} \mathrm{Th}$ with depth were observed in the upper $140 \mathrm{~m}$ when compared to profiles from all other stations $\left(85^{\circ} \mathrm{W}\right.$ to $\left.152^{\circ} \mathrm{W}\right)$. Both the density and total ${ }^{234} \mathrm{Th}$ data suggested that upwelling corrections would be most appropriate from the mixed layer to the approximate depth of the marker shown here, where the large gradients cease. 


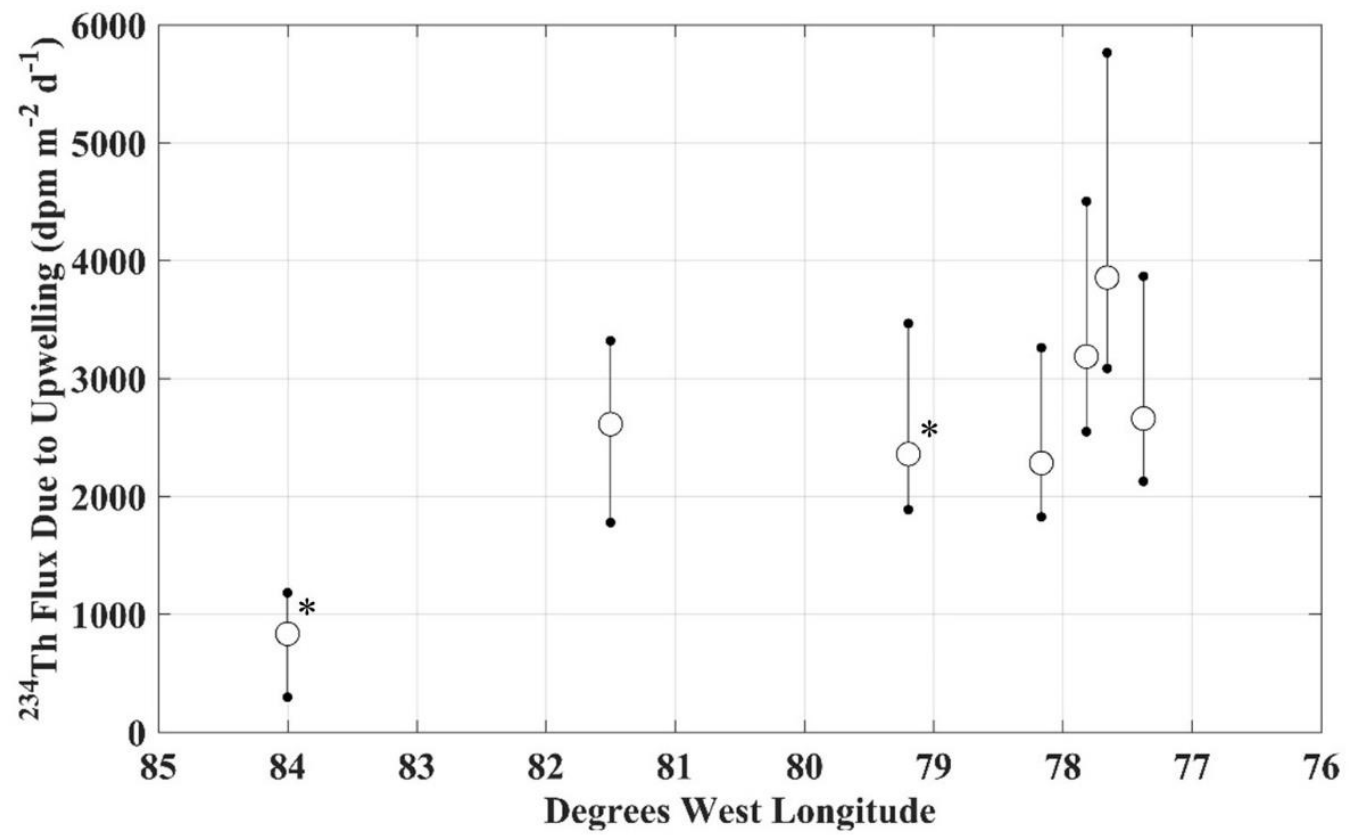

Supplemental Figure 7: Flux of ${ }^{234} \mathrm{Th}$ due to upwelling calculated at the base of the euphotic zone. The stations between $77^{\circ} \mathrm{W}$ to $84^{\circ} \mathrm{W}$ (1 to 7) showed large surface gradients in ${ }^{234} \mathrm{Th}$ with depth when compared to the profiles of all other stations. For these 7 stations, the upwelling component of the ${ }^{234} \mathrm{Th}$ flux from Eq. 5, which includes a surface gradient $\left(\partial^{234} \mathrm{Th} / \partial \mathrm{z}\right)$ and upwelling rate $(w)$, is shown here as calculated at Ez. Individual surface gradients were calculated for each station and multiplied by an upwelling rate derived from a constant upwelling model (Kadko, 2017) and these results are shown as open circles. The upwelling component of ${ }^{234} \mathrm{Th}$ flux was set to be non-zero in Eq. 5 at depths between the base of the mixed layer and the density gradient boundary (Supp. Table 3, Supp. Fig. 6). For these stations, Ez was shallower than the gradient boundary. Upwelling rates from Kadko (2017) were only determined for stations $1\left(79.2^{\circ}\right.$ $\mathrm{W}$ at $\left.3 \mathrm{~m} \mathrm{~d}^{-1}\right)$ and $7\left(84^{\circ} \mathrm{W}\right.$ at $\left.1.1 \mathrm{~m} \mathrm{~d}^{-1}\right)$, which are indicated by asterisks. The upwelling rate at station 1 was applied to the stations east of $79^{\circ} \mathrm{W}$ and the rate at $81.5^{\circ} \mathrm{W}$ was an average of the surrounding stations $\left(2.1 \mathrm{~m} \mathrm{~d}^{-1}\right)$. Vertical lines about the points represent the potential variability in derived fluxes due to the model used (constant upwelling with depth versus depth-dependent upwelling), $w$, and $u$ (horizontal advection). The variability shown incorporated a change in horizontal advection from $0 \mathrm{~m} \mathrm{~s}^{-1}$ to $0.2 \mathrm{~m} \mathrm{~s}^{-1}$, a change in upwelling from $2.4 \mathrm{~m} \mathrm{~d}^{-1}$ to $4.9 \mathrm{~m} \mathrm{~d}^{-1}$ for station 1 and $0.4 \mathrm{~m} \mathrm{~d}^{-1}$ to $2.0 \mathrm{~m} \mathrm{~d}^{-1}$ for station 7 , and the model was changed from constant to depth-dependent (Supp. Table 3). The maximum difference observed at each station between the open circles $\left(0 \mathrm{~m} \mathrm{~s}^{-1}\right.$ horizontal advection, constant model) and the outlier cases (endpoints) was used as the uncertainty on the upwelling flux components. 

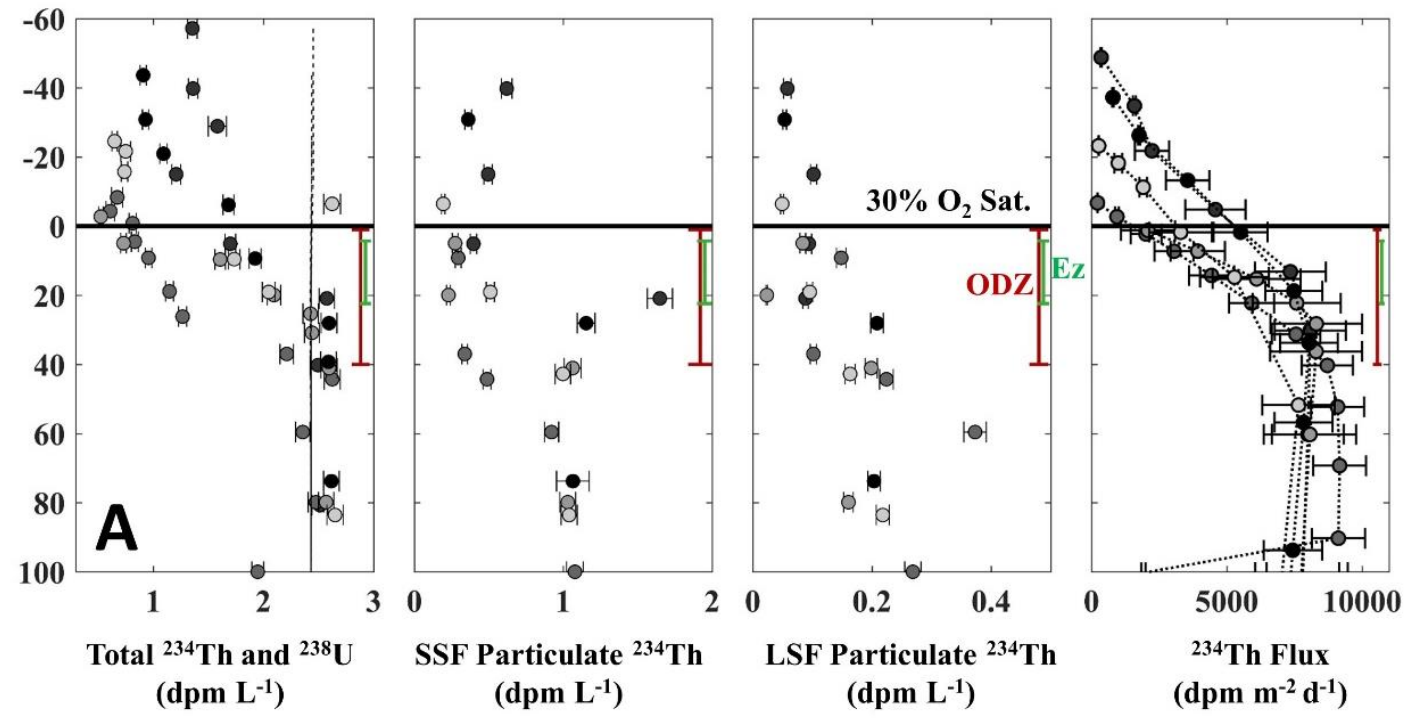

Supplemental Figure 8 (A to E): ${ }^{234} \mathrm{Th}$ results plotted relative to the upper $30 \%$ oxygen saturation boundary. Stations are grouped as follows: (A) Shelf Stations $1-5$ from $77^{\circ} \mathrm{W}$ to $80^{\circ}$ $\mathrm{W}$, (B) Offshore Stations 6-10 from $80^{\circ} \mathrm{W}$ to $92^{\circ} \mathrm{W}$, (C) Offshore Stations $11-15$ from $92^{\circ} \mathrm{W}$ to $105^{\circ} \mathrm{W}$, (D) Gyre Stations 16-26 from $105^{\circ} \mathrm{W}$ to $129^{\circ} \mathrm{W}$, (E) Gyre Stations 27 to 36 from $129^{\circ}$ $\mathrm{W}$ to $152^{\circ} \mathrm{W}$. All data points are shown as depth relative to the surface ocean depth where oxygen saturation levels first reach $30 \%$. This depth is shown as zero (black line). The range in the depth of the euphotic base (Ez) is indicated by the green bracket and the range in the depth of the upper ODZ boundary is indicated by the red bracket. Data shading (filled circles) indicates the location of stations within each region, with black points being the easternmost station and the lightest grey being the westernmost. Note the $\mathrm{x}$-axis change for the ${ }^{234} \mathrm{Th}$ flux panel. 

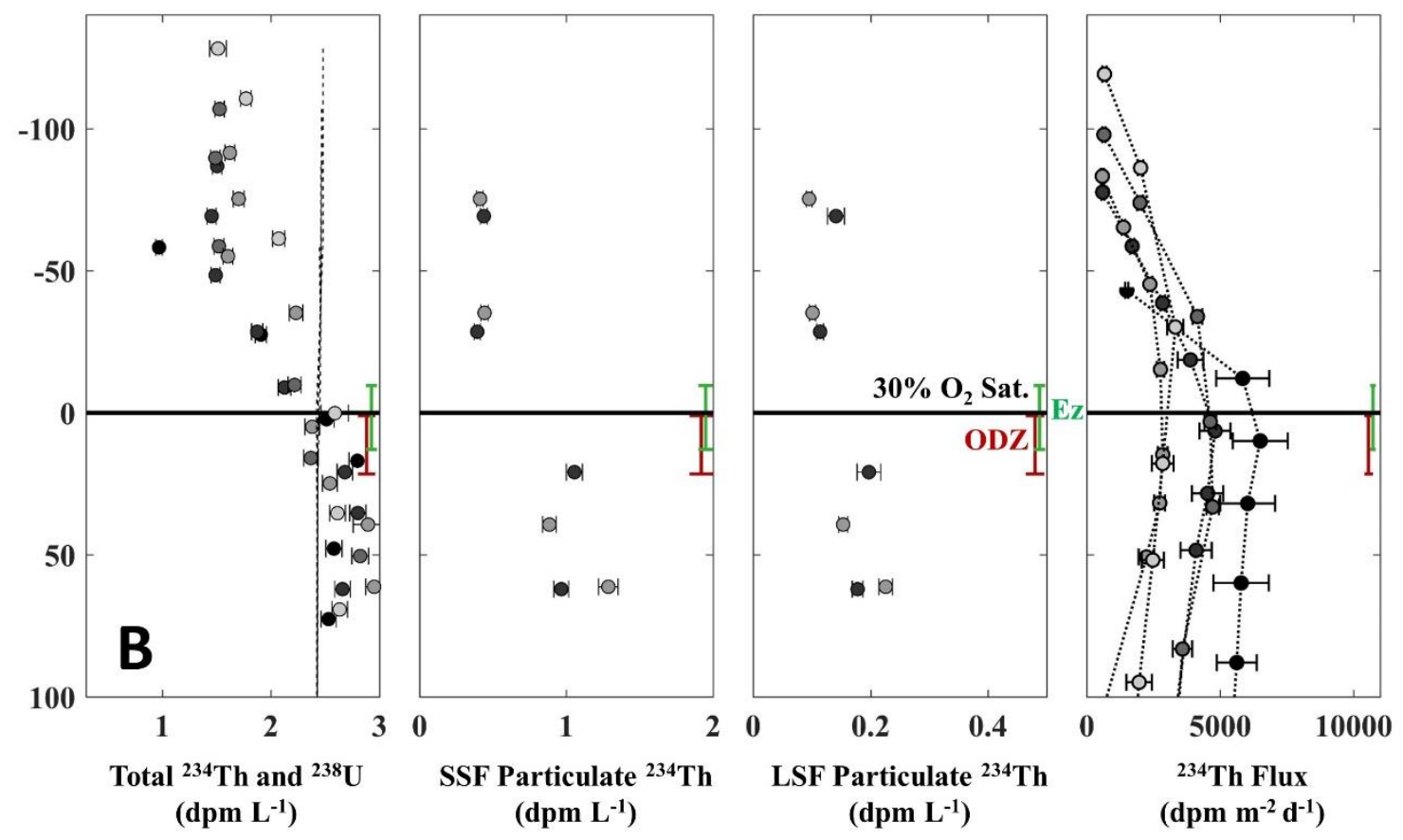

Supplemental Figure 8B: ${ }^{234}$ Th results plotted relative to the upper 30\% oxygen saturation boundary. Details listed with Supp. Fig. 8A. 

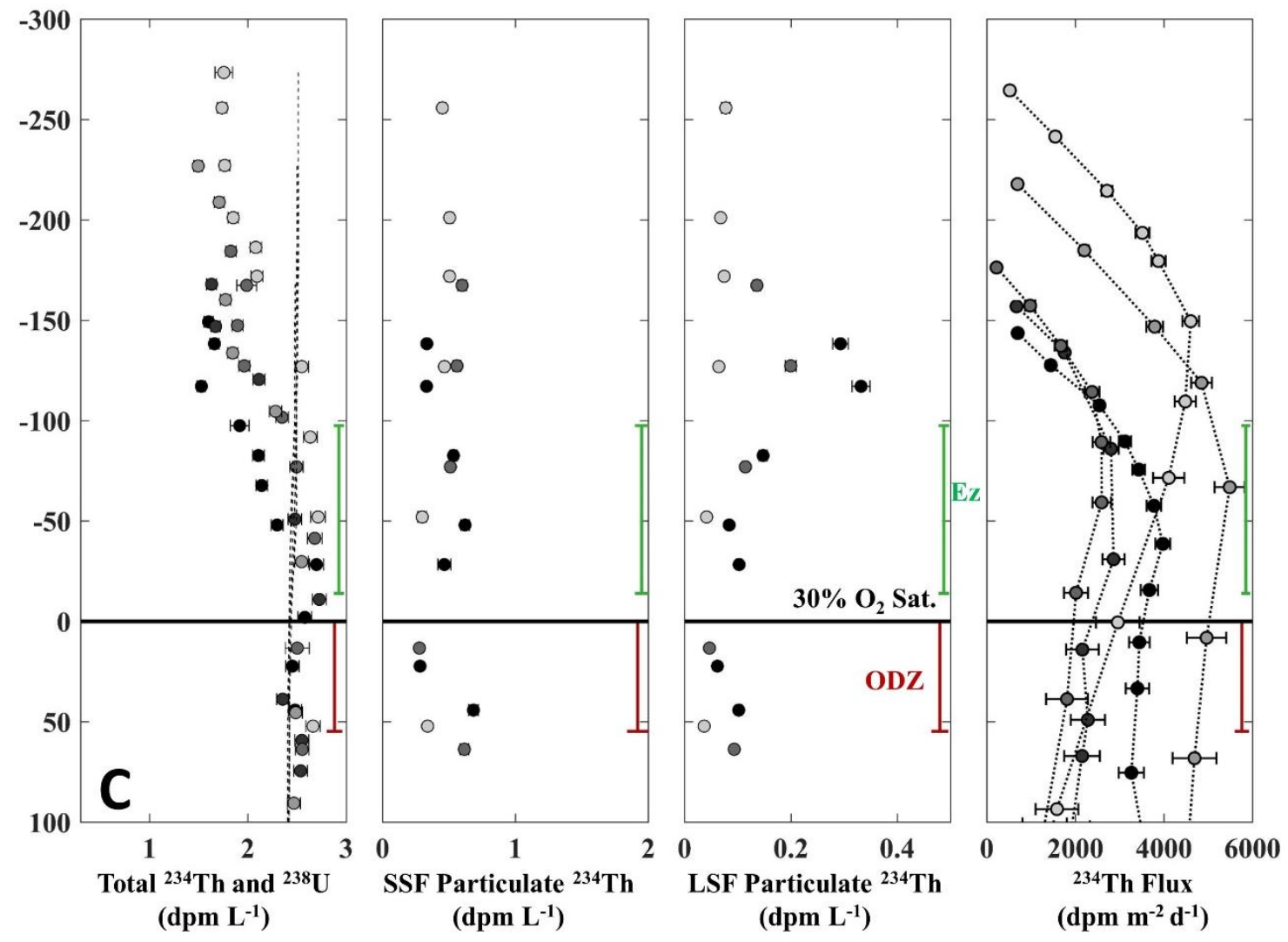

Supplemental Figure 8C: ${ }^{234}$ Th results plotted relative to the upper 30\% oxygen saturation boundary. Details listed with Supp. Fig. 8A. 

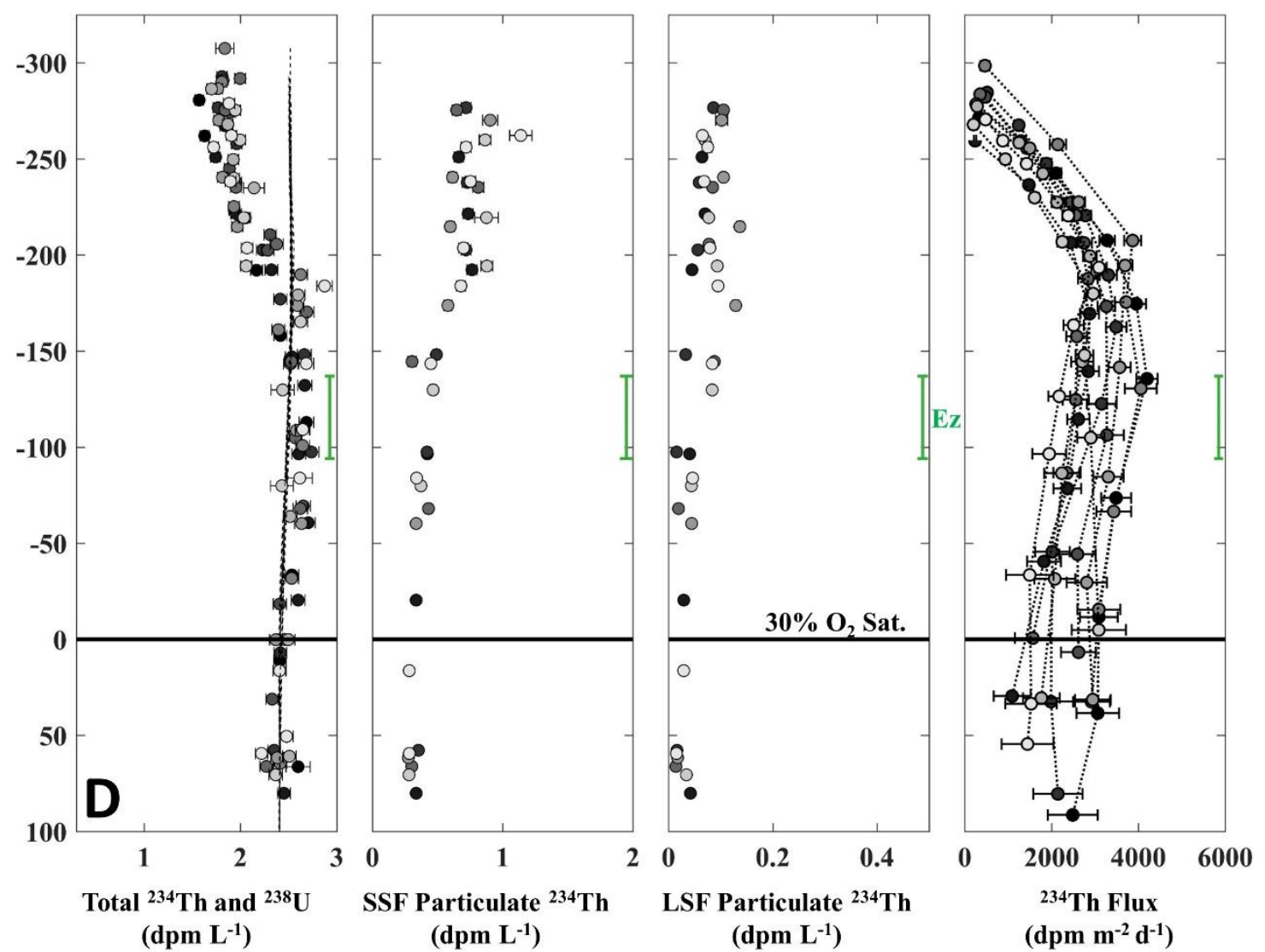

Supplemental Figure 8D: ${ }^{234}$ Th results plotted relative to the upper $30 \%$ oxygen saturation boundary. Details listed with Supp. Fig. 8A. 

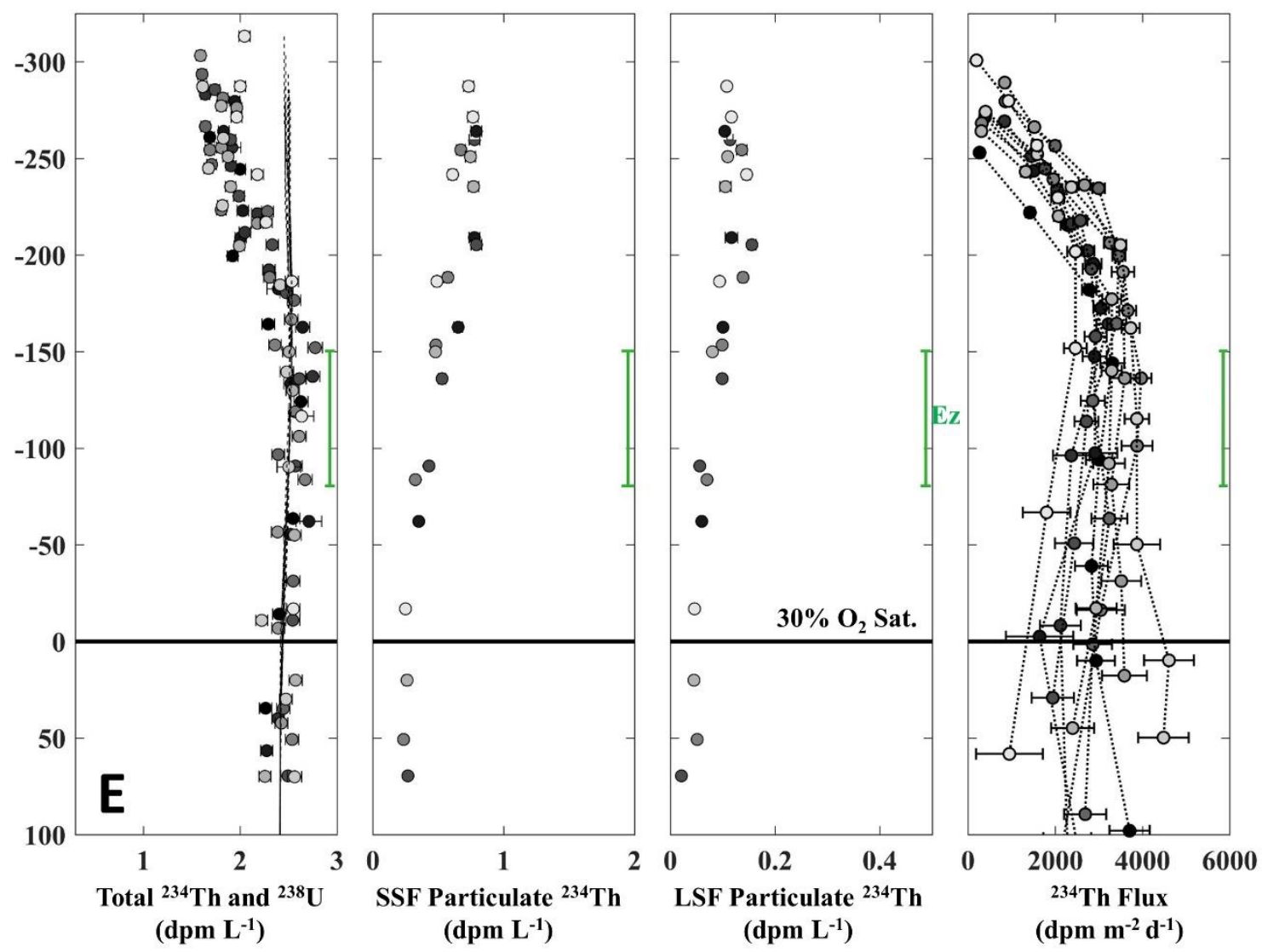

Supplemental Figure 8E: ${ }^{234} \mathrm{Th}$ results plotted relative to the upper $30 \%$ oxygen saturation boundary. Details listed with Supp. Fig. 8A. 


\section{Chapter 3:}

$\mathrm{A}^{234} \mathrm{Th}$-based method for deriving particulate export and upper ocean residence times of trace metals 


\begin{abstract}
Better constraints on the magnitude of surface export, the extent of remineralization, and the residence time of biologically-relevant elements are required in order to understand marine food web dynamics, track the transport of anthropogenic trace metals in the ocean, and improve global climate models. While prior studies have been successful in constructing basin-scale budgets of carbon in the surface ocean, the cycling of particulate trace metals is poorly understood. The ${ }^{234} \mathrm{Th}-$ ${ }^{238} \mathrm{U}$ method is used here with data from the GP-16 GEOTRACES transect to estimate the magnitude of the processes controlling the export of iron (Fe), cobalt (Co), manganese (Mn), and cadmium (Cd) in the upper $200 \mathrm{~m}$ of the southeastern tropical Pacific. These efforts and those from other GEOTRACES-based studies will help to close the budgets of trace metals in the upper ocean, which in turn will lead to improved estimates from global biogeochemical and climate models.

Flux profiles indicated that $\mathrm{Cd}$ and $\mathrm{Co}$ behave similarly to particulate phosphorus and organic carbon, with the highest fluxes in the productive shelf region and decreasing flux with depth due to remineralization. Mn flux was controlled by redox conditions at oxygen deficient stations and by precipitation and/or scavenging elsewhere. Fe inventories are maintained by dust and coastal inputs and the distribution of Fe fluxes in the upper ocean is governed by authigenic, lithogenic and biological processes. Residence times for the sinking pool of each element were on the order of days to weeks, supporting the use of ${ }^{234} \mathrm{Th}\left(\mathrm{t}^{1} \frac{1}{2}=24.1\right.$ days) as a tracer of surface export. Total residence times were the order of years for all elements, except Fe, which had values on the order of weeks to months. The more rapid cycling of $\mathrm{Fe}$ in this region is consistent with low dust input estimates, upwelling-driven high productivity, and the resulting propensity for this region to be iron-limited.
\end{abstract}




\subsection{Introduction}

\subsubsection{Constraining the particulate export of trace metals}

Although trace metals are present at concentrations in the ocean of $<10 \mu \mathrm{mol} \mathrm{kg}^{-1}$, they are essential to marine ecosystems, impact the exchange of nutrients to deeper waters, and can affect global trends in ocean productivity and climate change (Kumar et al., 1995; Martínez-garcía et al., 2014; Mills et al., 2004; Moore and Braucher, 2008; Morel et al., 2004; Morel and Price, 2003; Saito et al., 2010; Sunda and Huntsman, 1997; Watson et al., 2000). Iron (Fe) is critical to metabolic processes and can limit primary productivity in high-nitrate low-chlorophyll regions of the ocean (Boyd et al., 2004; Martin et al., 1994). Cobalt (Co) has been shown to be co-limiting to phytoplankton growth (Saito and Goepfert, 2008) and other trace metals, such as manganese (Mn) and cadmium (Cd), are utilized in enzymes and as electron acceptors (Moffett and Ho, 1996; Morel and Price, 2003; Tebo et al., 1984). Because metals like Cd are used as paleo-productivity proxies (Boyle, 1988; Elderfield and Rickaby, 2000), it is vital to understand the mechanisms of their delivery to the deep ocean. Lastly, the geochemical fates of anthropogenic metals, such as cesium, and mercury, influence the health of ocean ecosystems and our decision-making on the safe utilization of ocean resources (Buesseler et al., 2012; Lamborg et al., 2014).

Global biogeochemical models (GBMs) can improve our understanding of the impact of trace metals on past and present oceanic biogeochemical cycles, as well as aid in predicting the state of Earth's climate in the future. However, a recent comparison of thirteen Fe-incorporating GBMs found that 'all models struggle to reproduce many aspects of the observed spatial patterns' (Tagliabue et al., 2016). For other metals, such as Mn and Al, good agreement between observed and modeled values have been achieved in select parts of the global ocean (van Hulten et al., 2017, 2014, 2013). However, each GBM still struggles to replicate certain processes (e.g. surfacemidwater exchange of Mn in the Pacific oxygen minimum zone) and gradients in specific ocean basins (deep Atlantic Al).

To improve these GBMs and our overall understanding of trace metal cycling, better estimates of trace metal fluxes are needed. One of the most important fluxes to constrain is export from the surface to the deep ocean, because deep ocean waters and sediments can act as a long term sink. Like carbon, which reaches the deep ocean via two mechanisms, referred to as the solubility pump and the biological pump, trace elements can move from the surface to the deep ocean via large 
scale water mass mixing (centennial to millennial timescales) or by association with sinking particles (day to month timescales). Because particle cycling impacts the distribution of total concentrations within the ocean over relatively short timescales, particulate flux estimates are essential to interpreting observed patterns in metal distributions.

\subsubsection{Direct methods for quantifying particulate trace metal export in the upper ocean}

Prior studies have often used the upper ocean distributions of trace metals to speculate about what factors control surface budgets (e.g. Bruland et al., 1994; Johnson et al., 1997) and concentrations alone may be misleading. In a study of the Indian Ocean (Buesseler et al., 1998) a relative low in dissolved Fe was observed in 1995 during the late-SW Monsoon (Measures and Vink 1998). With dissolved Fe measurements only, an Fe low like this one could signify permanent metal-limitation in the region, a partitioning of the Fe into the particulate phase, or a recent loss of surface metals without replenishment. In this case, there was concurrent sampling of Fe, carbon, and a ${ }^{234} \mathrm{Th}$ 'flux gauge', and the cause of the Fe low was identified as a local diatom bloom. Similarly, trace metal residence times are sensitive to how inventory measurements are made and which flux estimates are applied. Many prior studies have calculated residence times using a combination of dissolved (or total) concentrations with a single dust sampling effort or historical deposition estimates (Croot et al., 2004; Saito and Moffett, 2002; Yeats et al., 1992). While these approaches do provide useful bounds, dust-based methods are limited by aerosol solubility and steady state assumptions, when dust events are by nature episodic.

One of the primary methods to directly determine surface export has been the utilization of sediment traps. Deep-moored trap studies are common, while only a few shallow trap (less than $500 \mathrm{~m}$ depth) measurements of trace metals, such as $\mathrm{Al}, \mathrm{Fe}$, and $\mathrm{Pb}$, had been made prior to the mid-2000s (Weinstein and Moran, 2005). These trap studies were also limited to distinct biogeochemical regions, such as the Mediterranean Sea (Migon et al., 2002; Quetel et al., 1993), the Ross Sea (Collier et al., 2000), and the Greenland Sea (Schüßler et al., 1997). With improvements in trace metal sampling techniques and the advent of neutrally buoyant traps, the number of surface ocean measurements of trace metal fluxes increased (e.g. Bowie et al., 2009; Pohl et al., 2004; Stanley et al., 2004), although often for a select group of metals, such as barium (Dehairs et al., 2000; Dymond and Collier, 1996; Jeandel et al., 2000; McManus et al., 2002) or 
lead (Alleman et al., 2000), and with relatively large uncertainties (Lamborg et al., 2008; Noble et al., 2012).

The other established technique for measuring surface export is the ${ }^{234} \mathrm{Th}-{ }^{238} \mathrm{U}$ disequilibrium method (e.g. Benitez-Nelson et al., 2001; Buesseler et al., 1992). ${ }^{234}$ Th, with a half-life of 24.1 days, is highly particle reactive and its disequilibrium with ${ }^{238} \mathrm{U}$ reflects processes acting on timescales of days to weeks. ${ }^{238} \mathrm{U}(\mathrm{t} 1 / 2=4.47$ billion years) behaves conservatively with respect to salinity in the ocean (Not et al., 2012; Owens et al., 2011). Although ${ }^{234} \mathrm{Th}$ is found to be at equilibrium with ${ }^{238} \mathrm{U}$ throughout much of the water column, rapidly sinking particles in the surface ocean create a quantifiable deficit in ${ }^{234} \mathrm{Th}$ when they carry attached ${ }^{234} \mathrm{Th}$ to depth more rapidly than ${ }^{234} \mathrm{Th}$ is created by ${ }^{238} \mathrm{U}$ decay. A deficit or excess of ${ }^{234} \mathrm{Th}$ relative to its parent is used with an element: ${ }^{234} \mathrm{Th}$ ratio on sinking particles at a given depth to determine a flux of that element (Buesseler et al., 2006, 1992). Most prior trace metal export studies utilizing the ${ }^{234}$ Th method (filled circles, Fig. 1) have been limited in their depth resolution (e.g. Weinstein and Moran, 2005), spatial extent (e.g. Smith et al., 2014), and/or their scope (e.g. Fe only studies: Lemaitre et al., 2016; Planquette et al., 2011). Recent campaigns through the GEOTRACES program have provided a unique opportunity for the simultaneous, high-resolution, basin-scale collection of total ${ }^{234} \mathrm{Th}$ and trace metal: ${ }^{234} \mathrm{Th}$ ratios via high volume pumping.

\subsubsection{Elements of interest}

The scope of this synthesis study includes an assessment of four biologically-relevant trace metals: Cd, Co, Mn, and Fe. Phosphorus (P) and particulate organic carbon (POC) have also been included in the analysis as biological endmembers. Collectively, these components of interest, $\mathrm{Cd}$, $\mathrm{Co}, \mathrm{Fe}, \mathrm{Mn}, \mathrm{P}$, and POC will be referred to as 'elements' or ' $\mathrm{E}$ ', with the understanding that other forms of carbon exist in the particulate phase (i.e. particulate inorganic carbon or PIC).

\subsection{Methods}

\subsubsection{Collection and analysis summary for ${ }^{234} \mathrm{Th},{ }^{238} \mathrm{U}$, and trace metals}

Samples were collected aboard the R/V Thomas G. Thompson between October $29^{\text {th }}$ and December $18^{\text {th }}, 2013$ on the U.S. GEOTRACES GP-16 cruise (referred to here as 'the transect'). Between $75^{\circ} \mathrm{W}$ and $155^{\circ} \mathrm{W}, 35$ stations were sampled for dissolved parameters and total ${ }^{234} \mathrm{Th}$. Full water column high-volume particulate samples were taken at 21 stations, but only the upper 
$400 \mathrm{~m}$ is discussed here (Fig. 1). Detailed methods for the measurement and analyses of total and particulate ${ }^{234}$ Th (Black et al., 2017), size-fractionated particulate trace metals (Lee et al., 2017), and particle composition (Lam et al., 2017) have been published previously. In summary, total ${ }^{234} \mathrm{Th}$ was sampled using a standard rosette. McLane in-situ pumps were to collect ${ }^{234} \mathrm{Th}, \mathrm{POC}, \mathrm{P}$, and trace metal particulates (Bishop et al., 2012; Lam et al., 2017). Each pump system contained two filter heads. One head containing a polyester pre-filter $(>51 \mu \mathrm{m})$ and a pair of QMA filters $(>1 \mu \mathrm{m})$ pumped $\sim 1100 \mathrm{~L}$ and the other with a pre-filter and a pair of $0.8 \mathrm{um}$ Supor filters pumped $\sim 400 \mathrm{~L}$ (Lee et al 2017). 'Small particle' ${ }^{234} \mathrm{Th}$ and POC (1-51 $\left.\mu \mathrm{m}\right)$ were measured on a $25 \mathrm{~mm}$ punch of the QMA. All of the collected material from the Supor-side pre-filter was used to quantify 'large particle' $(>51 \mu \mathrm{m}){ }^{234} \mathrm{Th}$ and POC. Small particle trace metal measurements were made on a 1/16 or $1 / 8$ wedge of the Supor filter and large particle analyses used material from a 1/8 wedge of the QMA-side pre-filter.

The detection limit for the particulate trace metal measurements, defined as three times the standard deviation (s.d.) of the dipped blanks, was used as the sample concentration in instances where the measured concentration was below the detection limit (BDL), but the result was oceanographically consistent and followed the pattern established by results from surrounding sample depths. For large particle Co and Cd, flux estimates for the upper $400 \mathrm{~m}$ were not impacted because only one instance of these BDL results occurred for either metal. For Mn, 18 out of the 121 useable samples in the upper $400 \mathrm{~m}$ were below the $3.03 \mathrm{pM}$ detection limit, with concentrations ranging from $0.99 \mathrm{pM}$ to $2.90 \mathrm{pM}$. The impact of these BDL results on the overall Mn flux estimates was considered negligible, since these results were within 3-fold of the detection limit and the range in BDL results was small compared to the full range in measured values (maximum Mn concentration in upper $400 \mathrm{~m}=148 \mathrm{pM}$ ). On the other hand, 31 of the 119 useable large particle Fe results were BDL (21.7 pM) and 11 of these were $>3$-fold lower than the detection limit. Of these 11 samples, all but one was found in the Gyre and 4 samples were at station 26. Most of these BDL samples were from $<80 \mathrm{~m}$. Therefore, the $100 \mathrm{~m}$ Fe flux estimates and residence times in the Gyre should be viewed as maximum possible values or upper limits.

Large particle E: ${ }^{234} \mathrm{Th}$ ratios are vital to the ${ }^{234} \mathrm{Th}$ method of flux calculation. Because ${ }^{234} \mathrm{Th}$ and POC were sequentially measured from the same pre-filter material and all other elements were determined from material collected with a separate filter head, the non-POC ratios have the potential to be affected by inter-head variability and/or poor particle distribution on a given filter. 
Based on the GEOTRACES inter-calibration study for small particle ${ }^{234}$ Th activities, inter-filter variability was low for quarters of the same QMA and Supor filters, with $2 \%$ and $2.5 \%$ s.d., respectively (Maiti et al., 2012). Smaller volume punches ( 3\% of total volume filters) had up to a s.d. of $17 \%$ between 20 punches of the same filter. This was equivalent to the inter-pump variability observed between 8 pumps on a single deployment ( $8 \%$ to 18\%), so inter-head variability would not be much greater than the variability observed within a single filter. Despite the possibility of occasional differences in the particle distribution on a given filter and the potential for large particle filter variability to be more than that for small particle filters, element concentrations and $\mathrm{E}:{ }^{234} \mathrm{Th}$ ratios along the transect consistently produced the same general patterns within each zone and with depth (Figs. 2 and 3). The strength of these patterns suggested that any uncertainties relating to filter distribution differences were minor. Moreover, a broad analysis of zonal patterns would average out any inter-head differences that could have occurred.

\subsubsection{Biogeochemical regions of the transect}

Three zones have been used here (Fig. 1) to discuss the study results: the Shelf $\left(77^{\circ} \mathrm{W}\right.$ to $80^{\circ}$ $\mathrm{W})$, the Offshore $\left(80^{\circ} \mathrm{W}\right.$ to $\left.100^{\circ} \mathrm{W}\right)$, and the Gyre $\left(100^{\circ} \mathrm{W}\right.$ to $\left.152^{\circ} \mathrm{W}\right)$. The Shelf is an upwellinginfluenced, highly productive coastal zone, while the Gyre is an open ocean, low nutrient, subtropical gyre. The local gyre has an oxygen minimum zone (OMZ) where dissolved $\mathrm{O}_{2}$ drops to a detectable minima ( $4 \%$ to $29 \%$ oxygen saturation) due to weak ventilation and the respiration of sinking organic matter (Karstensen et al., 2008). The average depth of these minima was 340 $\mathrm{m}$, which was well below the average depth of the base of the euphotic zone (Ez) of $170 \mathrm{~m}$ (Supp. Table 1). The Shelf and Offshore, on the other hand, had oxygen deficient waters starting an average depth of $50 \mathrm{~m}$ and $150 \mathrm{~m}$, respectively, where the average Ez were $40 \mathrm{~m}$ and $120 \mathrm{~m}$. Here oxygen deficiency means that the concentration of $\mathrm{O}_{2}$ dropped below detection using traditional methods $\left(\mathrm{O}_{2}<1 \mu \mathrm{M}\right)$ and the upper boundary of the ODZ (uODZ, Black et al., 2017) indicates the approximate depth of change to oxygen deficient redox conditions in the water column. The ODZ waters thinned from $\sim 700 \mathrm{~m}$ below the $\mathrm{uODZ}$ in the Shelf to $\sim 150 \mathrm{~m}$ in the Offshore.

\subsubsection{Flux determination for the elements of interest with the ${ }^{234} \mathrm{Th}-{ }^{238} \mathrm{U}$ disequilibrium method}

The particulate flux of ${ }^{234} \mathrm{Th}$ in $\mathrm{dpm} \mathrm{L}^{-1} \mathrm{~d}^{-1}$ (disintegrations per minute per liter) can be calculated at depth $z$ with the following relationship: 


$$
\left.P_{T h @ z}=\int_{0}^{z}\left(\lambda_{T h}\left({ }^{238} U-{ }^{234} T h\right) \pm w \frac{\partial^{234} T h}{\partial z} \pm u \frac{\partial^{234} T h}{\partial x} \pm v \frac{\partial^{234} T h}{\partial y}\right) d z \text { (Eq. } 1\right)
$$

where ${ }^{234} \mathrm{Th}$ is the activity of total ${ }^{234} \mathrm{Th}$ in $\mathrm{dpm} \mathrm{L} \mathrm{L}^{-1},{ }^{238} \mathrm{U}$ is the salinity-derived activity of uranium, $\lambda_{T h}$ is the decay constant for ${ }^{234} \mathrm{Th}\left(0.0288\right.$ day $\left.^{-1}\right), w$ is the upwelling velocity, $u$ is the zonal velocity, $v$ is the meridional velocity, and the gradients $\left(\partial^{234} \mathrm{Th}\right.$ terms) are vertical, west to east, and south to north, respectively. If the upwelling, zonal, and meridional terms are determined to be negligible and the system is assumed to be in steady state, the flux can be calculated using a simplified version of Eq. 1:

$$
P_{T h @ z}=\int_{0}^{z} \lambda_{T h}\left({ }^{238} U-{ }^{234} T h\right) d z \quad \text { (Eq. 2) }
$$

For most stations, the particulate flux of ${ }^{234} \mathrm{Th}$ was calculated using Eq. 2. However, the locations between $77^{\circ} \mathrm{W}$ and $84^{\circ} \mathrm{W}$ were determined to be impacted by upwelling (Kadko, 2017) and all ${ }^{234} \mathrm{Th}$ fluxes used here for this region have been corrected for these effects (Black et al 2017). The high-resolution measurements of total ${ }^{234} \mathrm{Th}$ allow for the calculation of ${ }^{234} \mathrm{Th}$ flux at any depth in the upper $400 \mathrm{~m}$ by linear extrapolation between sampled depths.

Although the ${ }^{234} \mathrm{Th}$ method has been used infrequently to calculate fluxes for elements other than nitrogen, carbon, and silica, the technique has the potential to be applied to a host of elements associated with marine particles. The particulate flux of an element at depth $z$ can be determined with the following equation:

$$
P_{E @ z}=\frac{[E]}{{ }^{234} T h} P_{T h @ z} \quad(\text { Eq. 3) }
$$

where the ratio of the element concentration to the ${ }^{234} \mathrm{Th}$ activity on sinking particles is multiplied by the particulate flux of ${ }^{234} \mathrm{Th}$ at depth $z$. It is assumed that the $\mathrm{E}:{ }^{.24} \mathrm{Th}$ on large particles $(>51$ $\mu \mathrm{m})$ measured at $z$ is representative of sinking particles (Buesseler et al., 2006). Eq. 3 can be used to calculate an increase or decrease in flux that results from production or remineralization processes. The sampling resolution on this transect (4 to 7 depths from $0 \mathrm{~m}$ to $300 \mathrm{~m}$ ) was sufficient at the majority of stations to capture general patterns in $\mathrm{E}:{ }^{234} \mathrm{Th}$ with depth. Fluxes have been calculated at $100 \mathrm{~m}, 200 \mathrm{~m}$, the uODZ where it existed, and at $100 \mathrm{~m}$ below the uODZ. The 100 $\mathrm{m}$ flux is of particular importance because this region of the Pacific is strongly stratified (strong 
halocline) and winter mixed layers do not generally reach deeper than $100 \mathrm{~m}$ (Fiedler and Talley, 2006). Therefore, any flux passing through $100 \mathrm{~m}$ depth is likely sequestered.

\subsubsection{Determination of the bio-authigenic and lithogenic fractions of element fluxes}

The fraction of the element concentrations that were attributable to a lithogenic origin or to a combination of biological processes and authigenic precipitation ('bio-authigenic') was determined. Average upper continental crust ratios were multiplied by the particle concentration of $\mathrm{Al}$ in a given sample to calculate the lithogenic fraction and the remainder was attributed to bioauthigenic processes. The ratios used included $\mathrm{Cd}: \mathrm{Al}=2.93 \times 10^{-7}, \mathrm{Co}: \mathrm{Al} 5.7 \times 10^{-5}, \mathrm{Fe}: \mathrm{Al}=0.211$,

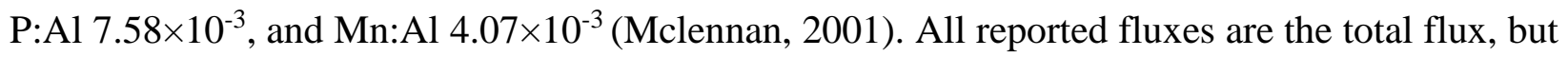
the percentage that was attributed to a lithogenic origin (Supp. Tables 1A to 1E) will be discussed where relevant, with the understanding that there can be some regional deviations from the average crustal ratios.

\subsubsection{Determination of upper ocean residence times}

The removal timescale or residence time of an element, $\tau$, can be determined from:

$$
\tau_{E}=\frac{I_{E}}{P_{E @ z}} \quad \text { (Eq. 4) }
$$

where $I$ is the water column inventory of a given element for a given depth region (e.g. $0 \mathrm{~m}$ to 50 $\mathrm{m})$, generally expressed in $\mathrm{dpm} \mathrm{m}^{-2}$. I can be expressed as a total water column inventory ( $I_{\text {Tot }}$; sum of dissolved and particulate phases), as a particulate inventory ( $I_{\text {Part }}$; large and small particles), or as a sinking inventory ( $I_{S i n k}$; large particle concentration). The application of specific values for $I$ in Eq. 4 yields removal timescales relative to the total $\left(\tau_{T o t}\right)$, particulate $\left(\tau_{\text {Part }}\right)$, and sinking $\left(\tau_{\text {Sink }}\right)$ pool of a given element. Dissolved phosphate and trace metal concentrations utilized here were obtained from BCO-DMO (See Website and Database References). Since the dissolved and particulate sampling equipment was not deployed shallower than $15 \mathrm{~m}$ to $20 \mathrm{~m}$, the inventory for the surface layer is assumed to be the same as the topmost sample and was integrated as such.

\subsection{Results}

This study focuses on the upper $400 \mathrm{~m}$ of the water column where the use of ${ }^{234} \mathrm{Th}$ to estimate element fluxes is well established (Le Moigne et al., 2013; Savoye et al., 2006). As the first basin- 
scale attempt at using ${ }^{234}$ Th to constrain surface fluxes for trace metals, where all parts of Eqs. 3 and 4 were measured together, the observed variability in each input parameter has been evaluated.

\subsubsection{Upper ocean ${ }^{234} \mathrm{Th}$ activities and element concentrations in the large particle phase}

While the largest amount of ${ }^{234} \mathrm{Th}$ in the ocean is usually found in the dissolved phase, the particulate activities can account for over $50 \%$ of the total ${ }^{234}$ Th present in the upper ocean (Black et al., 2017). Along the transect, small particle activities ranged from $0.2 \mathrm{dpm} \mathrm{L}^{-1}$ to $1.6 \mathrm{dpm} \mathrm{L}{ }^{-1}$, making up approximately 10 to $60 \%$ of the total ${ }^{234} \mathrm{Th}(0 \mathrm{~m}$ to $500 \mathrm{~m}$ : mean $=26 \%)$, and large particle activities ranged from $0.01 \mathrm{dpm} \mathrm{L}^{-1}$ to $0.37 \mathrm{dpm} \mathrm{L}^{-1}$, which constituted $<<10 \%$ to $20 \%$ of total ${ }^{234} \mathrm{Th}($ mean $=4 \%)$. The activity of ${ }^{234} \mathrm{Th}$ in the particulate phase generally goes down with depth as particle abundance drops through water column (Fig. 2, panels C and D in Fig.3).

Large particle POC and P decreased non-linearly with depth, on average, from the base of the mixed layer (MLD) to $400 \mathrm{~m}$ (Figs. 2 and 3). POC and P uptake in the surface and remineralization at depth translated to increasing dissolved concentrations and decreasing particulate concentrations with depth. Large particle concentrations just below the MLD varied by an order of magnitude from the Shelf to the Gyre, although the Offshore and Gyre values were very similar at equivalent depths and large particle concentrations in all three zones converged below $200 \mathrm{~m}$.

Of the four trace metals, large particle $\mathrm{Cd}$ and Co showed the most similarity in profile shape to the large particle POC and P. Concentrations of Cd and Co below the MLD varied by an order of magnitude from the Shelf to the Gyre. As expected from the tight coupling of global dissolved concentrations of Cd and P (de Baar et al., 1994), large particle Cd profiles closely paralleled those of P. Compared to POC, P, and Cd profiles, large particle Co showed a more gradual decrease with depth in the Shelf and Offshore (Fig. 2). In the Gyre, the observed values below the MLD decreased until around $200 \mathrm{~m}$, where large particle values began to increase with depth. A secondary maximum the total particulate Co concentrations (large and small particles) was noted by Lee et al. (2017) at stations west of $100^{\circ} \mathrm{W}$ and at depths ranging from $250 \mathrm{~m}$ to $500 \mathrm{~m}$.

Mn concentration profiles deviated the most from those of the other elements. The effects of the dissolution of $\mathrm{Mn}$ oxides by photo-produced $\mathrm{H}_{2} \mathrm{O}_{2}$ (insoluble $\mathrm{Mn}$ reduced to dissolvable forms of Mn) was visible in the low concentrations of large particle Mn in the surface (Lee et al., 2007). Below the MLD, particulate Mn increased in the Shelf and Offshore until the uODZ was reached (Fig. 2). This increase has been attributed to the precipitation of Mn-oxyhydroxides by Mn- 
oxidizing bacteria (Cowen and Bruland, 1985; Ohnemus et al., 2016; Lee et al., 2017). Low particulate Mn concentrations within the ODZ suggested that the particulate Mn (Mn oxyhydroxides) was reduced below the uODZ and this reduction is consistent with redox processes observed on this campaign (Cutter et al., 2017). In the Gyre, particulate Mn started low at the MLD and increased with depth. Individual station profiles showed a distinct increase from the MLD to $\sim 300 \mathrm{~m}$, except at $125^{\circ} \mathrm{W}, 128^{\circ} \mathrm{W}$, and $137^{\circ} \mathrm{W}$ where a maximum in Mn occurred just above Ez that was noticeably larger than the concentrations observed in the $200 \mathrm{~m}$ to $300 \mathrm{~m}$ depth range.

Large particle Fe concentration profiles showed the greatest variability in shape. Although the absolute range in Fe values observed in the Gyre was small ( $0 \mathrm{nM}$ and $0.3 \mathrm{nM})$, almost equal numbers of stations had profiles that increased with depth, decreased only, decreased with a peak above Ez, or decreased with peak below Ez (Fig. 2). Large particle Fe concentrations were an order of magnitude higher in the Shelf, generally increasing from the euphotic zone through the uODZ (Fig 2) and with a local maximum always below the uODZ. As the uODZ depth deepened in the Offshore, the peak in large particle Fe also moved deeper and decreased in absolute concentration from east to west (Fig. 2).

The location of non-surface maxima (peaks found at depths $>20 \mathrm{~m}$ ) was similar for many of the elements and ${ }^{234} \mathrm{Th}$. Two types of particulate, non-surface peaks were observed: those in the Shelf and Offshore associated with the uODZ boundary (Supp. Table 1) and those in the Gyre, which occurred in the zone of remineralization below Ez and above the local oxygen minima (280380 m; Black et al., 2017). All non-surface maxima in POC, P, Cd, Co, and Fe in the Shelf and Offshore were located at $( \pm 5 \mathrm{~m})$ or below the local uODZ, matching a unique feature in large and small particulate ${ }^{234}$ Th that often coincided with local minima in transmission and maxima in fluorescence (Black et al., 2017). The coincidence of ${ }^{234} \mathrm{Th}$ with peaks in Fe was most common, although a few maxima in POC, Co, and $\mathrm{P}$ were also observed with ${ }^{234} \mathrm{Th}$ highs. The Gyre contained relatively small non-surface maxima in ${ }^{234} \mathrm{Th}$ that generally matched with the fluorescence maxima above Ez and not with any of the trace metal peaks. Small non-surface peaks were present in concentration profiles for $\mathrm{P}$, and occasionally $\mathrm{Cd}$, in the depth region between $\mathrm{Ez}$ and the local oxygen minimum. Total ${ }^{234} \mathrm{Th}$ results indicated that net remineralization was occurring in this region of the water column. It should be noted that Fe and $\mathrm{P}$ peaks coincided at some Gyre stations (e.g. station 18 at $112.8^{\circ} \mathrm{W}$ and 21 at $115^{\circ} \mathrm{W}$ ) and not at others (e.g. station 26 at $128^{\circ} \mathrm{W}$, where the non-surface $\mathrm{P}$ peak is most pronounced). 


\subsubsection{Regional E: ${ }^{234} \mathrm{Th}$}

Large particle POC: ${ }^{234}$ Th decreased with depth in the upper $400 \mathrm{~m}$ (Fig. 2, Table 1). Because peaks in POC and ${ }^{234} \mathrm{Th}$ often coincided in the Shelf and Offshore, the resulting POC: ${ }^{234} \mathrm{Th}$ profiles had smaller and fewer peaks. The zonal means of large particle POC: ${ }^{234}$ Th ranged from $2 \mu$ mol $\mathrm{dpm}^{-1}$ to $5 \mu \mathrm{mol} \mathrm{dpm}{ }^{-1}$ in the 'surface layer' (SL, 0 to $100 \mathrm{~m}$ ) and $0.7 \mu \mathrm{mol} \mathrm{dpm}{ }^{-1}$ to $1.3 \mu \mathrm{mol} \mathrm{dpm}^{-}$ ${ }^{1}$ in the 'subsurface layer' (SSL, 100 to $400 \mathrm{~m}$ ), with the highest ratios in the shallow Shelf SL and

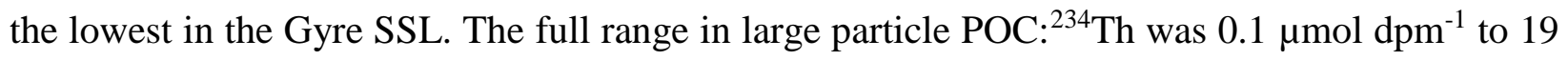
$\mu \mathrm{mol} \mathrm{dpm}{ }^{-1}$. Except in the Gyre SL, large particle POC: ${ }^{234} \mathrm{Th}$ had the smallest zonal ranges relative to all other elements. In the Shelf SL, the 19-fold difference between the lowest and highest value observed was much smaller than the 60- to 120 -fold differences seen for other elements.

Large particle P: ${ }^{234}$ Th profiles also decreased non-linearly with depth (Fig. 2). The occasional non-surface $\mathrm{P}$ peaks that were observed resulted in pronounced maxima in large particle $\mathrm{P}:{ }^{234} \mathrm{Th}$ at five locations in the Offshore and Gyre. The mean SL values ranged from $19 \mathrm{nmol} \mathrm{dpm}^{-1}$ to 150 nmol dpm${ }^{-1}$ and the total range in observed ratios was $2 \mathrm{nmol} \mathrm{dpm}^{-1}$ to $600 \mathrm{nmol} \mathrm{dpm}^{-1}$ (Table 1). The gradient from the Shelf SL to the Offshore SL was greater for P. ${ }^{234}$ Th than all other elements, except Fe: ${ }^{234} \mathrm{Th}$, and was reflected in the large decrease $(77 \%)$ between the mean values for the Shelf and Offshore SL.

Although the large particle $\mathrm{Cd}:{ }^{234} \mathrm{Th}$ were three orders of magnitude lower than those for $\mathrm{P}:{ }^{234} \mathrm{Th}$, the profiles in the Shelf and Offshore looked almost identical and followed a nutrient-like decrease with depth in the Shelf and Offshore (Table 1, Fig. 2). Next to Fe and P ratios, Cd: ${ }^{234} \mathrm{Th}$ had the largest relative range of values observed in the SL, with a $2 \mathrm{pmol} \mathrm{dpm}^{-1}$ to $560 \mathrm{pmol} \mathrm{dpm}^{-}$ ${ }^{1}$ difference from Gyre to Shelf. The means of $\mathrm{Cd}:{ }^{234} \mathrm{Th}$ for the SL were $7 \mathrm{pmol} \mathrm{dpm}{ }^{-1}, 44 \mathrm{pmol}$ $\mathrm{dpm}^{-1}$, and $140 \mathrm{pmol} \mathrm{dpm}^{-1}$ from the Gyre to Shelf, while the SSL averages were much closer at 3 pmol dpm ${ }^{-1}, 9 \mathrm{pmol} \mathrm{dpm}^{-1}$, and $18 \mathrm{pmol} \mathrm{dpm}^{-1}$, respectively. At most Gyre stations, Cd: ${ }^{234} \mathrm{Th}$ increased with depth below $150 \mathrm{~m}$.

Despite the nutrient-like decrease with depth in the upper $100 \mathrm{~m}$ (Fig. 2), large particle $\mathrm{Co}:{ }^{234} \mathrm{Th}$ were similar across the transect (Table 1). The means of the SL and SSL ratios were all between $2 \mathrm{pmol} \mathrm{dpm}^{-1}$ and $8 \mathrm{pmol} \mathrm{dpm}^{-1}$, except for in the Shelf SL (mean $=20 \mathrm{pmol} \mathrm{dpm}^{-1}$ ). Low SL ratios in the Gyre transitioned to elevated Co: ${ }^{234} \mathrm{Th}$ below $200 \mathrm{~m}$. Although still relatively small compared to differences observed in Shelf element ratios, the range in SSL Co: ${ }^{234}$ Th in the Gyre 
(1-21 pmol dpm ${ }^{-1}$ ) was much larger than the SL range of $1 \mathrm{pmol} \mathrm{dpm}^{-1}$ to $5 \mathrm{pmol} \mathrm{dpm}^{-1}$ and more pronounced than the increase in large particle Co concentration alone.

The large particle Mn: ${ }^{234} \mathrm{Th}$ profiles in the Shelf and Offshore generally followed similar patterns to the Mn concentration profiles, decreasing with depth in the Shelf and Offshore and increasing with depth in the Gyre. Ratios for the entire transect ranged from $30 \mathrm{pmol} \mathrm{dpm}^{-1}$ to 2800 pmol dpm ${ }^{-1}$. The Shelf SL $\left(550 \pm 760 \mathrm{pmol} \mathrm{dpm}^{-1}\right)$ and the Gyre SSL $\left(540 \pm 460 \mathrm{pmol} \mathrm{dpm}^{-1}\right)$ had almost identical means, albeit with large s.d. The remaining four water column regions all had

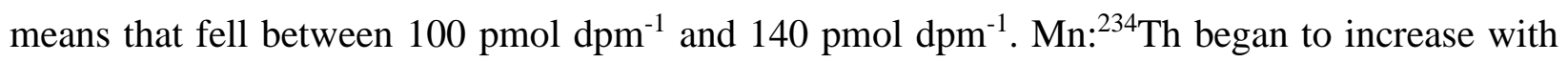
depth in the gyre at around $100 \mathrm{~m}$ depth, shallower than for Co: ${ }^{234} \mathrm{Th}$.

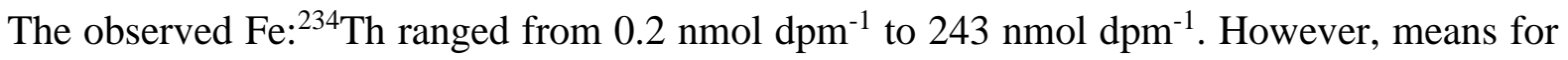
the Offshore and Gyre, ranging from only $1.0 \mathrm{nmol} \mathrm{dpm}^{-1}$ to $1.7 \mathrm{nmol} \mathrm{dpm}^{-1}$, showed that most of the variability in Fe: ${ }^{.234} \mathrm{Th}$ was in the Shelf. Indeed, the average ratio and s.d. of the Shelf SL was $36 \pm 71 \mathrm{nmol} \mathrm{dpm}^{-1}$. Although the Fe: ${ }^{234} \mathrm{Th}$ profiles were highly variable in shape, the observed patterns with depth were much more consistent than those for Fe concentration. With the exception of stations 15 and $17\left(104^{\circ} \mathrm{W}\right.$ and $\left.109^{\circ} \mathrm{W}\right)$, all other stations had increasing Fe: ${ }^{234} \mathrm{Th}$ from near Ez to $400 \mathrm{~m}$. Since Fe and ${ }^{234} \mathrm{Th}$ had frequent non-surface maxima that coincided, it was not surprising that the ratios produced had a more consistent pattern with depth.

\subsection{3 ${ }^{234}$ Th-derived flux estimates}

\subsubsection{1 ${ }^{234}$ Th flux}

Except the stations from the coast $\left(77^{\circ} \mathrm{W}\right.$ to $84^{\circ} \mathrm{W}$ ), whose ${ }^{234} \mathrm{Th}$ flux estimates were adjusted for the impact of upwelling, locations along the transect had relatively similar ${ }^{234} \mathrm{Th}$ fluxes at 100 $\mathrm{m}$ (Black et al., 2017). Stations west of $85^{\circ}$ had ${ }^{234} \mathrm{Th}$ fluxes ranging between $1100 \mathrm{dpm} \mathrm{m}^{-2} \mathrm{~d}^{-1}$ and $2000 \mathrm{dpm} \mathrm{m}^{-2} \mathrm{~d}^{-1}$ (mean $=1540 \mathrm{dpm} \mathrm{m}^{-2} \mathrm{~d}^{-1}$, Supp. Table 1). The Shelf stations had an average $100 \mathrm{~m}$ flux of $6500 \mathrm{dpm} \mathrm{m}^{-2} \mathrm{~d}^{-1}$. At $200 \mathrm{~m}$, the mean values for the Shelf, Offshore, and Gyre were $5700 \mathrm{dpm} \mathrm{m}^{-2} \mathrm{~d}^{-1}, 1250 \mathrm{dpm} \mathrm{m}^{-2} \mathrm{~d}^{-1}$, and $1450 \mathrm{dpm} \mathrm{m}^{-2} \mathrm{~d}^{-1}$, respectively, which reflected an overall decrease in ${ }^{234} \mathrm{Th}$ flux through the 100 to $200 \mathrm{~m}$ zone. Because of the relatively small differences

observed in ${ }^{234} \mathrm{Th}$ fluxes at a particular depth within a given transect zone, the sinking E: ${ }^{234} \mathrm{Th}$ became the most important factor governing the calculated element fluxes.

\subsubsection{Zonal trends in flux}


As expected, POC fluxes between the MLD and $300 \mathrm{~m}$ were generally decreasing with depth at all stations (Fig. 3, Supp. Fig. 1) and there was a decrease across the transect, with Shelf values being the highest (Fig. 4, Supp. Table 2A). The average POC flux at $100 \mathrm{~m}$ for each of the three zones was $9 \mathrm{mmol} \mathrm{m}^{-2} \mathrm{~d}^{-1}, 2.7 \mathrm{mmol} \mathrm{m}^{-2} \mathrm{~d}^{-1}$, and $1.6 \mathrm{mmol} \mathrm{m}^{-2} \mathrm{~d}^{-1}$, with relative standard deviations (RSDs) all below 50\% (Table 2). The fluxes at $100 \mathrm{~m}$ ranged from $1 \mathrm{mmol} \mathrm{m}^{-2} \mathrm{~d}^{-1}$ to $13 \mathrm{mmol} \mathrm{m}^{-}$ ${ }^{2} \mathrm{~d}^{-1}$, with the highest flux calculated at $77.4^{\circ} \mathrm{W}$. POC fluxes at $100 \mathrm{~m}$, determined using individual station POC: ${ }^{234} \mathrm{Th}$, matched within reported uncertainties those determined from a linear regression of POC: ${ }^{234}$ Th with depth for each of the three zones (Black et al., 2017).

The flux of particulate P also decreased with depth (Fig. 3, Supp. Fig. 1), except between $112.8^{\circ}$ $\mathrm{W}$ and $115^{\circ} \mathrm{W}$. The largest $\mathrm{P}$ flux at $100 \mathrm{~m}\left(900 \mu \mathrm{mol} \mathrm{m} \mathrm{m}^{-2} \mathrm{~d}^{-1}\right)$ was observed at $79^{\circ} \mathrm{W}$ and the smallest was in the Gyre at $152^{\circ} \mathrm{W}\left(8.2 \mu \mathrm{mol} \mathrm{m} \mathrm{d}^{-1}\right.$, Supp. Table $\left.2 \mathrm{~B}\right)$. The lowest $200 \mathrm{~m}$ flux was found at $89^{\circ} \mathrm{W}$ (station 9: $1.3 \mu \mathrm{mol} \mathrm{m} \mathrm{m}^{-2}$ ), which was an order of magnitude lower than at 200 $\mathrm{m}$ at the adjacent stations. A 9-fold decrease was observed between the average Shelf $\mathrm{P}$ flux at 100 $\mathrm{m}\left(\sim 300 \mu \mathrm{mol} \mathrm{m} \mathrm{m}^{-2}\right)$ and the average value for the Offshore $\left(33 \mu \mathrm{mol} \mathrm{m} \mathrm{m}^{-2}\right)$. Only Fe had a steeper gradient between these two zones. The RSDs of the mean P fluxes at $200 \mathrm{~m}$ were among the highest of all the elements in all three zones.

Cd fluxes followed the same patterns observed in P fluxes (Fig. 3, Supp. Fig. 1), decreasing with depth except between $112.8^{\circ} \mathrm{W}$ and $115^{\circ} \mathrm{W}$. The relative magnitude of flux change over a given depth interval was occasionally different for $\mathrm{Cd}$ and $\mathrm{P}$. For instance, at $112.8^{\circ} \mathrm{W}$ (station 18) $\mathrm{Cd}$ fluxes followed the depth increases of $\mathrm{Co}, \mathrm{Mn}$, and Fe more closely. Cd fluxes at $100 \mathrm{~m}$ ranged from $2.1 \mathrm{nmol} \mathrm{m} \mathrm{m}^{-1}\left(132.5^{\circ} \mathrm{W}\right)$ to $1000 \mathrm{nmol} \mathrm{m} \mathrm{d}^{-2}\left(79.2^{\circ} \mathrm{W}\right.$, Supp. Table 2C), with zonal averages for the Shelf, Offshore, and Gyre of $291 \mathrm{nmol} \mathrm{m}^{-2} \mathrm{~d}^{-1}, 40 \mathrm{nmol} \mathrm{m} \mathrm{d}^{-1}$, and $6 \mathrm{nmol} \mathrm{m}^{-2} \mathrm{~d}^{-}$ ${ }^{1}$, respectively. The RSDs for the upper $100 \mathrm{~m}(137 \%)$ and $100 \mathrm{~m}$ to $200 \mathrm{~m}(85 \%)$ zones in the Shelf were the largest relative to all other elements.

Co fluxes in the upper $300 \mathrm{~m}$ decreased with depth, similar to those for POC and P, from the coast until $100^{\circ} \mathrm{W}$, where the subsurface ODZ was no longer present (Fig. 3, Supp. Fig. 1). West of $110^{\circ} \mathrm{W}$, Co fluxes increased slightly with depth below $150 \mathrm{~m}$ (Supp. Table 2D). Co fluxes across the transect ranged from $1 \mathrm{nmol} \mathrm{m} \mathrm{d}^{-1}$ to $70 \mathrm{nmol} \mathrm{m}^{-2} \mathrm{~d}^{-1}$, with averages for both $100 \mathrm{~m}$ and $200 \mathrm{~m}$ depth varying by an order of magnitude from the Shelf to the Gyre (Table 2). The average $100 \mathrm{~m}$ fluxes in the Shelf and Offshore were $39 \mathrm{nmol} \mathrm{m}^{-2} \mathrm{~d}^{-1}$ and $10 \mathrm{nmol} \mathrm{m}^{-2} \mathrm{~d}^{-1}$. Although 
the $100 \mathrm{~m}$ and $200 \mathrm{~m}$ means were both $3 \mathrm{nmol} \mathrm{m}^{-2} \mathrm{~d}^{-1}$ in the Gyre, eight of twelve stations had a larger flux at $200 \mathrm{~m}$, reflecting a real increase with depth. RSDs were generally lower or about average compared to other elements for all parameters.

East of $100^{\circ} \mathrm{W}$, Mn fluxes increased from the surface until the uODZ and then the fluxes dropped significantly (Fig. 3, Supp. Fig. 1). Of the eight Shelf and Offshore stations that had quantifiable flux values at the uODZ and uODZ+100, seven showed a sharp decline in flux over this $100 \mathrm{~m}$ layer (Supp. Table 2E). The mean $100 \mathrm{~m}$ flux for the Shelf $\left(\sim 1000 \mathrm{nmol} \mathrm{m}^{-2} \mathrm{~d}^{-1}\right)$ was double the mean $200 \mathrm{~m}$ flux (Table 2). The corresponding values for the Offshore were $\sim 370 \mathrm{nmol}$ $\mathrm{m}^{-2} \mathrm{~d}^{-1}$ and $125 \mathrm{nmol} \mathrm{m} \mathrm{m}^{-2} \mathrm{~d}^{-1}$, respectively, reflecting a 3-fold decrease. The lowest flux observed was at $89^{\circ} \mathrm{W}\left(200 \mathrm{~m}: 10 \mathrm{nmol} \mathrm{m} \mathrm{m}^{-2} \mathrm{~d}^{-1}\right)$ and was an order of magnitude less than all other $200 \mathrm{~m}$ fluxes (Supp. Table 2E). In contrast, the Mn fluxes increased with depth west of $100^{\circ} \mathrm{W}$, with a mean $100 \mathrm{~m}$ flux of $187 \mathrm{nmol} \mathrm{m}^{-2} \mathrm{~d}^{-1}$ and a $200 \mathrm{~m}$ flux of $558 \mathrm{nmol} \mathrm{m}^{-2} \mathrm{~d}^{-1}$.

Fe fluxes were greater than those for all other trace metals (Fig. 4). At both $100 \mathrm{~m}$ and $200 \mathrm{~m}$, the gradient in the mean values from Shelf to Offshore was the largest of all of the elements, with a 23-fold decrease in the $100 \mathrm{~m}$ values and a 31-fold decrease at $200 \mathrm{~m}$ (Table 2). The Shelf, Offshore, and Gyre flux means at $100 \mathrm{~m}$ were $61 \mu \mathrm{mol} \mathrm{m} \mathrm{d}^{-1}, 3 \mu \mathrm{mol} \mathrm{m} \mathrm{m}^{-1}$, and $1.6 \mu \mathrm{mol} \mathrm{m}$ $\mathrm{d}^{-1}$ and the total range in $100 \mathrm{~m}$ fluxes of Fe ranged from $0.7 \mu \mathrm{mol} \mathrm{m} \mathrm{m}^{-2} \mathrm{~d}^{-1}$ to $100 \mu \mathrm{mol} \mathrm{m}^{-2} \mathrm{~d}^{-1}$. Similar to Mn, the lowest flux value found along the transect was $0.4 \mu \mathrm{mol} \mathrm{m}^{-2} \mathrm{~d}^{-1}$ at $89^{\circ} \mathrm{W}$ (Supp. Table 2F). Although the RSDs on the mean zonal values were high (Table 2), it appeared from individual station data (Supp. Table 2E) that fluxes generally increased with depth in the Shelf and Gyre. Locations that had rather large increases in flux with depth were in the Shelf at $79.2^{\circ} \mathrm{W}$, and in the Gyre at $112.8^{\circ} \mathrm{W}, 115^{\circ} \mathrm{W}, 147.5^{\circ} \mathrm{W}$ (stations $1,18,21$, and 34 ).

Although all elements had large fluxes at $100 \mathrm{~m}$ in the Shelf and decreasing values to the west, the across transect pattern was most similar for POC, Cd, P, and Co (Supp. Fig. 2). POC and P had a more gradual decrease to the west, whereas $\mathrm{Cd}$ and Co declined rapidly. When compared to the flux patterns of the other four elements, Mn and Fe fluxes at $100 \mathrm{~m}$ remained relatively consistent across the Offshore and Gyre. In the Shelf, POC fluxes at $100 \mathrm{~m}$ decreased gradually from the coast to $80^{\circ} \mathrm{W}$. P, Cd, Co, and $\mathrm{Mn}$ had decreasing fluxes until $78.2^{\circ} \mathrm{W}$ (station 5) and at $79.2^{\circ} \mathrm{W}$ (station 1), the fluxes at $100 \mathrm{~m}$ increased to the highest values observed at $100 \mathrm{~m}$ on the transect. Fe fluxes in the Shelf gradually decreased to $78.2^{\circ} \mathrm{W}$ and showed a small increase at the next 
station. In the Gyre, there were two regions where the $100 \mathrm{~m}$ fluxes were locally elevated (higher than the surrounding stations) for all elements, but Fe: $104^{\circ} \mathrm{W}$ to $125^{\circ} \mathrm{W}$ (stations 15 to 25 ) and $142^{\circ} \mathrm{W}$ to $147.5^{\circ} \mathrm{W}$ (stations 32 to 34 ). The patterns in Fe fluxes only matches these highs at some stations.

\subsubsection{Flux Ratios}

The ratio of the flux of a given element between two depth horizons can be used to describe how fluxes of elements may be transferred through or transformed between two depths in the water column. In this study, the flux ratio has been calculated by dividing the flux of an element at 200 $\mathrm{m}$ by the flux at $100 \mathrm{~m}$ (Fig. 5). As noted earlier, winter mixed layers do not generally reach deeper than $100 \mathrm{~m}$ (Fiedler and Talley, 2006) and the deepest Ez are slightly under $200 \mathrm{~m}$. For a strictly biological component like POC, the flux ratio can be looked as a carbon transfer efficiency over this $100 \mathrm{~m}$ depth zone. For elements like $\mathrm{Mn}$ and Fe, whose flux can be impacted by additional processes, such as scavenging, in-situ precipitation, and dissolution, the flux ratio is used as an indicator of whether there has been a loss from the sinking particle pool (flux ratio <1) or an increase in particulate flux (flux ratio $>1$ ) between these two depths.

The average flux ratio for POC in the Shelf was higher than in the other two zones and $\sim 1$, suggesting that there was no attenuation of POC through this zone (Table 2). In the Offshore and Gyre, all the flux ratios for POC were below 1, with zonal means of 0.5 and 0.7 , respectively, and a range of 0.1 to 0.9 (Supp. Table 2A). Other parameters were used to quantify transfer efficiencies with respect to surface productivity in Black et al. (2017), but an assessment of the flux ratios resulted in the same conclusion: there was substantial POC remineralization below Ez in this region of the Pacific and the Offshore had a lower mean transfer efficiency than the Gyre.

The flux ratios for $\mathrm{P}$ and $\mathrm{Cd}$ were almost always $<1$, reflecting that remineralization was the dominant process affecting particulate P and Cd from $100 \mathrm{~m}$ to $200 \mathrm{~m}$ (Fig. 5, Supp. Tables 2B and 2C). Occasional subsurface peaks, like at stations 11 and $18\left(94^{\circ} \mathrm{W}\right.$ and $\left.112.8^{\circ} \mathrm{W}\right)$, led to local flux ratios that were over 1. The range in flux ratios observed was 0 to 1.3 for $\mathrm{P}$ and 0 to 1.5 for $\mathrm{Cd}$, with the lowest values found at $89^{\circ} \mathrm{W}$. The mean flux ratios for $\mathrm{P}$ and $\mathrm{Cd}$ were all within 1 s.d. for all three zones (Table 2). Unlike POC, which had RSDs under $60 \%$ for these two zones, the variability in flux ratios for P and Cd translated to RSDs of $\sim 100 \%$ in the Shelf and Offshore. 
The patterns in flux ratios for Co were most similar to those for POC in the Shelf and Offshore and diverged in the Gyre (Fig. 5). The full range of flux ratios for Co was 0.1 to 2.1 (Supp. Table 2D). Flux ratios varied little in the Shelf with a mean and s.d. of $1.0 \pm 0.1$ (Table 2). Offshore ratios ranged from 0.1 to 0.7 , indicating that remineralization was dominant here. In the Gyre, the range in Co flux ratios was 0.8 to 2.1 and the mean was $1.4 \pm 0.4$. These results reflected a distinct shift in the processes governing the distributions of Co fluxes with depth in the Gyre. Although remineralization was occurring between $100 \mathrm{~m}$ and $200 \mathrm{~m}$, as indicated by decreasing POC fluxes, the cumulative impact of all processes produced a net addition of Co to the sinking pool (Fig. 5).

Flux ratios for Mn followed a similar pattern as for Co (Fig. 5). Although RSDs were high in the Shelf and Offshore, zonal means of $0.7 \pm 0.6$ and $0.4 \pm 0.4$, respectively, suggested that $\mathrm{Mn}$ was released from the large particle pool between $100 \mathrm{~m}$ and $200 \mathrm{~m}$. Above the uODZ, Mn(IIIIV)-oxyhydroxides could precipitate and below the uODZ these particulate phases were reductively dissolved (Ohnemus et al., 2016). The flux of Mn decreased from the uODZ to uODZ+100 at 7 of 8 stations between $77.7^{\circ} \mathrm{W}$ to $99^{\circ} \mathrm{W}$. For this reason, the flux ratio was also assessed between these two depths and the average flux ratio (flux at uODZ+100:flux at uODZ) was $0.5 \pm 0.4$ (Supp. Table 2E). In the Gyre, the average Mn flux ratio increased to $3.4 \pm 1.2$ and values ranged from 1.2 to 5.3. All Gyre flux ratios were a result of an increase in $\mathrm{Mn}$ in the large particle pool (Table 2, Supp. Table 2E).

Fe flux ratios were the most variable (range $=0.3$ to 5.0 ) and generally indicated an increasing large particle Fe flux with depth across the transect (Fig. 5, Supp. Table 2F). Zonal means for the Shelf, Offshore, and Gyre were 2.3, 0.9, and 1.9 (Table 2), suggesting that remineralization or a loss of Fe from the large particle pool was more prevalent in the Offshore zone. There was no clear pattern when considering the change in flux between the uODZ and $100 \mathrm{~m}$ below it, as with Mn, however this is consistent with findings that the redox potential was low enough for $\mathrm{Mn}$ in the ODZ, but not for dissimilatory Fe reduction (Supp. Table 2F; Cutter et al., 2017). The corresponding flux ratio for these depths was $1.0 \pm 0.7$.

\subsubsection{Bio-authigenic and lithogenic components of sinking flux}

$\mathrm{P}$ and $\mathrm{Cd}$ concentrations at $100 \mathrm{~m}$, and therefore $\mathrm{P}$ and $\mathrm{Cd}$ flux, were $\leq 1 \%$ lithogenic at all stations (Table 2, Supp. Table 2). The lithogenic fraction of Co flux at $100 \mathrm{~m}$ rose to $19 \%$ near the coast, with an average Shelf value of $9 \%$, but on average Co flux at $100 \mathrm{~m}$ across the entire transect 
was $96 \%$ bio-authigenic (Supp. Table 2D). Overall, the flux of P, Cd, and Co did not change with respect to the percent lithogenic fraction from $100 \mathrm{~m}$ to $200 \mathrm{~m}$, although Co flux was a few percent more lithogenic at $200 \mathrm{~m}$ (Supp. Table 2D) and the lithogenic percent at $99^{\circ} \mathrm{W}$ was $\sim 30 \%$ for $\mathrm{Cd}$ and $\mathrm{P}$ at $200 \mathrm{~m}$ (Supp. Tables 2B and 2C).

Mn and Fe fluxes, on the other hand, had substantial lithogenic fractions. Large particle Mn most likely originated from dust or coastal shelf fluxes east of $78^{\circ} \mathrm{W}$ because the lithogenic fraction of the large particle Mn was $>50 \%$ in the Shelf at $100 \mathrm{~m}$ (Supp. Table 2E). The average Mn flux at $100 \mathrm{~m}$ across the rest of the transect was 98\% bio-authigenic. Unlike Co and Mn, which had elevated lithogenic values in the Shelf only, the percentage of lithogenic Fe flux at $100 \mathrm{~m}$ was consistently high across the transect, ranging from 6 to $42 \%$ (mean $=$ of $17 \pm 10 \%$, Table 2, Supp. Table 2E). At $200 \mathrm{~m}$ the transect average was $19 \pm 7 \%$. These elevated values across the full transect suggested that direct dust inputs likely contributed around 10-20\% of the total sinking flux of particulate Fe.

\subsubsection{Residence time estimates}

Residence times are summarized here as they relate to the cycling of elements through the large particle pool $\left(\tau_{\text {Sink }}\right)$ and the total pool $\left(\tau_{\text {Tot }}\right)$. Residence times for the particulate pool, have been included for reference in Table 3 . All $\tau_{\text {Sink }}$ and $\tau_{\text {Tot }}$ were included in the statistical plots shown in Figs. 6A and 6B, however, the zonal means (Table 3) did not incorporate individual station estimates if they were flagged as outliers and far exceeded typical residence time for that pool. For example, since all other $\tau_{\text {Sink }}$ for Cd ranged from 0 days to 30 days, the $\tau_{\text {Sink }}$ of $\sim 120$ days at station 28 was considered anomalous. Three anomalous values, this one for $\mathrm{Cd}$ and two for Fe (Fig. 6A), were removed from all average $\tau_{\text {Sink }}$ and $\tau_{\text {Tot }}$ estimates reported in Table 3.

The majority of $\tau_{\text {Sink }}$ for all elements ranged from 0.5 days to 26 days (Fig. 6A). The mean $\tau_{\text {Sink }}$

for each zone was similar for POC, P, Cd, and Co (Table 3) and in the Shelf ranged from 5 days to 10 days for these four elements. In the Offshore, the mean $\tau_{\text {Sink }}$ were higher at 13 days to 16 days, while the Gyre residence times ranged from 8 days to 11 days. Mn and Fe, on the other hand, had shorter mean $\tau_{\text {Sink. The }} \tau_{\text {Sink }}$ for Fe was relatively consistent across the entire transect (3 days to 4 days, Table 3), while Mn $\tau_{\text {Sink }}$ values were higher, on average, in the Shelf and Offshore than in the Gyre. The longest $\tau_{\text {Sink }}$ for all elements were found in the Offshore. 
The $\tau_{\text {Tot }}$ were much longer than the $\tau_{\text {Sink }}$ due to the large dissolved inventories of most elements (Fig. 6B, Supp. Fig. 3). $\tau_{\text {Tot }}$ were generally on the order of years (Table 3), although Fe had a short $\tau_{\text {Tot }}$, ranging from 0.01 years to 0.1 years (5-47 days). The zonal means of $\tau_{\text {Tot }}$ for Fe were 0.03 years (Shelf) and 0.07 years (Offshore and Gyre). The particulate inventory of Fe was, on average, $65 \%$ of the total inventory and large particle fraction averaged $23 \%$. The $\tau_{\mathrm{Tot}}$ of $\mathrm{Cd}, \mathrm{Mn}$, and Co were much longer and ranged from 0.1 years to 5.1 years. Values for P ranged from 0.5 years to 12.9 years. For P, Co, and Mn, the mean of the Offshore residence times was the longest and for $\mathrm{Cd}$, the largest mean was found in the Shelf.

\subsection{Discussion}

\subsubsection{Regional trends in ${ }^{234} \mathrm{Th}$ variability}

${ }^{234} \mathrm{Th}$ production by ${ }^{238} \mathrm{U}$ and its short half-life limit the possible activities of ${ }^{234} \mathrm{Th}$ in the surface ocean. Total ${ }^{234} \mathrm{Th}$ dropped to $0.5 \mathrm{dpm} \mathrm{L}^{-1}$ in the Shelf and was elevated to $2.95 \mathrm{dpm} \mathrm{L} \mathrm{L}^{-1}$ just below Ez in the Offshore. Large particle ${ }^{234}$ Th activities in the upper $400 \mathrm{~m}$ ranged from 0.01 $\mathrm{dpm} \mathrm{L} \mathrm{L}^{-1}$ to $0.4 \mathrm{dpm} \mathrm{L} \mathrm{L}^{-1}$ (mean $=0.1 \mathrm{dpm} \mathrm{L}^{-1}$ ), which amounted to $<1 \%$ to $22 \%$ of total ${ }^{234} \mathrm{Th}$. This

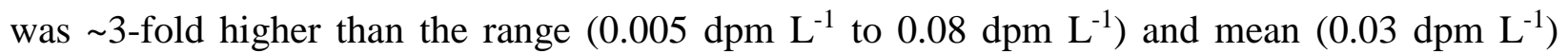
observed during the Atlantic GA03 cruise (Owens et al., 2015). Although inter-basin differences exist and seasonal changes will impact intra-basin variability, the range in total ${ }^{234} \mathrm{Th}$ values observed on these large campaigns is small compared to the orders of magnitude variations in trace metal concentrations observed on the Pacific cruise alone. This suggests that ${ }^{234} \mathrm{Th}$ activities are not the predominant source of variability in $\mathrm{E}:{ }^{234} \mathrm{Th}$ ratios and the subsequent flux calculations.

\subsubsection{Element concentrations in large particles}

Until the start of the GEOTRACES program, size-fractionated particulate measurements of trace elements were rare (e.g. Bishop et al., 1977; Frew et al., 2006; Weinstein and Moran, 2004) and often limited to a select few metals (e.g. Bishop and Fleisher, 1987; Lam et al., 2006) or a single location (e.g. Cullen and Sherrell, 1999). Methodological differences, such as the filter size for 'sinking' particles, can further complicate historical comparisons. However, prior studies will be utilized when possible to put the regional trends from this transect in a global context.

Large particle concentrations for POC (0 $\mu \mathrm{M}$ to $1.5 \mu \mathrm{M}$; Lam et al., 2015), P (0 nM to 40 nM; Twining et al., 2015), Cd (0 pM to 8 pM; Twining et al., 2015), and Co (0 pM to 5 pM; 
Twining et al., 2015) from the upper $400 \mathrm{~m}$ in the North Atlantic were similar in magnitude to the results from this transect (Fig. 2). Comparable concentrations of large particle Fe have been found in the waters of the subarctic Pacific (0.01 nM to $2.5 \mathrm{nM}$; Lam et al., 2006) and the Gulf of Maine, Scotian Shelf, and Labrador Sea (0 nM to $30 \mathrm{nM}$; Weinstein and Moran, 2004). The range observed for large particle $\mathrm{Mn}$ in the upper $400 \mathrm{~m}$ was the only one which did not encompass the general range observed elsewhere. Upper ocean samples from both historical (Bishop and Fleisher, 1987) and recent (Twining et al., 2015) measurements of the North Atlantic have shown large particle Mn surpassing $1000 \mathrm{pM}$. These values were much larger than the maximum large particle surface value from this transect $(150 \mathrm{pM})$, despite the similarity in dissolved surface concentrations $(1 \mathrm{nM}$ to $5 \mathrm{nM}$ ) that were measured along both the North Atlantic and Pacific campaigns (Hatta et al., 2015; Resing et al., 2015). This difference in large particle Mn concentrations might be attributed to a combination of the larger Mn inputs from dust and rivers in the Atlantic versus the Pacific (van Hulten et al., 2017), differing characteristics of coastal waters in the two basins (e.g. higher concentrations of humics in the North Atlantic promoting oxidation), and the reduction of $\mathrm{Mn}$ within the ODZ at the coastal Pacific stations, where particulate Mn would be expected to reach higher concentrations in the absence of an ODZ.

Large particle POC, $\mathrm{P}$, and Cd concentration profiles in all three zones reflect the production of particles in the sunlit upper ocean and remineralization of these constituents as particles sank downwards. There were small deviations near the uODZ from the typical decrease with depth, however, POC and P are useful biological endmembers because they are almost exclusively associated with biological matter in the upper ocean and the relationships of POC, PON, and P on this transect matched the general stoichiometry of plankton (Lam et al., 2017). The elevated levels of $\mathrm{P}, \mathrm{Cd}$, and Co near the uODZ have been shown to be in excess of those that would be expected as a result of Fe-oxyhydroxide scavenging leading to the suggestion that the uODZ-associated peaks in these elements resulted from luxury uptake by local communities (Ohnemus et al., 2016; Lee et al., 2017). Large particle Cd concentration profiles mirrored those of $\mathrm{P}$ and thus, while a trace metal, $\mathrm{Cd}$ in the large particle pool behaved like a biological component. The steeper decline in large particle $\mathrm{P}$ and $\mathrm{Cd}$ concentrations compared to $\mathrm{POC}$ also reflected the shorter remineralization length scale of $\mathrm{P}$ and Cd (Fig. 2; Lam et al., 2017).

$\mathrm{Co}, \mathrm{Mn}$, and $\mathrm{Fe}$ each had uniquely non-biological trends in one or all transect zones. Large particle Co profiles followed the nutrient-like pattern described above in all regions except below 
$150 \mathrm{~m}$ in the Gyre. The increase in large particle Co below $150 \mathrm{~m}$ here could result from scavenging onto large particles as a result of the co-oxidation of Co and Mn (Moffett and Ho, 1996) or the aggregation of smaller particles (Lam et al., 2017). Large particle Mn decreased with depth at stations east of $99^{\circ} \mathrm{W}$, where redox conditions below the uODZ led to the substantial decline in particulate Mn, and increased with depth in the Gyre, where Mn-oxyhydroxides were able to form with no ODZ. While this precipitation can be attributed to Mn-oxidizing bacteria (as can the co-precipitation of $\mathrm{Co}$ ), the resulting particulate concentration profiles do not follow the pattern of a biologically-utilized nutrient because of surface photo-inhibition. Similarly, Fe does not display the characteristics common to the biological elements and the observed variability in Fe profiles likely stems from the fact that large particle Fe distributions along the transect were governed by a combination of lithogenic sources (i.e. dust and coastal fluxes), internal biological particle cycling (i.e. production and remineralization), and authigenic precipitation (Lee et al., 2017).

\subsubsection{Large particle E: ${ }^{234} \mathrm{Th}$}

For the nutrient-like elements, the most variability in $\mathrm{E}:{ }^{234} \mathrm{Th}$ was observed in the shallow Shelf (Table 1). RSDs were lowest here for POC at 100\% and highest for Cd at 120\% (Supp. Table 2). While RSDs dropped between $45 \%$ and $60 \%$ in the surface layer Offshore and Gyre for POC, P, and $\mathrm{Co}, \mathrm{Cd}:{ }^{234} \mathrm{Th}$ RSDs ranged from $70 \%$ to $115 \%$ here. In general, POC and P had the most consistent profiles with depth and smallest $\mathrm{E}^{234} \mathrm{Th}$ ranges in the subsurface which demonstrated that biological processes were the major determinant (Fig. 2). Co: $:{ }^{234} \mathrm{Th}$ and $\mathrm{Cd}^{234} \mathrm{Th}$ showed less consistency, especially in the Gyre where $\mathrm{E}:{ }^{234} \mathrm{Th}$ tended to increase for both elements in the SSL. Greater variability in $\mathrm{Co}$ and $\mathrm{Cd}$ ratios supports some influence of scavenging, however, the $\mathrm{E}:{ }^{234} \mathrm{Th}$ profiles generally reflected production and remineralization processes.

The shape of the $\mathrm{Mn}:{ }^{234} \mathrm{Th}$ profiles in the Shelf and Offshore, like the particulate $\mathrm{Mn}$ concentration profiles, suggest an increased presence of Mn-oxidizing bacteria in the low-light surface waters (increased particulate $\mathrm{Mn}:{ }^{234} \mathrm{Th}$ with depth) and reflect the change in redox conditions at the uODZ (drop in particulate $\mathrm{Mn}:{ }^{234} \mathrm{Th}$ ). $\mathrm{Mn}:{ }^{234} \mathrm{Th}$ in the Gyre increased with depth, suggesting that the scavenging mechanisms impacting Co (and potentially $\mathrm{Cd}$ ) were also influencing Mn. Compared to the mean Mn: ${ }^{234} \mathrm{Th}$ in the Gyre SL, the SSL mean was 5-fold larger, 
demonstrating the constraints of photo-inhibition in the surface on the precipitation of Mn oxides and the influence of Mn-oxidizing bacterial processes through the SSL layer.

The variability in Fe: ${ }^{234}$ Th was among the highest observed in the SL and SSL. The Shelf RSDs, in particular, were the largest of all elements at 200\% in the SL and $86 \%$ in the SSL (Table 1). Despite the wide range in ratios, $\mathrm{Fe}:{ }^{234} \mathrm{Th}$ generally increased with depth, suggesting that similar processes govern the distribution of $\mathrm{Fe}$ in all zones. In contrast to the decrease observed for all other $\mathrm{E}:{ }^{234} \mathrm{Th}$ through the uODZ, Fe: ${ }^{234} \mathrm{Th}$ increased with depth. This consistent trend was not seen in the large particle Fe profiles alone. It is unclear whether Fe-oxyhydroxides directly scavenged the ${ }^{234} \mathrm{Th}$ or if additional biochemical processes were at work in the uODZ that would impact large particle Fe and ${ }^{234} \mathrm{Th}$ in the same fashion.

\subsubsection{On the use of large versus small particle $\mathrm{E}:{ }^{234} \mathrm{Th}$}

Differences in the $\mathrm{E}:{ }^{234} \mathrm{Th}$ ratios between the large particle and small particle pool could also impact flux estimates if the small particle pool $(1-51 \mu \mathrm{m})$ was sinking extremely slowly, but substantially contributing to the local particle flux. Recent sediment trap work has suggested that smaller, slow sinking particles could contribute to the overall flux in oligotrophic regions (Durkin et al., 2015; Hung et al., 2012; Puigcorbé et al., 2015). A previous study in the northwestern Pacific also showed elevated $\mathrm{Mn}$ in subsurface traps that could have resulted from small, slow sinking Mn-rich particles that originate from large lateral shelf plumes (Lam and Bishop, 2008; Lamborg et al., 2008). Further study in a variety of biogeochemical regions is necessary to determine (1) whether the small particles play a substantial role in the total regional flux for a give site and season and (2) whether the large and small particle $\mathrm{E}:{ }^{234}$ Th ratios differ enough that varying contributions of each could change the total flux derived from ${ }^{234} \mathrm{Th}$. The former question could be addressed by a sampling campaign employing both trap and ${ }^{234} \mathrm{Th}$ methods. The latter can be assessed here using a comparison of derived $\mathrm{E}:{ }^{234} \mathrm{Th}$ ratios for both size fractions.

Plotting the large particle $\mathrm{E}:{ }^{234} \mathrm{Th}$ versus the small particle $\mathrm{E}:{ }^{234} \mathrm{Th}$ from the same depth demonstrates the potential impact (if any) that using the small particle ratio or a combination of small and large particle ratios could have on the flux estimates for each element (Fig. 7). If a point falls above the 1:1 line, it suggests that a flux calculated at this depth with a large particle $\mathrm{E}:{ }^{234} \mathrm{Th}$ ratio would underestimate the total flux, but only if the small particles were in fact a significant contributor to the total flux. The inverse is true for points falling near the lower dotted line. Because 
the $100 \mathrm{~m}$ and $200 \mathrm{~m}$ data for POC, P, Cd, and Co generally fall about the solid black line (1:1), for the transect data as a whole, the large and small particle $\mathrm{E}:{ }^{234} \mathrm{Th}$ ratios would produce the same regional total flux estimates for these four elements. On the other hand, the large and small particle $\mathrm{E}:{ }^{234} \mathrm{Th}$ for $\mathrm{Mn}$ and Fe suggest that further study is needed to determine whether small particles could be a significant contribution to the flux of $\mathrm{Mn}$ and Fe. The data for these elements in Fig. 7 cluster closer to the 1:2 line, where the small particle $\mathrm{E}:{ }^{234} \mathrm{Th}$ ratios are $50 \%$ smaller than the large particle ratios. This ratio difference would be important if the small particles were a substantial contributor to the total flux.

\subsubsection{Global variability in $\mathrm{E}:{ }^{234} \mathrm{Th}$ ratios}

Although mixed layer POC: ${ }^{234}$ Th have been shown to vary substantially (Buesseler et al., 2009; Passow et al., 2006), this Pacific campaign and the Atlantic GEOTRACES cruises have illustrated that subsurface POC: ${ }^{234} \mathrm{Th}$ ratios in ocean gyres seem to converge into a relatively small range (Black et al., 2017; Owens et al., 2015). For example, in the North and South Atlantic gyres ratios

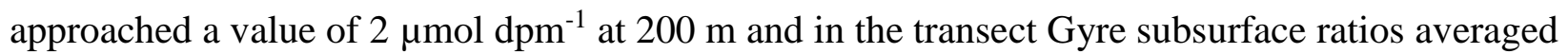
$0.7 \pm 0.1 \mu \mathrm{mol} \mathrm{dpm}{ }^{-1}$ (Ez to $400 \mathrm{~m}$ ). This suggests that for elements that behave like POC, it might be possible to sample at fewer intervals or to use equations to extrapolate $\mathrm{E}:{ }^{234} \mathrm{Th}$ in a given region. Conversely, the variability observed for the other $\mathrm{E}:{ }^{234} \mathrm{Th}$, whether across the entire transect (e.g. for $\mathrm{Fe}$ ) or in a single region (e.g. Cd in the Gyre), suggested that high resolution sampling is still needed for most elements that have not been as extensively studied as POC.

Our understanding of the processes that impact the partitioning of trace metals (including

${ }^{234} \mathrm{Th}$ ) into the various particulate size fractions is still developing (Lam et al., 2015; Ohnemus and Lam, 2015; Twining et al., 2015; Lee et al., 2017; Lam et al., 2017). Except for the literature on POC: ${ }^{.234} \mathrm{Th}$ (e.g. Buesseler et al., 2006), only a few other studies have published E: ${ }^{234} \mathrm{Th}$ (Lemaitre et al., 2016; Smith et al., 2014; Weinstein and Moran, 2005). Large particle Fe: ${ }^{234}$ Th from these studies cover limited areas, including the Kerguelen Plateau (3 to $59 \mathrm{nmol} \mathrm{dpm}^{-1}$ ), the Scotian Shelf and Gulf of Maine ( 0 to $800 \mathrm{nmol} \mathrm{dpm}{ }^{-1}$ ), and the Labrador Sea (0 to $40 \mathrm{nmol} \mathrm{dpm}^{-1}$ ), respectively. $\mathrm{Cd}:{ }^{234} \mathrm{Th}$, ranging from 0 to $350 \mathrm{pmol} \mathrm{dpm}^{-1}$, were also obtained for the Scotian Shelf and Gulf of Maine.

\subsubsection{Particulate fluxes in the eastern tropical South Pacific}


The discussion of particulate flux results is grouped in to three sections according to the most important factors governing the magnitude and distribution of fluxes for each element. Although Co data from the Gyre suggest that scavenging could also be important for increasing fluxes from $100 \mathrm{~m}$ to $200 \mathrm{~m}$, POC, P, Cd, and Co fluxes appear to be most strongly controlled by production and remineralization. Fe and $\mathrm{Mn}$ are discussed separately because the redox conditions in this region of the Pacific uniquely impact $\mathrm{Mn}$, even though both elements are strongly controlled by precipitation and/or scavenging. An element to element assessment of the relative importance of these factors is facilitated by the use of the flux ratio (Fig. 5), but it is important to note that with only three stations in the Shelf with depths $>200 \mathrm{~m}$, each value is weighted heavily.

Comparisons to other regional studies have been made when appropriate, however, only four studies have been published that simultaneously sampled total ${ }^{234} \mathrm{Th}$ fluxes and large particle E: ${ }^{234}$ Th ratios (Lemaitre et al., 2016; Planquette et al., 2011; Smith et al., 2014; Weinstein and Moran, 2005). These studies have reported only Cd and Fe fluxes (Fig. 1). Comparison to these studies and those utilizing traps is complicated by sampling differences, including the deployment depths. Therefore, although these previous studies have been used to put the Pacific fluxes in a global context, the methodological, seasonal, and regional differences may be responsible for some of the observed variability.

\subsubsection{1 'Biological' element fluxes}

The ${ }^{234}$ Th-derived POC fluxes have been shown to be consistent with model outputs for this region (Black et al., 2017) and P, Cd, and Co fluxes from the following prior studies suggest that our fluxes are reasonable. No comparable ${ }^{234} \mathrm{Th}$-based studies for $\mathrm{P}$ are presently published. The Offshore and Gyre fluxes of $\mathrm{P}$ at $100 \mathrm{~m}$ and $200 \mathrm{~m}\left(1.3 \mu \mathrm{mol} \mathrm{m} \mathrm{m}^{-2} \mathrm{~d}^{-1}\right.$ to $\left.57 \mu \mathrm{mol} \mathrm{m} \mathrm{m}^{-1}\right)$ were slightly higher than those observed in the North Atlantic gyre using traps at $150 \mathrm{~m}(0.95 \mu \mathrm{mol} \mathrm{m}$ ${ }^{2} \mathrm{~d}^{-1}$ to $4.7 \mu \mathrm{mol} \mathrm{m}{ }^{-2} \mathrm{~d}^{-1}$; Stanley et al., 2004) and the Shelf P fluxes (46 $\mu \mathrm{mol} \mathrm{m}^{-2} \mathrm{~d}^{-1}$ to $900 \mu \mathrm{mol}$ $\mathrm{m}^{-2} \mathrm{~d}^{-1}$ ) were much lower than $150 \mathrm{~m}$ trap fluxes observed in the Benguela upwelling region (800 $\mu \mathrm{mol} \mathrm{m} \mathrm{m}^{-1}$; to $2300 \mu \mathrm{mol} \mathrm{m}{ }^{-2} \mathrm{~d}^{-1}$; Noble et al., 2012). The only published ${ }^{234} \mathrm{Th}$-based Cd fluxes (Fig. 1; Smith et al., 2014) are relatively similar $\left(1 \mathrm{nmol} \mathrm{m}^{-2} \mathrm{~d}^{-1}\right.$ to $\left.31 \mathrm{nmol} \mathrm{m}^{-2} \mathrm{~d}^{-1}\right)$ at $50 \mathrm{~m}$ to those of the Offshore and Gyre regions. Shelf fluxes of Cd, ranging from $47 \mathrm{nmol} \mathrm{m}^{-2} \mathrm{~d}^{-1}$ to $1000 \mathrm{nmol}$ $\mathrm{m}^{-2} \mathrm{~d}^{-1}$, are high compared to all other previous studies using traps or ${ }^{234} \mathrm{Th}$ (Fig. 1; Migon et al., 2002; Pohl et al., 2004; Schüßler et al., 1997). Fluxes from a Co study using estimated large particle 
Co: ${ }^{234} \mathrm{Th}$ ratios from the western Atlantic (Dulaquais et al., 2014), traps from the Benguela Upwelling region, and traps from the northwestern Pacific (Lamborg et al., 2008) all fall in between $2 \mathrm{nmol} \mathrm{m} \mathrm{d}^{-1}$ and $30 \mathrm{nmol} \mathrm{m} \mathrm{m}^{-2} \mathrm{~d}^{-1}$. These Co flux ranges are close to those found on this transect at $100 \mathrm{~m}$ and $200 \mathrm{~m}\left(0.7 \mathrm{nmol} \mathrm{m}^{-2} \mathrm{~d}^{-1}\right.$ to $\left.70 \mathrm{nmol} \mathrm{m}{ }^{-2} \mathrm{~d}^{-1}\right)$.

POC, P, Cd, and Co all showed a strong offshore gradient in $100 \mathrm{~m}$ fluxes between the Shelf and the Gyre (Fig. 4, Supp. Fig. 2) paralleling the satellite-based 16-day NPP estimates that decreased from 188 to $34 \mathrm{mmolC} \mathrm{m}{ }^{-2} \mathrm{~d}^{-1}$ from the coast to $152^{\circ} \mathrm{W}$ (Black et al., 2017). The smallest gradient in $100 \mathrm{~m}$ fluxes was observed for POC, while the flux at $100 \mathrm{~m}$ for $\mathrm{P}, \mathrm{Cd}$, and $\mathrm{Co}$ decreased 30- to 50-fold from east to west (Supp. Fig. 2). For the trace metals, the relative difference between the mean for each zone was larger than what was observed for POC (Table 2). This suggested that although the flux of these four elements is governed by similar biological processes in the upper $100 \mathrm{~m}$, perhaps the remineralization of POC and the processes that govern the removal of POC from the large particle pool are more variable and/or intense for P, Cd, and Co. In addition, differences in these flux gradients could partly reflect differences E: ${ }^{234} \mathrm{Th}$ caused by variations in cell quotas in a given zone, which could be elevated for $\mathrm{P}, \mathrm{Cd}$, and Co with respect to a similar carbon demand.

The top panels in Fig. 5, showing the median flux ratio for POC, P, and Cd, illustrate the trend that would be expected for biological components of particulate matter. Aside from the few values $>1$ (whiskers, Fig. 5), POC, P, and Cd decrease in flux from $100 \mathrm{~m}$ to $200 \mathrm{~m}$. The mean values and their corresponding s.d. (Table 2) suggested that the average level of flux attenuation between 100 $\mathrm{m}$ and $200 \mathrm{~m}$ was indistinguishable amongst all three elements and for all three regions (except POC in the Shelf). Each of these flux ratio means fall between 0.5 and 0.7. P and $\mathrm{Cd}$ have lower medians in all three zones compared to those for POC (Fig. 5) and the data for $\mathrm{P}$ and $\mathrm{Cd}$ are also unevenly distributed, with the $25^{\text {th }}$ to $50^{\text {th }}$ percentile being concentrated in a smaller range. Without in-situ, incubation-based uptake rates for $\mathrm{P}$ and $\mathrm{Cd}$, the absolute percentage of surface production reaching depth cannot be calculated, as was done with POC (Black et al., 2017). However, the data indicate that attenuation was greater for $\mathrm{P}$ and $\mathrm{Cd}$, than for POC.

While the Co flux ratio statistics for the Shelf and Offshore closely match those of POC (Fig. 5) and the decreasing gradient in $100 \mathrm{~m}$ flux values across the Gyre follow the same pattern as fluxes of $\mathrm{Cd}$ and $\mathrm{P}$, the flux ratios of Co in the Gyre more closely matched those of Mn. Even 
though the mean fluxes for the Gyre at $100 \mathrm{~m}$ and $200 \mathrm{~m}$ are both $3 \mathrm{nmol} \mathrm{m}^{-2} \mathrm{~d}^{-1} \pm 2 \mathrm{nmol} \mathrm{m}^{-2} \mathrm{~d}^{-1}$ for Co, greater than $75 \%$ of the Co flux ratios were at or above 1, suggesting that Co flux increased with depth at individual stations within the Gyre. Without additional data it is difficult to say whether the elevated flux ratios in Co and Mn are linked, but it appears that the distribution of large particle $\mathrm{Co}$ and the flux of Co reflected a mostly biological patterns in the low oxygen regions where Mn oxidation cannot occur (co-oxidizing Co) and pattern that reflected scavenging in the SSL Gyre.

\subsubsection{Manganese fluxes}

This region of the Pacific boasts larger particulate fluxes than observed in most other areas of the world's ocean, but magnitudes matching those in this study (100-5000 $\left.\mathrm{nmol} \mathrm{m}^{-2} \mathrm{~d}^{-1}\right)$ were found in the low oxygen regions off California and Mexico in the early 1980s (Martin et al., 1985, 1980, Martin and Knauer, 1984, 1983). Excluding the outlier from station $1\left(3100 \mathrm{nmol} \mathrm{m}^{-2} \mathrm{~d}^{-1}\right)$, the range in Mn flux observed at $100 \mathrm{~m}$ depth was $10 \mathrm{nmol} \mathrm{m} \mathrm{d}^{-2}$ to $1400 \mathrm{nmol} \mathrm{m}^{-2} \mathrm{~d}^{-1}$ with a mean of $402 \pm 328 \mathrm{nmol} \mathrm{m}^{-2} \mathrm{~d}^{-1}$. While no ${ }^{234} \mathrm{Th}$-based upper ocean flux studies exist for Mn (Fig. 1), traps deployed between $100 \mathrm{~m}$ to $200 \mathrm{~m}$ have captured fluxes from $10 \mathrm{nmol} \mathrm{m}^{-2} \mathrm{~d}^{-1}$ to $280 \mathrm{nmol}$ $\mathrm{m}^{-2} \mathrm{~d}^{-1}$ in the open ocean, with the highest fluxes observed in the North-East Water Polynya (Schüßler et al., 1997) and the lowest in the Benguela Upwelling region (Noble et al., 2012).

The small decrease in the zonal medians for Mn flux at $100 \mathrm{~m}$ (Fig. 4) indicates that something other than the large Shelf to Gyre gradient in surface NPP was impacting the magnitude of $100 \mathrm{~m}$ fluxes observed and the smaller gradient in dissolved Mn (Supp. Fig. 3). The small relative difference in the mean flux of Mn at $100 \mathrm{~m}$ between the Shelf and in the Gyre and the lack of a $100 \mathrm{~m}$ gradient within the Gyre (Supp. Fig. 2) suggests that Mn flux is well constrained in the upper $100 \mathrm{~m}$ by processes resulting in Mn oxide dissolution in the sunlit upper ocean and oxidation by bacteria in the subsurface. The largest degree of variability in Mn flux was observed in the Gyre subsurface, where scavenging led to the largest increases with depth seen across the transect and flux ratios $>1$ (Table 2, Fig. 5). The large shift in flux ratios between the Offshore and the Gyre (Fig. 5), suggest that Mn is uniquely impacted by redox conditions in the ODZs, which lead to a decrease in flux with depth, and scavenging in the Gyre, which increases Mn flux with depth.

\subsubsection{Iron fluxes}


While many trace elements may be co-limiting surface productivity, global ocean studies suggest that nitrogen and iron abundance are two of the most significant controls on productivity in much of the world's ocean (Moore et al., 2013). As a result, Fe has been investigated to the greatest extent using the ${ }^{234}$ Th method (Fig. 1), although the regions studied are limited. Fe fluxes from the Shelf, ranging from $12 \mu \mathrm{mol} \mathrm{m} \mathrm{m}^{-2} \mathrm{~d}^{-1}$ to $100 \mu \mathrm{mol} \mathrm{m} \mathrm{m}^{-1}$ at $100 \mathrm{~m}$, were of a similar magnitude as those seen in the Kerguelen Plateau $\left(1-46 \mu \mathrm{mol} \mathrm{m}^{-2} \mathrm{~d}^{-1}\right.$ at $35 \mathrm{~m}$ to $123 \mathrm{~m}$ depth; Lemaitre et al., 2016), the Crozet Islands (3-67 $\mu \mathrm{mol} \mathrm{m} \mathrm{m}^{-2}$ at $80 \mathrm{~m}$ to $200 \mathrm{~m}$ depth; Planquette et al., 2011), and the Gulf of Maine/Scotian Shelf/Labrador Sea $\left(2-151 \mu \mathrm{mol} \mathrm{m}^{-2} \mathrm{~d}^{-1}\right.$ at $50 \mathrm{~m}$ to 100 m depth; Smith et al., 2014; Weinstein and Moran, 2005). Offshore and Gyre fluxes measured at $100 \mathrm{~m}$ and $200 \mathrm{~m}\left(0.4-8 \mu \mathrm{mol} \mathrm{m} \mathrm{m}^{-2} \mathrm{~d}^{-1}\right)$ match more closely with open ocean trap studies in the North Atlantic and North Pacific (2-16 $\mu \mathrm{mol} \mathrm{m}^{-2} \mathrm{~d}^{-1}$ at $150 \mathrm{~m}$; Lamborg et al., 2008; Stanley et al., 2004) and those found in iron-limited areas like the Benguela Upwelling region, southeast of New Zealand, and the Ross Sea (0.01-0.6 $\mu \mathrm{mol} \mathrm{m} \mathrm{m}^{-2} \mathrm{~d}^{-1}$ at $120 \mathrm{~m}$ to $200 \mathrm{~m}$; Collier et al., 2000; Frew et al., 2006; Noble et al., 2012).

As was observed for POC, P, Cd, and Co, Fe has a large cross-transect gradient in particulate flux at $100 \mathrm{~m}$. However, like $\mathrm{Mn}$, the flux of Fe at $100 \mathrm{~m}$ does not gradually decrease from the Shelf to the westernmost station (Supp. Fig. 2). The median flux ratio for Fe in the Shelf and Gyre is above 1, reflecting an increase in Fe flux with depth (Fig. 4), however, the average fluxes at 100 $\mathrm{m}$ and $200 \mathrm{~m}$ in the Gyre do not indicate an increase within calculated uncertainties (Table 2). The overall variability across the transect is highest for $\mathrm{Fe}$ at $100 \mathrm{~m}$, relative to the other elements, with a mean and standard deviation of $14 \pm 30 \mu \mathrm{mol} \mathrm{m} \mathrm{m}^{-2}$. The large variability in Fe fluxes in the surface ocean and within the Shelf and Offshore ODZ could be related to lithogenic inputs and the authigenic precipitation of Fe-oxyhydroxides within the ODZ (Heller et al., 2017).

\subsubsection{Sources, sinks, and internal cycling of trace elements in the eastern tropical South Pacific}

To fully understand the controls on the upper ocean export of trace metals, we must consider

${ }^{234} \mathrm{Th}$-derived export estimates alongside data on surface inputs and the timescales of removal for these elements. Summary schematics (Figs. 8A to 8D), incorporating results from this study, cell ratio- and NPP-derived uptake estimates, dust fluxes, and ${ }^{228}$ Ra-based offshore transport estimates for dissolved constituents (Sanial et al., 2017), help illustrate the relative magnitude of processes 
impacting element budgets in the upper $200 \mathrm{~m}$. Unless otherwise noted, bulk dust estimates were determined using an average input for this region of $0.5 \mathrm{~g} \mathrm{~m}^{-2} \mathrm{y}^{-1}$ (Mahowald et al., 2005) and

upper crust ratios for each element (Mclennan, 2001). ${ }^{228}$ Ra-derived estimates represent the dissolved trace metal constituents that originated from the shelf and are moving offshore between the zones indicated (Figs. 8A to 8D). This shelf-sourced flux could originate from a combination of riverine, sediment, and subterranean groundwater inputs.

\subsubsection{Cd}

The decrease in Cd flux from $100 \mathrm{~m}$ to $200 \mathrm{~m}$ across the entire transect reflected the dominance of remineralization processes and the attenuation of particulate $\mathrm{Cd}$ flux was greatest in the Shelf and smallest in the Gyre (Fig. 8A). The addition of NPP- and bulk plankton ratio-derived uptake estimates suggests that $\mathrm{Cd}$ export from the upper $100 \mathrm{~m}$ could represent $<5 \%$ of the $\mathrm{Cd}$ taken up by surface plankton in the Offshore and Gyre and almost $25 \%$ in the Shelf. This trend is similar to that seen for POC flux (Black et al., 2017) in that the absolute magnitude of Cd export is largest nearer to the coast due to high surface productivity, although flux attenuation below $100 \mathrm{~m}$ is greatest in the Shelf and Offshore.

The uptake of Cd by surface communities and the subsequent sinking of this organic material is the most likely source of the export of particulate $\mathrm{Cd}$ because the calculated bio-authigenic fraction of Cd flux at $100 \mathrm{~m}$ was $\geq 99 \%$ at all stations. Furthermore, dust estimates for this region of $0.0012 \mathrm{nmol} \mathrm{m}^{-2} \mathrm{~d}^{-1}$ amount to $<1 \%$ of the smallest $100 \mathrm{~m} \mathrm{Cd}$ flux observed here (Supp. Table 2C), confirming that lithogenic, aerosol sources are negligible. The Ra-derived flux estimate for the transport of dissolved $\mathrm{Cd}$ from the coastal region is about the same as the uptake rate estimated for the Gyre. Therefore, the supply of Cd from the coast would be enough to support surface communities in the Gyre and should not be a major limiting factor of productivity in the region.

\subsubsection{Co}

Similar to Cd flux, Co flux in the Shelf and Offshore decreased from 100 to $200 \mathrm{~m}$ due to remineralization (Figs $8 \mathrm{~A}$ and $8 \mathrm{~B}$ ). Fluxes for both elements in the Offshore reflected $\sim 60 \%$ attenuation from $100 \mathrm{~m}$ to $200 \mathrm{~m}$, while Co in the Shelf showed surprisingly little attenuation relative to that seen for $\mathrm{Cd}$ flux. Unique to $\mathrm{Co}$, uptake rates based on mixed layer plankton cell ratios from this cruise were higher for the Offshore region than the Shelf. The $100 \mathrm{~m}$ export fluxes of Co were $22 \%, 4 \%$ and $10 \%$ of the surface uptake rates in the Shelf, Offshore, and Gyre, 
respectively. The larger degree of attenuation of particulate Co in the Offshore was anticipated due to the observed attenuation of POC within this zone, however, the relative amount of Co exported in the Gyre was much less than that of $\mathrm{Cd}$. This difference directly reflects the low uptake rates of Co in the Gyre. In addition, Co scavenging, which may have been slower or unlikely in the ODZ due to the reduction of particulate Mn, could be occurring between $100 \mathrm{~m}$ and $200 \mathrm{~m}$ in the Gyre. The difference in the export fluxes at these depths is small, but suggestive of a switch from a remineralization-dominated system to one controlled by scavenging.

The lithogenic fraction of Co flux at 100 m was between $10 \%$ and $20 \%$ in the Shelf, but only $3 \%$ in the Offshore and Gyre, on average, suggesting that particulate inputs from dust and sediments are relatively small in this region. The estimated dust input was $0.4 \mathrm{nmol} \mathrm{m} \mathrm{d}^{-1}$, which would still be of little consequence ( $<15 \%$ of export) in the Gyre. Long term sources of the dissolved pool most likely originate from coastal shelf fluxes and upwelling (Hawco et al., 2016; Saito et al., 2004; Sanial et al., 2017). Ra-based estimates suggest that $59 \mathrm{nmolCo} \mathrm{m}^{-2} \mathrm{~d}^{-1}$ is being supplied from the Shelf to the Gyre. Like with $\mathrm{Cd}$, this flux provides enough dissolved Co to supply the estimated uptake rates in the Gyre and the export observed at both $100 \mathrm{~m}$ and $200 \mathrm{~m}$.

\subsubsection{Mn}

Approximately 50\% of the exported flux of Mn at $100 \mathrm{~m}$ was observed at $200 \mathrm{~m}$ in the Shelf, while the Offshore $200 \mathrm{~m}$ flux of Mn was only 35\% of the $100 \mathrm{~m}$ flux (Fig. 8C). Therefore, the greatest remineralization of $\mathrm{Mn}$ or the release of $\mathrm{Mn}$ from the large particle pool was in the Offshore zone, similar to the more biological elements. The reason for this decrease, however, is not biological, but rather an interaction between the residence time of large particles and aggregation/disaggregation rates in either region. Longer particle residence times mean more time for particle exchange and time for Mn dissolution. Conversely, in the Gyre the flux increases 3.5fold from $100 \mathrm{~m}$ to $200 \mathrm{~m}$, showing that Mn-reduction is no longer inhibiting the scavenging and precipitation of particulate $\mathrm{Mn}$. Zonal $\mathrm{Mn}: \mathrm{P}$ or $\mathrm{Mn}: \mathrm{C}$ were not reported for the transect and so uptake rate estimates were derived from the range in ratios observed for the Equatorial Pacific. Although these are rough estimations of regional Mn uptake, the ratio of export at $100 \mathrm{~m}$ relative to the uptake rate is always $>1$, reflecting the increase in the bacterially-mediated precipitation of Mn below the sunlit surface layer. 
Mn export fluxes are relatively high considering the low predicted average dust deposition to this region of the ocean (Jickells et al., 2005). In fact, Mn-specific dust estimates for the Pacific that are currently being employed in the first Mn GBM only total $20 \mathrm{nmol} \mathrm{m}^{-2} \mathrm{~d}^{-1}$ and the addition of sediment fluxes only increases the Mn sources in the region to $41 \mathrm{nmol} \mathrm{m}^{-2} \mathrm{~d}^{-1}$ (van Hulten et al., 2017). Although there was evidence in the particle composition data east of $78^{\circ} \mathrm{W}$, that suggested the lithogenic fraction dominated at $100 \mathrm{~m}$, particulate dust or sediment inputs could not explain the large Mn fluxes in the Offshore and Gyre where the composition of at $100 \mathrm{~m}$ was $98 \%$ bio-authigenic. Furthermore, authigenic or scavenging processes were the most likely source because average $\mathrm{Mn}: \mathrm{P}$ ratios $\left(\mathrm{mmol} \mathrm{mol}^{-1}\right)$ at $100 \mathrm{~m}$ in the Offshore and Gyre were 12, much higher than the plankton ratios (0.35-0.53) previously observed in the Equatorial Pacific (Twining and Baines, 2013). Ra-derived estimates for dissolved offshore transport of Mn totaled $1960 \mathrm{nmol}$ $\mathrm{m}^{-2} \mathrm{~d}^{-1}$ suggest that fluxes from the coastal shelf (e.g. shelf sediments) are much larger than predicted and would provide more than enough Mn to support the observed precipitation and/or scavenging. This may be one reason why the Mn GBM used by van Hulten et al. (2017) generally succeeded in reproducing global water column distributions, but was unable to match in-situ measurements in the Pacific OMZ.

\subsubsection{Fe}

Unlike the other trace metals, the zonal average for Fe export flux dropped substantially from the Shelf to the Offshore and then remained at a similar value in the Gyre (Fig. 8D). This parallels the trends observed in the dissolved distributions (Supp. Fig. 3), where Cd, Co, and Mn are elevated in both the Shelf and ODZ, while dissolved Fe is only high in concentration in the subsurface Shelf. The precipitation and/or scavenging of Fe appeared to be most important in the Shelf and Gyre, with remineralization dominating in the Offshore. However, the mean export fluxes at $100 \mathrm{~m}$ and $200 \mathrm{~m}$ in the Offshore and Gyre are within uncertainties and thus, separating the relative importance of remineralization and authigenic processes is difficult. Like Mn, uptake estimates are very close or smaller than export estimates at $100 \mathrm{~m}$. This suggests that Fe could be limiting productivity if dust and sediment inputs to the surface ocean are low.

Of all the elements, Fe is the most likely to originate with dust in this region, although larger coastal shelf sources are required to sustain the particulate fluxes observed in the Shelf (Fig. 8D). A flux of dissolved Fe of $\sim 1 \mu \mathrm{mol} \mathrm{m} \mathrm{m}^{-2} \mathrm{~d}^{-1}$ was predicted from coastal shelf sources (Sanial et al., 
2017) and Ra-estimates suggest that the dissolved Fe flux was attenuated more rapidly than for other elements as waters traveled westward. Dust estimates previously used in GBMs (0.01-0.2 $\mu \mathrm{mol} \mathrm{m} \mathrm{m}^{-2}$; Tagliabue et al., 2016) and those estimated from crustal ratios $\left(0.9 \mu \mathrm{mol} \mathrm{m}^{-2} \mathrm{~d}^{-1}\right.$; Mahowald et al., 2005; Mclennan, 2001) could account for 30\% to 50\% of Offshore and Gyre fluxes at $100 \mathrm{~m}$. Fe did have a sizeable lithogenic component at most stations (transect average $=$ $17 \% \pm 10 \%$ ), but percentages did not reach above $30 \%$ in the Gyre, where they would be most important. If lithogenics contributed $10 \%$ to $20 \%$ of the flux, on average, whether by direct dust deposition or lateral transport, the remainder could be linked to biological or authigenic processes. It is unlikely, however, that all of the remainder comes from sinking biological material because the range in observed Fe:P ratios for a variety of plankton types, including those from regions of the Pacific, does not surpass 31 ( $\mathrm{mmol} \mathrm{mol}^{-1}$; Twining and Baines, 2013), while the transect range at $100 \mathrm{~m}$ started at 31 (Offshore) and went to 917 (Shelf). Although luxury uptake cannot be ruled out, an average large particle Fe:P of 116, well in excess of expected cell ratios, favors an authigenic explanation, such as the precipitation of Fe-oxyhydroxides (Heller et al., 2017).

\subsubsection{Removal timescales of trace elements in the eastern tropical South Pacific}

The ${ }^{234}$ Th-derived removal timescales of these elements can provide insight into the processes controlling the distributions and fluxes of trace metals in the surface ocean. $\tau_{\text {Sink }}$ expresses how rapidly the large particle pool is cycling and indicates if the processes impacting the large particle pools are similar for all elements, including ${ }^{234} \mathrm{Th}$. The total residence time $\left(\tau_{\text {Tot }}\right)$, which is shown in Fig. 8 for 0-100 m and 0-200 m (lower left corners), shows how quickly particle flux is removing the total inventory of the element. These timescales are useful when thinking about the biological accessibility of trace metals, dust solubility, and limitations on productivity.

\subsubsection{1 $\tau_{\text {Sink }}$}

Most elements had rapid $\tau_{\text {Sink }}$ values on the order of a few days to a few weeks (Table 3, Fig. 6A). The more biological elements, had longer median and mean $\tau_{\text {Sink }}$ values in the upper $100 \mathrm{~m}$ than those of Mn and Fe. The shortest $\tau_{\text {Sink }}$ were generally found in the Shelf, although $\mathrm{Cd}$ and Fe had a large range in $\tau_{\text {Sink }}$ here. For the biological elements, $\tau_{\text {Sink }}$ was rapid in the Shelf because fluxes were high here (Figs. 4 and 8). Offshore $\tau_{\text {Sink }}$ were elevated above the other two zones because while fluxes dropped precipitously from the Shelf, large particle inventories for the upper $100 \mathrm{~m}$ did not drop drastically until the Gyre. Fe was the exception, having a small large particle 
pool in both the Offshore and Gyre, and this was reflected in the low Offshore $\tau_{\text {Sink. All zonal }}$ averages of $\tau_{\text {Sink }}$ for $\mathrm{Mn}$ and Fe were 6 days, suggesting that the large particle pool of Fe and Mn cycled more rapidly than those of the other elements and that $\mathrm{Mn}$ and Fe loss via the sinking of particles was relatively large compared to that of other elements.

Not much data exists for a comparison of trace metals residence times within the large particle pool. However, a study of P cycling in the Gulf of Maine found that, while variable, the cycling of particulate $\mathrm{P}$ in the euphotic zone appeared to be on the order of days to just over a week in the coastal ocean (10 - $102 \mu \mathrm{m}$ size range; Benitez-Nelson and Buesseler, 1999). This range fits with the results from our study and support a relatively consistent timescale for large particle element cycling in the upper ocean. ${ }^{234} \mathrm{Th}$, with a half-life of 24.1 days, is well suited for tracking elements in the particulate phase over these timescales.

\subsubsection{2 $\tau_{\text {Tot }}$}

Although successful in determining trace element fluxes, the few ${ }^{234} \mathrm{Th}$ studies noted here did not attempt residence time estimations (Lemaitre et al., 2016; Planquette et al., 2011; Smith et al., 2014), except for Weinstein and Moran (2005) where $\tau_{\text {Tot }}$ were determined for $\mathrm{Al}$ and Pb only. The results of this study fell within estimates for the global ocean utilizing other methods for most elements. Co $\tau_{\text {Tot }}$ for the upper $100 \mathrm{~m}$ in the Atlantic has been estimated between 0.32 years (particulate export-derived) to 7.6 years (dust estimate-derived, Saito and Moffett, 2002). Previous Mn estimates for the Pacific and Atlantic have ranged from a few years to 20 years (Martin et al., 1985, 1980; Martin and Knauer, 1984; Shiller, 1997). Low-end dust-based estimates for Fe in the equatorial Atlantic match more closely with results from this study at 6 days to 62 days (Croot et al., 2004), whereas an estimate for the North Pacific Gyre for the upper $100 \mathrm{~m}$ to $200 \mathrm{~m}$ were estimated at 6 months to 12 months using a ${ }^{230} \mathrm{Th}^{-232} \mathrm{Th}$ method and Fe:Th solubility data (Hayes et al., 2015). Cd and $\mathrm{P}$ values were difficult to find, but a single trap study form the South China Sea reported $\tau_{\text {Tot }}$ on the order of 1 month to 3 months, much shorter than what was estimated on this transect. The broad agreement in most of these numbers is promising.

The $\tau_{\text {Tot }}$ results showed that $\mathrm{P}$ remained in the upper $100 \mathrm{~m}$ a bit longer ( 0.5 years to 13 years) than Co and Cd (0.1 years to 5 years), but overall these biological elements had similar $\tau_{\text {Tot }}$ (Figs. 6B, 8A, and 8B). Mn $\tau_{\text {Tot, }}$ despite being controlled by different processes, also fell in this range. The similarity in these $\tau_{\text {Tot }}$ was unsurprising considering the partitioning of the total inventory of 
each element in the upper $100 \mathrm{~m}$. The large particle pool composed, on average, $1 \%$ to $2 \%$ of the total pool for $\mathrm{Mn}, \mathrm{Co}$, and $\mathrm{P}$ and $\mathrm{Cd}$ and $\mathrm{Fe}$ percentages were higher at $6 \%$ and $23 \%$, respectively. Because the large particle pool is a greater contributor for $\mathrm{Cd}$ and $\mathrm{Fe}$, sinking particle fluxes have a greater impact and $\tau_{\text {Tot }}$ are shorter. $\mathrm{Cd} \tau_{\text {Tot }}$ were slightly shorter in the Offshore and Gyre than those for $\mathrm{Co}$ and $\mathrm{Mn}$. Fe was the extreme case with a rapid turnover of 5 days to 47 days at $100 \mathrm{~m}$ across the transect. With Fe predicted to be the limiting nutrient for this region, it was not surprising to find such low $\tau_{\text {Tot }}$ relative to all other elements.

The total residence time in the upper $200 \mathrm{~m}$ reflects the balance of processes removing each element from the particulate pool, like remineralization, and the affinity of particles for an element combined with the propensity of these elements to stay in the particulate phase. Long $\tau_{\text {Tot }}$ indicate the dominance of remineralization over processes like precipitation or scavenging. In this region, the biological elements have the largest dissolved inventories (relative to flux magnitude, Supp. Fig. 3), the greatest importance of remineralization processes in the overall determination of export flux (low flux ratio), and the longest residence times (Fig. 8). Mn behaved similarly in the Shelf and Offshore where the ODZ was present (giving flux profiles a pseudo-biological shape), but scavenging dominated the subsurface Gyre and this drove $\tau_{\text {Tot }}$ down (Fig. 8C). Fe had short $\tau_{\text {Tot }}$ everywhere due to a combination of low dissolved inventories (Supp. Fig. 3) and a dominance of scavenging and/or authigenic precipitation in all zones of the transect (Fig. 8D).

\subsection{Conclusions}

Despite the large variability in flux estimates observed for trace metal elements compared to those for purely biological components, like POC, key patterns have emerged within the three biogeochemical zones of the transect that group the elements of interest into two main categories. These groups, reflecting the major processes controlling the distributions and flux of these elements in the southeastern Pacific, are biological (Cd, Co, P) and authigenic-lithogenic (Mn, Fe). Depending on the local processes, Co could be considered a hybrid element with the properties of both groups. These elements may fall partially into different categories in other regions of the ocean, for instance, in the North Atlantic, where dust fluxes can be large, or at depths $>400 \mathrm{~m}$, where scavenging may play a more important role than biological processes for an element like Co. The flux ratio employed here can aid in evaluating regional trends in the relative importance of these processes and in characterizing groups of elements with similar characteristics. 
Dissolved inventories, the low ${ }^{228} \mathrm{Ra}$-based offshore transport estimates, and the large ${ }^{234} \mathrm{Th}-$ based export fluxes in the Shelf relative to the other two zones, show that Fe is rapidly stripped from the water column in the Shelf, while other trace metals are transported much farther into the open ocean. In this region of the Pacific, the release of $\mathrm{Cd}, \mathrm{Co}$, and $\mathrm{Mn}$ from shelf sediments can be an important source for the open ocean and the communities that reside there. The importance of dust inputs is minimal for the surface budgets of these elements. The offshore transport of Fe, on the other hand, matches the magnitude estimated for dust fluxes and thus, both sources are vital for sustaining open ocean communities. As the combined dust and lateral flux estimates fall just under and over uptake estimates for the Offshore and Gyre, Fe is likely the micronutrient limiting productivity in this region.

Although it has been suggested that whole-ocean residence times could be 'governed largely by processes occurring either in near-surface waters or at ocean margins' (Sherrell and Boyle, 1992), non-aerosol-based flux and residence time studies for trace metals in upper $400 \mathrm{~m}$ of the ocean are scarce. This study of the southeastern tropical Pacific has illustrated the benefit of cosampling for particulate trace metals and ${ }^{234} \mathrm{Th}$ and produced some of the first large-scale ${ }^{234} \mathrm{Th}$ derived flux and surface residence time estimates for trace metals in the global ocean. These flux and residence time estimates will help to close trace metal budgets for the Pacific Ocean and we anticipate that these data will aid in the improvement of current GBMs used to model dissolve and particulate trace metal distributions, especially in the Pacific OMZ, where GBMs struggle to reproduce in-situ distributions (van Hulten et al., 2017). The ${ }^{234}$ Th-method for determining the residence time of trace metals in the surface ocean provides a useful alternative to dust-based estimates, where observation-based fluxes have been shown to disagree by two orders of magnitude in some cases (Anderson et al., 2016).

There are many opportunities to carry this ${ }^{234} \mathrm{Th}$-based technique forward. As of this thesis, the GEOTRACES program has already provided a platform for paired ${ }^{234}$ Th-trace metal sampling, collected via high volume in-situ pumping, in the North Atlantic (US GA03; France GA01), Southern Pacific (this transect GP-16), the western Arctic (US GN01), the Mediterranean Sea (Spain GA04), and the Kerguelen Plateau (France GIpr01). In addition, although only four trace metals were the focus of this study, many more have been measured during these cruises and the ${ }^{234} \mathrm{Th}$-method could provide the first estimate of particulate surface flux ever for some of the rarer trace metals. Further insight will be attained by the use of other flux gages in tandem with ${ }^{234} \mathrm{Th}$, 
as has been done here with ${ }^{228} \mathrm{Ra}$. Recommendations for future GEOTRACES campaigns or individual studies include: (1) sampling E: ${ }^{234} \mathrm{Th}$ at a minimum of 6 depths in the upper $300 \mathrm{~m}$, spaced in order to capture both relevant features (like an ODZ or deep chlorophyll maxima) and to maintain evenly spaced intervals, (2) utilizing multiple tracer systems to capture processes over varying timescales to integrate estimates for both dissolved and particulate pools, and (3) incorporating a paired trap and ${ }^{234} \mathrm{Th}$ flux process study to determine what types of particles contribute to the sinking flux of trace metals.

\section{Acknowledgements}

This work was supported by the National Science Foundation (OCE-1232669) to Woods Hole Oceanographic Institution and the University of South Carolina. E. Black was also funded by a NASA Earth and Space Science Graduate Fellowship (NNX13AP31H). POC and particulate trace element work was supported by NSF OCE-1518110 to USSC. The authors would like to thank the captain, crew, and scientists aboard the R/V Thomas G. Thompson and the chief scientists, James Moffett and Chris German. This work would not have been possible without the members of the pump group including Matt Charette, William Moore, Dan Ohnemus, and Virginie Sanial. A special thanks to Virginie Sanial for providing additional ${ }^{228} \mathrm{Ra}$-based estimates for $\mathrm{Cd}$ and input on the offshore transport of trace metals. 


\section{References}

Alleman, L.Y., Hamelin, B., Véron, A.J., Miquel, J.C., Heussner, S., 2000. Lead sources and transfer in the coastal Mediterranean: Evidence from stable lead isotopes in marine particles. Deep. Res. II 47, 2257-2279. doi:10.1016/S0967-0645(00)00024-2

Anderson, R.F., Cheng, H., Edwards, R.L., Fleisher, M.Q., Hayes, C.T., Huang, K., Kadko, D., Lam, P.J., Landing, W.M., Lao, Y., Lu, Y., Measures, C.I., Moran, S.B., Morton, P.L., Ohnemus, D.C., Robinson, L.F., Shelley, R.U., 2016. How well can we quantify dust deposition to the ocean? Philos. Trans. R. Soc. A 374. doi:10.1098/rsta.2015.0285

Benitez-Nelson, C., Buesseler, K.O., Karl, D.M., Andrews, J., 2001. A time-series study of particulate matter export in the North Pacific Subtropical Gyre based on 234Th:238U disequilibrium. Deep. Res. I 48, 2595-2611.

Benitez-Nelson, C.R., Buesseler, K.O., 1999. Variability of inorganic and organic phosphorus turnover rates in the coastal ocean. Nature 398, 502-505.

Bishop, J.K., Edmond, J.M., Ketten, D.R., Bacon, M.P., Silker, W.B., 1977. The chemistry, biology, and vertical flux of particulate matter from the upper $400 \mathrm{~m}$ of the equatorial Atlantic Ocean. Deep. Res. 24, 511-548.

Bishop, J.K.B., Fleisher, M.Q., 1987. Particulate manganese dynamics in Gulf Stream warm-core rings and surrounding waters of the N.W. Atlantic. Geochim. Cosmochim. Acta 51, 2807-2825. doi:10.1016/0016-7037(87)90160-8

Bishop, J.K.B., Lam, P.J., Wood, T.J., 2012. Getting good particles: Accurate sampling of particles by large volume in-situ filtration. Limnol. Oceanogr. Methods 10, 681-710. doi:10.4319/lom.2012.10.681

Black, E.E., Buesseler, K.O., Pike, S.M., Lam, P.J., 2017. ${ }^{234}$ Th as a tracer of particulate export and remineralization in the southeastern tropical Pacific. Mar. Chem. In Press, Corrected Proof. doi:10.1016/j.marchem.2017.06.009

Bowie, A.R., Lannuzel, D., Remenyi, T.A., Wagener, T., Lam, P.J., Boyd, P.W., Guieu, C., Townsend, A.T., Trull, T.W., 2009. Biogeochemical iron budgets of the Southern Ocean south of Australia: Decoupling of iron and nutrient cycles in the subantarctic zone by the summertime supply. Global Biogeochem. Cycles 23, 1-14. doi:10.1029/2009GB003500

Boyd, P.W., Law, C.S., Wong, C.S., Nojiri, Y., Tsuda, A., Levasseur, M., Takeda, S., Rivkin, R., Harrison, P., Strzepek, R., Gower, J., McKay, R.M., Abraham, E., Arychuk, M., Barwell-Clarke, J., Crawford, W., Crawford, D., Hale, M., Harada, K., Johnson, K., Kiyosawa, H., Kudo, I., Marchetti, A., Miller, W., Needoba, J., Nishioka, J., Ogawa, H., Page, J., Robert, M., Saito, H., Sastri, A., Sherry, N., Soutar, T., Sutherland, N., Taira, Y., Whitney, F., Wong, S.-K.E., Yoshimura, T., 2004. The decline and fate of an iron-induced subarctic phytoplankton bloom. Nature 428, 549-553.

Boyle, E.A., 1988. Cadmium: Chemical tracer of deepwater paleoceanography. Paleoceanography 3, 471-489. doi:10.1029/PA003i004p00471

Bruland, K.W., Orians, K.J., Cowen, J.P., 1994. Reactive trace metals in the stratified central North Pacific. Geochim. Cosmochim. Acta 58, 3171-3182. doi:10.1016/0016-7037(94)90044-2

Buesseler, K.O., Bacon, M.P., Cochran, J.K., Livingston, H.D., 1992. Carbon and nitrogen export during the JGOFS North Atlantic Bloom Experiment estimated from ${ }^{234} \mathrm{Th}:{ }^{238} \mathrm{U}$ disequilibria. Deep. Res. $39,1115-1137$. 
Buesseler, K.O., Ball, L., Andrews, J., Benitez-Nelson, C., Belastock, R., Chai, F., Chao, Y., 1998. Upper ocean export of particulate organic carbon in the Arabian Sea derived from thorium-234. Deep.

Res. II 45, 2461-2487.

Buesseler, K.O., Benitez-Nelson, C.R., Moran, S.B., Burd, A., Charette, M., Cochran, J.K., Coppola, L., Fisher, N.S., Fowler, S.W., Gardner, W.D., Guo, L.D., Gustafsson, O., Lamborg, C., Masque, P., Miquel, J.C., Passow, U., Santschi, P.H., Savoye, N., Stewart, G., Trull, T., 2006. An assessment of particulate organic carbon to thorium-234 ratios in the ocean and their impact on the application of 234Th as a POC flux proxy. Mar. Chem. 100, 213-233. doi:10.1016/j.marchem.2005.10.013

Buesseler, K.O., Jayne, S.R., Fisher, N.S., Rypina, I.I., Baumann, H., Baumann, Z., Breier, C.F., Douglass, E.M., George, J., Macdonald, A.M., Miyamoto, H., Nishikawa, J., Pike, S.M., Yoshida, S., 2012. Fukushima-derived radionuclides in the ocean and biota off Japan. Proc. Natl. Acad. Sci. 109, 5984-5988. doi:10.1073/pnas.1120794109

Buesseler, K.O., Pike, S., Maiti, K., Lamborg, C.H., Siegel, D.A., Trull, T.W., 2009. Thorium-234 as a tracer of spatial, temporal and vertical variability in particle flux in the North Pacific. Deep. Res. I 56, 1143-1167. doi:10.1016/j.dsr.2009.04.001

Collier, R., Dymond, J., Honjo, S., Manganini, S., Francois, R., Dunbar, R., 2000. The vertical flux of biogenic and lithogenic material in the Ross Sea: moored sediment trap observations 1996-1998. Deep. Res. II 47, 3491-3520. doi:10.1016/S0967-0645(00)00076-X

Cowen, J.P., Bruland, K.W., 1985. Metal deposits associated with bacteria: implications for Fe and Mn marine biogeochemistry. Deep Sea Res. 32, 253-272. doi:10.1016/0198-0149(85)90078-0

Croot, P.L., Streu, P., Baker, A.R., 2004. Short residence time for iron in surface seawater impacted by atmospheric dry deposition from Saharan dust events. Geophys. Res. Lett. 31, 1-4. doi:10.1029/2004GL020153

Cullen, J.T., Sherrell, R.M., 1999. Techniques for determination of trace metals in small samples of sizefractionated particulate matter: Phytoplankton metals off central California. Mar. Chem. 67, 233247. doi:10.1016/S0304-4203(99)00060-2

Cutter, G.A., Moffett, J.W., Nielsdottir, M., Sanial, V., 2017. Multiple oxidation state trace elements in low oxygen waters of Peru: in situ redox processes and horizontal advective/diffusive transport. Mar. Chem. Submitted.

de Baar, H.J.W., Saager, P.M., Nolting, R.F., van der Meer, J., 1994. Cadmium versus phosphate in the world ocean. Mar. Chem. 46, 261-281.

Dehairs, F., Fagel, N., Antia, a N., Peitner, R., Elskens, M., Goeyens, L., 2000. Export production in the Bay of Biscay as estimated from barium-barite in settling material: a comparison with new production. Deep. Res. I 47, 583-601. doi:10.1016/S0967-0637(99)00072-2

Dulaquais, G., Boye, M., Middag, R., Owens, S., Puigcorbé, V., Buesseler, K.O., Masqué, P., de Baar, H.J.W., Carton, X., 2014. Contrasting biochemical cycles of cobalt in the surface western Atlantic Ocean. Global Biogeochem. Cycles 28, 1387-1412. doi:10.1002/2014GB004903

Durkin, C.A., Estapa, M.L., Buesseler, K.O., 2015. Observations of carbon export by small sinking particles in the upper mesopelagic. Mar. Chem. 175, 72-81. doi:10.1016/j.marchem.2015.02.011

Dymond, J., Collier, R., 1996. Particulate barium fluxes and their relationships to biological productivity. Deep Sea Res. II 43, 1283-1308. doi:10.1016/0967-0645(96)00011-2

Elderfield, H., Rickaby, R., 2000. Oceanic Cd/P ratio and nutrient utilization in the glacial Southern Ocean. Nature 405, 305-310. doi:10.1038/35012507 
Fiedler, P.C., Talley, L.D., 2006. Hydrography of the eastern tropical Pacific : A review. Prog. Oceanogr. 69, 143-180. doi:10.1016/j.pocean.2006.03.008

Frew, R.D., Hutchins, D.A., Nodder, S., Sanudo-Wilhelmy, S., Tovar-Sanchez, A., Leblanc, K., Hare, C.E., Boyd, P.W., 2006. Particulate iron dynamics during FeCycle in subantarctic waters southeast of New Zealand. Global Biogeochem. Cycles 20, 1-15. doi:10.1029/2005GB002558

Hatta, M., Measures, C.I., Wu, J., Roshan, S., Fitzsimmons, J.N., Sedwick, P., Morton, P., 2015. An overview of dissolved Fe and Mn distributions during the 2010-2011 U.S. GEOTRACES north Atlantic cruises: GEOTRACES GA03. Deep. Res. II 116, 117-129. doi:10.1016/j.dsr2.2014.07.005

Hawco, N.J., Ohnemus, D.C., Resing, J.A., Twining, B.S., Saito, M.A., 2016. A dissolved cobalt plume in the oxygen minimum zone of the eastern tropical South Pacific. Biogeosciences 13, 5697-5717. doi:10.5194/bg-13-5697-2016

Hayes, C.T., Fitzsimmons, J.N., Boyle, E.A., Mcgee, D., Anderson, R.F., Weisend, R., Morton, P.L., 2015. Thorium isotopes tracing the iron cycle at the Hawaii Ocean Time-series Station ALOHA. Geochim. Cosmochim. Acta 169, 1-16. doi:10.1016/j.gca.2015.07.019

Heller, M.I., Lam, P.J., Moffett, J.W., Till, C.P., Lee, J.-M., Toner, B.M., Marcus, M.A., 2017. Accumulation of Fe oxides in the Peruvian oxygen deficient zone implies non-oxygen dependent Fe oxidation. Geochim. Cosmochim. Acta 211, 174-193. doi:10.1016/j.gca.2017.05.019

Hung, C.C., Gong, G.C., Santschi, P.H., 2012. 234Th in different size classes of sediment trap collected particles from the Northwestern Pacific Ocean. Geochim. Cosmochim. Acta 91, 60-74. doi:10.1016/j.gca.2012.05.017

Jeandel, C., Tachikawa, K., Bory, A., Dehairs, F., 2000. Biogenic barium in suspended and trapped material as a tracer of export production in the tropical NE Atlantic (EUMELI sites). Mar. Chem. 71, 125-142. doi:10.1016/S0304-4203(00)00045-1

Jickells, T.D., An, Z.S., Andersen, K.K., Baker, A.R., Bergametti, G., Brooks, N., Cao, J.J., Boyd, P.W., Duce, R.A., Hunter, K.A., Kawahata, H., Kubilay, N., Liss, P.S., Mahowald, N., Prospero, J.M., Ridgewell, A.J., Tegen, I., Torres, R., 2005. Global iron connections between desert dust, ocean biogeochemistry, and climate. Science 308, 67-72.

Johnson, K.S., Michael Gordon, R., Coale, K.H., 1997. What controls dissolved iron concentrations in the world ocean? Mar. Chem. 57, 137-161. doi:10.1016/S0304-4203(97)00043-1

Kadko, D., 2017. Upwelling and primary production during the U.S. GEOTRACES East Pacific Zonal Transect. Global Biogeochem. Cycles 31, 1-15. doi:10.1002/2016GB005554

Karstensen, J., Stramma, L., Visbeck, M., 2008. Oxygen minimum zones in the eastern tropical Atlantic and Pacific oceans. Prog. Oceanogr. 77, 331-350. doi:10.1016/j.pocean.2007.05.009

Kumar, N., Anderson, R.F., Mortlock, R.A., Froelich, P.N., Kubik, P., Dittrich-Hannen, B., Suter, M., 1995. Increased biological productivity and export production in the glacial Southern Ocean. Nature 378, 675-680. doi:10.1038/378675a0

Lam, P.J., Bishop, J.K.B., 2008. The continental margin is a key source of iron to the HNLC North Pacific Ocean. Geophys. Res. Lett. 35, 1-5. doi:10.1029/2008GL033294

Lam, P.J., Bishop, J.K.B., Henning, C.C., Marcus, M.A., Waychunas, G.A., Fung, I.Y., 2006. Wintertime phytoplankton bloom in the subarctic Pacific supported by continental margin iron. Global Biogeochem. Cycles 20, 1-12. doi:10.1029/2005GB002557

Lam, P.J., Ohnemus, D.C., Auro, M.E., 2015. Size-fractionated major particle composition and concentrations from the US GEOTRACES North Atlantic Zonal Transect. Deep. Res. II 116, 303320. doi:10.1016/j.dsr2.2014.11.020 
Lamborg, C.H., Buesseler, K.O., Lam, P.J., 2008. Sinking fluxes of minor and trace elements in the North Pacific Ocean measured during the VERTIGO program. Deep. Res. Part II Top. Stud. Oceanogr. 55, 1564-1577. doi:10.1016/j.dsr2.2008.04.012

Lamborg, C.H., Hammerschmidt, C.R., Bowman, K.L., Swarr, G.J., Munson, K.M., Ohnemus, D.C., Lam, P.J., Heimbürger, L.-E., Rijkenberg, M.J.A., Saito, M.A., 2014. A global ocean inventory of anthropogenic mercury based on water column measurements. Nature 512, 65-68. doi:10.1038/nature 13563

Le Moigne, F.A.C., Henson, S.A., Sanders, R.J., Madsen, E., 2013. Global database of surface ocean particulate organic carbon export fluxes diagnosed from the 234Th technique. Earth Syst. Sci. Data 5, 295-304. doi:10.5194/essd-5-295-2013

Lemaitre, N., Planquette, H., Dehairs, F., van der Merwe, P., Bowie, A.R., Trull, T.W., LaurenceauCornec, E.C., Davies, D., Bollinger, C., Le Goff, M., Grossteffan, E., Planchon, F., 2016. Impact of the natural Fe-fertilization on the magnitude, stoichiometry and efficiency of particulate biogenic silica, nitrogen and iron export fluxes. Deep. Res. I 117, 11-27. doi:10.1016/j.dsr.2016.09.002

Mahowald, N.M., Baker, A.R., Bergametti, G., Brooks, N., Duce, R.A., Jickells, T.D., Prospero, J.M., Tegen, I., 2005. Atmospheric global dust cycle and iron inputs to the ocean. Global Biogeochem. Cycles 19. doi:10.1029/2004GB002402

Maiti, K., Buesseler, K.O., Pike, S.M., Benitez-Nelson, C., Cai, P., Chen, W., Cochran, K., Dai, M., Dehairs, F., Gasser, B., 2012. Intercalibration studies of short-lived thorium-234 in the water column and marine particles. Limnol. Oceanogr. Methods 10, 631-644.

doi:10.4319/lom.2012.10.631

Martin, J.H., Coale, K.H., Johnson, K.S., Fitzwater, S.E., Gordon, R.M., Tanner, S.J., Hunter, C.N., Elrod, V.A., Nowicki, J.L., Coley, T.L., Barber, R.T., Lindley, S., Watson, A.J., Van Scoy, K., Law, C.S., Liddicoat, M.I., Ling, R., Stanton, T., Stockel, J., Collins, C., Anderson, A., Bidigare, R., Ondrusek, M., Latasa, M., Millero, F.J., Lee, K., Yao, W., Zhang, J.Z., Friederich, G., Sakamoto, C., Chavez, F., Buck, K., Kolber, Z., Greene, R., Falkowski, P., Chisholm, S.W., Hoge, F., Swift, R., Yungel, J., Turner, S., Nightingale, P., Hatton, A., Liss, P., Tindale, N.W., 1994. Testing the iron hypothesis in ecosystems of the equatorial Pacific Ocean. Nature. doi: $10.1038 / 371123 \mathrm{a} 0$

Martin, J.H., Knauer, G.A., 1984. VERTEX: manganese transport through oxygen minima. Earth Planet. Sci. Lett. 67, 35-47.

Martin, J.H., Knauer, G.A., 1983. VERTEX: Manganese transport with $\mathrm{CaCO}_{3}$. Deep. Res. 30, 411-425.

Martin, J.H., Knauer, G.A., Broenkow, W.W., 1985. VERTEX: the lateral transport of manganese in the northeast Pacific. Deep Sea Res. Part A, Oceanogr. Res. Pap. 32. doi:10.1016/01980149(85)90056-1

Martin, J.H., Knauer, G.A., Landing, M., Laboratories, M., Landing, M., Ca, U.S.A., 1980. Manganese cycling in Northeast Pacific waters. Earth Planet. Sci. Lett. 51, 266-274.

Martínez-garcía, A., Sigman, D.M., Ren, H., Anderson, R.F., Straub, M., Hodell, D.A., Jaccard, S.L., Eglinton, T.I., Haug, G.H., 2014. Iron Fertilization of the Subantarctic Ocean During the Last Ice Age 1347. doi:10.1126/science. 1246848

Mclennan, S.M., 2001. Relationships between the trace element composition of sedimentary rocks and upper continental crust. Geochemistry Geophys. Geosystems 2.

McManus, J., Dymond, J., Dunbar, R.B., Collier, R.W., 2002. Particulate barium fluxes in the Ross Sea. Mar. Geol. 184, 1-15. doi:10.1016/S0025-3227(01)00300-0 
Migon, C., Sandroni, V., Marty, J.C., Gasser, B., Miquel, J.C., 2002. Transfer of atmospheric matter through the euphotic layer in the northwestern Mediterranean: Seasonal pattern and driving forces. Deep. Res. II 49, 2125-2141. doi:10.1016/S0967-0645(02)00031-0

Mills, M.M., Ridame, C., Davey, M., La Roche, J., Geider, R.J., 2004. Iron and phosphorus co-limit nitrogen fixation in the eastern tropical North Atlantic. Nature 429, 292-294. doi:10.1038/nature03632

Moffett, J.W., Ho, J., 1996. Oxidation of cobalt and manganese in seawater via a common microbially catalyzed pathway. Geochim. Cosmochim. Acta 60, 3415-3424. doi:10.1016/0016-7037(96)001767

Moore, C.M., Mills, M.M., Arrigo, K.R., Berman-Frank, I., Bopp, L., Boyd, P.W., Galbraith, E.D., Geider, R.J., Guieu, C., Jaccard, S.L., Jickells, T.D., Roche, J. La, Lenton, T.M., Mahowald, N.M., Marañón, E., Marinov, I., Moore, J.K., Nakatsuka, T., Oschlies, A., Saito, M.A., Thingstad, T.F., Tsuda, A., Ulloa, O., 2013. Processes and patterns of oceanic nutrient limitation. Nat. Geosci. 6, 701-710. doi:10.1038/ngeo1765

Moore, J.K., Braucher, O., 2008. Sedimentary and mineral dust sources of dissolved iron to the world ocean. Biogeosciences 5, 631-656.

Morel, F.M.M., Milligan, A.J., Saito, M.A., 2004. Marine bioinorganic chemistry: The role of trace metals in the oceanic cycles of major nutrients, in: Elderfield, H. (Ed.), Treatise on Geochemistry. San Diego, pp. 113-143.

Morel, F.M.M., Price, N.M., 2003. The Biogeochemical Cycles of Trace Metals in the Oceans. Science 300, 944-947.

Noble, A.E., Lamborg, C.H., Ohnemus, D.C., Lam, P.J., Goepfert, T.J., Measures, C.I., Frame, C.H., Casciotti, K.L., DiTullio, G.R., Jennings, J., Saito, M.A., 2012. Basin-scale inputs of cobalt, iron, and manganese from the Benguela-Angola front to the South Atlantic Ocean. Limnol. Oceanogr. 57, 989-1010. doi:10.4319/lo.2012.57.4.0989

Not, C., Brown, K.A., Ghaleb, B., Hillaire-Marcel, C., 2012. Conservative behavior of uranium vs. salinity in Arctic sea ice and brine. Mar. Chem. 130-131, 33-39. doi:10.1016/j.marchem.2011.12.005

Ohnemus, D.C., Lam, P.J., 2015. Cycling of lithogenic marine particles in the US GEOTRACES North Atlantic transect. Deep. Res. Part II Top. Stud. Oceanogr. 116, 283-302. doi:10.1016/j.dsr2.2014.11.019

Ohnemus, D.C., Rauschenberg, S., Cutter, G.A., Fitzsimmons, J.N., Sherrell, R.M., Twining, B.S., 2016. Elevated trace metal content of prokaryotic plankton communities associated with marine oxygen deficient zones. Limnol. Oceanogr. 62, 3-25. doi:10.1002/lno.10363

Owens, S.A., Buesseler, K.O., Sims, K.W.W., 2011. Re-evaluating the ${ }^{238}$ U-salinity relationship in seawater: Implications for the ${ }^{238} \mathrm{U}-234 \mathrm{Th}$ disequilibrium method. Mar. Chem. 127, 31-39. doi:10.1016/j.marchem.2011.07.005

Owens, S.A., Pike, S., Buesseler, K.O., 2015. Thorium-234 as a tracer of particle dynamics and upper ocean export in the Atlantic Ocean. Deep. Res. II 116, 42-59. doi:10.1016/j.dsr2.2014.11.010

Passow, U., Dunne, J., Murray, J.W., Balistrieri, L., Alldredge, A.L., 2006. Organic carbon to ${ }^{234}$ Th ratios of marine organic matter. Mar. Chem. 100, 323-336. doi:10.1016/j.marchem.2005.10.020

Planquette, H., Sanders, R.R., Statham, P.J., Morris, P.J., Fones, G.R., 2011. Fluxes of particulate iron from the upper ocean around the Crozet Islands: A naturally iron-fertilized environment in the Southern Ocean. Global Biogeochem. Cycles 25. doi:10.1029/2010GB003789 
Pohl, C., Löffler, A., Hennings, U., 2004. A sediment trap flux study for trace metals under seasonal aspects in the stratified Baltic Sea (Gotland Basin; $57^{\circ} 19.20^{\prime} \mathrm{N} ; 20^{\circ} 03.00^{\prime} \mathrm{E}$ ). Mar. Chem. 84, $143-$ 160. doi:10.1016/j.marchem.2003.07.002

Puigcorbé, V., Benitez-nelson, C.R., Masqué, P., Verdeny, E., White, A.E., Popp, B.N., Prahl, F.G., Lam, P.J., 2015. Small phytoplankton drive high summertime carbon and nutrient export in the Gulf of California and Eastern Tropical North Pacific. Global Biogeochem. Cycles 1309-1332. doi:10.1002/2015GB005134

Quetel, C.R., Remoudaki, E., Davies, J.E., Miquel, J.C., Fowler, S.W., Lambert, C.E., Bergametti, G., Buat-Menard, P., 1993. Impact of atmospheric deposition on particulate iron flux and distribution in northwestern Mediterranean waters. Deep. Res. I 40, 989-1002. doi:10.1016/0967-0637(93)90085$\mathrm{H}$

Resing, J.A., Sedwick, P.N., German, C.R., Jenkins, W.J., Moffett, J.W., Sohst, B.M., Tagliabue, A., 2015. Basin-scale transport of hydrothermal dissolved metals across the South Pacific Ocean. Nature 523, 200-203. doi:10.1038/nature14577

Saito, M.A., Bertrand, E.M., Dutkiewicz, S., Bulygin, V. V, Moran, D.M., Monteiro, F.M., Follows, M.J., Valois, F.W., Waterbury, J.B., 2010. Iron conservation by reduction of metalloenzyme inventories in the marine diazotroph Crocosphaera watsonii. doi:10.1073/pnas.1006943108

Saito, M.A., Goepfert, T.J., 2008. Zinc-cobalt colimitation of Phaeocystis antarctica. Limnol. Oceanogr. 53, 266-275. doi:10.4319/1o.2008.53.1.0266

Saito, M.A., Moffett, J.W., 2002. Temporal and spatial variability of cobalt in the Atlantic Ocean. Geochim. Cosmochim. Acta 66, 1943-1953. doi:10.1016/s0016-7037(02)00829-3

Saito, M.A., Moffett, J.W., Ditullio, G.R., 2004. Cobalt and nickel in the Peru upwelling region: A major flux of labile cobalt utilized as a micronutrient. Glob. Biogeochem. Cycles 18, 1-14. doi:10.1029/2003GB002216

Sanial, V., Kipp, L.E., Henderson, P.B., Beek, P. Van, Reyss, J., Hammond, D.E., Hawco, N.J., Saito, M.A., Resing, J.A., Sedwick, P., Moore, W.S., Charette, M.A., 2017. Radium-228 as a tracer of dissolved trace element inputs from the Peruvian continental margin. Mar. Chem. doi:10.1016/j.marchem.2017.05.008

Savoye, N., Benitez-Nelson, C., Burd, A.B., Cochran, J.K., Charette, M., Buesseler, K.O., Jackson, G.A., Roy-Barman, M., Schmidt, S., Elskens, M., 2006. ${ }^{234}$ Th sorption and export models in the water column: A review. Mar. Chem. 100, 234-249. doi:10.1016/j.marchem.2005.10.014

Schüßler, U., Schulz-Bull, D.E., Bauerfeind, E., 1997. Annual fluxes of particulate chemical trace compounds during the North-East Water Polynya Experiment. J. Mar. Syst. 10, 391-400. doi:10.1016/S0924-7963(96)00077-2

Sherrell, R.M., Boyle, A., 1992. The trace metal composition of suspended particles in the oceanic water column near Bermuda. Earth Planet. Sci. Lett. 111, 155-174.

Shiller, A.M., 1997. Manganese in surface waters of the Atlantic Ocean. Geophys. Res. Lett. 24, 14951498.

Smith, J.N., Yeats, P.A., Knowlton, S.E., Moran, S.B., 2014. Comparison of ${ }^{234} \mathrm{Th} /{ }^{238} \mathrm{U}$ and mass balance models for estimating metal removal fluxes in the Gulf of Maine and Scotian Shelf. Cont. Shelf Res. 77, 107-117. doi:10.1016/j.csr.2014.01.008

Stanley, R.H.R., Buesseler, K.O., Manganini, S.J., Steinberg, D.K., Valdes, J.R., 2004. A comparison of major and minor elemental fluxes collected in neutrally buoyant and surface-tethered sediment traps. Deep. Res. I 51, 1387-1395. doi:10.1016/j.dsr.2004.05.010 
Sunda, W.G., Huntsman, S.A., 1997. Interrelated influence of iron, light and cell size on marine phytoplankton growth. Nature 2051, 389-392.

Tagliabue, A., Aumont, O., Death, R., Dunne, J.P., Dutkiewicz, S., Galbraith, E., Misumi, K., Moore, J.K., Ridgwell, A., Sherman, E., Stock, C., Vichi, M., Völker, C., Yool, A., 2016. How well do global ocean biogeochemistry models simulate dissolved iron distributions? Global Biogeochem. Cycles 30, 149-174. doi:10.1002/2015GB005289

Tebo, B.M., Nealson, K.H., Emerson, S., Jacobs, L., 1984. Microbial mediation of Mn(II) and Co(II) precipitation at the $\mathrm{O}_{2} / \mathrm{H}_{2} \mathrm{~S}$ interfaces in two anoxic fjords. Limnol. Oceanogr. 29, 1247-1258.

Twining, B.S., Baines, S.B., 2013. The trace metal composition of marine phytoplankton. Ann. Rev. Mar. Sci. 5, 191-215. doi:10.1146/annurev-marine-121211-172322

Twining, B.S., Rauschenberg, S., Morton, P.L., Ohnemus, D.C., Lam, P.J., 2015. Comparison of particulate trace element concentrations in the North Atlantic Ocean as determined with discrete bottle sampling and in situ pumping. Deep. Res. II 116, 273-282. doi:10.1016/j.dsr2.2014.11.005

van Hulten, M.M.P., Middag, R., Dutay, J.-C., de Baar, H.J.W., Roy-Barman, M., Gehlen, M., Tagliabue, A., Sterl, A., 2017. Manganese in the West Atlantic Ocean in context of the first global ocean circulation model of manganese. Biogeosciences 14, 1123-1152. doi:10.5194/bg-14-1123-2017

van Hulten, M.M.P., Sterl, A., Middag, R., De Baar, H.J.W., Gehlen, M., Dutay, J.C., Tagliabue, A., 2014. On the effects of circulation, sediment resuspension and biological incorporation by diatoms in an ocean model of aluminium. Biogeosciences 11, 3757-3779. doi:10.5194/bg-11-3757-2014

van Hulten, M.M.P., Sterl, A., Tagliabue, A., Dutay, J.C., Gehlen, M., de Baar, H.J.W., Middag, R., 2013. Aluminium in an ocean general circulation model compared with the West Atlantic Geotraces cruises. J. Mar. Syst. 126, 3-23. doi:10.1016/j.jmarsys.2012.05.005

Watson, A.J., Bakker, D.C.E., Ridgewell, A.J., Boyd, P.W., Law, C.S., 2000. Effect of iron supply on Southern Ocean CO2 uptake and implications for glacial atmospheric CO2. Nature 407, 730-733.

Weinstein, S.E., Moran, S.B., 2005. Vertical flux of particulate $\mathrm{Al}, \mathrm{Fe}, \mathrm{Pb}$, and $\mathrm{Ba}$ from the upper ocean estimated from ${ }^{234} \mathrm{Th} /{ }^{238} \mathrm{U}$ disequilibria. Deep. Res. I 52, 1477-1488. doi:10.1016/j.dsr.2005.03.008

Weinstein, S.E., Moran, S.B., 2004. Distribution of size-fractionated particulate trace metals collected by bottles and in-situ pumps in the Gulf of Maine-Scotian Shelf and Labrador Sea. Mar. Chem. 87, 121-135. doi:10.1016/j.marchem.2004.02.004

Yeats, P.A., Dalziel, J.A., Moran, S.B., 1992. A Comparison of Dissolved and Particulate Mn and Al Distributions in the Western North-Atlantic. Oceanol. Acta 15, 609-619.

\section{Website and Database References}

Bates, Nicholas, 2017. Seawater Inorganic Carbon Data (DIC) from the 2013 R/V Thomas G. Thompson TN303 cruise in the Eastern Tropical Pacific from October to December 2013 (U.S. GETORACES EPZT project). Biological and Chemical Oceanography Data Management Office (BCO-DMO). Dataset version 2017-05-03. http://lod.bco-dmo.org/id/dataset/699295 [access date: 29 June 2017].

Carlson, Craig, 2016. Dissolved Organic Carbon (DOC) measurements from R/V Thomas G. Thompson cruise TN303 in the Eastern Tropical Pacific in 2013 (U.S. GEOTRACES EPZT project). Biological and Chemical Oceanography Data Management Office (BCO-DMO). Dataset version 2016-06-08. http://lod.bco-dmo.org/id/dataset/643592 [access date: 29 June 2017].

John, Seth, 2015. Dissolved (0.4 micron filtered) Fe, $\mathrm{Zn}$ and $\mathrm{Cd}$ isotope ratios and concentrations across GEOTRACES-EPZT, from cruise TN303, 2013. Biological and Chemical Oceanography Data 
Management Office (BCO-DMO). Dataset version 2016-06-21. http://lod.bcodmo.org/id/dataset/643809 [access date: 29 June 2017].

Moffett, James, German, Christopher and Cutter, Gregory (2014) ODF bottle data along the US GEOTRACES East Pacific Zonal Transect from the R/V Thomas G. Thompson TN303 cruise in the tropical Pacific from Peru to Tahiti during 2013 (U.S. GEOTRACES EPZT project). Biological and Chemical Oceanography Data Management Office (BCO-DMO). Dataset version 2014-10-30. http://lod.bco-dmo.org/id/dataset/503145 [access date: 29 June 2017].

Resing, Joseph and Sedwick, Peter, 2016. Concentrations of dissolved manganese (Mn) in water-column samples from major stations that were occupied during the U.S. GEOTRACES EPZT cruise (R/V Thomas G. Thompson TN303) from October to December 2013. Biological and Chemical Oceanography Data Management Office (BCO-DMO). Dataset version 2016-12-20. http://lod.bcodmo.org/id/dataset/670692 [access date: 29 June 2017].

Saito, Mak, 2016. Total and labile dissolved Cobalt from the GEOTRACES EPZT cruise. Biological and Chemical Oceanography Data Management Office (BCO-DMO). Dataset version 2016-07-01. http://lod.bco-dmo.org/id/dataset/642974 [access date: 29 June 2017].

Sedwick, Peter and Resing, Joseph, 2016. Water-column dissolved iron (Fe) concentrations for major stations occupied during U.S. GEOTRACES EPZT cruise (R/V Thomas G. Thompson TN303) from October to December 2013; post-cruise analyses conducted at Old Dominion University. Biological and Chemical Oceanography Data Management Office (BCO-DMO). Dataset version 2016-12-19. http://lod.bco-dmo.org/id/dataset/670285 [access date: 29 June 2017]. 
Table 1: Large and small particle E: $:^{234}$ Th ratios. Element: ${ }^{.34}$ Th statistics (means, standard deviations, and ranges) are grouped by zone and into the 'surface layer' (SL, 0-100 m, with mixed layer) and the subsequent $300 \mathrm{~m}$ (SSL, 'subsurface layer', 100-400 m).

\begin{tabular}{|c|c|c|c|c|c|c|c|c|c|c|c|c|c|c|c|c|c|}
\hline \multirow{3}{*}{$\begin{array}{c}\text { Large particle ratios } \\
\text { POC: }:{ }^{234} \mathrm{Th}(\mu \mathrm{mol}: \mathrm{dpm}) \mathrm{SL}\end{array}$} & \multicolumn{5}{|c|}{ Shelf } & \multicolumn{6}{|c|}{ Offshore } & \multicolumn{6}{|c|}{ Gyre } \\
\hline & \multicolumn{2}{|c|}{ Mean \pm s.d. } & \multicolumn{3}{|c|}{ Range } & \multicolumn{3}{|c|}{ Mean \pm s.d. } & \multicolumn{3}{|c|}{ Range } & \multicolumn{3}{|c|}{ Mean \pm s.d. } & \multicolumn{3}{|c|}{ Range } \\
\hline & 5 & \pm 5 & 1 & - & 19 & 2 & \pm & 1 & 1 & & 3 & 2 & \pm & 1 & 0.1 & & 3 \\
\hline POC: ${ }^{234} \mathrm{Th}(\mu \mathrm{mol}: \mathrm{dpm}) \mathrm{SSL}$ & 1.3 & \pm 0.3 & 1 & - & 2 & 1 & \pm & 0.2 & 0.7 & - & 2 & 0.7 & \pm & 0.2 & 0.3 & & 2 \\
\hline $\mathrm{P}:{ }^{.234} \mathrm{Th}$ (nmol:dpm) SL & 140 & \pm 170 & 5 & - & 600 & 35 & \pm & 20 & 11 & - & 72 & 19 & \pm & 9 & 2 & & 35 \\
\hline $\mathrm{P}:{ }^{.234} \mathrm{Th}$ (nmol:dpm) SSL & 23 & \pm 16 & 8 & - & 54 & 8 & \pm & 6 & 3 & - & 27 & 6 & \pm & 5 & 2 & & 28 \\
\hline $\mathrm{Cd}:{ }^{234} \mathrm{Th}$ (pmol:dpm) SL & 130 & \pm 160 & 6 & - & 560 & 44 & \pm & 32 & 5 & - & 93 & 7 & \pm & 8 & 2 & - & 49 \\
\hline $\mathrm{Cd}:{ }^{234} \mathrm{Th}$ (pmol:dpm) SSL & 18 & \pm 14 & 5 & - & 45 & 9 & \pm & 10 & 3 & - & 41 & 3 & \pm & 2 & 1 & - & 8 \\
\hline Co: ${ }^{234} \mathrm{Th}$ (pmol:dpm) SL & 20 & \pm 22 & 1 & - & 78 & 8 & \pm & 4 & 2 & - & 13 & 2 & \pm & 1 & 1 & - & 5 \\
\hline Co: ${ }^{234} \mathrm{Th}$ (pmol:dpm) SSL & 7 & \pm 4 & 3 & - & 15 & 3 & \pm & 1 & 2 & - & 6 & 4 & \pm & 5 & 1 & - & 21 \\
\hline $\mathrm{Mn}:{ }^{234} \mathrm{Th}$ (pmol:dpm) SL & 520 & \pm 740 & 40 & - & 2800 & 140 & \pm & 80 & 50 & - & 280 & 100 & \pm & 30 & 50 & - & 170 \\
\hline Mn: ${ }^{234} \mathrm{Th}$ (pmol:dpm) SSL & 110 & \pm 70 & 50 & - & 210 & 140 & \pm & 140 & 30 & - & 590 & 540 & \pm & 460 & 70 & - & 1800 \\
\hline $\mathrm{Fe}:{ }^{234} \mathrm{Th}$ (nmol:dpm) SL & 36 & \pm 71 & 4 & - & 243 & 1.1 & \pm & 1.5 & 0.2 & - & 4 & 1.0 & \pm & 0.9 & 0.4 & & 4 \\
\hline $\mathrm{Fe}:{ }^{234} \mathrm{Th}$ (nmol:dpm) SSL & 14 & \pm 12 & 0.5 & - & 38 & 1.3 & \pm & 1.1 & 0.4 & - & 4 & 1.7 & \pm & 1.3 & 0.4 & - & 5 \\
\hline
\end{tabular}


Shelf

Offshore

Gyre

\begin{tabular}{|c|c|c|c|c|c|c|c|c|c|c|c|c|c|c|c|c|c|c|}
\hline \multirow{3}{*}{$\begin{array}{c}\text { Small particle ratios } \\
\text { POC: }{ }^{234} \mathrm{Th}(\mu \mathrm{mol}: \mathrm{dpm}) \mathrm{SL}\end{array}$} & & & \\
\hline & \multicolumn{3}{|c|}{ Mean \pm s.d. } & \multicolumn{3}{|c|}{ Range } & \multicolumn{3}{|c|}{ Mean \pm s.d. } & \multicolumn{3}{|c|}{ Range } & \multicolumn{3}{|c|}{ Mean \pm s.d. } & \multicolumn{3}{|c|}{ Range } \\
\hline & 4 & \pm & 3 & 1 & & 12 & 3 & \pm & 1 & 2 & & 5 & 2 & \pm & 0.4 & 1.0 & & 2 \\
\hline POC: ${ }^{234} \mathrm{Th}(\mu \mathrm{mol}: \mathrm{dpm}) \mathrm{SSL}$ & 1.1 & \pm & 0.3 & 0.8 & & 2 & 0.8 & \pm & 0.2 & 0.5 & & 1.2 & 0.6 & \pm & 0.2 & 0.3 & & 1.1 \\
\hline $\mathrm{P}:{ }^{234} \mathrm{Th}$ (nmol:dpm) SL & 70 & \pm & 60 & 6 & 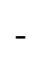 & 190 & 41 & \pm & 19 & 25 & & 81 & 21 & \pm & 7 & 11 & 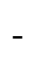 & 35 \\
\hline $\mathrm{P}:{ }^{234} \mathrm{Th}$ (nmol:dpm) SSL & 13 & \pm & 4 & 10 & & 21 & 9 & \pm & 3 & 4 & & 17 & 7 & \pm & 3 & 4 & & 16 \\
\hline Cd: ${ }^{234} \mathrm{Th}$ (pmol:dpm) SL & 63 & \pm & 55 & 5 & & 180 & 34 & \pm & 18 & 13 & & 61 & 3 & \pm & 1 & 2 & & 6 \\
\hline $\mathrm{Cd}:{ }^{234} \mathrm{Th}$ (pmol:dpm) SSL & 8 & \pm & 3 & 5 & & 16 & 9 & \pm & 6 & 3 & & 22 & 6 & \pm & 3 & 2 & & 17 \\
\hline Co: ${ }^{234} \mathrm{Th}$ (pmol:dpm) SL & 8 & - & 7 & 1 & & 23 & 12 & \pm & 4 & 7 & & 18 & 3 & \pm & 1 & 2 & & 6 \\
\hline Co: ${ }^{234} \mathrm{Th}$ (pmol:dpm) SSL & 2 & - & 0.7 & 1 & & 4 & 4 & \pm & 2 & 1 & & 8 & 3 & \pm & 2 & 1 & & 13 \\
\hline Mn: ${ }^{234} \mathrm{Th}$ (pmol:dpm) SL & 160 & \pm & 200 & 10 & & 700 & 120 & \pm & 90 & 50 & - & 300 & 27 & \pm & 9 & 16 & - & 52 \\
\hline Mn: ${ }^{234} \mathrm{Th}$ (pmol:dpm) SSL & 22 & $\underline{-}$ & 14 & 11 & & 54 & 67 & \pm & 70 & 8 & - & 220 & 260 & \pm & 170 & 30 & - & 800 \\
\hline $\mathrm{Fe}:{ }^{234} \mathrm{Th}$ (nmol:dpm) SL & 3 & \pm & 3 & 0.8 & & 12 & 0.3 & \pm & 0.3 & 0.1 & - & 0.9 & 0.2 & \pm & 0.3 & 0.03 & - & 2 \\
\hline $\mathrm{Fe}:{ }^{234} \mathrm{Th}$ (nmol:dpm) SSL & 4 & \pm & 2 & 2.0 & - & 7 & 0.8 & \pm & 0.6 & 0.1 & - & 2 & 0.3 & \pm & 0.4 & 0.03 & - & 1.4 \\
\hline
\end{tabular}


Table 2: Fluxes, flux ratios, and \% lithogenic fraction. Flux averages and their respective standard deviations for the elements of interest are reported at $100 \mathrm{~m}$ and $200 \mathrm{~m}$ for each zone. Flux ratios are equivalent to the flux at $200 \mathrm{~m}$ divided by the flux at $100 \mathrm{~m}$. The average percent of the total flux at $100 \mathrm{~m}$ that was composed of lithogenics is reported for each zone.

\begin{tabular}{|c|c|c|c|c|c|c|c|c|c|}
\hline \multirow{3}{*}{$\begin{array}{l}\text { Flux at } 100 \text { m } \\
\text { POC }\left(\mathrm{mmol} \mathrm{m}^{-2} \mathrm{~d}^{-1}\right)\end{array}$} & \multicolumn{3}{|c|}{ Shelf } & \multicolumn{3}{|c|}{ Offshore } & \multicolumn{3}{|c|}{ Gyre } \\
\hline & \multicolumn{3}{|c|}{ Mean \pm s.d. } & \multicolumn{3}{|c|}{ Mean \pm s.d. } & \multicolumn{3}{|c|}{ Mean \pm s.d. } \\
\hline & 9 & \pm & 3 & 2.7 & \pm & 1.3 & 1.6 & \pm & 0.6 \\
\hline $\mathrm{P}\left(\mu \mathrm{mol} \mathrm{m} \mathrm{m}^{-2} \mathrm{~d}^{-1}\right)$ & 302 & \pm & 342 & 33 & \pm & 17 & 17 & \pm & 8 \\
\hline $\mathrm{Cd}\left(\mathrm{nmol} \mathrm{m} \mathrm{m}^{-2} \mathrm{~d}^{-1}\right)$ & 291 & \pm & 400 & 40 & \pm & 25 & 6 & \pm & 3 \\
\hline Co $\left(n m o l m^{-2} d^{-1}\right)$ & 39 & \pm & 21 & 10 & \pm & 6 & 3 & \pm & 2 \\
\hline $\operatorname{Mn}\left(n m o l m^{-2} d^{-1}\right)$ & 1020 & \pm & $\begin{array}{l}117 \\
3\end{array}$ & 368 & \pm & 313 & 187 & \pm & 74 \\
\hline $\mathrm{Fe}\left(\mu \mathrm{mol} \mathrm{m} \mathrm{m}^{-2} \mathrm{~d}^{-1}\right)$ & 61 & \pm & 33 & 3 & \pm & 4 & 1.6 & \pm & 0.7 \\
\hline \multicolumn{10}{|l|}{ Flux at $200 \mathrm{~m}$} \\
\hline POC $\left(\mathrm{mmol} \mathrm{m}^{-2} \mathrm{~d}^{-1}\right)$ & 8 & \pm & 1 & 1 & \pm & 1 & 1.0 & \pm & 0.3 \\
\hline $\mathrm{P}\left(\mu \mathrm{mol} \mathrm{m} \mathrm{m}^{-2} \mathrm{~d}^{-1}\right)$ & 105 & \pm & 83 & 14 & \pm & 14 & 11 & \pm & 9 \\
\hline $\mathrm{Cd}\left(\mathrm{nmol} \mathrm{m} \mathrm{m}^{-2} \mathrm{~d}^{-1}\right)$ & 81 & \pm & 69 & 17 & \pm & 22 & 4 & \pm & 2 \\
\hline Co $\left(n m o l m^{-2} d^{-1}\right)$ & 35 & \pm & 23 & 4 & \pm & 3 & 3 & \pm & 2 \\
\hline $\operatorname{Mn}\left(\operatorname{nmol~m}{ }^{-2} d^{-1}\right)$ & 520 & \pm & 336 & 125 & \pm & 87 & 658 & \pm & 388 \\
\hline $\mathrm{Fe}\left(\mu \mathrm{mol} \mathrm{m}{ }^{-2} \mathrm{~d}^{-1}\right)$ & 71 & \pm & 52 & 2 & \pm & 3 & 2 & \pm & 2 \\
\hline \multicolumn{10}{|l|}{ Flux ratio } \\
\hline $\mathrm{POC}$ & 1.1 & \pm & 0.4 & 0.5 & \pm & 0.3 & 0.7 & \pm & 0.2 \\
\hline $\mathrm{P}$ & 0.5 & \pm & 0.5 & 0.5 & \pm & 0.5 & 0.6 & \pm & 0.3 \\
\hline $\mathrm{Cd}$ & 0.5 & \pm & 0.5 & 0.6 & \pm & 0.7 & 0.7 & \pm & 0.2 \\
\hline $\mathrm{Co}$ & 1.0 & \pm & 0.1 & 0.5 & \pm & 0.3 & 1.4 & \pm & 0.4 \\
\hline $\mathrm{Mn}$ & 0.7 & \pm & 0.6 & 0.4 & \pm & 0.4 & 3.4 & \pm & 1.2 \\
\hline $\mathrm{Fe}$ & 2.3 & \pm & 1.7 & 0.9 & \pm & 0.5 & 1.9 & \pm & 1.7 \\
\hline \multicolumn{10}{|c|}{ \% Lithogenic flux at $100 \mathrm{~m}$} \\
\hline $\mathrm{POC}$ & & -- & & & -- & & & -- & \\
\hline $\mathrm{P}$ & $0 \%$ & \pm & $0 \%$ & $0 \%$ & \pm & $0 \%$ & $0 \%$ & \pm & $0 \%$ \\
\hline $\mathrm{Cd}$ & $0 \%$ & \pm & $0 \%$ & $0 \%$ & \pm & $0 \%$ & $0 \%$ & \pm & $0 \%$ \\
\hline Co & $9 \%$ & \pm & $8 \%$ & $1 \%$ & \pm & $1 \%$ & $2 \%$ & \pm & $1 \%$ \\
\hline $\mathrm{Mn}$ & $51 \%$ & \pm & $48 \%$ & $3 \%$ & \pm & $4 \%$ & $2 \%$ & \pm & $1 \%$ \\
\hline $\mathrm{Fe}$ & $17 \%$ & \pm & $10 \%$ & $24 \%$ & \pm & $13 \%$ & $14 \%$ & \pm & $7 \%$ \\
\hline
\end{tabular}


Table 3: Upper ocean residence times. Removal timescales were calculated by dividing either the total element inventory, the total particulate inventory, or the inventory on sinking particles only, by the particulate flux of an element to get $\tau_{T o t}, \tau_{\text {Part }}$, and $\tau_{S i n k}$, respectively. Zonal averages of residence times and their respective standard deviations for the elements of interest are reported for the upper $100 \mathrm{~m}$ in days (particulate pools) and years (total pool).

\begin{tabular}{|c|c|c|c|c|c|c|c|c|}
\hline & \multicolumn{2}{|c|}{ Shelf } & \multicolumn{3}{|c|}{ Offshore } & \multicolumn{3}{|c|}{ Gyre } \\
\hline$\tau_{\text {Sink }}($ days $)$ & \multicolumn{2}{|c|}{ Mean \pm s.d. } & \multicolumn{3}{|c|}{ Mean \pm s.d. } & \multicolumn{3}{|c|}{ Mean \pm s.d. } \\
\hline POC & 7 & \pm 4 & 15 & \pm & 7 & 10 & \pm & 4 \\
\hline $\mathrm{P}$ & 7 & \pm 8 & 16 & \pm & 5 & 12 & \pm & 6 \\
\hline $\mathrm{Cd}$ & 10 & \pm 10 & 16 & \pm & 3 & 9 & \pm & 4 \\
\hline $\mathrm{Co}$ & 5 & \pm 3 & 13 & \pm & 4 & 11 & \pm & 5 \\
\hline $\mathrm{Mn}$ & 5 & \pm 4 & 6 & \pm & 2 & 2 & \pm & 2 \\
\hline $\mathrm{Fe}$ & 3 & \pm 3 & 4 & \pm & 1 & 5 & \pm & 3 \\
\hline \multicolumn{9}{|l|}{$\tau_{\text {Part }}($ days $)$} \\
\hline POC & 36 & \pm 8 & 68 & \pm & 27 & 77 & \pm & 23 \\
\hline $\mathrm{P}$ & 24 & \pm 16 & 77 & \pm & 30 & 101 & \pm & 55 \\
\hline $\mathrm{Cd}$ & 30 & \pm 24 & 61 & \pm & 27 & 48 & \pm & 22 \\
\hline Co & 20 & \pm 15 & 80 & \pm & 45 & 105 & \pm & 35 \\
\hline $\mathrm{Mn}$ & 17 & \pm 9 & 28 & \pm & 15 & 14 & \pm & 6 \\
\hline $\mathrm{Fe}$ & 7 & \pm 4 & 18 & \pm & 11 & 15 & \pm & 9 \\
\hline \multicolumn{9}{|l|}{$\tau_{\text {Tot }}($ years $)$} \\
\hline POC w.r.t. DIC & 70 & \pm 21 & 251 & \pm & 102 & 397 & \pm & 121 \\
\hline POC w.r.t. DOC & 2 & \pm 1 & 8 & \pm & 4 & 14 & \pm & 5 \\
\hline $\mathrm{P}$ & 4 & \pm 3 & 9 & \pm & 2 & 7 & \pm & 3 \\
\hline $\mathrm{Cd}$ & 2.2 & \pm 2.2 & 1.0 & \pm & 0.6 & 0.6 & \pm & 0.6 \\
\hline Co & 1.3 & \pm 0.7 & 2.4 & \pm & 0.9 & 2.1 & \pm & 1.2 \\
\hline $\mathrm{Mn}$ & 1.2 & \pm 0.7 & 3.3 & \pm & 1.9 & 1.5 & \pm & 0.4 \\
\hline $\mathrm{Fe}$ & 0.03 & \pm 0.01 & 0.07 & \pm & 0.04 & 0.07 & \pm & 0.03 \\
\hline
\end{tabular}



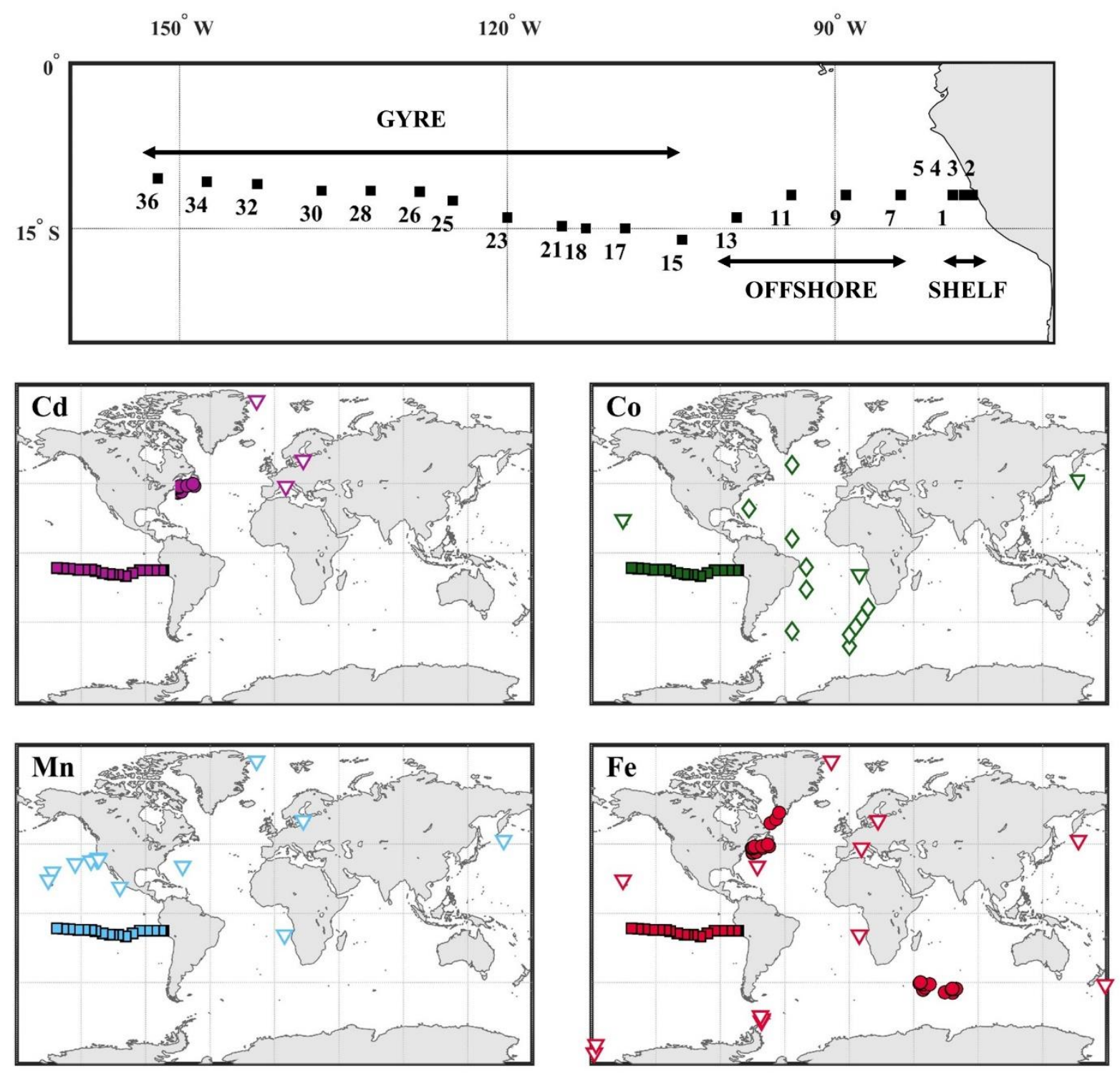

Figure 1: Station locations with prior 0 to $400 \mathrm{~m}$ study sites for trap- and ${ }^{234} \mathrm{Th}$-based flux estimates. GP-16 transect locations where particulate ${ }^{234} \mathrm{Th}$, trace metal, and particle composition measurements were taken are shown in the top panel. In the lower panels, sites where simultaneous total ${ }^{234} \mathrm{Th}$, large particle ${ }^{234} \mathrm{Th}$, and large particle element measurements have been made are shown as filled squares (this study) and circles (Lemaitre et al., 2016; Planquette et al., 2011; Smith et al., 2014; Weinstein and Moran, 2005). Prior ${ }^{234}$ Th studies where large particle E: ${ }^{234}$ Th values were extrapolated from other data are indicated by diamonds (Bown et al., 2011; Dulaquais et al., 2014) and trap-only studies by nabla (Collier et al., 2000; Frew et al., 2006; Lamborg et al., 2008; Martin, 1990; Martin et al., 1985, 1980, Martin and Knauer, 1984, 1983; Migon et al., 2002; Noble et al., 2012; Pohl et al., 2004; Quetel et al., 1993; Schüßler et al., 1997; Stanley et al., 2004). 

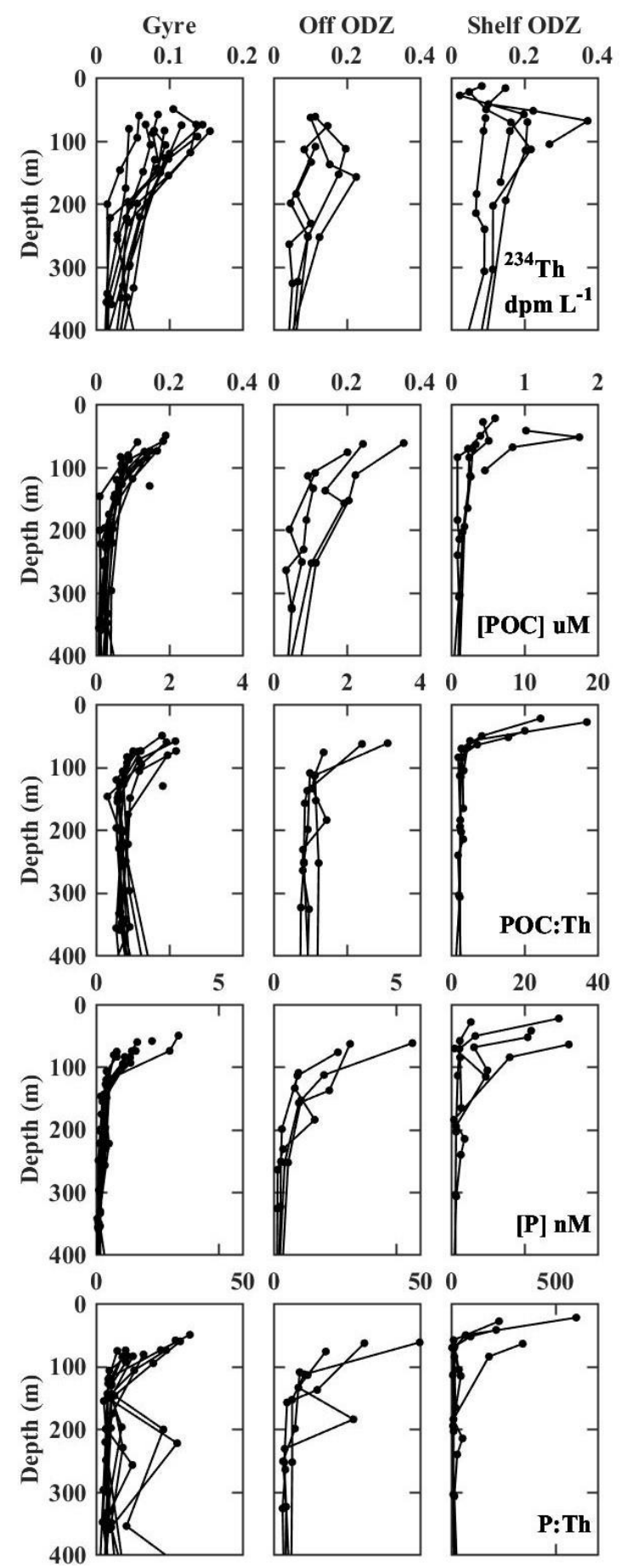

Figure 2: Element concentration and E: ${ }^{234} \mathbf{T h}$. Station profiles of ${ }^{234} \mathrm{Th}$ large particle activity (top panels) are shown with the concentration profiles for the elements and the E: ${ }^{234} \mathrm{Th}$ profiles. All profiles are shown versus depth and grouped by zone. Mixed layer samples are not shown. The units for $\mathrm{E}:{ }^{234} \mathrm{Th}$ are the concentration units indicated for each element to dpm (e.g. POC: ${ }^{234} \mathrm{Th}$ in $\mu \mathrm{mol}: \mathrm{dpm})$. 

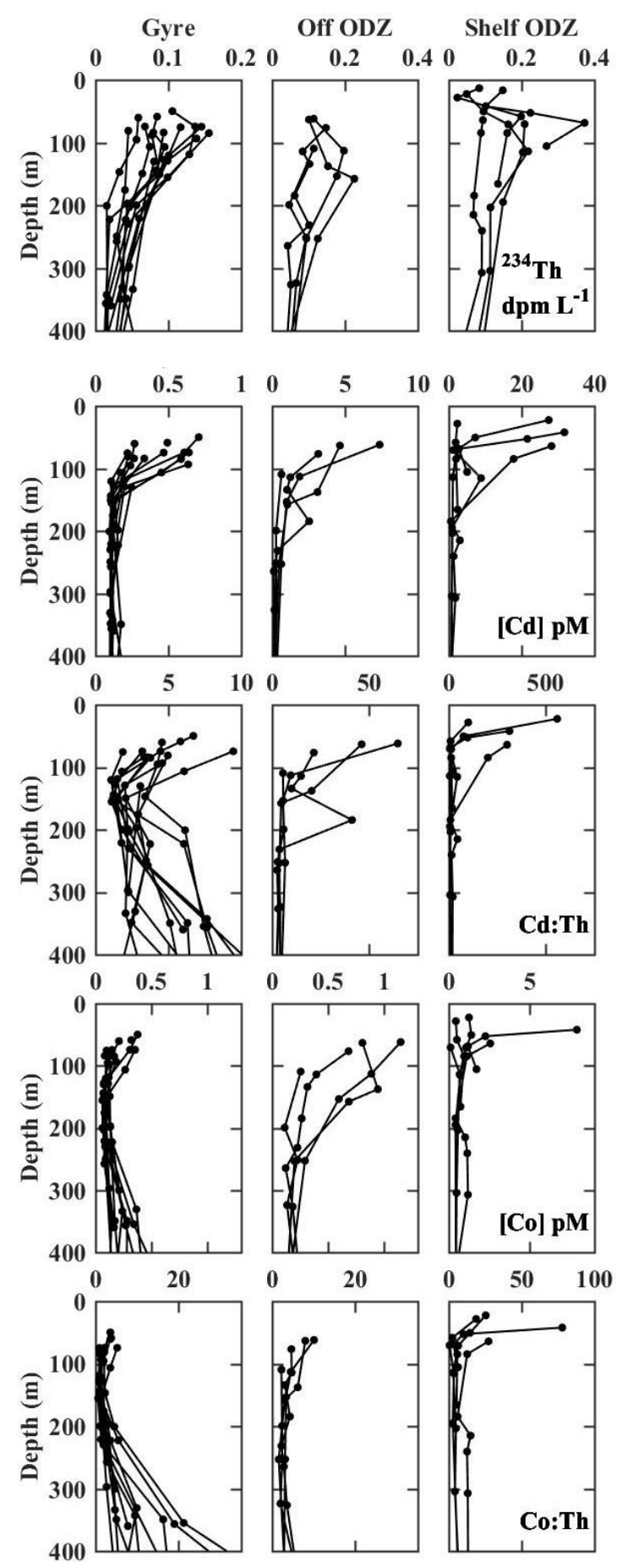

Figure 2: Element concentration and E: ${ }^{234} \mathbf{T h}$. Station profiles of ${ }^{234} \mathrm{Th}$ large particle activity (top panels) are shown with the concentration profiles for the elements and the E: ${ }^{234} \mathrm{Th}$ profiles. All profiles are shown versus depth and grouped by zone. Mixed layer samples are not shown. The units for $\mathrm{E}:{ }^{234} \mathrm{Th}$ are the concentration units indicated for each element to dpm (e.g. POC: ${ }^{.234} \mathrm{Th}$ in $\mu$ mol:dpm). 

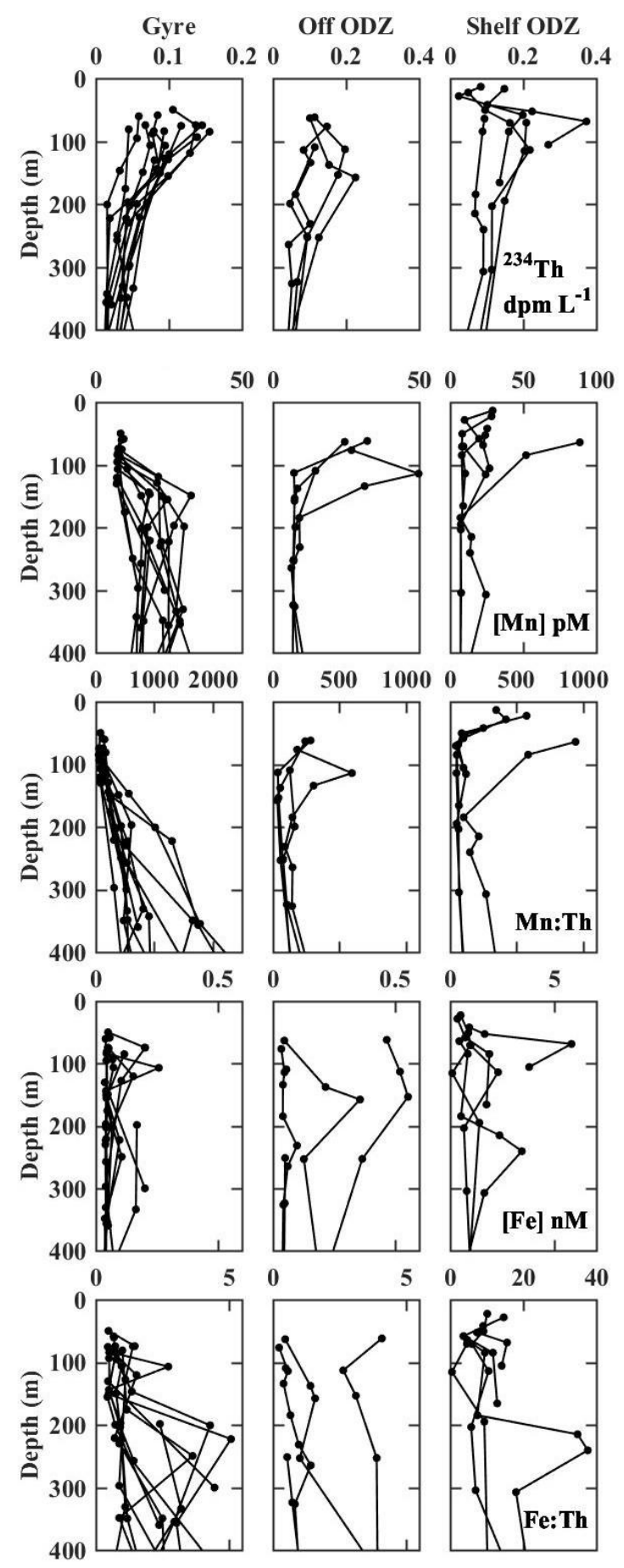

Figure 2: Element concentration and E: ${ }^{234} \mathbf{T h}$. Station profiles of ${ }^{234} \mathrm{Th}$ large particle activity (top panels) are shown with the concentration profiles for the elements and the E: ${ }^{234} \mathrm{Th}$ profiles. All profiles are shown versus depth and grouped by zone. Mixed layer samples are not shown. The units for $\mathrm{E}:{ }^{234} \mathrm{Th}$ are the concentration units indicated for each element to dpm (e.g. POC: ${ }^{234} \mathrm{Th}$ in $\mu$ mol:dpm). 

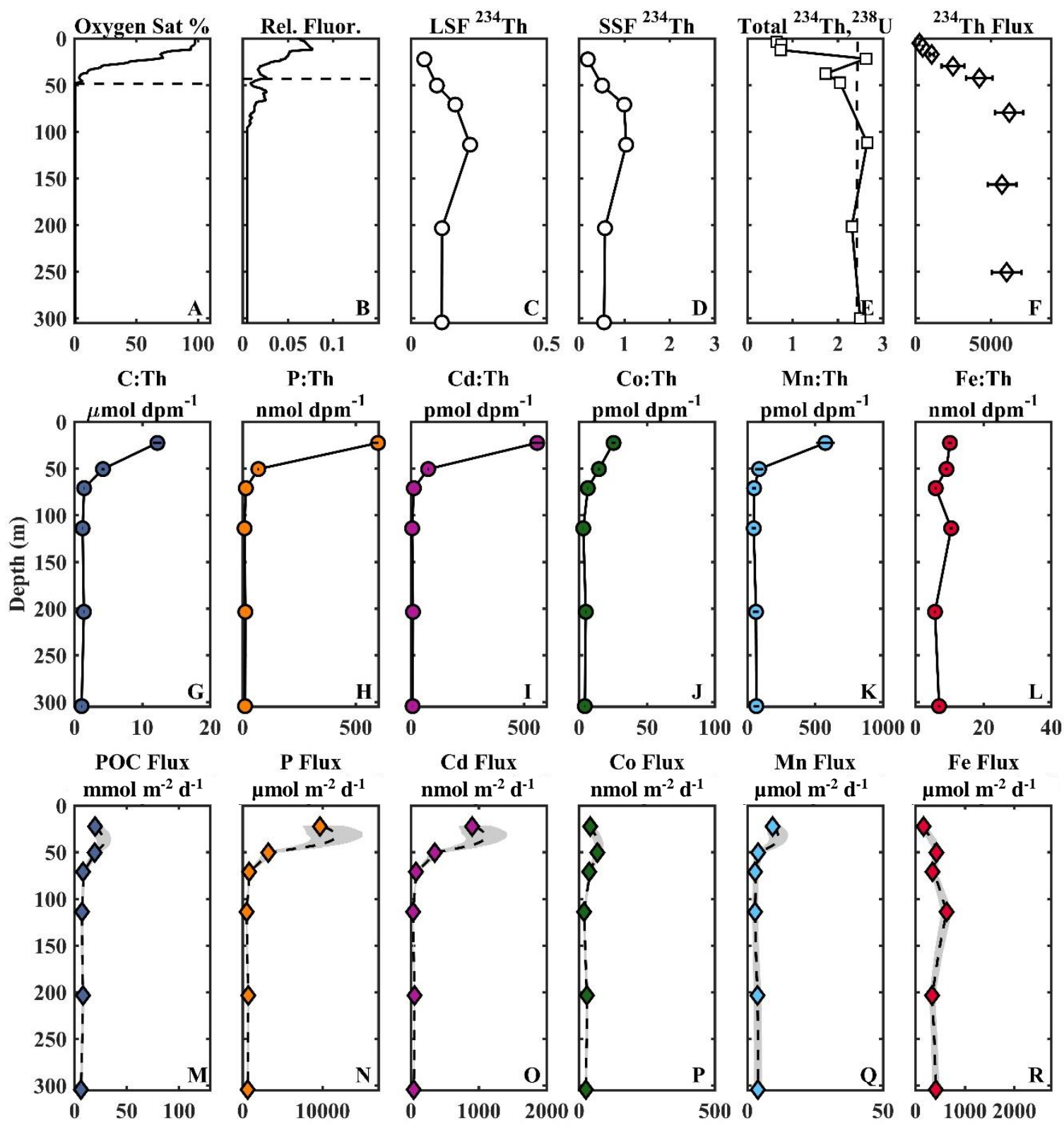

Figure 3: Element ratios and fluxes for characteristic stations in the Shelf (4-Above), Offshore (9), and Gyre (26). Each zone is represented by a station that generally follows the regional trends in element parameters. Oxygen saturation \% $(A)$ and relative florescence $(B)$ are shown with dashed lines indicating the depth of the uODZ boundary (panel $A$ ), when present, and Ez (panel $B)$. Particulate ${ }^{234} \mathrm{Th}(C$ and $D)$, total ${ }^{234} \mathrm{Th}\left(E\right.$, squares), and total ${ }^{238} \mathrm{U}$ activities $(E$, dashed line) are shown in dpm $\mathrm{L}^{-1}$. The derived ${ }^{234} \mathrm{Th}$ flux with depth $(F)$ is shown in dpm $\mathrm{m}^{-2} \mathrm{~d}^{-}$ ${ }^{1}$. The second row of panels ( $G$ to $L$ ) shows $\mathrm{E}:{ }^{234} \mathrm{Th}$ in the large particle fraction with the error on individual measurements indicated by the horizontal black lines. The last row of panels $(M$ to $R)$ shows the derived element flux with depth. The elemental fluxes are calculated at a given depth by multiplying the ${ }^{234} \mathrm{Th}$ flux $(F)$ by the element: ${ }^{234} \mathrm{Th}$ ratio $(G$ to $L)$. Extrapolated errors from the element ratios and ${ }^{234} \mathrm{Th}$ fluxes are indicated by the grey shading. 

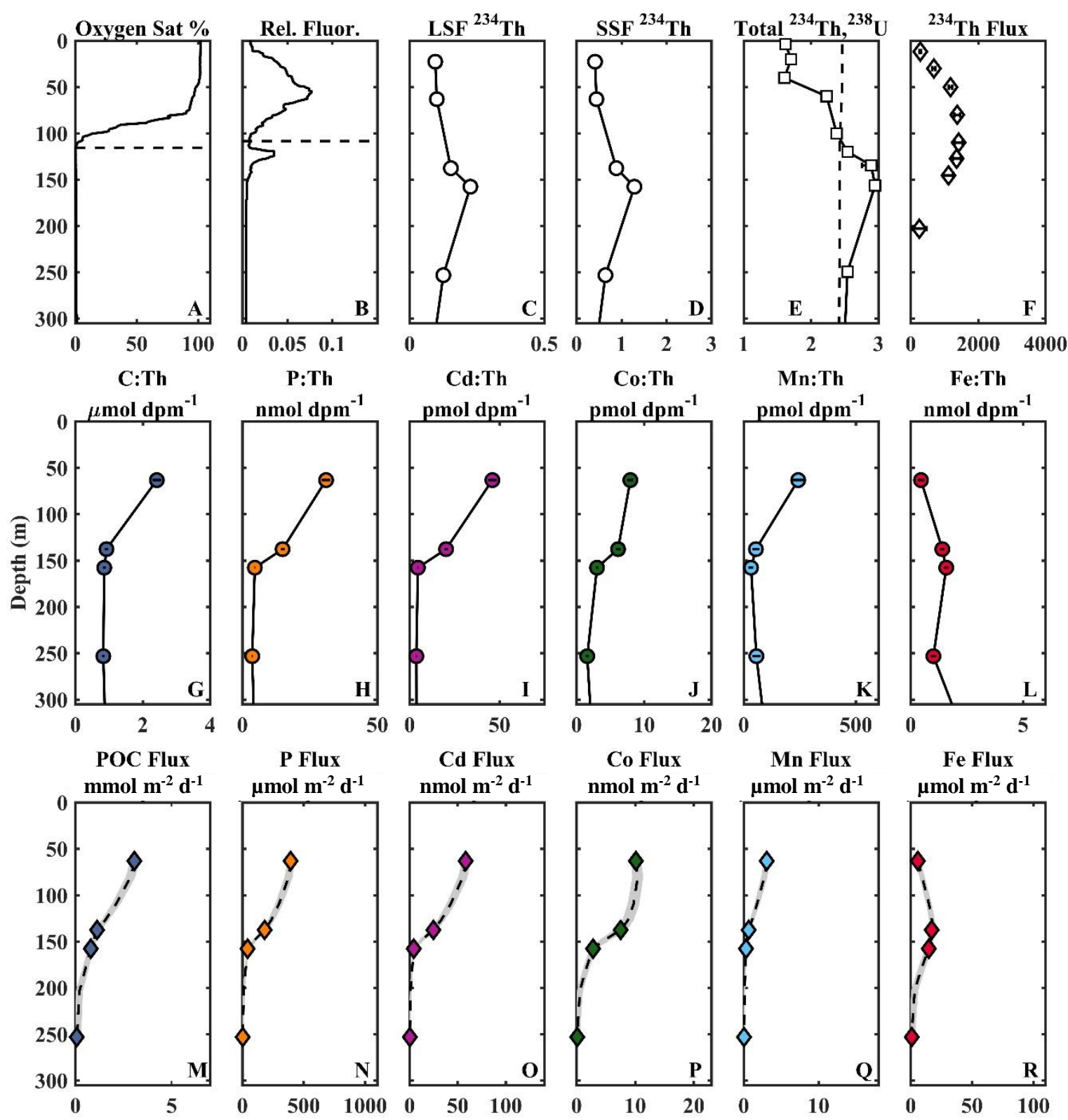

Fe Flux

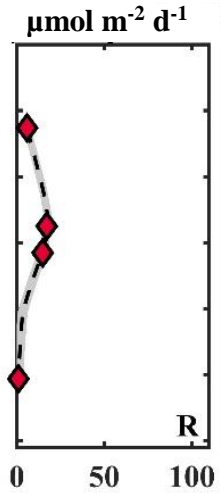

Figure 3: Element ratios and fluxes for characteristic stations in the Shelf (4), Offshore (9Above), and Gyre (26). Each zone is represented by a station that generally follows the regional trends in element parameters. Oxygen saturation \% $(A)$ and relative florescence $(B)$ are shown with dashed lines indicating the depth of the uODZ boundary (panel $A$ ), when present, and Ez (panel $B$ ). Particulate ${ }^{234} \mathrm{Th}(C$ and $D)$, total ${ }^{234} \mathrm{Th}\left(E\right.$, squares), and total ${ }^{238} \mathrm{U}$ activities ( $E$, dashed line) are shown in dpm $\mathrm{L}^{-1}$. The derived ${ }^{234} \mathrm{Th}$ flux with depth $(F)$ is shown in $\mathrm{dpm} \mathrm{m}^{-2} \mathrm{~d}^{-1}$. The second row of panels ( $G$ to $L$ ) shows $\mathrm{E}:{ }^{234} \mathrm{Th}$ in the large particle fraction with the error on individual measurements indicated by the horizontal black lines. The last row of panels ( $M$ to $R$ ) shows the derived element flux with depth. The elemental fluxes are calculated at a given depth by multiplying the ${ }^{234} \mathrm{Th}$ flux $(F)$ by the element: ${ }^{234} \mathrm{Th}$ ratio $(G$ to $L)$. Extrapolated errors from the element ratios and ${ }^{234} \mathrm{Th}$ fluxes are indicated by the grey shading. 

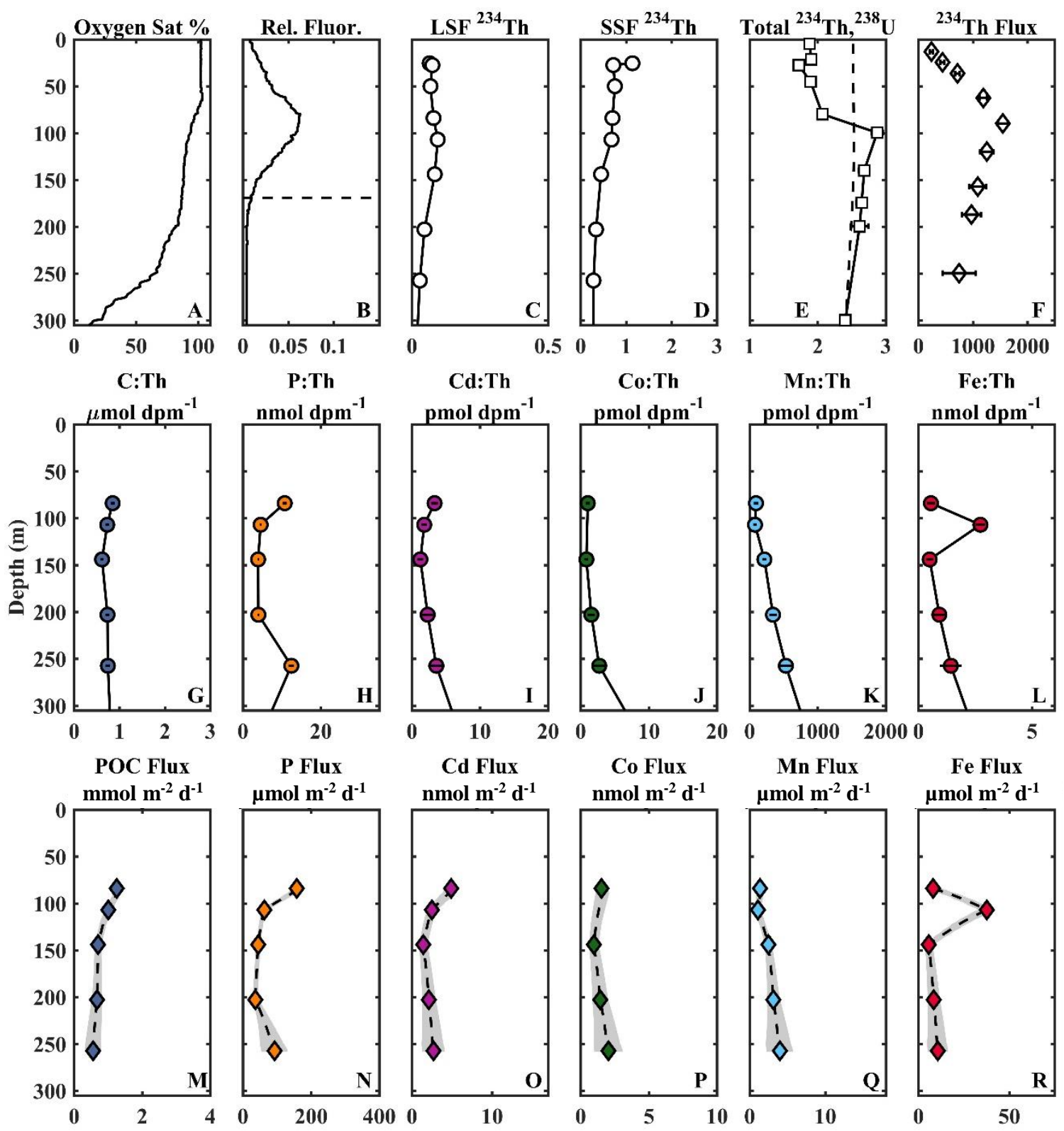

Fe Flux

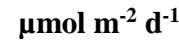

Figure 3: Element ratios and fluxes for characteristic stations in the Shelf (4), Offshore (9), and Gyre (26-Above). Each zone is represented by a station that generally follows the regional trends in element parameters. Oxygen saturation \% $(A)$ and relative florescence $(B)$ are shown with dashed lines indicating the depth of the uODZ boundary (panel $A$ ), when present, and Ez (panel $B$ ). Particulate ${ }^{234} \mathrm{Th}(C$ and $D)$, total ${ }^{234} \mathrm{Th}\left(E\right.$, squares), and total ${ }^{238} \mathrm{U}$ activities ( $E$, dashed line) are shown in dpm $\mathrm{L}^{-1}$. The derived ${ }^{234} \mathrm{Th}$ flux with depth $(F)$ is shown in $\mathrm{dpm} \mathrm{m}^{-2} \mathrm{~d}^{-1}$. The second row of panels ( $G$ to $L$ ) shows $\mathrm{E}:{ }^{234} \mathrm{Th}$ in the large particle fraction with the error on individual measurements indicated by the horizontal black lines. The last row of panels ( $M$ to $R$ ) shows the derived element flux with depth. The elemental fluxes are calculated at a given depth by multiplying the ${ }^{234} \mathrm{Th}$ flux $(F)$ by the element: ${ }^{234} \mathrm{Th}$ ratio $(G$ to $L)$. Extrapolated errors from the element ratios and ${ }^{234} \mathrm{Th}$ fluxes are indicated by the grey shading. 

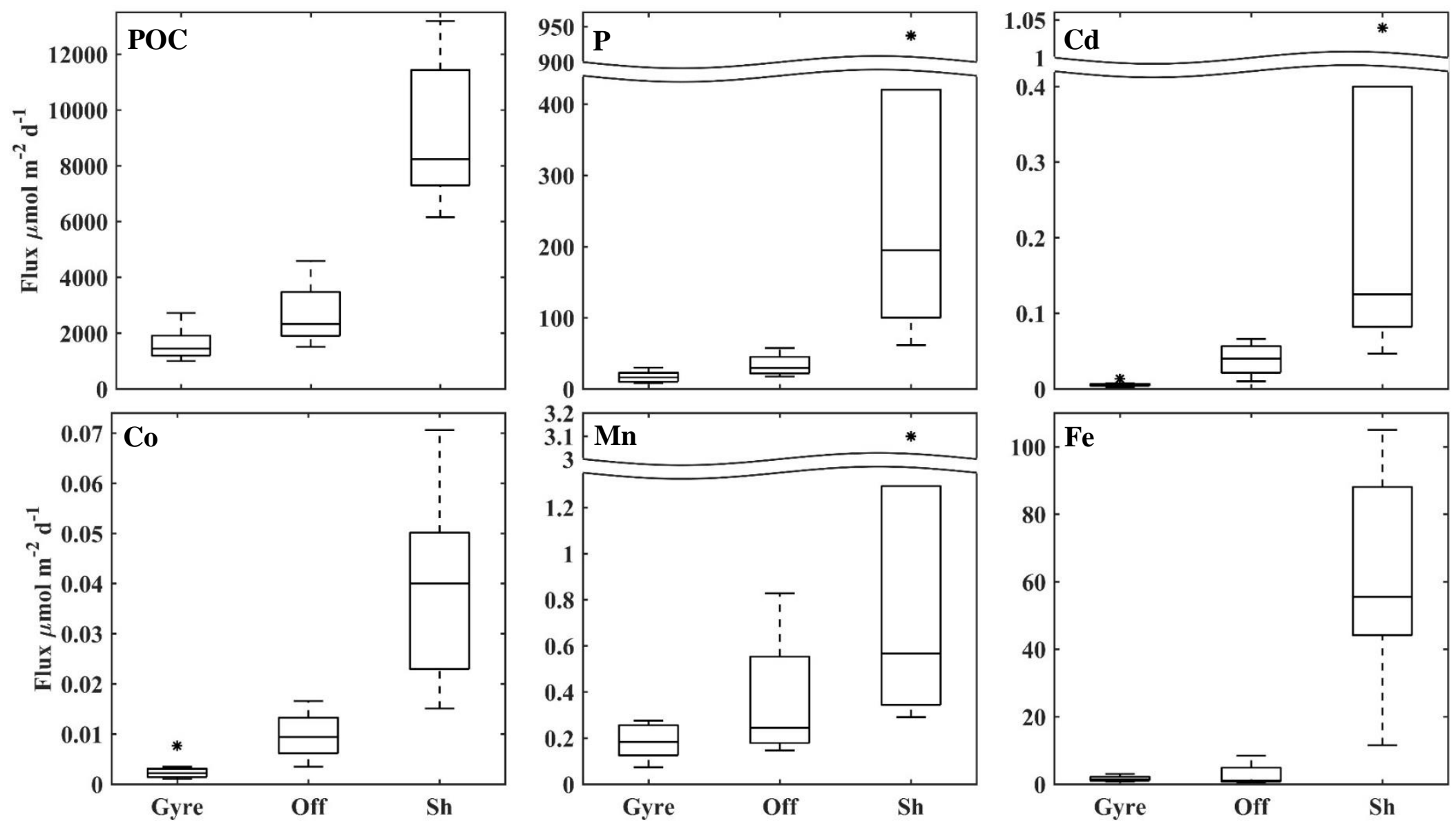

Figure 4: Zonal fluxes. Box plots are shown with central horizontal lines representing the zonal median for the Shelf (Sh), Offshore (Off), and Gyre. The bottom and the top edges of the boxes show the $25^{\text {th }}$ and $75^{\text {th }}$ percentiles. The whiskers extend to the highest and lowest points, with the exception of any data outliers, which are shown as asterisks. Outliers in this study fell outside of the $99.65^{\text {th }}$ percentile. 

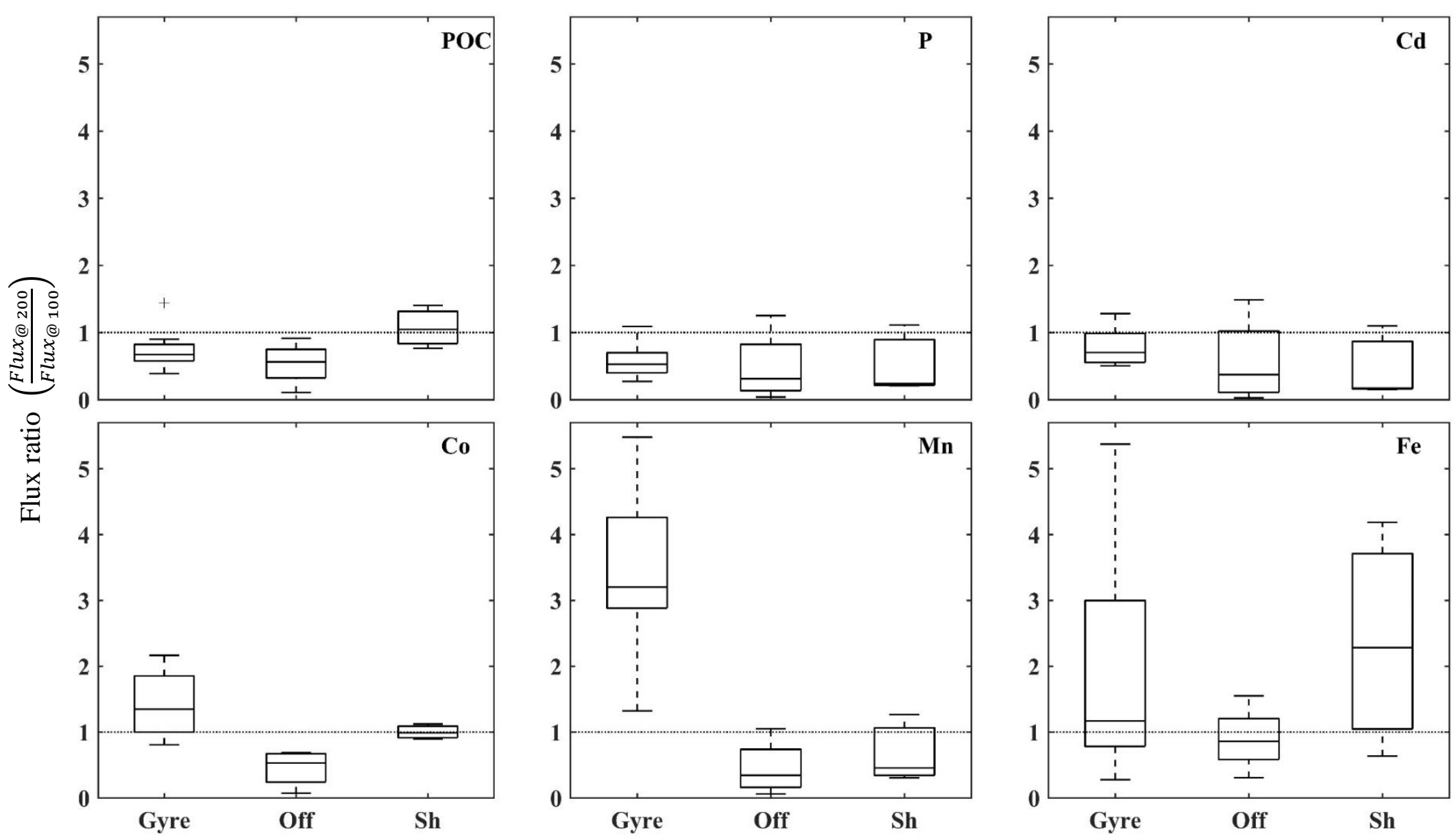

Figure 5: Zonal flux ratios. Median, $25^{\text {th }}$ percentile, $75^{\text {th }}$ percentile, and outlier flux ratios are reported as describe in Fig. 4 . Flux ratios are equivalent to the flux at $200 \mathrm{~m}$ divided by the flux at $100 \mathrm{~m}$. Flux ratios $>1$ indicate that flux has increased with depth between 100 $\mathrm{m}$ and $200 \mathrm{~m}$, whereas values $<1$ indicate a decrease over the same interval. 

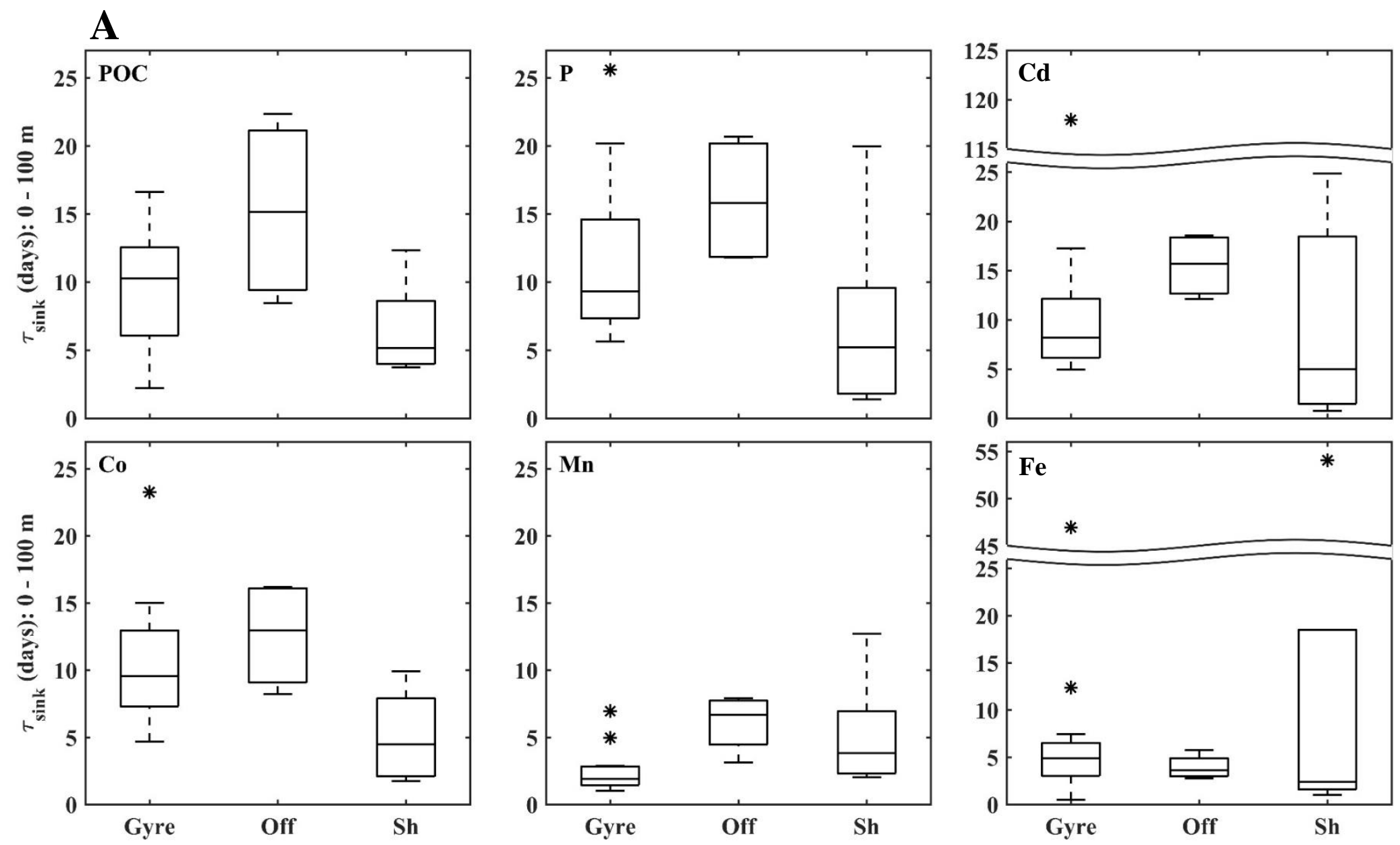

Figure 6: Zonal residence times. Figure $6 \mathrm{~A}$ contains $\tau_{\text {Sink }}$ data as calculated using the large particle flux and large particle inventory for the upper $100 \mathrm{~m}$. Figure $6 \mathrm{~B}$ shows the $\tau_{\text {Tot }}$ as calculated using the large particle flux and total inventory. Box plot structure is as described in Fig. 4. 

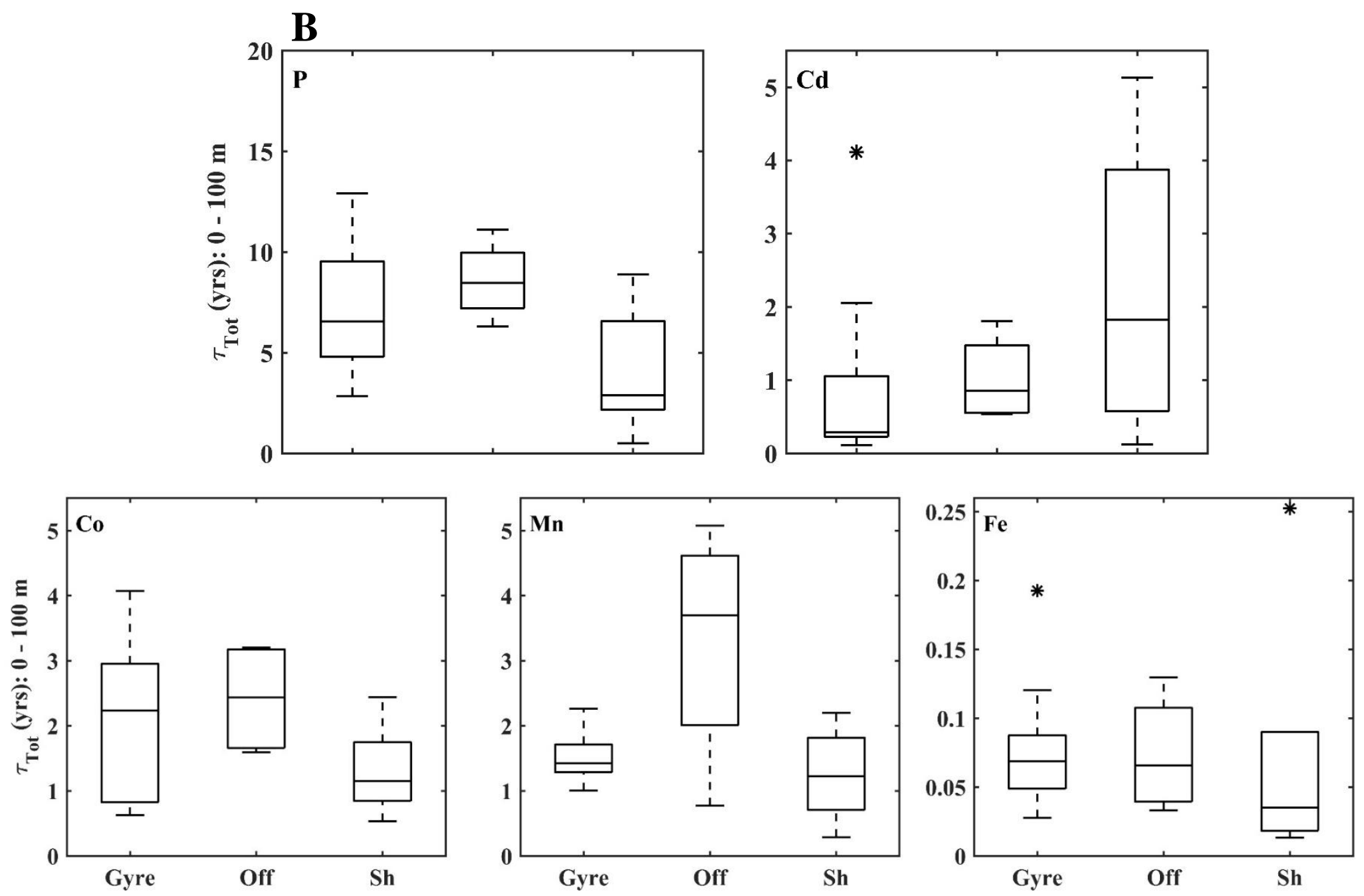

Figure 6: Zonal residence times. Figure $6 \mathrm{~A}$ contains $\tau_{\text {Sink }}$ data as calculated using the large particle flux and large particle inventory for the upper $100 \mathrm{~m}$. Figure $6 \mathrm{~B}$ shows the $\tau_{\text {Tot }}$ as calculated using the large particle flux and total inventory. Box plot structure is as described in Fig. 4. 

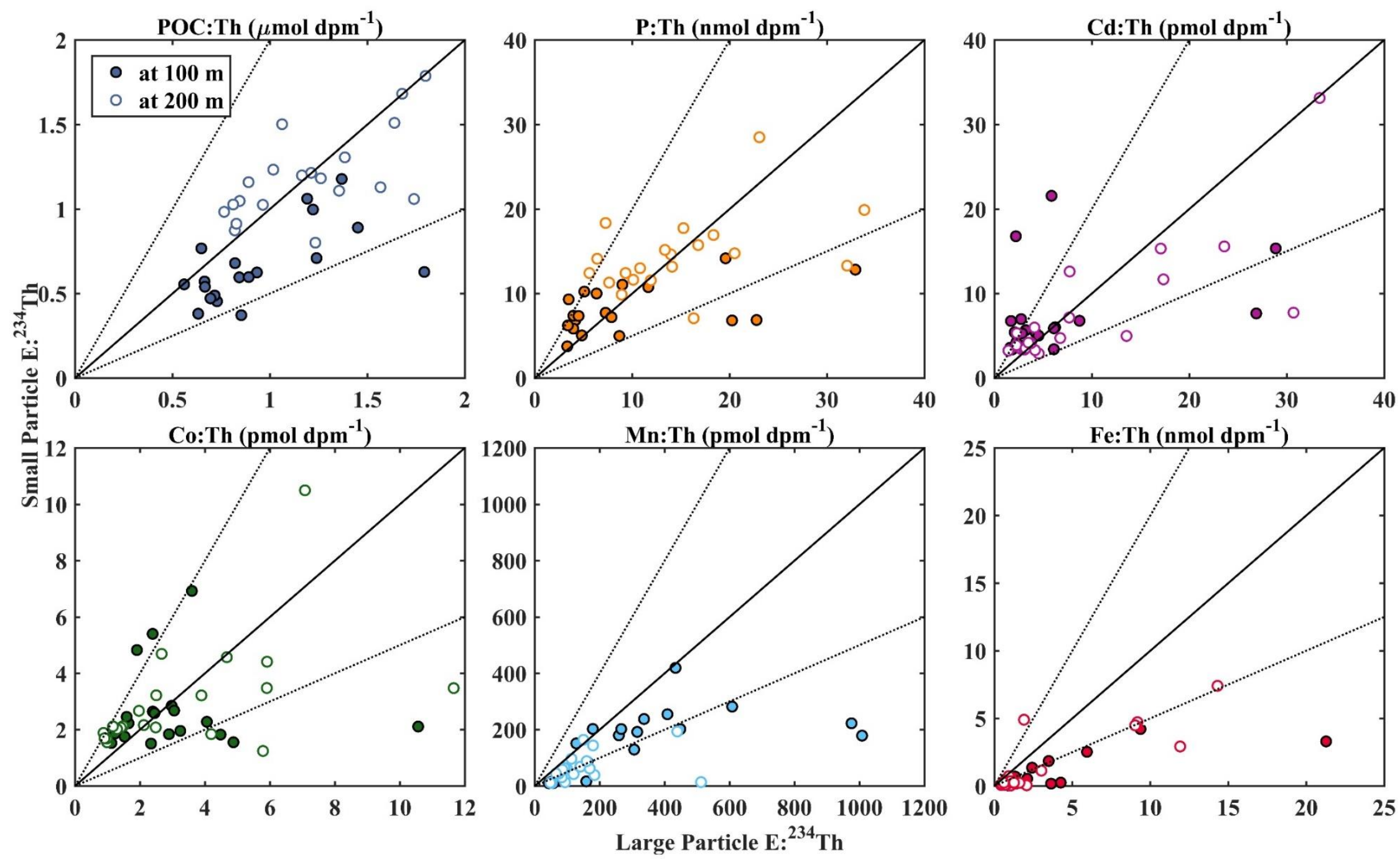

Figure 7: Comparison of large and small particle $\mathbf{E}:{ }^{234} \mathbf{T h}$. The relationship between the $\mathrm{E}:{ }^{234} \mathrm{Th}$ values in the large particle pool and those found in the small particle pool at $100 \mathrm{~m}$ and 200 meters are shown. A 1:1 line indicates when the E: ${ }^{234} \mathrm{Th}$ is equivalent for both particle size fractions, while the dotted lines show 1:2 and 2:1 relationships. Fluxes derived from values near the 1:1 line could have varying contributions of large and small particles without changing the flux estimate at this depth. 


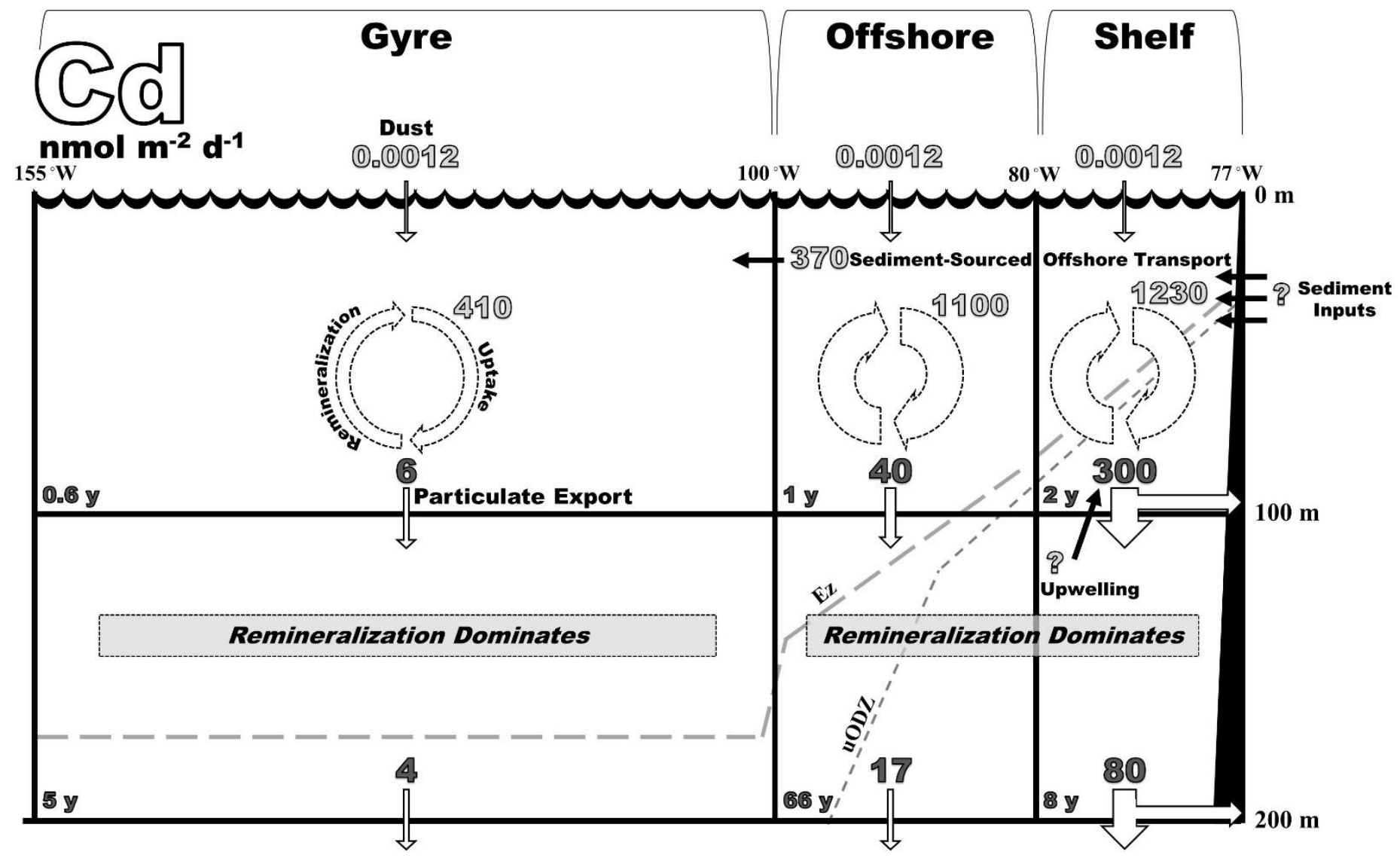

Figure 8: Particulate cycling of Cd, Co, Mn, and Fe in the Southeastern Tropical Pacific. Particulate export determined for each region (dark grey) is shown with uptake and dust flux estimates (light grey) in the units indicated (upper left). Potential pathways for the advection of dissolved constituents are shown (black arrows). Total residence times are reported for 0-100 $\mathrm{m}$ and 0-200 $\mathrm{m}$ sections of the water column in years. The offshore transport of dissolved constituents was derived using ${ }^{228} \mathrm{Ra}$ measurements from this campaign (Sanial et al., 2017). Uptake values were determined for each element in each zone by multiplying MODIS-derived averages of NPP $\left(\mathrm{mmolC} \mathrm{m}^{-2} \mathrm{~d}^{-1}\right)$ by the estimated ratio of that element to carbon in bulk phytoplankton. Previously reported NPP values were 114, 99, and $96 \mathrm{mmolC} \mathrm{m} \mathrm{m}^{-2} \mathrm{~d}^{-1}$ for the Shelf, Offshore, and Gyre (Black et al., 2017). Co:P and Cd:P were measured on bulk particulate matter $(>0.45 \mu \mathrm{m})$ within the mixed layer of the Shelf and Offshore stations (Ohnemus et al., 2016). A bulk particle C:P of $~ 50 \mathrm{~mol}: \mathrm{mol}$ for these zones was used to determine the corresponding Co:C and $\mathrm{Cd}: \mathrm{C}$ ratios needed to determine Co and Cd uptake (Lam et al., 2017). Mn:C, Fe:C, and Co:C from bulk particle analyses in the Equatorial Pacific (20-25 m; Twining et al., 2011) were used to estimate $\mathrm{Mn}$ and $\mathrm{Fe}$ uptake for all zones and Co uptake in the Gyre because these measurements had not been made here. 


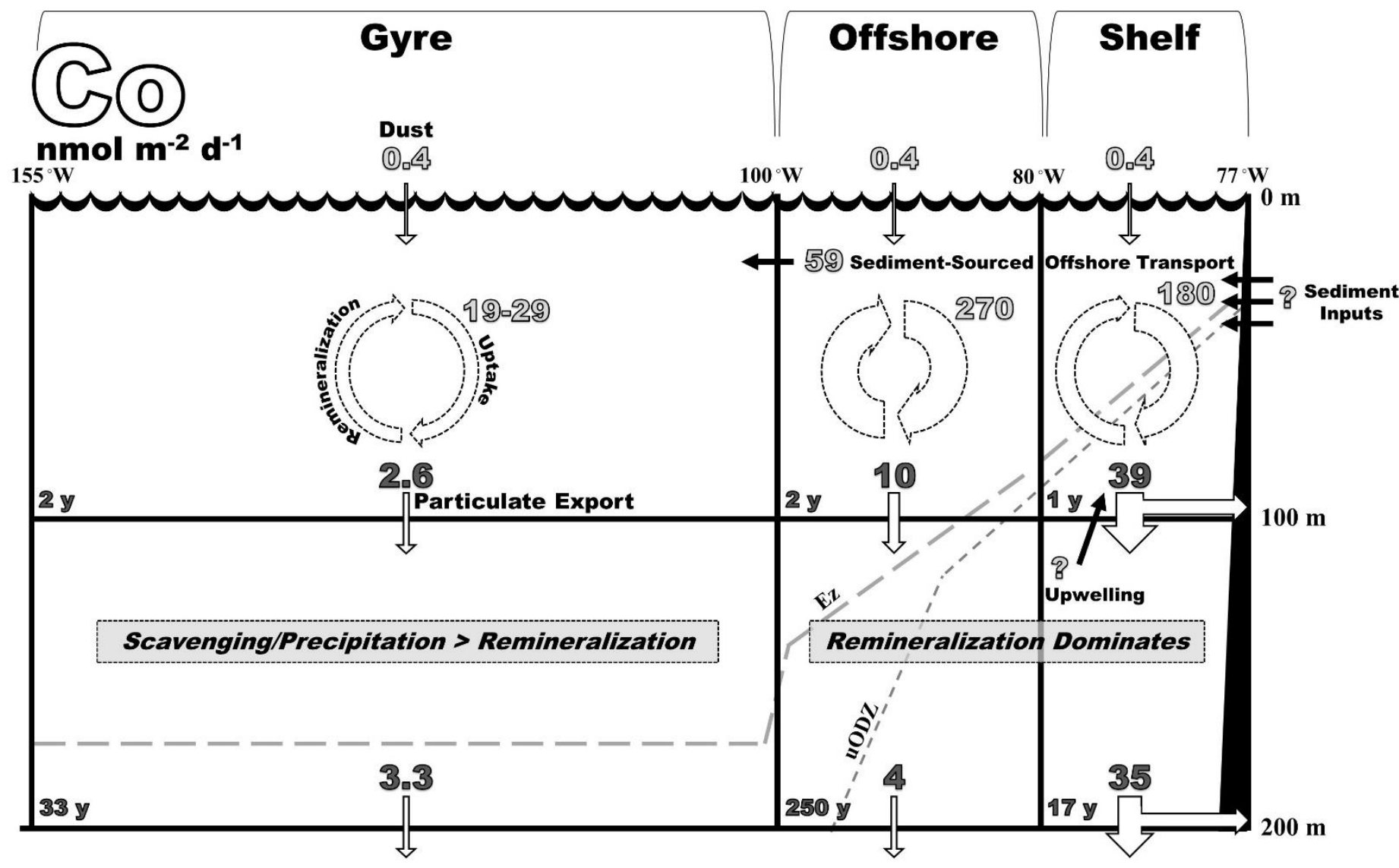

Figure 8: Particulate cycling of Cd, Co, Mn, and Fe in the Southeastern Tropical Pacific. Particulate export determined for each region (dark grey) is shown with uptake and dust flux estimates (light grey) in the units indicated (upper left). Potential pathways for the advection of dissolved constituents are shown (black arrows). Total residence times are reported for 0-100 m and 0-200 $\mathrm{m}$ sections of the water column in years. The offshore transport of dissolved constituents was derived using ${ }^{228}$ Ra measurements from this campaign (Sanial et al., 2017). Uptake values were determined for each element in each zone by multiplying MODIS-derived averages of NPP $\left(\mathrm{mmolC} \mathrm{m}^{-2} \mathrm{~d}^{-1}\right)$ by the estimated ratio of that element to carbon in bulk phytoplankton. Previously reported NPP values were 114, 99, and $96 \mathrm{mmolC} \mathrm{m} \mathrm{d}^{-1}$ for the Shelf, Offshore, and Gyre (Black et al., 2017). Co:P and Cd:P were measured on bulk particulate matter $(>0.45 \mu \mathrm{m})$ within the mixed layer of the Shelf and Offshore stations $(\mathrm{Ohnemus}$ et al., 2016). A bulk particle C:P of $\sim 50 \mathrm{~mol}: \mathrm{mol}$ for these zones was used to determine the corresponding Co:C and Cd:C ratios needed to determine Co and Cd uptake (Lam et al., 2017). Mn:C, Fe:C, and Co:C from bulk particle analyses in the Equatorial Pacific (20-25 m; Twining et al., 2011) were used to estimate $\mathrm{Mn}$ and Fe uptake for all zones and Co uptake in the Gyre because these measurements had not been made here. 


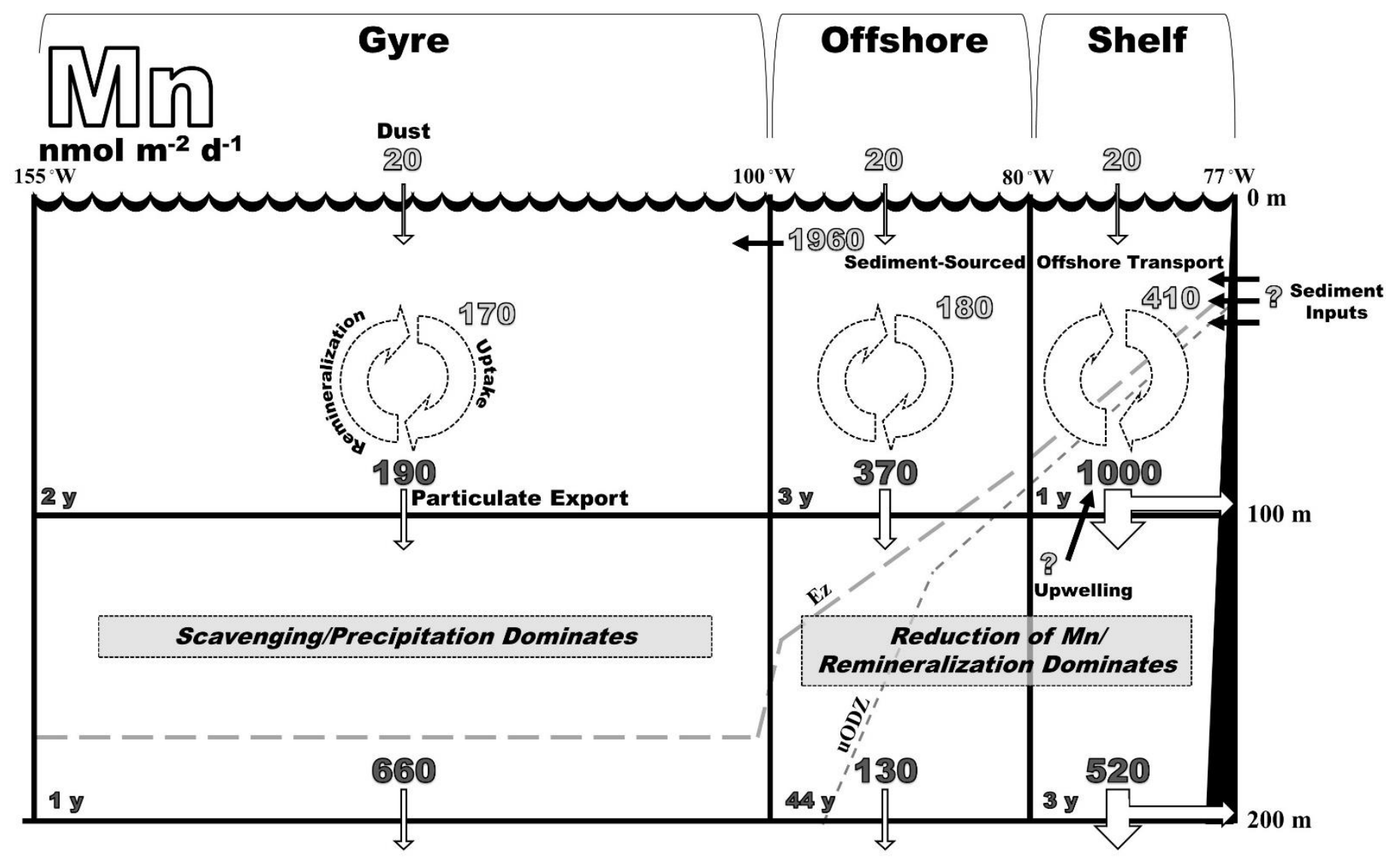

Figure 8: Particulate cycling of Cd, Co, Mn, and Fe in the Southeastern Tropical Pacific. Particulate export determined for each region (dark grey) is shown with uptake and dust flux estimates (light grey) in the units indicated (upper left). Potential pathways for the advection of dissolved constituents are shown (black arrows). Total residence times are reported for 0-100 m and 0-200 $\mathrm{m}$ sections of the water column in years. The offshore transport of dissolved constituents was derived using ${ }^{228} \mathrm{Ra}$ measurements from this campaign (Sanial et al., 2017). Uptake values were determined for each element in each zone by multiplying MODIS-derived averages of NPP $\left(\mathrm{mmolC} \mathrm{m}^{-2} \mathrm{~d}^{-1}\right)$ by the estimated ratio of that element to carbon in bulk phytoplankton. Previously reported NPP values were 114, 99, and $96 \mathrm{mmolC} \mathrm{m} \mathrm{d}^{-1}$ for the Shelf, Offshore, and Gyre (Black et al., 2017). Co:P and Cd:P were measured on bulk particulate matter $(>0.45 \mu \mathrm{m})$ within the mixed layer of the Shelf and Offshore stations $(\mathrm{Ohnemus}$ et al., 2016). A bulk particle C:P of $\sim 50 \mathrm{~mol}: \mathrm{mol}$ for these zones was used to determine the corresponding Co:C and $\mathrm{Cd}: \mathrm{C}$ ratios needed to determine Co and Cd uptake (Lam et al., 2017). Mn:C, Fe:C, and Co:C from bulk particle analyses in the Equatorial Pacific (20-25 m; Twining et al., 2011) were used to estimate $\mathrm{Mn}$ and $\mathrm{Fe}$ uptake for all zones and Co uptake in the Gyre because these measurements had not been made here. 


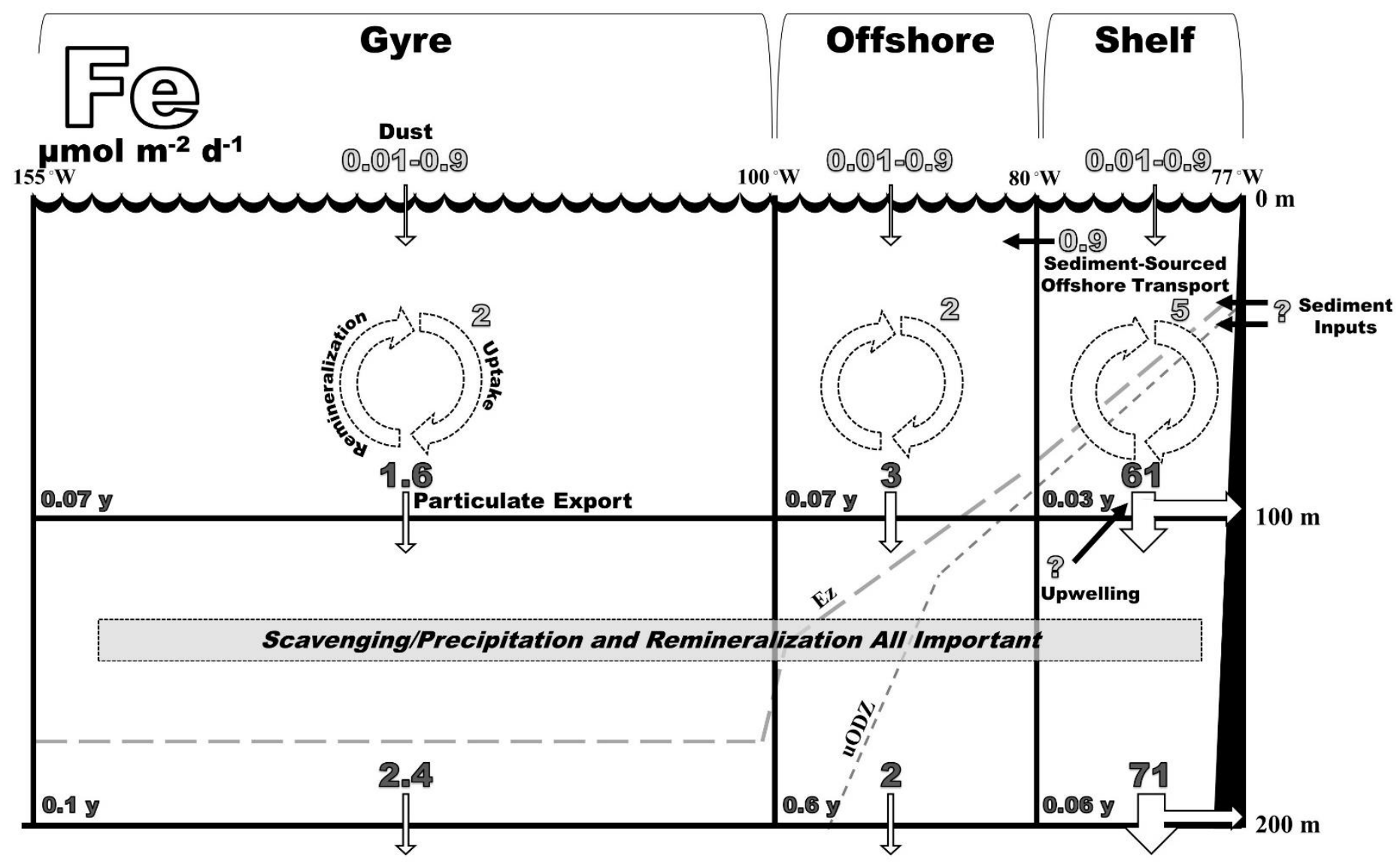

Figure 8: Particulate cycling of Cd, Co, Mn, and Fe in the Southeastern Tropical Pacific. Particulate export determined for each region (dark grey) is shown with uptake and dust flux estimates (light grey) in the units indicated (upper left). Potential pathways for the advection of dissolved constituents are shown (black arrows). Total residence times are reported for 0-100 m and 0-200 $\mathrm{m}$ sections of the water column in years. The offshore transport of dissolved constituents was derived using ${ }^{228} \mathrm{Ra}$ measurements from this campaign (Sanial et al., 2017). Uptake values were determined for each element in each zone by multiplying MODIS-derived averages of NPP $\left(\mathrm{mmolC} \mathrm{m}^{-2} \mathrm{~d}^{-1}\right)$ by the estimated ratio of that element

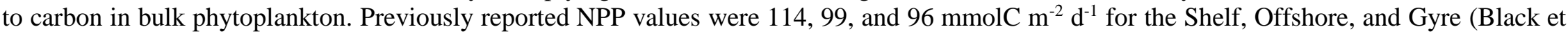
al., 2017). Co:P and Cd:P were measured on bulk particulate matter $(>0.45 \mu \mathrm{m})$ within the mixed layer of the Shelf and Offshore stations (Ohnemus et al., 2016). A bulk particle C:P of $\sim 50 \mathrm{~mol}: \mathrm{mol}$ for these zones was used to determine the corresponding Co:C and $\mathrm{Cd}: \mathrm{C}$ ratios needed to determine Co and Cd uptake (Lam et al., 2017). Mn:C, Fe:C, and Co:C from bulk particle analyses in the Equatorial Pacific (20-25 m; Twining et al., 2011) were used to estimate $\mathrm{Mn}$ and $\mathrm{Fe}$ uptake for all zones and Co uptake in the Gyre because these measurements had not been made here. 
Supplemental Table 1: Zonal boundaries and ${ }^{234}$ Th flux results. ${ }^{234} \mathrm{Th}$ flux values are reported in $\mathrm{dpm}^{-2} \mathrm{~d}^{-1}$ for the Shelf, Offshore, and Gyre zones at four depths of interest: 100 m, $200 \mathrm{~m}$ the upper boundary of the ODZ (uODZ; Black et al., 2017), and 100 m below the uODZ. Previously reported Ez and uODZ depths have been included, however, any zonal averages stated in the text include all stations sampled on the campaign and not just those discussed in this study (Black et al., 2017; Ohnemus et al., 2016).

\begin{tabular}{|c|c|c|c|c|c|c|c|c|c|c|c|c|c|c|c|c|c|}
\hline \multirow[t]{2}{*}{ Zone } & \multirow{2}{*}{$\begin{array}{l}\text { St } \\
2\end{array}$} & \multirow{2}{*}{$\begin{array}{c}\begin{array}{c}\text { Lat } \\
\mathbf{S}\end{array} \\
12.0\end{array}$} & \multirow{2}{*}{$\begin{array}{c}\begin{array}{c}\text { Lon } \\
\text { W }\end{array} \\
77.4\end{array}$} & \multicolumn{3}{|c|}{ 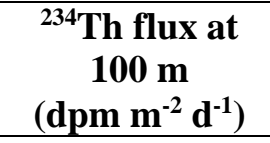 } & \multicolumn{3}{|c|}{$\begin{array}{c}{ }^{234} \text { Th flux at } 200 \\
\text { m } \\
\left(\mathrm{dpm} \mathrm{m} \mathrm{m}^{-2} \mathrm{~d}^{-1}\right) \\
\end{array}$} & \multirow{2}{*}{$\begin{array}{c}\begin{array}{c}\text { Average } \\
\text { Ez } \\
(\mathbf{m})\end{array} \\
30\end{array}$} & \multirow{2}{*}{$\begin{array}{c}\begin{array}{c}\mathbf{u O D Z} \\
(\mathbf{m})\end{array} \\
48\end{array}$} & \multicolumn{3}{|c|}{$\begin{array}{c}{ }^{234} \text { Th flux at } \\
\text { uODZ } \\
\left(\mathrm{dpm} \mathrm{m}^{-2} \mathrm{~d}^{-1}\right) \\
\end{array}$} & \multicolumn{3}{|c|}{$\begin{array}{c}{ }^{234} \text { Th flux at } \\
\text { uODZ +100 } \\
\left(\text { dpm m }^{-2} d^{-1}\right)\end{array}$} \\
\hline & & & & 7300 & \pm & 700 & & -- & & & & 6500 & & 700 & & -- & \\
\hline \multirow{4}{*}{ Shelf } & 3 & 12.0 & 77.7 & 7000 & \pm & 1000 & & -- & & 25 & 41 & 7000 & \pm & 1000 & & -- & \\
\hline & 4 & 12.0 & 77.8 & 6100 & \pm & 900 & 6000 & \pm & 1000 & 43 & 49 & 4600 & \pm & 900 & 6000 & \pm & 1000 \\
\hline & 5 & 12.0 & 78.2 & 6100 & \pm & 800 & 5200 & \pm & 700 & 50 & 61 & 5100 & \pm & 700 & 5800 & \pm & 800 \\
\hline & 1 & 12.0 & 79.2 & 6100 & \pm & 900 & 6000 & \pm & 900 & 66 & 72 & 5100 & \pm & 900 & 5900 & \pm & 900 \\
\hline \multirow{4}{*}{ Offshore } & 7 & 12.0 & 84.0 & 2800 & \pm & 400 & 2100 & \pm & 400 & 94 & 99 & 2700 & \pm & 400 & 2100 & \pm & 400 \\
\hline & 9 & 12.0 & 89.0 & 1400 & \pm & 100 & 300 & \pm & 200 & 109 & 116 & 1400 & \pm & 100 & 200 & \pm & 200 \\
\hline & 11 & 12.0 & 94.0 & 1900 & \pm & 90 & 1700 & \pm & 100 & 130 & 190 & 1700 & \pm & 100 & 1900 & \pm & 200 \\
\hline & 13 & 14.0 & 99.0 & 1300 & \pm & 100 & 900 & \pm & 200 & 139 & 234 & 900 & \pm & 300 & 500 & \pm & 200 \\
\hline \multirow{13}{*}{ Gyre } & 15 & 16.0 & 104.0 & 2000 & \pm & 100 & 2100 & \pm & 200 & 168 & & & & & & & \\
\hline & 17 & 15.0 & 109.2 & 1400 & \pm & 100 & 1100 & \pm & 200 & 163 & & & & & & & \\
\hline & 18 & 15.0 & 112.8 & 1600 & \pm & 100 & 1400 & \pm & 200 & 174 & & & & & & & \\
\hline & 20 & 15.0 & 113.5 & 1500 & \pm & 100 & 1600 & \pm & 200 & 191 & & & & & & & \\
\hline & 21 & 14.8 & 115.0 & 1400 & \pm & 100 & 1200 & \pm & 200 & 167 & & & & & & & \\
\hline & 23 & 14.0 & 120.0 & 1800 & \pm & 100 & 1700 & \pm & 200 & 169 & & & & & & & \\
\hline & 25 & 12.5 & 125.0 & 1500 & \pm & 100 & 1500 & \pm & 300 & 163 & & & & & & & \\
\hline & 26 & 11.7 & 128.0 & 1400 & \pm & 100 & 900 & \pm & 200 & 169 & & & & & & & \\
\hline & 28 & 11.6 & 132.5 & 1500 & \pm & 100 & 1400 & \pm & 200 & 162 & & & & & & & \\
\hline & 30 & 11.6 & 137.0 & 1400 & \pm & 100 & 1300 & \pm & 200 & 168 & & & & & & & \\
\hline & 32 & 11.0 & 142.9 & 1800 & \pm & 100 & 1900 & \pm & 200 & 153 & & & & & & & \\
\hline & 34 & 10.8 & 147.5 & 1600 & \pm & 100 & 1600 & \pm & 200 & 175 & & & & & & & \\
\hline & 36 & 10.5 & 152.0 & 1100 & \pm & 100 & 1100 & \pm & 200 & 179 & & & & & & & \\
\hline
\end{tabular}


Supplemental Table 2: Fluxes by station. Supp. Tables 1A to 1F correspond to the particulate sinking fluxes of POC, P, Cd, Mn, Co, and Fe, respectively. Fluxes determined for the elements of interest are reported at the five depths of interest following Supp. Table 1. Flux ratios and the percent of flux at $100 \mathrm{~m}$ and $200 \mathrm{~m}$ that was composed of lithogenic material (\%) are also reported for each station.

\section{Supplemental Table 2A}

\begin{tabular}{|c|c|c|c|c|c|c|c|c|c|c|c|c|c|c|}
\hline \multirow{2}{*}{$\begin{array}{l}\mathbf{S t} \\
2\end{array}$} & \multicolumn{3}{|c|}{ 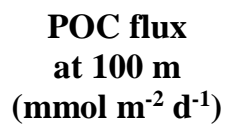 } & \multicolumn{3}{|c|}{ 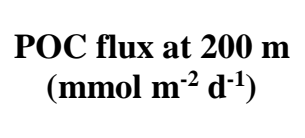 } & \multirow{2}{*}{$\begin{array}{c}\text { Flux ratio } \\
\text { (100 to } 200 \mathrm{~m})\end{array}$} & \multicolumn{3}{|c|}{ 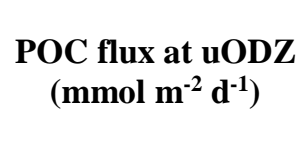 } & \multicolumn{3}{|c|}{$\begin{array}{l}\text { POC flux at uODZ + } \\
100\left(\mathrm{mmol} \mathrm{m}^{-2} \mathrm{~d}^{-1}\right)\end{array}$} & \multirow{2}{*}{$\begin{array}{c}\begin{array}{c}\text { Flux ratio } \\
\text { (uODZ to } \\
\text { uODZ+100) }\end{array} \\
--\end{array}$} \\
\hline & 13 & \pm & 1 & & -- & & & 57 & \pm & 7 & & -- & & \\
\hline 3 & 11 & \pm & 2 & & -- & & -- & 80 & \pm & 10 & 11 & \pm & 2 & 0.1 \\
\hline 4 & 8 & \pm & 1 & 8 & \pm & 1 & 1.0 & 21 & \pm & 4 & 7 & \pm & 1 & 0.3 \\
\hline 5 & 8 & \pm & 1 & 6.3 & \pm & 0.9 & 0.8 & 11 & \pm & 2 & 7 & \pm & 1 & 0.6 \\
\hline 1 & 6 & \pm & 1 & 10 & \pm & 1 & 1.7 & 13 & \pm & 2 & 7 & \pm & 1 & 0.5 \\
\hline 7 & 4.6 & \pm & 0.7 & 2.5 & \pm & 0.5 & 0.5 & 4.7 & \pm & 0.8 & 2.5 & \pm & 0.5 & 0.5 \\
\hline 9 & 2.4 & \pm & 0.2 & 0.3 & \pm & 0.2 & 0.1 & 1.9 & \pm & 0.2 & 0.2 & \pm & 0.2 & 0.1 \\
\hline 11 & 2.3 & \pm & 0.2 & 2.1 & \pm & 0.2 & 0.9 & 2.3 & \pm & 0.2 & 1.6 & \pm & 0.2 & 0.7 \\
\hline 13 & 1.5 & \pm & 0.1 & 0.9 & \pm & 0.2 & 0.6 & 0.7 & \pm & 0.2 & 0.4 & \pm & 0.2 & 0.6 \\
\hline 15 & 2.7 & \pm & 0.2 & 1.9 & \pm & 0.2 & 0.7 & & & & & & & \\
\hline 17 & 2.5 & \pm & 0.2 & 1.0 & \pm & 0.2 & 0.4 & & & & & & & \\
\hline 18 & & -- & & 0.9 & \pm & 0.2 & -- & & & & & & & \\
\hline 20 & & -- & & & -- & & -- & & & & & & & \\
\hline 21 & 1.5 & \pm & 0.1 & 0.8 & \pm & 0.1 & 0.5 & & & & & & & \\
\hline 23 & 1.8 & \pm & 0.1 & 1.1 & \pm & 0.2 & 0.6 & & & & & & & \\
\hline 25 & 1.2 & \pm & 0.1 & 0.8 & \pm & 0.2 & 0.7 & & & & & & & \\
\hline 26 & 1.1 & \pm & 0.1 & 0.7 & \pm & 0.2 & 0.6 & & & & & & & \\
\hline 28 & 1.2 & \pm & 0.1 & 1.0 & \pm & 0.2 & 0.8 & & & & & & & \\
\hline 30 & 1.2 & \pm & 0.1 & 0.9 & \pm & 0.1 & 0.8 & & & & & & & \\
\hline 32 & 1.4 & \pm & 0.1 & 1.2 & \pm & 0.1 & 0.9 & & & & & & & \\
\hline 34 & & -- & & & -- & & -- & & & & & & & \\
\hline 36 & 1.0 & \pm & 0.1 & 0.9 & \pm & 0.2 & 0.9 & & & & & & & \\
\hline
\end{tabular}




\section{Supplemental Table 2B}

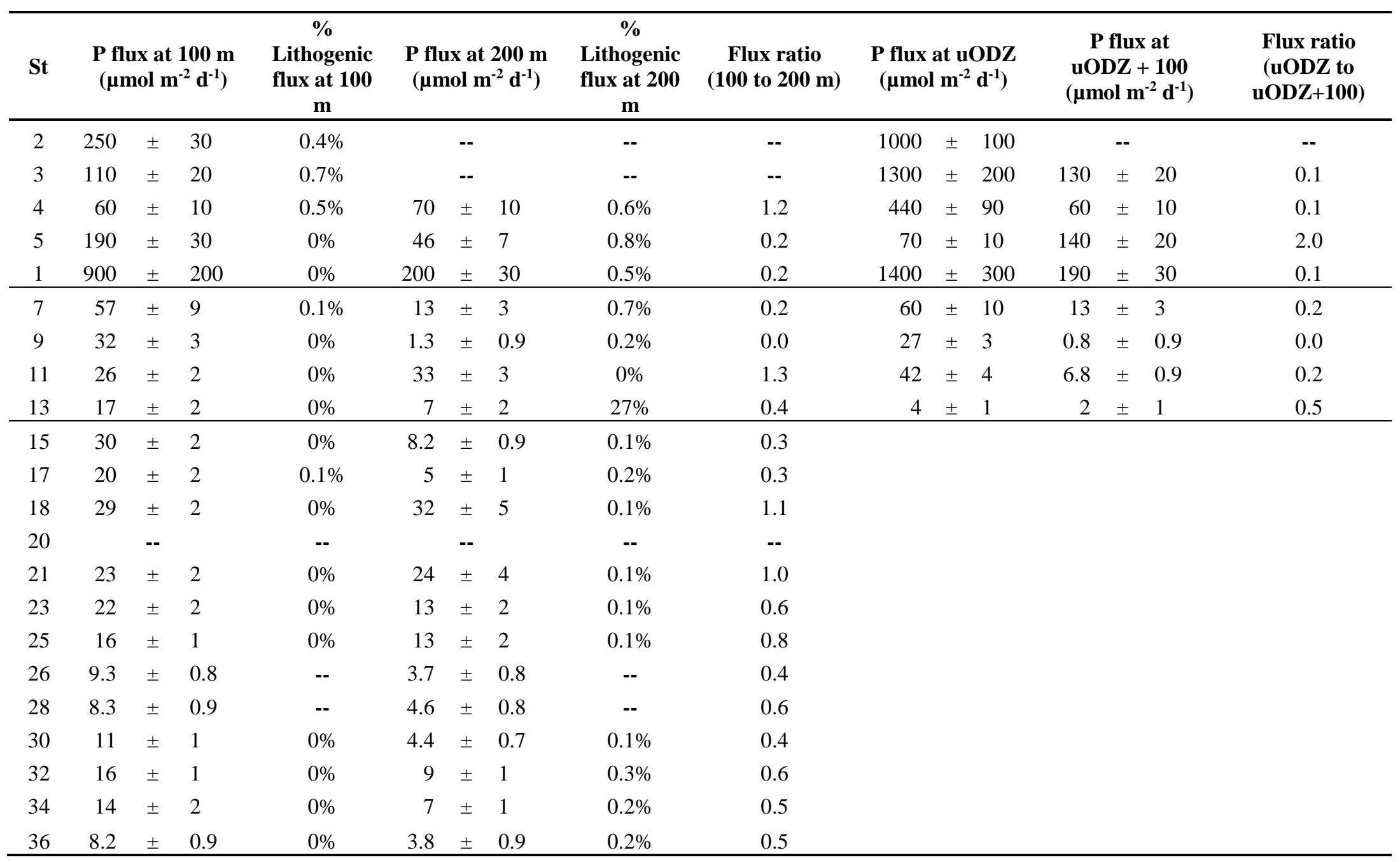


Supplemental Table 2C

\begin{tabular}{|c|c|c|c|c|c|c|c|c|c|c|c|c|c|c|c|c|}
\hline \multirow{2}{*}{$\begin{array}{l}\text { St } \\
2\end{array}$} & \multicolumn{3}{|c|}{ 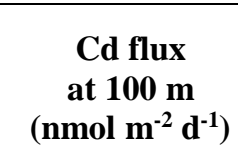 } & \multirow{2}{*}{$\begin{array}{c}\text { \% Lithogenic } \\
\text { flux at } \mathbf{1 0 0 ~} \mathbf{~ m} \\
0 \%\end{array}$} & \multicolumn{3}{|c|}{ 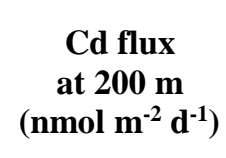 } & \multirow{2}{*}{$\begin{array}{c}\% \\
\begin{array}{c}\text { Lithogenic } \\
\text { flux at } 200 \\
\text { m }\end{array} \\
--\end{array}$} & \multirow{2}{*}{$\begin{array}{c}\text { Flux ratio } \\
\text { (100 to } 200 \mathrm{~m})\end{array}$} & \multicolumn{3}{|c|}{$\begin{array}{c}\text { Cd flux at } \\
\text { uODZ } \\
\left(\mathrm{nmol} \mathrm{m}^{-2} \mathbf{d}^{-1}\right)\end{array}$} & \multicolumn{3}{|c|}{$\begin{array}{c}\text { Cd flux at } \\
\text { uODZ }+100 \\
\left(\mathbf{n m o l ~ m}^{-2} \mathbf{d}^{-1}\right)\end{array}$} & \multirow{2}{*}{$\begin{array}{r}\begin{array}{r}\text { Flux ratio } \\
\text { (uODZ to }\end{array} \\
\mathbf{u O D Z + 1 0 0}) \\
--\end{array}$} \\
\hline & 130 & \pm & 10 & & & -- & & & & 1200 & \pm & 100 & & -- & & \\
\hline 3 & 90 & \pm & 20 & $0 \%$ & & -- & & -- & -- & 600 & \pm & 100 & 100 & \pm & 20 & 0.2 \\
\hline 4 & 47 & \pm & 8 & $0 \%$ & 51 & \pm & 9 & $0 \%$ & 1.1 & 470 & \pm & 90 & 39 & \pm & 7 & 0.1 \\
\hline 5 & 190 & \pm & 30 & $0 \%$ & 32 & \pm & 5 & $0 \%$ & 0.2 & 33 & \pm & 6 & 130 & \pm & 20 & 3.9 \\
\hline 1 & 1000 & \pm & 200 & $0 \%$ & 160 & \pm & 30 & $0 \%$ & 0.2 & 1300 & \pm & 200 & 200 & \pm & 30 & 0.2 \\
\hline 7 & 70 & \pm & 10 & $0 \%$ & 13 & \pm & 3 & $0 \%$ & 0.2 & 70 & \pm & 10 & 13 & \pm & 3 & 0.2 \\
\hline 9 & 47 & \pm & 4 & $0 \%$ & 1.3 & \pm & 0.9 & $0 \%$ & 0.0 & 39 & \pm & 4 & 0.9 & \pm & 0.9 & 0.0 \\
\hline 11 & 33 & \pm & 2 & $0 \%$ & 49 & \pm & 5 & $0 \%$ & 1.5 & 63 & \pm & 6 & 5 & \pm & 1 & 0.1 \\
\hline 13 & 10 & \pm & 1 & $0 \%$ & 6 & \pm & 2 & $34 \%$ & 0.6 & 3 & \pm & 1 & 2 & \pm & 1 & 0.7 \\
\hline 15 & 13 & \pm & 1 & $0 \%$ & 7 & \pm & 2 & $0 \%$ & 0.5 & & & & & & & \\
\hline 17 & 7 & \pm & 1 & -- & 4 & \pm & 1 & -- & 0.6 & & & & & & & \\
\hline 18 & 7 & $\begin{array}{l} \pm \\
-\end{array}$ & 1 & $0 \%$ & 8 & \pm & 3 & $0 \%$ & 1.1 & & & & & & & \\
\hline 20 & & - & & -- & & -- & & -- & -- & & & & & & & \\
\hline 21 & 5.2 & \pm & 0.9 & $0 \%$ & 5 & \pm & 2 & $0 \%$ & 1.0 & & & & & & & \\
\hline 23 & 5.1 & \pm & 0.7 & $0 \%$ & 3 & \pm & 1 & $0 \%$ & 0.6 & & & & & & & \\
\hline 25 & 4.5 & \pm & 0.7 & $0 \%$ & 4 & \pm & 1 & $0 \%$ & 0.9 & & & & & & & \\
\hline 26 & 3.3 & \pm & 0.7 & -- & 2.1 & \pm & 0.8 & -- & 0.6 & & & & & & & \\
\hline 28 & 2.1 & \pm & 0.6 & -- & 2.2 & \pm & 0.8 & -- & 1.0 & & & & & & & \\
\hline 30 & 4.5 & \pm & 0.6 & $0 \%$ & 2.4 & \pm & 0.9 & $0 \%$ & 0.5 & & & & & & & \\
\hline 32 & 7.2 & \pm & 0.8 & $0 \%$ & 4 & \pm & 1 & $0 \%$ & 0.6 & & & & & & & \\
\hline 34 & 5.6 & \pm & 0.9 & $0 \%$ & 4 & \pm & 1 & $0 \%$ & 0.7 & & & & & & & \\
\hline 36 & 2.6 & \pm & 0.5 & $0 \%$ & 1.9 & \pm & 0.7 & $0 \%$ & 0.7 & & & & & & & \\
\hline
\end{tabular}




\section{Supplemental Table 2D}

\begin{tabular}{|c|c|c|c|c|c|c|c|c|c|c|c|c|c|c|c|}
\hline \multirow{2}{*}{$\begin{array}{l}\text { St } \\
2\end{array}$} & \multicolumn{3}{|c|}{ 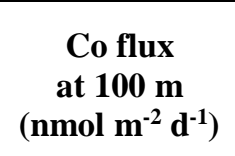 } & \multirow{2}{*}{$\begin{array}{c}\% \\
\begin{array}{c}\text { Lithogenic } \\
\text { flux at } \mathbf{1 0 0} \\
\mathbf{m}\end{array} \\
19 \%\end{array}$} & \multicolumn{3}{|c|}{ 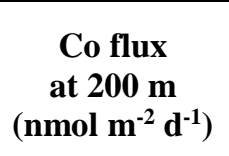 } & \multirow{2}{*}{$\begin{array}{c}\% \\
\begin{array}{c}\text { Lithogenic } \\
\text { flux at } 200 \\
\text { m }\end{array} \\
--\end{array}$} & \multirow{2}{*}{$\begin{array}{c}\begin{array}{c}\text { Flux ratio } \\
(100 \text { to } 200 \\
\text { m) }\end{array} \\
--\end{array}$} & \multicolumn{2}{|c|}{$\begin{array}{c}\text { Co flux at } \\
\text { uODZ } \\
\left(\text { nmol m}^{-2} \mathbf{d}^{-1}\right)\end{array}$} & \multicolumn{3}{|c|}{$\begin{array}{c}\text { Co flux at } \\
\text { uODZ + 100 } \\
\left(\text { nmol m }^{-2} d^{-1}\right)\end{array}$} & \multirow{2}{*}{$\begin{array}{c}\begin{array}{c}\text { Flux ratio } \\
(\text { uODZ to }\end{array} \\
\text { uODZ+100) } \\
--\end{array}$} \\
\hline & 43 & \pm & 5 & & & -- & & & & 260 & \pm 30 & & -- & & \\
\hline 3 & 40 & \pm & 7 & $14 \%$ & & -- & & -- & -- & 90 & \pm 20 & 38 & \pm & 7 & 0.4 \\
\hline 4 & 26 & \pm & 4 & $9 \%$ & 29 & \pm & 5 & $10 \%$ & 1.1 & 70 & \pm 10 & 22 & \pm & 4 & 0.3 \\
\hline 5 & 15 & \pm & 2 & $1 \%$ & 15 & \pm & 2 & $18 \%$ & 1.0 & 49 & \pm 8 & 18 & \pm & 3 & 0.4 \\
\hline 1 & 70 & \pm & 10 & $2 \%$ & 60 & \pm & 10 & $12 \%$ & 0.9 & 110 & \pm 20 & 41 & \pm & 7 & 0.4 \\
\hline 7 & 17 & \pm & 3 & $2 \%$ & 7 & \pm & 2 & $10 \%$ & 0.4 & 17 & \pm 3 & 7 & \pm & 2 & 0.4 \\
\hline 9 & 10 & \pm & 1 & $0 \%$ & 0.7 & \pm & 0.5 & $3 \%$ & 0.1 & 9.4 & \pm 0.9 & 0.5 & \pm & 0.5 & 0.1 \\
\hline 11 & 9 & \pm & 1 & $1 \%$ & 6 & \pm & 1 & $1 \%$ & 0.7 & 6.9 & \pm 0.9 & 6 & \pm & 1 & 0.9 \\
\hline 13 & 3.5 & \pm & 0.4 & $2 \%$ & 2.3 & \pm & 0.7 & $3 \%$ & 0.7 & 2.1 & \pm 0.7 & 1.1 & \pm & 0.6 & 0.5 \\
\hline 15 & 7.6 & \pm & 0.9 & $1 \%$ & 6 & \pm & 1 & $1 \%$ & 0.8 & & & & & & \\
\hline 17 & 3.0 & \pm & 0.9 & -- & 2.6 & \pm & 0.9 & -- & 0.9 & & & & & & \\
\hline 18 & 3.1 & \pm & 0.8 & $3 \%$ & 6 & \pm & 2 & $2 \%$ & 1.9 & & & & & & \\
\hline 20 & & -- & & -- & & -- & & -- & -- & & & & & & \\
\hline 21 & 3.5 & \pm & 0.6 & $1 \%$ & 5 & \pm & 1 & $4 \%$ & 1.4 & & & & & & \\
\hline 23 & 2.7 & \pm & 0.4 & $1 \%$ & 2.7 & \pm & 0.8 & $2 \%$ & 1.0 & & & & & & \\
\hline 25 & 2.1 & \pm & 0.5 & $2 \%$ & 4 & \pm & 1 & $2 \%$ & 1.9 & & & & & & \\
\hline 26 & 1.4 & \pm & 0.5 & -- & 1.4 & \pm & 0.6 & -- & 1.0 & & & & & & \\
\hline 28 & 1.3 & \pm & 0.4 & -- & 1.7 & \pm & 0.6 & -- & 1.3 & & & & & & \\
\hline 30 & 1.4 & \pm & 0.3 & $2 \%$ & 1.5 & \pm & 0.6 & $2 \%$ & 1.1 & & & & & & \\
\hline 32 & 2.3 & \pm & 0.4 & $1 \%$ & 3.5 & \pm & 0.7 & $5 \%$ & 1.5 & & & & & & \\
\hline 34 & 1.9 & \pm & 0.5 & $2 \%$ & 3.9 & \pm & 0.8 & $3 \%$ & 2.1 & & & & & & \\
\hline 36 & 1.0 & \pm & 0.3 & $3 \%$ & 1.7 & \pm & 0.6 & $3 \%$ & 1.7 & & & & & & \\
\hline
\end{tabular}




\section{Supplemental Table 2E}

\begin{tabular}{|c|c|c|c|c|c|c|c|c|c|c|c|c|c|c|c|c|}
\hline \multirow{2}{*}{$\begin{array}{l}\text { St } \\
2\end{array}$} & \multicolumn{3}{|c|}{$\begin{array}{l}\text { Mn flux at } 100 \mathrm{~m} \\
\quad\left(\mathrm{nmol} \mathrm{m}^{-2} \mathrm{~d}^{-1}\right)\end{array}$} & \multirow{2}{*}{$\begin{array}{c}\% \\
\text { Lithogenic } \\
\text { flux at } \mathbf{1 0 0} \\
\mathbf{m} \\
85 \%\end{array}$} & \multicolumn{3}{|c|}{$\begin{array}{l}\text { Mn flux at } 200 \mathrm{~m} \\
\quad\left(\mathrm{nmol} \mathrm{\textrm {m } ^ { - 2 }} \mathbf{d}^{-1}\right)\end{array}$} & \multirow{2}{*}{$\begin{array}{c}\% \\
\begin{array}{c}\text { Lithogenic } \\
\text { flux at } 200 \\
\text { m }\end{array} \\
--\end{array}$} & \multirow{2}{*}{$\begin{array}{c}\begin{array}{c}\text { Flux } \\
\text { ratio } \\
(100 \text { to } \\
200 \mathrm{~m})\end{array} \\
--\end{array}$} & \multicolumn{3}{|c|}{$\begin{array}{c}\text { Mn flux at } \\
\text { uODZ (nmol m- } \\
\left.{ }^{2} \mathbf{d}^{-1}\right)\end{array}$} & \multicolumn{3}{|c|}{$\begin{array}{c}\text { Mn flux at } \\
\text { uODZ + 100 } \\
\left(\mathbf{n m o l ~ m}^{-2} \mathbf{d}^{-1}\right)\end{array}$} & \multirow{2}{*}{$\begin{array}{c}\begin{array}{c}\text { Flux ratio } \\
\text { (uODZ to }\end{array} \\
\mathbf{u O D Z + 1 0 0 )}\end{array}$} \\
\hline & 700 & \pm & 100 & & & -- & & & & 1100 & \pm & 200 & & -- & & \\
\hline 3 & 400 & \pm & 100 & $110 \%$ & & -- & & -- & -- & 2400 & \pm & 900 & 400 & \pm & 100 & 0.2 \\
\hline 4 & 300 & \pm & 100 & $56 \%$ & 400 & \pm & 100 & $56 \%$ & 1.3 & 500 & \pm & 200 & 300 & \pm & 100 & 0.6 \\
\hline 5 & 600 & \pm & 100 & $2 \%$ & 260 & \pm & 90 & $74 \%$ & 0.4 & 2400 & \pm & 400 & 500 & \pm & 100 & 0.2 \\
\hline 1 & 3100 & \pm & 600 & $4 \%$ & 900 & \pm & 300 & $56 \%$ & 0.3 & 4100 & \pm & 800 & 1000 & \pm & 300 & 0.2 \\
\hline 7 & 280 & \pm & 60 & $8 \%$ & 120 & \pm & 50 & $39 \%$ & 0.4 & 290 & \pm & 60 & 120 & \pm & 50 & 0.4 \\
\hline 9 & 210 & \pm & 30 & $2 \%$ & 10 & \pm & 10 & $11 \%$ & 0.0 & 150 & \pm & 30 & 10 & \pm & 10 & 0.1 \\
\hline 11 & 830 & \pm & 80 & $0 \%$ & 220 & \pm & 60 & $2 \%$ & 0.3 & 240 & \pm & 60 & 300 & \pm & 100 & 1.3 \\
\hline 13 & 150 & \pm & 30 & $3 \%$ & 150 & \pm & 60 & $44 \%$ & 1.0 & 90 & \pm & 40 & 60 & \pm & 30 & 0.7 \\
\hline 15 & 280 & \pm & 70 & $2 \%$ & 900 & \pm & 100 & $1 \%$ & 3.2 & & & & & & & \\
\hline 17 & 260 & \pm & 90 & $3 \%$ & 300 & \pm & 100 & $2 \%$ & 1.2 & & & & & & & \\
\hline 18 & 270 & \pm & 80 & $2 \%$ & 1400 & \pm & 300 & $1 \%$ & 5.2 & & & & & & & \\
\hline 20 & & -- & & -- & & -- & & -- & -- & & & & & & & \\
\hline 21 & 250 & \pm & 50 & $1 \%$ & 1200 & \pm & 200 & $1 \%$ & 4.8 & & & & & & & \\
\hline 23 & 220 & \pm & 40 & $1 \%$ & 700 & \pm & 100 & $1 \%$ & 3.2 & & & & & & & \\
\hline 25 & 240 & \pm & 50 & $1 \%$ & 900 & \pm & 200 & $1 \%$ & 3.8 & & & & & & & \\
\hline 26 & 120 & \pm & 40 & -- & 310 & \pm & 80 & -- & 2.6 & & & & & & & \\
\hline 28 & 110 & \pm & 40 & -- & 350 & \pm & 80 & -- & 3.2 & & & & & & & \\
\hline 30 & 130 & \pm & 30 & $2 \%$ & 410 & \pm & 80 & $0 \%$ & 3.2 & & & & & & & \\
\hline 32 & 150 & \pm & 40 & $1 \%$ & 800 & \pm & 100 & $2 \%$ & 5.3 & & & & & & & \\
\hline 34 & 140 & \pm & 50 & $2 \%$ & 420 & \pm & 80 & $2 \%$ & 3.0 & & & & & & & \\
\hline 36 & 70 & \pm & 30 & $3 \%$ & 200 & \pm & 60 & $2 \%$ & 2.9 & & & & & & & \\
\hline
\end{tabular}




\section{Supplemental Table 2F}

\begin{tabular}{|c|c|c|c|c|c|c|c|c|c|c|c|c|c|c|c|}
\hline \multirow{2}{*}{$\begin{array}{l}\text { St } \\
2\end{array}$} & \multicolumn{3}{|c|}{$\begin{array}{l}\text { Fe flux at } 100 \mathrm{~m} \\
\left(\mu \mathrm{mol} \mathrm{m^{-2 }} \mathbf{d}^{-1}\right)\end{array}$} & \multirow{2}{*}{$\begin{array}{c}\begin{array}{c}\% \\
\text { Lithogenic } \\
\text { flux at } \mathbf{1 0 0} \\
\mathbf{m}\end{array} \\
29 \%\end{array}$} & \multicolumn{3}{|c|}{$\begin{array}{l}\text { Fe flux at } 200 \mathrm{~m} \\
\left(\mu \mathrm{mol} \mathrm{m^{-2 }} \mathbf{d}^{-1}\right)\end{array}$} & \multirow{2}{*}{$\begin{array}{c}\% \\
\text { Lithogenic } \\
\text { flux at } 200 \\
\text { m } \\
--\end{array}$} & \multirow{2}{*}{$\begin{array}{l}\text { Flux ratio } \\
(100 \text { to } \\
200 \mathrm{~m}) \\
--\end{array}$} & \multicolumn{2}{|c|}{$\begin{array}{c}\text { Fe flux at } \\
\text { uODZ } \\
\left(\mu \mathrm{mol} \mathrm{m}^{-2} \mathbf{d}^{-1}\right)\end{array}$} & \multicolumn{3}{|c|}{$\begin{array}{c}\text { Fe flux at } \\
\text { uODZ }+100 \\
\left(\mu \mathrm{mol} \mathrm{m^{-2 }} \mathbf{d}^{-1}\right)\end{array}$} & \multirow{2}{*}{$\begin{array}{c}\begin{array}{c}\text { Flux ratio } \\
\text { (uODZ to } \\
\text { uODZ+100) }\end{array} \\
--\end{array}$} \\
\hline & 100 & \pm & 10 & & & -- & & & & 53 & \pm 6 & & -- & & \\
\hline 3 & 80 & \pm & 10 & $25 \%$ & & -- & & -- & -- & 70 & \pm 10 & 90 & \pm & 20 & 1.3 \\
\hline 4 & 55 & \pm & 9 & $15 \%$ & 35 & \pm & 6 & $31 \%$ & 0.6 & 42 & \pm 8 & 50 & \pm & 9 & 1.2 \\
\hline 5 & 12 & \pm & 2 & $6 \%$ & 48 & \pm & 7 & $20 \%$ & 4.0 & 220 & $\pm \quad 30$ & 32 & \pm & 5 & 0.1 \\
\hline 1 & 56 & \pm & 9 & $11 \%$ & 130 & \pm & 20 & $22 \%$ & 2.3 & 33 & \pm 6 & 46 & \pm & 8 & 1.4 \\
\hline 7 & 8 & \pm & 1 & $14 \%$ & 7 & \pm & 2 & $33 \%$ & 0.9 & 9 & \pm 1 & 7 & \pm & 2 & 0.8 \\
\hline 9 & 1.3 & \pm & 0.2 & $14 \%$ & 0.4 & \pm & 0.3 & $19 \%$ & 0.3 & 1.6 & $\pm \quad 0.2$ & 0.3 & \pm & 0.3 & 0.2 \\
\hline 11 & 0.8 & \pm & 0.3 & $25 \%$ & 1.3 & \pm & 0.3 & $20 \%$ & 1.6 & 1.2 & $\pm \quad 0.4$ & 2.5 & \pm & 0.6 & 2.1 \\
\hline 13 & 0.6 & \pm & 0.2 & $42 \%$ & 0.5 & \pm & 0.2 & $32 \%$ & 0.8 & 0.4 & $\pm \quad 0.2$ & & -- & & -- \\
\hline 15 & 1.8 & \pm & 0.4 & $15 \%$ & 1.9 & \pm & 0.7 & $18 \%$ & 1.1 & & & & & & \\
\hline 17 & 1.5 & \pm & 0.5 & $27 \%$ & 2.2 & \pm & 0.6 & $14 \%$ & 1.5 & & & & & & \\
\hline 18 & 1.3 & \pm & 0.4 & $23 \%$ & 6 & \pm & 1 & $8 \%$ & 4.6 & & & & & & \\
\hline 20 & & -- & & -- & & -- & & -- & -- & & & & & & \\
\hline 21 & 0.8 & \pm & 0.3 & $12 \%$ & 4 & \pm & 1 & $15 \%$ & 5.0 & & & & & & \\
\hline 23 & 2.5 & \pm & 0.3 & $6 \%$ & 1.7 & \pm & 0.5 & $15 \%$ & 0.7 & & & & & & \\
\hline 25 & 0.7 & \pm & 0.3 & $20 \%$ & 1.4 & \pm & 0.5 & $19 \%$ & 2.0 & & & & & & \\
\hline 26 & 3.0 & \pm & 0.4 & -- & 0.8 & \pm & 0.3 & -- & 0.3 & & & & & & \\
\hline 28 & 1.5 & \pm & 0.3 & -- & 1.2 & \pm & 0.4 & -- & 0.8 & & & & & & \\
\hline 30 & 1.0 & \pm & 0.2 & $11 \%$ & 1.0 & \pm & 0.4 & $10 \%$ & 1.0 & & & & & & \\
\hline 32 & 1.1 & \pm & 0.2 & $10 \%$ & 4.5 & \pm & 0.6 & $15 \%$ & 4.1 & & & & & & \\
\hline 34 & 2.6 & \pm & 0.5 & $6 \%$ & 3.4 & \pm & 0.6 & $13 \%$ & 1.3 & & & & & & \\
\hline 36 & 1.4 & \pm & 0.2 & $8 \%$ & 1.1 & \pm & 0.3 & $15 \%$ & 0.8 & & & & & & \\
\hline
\end{tabular}



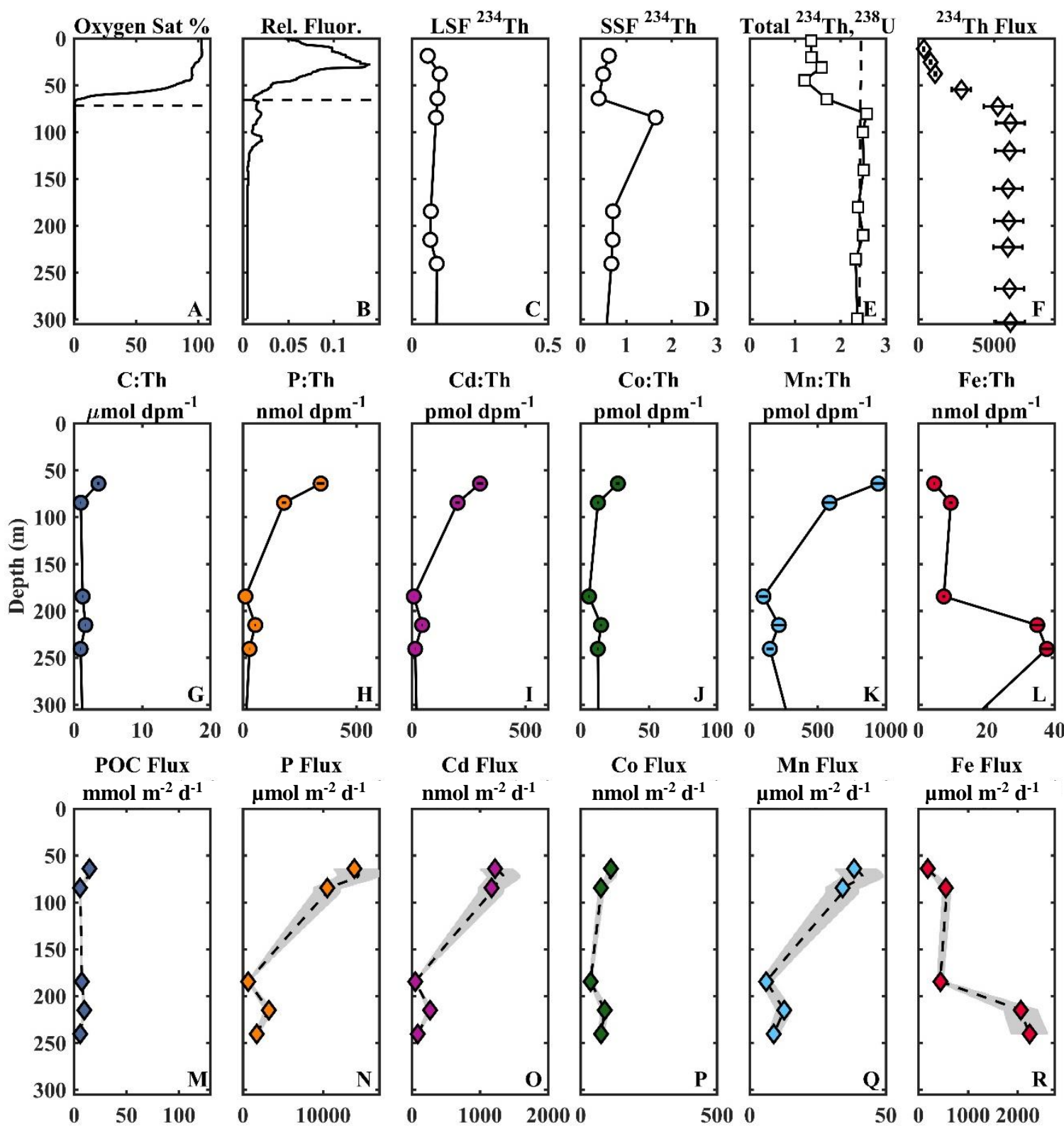

Fe Flux

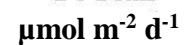

Supplemental Figure 1: Element ratios and fluxes for Shelf Station 1. The stations shown here are those not included in Fig. 3. Oxygen saturation \% $(A)$ and relative florescence $(B)$ are shown with dashed lines indicating the depth of the uODZ boundary (panel $A$ ), when present, and Ez (panel $B)$. Particulate ${ }^{234} \mathrm{Th}(C$ and $D)$, total ${ }^{234} \mathrm{Th}\left(E\right.$, squares), and total ${ }^{238} \mathrm{U}$ activities ( $E$, dashed line) are shown in dpm $\mathrm{L}^{-1}$. The derived ${ }^{234} \mathrm{Th}$ flux with depth $(F)$ is shown in $\mathrm{dpm} \mathrm{m}^{-2} \mathrm{~d}^{-1}$. The second row of panels ( $G$ to $L$ ) shows $E:^{234} \mathrm{Th}$ in the large particle fraction with the error on individual measurements indicated by the horizontal black lines. The last row of panels $(M$ to $R)$ shows the derived element flux with depth. The elemental fluxes are calculated at a given depth by multiplying the ${ }^{234} \mathrm{Th}$ flux $(F)$ by the element: ${ }^{234} \mathrm{Th}$ ratio $(G$ to $L)$. Extrapolated errors from the element ratios and ${ }^{234} \mathrm{Th}$ fluxes are indicated by the grey shading. 

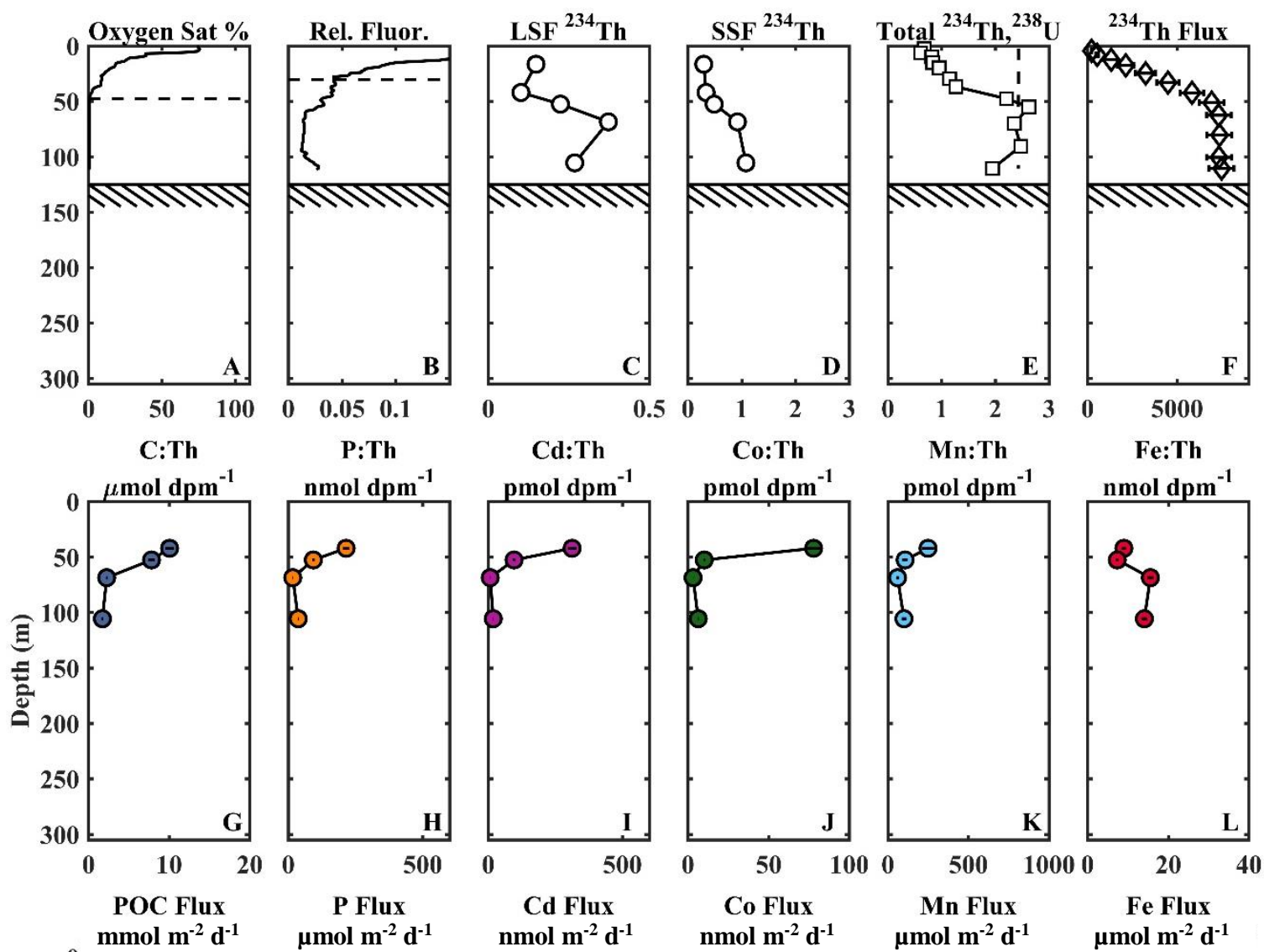

Fe:Th
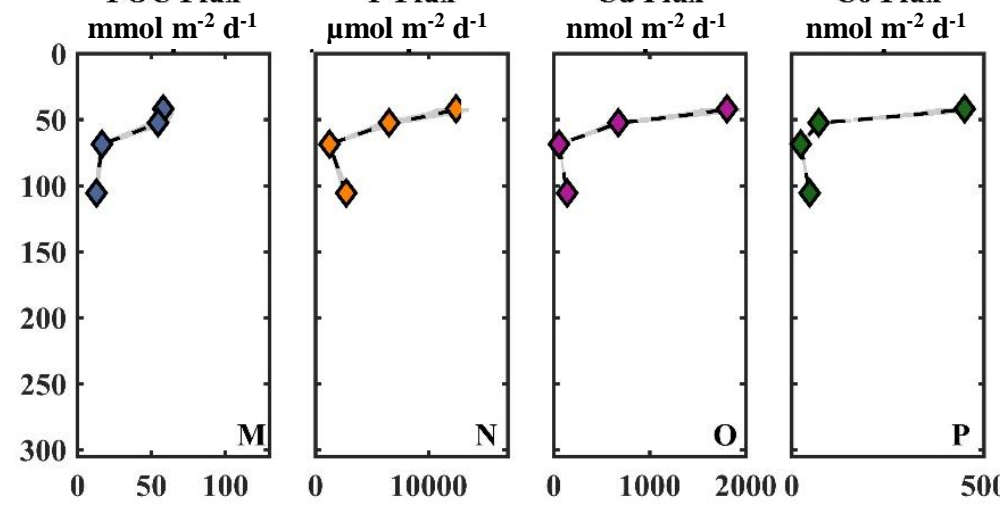

Mn Flux

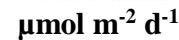

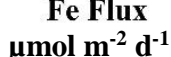

Supplemental Figure 1: Element ratios and fluxes for Shelf Station 2. The stations shown here are those not included in Fig. 3. Oxygen saturation \% $(A)$ and relative florescence $(B)$ are shown with dashed lines indicating the depth of the uODZ boundary (panel $A$ ), when present, and Ez (panel $B)$. Particulate ${ }^{234} \mathrm{Th}(C$ and $D)$, total ${ }^{234} \mathrm{Th}\left(E\right.$, squares), and total ${ }^{238} \mathrm{U}$ activities ( $E$, dashed line) are shown in dpm $\mathrm{L}^{-1}$. The derived ${ }^{234} \mathrm{Th}$ flux with depth $(F)$ is shown in dpm $\mathrm{m}^{-2} \mathrm{~d}^{-1}$. The second row of panels $\left(G\right.$ to $L$ ) shows $E:^{234} \mathrm{Th}$ in the large particle fraction with the error on individual measurements indicated by the horizontal black lines. The last row of panels $(M$ to $R$ ) shows the derived element flux with depth. The elemental fluxes are calculated at a given depth by multiplying the ${ }^{234} \mathrm{Th}$ flux $(F)$ by the element: ${ }^{234} \mathrm{Th}$ ratio $(G$ to $L)$. Extrapolated errors from the element ratios and ${ }^{234} \mathrm{Th}$ fluxes are indicated by the grey shading. 

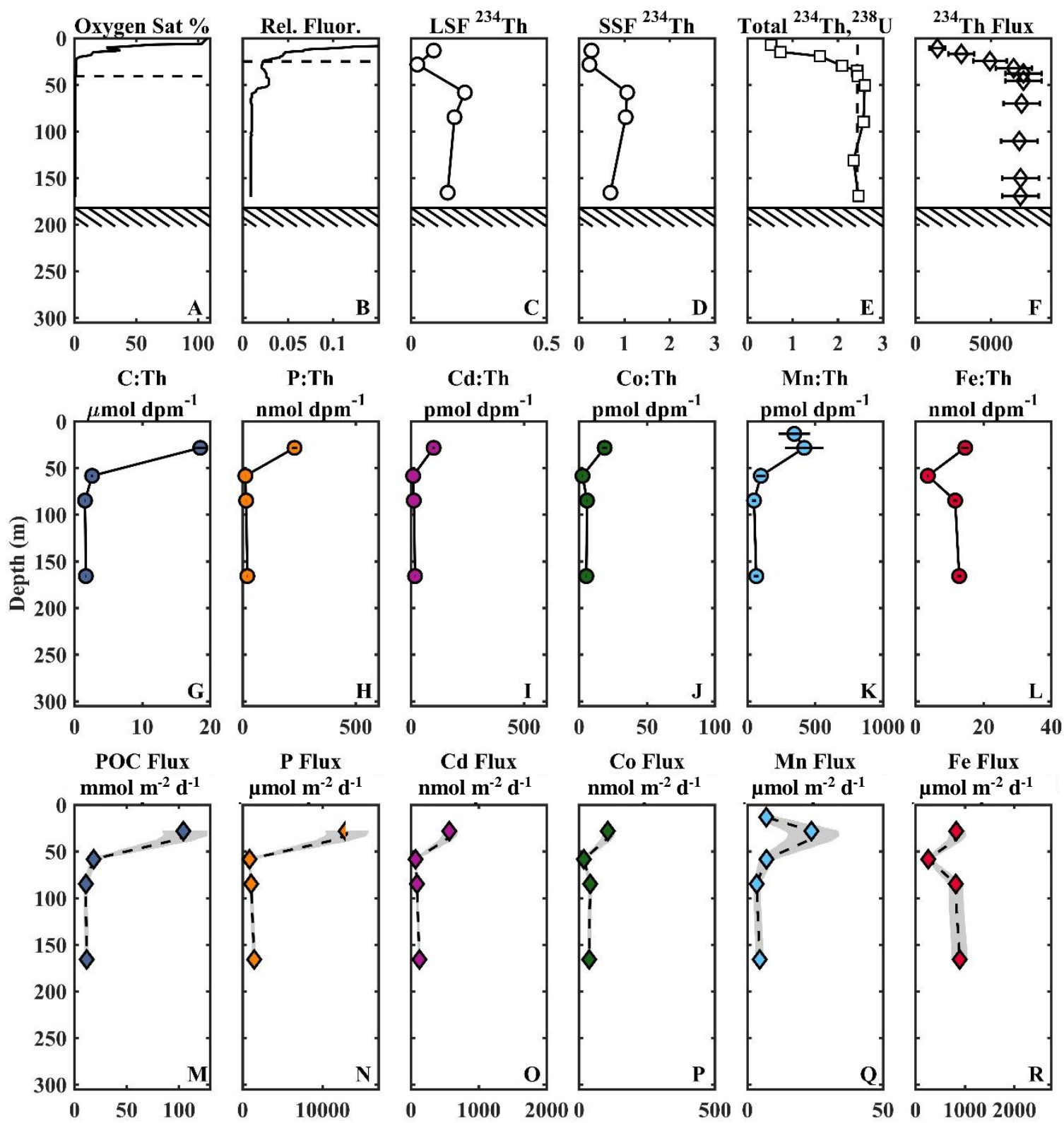

umol m $\mathrm{m}^{-2} \mathrm{~d}^{-1}$

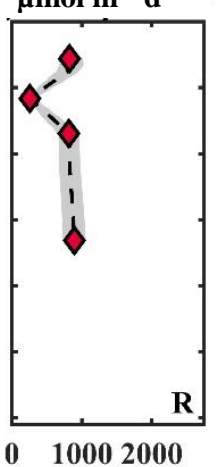

Supplemental Figure 1: Element ratios and fluxes for Shelf Station 3. The stations shown here are those not included in Fig. 3. Oxygen saturation $\%(A)$ and relative florescence $(B)$ are shown with dashed lines indicating the depth of the uODZ boundary (panel $A$ ), when present, and Ez (panel $B)$. Particulate ${ }^{234} \mathrm{Th}(C$ and $D)$, total ${ }^{234} \mathrm{Th}\left(E\right.$, squares), and total ${ }^{238} \mathrm{U}$ activities ( $E$, dashed line) are shown in dpm $\mathrm{L}^{-1}$. The derived ${ }^{234} \mathrm{Th}$ flux with depth $(F)$ is shown in dpm m $\mathrm{m}^{-2} \mathrm{~d}^{-1}$. The second row of panels ( $G$ to $L$ ) shows $\mathrm{E}:{ }^{234} \mathrm{Th}$ in the large particle fraction with the error on individual measurements indicated by the horizontal black lines. The last row of panels ( $M$ to $R$ ) shows the derived element flux with depth. The elemental fluxes are calculated at a given depth by multiplying the ${ }^{234} \mathrm{Th}$ flux $(F)$ by the element: ${ }^{234} \mathrm{Th}$ ratio $(G$ to $L)$. Extrapolated errors from the element ratios and ${ }^{234} \mathrm{Th}$ fluxes are indicated by the grey shading. 

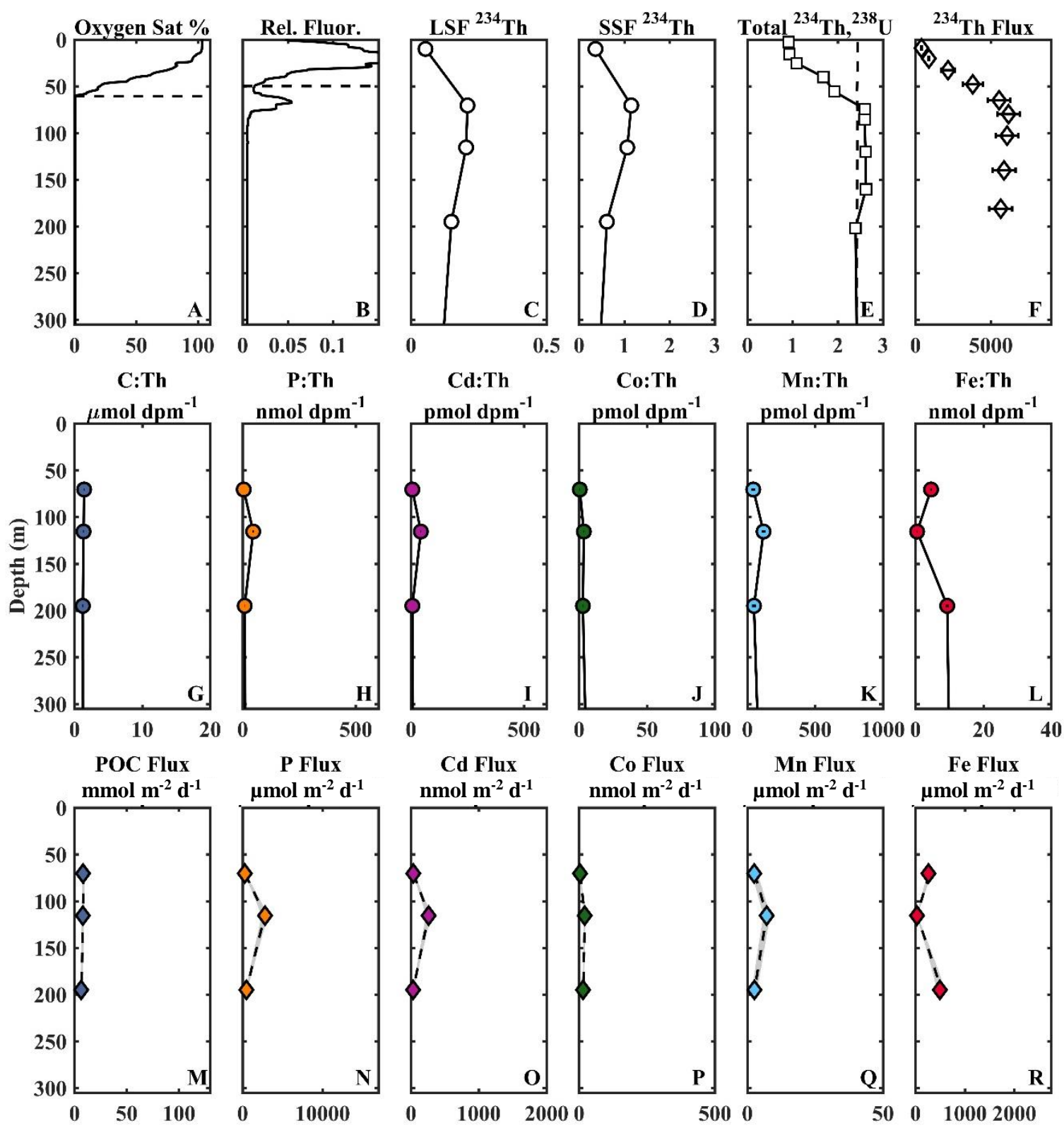

Supplemental Figure 1: Element ratios and fluxes for Shelf Station 5. The stations shown here are those not included in Fig. 3. Oxygen saturation \% $(A)$ and relative florescence $(B)$ are shown with dashed lines indicating the depth of the uODZ boundary (panel $A$ ), when present, and Ez (panel $B)$. Particulate ${ }^{234} \mathrm{Th}(C$ and $D)$, total ${ }^{234} \mathrm{Th}\left(E\right.$, squares), and total ${ }^{238} \mathrm{U}$ activities ( $E$, dashed line) are shown in dpm $\mathrm{L}^{-1}$. The derived ${ }^{234} \mathrm{Th}$ flux with depth $(F)$ is shown in $\mathrm{dpm} \mathrm{m}^{-2} \mathrm{~d}^{-1}$. The second row of panels $\left(G\right.$ to $L$ ) shows $E:{ }^{234} \mathrm{Th}$ in the large particle fraction with the error on individual measurements indicated by the horizontal black lines. The last row of panels $(M$ to $R$ ) shows the derived element flux with depth. The elemental fluxes are calculated at a given depth by multiplying the ${ }^{234} \mathrm{Th}$ flux $(F)$ by the element: ${ }^{234} \mathrm{Th}$ ratio $(G$ to $L)$. Extrapolated errors from the element ratios and ${ }^{234} \mathrm{Th}$ fluxes are indicated by the grey shading. 

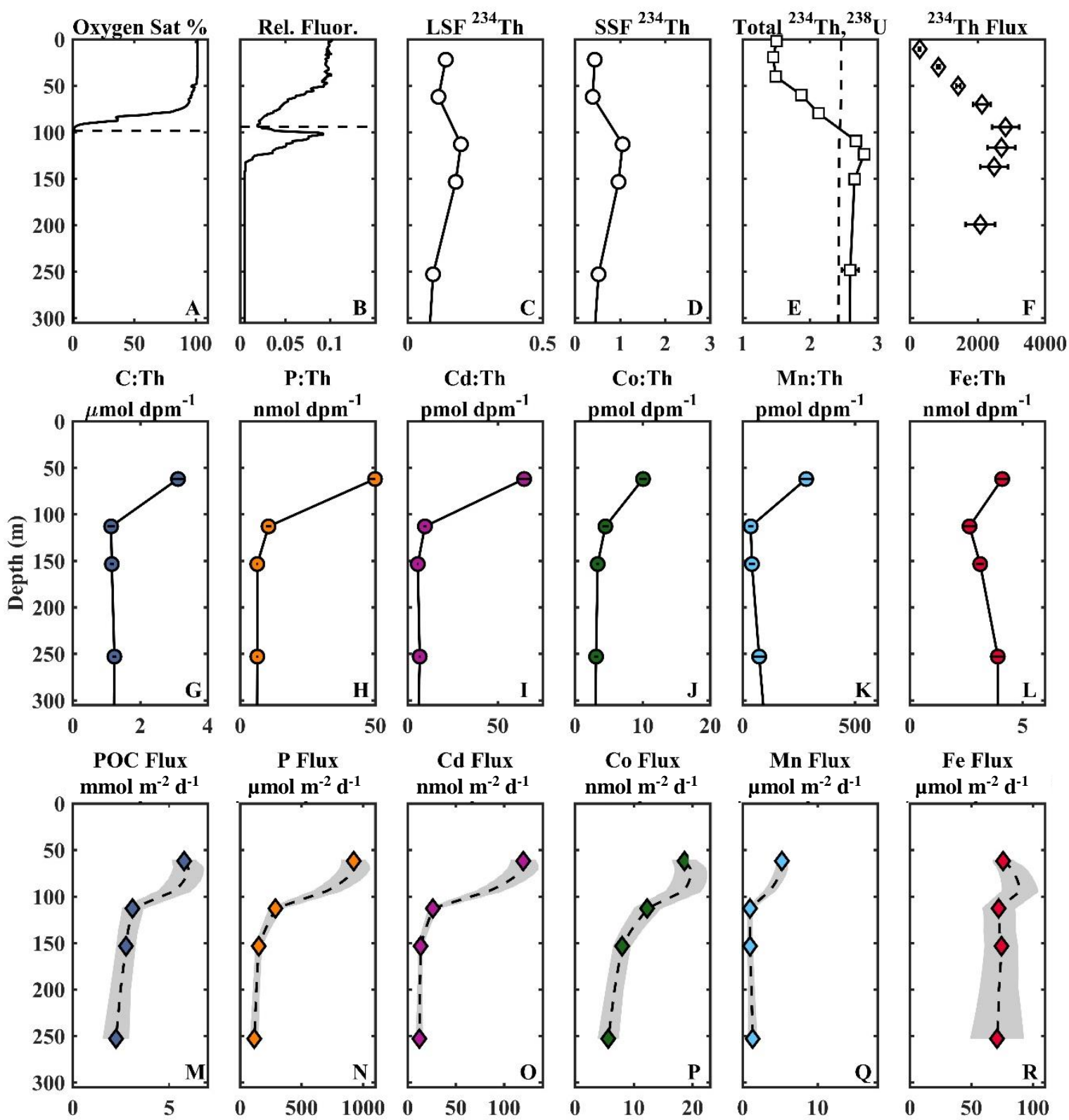

Fe Flux
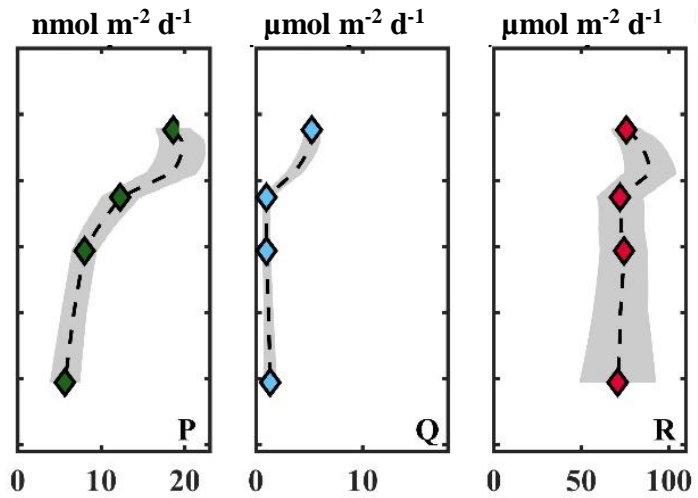

Supplemental Figure 1: Element ratios and fluxes for Offshore Station 7. The stations shown here are those not included in Fig. 3. Oxygen saturation $\%(A)$ and relative florescence $(B)$ are shown with dashed lines indicating the depth of the uODZ boundary (panel $A$ ), when present, and Ez (panel $B)$. Particulate ${ }^{234} \mathrm{Th}(C$ and $D)$, total ${ }^{234} \mathrm{Th}\left(E\right.$, squares), and total ${ }^{238} \mathrm{U}$ activities $(E$, dashed line) are shown in dpm $\mathrm{L}^{-1}$. The derived ${ }^{234}$ Th flux with depth $(F)$ is shown in $\mathrm{dpm} \mathrm{m}^{-2} \mathrm{~d}^{-}$ 1 . The second row of panels ( $G$ to $L$ ) shows $\mathrm{E}:{ }^{234} \mathrm{Th}$ in the large particle fraction with the error on individual measurements indicated by the horizontal black lines. The last row of panels ( $M$ to $R$ ) shows the derived element flux with depth. The elemental fluxes are calculated at a given depth by multiplying the ${ }^{234} \mathrm{Th}$ flux $(F)$ by the element: ${ }^{234} \mathrm{Th}$ ratio $(G$ to $L)$. Extrapolated errors from the element ratios and ${ }^{234} \mathrm{Th}$ fluxes are indicated by the grey shading. 

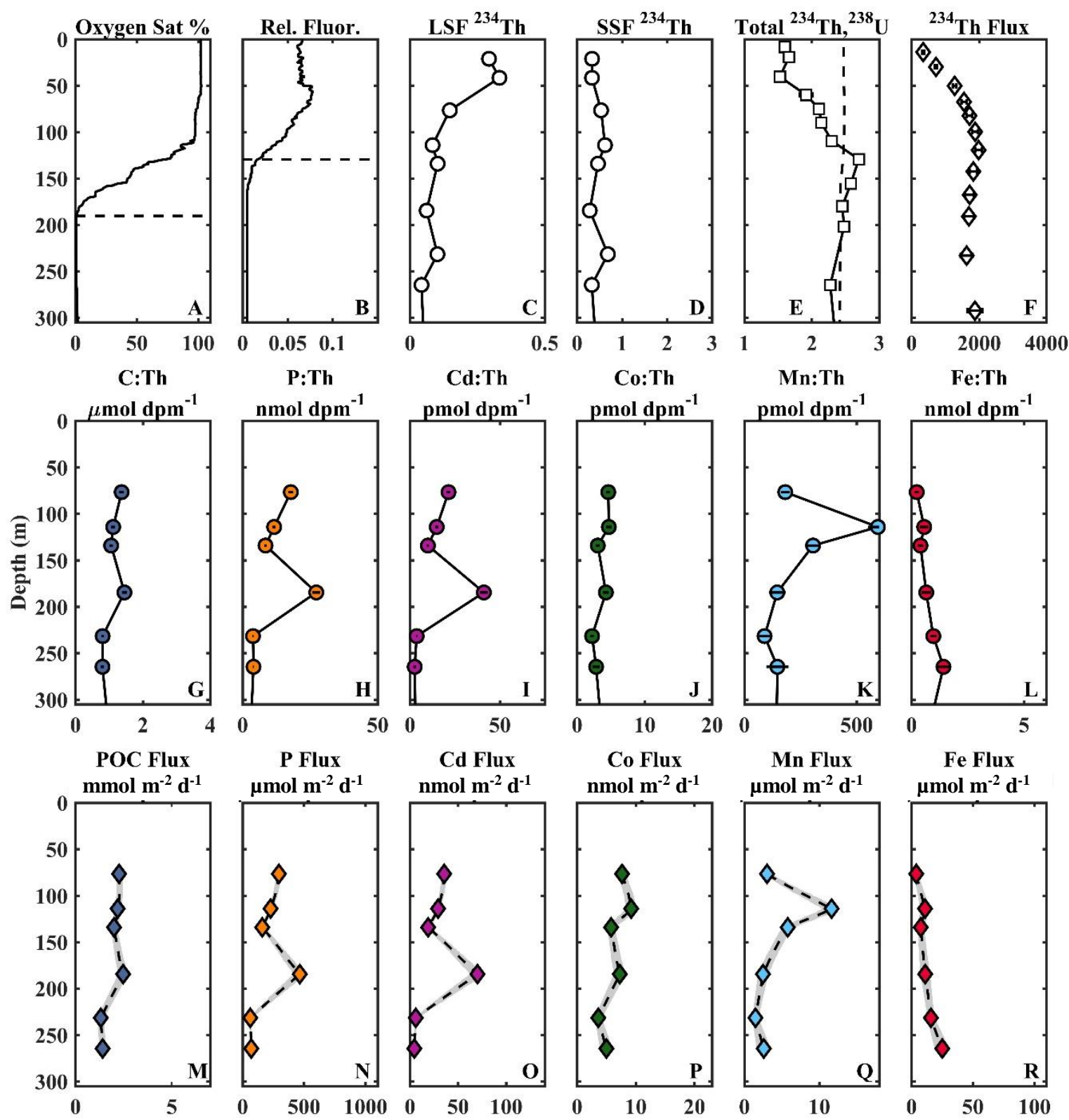

Fe Flux

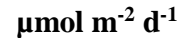

Supplemental Figure 1: Element ratios and fluxes for Offshore Station 11. The stations shown here are those not included in Fig. 3. Oxygen saturation $\%(A)$ and relative florescence $(B)$ are shown with dashed lines indicating the depth of the uODZ boundary (panel $A$ ), when present, and Ez (panel $B)$. Particulate ${ }^{234} \mathrm{Th}(C$ and $D)$, total ${ }^{234} \mathrm{Th}\left(E\right.$, squares), and total ${ }^{238} \mathrm{U}$ activities $(E$, dashed line) are shown in dpm $\mathrm{L}^{-1}$. The derived ${ }^{234} \mathrm{Th}$ flux with depth $(F)$ is shown in $\mathrm{dpm} \mathrm{m}^{-2} \mathrm{~d}^{-}$ ${ }^{1}$. The second row of panels $(G$ to $L)$ shows $\mathrm{E}:{ }^{234} \mathrm{Th}$ in the large particle fraction with the error on individual measurements indicated by the horizontal black lines. The last row of panels $(M$ to $R)$ shows the derived element flux with depth. The elemental fluxes are calculated at a given depth by multiplying the ${ }^{234} \mathrm{Th}$ flux $(F)$ by the element: ${ }^{234} \mathrm{Th}$ ratio $(G$ to $L)$. Extrapolated errors from the element ratios and ${ }^{234} \mathrm{Th}$ fluxes are indicated by the grey shading. 

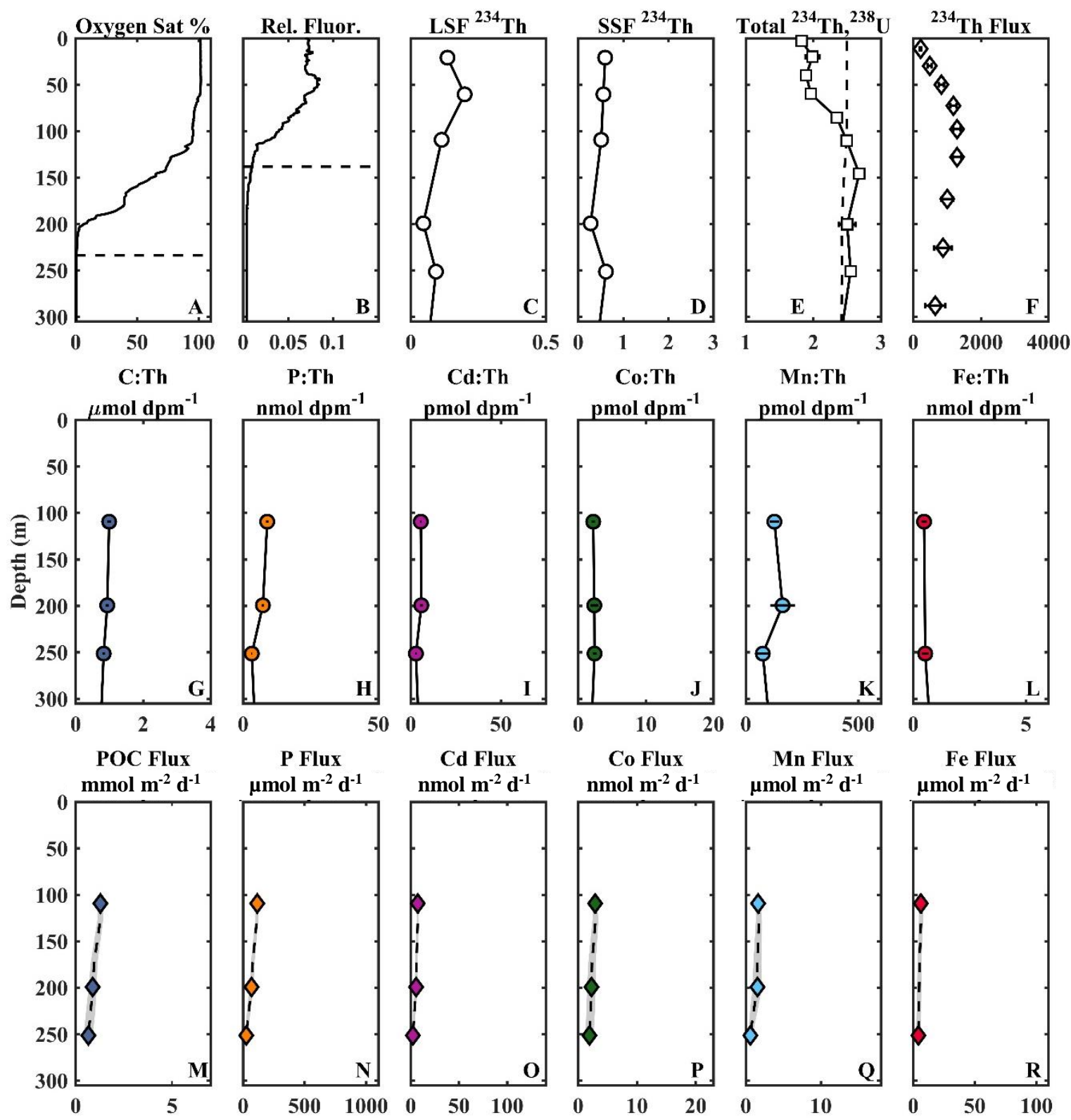

Supplemental Figure 1: Element ratios and fluxes for Offshore Station 13. The stations shown here are those not included in Fig. 3. Oxygen saturation $\%(A)$ and relative florescence $(B)$ are shown with dashed lines indicating the depth of the uODZ boundary (panel $A$ ), when present, and Ez (panel $B)$. Particulate ${ }^{234} \mathrm{Th}(C$ and $D)$, total ${ }^{234} \mathrm{Th}\left(E\right.$, squares), and total ${ }^{238} \mathrm{U}$ activities $(E$, dashed line) are shown in dpm $\mathrm{L}^{-1}$. The derived ${ }^{234} \mathrm{Th}$ flux with depth $(F)$ is shown in $\mathrm{dpm} \mathrm{m}^{-2} \mathrm{~d}^{-}$ ${ }^{1}$. The second row of panels ( $G$ to $L$ ) shows $\mathrm{E}:{ }^{234} \mathrm{Th}$ in the large particle fraction with the error on individual measurements indicated by the horizontal black lines. The last row of panels ( $M$ to $R$ ) shows the derived element flux with depth. The elemental fluxes are calculated at a given depth by multiplying the ${ }^{234} \mathrm{Th}$ flux $(F)$ by the element: ${ }^{234} \mathrm{Th}$ ratio $(G$ to $L)$. Extrapolated errors from the element ratios and ${ }^{234} \mathrm{Th}$ fluxes are indicated by the grey shading. 

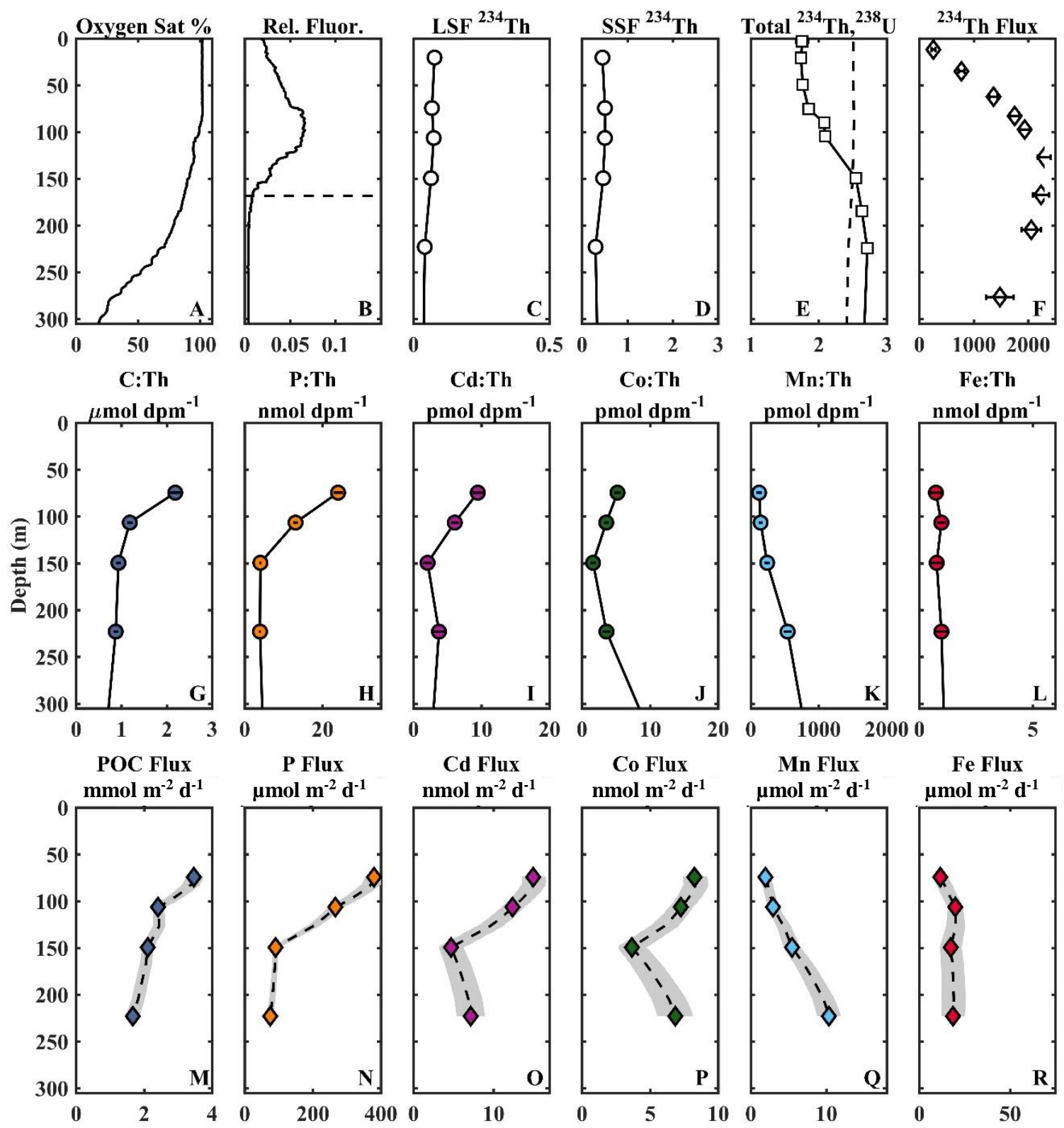

Fe Flux
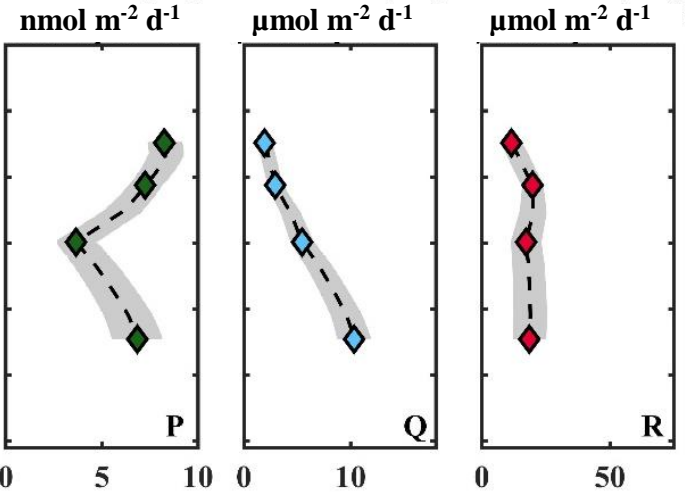

Supplemental Figure 1: Element ratios and fluxes for Gyre Station 15. The stations shown here are those not included in Fig. 3. Oxygen saturation $\%(A)$ and relative florescence $(B)$ are shown with dashed lines indicating the depth of the uODZ boundary (panel $A$ ), when present, and Ez (panel $B)$. Particulate ${ }^{234} \mathrm{Th}(C$ and $D)$, total ${ }^{234} \mathrm{Th}\left(E\right.$, squares), and total ${ }^{238} \mathrm{U}$ activities $(E$, dashed line) are shown in dpm $\mathrm{L}^{-1}$. The derived ${ }^{234} \mathrm{Th}$ flux with depth $(F)$ is shown in $\mathrm{dpm} \mathrm{m}^{-2} \mathrm{~d}^{-}$ ${ }^{1}$. The second row of panels ( $G$ to $L$ ) shows $\mathrm{E}:{ }^{234} \mathrm{Th}$ in the large particle fraction with the error on individual measurements indicated by the horizontal black lines. The last row of panels $(M$ to $R)$ shows the derived element flux with depth. The elemental fluxes are calculated at a given depth by multiplying the ${ }^{234} \mathrm{Th}$ flux $(F)$ by the element: ${ }^{234} \mathrm{Th}$ ratio $(G$ to $L)$. Extrapolated errors from the element ratios and ${ }^{234} \mathrm{Th}$ fluxes are indicated by the grey shading. 

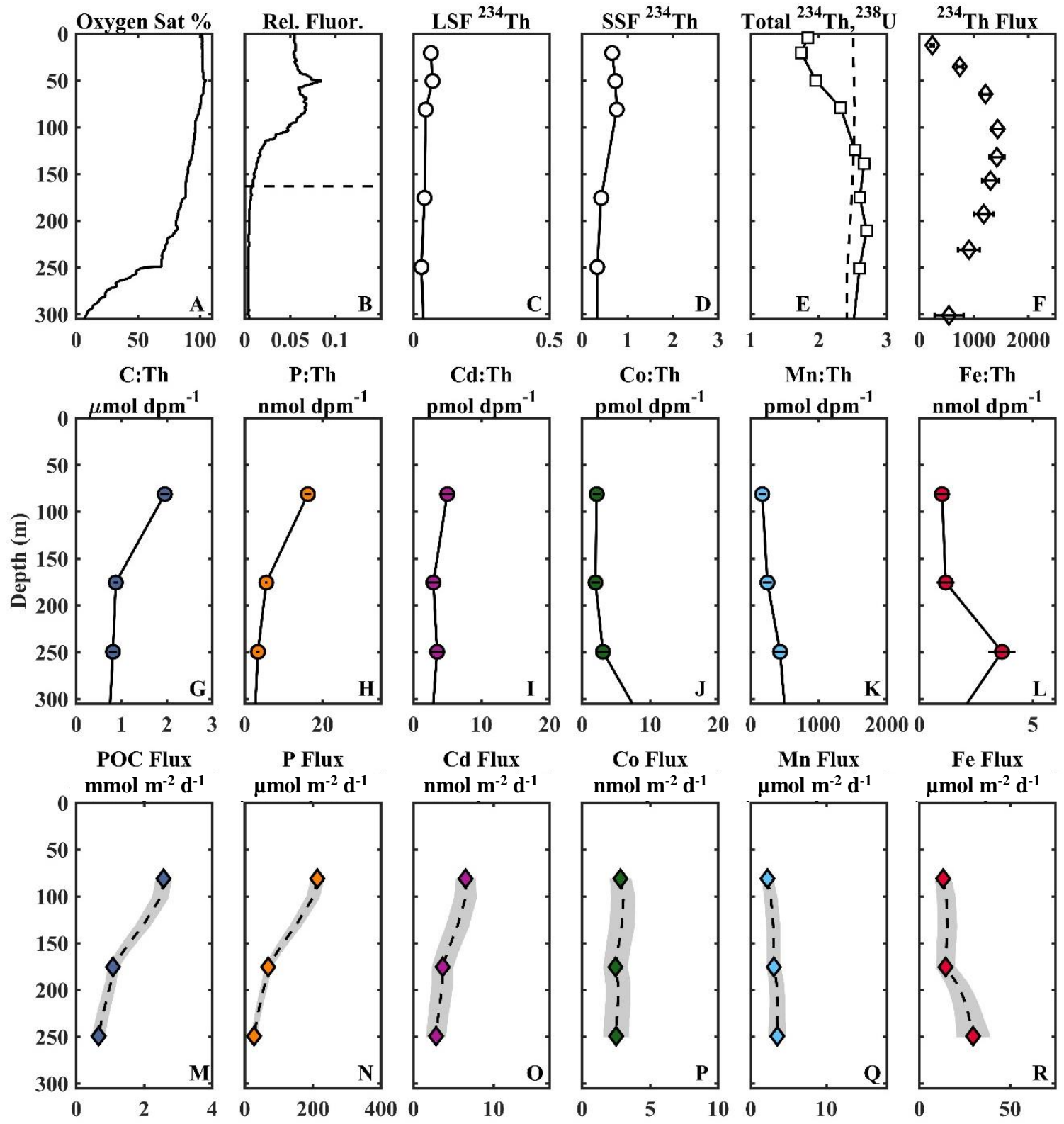

Supplemental Figure 1: Element ratios and fluxes for Gyre Station 17. The stations shown here are those not included in Fig. 3. Oxygen saturation $\%(A)$ and relative florescence $(B)$ are shown with dashed lines indicating the depth of the uODZ boundary (panel $A$ ), when present, and Ez (panel $B$ ). Particulate ${ }^{234} \mathrm{Th}(C$ and $D)$, total ${ }^{234} \mathrm{Th}\left(E\right.$, squares), and total ${ }^{238} \mathrm{U}$ activities $(E$, dashed line) are shown in dpm $\mathrm{L}^{-1}$. The derived ${ }^{234} \mathrm{Th}$ flux with depth $(F)$ is shown in $\mathrm{dpm} \mathrm{m}^{-2} \mathrm{~d}^{-}$ ${ }^{1}$. The second row of panels ( $G$ to $L$ ) shows $\mathrm{E}:{ }^{234} \mathrm{Th}$ in the large particle fraction with the error on individual measurements indicated by the horizontal black lines. The last row of panels $(M$ to $R$ ) shows the derived element flux with depth. The elemental fluxes are calculated at a given depth by multiplying the ${ }^{234} \mathrm{Th}$ flux $(F)$ by the element: ${ }^{234} \mathrm{Th}$ ratio $(G$ to $L)$. Extrapolated errors from the element ratios and ${ }^{234} \mathrm{Th}$ fluxes are indicated by the grey shading. 

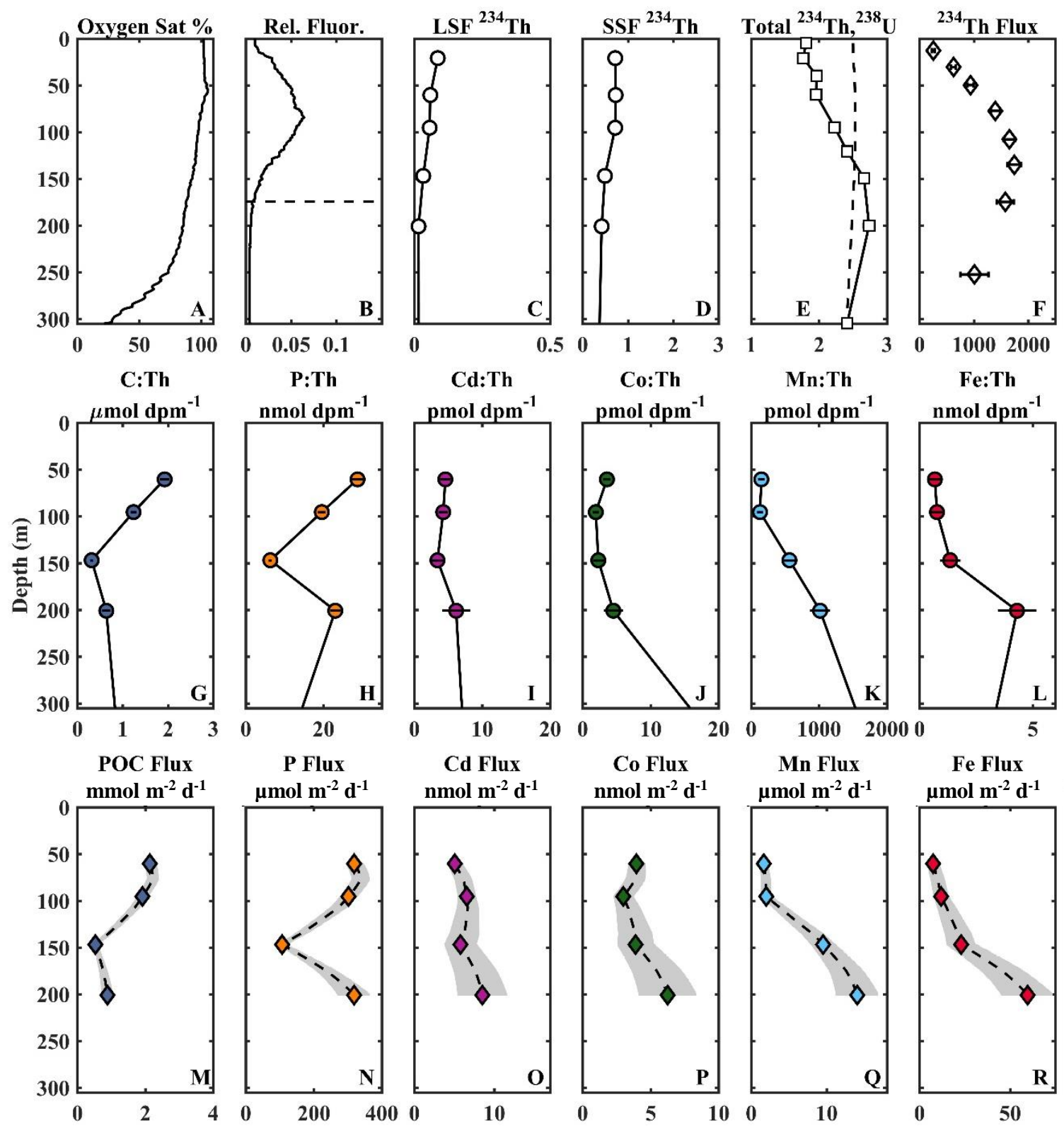

Supplemental Figure 1: Element ratios and fluxes for Gyre Station 18. The stations shown here are those not included in Fig. 3. Oxygen saturation $\%(A)$ and relative florescence $(B)$ are shown with dashed lines indicating the depth of the uODZ boundary (panel $A$ ), when present, and Ez (panel $B)$. Particulate ${ }^{234} \mathrm{Th}(C$ and $D)$, total ${ }^{234} \mathrm{Th}\left(E\right.$, squares), and total ${ }^{238} \mathrm{U}$ activities $(E$, dashed line) are shown in dpm $\mathrm{L}^{-1}$. The derived ${ }^{234} \mathrm{Th}$ flux with depth $(F)$ is shown in $\mathrm{dpm} \mathrm{m}^{-2} \mathrm{~d}^{-}$ ${ }^{1}$. The second row of panels $\left(G\right.$ to $L$ ) shows $\mathrm{E}:{ }^{234} \mathrm{Th}$ in the large particle fraction with the error on individual measurements indicated by the horizontal black lines. The last row of panels $(M$ to $R)$ shows the derived element flux with depth. The elemental fluxes are calculated at a given depth by multiplying the ${ }^{234} \mathrm{Th}$ flux $(F)$ by the element: ${ }^{234} \mathrm{Th}$ ratio $(G$ to $L)$. Extrapolated errors from the element ratios and ${ }^{234} \mathrm{Th}$ fluxes are indicated by the grey shading. 

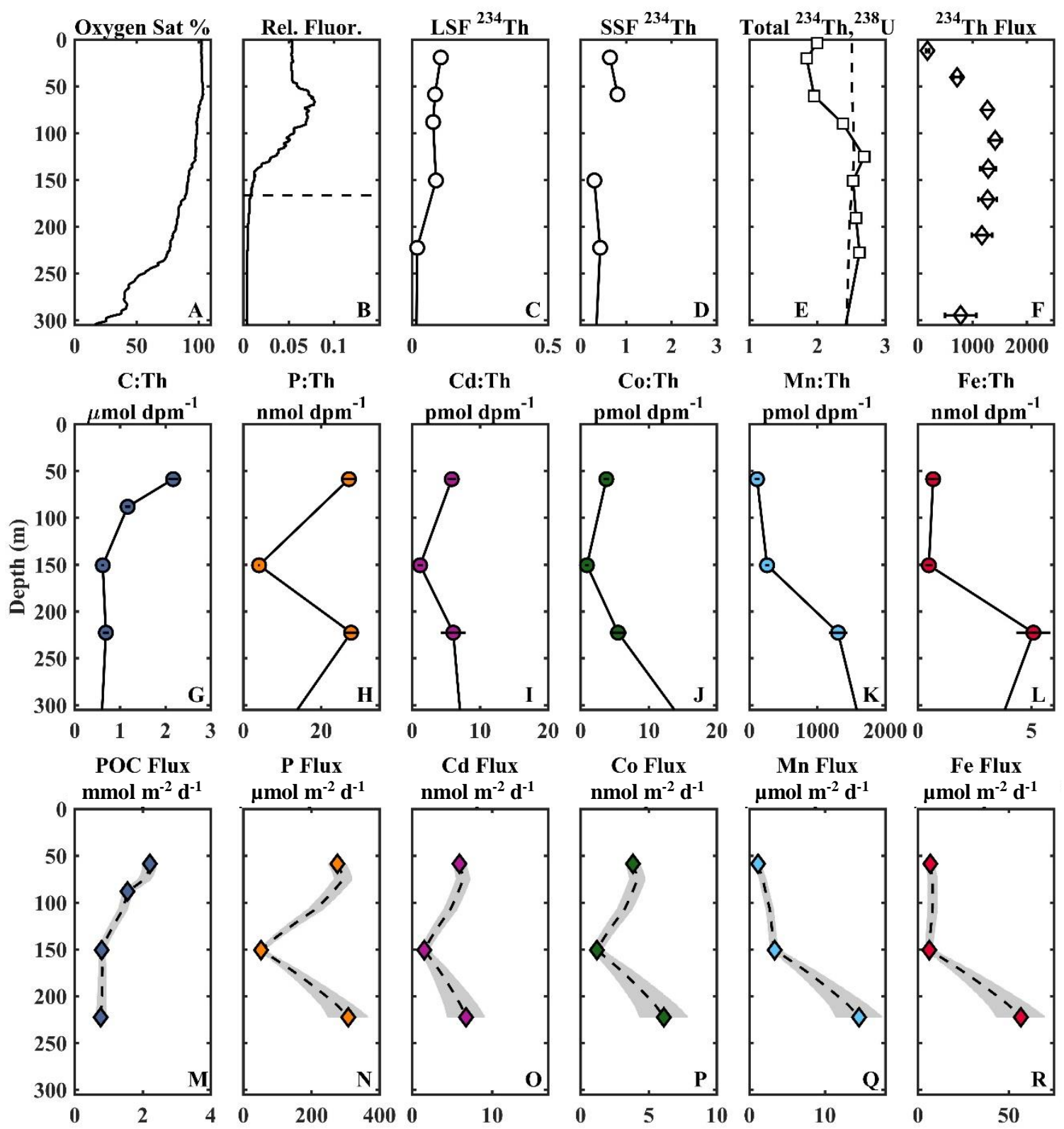

Supplemental Figure 1: Element ratios and fluxes for Gyre Station 21. The stations shown here are those not included in Fig. 3. Oxygen saturation $\%(A)$ and relative florescence $(B)$ are shown with dashed lines indicating the depth of the uODZ boundary (panel $A$ ), when present, and Ez (panel $B$ ). Particulate ${ }^{234} \mathrm{Th}(C$ and $D)$, total ${ }^{234} \mathrm{Th}\left(E\right.$, squares), and total ${ }^{238} \mathrm{U}$ activities $(E$, dashed line) are shown in dpm $\mathrm{L}^{-1}$. The derived ${ }^{234} \mathrm{Th}$ flux with depth $(F)$ is shown in $\mathrm{dpm} \mathrm{m}^{-2} \mathrm{~d}^{-}$ ${ }^{1}$. The second row of panels $\left(G\right.$ to $L$ ) shows $E:{ }^{234} \mathrm{Th}$ in the large particle fraction with the error on individual measurements indicated by the horizontal black lines. The last row of panels $(M$ to $R)$ shows the derived element flux with depth. The elemental fluxes are calculated at a given depth by multiplying the ${ }^{234} \mathrm{Th}$ flux $(F)$ by the element: ${ }^{234} \mathrm{Th}$ ratio $(G$ to $L)$. Extrapolated errors from the element ratios and ${ }^{234} \mathrm{Th}$ fluxes are indicated by the grey shading. 

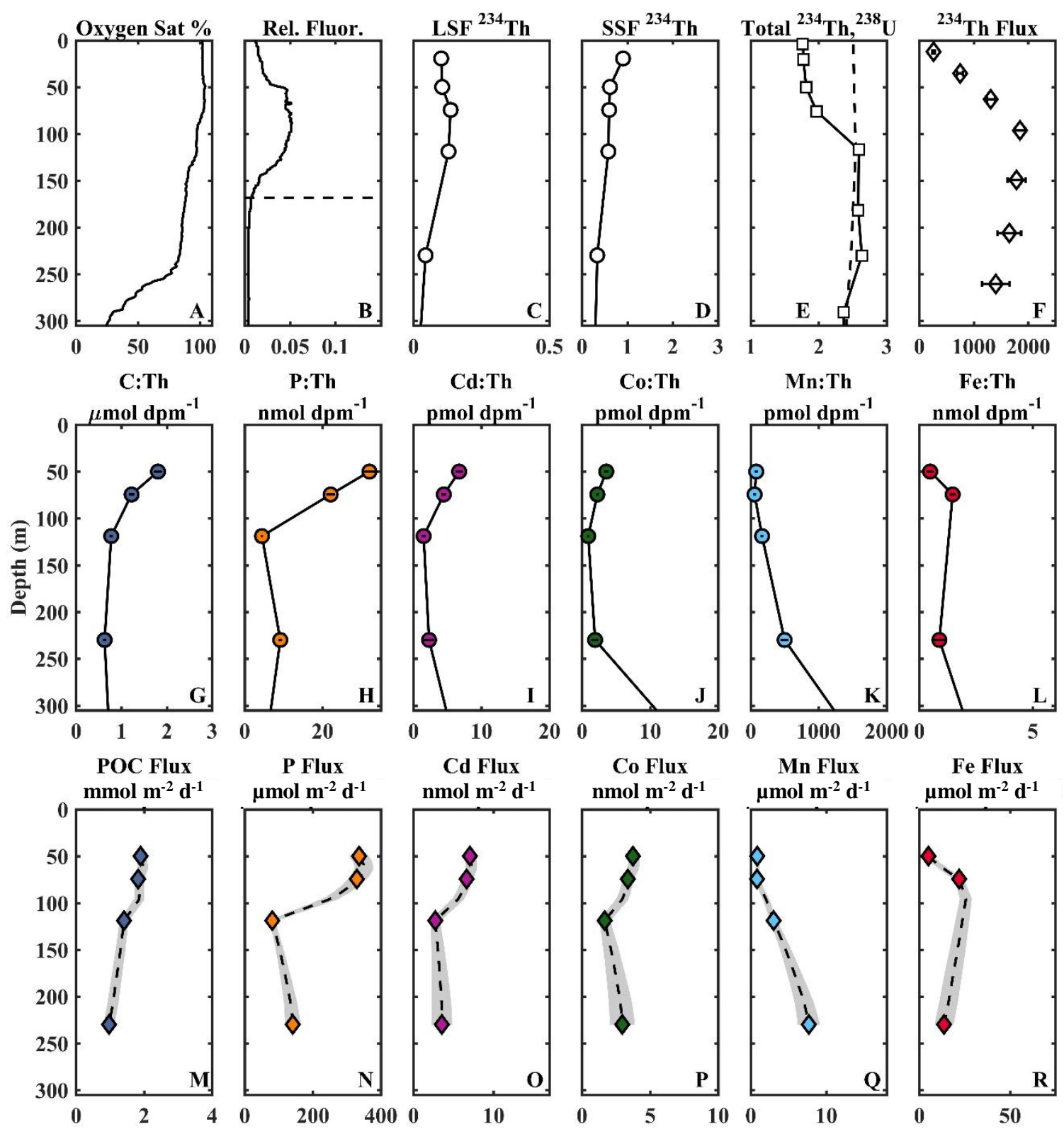

Supplemental Figure 1: Element ratios and fluxes for Gyre Station 23. The stations shown here are those not included in Fig. 3. Oxygen saturation $\%(A)$ and relative florescence $(B)$ are shown with dashed lines indicating the depth of the uODZ boundary (panel $A$ ), when present, and Ez (panel $B$ ). Particulate ${ }^{234} \mathrm{Th}(C$ and $D)$, total ${ }^{234} \mathrm{Th}\left(E\right.$, squares), and total ${ }^{238} \mathrm{U}$ activities $(E$, dashed line) are shown in dpm $\mathrm{L}^{-1}$. The derived ${ }^{234} \mathrm{Th}$ flux with depth $(F)$ is shown in $\mathrm{dpm} \mathrm{m}^{-2} \mathrm{~d}^{-}$ ${ }^{1}$. The second row of panels ( $G$ to $L$ ) shows E: ${ }^{234} \mathrm{Th}$ in the large particle fraction with the error on individual measurements indicated by the horizontal black lines. The last row of panels $(M$ to $R$ ) shows the derived element flux with depth. The elemental fluxes are calculated at a given depth by multiplying the ${ }^{234} \mathrm{Th}$ flux $(F)$ by the element: ${ }^{234} \mathrm{Th}$ ratio $(G$ to $L)$. Extrapolated errors from the element ratios and ${ }^{234} \mathrm{Th}$ fluxes are indicated by the grey shading. 

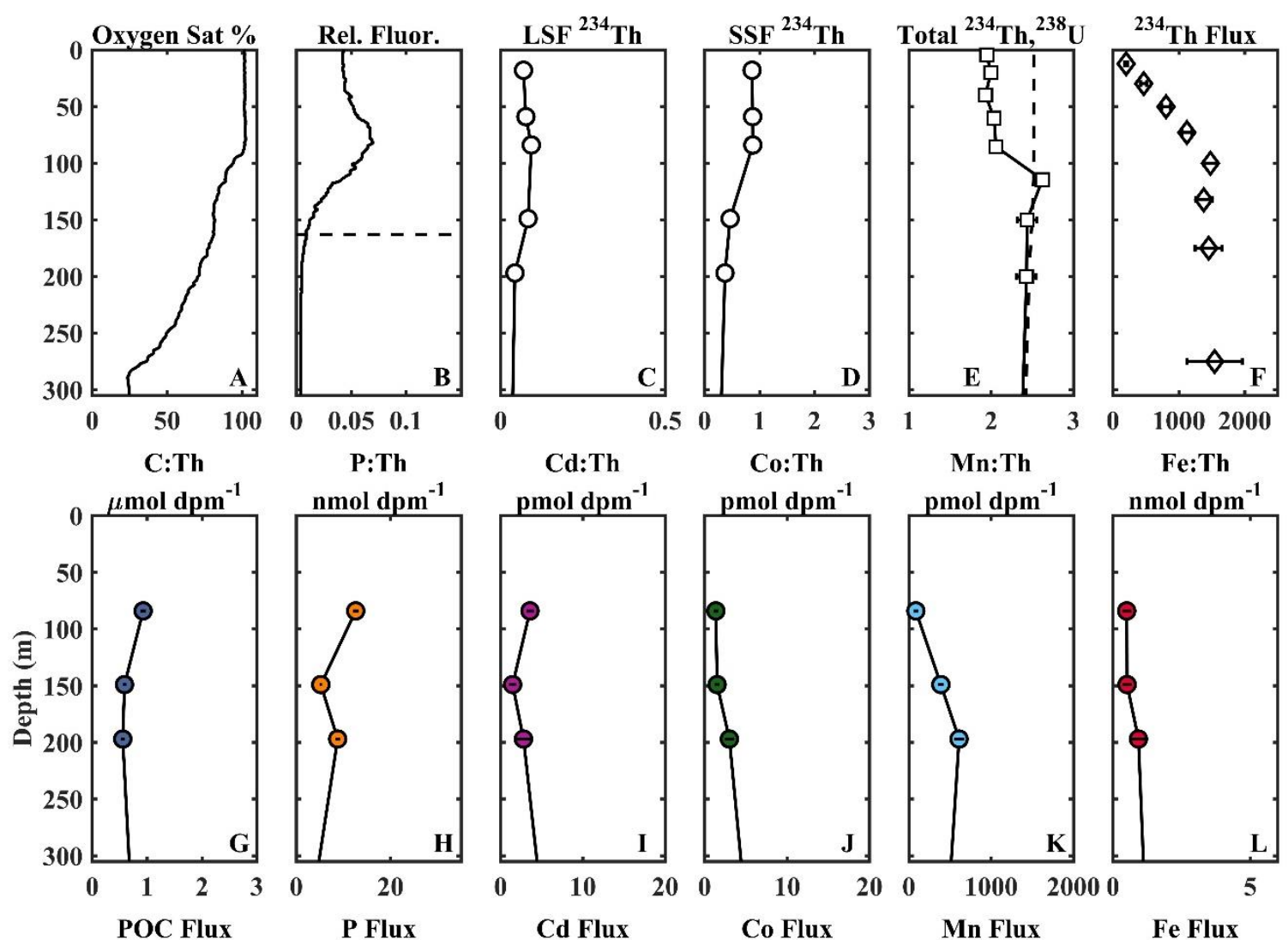

pmol dpm ${ }^{-1}$
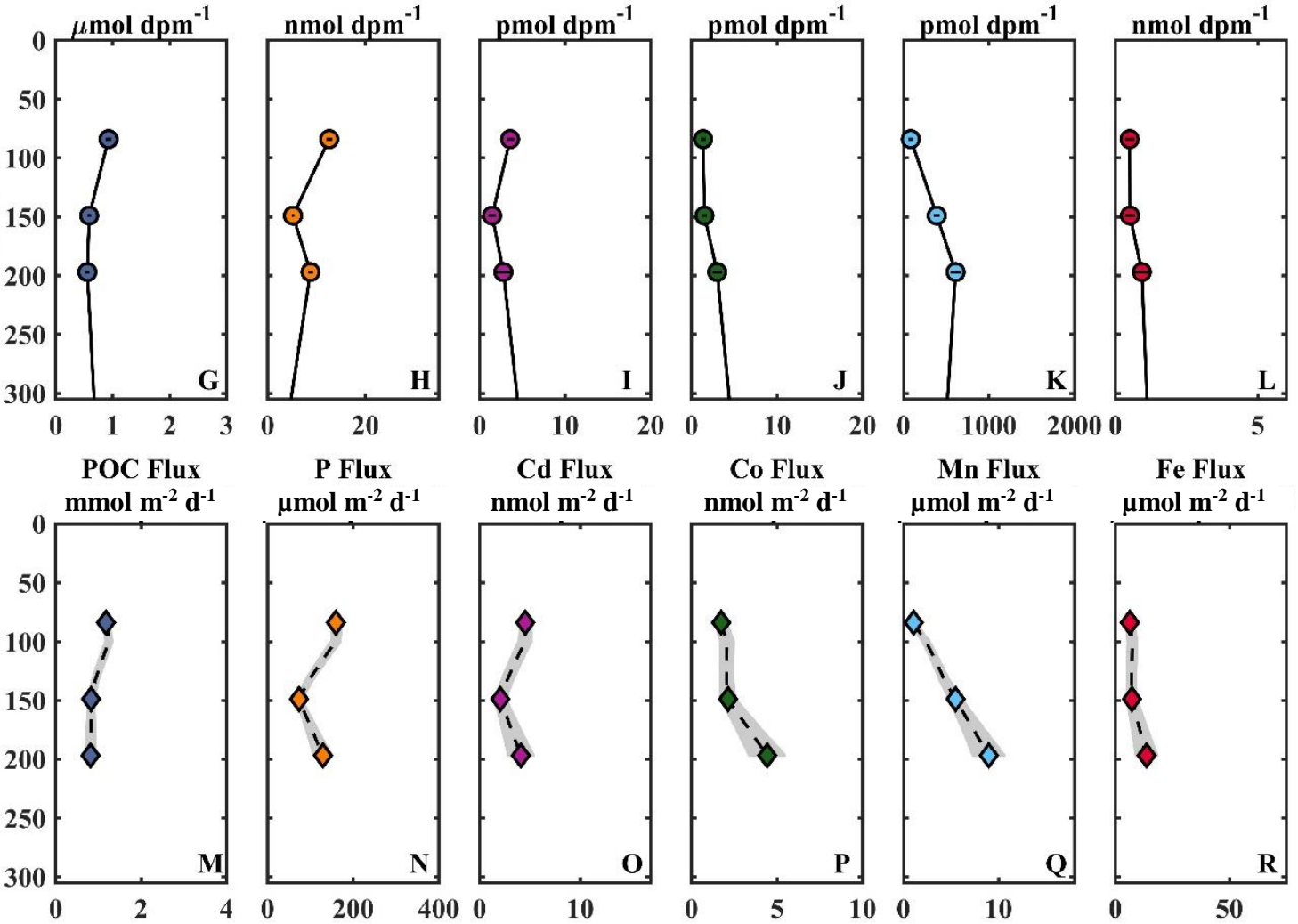

Supplemental Figure 1: Element ratios and fluxes for Gyre Station 25. The stations shown here are those not included in Fig. 3. Oxygen saturation $\%(A)$ and relative florescence $(B)$ are shown with dashed lines indicating the depth of the uODZ boundary (panel $A$ ), when present, and Ez (panel $B)$. Particulate ${ }^{234} \mathrm{Th}(C$ and $D)$, total ${ }^{234} \mathrm{Th}\left(E\right.$, squares), and total ${ }^{238} \mathrm{U}$ activities $(E$, dashed line) are shown in dpm $\mathrm{L}^{-1}$. The derived ${ }^{234} \mathrm{Th}$ flux with depth $(F)$ is shown in $\mathrm{dpm} \mathrm{m}^{-2} \mathrm{~d}^{-}$ ${ }^{1}$. The second row of panels ( $G$ to $L$ ) shows $\mathrm{E}:{ }^{234} \mathrm{Th}$ in the large particle fraction with the error on individual measurements indicated by the horizontal black lines. The last row of panels $(M$ to $R)$ shows the derived element flux with depth. The elemental fluxes are calculated at a given depth by multiplying the ${ }^{234} \mathrm{Th}$ flux $(F)$ by the element: ${ }^{234} \mathrm{Th}$ ratio $(G$ to $L)$. Extrapolated errors from the element ratios and ${ }^{234} \mathrm{Th}$ fluxes are indicated by the grey shading. 

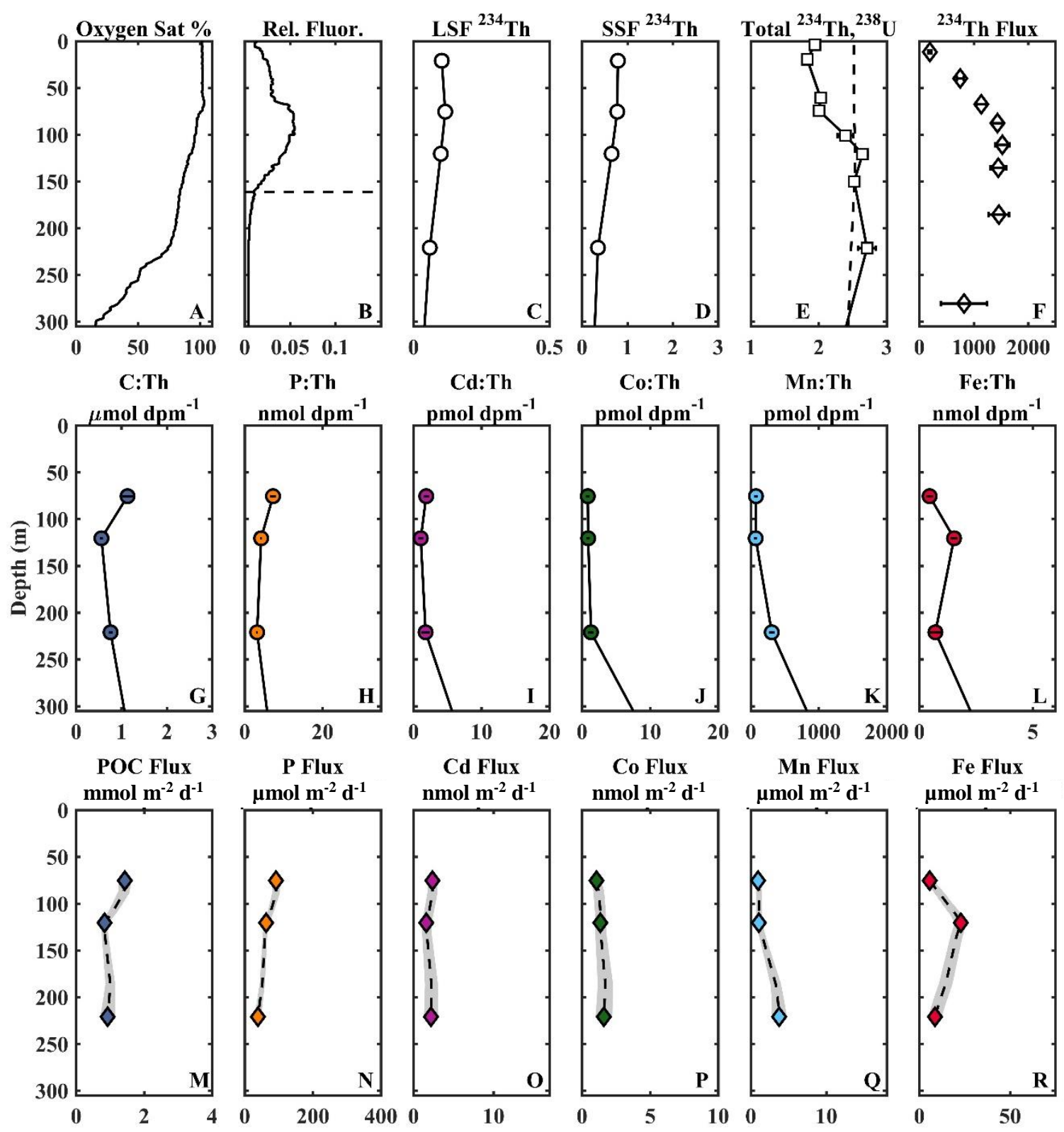

Supplemental Figure 1: Element ratios and fluxes for Gyre Station 28. The stations shown here are those not included in Fig. 3. Oxygen saturation $\%(A)$ and relative florescence $(B)$ are shown with dashed lines indicating the depth of the uODZ boundary (panel $A$ ), when present, and Ez (panel $B)$. Particulate ${ }^{234} \mathrm{Th}(C$ and $D)$, total ${ }^{234} \mathrm{Th}\left(E\right.$, squares), and total ${ }^{238} \mathrm{U}$ activities $(E$, dashed line) are shown in dpm $\mathrm{L}^{-1}$. The derived ${ }^{234} \mathrm{Th}$ flux with depth $(F)$ is shown in $\mathrm{dpm} \mathrm{m}^{-2} \mathrm{~d}^{-}$ ${ }^{1}$. The second row of panels ( $G$ to $L$ ) shows $\mathrm{E}:{ }^{234} \mathrm{Th}$ in the large particle fraction with the error on individual measurements indicated by the horizontal black lines. The last row of panels $(M$ to $R)$ shows the derived element flux with depth. The elemental fluxes are calculated at a given depth by multiplying the ${ }^{234} \mathrm{Th}$ flux $(F)$ by the element: ${ }^{234} \mathrm{Th}$ ratio $(G$ to $L)$. Extrapolated errors from the element ratios and ${ }^{234} \mathrm{Th}$ fluxes are indicated by the grey shading. 

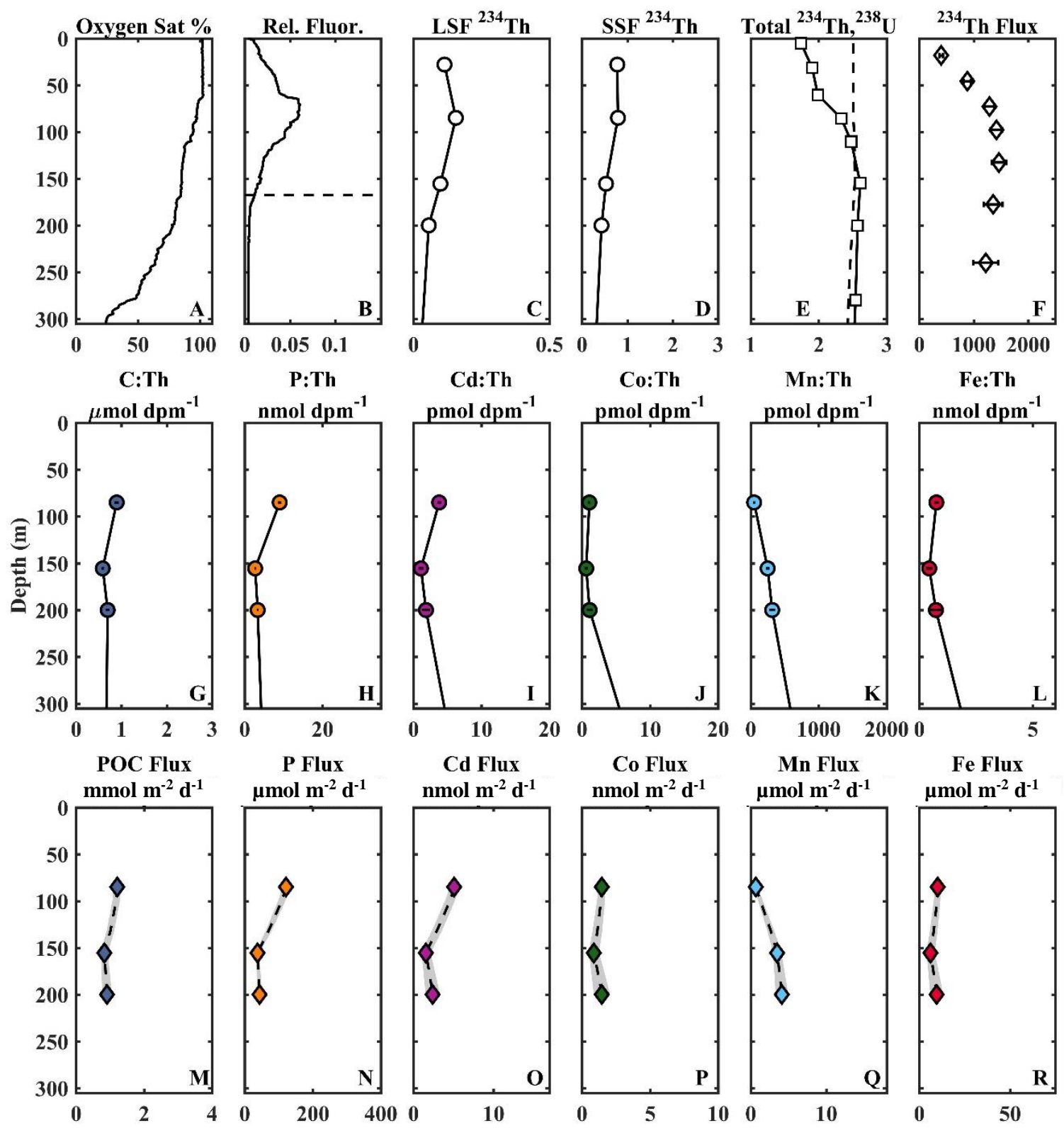

Supplemental Figure 1: Element ratios and fluxes for Gyre Station 30. The stations shown here are those not included in Fig. 3. Oxygen saturation $\%(A)$ and relative florescence $(B)$ are shown with dashed lines indicating the depth of the uODZ boundary (panel $A$ ), when present, and Ez (panel $B$ ). Particulate ${ }^{234} \mathrm{Th}(C$ and $D)$, total ${ }^{234} \mathrm{Th}\left(E\right.$, squares), and total ${ }^{238} \mathrm{U}$ activities $(E$, dashed line) are shown in dpm $\mathrm{L}^{-1}$. The derived ${ }^{234} \mathrm{Th}$ flux with depth $(F)$ is shown in $\mathrm{dpm} \mathrm{m}^{-2} \mathrm{~d}^{-}$ ${ }^{1}$. The second row of panels ( $G$ to $L$ ) shows $\mathrm{E}:{ }^{234} \mathrm{Th}$ in the large particle fraction with the error on individual measurements indicated by the horizontal black lines. The last row of panels $(M$ to $R$ ) shows the derived element flux with depth. The elemental fluxes are calculated at a given depth by multiplying the ${ }^{234} \mathrm{Th}$ flux $(F)$ by the element: ${ }^{234} \mathrm{Th}$ ratio $(G$ to $L)$. Extrapolated errors from the element ratios and ${ }^{234} \mathrm{Th}$ fluxes are indicated by the grey shading. 

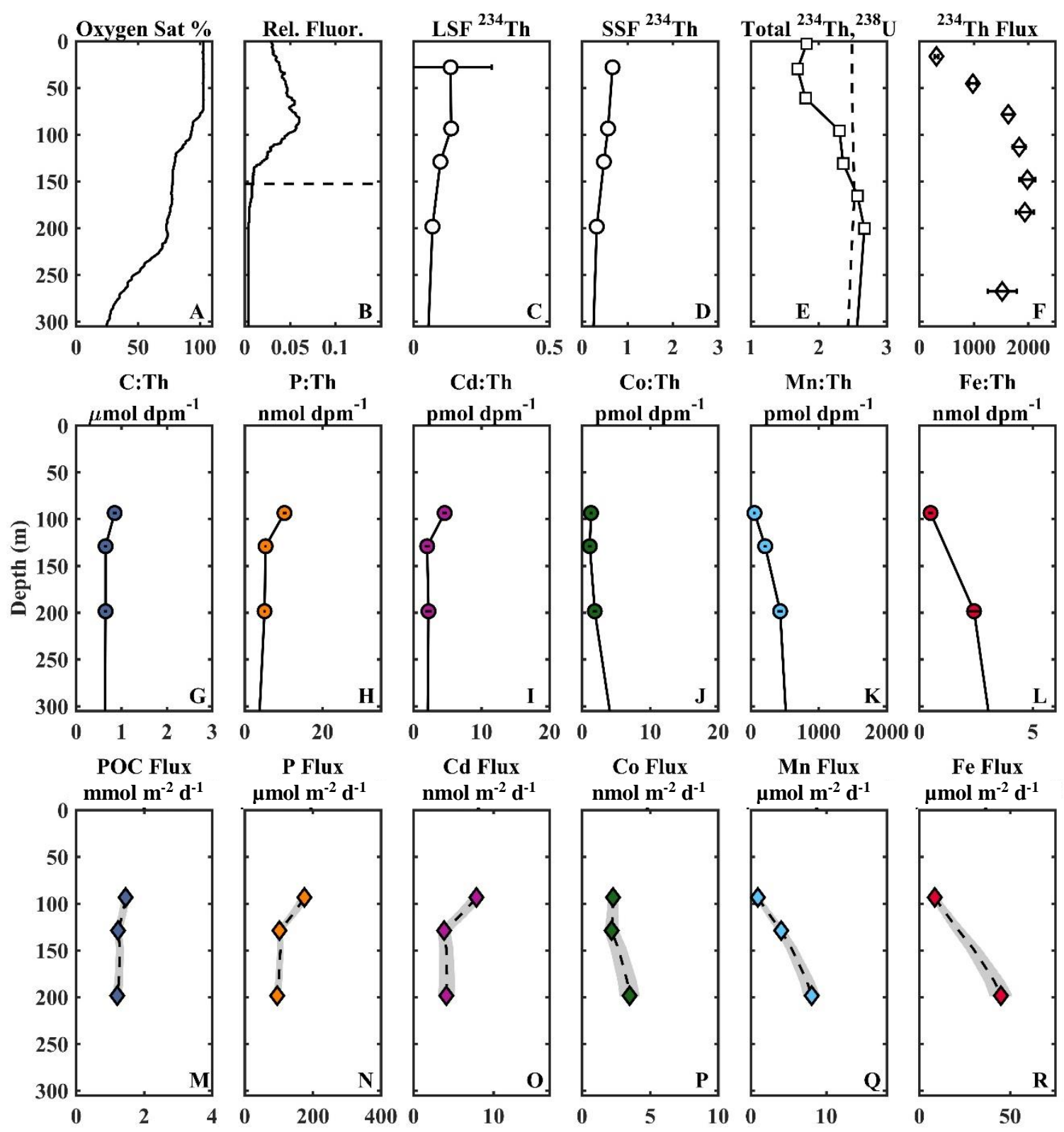

Fe Flux

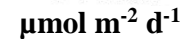

Supplemental Figure 1: Element ratios and fluxes for Gyre Station 32. The stations shown here are those not included in Fig. 3. Oxygen saturation $\%(A)$ and relative florescence $(B)$ are shown with dashed lines indicating the depth of the uODZ boundary (panel $A$ ), when present, and Ez (panel $B)$. Particulate ${ }^{234} \mathrm{Th}(C$ and $D)$, total ${ }^{234} \mathrm{Th}\left(E\right.$, squares), and total ${ }^{238} \mathrm{U}$ activities $(E$, dashed line) are shown in dpm $\mathrm{L}^{-1}$. The derived ${ }^{234} \mathrm{Th}$ flux with depth $(F)$ is shown in $\mathrm{dpm} \mathrm{m}^{-2} \mathrm{~d}^{-}$ ${ }^{1}$. The second row of panels ( $G$ to $L$ ) shows $\mathrm{E}:{ }^{234} \mathrm{Th}$ in the large particle fraction with the error on individual measurements indicated by the horizontal black lines. The last row of panels $(M$ to $R)$ shows the derived element flux with depth. The elemental fluxes are calculated at a given depth by multiplying the ${ }^{234} \mathrm{Th}$ flux $(F)$ by the element: ${ }^{234} \mathrm{Th}$ ratio $(G$ to $L)$. Extrapolated errors from the element ratios and ${ }^{234} \mathrm{Th}$ fluxes are indicated by the grey shading. 

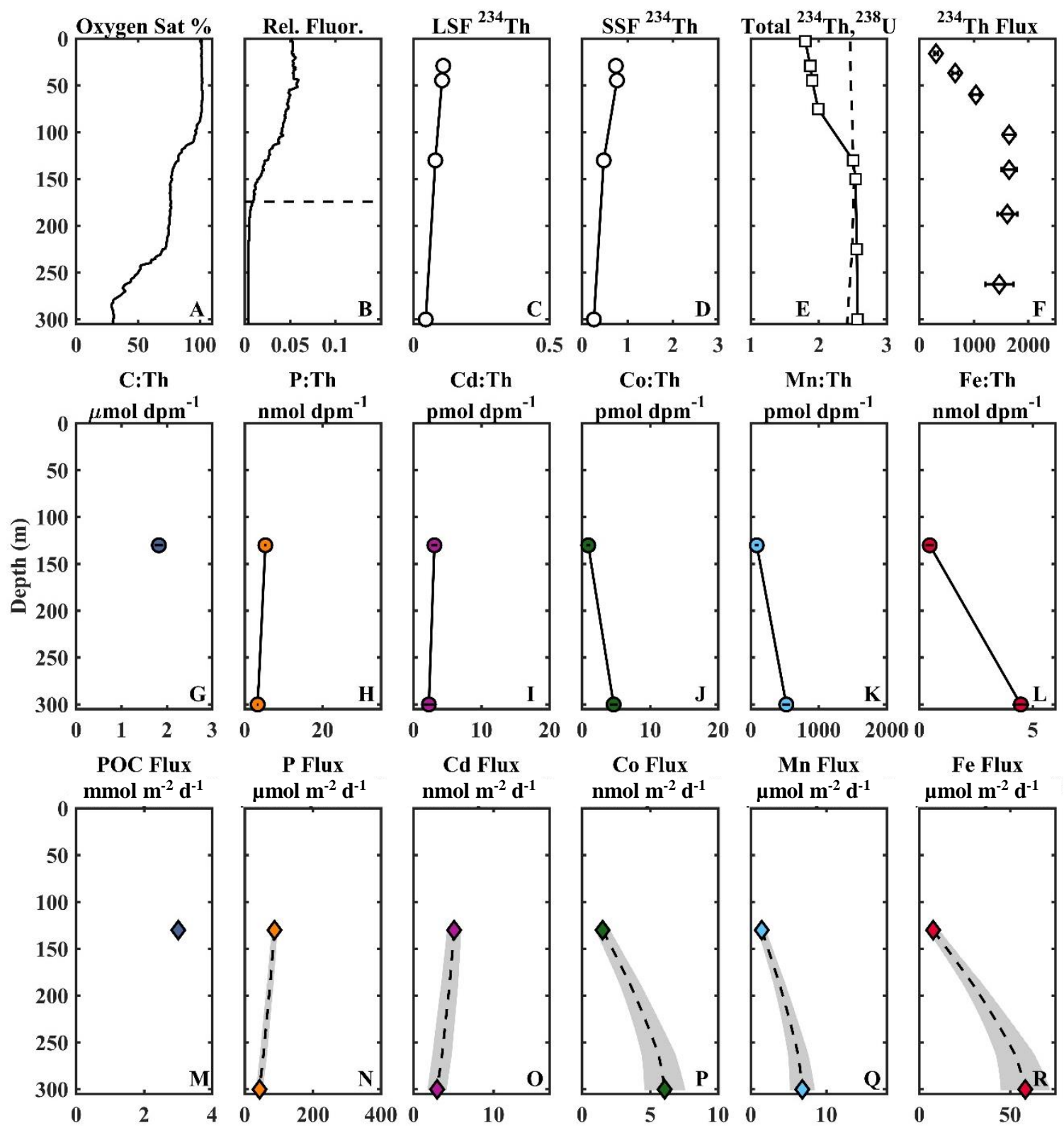

Supplemental Figure 1: Element ratios and fluxes for Gyre Station 34. The stations shown here are those not included in Fig. 3. Oxygen saturation $\%(A)$ and relative florescence $(B)$ are shown with dashed lines indicating the depth of the uODZ boundary (panel $A$ ), when present, and Ez (panel $B)$. Particulate ${ }^{234} \mathrm{Th}(C$ and $D)$, total ${ }^{234} \mathrm{Th}\left(E\right.$, squares), and total ${ }^{238} \mathrm{U}$ activities $(E$, dashed line) are shown in dpm $\mathrm{L}^{-1}$. The derived ${ }^{234}$ Th flux with depth $(F)$ is shown in $\mathrm{dpm} \mathrm{m}^{-2} \mathrm{~d}^{-}$ ${ }^{1}$. The second row of panels ( $G$ to $L$ ) shows E: ${ }^{234} \mathrm{Th}$ in the large particle fraction with the error on individual measurements indicated by the horizontal black lines. The last row of panels ( $M$ to $R$ ) shows the derived element flux with depth. The elemental fluxes are calculated at a given depth by multiplying the ${ }^{234} \mathrm{Th}$ flux $(F)$ by the element: ${ }^{234} \mathrm{Th}$ ratio $(G$ to $L)$. Extrapolated errors from the element ratios and ${ }^{234} \mathrm{Th}$ fluxes are indicated by the grey shading. 

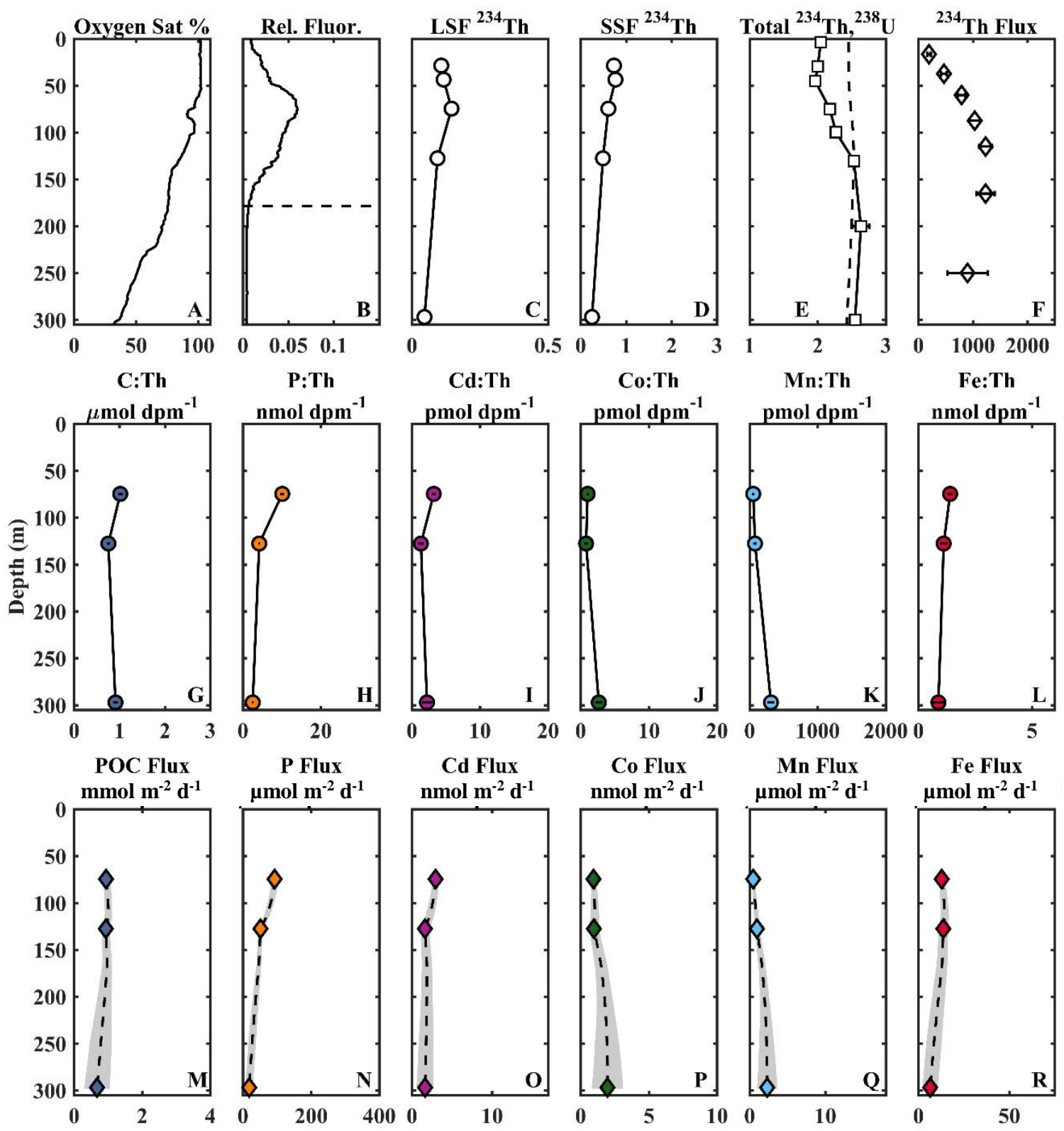

Supplemental Figure 1: Element ratios and fluxes for Gyre Station 36. The stations shown here are those not included in Fig. 3. Oxygen saturation $\%(A)$ and relative florescence $(B)$ are shown with dashed lines indicating the depth of the uODZ boundary (panel $A$ ), when present, and Ez (panel $B$ ). Particulate ${ }^{234} \mathrm{Th}(C$ and $D)$, total ${ }^{234} \mathrm{Th}\left(E\right.$, squares), and total ${ }^{238} \mathrm{U}$ activities $(E$, dashed line) are shown in dpm $\mathrm{L}^{-1}$. The derived ${ }^{234} \mathrm{Th}$ flux with depth $(F)$ is shown in dpm $\mathrm{m}^{-2} \mathrm{~d}^{-}$ ${ }^{1}$. The second row of panels ( $G$ to $L$ ) shows E: ${ }^{234} \mathrm{Th}$ in the large particle fraction with the error on individual measurements indicated by the horizontal black lines. The last row of panels $(M$ to $R$ ) shows the derived element flux with depth. The elemental fluxes are calculated at a given depth by multiplying the ${ }^{234} \mathrm{Th}$ flux $(F)$ by the element: ${ }^{234} \mathrm{Th}$ ratio $(G$ to $L)$. Extrapolated errors from the element ratios and ${ }^{234} \mathrm{Th}$ fluxes are indicated by the grey shading. 

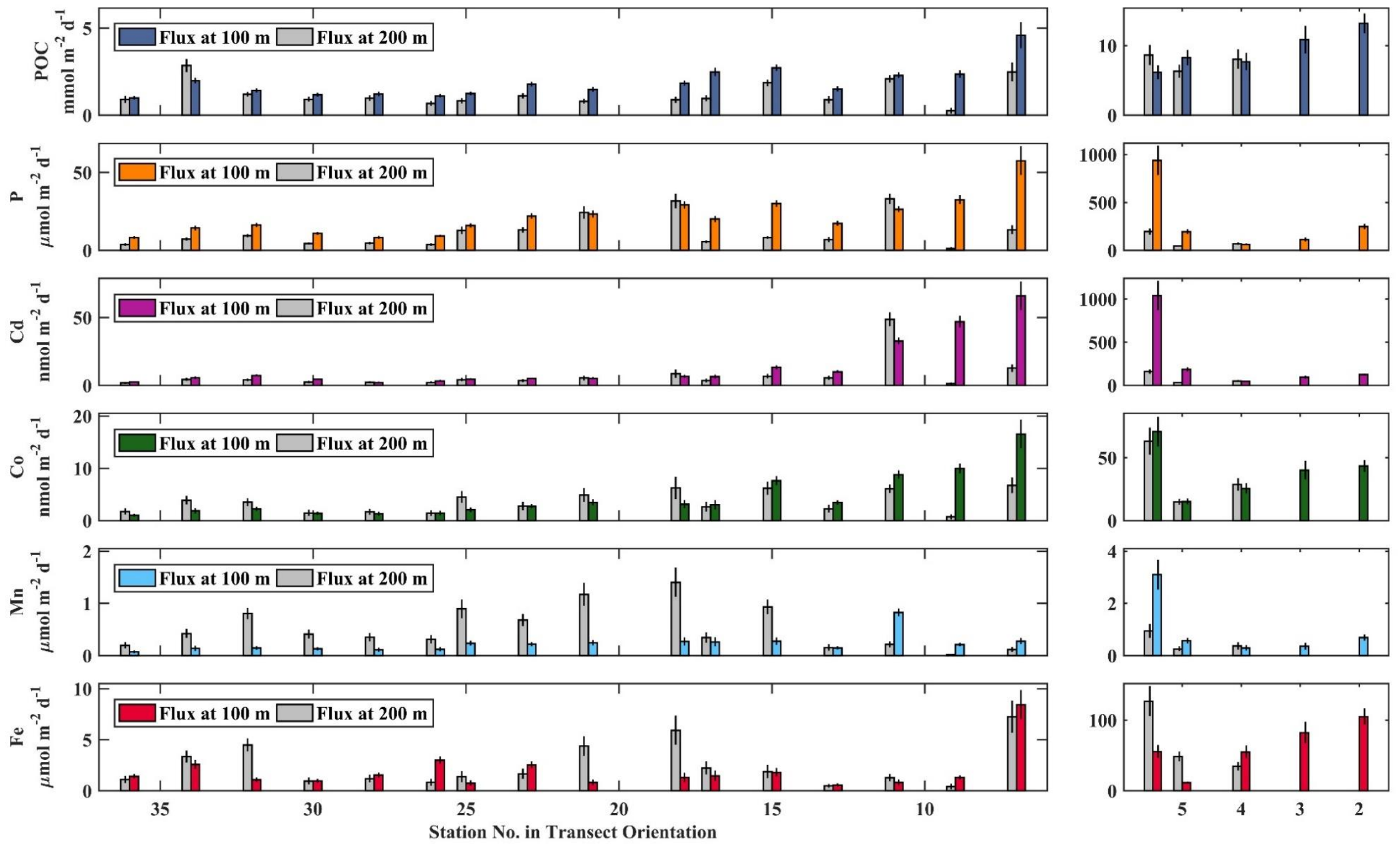

Supplemental Figure 2: Element fluxes at $100 \mathrm{~m}$ and $200 \mathrm{~m}$ by station. Fluxes are shown for all elements with station 1 as location ' 5.5 ' to be consistent with its relative location along the transect. Geographic information for each station can be found in Table 1. 

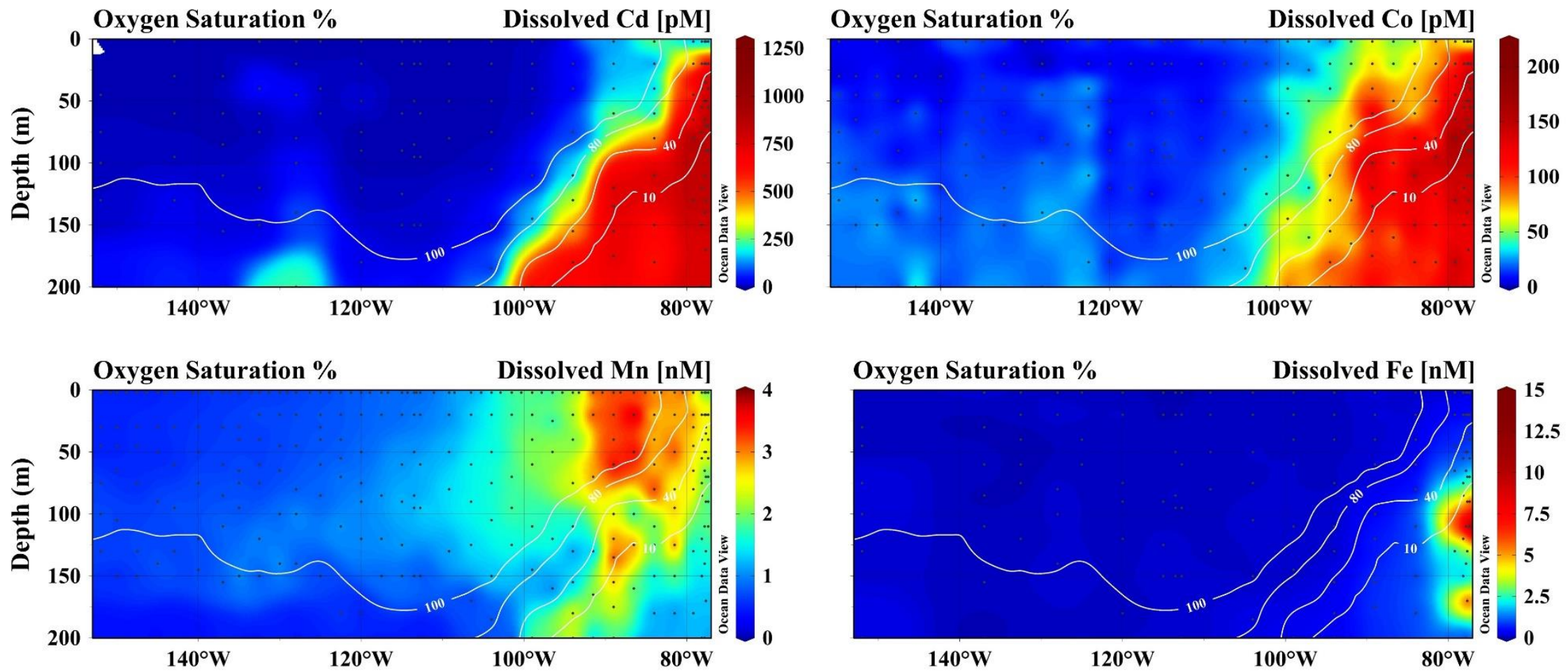

Supplemental Figure 3: Dissolved metal concentrations in the upper $200 \mathrm{~m}$. See data references for sources. 
Chapter 4:

Particulate export and remineralization in the Western Arctic Ocean: insights from the ${ }^{234} \mathbf{T h}-{ }^{238} \mathrm{U}$ method 


\begin{abstract}
The Arctic Ocean, a historically nutrient- and light-limited region of the global ocean, is experiencing unprecedented shifts in sea surface temperature, ice cover, ice thickness, and productivity as a result of climate change. Upper ocean particle export and remineralization estimates based on in-situ measurements are an important means of assessing potential changes to the efficiency and strength of the regional biological carbon pump (BCP). High-resolution sampling of ${ }^{234} \mathrm{Th}\left(\mathrm{t}_{1 / 2} \sim 24\right.$ days) and particulate organic carbon (POC) was performed across the Western Arctic Ocean in order to constrain upper ocean fluxes of POC as a part of the 2015 U.S. GEOTRACES program. POC fluxes over the shelf-slope were higher than in the Arctic basin (>1000 m water depth), but results were well within observed seasonal and annual variability. POC fluxes from the upper $150 \mathrm{~m}$ in the basin were negligible and consistent with prior study results, indicating that the export and efficiency of the BCP are low and have not changed substantially over the past three decades. With high resolution sampling of ${ }^{234} \mathrm{Th}$ and POC over the entire water column, two unique features were identified in mid- to deep waters: a subsurface maximum in particulate ${ }^{234}$ Th between $100 \mathrm{~m}$ and $200 \mathrm{~m}$ and an excess in total ${ }^{234}$ Th that persisted below 1000 $\mathrm{m}$ at three successive stations. Both features suggest a greater importance of rapid shelf-basin lateral transport than previously thought and the extensive ${ }^{234} \mathrm{Th}$ excesses indicate that greater export of POC to the deep basin via the BCP is possible.
\end{abstract}




\subsection{Introduction}

The Arctic Ocean is unlike other basins in the world's ocean. It is the smallest and shallowest, with $\sim 50 \%$ of its area underlain by continental shelves (Jakobsson, 2002). Unlike the Southern Ocean, the Arctic Ocean is almost completely surrounded by land and retains much of its permanent sea ice in the summer. Although the central Arctic has generally been characterized as a low productivity, oligotrophic basin (Arrigo and Van Dijken, 2011), marine ecosystems in the Arctic are highly episodic in nature and controlled by factors, such as ice cover, that have experienced dramatic shifts in the past few decades (Comiso et al., 2008). The biological carbon pump (BCP, see Chapter 1) is uniquely influenced in the Arctic by high latitude light level restrictions; short summers; ice advancement and retreat; seasonally-variant nutrient delivery via rivers, the Fram Strait to the east of Greenland, and the Bering Strait between Alaska and Russia; and sporadic inputs of ice-rafted sediments. During a short period in the spring and early summer, production in the coastal shelves can rival that of any other ocean basin (Arrigo and Van Dijken, 2011; Hill and Cota, 2005). The lengthening of these episodic blooms could lead to a variety of outcomes, one being a larger seasonal export of carbon from the surface ocean and a highly efficient biological pump (Wassmann et al., 2004).

Climate change is affecting the Arctic more rapidly than any other basin and recent studies indicate that ice-covered areas previously thought to contribute little to overall production can add 10-fold more than expected due to blooms beneath sea ice (Arrigo et al., 2014). Historically, the expanse of thick multiyear and seasonal ice cover has largely limited the productivity of the Arctic Ocean (Moore et al., 2004), however, in the past few decades the basin has experienced unparalleled changes in sea ice extent (Perovich and Richter-Menge, 2009) and productivity (Arrigo et al., 2014). Thinning ice and decreasing ice coverage could translate to larger blooms and higher export to the sediments (Arrigo et al., 2012; Boetius et al., 2013), but no consensus exists on how long term climate change will ultimately affect the Arctic BCP (Anderson and Macdonald, 2015; Honjo et al., 2010). Another complicating factor is the $\sim 1000 \mathrm{PgC}$ stored in permafrost soils that could be released via rivers as the Arctic warms (Zimov et al., 2006). The speed and timing of the release of this 'aged' carbon will further determine how the basin's BCP will be impacted in the coming decades. Satellite-derived estimates for the ice-free regions of the Arctic total only $1 \%$ or $0.5 \mathrm{PgC}$ of the global ocean's annual primary production (Arrigo and Van Dijken, 2011; Buitenhuis et al., 2013), however, the rapid changes occurring in the Arctic could 
mean a dramatic increase in local primary production, especially when under-ice regions are included.

The Arctic Ocean has historically been poorly represented in studies assessing the global BCP, primarily because of difficulties in performing field-based research, the lack of satellite data for ice-covered (and cloud-prone) regions, and inability of models to reproduce the unique conditions of the Arctic. Most global estimates, maps, and models stop at the Bering Strait, around $60^{\circ} \mathrm{N}$ (e.g. Henson et al., 2011; Siegel et al., 2014), although there have been recent efforts to adapt ocean color models to accurately quantify net primary production (NPP) in the Arctic Ocean (Ardyna et al., 2013; Arrigo et al., 2014). NPP is just one of the parameters used to assess changes to the BCP. Export, the magnitude of POC leaving the surface ocean, and the strength of the BCP (Export/NPP) are also important quantities to constrain because they indicate how much of the production occurring in the surface ocean is actually reaching a depth where the POC can be sequestered away from atmospheric interaction on timescales of centuries to millennia. NPP has been shown to be increasing the eastern Arctic (Arrigo and van Dijken, 2015; Arrigo and Van Dijken, 2011), but it's unclear whether increasing NPP has and will translate to an increase in export and/or a greater strength and efficiency of the BCP in the Arctic Ocean as a whole.

Field-based measurements of export are vital in the Arctic to track changes in the BCP. One of the most established means for studying carbon export and the state of the BCP is the ${ }^{234} \mathrm{Th}-$ ${ }^{238} \mathrm{U}$ disequilibrium method. Thorium is highly particle reactive, while its ${ }^{238} \mathrm{U}$ parent is conservative. As particles sink out of the surface ocean, a deficit between ${ }^{234} \mathrm{Th}$ and its parent is created if the particles carrying ${ }^{234} \mathrm{Th}$ sink faster than the production from ${ }^{238} \mathrm{U}$ decay occurs. If both ${ }^{234} \mathrm{Th}$ and carbon, for instance, are measured on the sinking particles, a flux of particulate carbon can be calculated (Buesseler et al., 1995; Benitez-Nelson et al., 2001). If high resolution profiles are used, the flux can be calculated at any depth in the water column. Only a few largescale, ${ }^{234}$ Th-based carbon export studies exist for the Western Arctic Ocean. Many studies in this region focus on the Chukchi shelf and slope areas (e.g. Ma et al., 2005; Moran et al., 2005). However, a few have traversed from the shelf to the central Arctic Ocean (Cai et al., 2010; Moran et al., 1997). Due to the uncertain and, perhaps, rapid nature of the changes occurring in this basin, there is a need for continued work that links shifts in the BCP in the Bering Strait and the coastal shelves, to those in the ice-free and ice-covered basin regions of the central Arctic (Bates et al., 2011). 


\subsection{Methods}

\subsubsection{Sample collection}

Samples were collected on the USCGC Healy during the GN01 U.S. GEOTRACES campaign from August $9^{\text {th }}$ to October $12^{\text {th }}, 2015$. The Dutch Harbor, Alaska to North Pole $\left(90^{\circ} \mathrm{N}\right)$ section will be referred to here as the 'northbound' or 'NB' transect and the return trip as the 'southbound' or 'SB' transect (Fig. 1). The North Pole station was reached on September $5^{\text {th }}$. Total ${ }^{234}$ Th samples were taken at 31 of the 66 stations occupied on the campaign using single Niskin bottles attached to a wire or in a standard rosette configuration at most stations. Size-fractionated particulate ${ }^{234} \mathrm{Th}$ samples were taken at 20 of these 31 stations (Table 1). Stations characterized as 'Shelf' had bottom depths $<600 \mathrm{~m}$, 'Slope' stations had depths $<1000 \mathrm{~m}$, and 'Basin' stations had depths $>1000 \mathrm{~m}$. Whereas the shelf, slope, and basin designations indicate the range in bottom depths, MIZ and ice designations indicate the degree of ice cover. Ice stations 31 and 33 had 100\% ice cover, while the marginal ice zone (MIZ) stations captured various states of the transition region between $1 \%$ to $100 \%$ ice cover. A submersible pump was used at MIZ stations 7 to 17 to collect total ${ }^{234} \mathrm{Th}$ samples and a standard rosette was used for the rest of the MIZ stations. A surface pump was used to obtain samples at the ice stations, where a hole was made using a gas-powered ice auger. At most, four depths were sampled at MIZ and ice stations.

\subsection{2 ${ }^{238} \mathrm{U}$ determination}

Total ${ }^{238} \mathrm{U}$ activities were calculated using bottle salinities from the depth of total ${ }^{234} \mathrm{Th}$ sampling and the relationship derived by Owens et al. (2011):

$$
\left.{ }^{238} U( \pm 0.047)=(0.0786 \pm 0.00446) * \text { Salinity }-(0.315 \pm 0.158) \quad \text { (Eq. } 1\right)
$$

where ${ }^{238} \mathrm{U}$ activity is reported in $\mathrm{dpm} \mathrm{L}^{-1}$. Although the presence of trace metals in ice-rafted sediments (Measures, 1999) and the process of ice formation, including brine rejection, might bring in to question whether a conservative ${ }^{238} \mathrm{U}$-salinity relationship would hold in the polar regions, numerous ${ }^{234} \mathrm{Th}^{-238} \mathrm{U}$ studies (e.g. Moran et al., 2005 in the Chukchi Sea) have concluded that direct ${ }^{238} \mathrm{U}$ seawater measurements from the Arctic Ocean are consistent with ${ }^{238} \mathrm{U}$ estimates calculated using standard salinity relationships (salinity from 27 to 35). In addition, Not et al. (2012) showed that the relationship held for sea ice, sea ice brine, and subsurface water samples 
from the Arctic ranging in salinity from $\sim 0$ to 135 . Salinities measured on this campaign ranged from 24.4 to 35.1 and calculated ${ }^{238} \mathrm{U}$ activities from $1.60 \mathrm{dpm} \mathrm{L}^{-1}$ to $2.44 \mathrm{dpm} \mathrm{L}{ }^{-1}$.

\subsubsection{Total ${ }^{234} \mathrm{Th}$ and particulate ${ }^{234} \mathrm{Th}$ analyses}

Four-liter samples were processed for total ${ }^{234} \mathrm{Th}$ using the method developed in Buesseler et al. (2001) and further amended by recent GEOTRACES campaigns (Black et al., 2017; Owens et al., 2015). All methods used here were the same as reported in Black et al. (2017), with the exception of the amounts and activities of thorium tracers. An exact 1-mL aliquot of a $50.39 \mathrm{dpm}$ $\mathrm{g}^{-1}{ }^{230} \mathrm{Th}$ yield monitor was added to the $4 \mathrm{~L}$ samples at sea. Two batches of ${ }^{229} \mathrm{Th}$ tracers were used in the lab, at activities of $68.87 \mathrm{dpm} \mathrm{g}^{-1}$ and $76.27 \mathrm{dpm} \mathrm{g}^{-1}$, and an $\sim 2 \mathrm{~g}$ aliquot of one of the tracers was added to each sample. The chemical recovery $\left({ }^{230} \mathrm{Th}\right)$ was determined using ICP-MS for 337 total ${ }^{234}$ Th samples. The mean chemical recovery for all reported values was $88.7 \%$ and the median recovery was $92 \%$. Four samples had recoveries below $40 \%$ and have not been reported. A few samples had identifiable auto-pipette issues and the mean recovery was applied to these data with a 1 s.d. uncertainty (10\%). Additional recovery analysis is discussed in the next section.

Particulate ${ }^{234}$ Th sampling and measurements were performed as detailed in Black et al. (2017). In short, large particle $(>51 \mu \mathrm{m})$ samples were collected using $51 \mu \mathrm{m}$ pore size pre-filter and small particle $(1$ to $51 \mu \mathrm{m})$ samples were collected using a pre-combusted and acid-leached QMA filter with a nominal pore size of $1 \mu \mathrm{m}$ (Lam et al., 2015b). The filters used for ${ }^{234} \mathrm{Th}$ analysis were located on separate heads attached to a McLane in-situ pumping system (Bishop et al., 2012). The QMA filter was punched and used directly for ${ }^{234} \mathrm{Th}$ and particulate organic carbon and nitrogen (POC and PON) analysis, while the particle material on the $51 \mu \mathrm{m}$ pre-filter was first rinsed onto a $25 \mathrm{~mm}$ silver filter using $0.1 \mu \mathrm{m}$-filtered seawater. Intercalibration efforts for these methods have shown good agreement and relatively even distributions of ${ }^{234} \mathrm{Th}$ on filters from these sampling systems (Maiti et al., 2012).

The efficiency of the beta detectors was determined by minimizing the ${ }^{234}$ Th deviation from ${ }^{238} \mathrm{U}$ for samples collected from below $1000 \mathrm{~m}$ and above $500 \mathrm{~m}$ off the seafloor that were not near a shelf or slope $(n=30)$. These regions of the water column are where ${ }^{234} \mathrm{Th}$ and ${ }^{238} \mathrm{U}$ are expected to be at equilibrium. Changes in the detector measurements were tracked using five 'low-level' uranium standards, with activities close to those measured for total and particulate samples, and 
five 'high-level' standards ranging from $230 \mathrm{dpm}$ to $370 \mathrm{dpm}$. Analysis of the lower activity uranium standard data suggested that a minimum 5\% detector uncertainty should be used. Since the counting uncertainty for total ${ }^{234} \mathrm{Th}$ samples was always below $5 \%$ (square root of the number of counts), the uncertainty on each total ${ }^{234} \mathrm{Th}$ measurement was set at $5 \%$. Some of the particulate ${ }^{234} \mathrm{Th}$ samples with relatively low activities had counting uncertainties above $5 \%$ and in these cases the counting uncertainty was used as the final measurement uncertainty.

\subsubsection{Total ${ }^{234}$ Th chemical recoveries}

Of the $\sim 340$ total ${ }^{234} \mathrm{Th}$ samples collected, $16 \%$ had chemical recoveries below $80 \%$. In comparison, the North Atlantic GA03 ( 600 samples) and Pacific GP16 ( 630 samples) GEOTRACES cruises had only $5 \%$ and $3 \%$ of the samples below the $80 \%$ threshold, respectively. Processing differences (e.g. different equipment) could not be linked to the trend in lower recoveries observed in the Arctic and lower recoveries did not appear to consistently correlate with any total or particulate ${ }^{234} \mathrm{Th}$ features. Total ${ }^{234} \mathrm{Th}$ samples with recoveries below $80 \%$ are not inherently of poor quality; they are, however, uncommon with this $4 \mathrm{~L}$ method if filters remain intact. It was determined that samples below $40 \%$ did not meet quality control standards, but those between $40 \%$ and $80 \%$ were oceanographically consistent and showed no signs that the recoveries adversely impacted the final ${ }^{234} \mathrm{Th}$ results.

If there were no methodological or processing issues (e.g. a failure of the auto pipette or a damaged filter), there should be no relationship between sample recovery and unadjusted cpm or final dpm of ${ }^{234} \mathrm{Th}$. However, after samples with known filter issues were removed ( 10), a relationship was still observed for $\sim 50$ samples on the Arctic cruise (Supp. Fig. 1). The percentage of filters damaged during the last filtration step aboard ship (noted when a crack in the filter is visible or Mn precipitate is observed on the frit below the filter) was not any higher for the Arctic than the other two cruises, but many samples from the Arctic appear to follow a pattern that mimicked a damaged filter or loss of volume in sample (Supp. Fig. 1). These samples appeared to be randomly distributed among the stations, with the except of station 19, which had most samples with recoveries below $80 \%$ (section distance $\sim 2750$ km, Supp. Fig. 2).

While the lower recoveries were spread over both transects, these values were most common in the upper $200 \mathrm{~m}$, where temperatures were the lowest. Elevating the sample temperature over the 8- to 12-hour equilibration step could be one avenue to test whether these lower recoveries 
were temperature-related in the Arctic. Prior Arctic studies have sped up their precipitation process by heating samples (Cai et al., 2010; Yu et al., 2012). That said, there was not a significant correlation between the in-situ temperature and recovery \% in the upper $200 \mathrm{~m}$, although samples were not kept at a consistent temperature from collection to processing and the time to initial processing varied. No further assessment is needed for this study, as chemical recoveries were determined for all samples, however the abundance of lower recoveries in this basin should be considered for future studies if the intention is to measure a handful of recoveries and apply the average value to all samples taken (Cai et al., 2006).

\subsubsection{POC and PON analyses}

The large particle silver filter and small particle QMA were analyzed for POC and PON following the methods in Lam et al. (2017). POC and PON analyses were completed after the final ${ }^{234}$ Th measurements using a Flash EA1112 Carbon/Nitrogen Analyzer with a Dynamic Flash Combustion technique. Measurements were blank corrected using dipped blanks housed near the filter heads on 1 pump from each cast. The s.d. of the blank filters was used to estimate the error in the POC and PON measurements.

\subsubsection{Particulate export flux of ${ }^{234} \mathrm{Th}, \mathrm{POC}$, and PON}

The balance between the production of ${ }^{234} \mathrm{Th}$ from the decay of the ${ }^{238} \mathrm{U}$ parent, the sinking flux of ${ }^{234} \mathrm{Th}$ on particles, and transport controls ${ }^{234} \mathrm{Th}$ activities in the upper ocean and can be expressed by the following relationship:

$$
\frac{\partial^{234} T h}{\partial t}={ }^{238} U \lambda_{T h}-{ }^{234} T h \lambda_{T h}-P+V \quad \text { (Eq. 2) }
$$

where ${ }^{234} \mathrm{Th}$ is the activity of total ${ }^{234} \mathrm{Th}$ in $\mathrm{dpm} \mathrm{L} \mathrm{L}^{-1},{ }^{238} U$ is the salinity-derived activity of uranium, $\lambda_{T h}$ is the decay constant for ${ }^{234} \mathrm{Th}\left(0.0288 \mathrm{day}^{-1}\right), P$ is the net removal of ${ }^{234} \mathrm{Th}$ on sinking particles $\left(\mathrm{dpm} \mathrm{L}^{-1} \mathrm{~d}^{-1}\right)$, and $V$ is the sum of the advective and vertical diffusive fluxes $\left(\mathrm{dpm} \mathrm{L}^{-1} \mathrm{~d}^{-1}\right)$. If the horizontal and vertical transport of ${ }^{234} \mathrm{Th}$ is negligible over the timescales relevant to the sinking flux of ${ }^{234} \mathrm{Th}$ and the uncertainties of the flux estimates, the system is assumed to be at steady state and Eq. 2 can be set equal to zero. The advective and diffusive fluxes are considered small if they constitute a flux <10\% of total flux contribution (Cai et al., 2010, 2008; Savoye et al., 2004) and in this case particulate ${ }^{234} \mathrm{Th}$ export can be determined using the following equation: 


$$
P_{T h @ z}=\lambda_{T h} \int_{0}^{z}\left({ }^{238} U-{ }^{234} T h\right) d z \quad \text { (Eq.3). }
$$

$P$, the particulate flux of ${ }^{234} \mathrm{Th}$ in $\mathrm{dpm} \mathrm{m}^{-2} \mathrm{~d}^{-1}$ is integrated to a depth $\mathrm{z}$.

If local lateral advection and/or upwelling is not negligible then these physical factors need to be incorporated as follows:

$$
P_{T h @ z}=\int_{0}^{z}\left(\lambda_{T h}\left({ }^{238} U-{ }^{234} T h\right) \pm w \frac{\partial^{234} T h}{\partial z} \pm u \frac{\partial^{234} T h}{\partial x} \pm v \frac{\partial^{234} T h}{\partial y}\right) d z
$$

where $w$ is the upwelling velocity, $u$ is the zonal velocity, and $v$ is the meridional velocity. $\partial^{234} \mathrm{Th}$, or the observed gradients in ${ }^{234} \mathrm{Th}$, are vertical (with depth), west to east, and south to north, respectively. Without repeated sampling campaigns to address temporal changes in ${ }^{234} \mathrm{Th}$ activities and additional East-West sampling crossing the NB and SB transects it is difficult to assess the assumptions of steady state and the 1-dimensional (1-D) flux model (Eq. 3). These assumptions are addressed in more detail in the Discussion.

Once $\mathrm{a}^{234} \mathrm{Th}$ flux has been determined, the particulate flux of other elements can also be found if the particulate concentration ratio of that element to ${ }^{234} \mathrm{Th}$ activity measured in the large particle fraction has been measured at that depth $z$. The following equation can be used:

$$
\text { Element Flux }=\frac{[\text { Element }]}{{ }^{234} \mathrm{Th}} * \text { Flux }{ }^{234} \mathrm{Th} \quad \text { (Eq. 5) }
$$

assuming that the element: ${ }^{234} \mathrm{Th}$ ratio measured at $z$ is representative of the sinking particles at this depth (Buesseler et al., 2006). This equation can be used to calculate increases or decreases in flux, depending on the relative magnitude of production or remineralization processes at a given depth. Three to seven large particle POC: ${ }^{234} \mathrm{Th}$ and PON: ${ }^{234}$ Th spanning the upper $200 \mathrm{~m}$ were used to determine POC and PON fluxes at each station at depths from $50 \mathrm{~m}$ to $150 \mathrm{~m}$. Fluxes were not reported for shelf stations where the water column was $\leq 50 \mathrm{~m}$.

\subsubsection{Removal rates and residence times of ${ }^{234} \mathrm{Th}$ in in shelf and slope waters}

For shelf and slope locations, where suspended particulate matter concentrations (SPM) are generally higher than in the basin (Moran and Smith, 2000; Trimble and Baskaran, 2005), scavenging can play an important role in decreasing total ${ }^{234} \mathrm{Th}$ activities in the upper $200 \mathrm{~m}$. Similar to the rate of removal of particulate ${ }^{234} \mathrm{Th}$ due to particle sinking ( $P_{T h}$, Eq. 3$)$, the net rate 
of removal of dissolved ${ }^{234} \mathrm{Th}$ onto particles $\left(J_{T h}\right)$ can be calculated, assuming steady state and negligible advection and diffusion, with the following equation:

$$
J_{T h @ z}=\lambda_{T h} \int_{0}^{z}\left({ }^{238} U-{ }^{234} T h_{\text {Diss }}\right) d z \text { (Eq. 6) }
$$

where the removal flux is in dpm m $\mathrm{m}^{-2} \mathrm{~d}^{-1}$ and the dissolved activity of ${ }^{234} \mathrm{Th}$ has been substituted into Eq. 3 for the total activity (Coale and Bruland, 1985). The integrated inventories of the dissolved $\left(I_{\text {Diss }}\right)$ and particulate $\left(I_{\text {Part }}\right)$ pools of ${ }^{234} \mathrm{Th}$ for a given depth range (e.g. $0 \mathrm{~m}$ to $\left.50 \mathrm{~m}\right)$ can be divided by their respective removal rates at the base of that layer, $J_{T h}$ and $P_{T h}$, to yield the residence times of dissolved $\left(\tau_{\text {Diss }}\right)$ and particulate $\left(\tau_{\text {Part }}\right){ }^{234}$ Th relative to these removal fluxes.

\subsubsection{Euphotic zone}

Data from a Sea-Bird SBE 911plus PAR sensor was used to estimate the base of the euphotic zone (Ez) or the depth of $1 \%$ light level of photosynthetically active radiation (PAR, Lee et al., 2007) along the transects. These determinations were not possible at half of the stations occupied because the bottom depths were too shallow, the CTD was not deployed to at least Ez, or the deployments were at night. In general, conditions in the Arctic make Ez estimations rather difficult in comparison to other ocean basins. Ice, snow, ice rafted sediments, and sea ice algae attenuate light transmission to surface waters and the spatial heterogeneity of each of these features (e.g. ice thickness) will alter the light field locally (Laney et al., 2017). In addition, the times and duration of daylight hours is highly variable throughout the year and the solar angle of incidence at these high latitudes means that the $1 \%$ light level used to mark Ez may be below the sensitivity limit of the PAR sensor. Lastly, any ice-covered location where the ice must be broken to attempt shipboard sampling will have an altered PAR field. When PAR-derived Ez depths were compared with those from a fluorescence-based method (Ohnemus et al., 2016; Owens et al., 2015), the average deviation in the depths was $33 \mathrm{~m} \pm 26 \mathrm{~m}$. For these reasons, we do not report station specific Ez depths here, however, PAR-based estimates for stations 7 to 10 and 46 to 57 averaged $\sim 125 \mathrm{~m}$. These results fall within the range of Ez observed in this region in prior years (Laney et al., 2017). Ez in the Central Arctic Ocean (100\% ice cover) have been found to be much shallower (0 to $50 \mathrm{~m}$, Gosselin et al., 1997; Laney et al., 2017) and thus, we report fluxes at $50 \mathrm{~m}$ and $125 \mathrm{~m}$ to approximate export from Ez at both open-water and ice-covered basin stations. 


\subsection{Results}

Here we present total and size-fractionate particulate ${ }^{234} \mathrm{Th}$ results from the GN01 Arctic Ocean campaign. The majority of this work focuses on the upper $500 \mathrm{~m}$, where the ${ }^{234} \mathrm{Th}^{238} \mathrm{U}$ disequilibrium method is most effective for determining surface export and remineralization fluxes. The ${ }^{234} \mathrm{Th}$ data from the rest of the water column have been briefly summarized here, with the exception of the mid-deep water column total ${ }^{234}$ Th activities from the NB stations that deviated from those observed in the rest of the Arctic basin.

\subsubsection{Hydrographic and biogeochemical features}

The study area has a complex, stratified water column with stark differences in temperature and salinity between the melt-influence surface waters, those originating in the Pacific and Atlantic, and those in the deep Arctic. The polar mixed layer is composed of low density, low salinity, high oxygen waters. At $76.5^{\circ} \mathrm{N}$ (station 14) this layer is slightly deeper than $50 \mathrm{~m}$ (Fig. 2). Included in the polar mixed layer is the extremely low salinity surface mixed layer, which ranges in thickness from $6 \mathrm{~m}$ to $31 \mathrm{~m}$ (Table 1). The base of this surface mixed layer will be referred to as the mixed layer depth or MLD. The polar layer is underlain by the Pacific halocline layer, waters which have lower oxygen, lower temperatures, and higher salinities. Although these waters originated in Pacific, they are significantly modified over the Arctic shelves before reaching the Arctic basin and the residence time of water in this layer is estimated to be $\sim 10$ years (Macdonald et al., 2005). Below the Pacific layer is the Atlantic halocline (210 $\mathrm{m}$ to $370 \mathrm{~m}$ at station 14, Fig. 2), which forms the boundary between the Pacific and Atlantic layers. The Atlantic layer residence time is $\sim 30$ years and these waters are higher in oxygen and warmer. The boundary between the Atlantic layer and the underlying deep Canada Basin bottom water is generally between $800 \mathrm{~m}$ and $1000 \mathrm{~m}$.

The boundary between the polar mixed layer and Pacific layer at the time of sampling marked the transition from oligotrophic surface conditions $(<1 \mu \mathrm{M}$ nitrate) to more nutrient-rich waters and a relatively large decrease in light transmission and a subsurface maximum in fluorescence was found here on the NB transect (Fig. 2, Supp. Fig. 3). The depth of the fluorescence maximum shows that the local communities must balance the need for incident radiation and nutrients and so locate themselves in this transition zone (Hill et al., 2005). This feature also appeared on the SB transect, but the peak was less pronounced relative to the fluorescence levels found in the overlying 
and underlying waters, and was much smaller in magnitude compared to that which was observed on the NB transect (Supp. Fig. 3). A smaller decrease in transmission was also observed occasionally at the base of the surface mixed layer (Fig. 2).

Satellite-derived, Chlorophyll-a (Chl-a) estimates were available for stations 1 to 6 and for stations 61 to 66 (Supp. Fig. 4), limiting this analysis to the coastal shelf regions. These estimates represent a $0.25^{\circ} \times 0.25^{\circ}$ MODIS-Aqua region $\left(24 \mathrm{~km}^{2}\right.$ to $\left.43 \mathrm{~km}^{2}\right)$ at each station. NB concentrations ([Chl-a]) suggest the largest spring bloom occurred at station 2, peaking in May. Smaller blooms occurred at stations 1, 3, and 6, peaking in June, June, and May, respectively. Between the month prior to sampling and the month of sampling, stations 1 and 2 had low and stable [Chl-a], suggesting no bloom was occurring. For stations 3 and 6, [Chl-a] increased from $\sim 1$ to $3 \mathrm{mg} \mathrm{m}^{-3}$. Data was not available for the month prior or month of sampling at stations 61 and 66, however, the July to August data was similar in [Chl-a] to stations 1 and 2. Most of the stations sampled had no Chl-a data from April to November, reflecting the limitations on the use of ocean color algorithms in the Arctic due to complications from ice and cloud cover (Supp. Fig. 5).

The low surface nutrient concentrations (Fig 2) and the depth of the fluorescence maximum once in the Arctic basin (Supp. Fig. 3) suggested that the basin locations with $<100 \%$ ice cover were sampled after the peak seasonal blooms had happened. Open water blooms of phytoplankton in the Arctic are generally thought to occur between $70^{\circ} \mathrm{N}$ to $80^{\circ} \mathrm{N}$ in the months of April through July, with the peak bloom at $80^{\circ} \mathrm{N}$ in July (tailing into August), depending on the pattern of ice retreat (Wassmann and Reigstad, 2011). Although shelf edge locations and ice cover are not consistent with latitude around the entire Arctic basin, based on this general timing, the elevated fluorescence on the NB transect could be from the tail end of a bloom and the SB open-ocean stations showed no biogeochemical remnants of a bloom, having been sampled over a month later.

\subsubsection{Total ${ }^{234} \mathrm{Th}$}

Total ${ }^{234} \mathrm{Th}$ activities ranged from $0.36 \mathrm{dpm} \mathrm{L} \mathrm{L}^{-1}$ to $2.85 \mathrm{dpm} \mathrm{L} \mathrm{L}^{-1}$. The lowest activities were found on the shelf and the highest activity was found at $\sim 500 \mathrm{~m}\left(79.98^{\circ} \mathrm{N}, 175.02^{\circ} \mathrm{W}\right)$. Generally, the higher total ${ }^{234} \mathrm{Th}$ values corresponded to depths with high salinities, which were located below $300 \mathrm{~m}$. Prior studies of the Chukchi Shelf have reported similar activities, with a maximum and minimum total ${ }^{234} \mathrm{Th}$ of $0.12 \mathrm{dpm} \mathrm{L}^{-1}$ in May and $3.95 \mathrm{dpm} \mathrm{L} \mathrm{L}^{-1}$ in August (Moran et al., 2005). 
The only station in the Bering Sea, station $1\left(60.22^{\circ} \mathrm{N}, 179.05^{\circ} \mathrm{W}\right)$, had mixed layer ${ }^{234} \mathrm{Th}$ as low as $1.35 \mathrm{dpm} \mathrm{L}^{-1}$. Surface deficits, subsurface excesses (starting at $90 \mathrm{~m}$ ), and ${ }^{234} \mathrm{Th}^{238} \mathrm{U}$ equilibrium (between $300 \mathrm{~m}$ and $400 \mathrm{~m}$ ) were all observed (Fig. 3). The ${ }^{234} \mathrm{Th}$ and ${ }^{238} \mathrm{U}$ profiles resembled those from other areas of the Pacific (Black et al., 2017; Maiti et al., 2010) and these more typical ${ }^{234} \mathrm{Th}$ profile sequences (deficit-excess-equilibrium) were not observed again along either Arctic transect. This characteristic profile supports the reliability of the method used here, despite differences between the Arctic profiles and those from other ocean basins (Black et al., 2017; Buesseler et al., 2005; Owens et al., 2015; Subha Anand et al., 2017).

The largest deficits of ${ }^{234} \mathrm{Th}$ were observed at the shelf stations (Table 1, Fig. 3), which were reached in mid-August (NB) and early October (SB). The SB shelf stations had slightly higher values of ${ }^{234} \mathrm{Th}$ with respect to ${ }^{238} \mathrm{U}$ (i.e. smaller deficits), on average, (Fig. 4). The range in ${ }^{234} \mathrm{Th}:{ }^{238} \mathrm{U}$ activities, 0.2 to 1.0 , showed no excesses of ${ }^{234} \mathrm{Th}$. Although there was variability in the profile shapes of total ${ }^{234} \mathrm{Th}$ activity at these shelf locations, a total ${ }^{234} \mathrm{Th}$ decrease with depth was generally observed and the minimum activities were found at the deepest depth sampled (Figs. 3 and 4). Station 2 was uniquely lacking a deficit in the mixed layer, but ${ }^{234} \mathrm{Th}$ decreased with depth below the MLD.

The slope stations (10 and 60) represent the boundary between the shelf and the western Arctic basin. Deficits of ${ }^{234} \mathrm{Th}$ were still present, but smaller than those on the shelf (Fig. 3). ${ }^{234} \mathrm{Th}:{ }^{238} \mathrm{U}$ ranged from 0.5 to 1.1 in the upper $450 \mathrm{~m}$. Although station 10 was much shallower at $\sim 270 \mathrm{~m}$, versus the $800 \mathrm{~m}$ depth of station 60, both slope locations reached equilibrium between $200 \mathrm{~m}$ and $300 \mathrm{~m}$. Neither station had a pronounced excess of ${ }^{234} \mathrm{Th}$ (Figs. 3 and 4). Interestingly, these stations had one larger deficit feature in or just below the MLD and another ${ }^{234} \mathrm{Th}$ deficit deeper in the water column. The deficits were separated by waters at equilibrium (>50 m layer at station 60 ).

The ice (31 and 33) and MIZ (7 to 19 and 46 to 57) stations were sampled to target features related to variations in ice cover. The maximum difference observed between ${ }^{234} \mathrm{Th}$ and ${ }^{238} \mathrm{U}$ activities at the ice stations was $0.17 \mathrm{dpm} \mathrm{L}^{-1}$ (depth of $5 \mathrm{~m}$ at station 31, Fig. 5) and the ${ }^{234} \mathrm{Th}:{ }^{238} \mathrm{U}$ remained between 0.92 and 1.08. Four out of five of the samples taken at these ice stations had ${ }^{234} \mathrm{Th}$ deficits, but the relative magnitudes of these deficits compared to those found over the shelf and slope were small (Fig. 3). Sinking particles leaving this $6 \mathrm{~m}$ layer, originating from the release of ice rafted sediment or from biological production, could have created the slight deficit in ${ }^{234} \mathrm{Th}$. 
A few of the NB MIZ stations occupied shallower, slope locations, while the majority of MIZ stations were located over deep basin waters. The compilation of all the $\mathrm{MIZ}^{234} \mathrm{Th}:{ }^{238} \mathrm{U}$ data show that NB slope MIZ (stations 7 to 10) have similar deficits of ${ }^{234} \mathrm{Th}$ in the surface. The SB MIZ data from $0 \mathrm{~m}$ to $450 \mathrm{~m}$ did show a substantial amount of variability, but fluctuated about the point of equilibrium $\left({ }^{234} \mathrm{Th}:{ }^{238} \mathrm{U}=1\right.$, Fig. 4). On the other hand, over $70 \%$ of the total ${ }^{234} \mathrm{Th}$ data was in excess of ${ }^{238} \mathrm{U}$ in the upper $450 \mathrm{~m}$ at the NB MIZ-basin stations.

The MIZ-basin and full ice cover basin stations, basin stations being those with bottom depths $>1000 \mathrm{~m}$, had a much smaller range in ${ }^{234} \mathrm{Th}$ activities in the upper $450 \mathrm{~m}$ than was seen in the shelf and slope regions (Figs. 3 and 4). In addition, while the MIZ-basin stations had slightly higher ${ }^{234} \mathrm{Th}:{ }^{238} \mathrm{U}$ than the ice-covered basin stations, the full range of ${ }^{234} \mathrm{Th}:{ }^{238} \mathrm{U}$ at all these locations, 0.88 to 1.20 , was still much smaller than the ranges elsewhere. Similar to what was observed at the MIZ-basin stations, the ice-covered basin stations on the NB transect had total ${ }^{234} \mathrm{Th}$ activities that were more often in excess of the ${ }^{238} \mathrm{U}$ activities (Figs. 3 and 4). These excesses were particularly large and extensive at stations 19 and 26, continuing below $400 \mathrm{~m}$.

The magnitude of ${ }^{234} \mathrm{Th}$ deficits and excesses at depths $>400 \mathrm{~m}$ in the Arctic basin is small $( \pm$ $0.2 \mathrm{dpm} \mathrm{L}^{-1}$ ), on average (Fig. 6), on the SB transect. It is generally expected that deeper waters reach equilibrium in the upper $500 \mathrm{~m}$ in all ocean basins, although known (e.g. at a hydrothermal vent, Owens et al., 2015) exceptions have been noted. A few single depth deviations from equilibrium activities occurred on the SB transect at depths below $200 \mathrm{~m}$, but the surrounding depths did not reflect a consistent trend and stayed within reported uncertainties of equilibrium (Fig. 6, Supp. Fig. 6D). On the other hand, larger magnitude, multi-depth ${ }^{234}$ Th excess features appeared between $75^{\circ} \mathrm{N}$ and $85^{\circ} \mathrm{N}$ (stations 14, 19, and 26) on the $\mathrm{NB}$ transect starting as shallow as $100 \mathrm{~m}$ and continuing below $1500 \mathrm{~m}$ (Fig. 6, Supp. Figs. 6A to 6C). The ${ }^{234}$ Th excess features were mostly uninterrupted with depth, although stations 14 and 26 both had ${ }^{234} \mathrm{Th}$ activities close to equilibrium breaking this pattern of elevated ${ }^{234} \mathrm{Th}$ with respective to ${ }^{238} \mathrm{U}$ below the subsurface temperature maximum ( $700 \mathrm{~m}$ to $800 \mathrm{~m}$ ). The ${ }^{234} \mathrm{Th}$ excess features at these three stations started deeper in the water column than the fluorescence maximum and transmission minimum, but near the base of the low temperature Pacific water layer. Three hypotheses for the source of this excess feature are discussed in Section 4.4.5.

\subsubsection{Particulate and dissolved ${ }^{234} \mathrm{Th}$}


Small particle ${ }^{234} \mathrm{Th}$ activities ranged from $0.05 \mathrm{dpm} \mathrm{L}^{-1}$ to $0.9 \mathrm{dpm} \mathrm{L}^{-1}(\mathrm{n}=217)$ and large particle ${ }^{234} \mathrm{Th}$ from $0.002 \mathrm{dpm} \mathrm{L}^{-1}$ to $0.3 \mathrm{dpm} \mathrm{L}^{-1}\left(\mathrm{n}=214\right.$, Fig. 8). The average ( \pm s.d.) ${ }^{234} \mathrm{Th}$ activities of small $\left(0.4 \pm 0.2 \mathrm{dpm} \mathrm{L}^{-1}\right)$ and large $\left(0.11 \pm 0.8 \mathrm{dpm} \mathrm{L}^{-1}\right)$ particles in the shelf-slope region were higher than the averages for the basin (upper $500 \mathrm{~m}$ ), which were $0.2 \pm 0.1 \mathrm{dpm} \mathrm{L}^{-1}$ and $0.02 \pm 0.03 \mathrm{dpm} \mathrm{L} \mathrm{L}^{-1}$, respectively (Fig. 7). The upper $500 \mathrm{~m}$ of the basin locations also had higher activities for both particle fractions than the waters below, which is typical for higher productivity surface waters. Aside from samples taken close to the shelves, slopes, or the sediments in the deeper Arctic Ocean, most of the water column had particulate ${ }^{234} \mathrm{Th}$ activities less than $0.025 \mathrm{dpm} \mathrm{L}^{-1}$ for large particles and $0.2 \mathrm{dpm} \mathrm{L}^{-1}$ for small particles. This included the under-ice surface waters of the North Pole (Fig. 7).

In addition to the particulate ${ }^{234} \mathrm{Th}$ activities being higher, on average, in the shelf-slope region and the upper $500 \mathrm{~m}$, since total ${ }^{234} \mathrm{Th}$ activities were lower here the fraction of particulate ${ }^{234} \mathrm{Th}$ was also higher (Fig. 7). While large particle ${ }^{234} \mathrm{Th}$ made up $\sim 11 \%$ and small particle ${ }^{234} \mathrm{Th} \sim 32 \%$ of total ${ }^{234} \mathrm{Th}$ at the shelf-slope stations, the two combined were less than $10 \%$ of the total ${ }^{234} \mathrm{Th}$ at all other stations and depths. Below $500 \mathrm{~m}$, the large particle fraction was $0.5 \%$ and the small particle fraction was $4 \%$ of the total ${ }^{234} \mathrm{Th}$, on average.

A distinct particulate peak was observed in both small and large particle ${ }^{234} \mathrm{Th}$ activities in the upper $500 \mathrm{~m}$ coinciding with the low temperature, low oxygen Pacific halocline layer (Fig. 7, Supp. Fig. 6). The maximum particulate activities on the SB transect were found within the Pacific halocline layer where temperatures less than $-1^{\circ} \mathrm{C}$ and on the NB transect the small particle activity peak was not as well constrained by this layer, although most of the high particulate activities were found at temperatures less than $-1^{\circ} \mathrm{C}$. In general, there was a gradual increase in particulate ${ }^{234} \mathrm{Th}$ activities from the surface to the depth of the particulate maximum and then a similar gradual decrease to the low activities observed for most of the basin samples $>400 \mathrm{~m}$ (Fig. 7, Supp. Fig. 6). This region where particulate activities were elevated over the rest of the water column was $>200 \mathrm{~m}$ thick near the shelf and $\sim 100 \mathrm{~m}$ near $87^{\circ} \mathrm{N}$, thinning as density contours shallowed and converged towards the pole. This peak was not discernable at the pole (station 32) in either size fraction. The depth of maximum particulate activities is not associated with the much shallower peak in fluorescence, which occurs within the water column between $30 \mathrm{~m}$ and $70 \mathrm{~m}$ (Supp. Fig. 3), although relative fluorescence and large particle ${ }^{234} \mathrm{Th}$ activities are both greater on the NB 
transect (Fig. 7, Supp. Fig. 3). The elevated particulate activities did, however, coincide with the bulk decrease in the fraction of dissolved ${ }^{234} \mathrm{Th}$ (Fig. 7).

\subsubsection{POC and PON}

Like particulate ${ }^{234}$ Th activities, POC and PON concentrations were high over the shelf-slope and lowest below $100 \mathrm{~m}$ in the Arctic basin (Fig. 8). On average, POC in the deep basin was 0.04 $\mu \mathrm{M}$ and $0.006 \mu \mathrm{M}$ for the small and large particle fractions, respectively. Deep Arctic concentrations are typically much lower than other basins, for example, compared to the southeastern Pacific where the mean POC concentration below 500 was an order of magnitude higher for both the small $(0.12 \mu \mathrm{M})$ and large $(0.03 \mu \mathrm{M})$ particle fractions. The deep water PON concentrations in the Arctic were also low at $8 \mathrm{nM}$ and $0.6 \mathrm{nM}$, respectively. The POC and PON concentrations in the upper $500 \mathrm{~m}$ of the Arctic basin were 6- to 8-fold larger than the deep water concentrations and over the shelf-slope were higher still. Small particles had average concentration of POC and PON of $2.6 \mu \mathrm{M}$ and $0.4 \mu \mathrm{M}$, while the large particle average concentrations were 1.9 $\mu \mathrm{M}$ and $0.3 \mu \mathrm{M}$, respectively. In the upper $500 \mathrm{~m}$, maxima in POC and PON coincided most often with the fluorescence feature between $30 \mathrm{~m}$ and $70 \mathrm{~m}$ (Supp. Fig. 6) and only occasionally would a secondary, smaller peak be observed that aligned with the particulate ${ }^{234} \mathrm{Th}$, low oxygen water feature (Section 4.3.3). Large particle POC and PON concentrations from the upper $500 \mathrm{~m}$ fit well within the range observed during other large volume pumping studies in and around the Chukchi Sea (e.g. Lalande et al., 2007; Lepore et al., 2007; Moran et al., 2005).

\subsubsection{POC: $:^{234} \mathrm{Th}$ and PON: ${ }^{234} \mathrm{Th}$}

The distributions of POC: ${ }^{234} \mathrm{Th}$ and PON: ${ }^{234} \mathrm{Th}$ more closely matched the profiles for POC and PON than those for particulate ${ }^{234} \mathrm{Th}$ activities, with the highest values from $0 \mathrm{~m}$ to $100 \mathrm{~m}$ (Fig. 8). Shelf-slope large particle ratios were the highest observed with average ( \pm s.d.) POC: ${ }^{.234} \mathrm{Th}$ and PON: ${ }^{234} \mathrm{Th}$ of $14 \pm 17 \mu \mathrm{mol} \mathrm{dpm}{ }^{-1}$ and $2.1 \pm 2.9 \mu \mathrm{mol} \mathrm{dpm}{ }^{-1}$, respectively. The average large particle POC: ${ }^{234} \mathrm{Th}$ and PON: ${ }^{234} \mathrm{Th}$ were double those for the small particles, but the means were within 1 s.d. In the upper $500 \mathrm{~m}$ of the Arctic basin, POC: ${ }^{.34} \mathrm{Th}$ and PON: ${ }^{234} \mathrm{Th}$ were 3- to 6-fold higher than those in deeper waters and the large and small particle ratios were also within 1 s.d. for both POC and PON. The mean large particle POC: ${ }^{234} \mathrm{Th}$ and PON: ${ }^{234} \mathrm{Th}$ in the basin's upper $500 \mathrm{~m}$ were $2.5 \pm 2.6 \mu \mathrm{mol} \mathrm{dpm}{ }^{-1}$ and $0.3 \pm 0.3 \mu \mathrm{mol} \mathrm{dpm}^{-1}$, and the larger s.d. illustrate the greater variability at these shallower depths. Lastly, below $500 \mathrm{~m}$, the ratios were relatively consistent and 
the average large particle ratios were indistinguishable (with uncertainties) from the small particle ratios. The average for large particle POC: ${ }^{234} \mathrm{Th}$ was $0.5 \pm 0.3 \mu \mathrm{mol} \mathrm{dpm}{ }^{-1}$ and for PON: ${ }^{234} \mathrm{Th}$ was $0.05 \pm 0.03 \mu \mathrm{mol} \mathrm{dpm}^{-1}$. Large particle ratios from this study matched ranges from prior studies utilizing high volume pumping methods and prior results showed that average POC: ${ }^{234} \mathrm{Th}$ and PON: ${ }^{234}$ Th were similar ( \pm 1 s.d.) between the large and small particle fractions in the upper 500 m. This similarity suggests that the ${ }^{234}$ Th-derived POC and PON fluxes here can be compared to those from studies utilizing both large particle ratios and total particulate ratios $(>1 \mu \mathrm{m}$, e.g. Chen et al., 2003), which doubles the number of historical points to 400 .

\subsection{Discussion}

\subsubsection{Assessment of advection and steady state assumptions}

In order to determine ${ }^{234} \mathrm{Th}$ export fluxes using Eq. 3, the vertical and lateral advection must be determined to be negligible relative to the magnitude of the calculated ${ }^{234} \mathrm{Th}$ export flux. The influence of upwelling or vertical advection should be negligible in most non-shelf regions of the Arctic at this time of year (Cai et al., 2010; Carmack and Chapman, 2003), especially in areas with more stratification, like the western Arctic, and areas with strong cross-shelf flows, like the Bering Strait (Woodgate et al., 2012, 2005). This is supported by the lack of a vertical gradient in total ${ }^{234} \mathrm{Th}$ with respect to the parent ${ }^{238} \mathrm{U}$ in the upper $30 \mathrm{~m}$ to $50 \mathrm{~m}$ at most basin stations (Fig 3). There were not sufficient total ${ }^{234} \mathrm{Th}$ data in the East-West $(\mathrm{E}-\mathrm{W})$ direction to evaluate whether lateral transport perpendicular to the transect direction had a significant impact on ${ }^{234} \mathrm{Th}$ fluxes. However, for the basin stations at similar latitudes the total ${ }^{234} \mathrm{Th}$ activities in the upper $30 \mathrm{~m}$ did not differ enough that average E-W current speeds over the distance between stations would produce a substantial contribution to the local ${ }^{234}$ Th flux.

Substantial current speeds in the along transect or North-South $(\mathrm{N}-\mathrm{S})$ direction were observed at basin stations using a shipboard ADCP (Supp. Fig. 8), which suggested that N-S lateral advection could impact ${ }^{234}$ Th fluxes here. Speeds for the upper $30 \mathrm{~m}$ averaged $4.1 \mathrm{~cm} \mathrm{~s}^{-1}$ at basin stations. N-S gradients from stations 12 to $32\left(7 \times 10^{-6} \mathrm{dpm} \mathrm{L}^{-1} \mathrm{~km}^{-1}\right)$ and stations 32 to $57\left(8 \times 10^{-}\right.$ ${ }^{5} \mathrm{dpm} \mathrm{L} \mathrm{Lm}^{-1} \mathrm{~km}^{-1}$ were determined using the mean total ${ }^{234} \mathrm{Th}$ in the upper $30 \mathrm{~m}$ at each station. These gradients were multiplied by the N-S current speeds (range of $0.1-8.0 \mathrm{~cm} \mathrm{~s}^{-1}$ ) to determine the potential contribution of lateral transport to the ${ }^{234} \mathrm{Th}$ flux at each basin station. Stations with poor $\mathrm{ADCP}$ data near $90^{\circ} \mathrm{N}$ (under $100 \%$ ice cover) were given a maximum estimate of $5 \mathrm{~cm} \mathrm{~s}^{-1}$. The 
resulting contributions to the ${ }^{234} \mathrm{Th}$ flux for stations 12 to 57 ranged from $0.2 \mathrm{dpm} \mathrm{m}^{-2} \mathrm{~d}^{-1}$ to 16 $\mathrm{dpm} \mathrm{m} \mathrm{m}^{-2} \mathrm{~d}^{-1}$, which was within the uncertainties for all ${ }^{234} \mathrm{Th}$ fluxes at these stations calculated at $50 \mathrm{~m}$.

Even greater current speeds were measured at the shelf and slope stations, with an average velocity of $11.6 \mathrm{~cm} \mathrm{~s}^{-1}$ and a range of $0.8 \mathrm{~cm} \mathrm{~s}^{-1}$ to $17.9 \mathrm{~cm} \mathrm{~s}^{-1}$ (Supp. Fig. 8). These shelf and shelf-break horizontal water velocities are within the typical range observed for this region (Ashjian et al., 2005). The N-S surface gradient in ${ }^{234} \mathrm{Th}$ was the same $\left(0.001 \mathrm{dpm} \mathrm{L}^{-1} \mathrm{~km}^{-1}\right)$ for both the NB and SB shelf-slope regions and when multiplied by the current speeds, resulted in an average flux component of $\sim 300 \mathrm{dpm} \mathrm{m}^{-2} \mathrm{~d}^{-1}$ for stations 1 to 10 and 60 to 66 . At station 6 , the lateral component would only total $14 \%$ of the calculated ${ }^{234} \mathrm{Th}$ flux at $50 \mathrm{~m}$, but could be as much as $87 \%$ at the same depth for station 60 . The transport of low ${ }^{234} \mathrm{Th}$ waters from the central shelf to more offshore locations would lead to an overestimate of ${ }^{234} \mathrm{Th}$ flux away from the shelf and an underestimate on shelf, although this would also depend on the activity of the incoming water from the Bering Strait.

In addition to adjustments for physical influences on ${ }^{234} \mathrm{Th}$ flux, it is also important to consider temporal or non-steady state effects. Without repeated measurements over the weeks prior to sampling, it is difficult to quantify the degree to which ${ }^{234} \mathrm{Th}$ activities might be changing in time. As blooms can create non-steady state conditions, we evaluated this possibility with the available satellite ocean color-derived [Chl-a] from the months prior to sampling. It is unlikely that a bloom within the water column occurred within the last couple weeks in the central basin because the ${ }^{234} \mathrm{Th}:{ }^{238} \mathrm{U}$ are so close to equilibrium at all basin stations in the upper $50 \mathrm{~m}$. In addition, steadystate assumptions are generally considered sufficient if ${ }^{234} \mathrm{Th}$ fluxes are below $800 \mathrm{dpm} \mathrm{m}^{-2} \mathrm{~d}^{-1}$ (Savoye et al., 2006). On the other hand, [Chl-a] data suggests that stations 3 and 6 could have been sampled during a bloom period and therefore, a steady state assumption would result in an underestimate or overestimate of the ${ }^{234} \mathrm{Th}$ flux (Supp. Fig. 4) depending on the stage of the bloom sampled. Not enough data was available for stations 60 to 66 to evaluate steady state conditions on the shelf-slope for the SB transect.

Based on these analyses, we concluded that vertical and horizontal advection had a negligible effect on ${ }^{234} \mathrm{Th}$ fluxes in the upper $150 \mathrm{~m}$ for the basin stations and steady state assumptions were reasonable in this region. Conversely, ${ }^{234} \mathrm{Th}$ fluxes determined for the shelf-slope stations could be 
significantly impacted by lateral advection and non-steady state conditions, but the absolute value of each of these impacts on the total flux of ${ }^{234} \mathrm{Th}$ is difficult to evaluate without greater spatial resolution of ${ }^{234} \mathrm{Th}$ sampling and additional temporal measurements. Therefore, we have reported ${ }^{234}$ Th fluxes for all stations assuming steady state and no lateral advection component (Table 2). We have noted those stations where lateral advection could play a significant role with an asterisk. Although most prior ${ }^{234} \mathrm{Th}$ studies from the Arctic have followed these assumptions (Cai et al., 2006; Moran et al., 2005, 1997; Moran and Smith, 2000; Roca-marti et al., 2016) or concluded that steady state or lateral inputs would not change ${ }^{234} \mathrm{Th}$ flux estimates more than a factor of two to four (Amiel and Cochran, 2008; Lepore et al., 2007), flux estimates from these shelf-slope stations should be used cautiously. No fluxes have been report for stations 2,3 , and 66, which were too shallow and potentially influenced by resuspension processes.

\subsubsection{High ${ }^{234}$ Th export and removal over the Western Arctic shelf-slope}

Unsurprisingly, the lowest ${ }^{234} \mathrm{Th}$ activities observed were over the shelf. Deficits of similar magnitudes have been found in prior studies of the Chukchi Shelf and slope region and attributed to intense particle scavenging that results from the higher productivity of the shelf-slope region (Bates et al., 2005; Hill et al., 2005) and sediment resuspension (Moran et al., 2005, 1997; Yu et al., 2012). At shelf stations $2,3,6$, and $61,{ }^{234} \mathrm{Th}$ activities decreased in the few samples taken just above the sediments, indicating the influence of resuspension here (Fig. 3). This was also supported by an increase in small particle ${ }^{234} \mathrm{Th}$.

At the shelf stations where $50 \mathrm{~m}$ flux estimates were possible, stations 6 and 61, the fluxes were $2370 \pm 40 \mathrm{dpm} \mathrm{m}^{-2} \mathrm{~d}^{-1}$ and $1180 \pm 60 \mathrm{dpm} \mathrm{m}^{-2} \mathrm{~d}^{-1}$, respectively (Table 2). These fluxes are within the range observed for the Chukchi Shelf (Fig. 9; e.g. Lepore et al., 2007; Moran et al., 2005). The ${ }^{234} \mathrm{Th}$ flux over the slope increased with depth from $50 \mathrm{~m}$ to $150 \mathrm{~m}$, with a $150 \mathrm{~m}$ flux of $2100 \pm 200 \mathrm{dpm} \mathrm{m}^{-2} \mathrm{~d}^{-1}$ at station 10 and $1400 \pm 200 \mathrm{dpm} \mathrm{m}^{-2} \mathrm{~d}^{-1}$ at station 60 . The flux increased more dramatically at station 10 , where the bottom depth was much shallower $(270 \mathrm{~m}) .{ }^{234} \mathrm{Th}$ fluxes observed at the Pacific endmember station in August (Table 2) were comparable to fluxes reported for the Bering Sea from August 1999 (Chen et al., 2003).

The net rate of removal of particulate ${ }^{234} \mathrm{Th}$ due to particle sinking $\left(P_{T h}\right)$ and the removal of dissolved ${ }^{234} \mathrm{Th}$ onto particles $\left(J_{T h}\right)$ were calculated for the shelf and slope stations at depths between $25 \mathrm{~m}$ and $200 \mathrm{~m}$ to facilitate comparison with prior studies of this region (Table 3), 
however, resuspension likely plays a role at some or all of these stations (Lin et al., 2016) and the shallow $P_{T h}$ should not be looked at as an estimate of permanent export to the sediments. Shallow $P_{T h}(25 \mathrm{~m})$ ranged from $330 \mathrm{dpm} \mathrm{m}^{-2} \mathrm{~d}^{-1}$ at station 66 to $1260 \mathrm{dpm} \mathrm{m}^{-2} \mathrm{~d}^{-1}$ at station 6 . The maximum values in both $P_{T h}$ and $J_{T h}$ were found at depths of $200 \mathrm{~m}$ at the slope stations and the range in $P_{T h}$ and $J_{T h}$ reported here were consistent with prior results (Cochran et al., 1995; Moran et al., 2005; Moran and Smith, 2000; Trimble and Baskaran, 2005; Yu et al., 2012).

Shelf-slope residence times generally fell in the range of a few days to 50 days for particulate

${ }^{234} \mathrm{Th}$ and 10 days to 200 days for the dissolved ${ }^{234} \mathrm{Th}$. These ranges were consistent with values previously reported for the Greenland Sea, Chukchi Sea, Barents Sea, and the Beaufort Sea (Cochran et al., 1995; Coppola et al., 2005; Moran and Smith, 2000; Yu et al., 2012). There was no clear increasing or decreasing trend in residence times from the shelf to the slope, suggesting that intra-shelf variability in scavenging and resuspension processes is similar to differences that could occur along the shelf-slope transition. However, the lack of upper ocean ${ }^{234} \mathrm{Th}$ deficits at the basin stations suggest that $\tau_{\text {Diss }}$ are much longer once away from the shelf-slope region and that the effects of resuspension and scavenging decline precipitously north of stations 10 and 60 .

\subsubsection{Low ${ }^{234}$ Th fluxes at open water and ice covered basin locations}

The first paired dissolved and particulate ${ }^{234} \mathrm{Th}$ measurements from under the central Arctic ice cap indicated that most depths in the upper $450 \mathrm{~m}$ had ${ }^{234} \mathrm{Th}:{ }^{238} \mathrm{U}$ ranging from 0.9 to 1.1 (Moran et al., 1997). Twenty years later, similar results were found, with limited ${ }^{234} \mathrm{Th}$ deficits in the upper $25 \mathrm{~m}$ and ${ }^{234} \mathrm{Th}:{ }^{238} \mathrm{U}$ from $50 \mathrm{~m}$ to $200 \mathrm{~m}$ that were indistinguishable from 1 (Cai et al., 2010). On this 2015 campaign, central Arctic ${ }^{234} \mathrm{Th}:{ }^{238} \mathrm{U}$ remained between 0.8 and 1.2 (Fig. 4). There was no indication of scavenging or export processes for this time of year compared to prior expeditions to the central Arctic Ocean and therefore, we have come to a similar conclusion as these prior studies, namely that there is limited export created by under-ice water column production.

Although still within the 0.8 to 1.2 range of ${ }^{234} \mathrm{Th}:{ }^{238} \mathrm{U}$ cited above, distinct excesses in ${ }^{234} \mathrm{Th}$ without overlying deficits were apparent in the upper $450 \mathrm{~m}$ of the NB transect MIZ, while the average ${ }^{234} \mathrm{Th}:{ }^{238} \mathrm{U}$ in similar regions on the SB transect remained near equilibrium. Previous ${ }^{234} \mathrm{Th}$ studies that traversed MIZs or partially ice-covered regions in the Southern Ocean (Buesseler et al., 2003; Rutgers van der Loeff et al., 2002; Savoye et al., 2004) and the Arctic (Amiel and 
Cochran, 2008; Cai et al., 2010; Ma et al., 2005) have suggested that processes at the ice-water boundary could lead to excesses and/or deficits of ${ }^{234} \mathrm{Th}$ with respect to ${ }^{238} \mathrm{U}$ that are atypical compared to those observed at low latitudes. In some cases, only excesses have been observed with no deficits (e.g. Cai et al., 2010), and in others both features were observed but the excesses far exceeded the deficits in the surface ocean (e.g. Savoye et al., 2004). Processes creating these features might include the release of ice-bound particles that could directly sink or the scavenging and release of ${ }^{234} \mathrm{Th}$ by ice-attached or sinking algae.

The shallow under-ice samples taken near the pole with close to equilibrium ${ }^{234} \mathrm{Th}:{ }^{238} \mathrm{U}$ (Fig. 5) support the assertion that the presence of the ice alone is not the driver of the observed excesses seen in other ice-covered regions. Furthermore, the inter-transect difference in the ${ }^{234} \mathrm{Th}:{ }^{238} \mathrm{U}$ observed through the MIZ region also suggests that factors besides the presence of ice play a more important role in governing ${ }^{234} \mathrm{Th}$ activities. For instance, the timing of the ice retreat relative to the date of sampling could mean that the NB transect was visited during a phase where ice retreat and prior processes had left behind excesses in ${ }^{234} \mathrm{Th}$ in the upper $500 \mathrm{~m}$, but the ice minimum had already been reached when the SB transect was begun. We did not have the means to definitively link the observed ${ }^{234} \mathrm{Th}:{ }^{238} \mathrm{U}$ surface excess features with particular processes, but potential sources of excess features are examined more in detail in Section 4.4.5.

All calculated ${ }^{234} \mathrm{Th}$ fluxes north of $76.5^{\circ} \mathrm{N}$ on way to the pole and north of $73^{\circ} \mathrm{N}$ on the return transect were either positive, but within uncertainties of zero, or negative (Table 2, Fig. 9). Of the 12 basin stations, 4 had positive fluxes at $50 \mathrm{~m}$, but these were all within uncertainties of zero flux. The average flux for all stations above $75^{\circ} \mathrm{N}$ and depths $25 \mathrm{~m}$ to $150 \mathrm{~m}$ was $-157 \pm 161 \mathrm{dpm} \mathrm{m}^{-2}$ $\mathrm{d}^{-1}$. These low fluxes are similar to those found in recent campaigns through the central Arctic (Cai et al., 2010; Roca-marti et al., 2016) and suggest that a scavenging had not occurred at these locations in the last few weeks (i.e. no deficits were present).

\subsubsection{Particulate ${ }^{234}$ Th maxima: an indicator of shelf to basin offshore transport?}

Although the subsurface peak in particulate ${ }^{234} \mathrm{Th}$ was pronounced, the mean large and small particle ${ }^{234}$ Th activities measured here fit well with the ranges and average of prior studies in all regions of the Western Arctic (Cai et al., 2010; Lalande et al., 2007; Lepore et al., 2007; Moran et al., 2005, 1997; Roca-marti et al., 2016). In addition, similar magnitudes in the partitioning of

${ }^{234} \mathrm{Th}$ were observed relative to prior studies, with the historical results showing small particle 
${ }^{234} \mathrm{Th}$ making up $\sim 22 \%$ of the total ${ }^{234} \mathrm{Th}$ at stations shallower than $1000 \mathrm{~m}$ and $\sim 15 \%$ at deeper stations, on average. Few historical particulate ${ }^{234}$ Th measurements have been made north of the shelf-slope region in the Western Arctic and thus, could have missed a subsurface particulate maximum if it was present in the basin (Ma et al., 2005; Moran et al., 1997). The feature was noted just one other time at a location near $75^{\circ} \mathrm{N}\left(145.9^{\circ} \mathrm{W}\right)$, where a peak in total particulate ${ }^{234} \mathrm{Th}$ at $200 \mathrm{~m}$ (activity $=0.53 \mathrm{dpm} \mathrm{L}^{-1}$ ) was found to be well above the activity of rest of the water column from 0 to $2860 \mathrm{~m}$ ( $0.2 \mathrm{dpm} \mathrm{L}^{-1}$; Baskaran et al., 2003). This location was also sampled during the late summer-early fall. SPM concentrations higher than overlying waters have been observed near the shelf-slope region between $100 \mathrm{~m}$ and $300 \mathrm{~m}$ and this could lead to elevated ${ }^{234} \mathrm{Th}$ activities in this depth horizon over the basin (Lepore et al., 2007; Moran et al., 2005).

Various combinations of the following hypotheses could explain the presence of the particulate ${ }^{234}$ Th maxima: an increase number of particles and/or SPM specifically, a local difference in particle composition that favored the attachment of more ${ }^{234} \mathrm{Th}$ atoms to each particle, or an allochthonous supply of ${ }^{234} \mathrm{Th}$-rich particles (i.e. higher ${ }^{234} \mathrm{Th}$ particle activities than the overlying and underlying waters). First, there was generally no appreciable decrease in transmission where ${ }^{234}$ Th activities peaked (Supp. Fig. 6). This suggested that that number of particles had not increased in this layer, however, lithogenics are not as well represented in transmissometry data as compared to organic particles (pers. comm. Phoebe Lam). Preliminary data show elevated concentrations of SPM and manganese oxides at similar depths as the ${ }^{234}$ Th maximum, although SPM was highest at the surface (pers. comm. Yang Xiang). There was not enough of a density difference between these layers to slow particles at this boundary that were already sinking and there was no evidence of surface export, so it is unlikely that the ${ }^{234} \mathrm{Th}$ in this layer originated from particles sinking directly from the overlying mixed layer. In summary, the available data indicated that Mn-oxide-rich SPM originated from shelf-slope processes could have scavenged and/or be scavenging ${ }^{234} \mathrm{Th}$ in this maxima layer.

The coincidence of the low oxygen Pacific layer with the peak in the particulate ${ }^{234} \mathrm{Th}$ activities suggests that the feature may have originated from particles entrained into Pacific water passing over the nearby East Siberian and/or Chukchi shelf-slope regions. At the slope stations (10 and 60) both large and small particle ${ }^{234} \mathrm{Th}$ activities are higher $>200 \mathrm{~m}$. Waters originating here would have particulate fractions making up $\sim 30 \%$ of the total ${ }^{234} \mathrm{Th}$. We cannot determine the exact direction and magnitude of flow along and across the transect over the weeks prior to sampling, 
but the ADCP data averaged over the upper $400 \mathrm{~m}$ indicated that velocities of $>5 \mathrm{~cm} \mathrm{~s}^{-1}$ were possible at the near-shelf stations. In some locations, current speeds reached $\pm 40 \mathrm{~cm} \mathrm{~s}^{-1}$ at $100 \mathrm{~m}$ (Supp. Fig. 8). If we assume the particles originated over the closest shelf, this would mean that at $0.05 \mathrm{~m} \mathrm{~s}^{-1}$ the particles would need $\sim 40$ days to reach station 14 ( $\sim 175 \mathrm{~km}$ to nearest shelf), 70 days to reach station $19(\sim 300 \mathrm{~km})$, and 125 days to reach station $26(\sim 550 \mathrm{~km})$. At this rate particulate ${ }^{234}$ Th would undergo substantial decay from its shelf origin to the stations on the NB transect, which is supported by the lowest particulate ${ }^{234} \mathrm{Th}$ activities being found at station 26 (Supp. Fig. 6). However, 125 days is equivalent to 5 half-lives of ${ }^{234} \mathrm{Th}$ and all of the measureable excess ${ }^{234} \mathrm{Th}$ on particles would have decayed upon reaching station 26 . Faster currents would be the simplest solution to the issue of ${ }^{234} \mathrm{Th}$ decay. The ADCP data did show regions with velocities well over $5 \mathrm{~cm} \mathrm{~s}^{-1}$ and eastward currents from the Chukchi slope at $185 \mathrm{~m}$ have been recorded at speeds $>30 \mathrm{~cm} \mathrm{~s}^{-1}$ for sustained periods (days) and with maximum speeds over $80 \mathrm{~cm} \mathrm{~s}^{-1}$ (Forest et al., 2007). Waters escaping the shelves via Barrow and/or Herald Canyons, two established flow paths to the basin, have an average velocity of $12 \mathrm{~cm} \mathrm{~s}^{-1}$ to $15 \mathrm{~cm} \mathrm{~s}^{-1}$ (Woodgate et al., 2005).

The same sequence of features was observed at NB stations 14, 19, and 26 (Supp. Figs. 6A to 6C): first, a shallow peak in small and large particle POC concentrations that coincided with a minimum in transmission and a maximum in florescence was present; second, a deeper subsurface peak in particulate ${ }^{234} \mathrm{Th}$ was observed in the low oxygen, low temperature Pacific layer; and third, $\mathrm{a}^{234} \mathrm{Th}$ excess feature starting at $\sim 150 \mathrm{~m}$ was found to extend to depths below $1600 \mathrm{~m}$. On the SB transect at stations 30, 38, 43, 46, 48, 52, and 56, only the first two features were apparent (Supp. Fig. 6D). The separation of the POC and ${ }^{234}$ Th particulate maxima suggest that these features have distinct origins. The upper POC peak would have resulted from in-situ production. The ${ }^{234} \mathrm{Th}$ maxima, if originating on-shelf, could have been composed of degraded material (low POC) or could have lost organic carbon during transit. Previous estimates of carbon concentration and carbon flux in the upper $350 \mathrm{~m}$ of the eastern Chukchi shelf showed the highest values for both parameters between 150 and $200 \mathrm{~m}$, which aligned with the shelf break (Ashjian et al., 2005).

The real importance and implication of this feature is that it suggests the possibility of extensive offshore transport of shelf materials to the central Arctic. Many shelf-slope studies focused on the Chukchi region have shown a mismatch between estimated export of POC from the water column and the remineralization, burial, and benthic respiration of this material in shelf sediments (Amiel and Cochran, 2008; Lepore et al., 2007; Moran et al., 2005). These studies do 
not prove offshore transport to the central Arctic basin and could represent a timescale offset between pulsed export events and gradual benthic respiration, but indicates that further study specifically targeting these transport processes is warranted. Sediment trap data from the Canada and Amundsen Basins suggest that lateral transport of allochthonous matter is likely the source of most trap material and not production from the overlying waters (Fahl and Nöthig, 2007; Hwang et al., 2008). In addition, fluxes at $3000 \mathrm{~m}$ in the Canada Basin increased in months when the region was completely ice covered and productivity was limited, further supporting the dominance of lateral over vertical transport of organic matter. While POC and PON were only occasionally elevated within this feature here, additional data from trace metal and isotope studies on this campaign may shed light on whether this ${ }^{234} \mathrm{Th}$ feature coincides with the transport of any other elements to the central basin.

\subsubsection{Potential sources for ${ }^{234} \mathrm{Th}$ excesses in the deep basin}

Shallow excess of ${ }^{234}$ Th are not uncommon in the Southern Ocean and the Arctic (Cai et al., 2010; Roca-marti et al., 2016; Rutgers van der Loeff et al., 2011; Savoye et al., 2004; Yu et al., 2012). A slope edge, MIZ station at $75^{\circ} \mathrm{N}$ that was sampled in August and September of 2008 changed from equilibrium activities of ${ }^{234} \mathrm{Th}$ in the upper to an excess equivalent to a ${ }^{234} \mathrm{Th}$ flux of $-1166 \mathrm{dpm} \mathrm{m}^{-2} \mathrm{~d}^{-1}$ or an inventory excess of $4.1 \times 10^{4} \mathrm{dpm} \mathrm{m}^{-2}$ over the upper $80 \mathrm{~m}$ of the water column (Yu et al., 2012). This suggests that excesses without export signals are possible, but we do not know if the feature continued deeper than $100 \mathrm{~m}$. Equivalent excess features starting at 75 $\mathrm{m}$ and continuing at least to $400 \mathrm{~m}$ (where sampling stopped) were seen in ${ }^{210} \mathrm{Po}-{ }^{210} \mathrm{~Pb}$ data from the central Arctic (Roca-marti et al., 2016). These stations with moderate ice cover (50\% to 80\%) had excesses much larger than the surface deficits that were present, if at all. These prior results suggest that surface features in the Arctic may not always follow the same 1-D patterns (deficits then excesses) that are observed elsewhere and that a comprehensive study of the relationship between ice, ice-associated food webs, ${ }^{234} \mathrm{Th}$ in the water column, and horizontal advection are needed.

The vertical $(>1000 \mathrm{~m})$ and lateral $(\sim 820 \mathrm{~km})$ extent of the deep ${ }^{234}$ Th excesses observed on the NB transect from $76.5^{\circ} \mathrm{N}$ to $84^{\circ} \mathrm{N}$ (stations 14 to 26 , Fig. 6), as well as the magnitude of the negative flux estimates ranging from $-8,000 \mathrm{dpm} \mathrm{m}^{-2} \mathrm{~d}^{-1}$ to $-11,500 \mathrm{dpm} \mathrm{m}^{-2} \mathrm{~d}^{-1}$, suggest that this feature is reflective of widespread processes. However, the depth and extent is atypical when 
compared to results from open ocean locations in ice-free regions of the world's ocean (e.g. Black et al., 2017; Owens et al., 2015). Excesses of ${ }^{234} \mathrm{Th}$ spanning 100s of meters have previously been found in and just north of the seasonal ice zone in the Southern Ocean from $\sim 125 \mathrm{~m}$ to $500 \mathrm{~m}$ at the deepest point sampled (Savoye et al., 2004). The excess features here were overlain by deficits, although the magnitude of the deficits was often much smaller than the excesses below. The maximum remineralization flux measured was $3800 \mathrm{dpm} \mathrm{m}^{-2} \mathrm{~d}^{-1}$, but the excess features could have extended deeper than the last sampling depth. Most Arctic ${ }^{234} \mathrm{Th}$-based studies have sampled the deeper basin at depths no greater than $400 \mathrm{~m}$, potentially missing features similar to those seen on this campaign. A deep excess $>1000 \mathrm{~m}$ in width was observed in the Canada Basin $\left(75^{\circ} \mathrm{N}\right.$, $146^{\circ} \mathrm{W}$ ), that would represent a flux of $-11,250 \mathrm{dpm} \mathrm{m}^{-2} \mathrm{~d}^{-1}$ (Baskaran et al., 2003). However, this feature was accompanied by a $1000 \mathrm{~m}$ of deficit above it, an increase in SPM to over $80 \mathrm{\mu g} \mathrm{L}^{-1}$ at $1500 \mathrm{~m}$, an increase in POC from $0.4 \mu \mathrm{M}$ to $\sim 3 \mu \mathrm{M}$ in the region of excess, and a POC: $:^{234} \mathrm{Th}$ that went above $15 \mu \mathrm{mol} \mathrm{dpm}^{-1}$ in this zone.

To explain the deep excesses observed on the NB transect, we present three potential hypotheses (Fig. 10) and address their likelihood based on prior research and related ancillary data. The hypotheses are as follows:

(H1) The canonical salinity- ${ }^{238} \mathrm{U}$ relationship is not valid due to an input of dissolved or particulate ${ }^{238} \mathrm{U}$.

(H2) Under-ice algae scavenging ${ }^{234} \mathrm{Th}$ from the upper water column were transported while ice-attached to these locations and upon ice melt the algae sank, releasing ${ }^{234} \mathrm{Th}$ to the water column.

(H3) Particulate matter with high ${ }^{234} \mathrm{Th}$ activities was rapidly transported from a nearby shelf or adjacent waters and ${ }^{234} \mathrm{Th}$ was released to the water column when this material sank.

Deep water deviations from the canonical salinity- ${ }^{238} \mathrm{U}$ relationship that are this pervasive have not been observed in the Arctic or elsewhere. High ${ }^{234} \mathrm{Th}$ relative to the salinity-derived ${ }^{238} \mathrm{U}$ could result from persistent regional differences in the salinity- ${ }^{238} \mathrm{U}$ relationship or the local supply of either dissolved or particulate ${ }^{238} \mathrm{U}$ from a nearby shelf. One of the primary reasons that (H1) is unlikely is that the salinity- ${ }^{238} \mathrm{U}$ relationship has been verified in the Arctic at a wide range of salinities (Not et al., 2012) and in general, studies from non-estuary, open ocean regions rarely 
show deviations from this relationship (Owens et al., 2011; Pates and Muir, 2007; Rengarajan et al., 2003; Toole et al., 1987). An Arctic-specific difference in the salinity relationship is also unlikely because we do not see the same magnitude of ${ }^{234}$ Th excess on the SB transect and the difference would have to exist only in the waters on the western side of the Canada Basin.

A transitory source of particulate or dissolved uranium to the water column on the NB transect would elevate ${ }^{234} \mathrm{Th}$ and ${ }^{238} \mathrm{U}$ activities, while the salnity- ${ }^{238} \mathrm{U}$ relationship would predict a lower ${ }^{238} \mathrm{U}$ activity. Dissolved ${ }^{238} \mathrm{U}$ is lost to sediments under low oxygen conditions (Klinkhammer and Palmer, 1991) and thus, an excess of dissolved ${ }^{238} \mathrm{U}$ unlikely. Resuspension and transport of particulate ${ }^{238} \mathrm{U}$ from these sediments could, in theory, provide an excess of ${ }^{238} \mathrm{U}$ if resuspension into oxic waters did not immediately cause particulate ${ }^{238} \mathrm{U}$ to transfer to the dissolved phase. This is the only scenario included for (H1) in Fig. 10, however, we were able to rule out this option using the background (>6 month) beta counts from the ${ }^{234} \mathrm{Th}$ analyses. Lin et al. (2016) have suggested that the residual beta activity of the ${ }^{234} \mathrm{Th}$ particulate counts at shelf stations in the Arctic are due to ${ }^{212} \mathrm{Bi}$ supported by ${ }^{228} \mathrm{Th}$ and ${ }^{234} \mathrm{Th}$ supported by ${ }^{238} \mathrm{U}$. We measured ${ }^{228} \mathrm{Th}$ on the large and small particle filters and therefore, were able to determine a rough estimate of the potential ${ }^{238} \mathrm{U}$ present on these filters. (H1) was ruled out because the ${ }^{228} \mathrm{Th}$ results suggest that the particulate ${ }^{238} \mathrm{U}$ activities are not disproportionately higher on the NB transect and the average estimated particulate ${ }^{238} \mathrm{U}$ contribution at stations 14,19 , and 26 was below $10 \%$.

The mechanisms required for $(\mathrm{H} 2)$, under-ice algae scavenging ${ }^{234} \mathrm{Th}$ from the upper water column (Amiel and Cochran, 2008) and the en masse sinking of ice-associated organic matter (Boetius et al., 2013), have been observed in the Arctic Ocean. However, the single published measurement for ${ }^{234} \mathrm{Th}$ in ice-associated algae showed an excess of $\sim 2.25 \mathrm{dpm} \mathrm{L}^{-1}$ of ${ }^{234}$ Th over ${ }^{238} \mathrm{U}$, which would equate to $<500 \mathrm{dpm} \mathrm{m}^{-2}$ added to the water column (30 $\mathrm{cm}$ core section). This amount is negligible next to the $1000 \mathrm{~s}$ of $\mathrm{dpm} \mathrm{m} \mathrm{m}^{-2} \mathrm{~d}^{-1}$ required to create the observed excess. Unpublished ${ }^{234} \mathrm{Th}$ activities from the $>1.2 \mu \mathrm{m}$ fraction of an ice core reached up to $240 \mathrm{dpm} \mathrm{L}{ }^{-1}$, but this elevated activity was only observed in a $0.5 \mathrm{~cm}$ layer at the base of the core (pers. comm. K.O. Buesseler and T. Trull). When this activity is converted to an inventory of ${ }^{234} \mathrm{Th}$, it represents less than $200 \mathrm{dpm} \mathrm{m}^{-2}$ that could be added to the water column below upon ice melt. Additional study is needed to gauge how representative these two cores are of the magnitude and extent of elevated ${ }^{234} \mathrm{Th}$ activities in average bottom ice conditions, but the results suggest that the sinking of ice-attached algae alone cannot cause the excesses in ${ }^{234} \mathrm{Th}$ that were found on the NB transect. 
Furthermore, the effects of ${ }^{234} \mathrm{Th}$ scavenging by ice-associated communities on water column distributions of ${ }^{234} \mathrm{Th}$ are not well understood and the ${ }^{234} \mathrm{Th}$ needs to be removed at some point, creating a deficit of equal proportion to the excess created by the release of ${ }^{234} \mathrm{Th}$ by algae. Rodriguez y Baena et al. (2008) found a ${ }^{234} \mathrm{Th}$ excess near one sea-ice edge station in the Southern Ocean that persisted 10s of $\mathrm{m}$ through the surface mixed layer and hypothesized that this feature was created by the release of ${ }^{234} \mathrm{Th}$ from ice-algae that had accumulated the ${ }^{234} \mathrm{Th}$ earlier in the season. The $\sim 40 \mathrm{~m}$ excess they observed could lead to $\sim 17,000 \mathrm{dpm} \mathrm{m}^{-2}$ added to the underlying water column if the ${ }^{234} \mathrm{Th}$ could be transported vertically by sinking matter. The prevalence of these features is unclear, however, and a process study would be needed to track water column and algae activities from initial uptake to release in the deep water column in order to confirm the validity of (H2). We did not observe any significant deficits at any of the basin stations, while Rodriguez y Baena et al. (2008) found deficits at adjacent stations and at the excess location when sampled 2 weeks later.

Based on the data available and evidence from the literature, we think (H3) is the most likely cause of the deep ${ }^{234} \mathrm{Th}$ excess (Fig. 10). The timescales of the physical processes responsible must be examined a bit more in detail in conjunction with other tracer and radionuclide data, however, (H3) is consistent with both the subsurface particulate ${ }^{234} \mathrm{Th}$ feature and the deep excesses in total ${ }^{234} \mathrm{Th}$. As detailed in Section 4.4.4, lateral transport of substantial particulate material originating near the shelf-slope break has been supported by shelf-slope studies, as well as trap studies in the deep basin. The fraction of particulate ${ }^{234} \mathrm{Th}$ is elevated above the surrounding water column at our slope stations and through the particulate ${ }^{234} \mathrm{Th}$ feature persisting between 100 to $250 \mathrm{~m}$ throughout both transects. High particulate ${ }^{234} \mathrm{Th}$ waters originating from the Chukchi or the East Siberian Shelves would need to move rather rapidly out into the central basin and then some of this material would need to (aggregate and) sink along the way. Lateral transport specific to this depth horizon could explain why surface activities at stations 14, 19, and 26 were not much above equilibrium, but the activities in the deeper Pacific and Atlantic layers were in excess. It would seem, based on the lower fraction of dissolved ${ }^{234} \mathrm{Th}$, the higher activities of the large and small particles, and the greater magnitude of the excesses at depth on the NB transect (Fig. 7), that the lateral export event happened more recently at this location with respect to sampling. With time, the dissolved fraction would increase (dissolved ${ }^{238} \mathrm{U}$ decay to ${ }^{234} \mathrm{Th}$ ), the 'extra' particulate ${ }^{234} \mathrm{Th}$ from the shelf-slope would decay, and excesses present in the deep water column would also decay, which would be 
consistent with what was observed on the SB transect. However, if the origin of these particulates was mostly from the west, where the shelf waters are closer to the NB locations, then transit time to the SB locations could also explain the lack of excess observed here.

\subsection{6 ${ }^{234}$ Th-derived POC and PON flux estimates}

From the ${ }^{234} \mathrm{Th}$ fluxes, POC and PON fluxes were determined using the ratio of these components to ${ }^{234} \mathrm{Th}$ on sinking particles. POC fluxes ranged from $-1.4 \mathrm{mmolC} \mathrm{m} \mathrm{m}^{-2}$ at the north pole $(125 \mathrm{~m})$ to a maximum of $83 \mathrm{mmolC} \mathrm{m} \mathrm{m}^{-2} \mathrm{~d}^{-1}$ over the shelf $\left(68^{\circ} \mathrm{N}, 50 \mathrm{~m}\right.$, Fig. 11, Table 2$)$. PON fluxes ranged from -0.2 mmolN m $\mathrm{m}^{-1}$ to $13 \mathrm{mmolN} \mathrm{m}^{-2} \mathrm{~d}^{-1}$. Over the shelf and slope, the largest fluxes were found in the upper $75 \mathrm{~m}$ and in the basin fluxes were mostly negative. These negative values reflected the negative ${ }^{234} \mathrm{Th}$ fluxes from which they were derived and represent the lack of net export in the basin. Prior ${ }^{234}$ Th-based flux studies have found similar magnitudes and distributions of POC fluxes (Table 4, Fig. 11). A large range in shelf-slope POC fluxes have been observed throughout the Arctic, which can be attributed, in part, to seasonal and annual variability. As was observed on this campaign, differences in local shelf dynamics and productivity during a single season can create additional variability. The results reported here fell within the ranges of prior sampling campaigns, although POC fluxes from the central basin during this campaign were on the lower end of the historical range. Nevertheless, numerous instances of negative fluxes, some as low as -6 mmolC $\mathrm{m}^{-2} \mathrm{~d}^{-1}$, have been reported in the most recent central Arctic studies (Cai et al., 2010; Roca-marti et al., 2016).

Although no in-situ NPP measurements were made on this campaign and satellite estimates were lacking (Supp. Fig. 5), prior NPP results can be used to make a general assessment of the efficiency of the BCP in the Arctic basin. Studies from mid-summer 1994 found NPP ranging from $1 \mathrm{mmolC} \mathrm{m}^{-2} \mathrm{~d}^{-1}$ to $214 \mathrm{mmolC} \mathrm{m}^{-2} \mathrm{~d}^{-1}$ in the Western and Central Arctic (Gosselin et al., 1997; Wheeler et al., 1996). Prior August and September NPP estimates were much lower, ranging from $<2 \mathrm{mmolC} \mathrm{m} \mathrm{d}^{-1}$ to $8 \mathrm{mmolC} \mathrm{m} \mathrm{d}^{-2}$ (Coupel et al., 2015; Fernández-Méndez et al., 2014). Since this campaign started in mid-August and nutrient, fluorescence, and ${ }^{234} \mathrm{Th}$ data all suggested that there was not a bloom in the few weeks prior to sampling the basin stations, the lower range was the most appropriate for comparison to export. Using POC fluxes from basin stations only, the highest POC flux in the upper $150 \mathrm{~m}\left(0.3 \mathrm{mmolC} \mathrm{m}^{-2} \mathrm{~d}^{-1}\right)$ divided by the lower NPP estimate (2 mmolC m $\left.\mathrm{m}^{-2} \mathrm{~d}^{-1}\right)$ produced a surface export efficiency of 0.15 . The lowest non-zero POC flux $(0.1$ 
mmolC $\left.\mathrm{m}^{-2} \mathrm{~d}^{-1}\right)$ divided by the higher NPP estimate $\left(8 \mathrm{mmolC} \mathrm{m}^{-2} \mathrm{~d}^{-1}\right)$ equated to an efficiency of 0.01. These efficiencies are comparable to those found in the oligotrophic regions of the Pacific Ocean (Black et al., 2017; Buesseler and Boyd, 2009) and show that in non-bloom periods, the Arctic has an extremely low efficiency BCP.

Despite the low basin efficiencies predicted above, the ${ }^{234} \mathrm{Th}$ excesses at depth suggested that recent remineralization of sinking organic material had occurred. We can estimate the prior flux of carbon and nitrogen from the sinking pool to the surrounding water column at stations 14,19 , and $26\left(76.5^{\circ} \mathrm{N}\right.$ to $\left.84^{\circ} \mathrm{N}\right)$ by integrating the excess ${ }^{234} \mathrm{Th}$ activities (negative ${ }^{234} \mathrm{Th}$ flux) and multiplying by the average POC: ${ }^{234} \mathrm{Th}$ and PON: ${ }^{234} \mathrm{Th}$ within the excess features at each station. The carbon flux estimates totaled $6 \mathrm{mmol} \mathrm{m}^{-2} \mathrm{~d}^{-1}, 5 \mathrm{mmol} \mathrm{m} \mathrm{d}^{-1}$, and $3 \mathrm{mmol} \mathrm{m} \mathrm{d}^{-1}$ when integrated to the depths indicated for stations $14(2194 \mathrm{~m}), 19(1395 \mathrm{~m})$, and $26(1523 \mathrm{~m})$, respectively. The corresponding nitrogen fluxes were 0.4 mmolN m $\mathrm{d}^{-1}, 0.5$ mmolN m $\mathrm{d}^{-1}$, and $0.2 \mathrm{mmolN} \mathrm{m} \mathrm{m}^{-2} \mathrm{~d}^{-1}$. These estimates require that all sinking material was converted completely to non-sinking material or that any partial remineralization processes affecting particulate POC, PON, and ${ }^{234} \mathrm{Th}$ occurred at the same ratio as the large particle fraction. It is likely that material originating over the shelf and in surface water had higher initial POC: ${ }^{234}$ Th ratios than those that were observed at depth at the time of sampling and thus, the POC remineralization fluxes predicted represent a low estimate for possible deep remineralization fluxes. Over the region of the water column where excesses were observed, the input of carbon from this remineralization would average $2 \mathrm{nM}$ to $4 \mathrm{nM}$ per day, a change which would not be noticeable in the short term when compared to the $>40 \mu \mathrm{M}$ in-situ dissolved concentrations.

Time-series sediment trap data from multiple locations in the Canada Basin (2000 m to 3750 $\mathrm{m}$ sampling depths) have recorded POC fluxes to the deep ocean ranging from $0.002 \mathrm{mmolC} \mathrm{m}^{-2}$ $\mathrm{d}^{-1}$ to $0.3 \mathrm{mmolC} \mathrm{m} \mathrm{m}^{-2} \mathrm{~d}^{-1}$ over a period of 7 years (Hwang et al., 2015). These POC fluxes and PON fluxes from the Canada Abyssal Plain (trap depth >3000 m; Honjo et al., 2010) were on par with the corresponding basin ${ }^{234}$ Th-derived fluxes at $150 \mathrm{~m}$ from this campaign. If most of the sinking material that created the ${ }^{234}$ Th excesses was broken down by $2000 \mathrm{~m}$, then these deep traps would not record this event or similar ones. This would seem to support (H3) over $(\mathrm{H} 2)$, although the mechanism for ${ }^{234}$ Th-delivery is still uncertain (Fig. 10). A massive deposition of algae was observed at depths $>3500 \mathrm{~m}$ in the eastern and central Arctic in 2012 that brought $9 \mathrm{gC} \mathrm{m}^{-2}$ to the seafloor. This deposition would average $25 \mathrm{mmol} \mathrm{m}^{-2} \mathrm{~d}^{-1}$ if it occurred over a period of a month 
and would provide much more carbon than we would need to match our estimates (Boetius et al., 2013). Nevertheless, it is unclear what effect this type of massive algae fall would have on the overlying water column, what fraction of POC and PON might be degraded along its transit to the seafloor, and if the feature we observed could be produced by this type of event. Here we have proposed that (H2), a massive algae fall, was less likely to create the ${ }^{234}$ Th excess than (H3). Deep sediment traps have yet to capture algae deposition of this magnitude, however, we cannot conclude that these massive algae falls are a new mechanism in the Arctic or make conclusions about their frequency and spatial distribution. If these events are more common or widespread than previous thought, it could have important implications for the efficiency of the biological pump and our understanding of carbon sequestration in the Arctic Ocean, but corroborating evidence from additional tracers sampled on this campaign and/or future work in the Arctic is needed to precede with the investigation of these excess features.

\subsection{Conclusions}

As multi-year sea ice has thinned and the annual extent of ice cover has declined, NPP has increased since the 1990s in many regions of the Arctic Ocean that are seasonally ice-covered (Arrigo and van Dijken, 2015). Our understanding of the potential importance of large under-ice blooms to annual carbon export is improving with more in-situ measurements in the central Arctic, however, more work is still needed to accurately represent the magnitude, extent, frequency, and the controls of these under-ice export events in biogeochemical models (Arrigo et al., 2014, 2012; Boetius et al., 2013). The results from the upper $150 \mathrm{~m}$ of this study suggest that the magnitude of export and low efficiency of the BCP has not changed substantially from historical estimates in the basins (>1000 m water depth) of the Western and Central Arctic Ocean and that this region of the Arctic still has one of the least efficient BCP in the world's ocean (Buesseler and Boyd, 2009; Honjo et al., 2010). A lack of sufficient satellite-derived NPP data and the inter-annual and spatial variability in export over the shelf and slope region make it difficult to put bounds on any changes in the BCP here, but POC and PON fluxes were consistent with the seasonal and annual variability observed in historical measurements.

Due to the exceptional high resolution sampling performed under the international GEOTRACES program, two unique features were able to be characterized in the Western Arctic: a shallow, subsurface maxima in particulate ${ }^{234} \mathrm{Th}$ and deep excesses in total ${ }^{234} \mathrm{Th}$. These features 
have important implications for rapid shelf-basin lateral transport and deep export in the basin via the BCP, but additional study is warranted to confirm whether these features collectively signify an increased efficiency of the Arctic BCP. In particular, process studies are needed to improve our understanding of how under ice communities and/or the release of ice rafted sediments impact the

${ }^{234} \mathrm{Th}$ activities in bottom ice, ice-attached algae, and the underlying water column so that the ${ }^{234} \mathrm{Th}$ method can be fully utilized in the basin. Other tracers from this GETORACES campaign and the German expedition, which was also performed in 2015, will be vital to the further interpretation of this dataset and determining whether similar features are common in the Eastern Arctic.

\section{Acknowledgements}

This work was supported by the National Science Foundation (OCE- 1458305). E. Black was also funded by a NASA Earth and Space Science Graduate Fellowship (NNX13AP31H). The authors would like to thank the captain, service members, crew, and scientists aboard the Coast Guard Cutter HEALY (WAGB-20). This work would not have been possible without help from Matt Charette, Steven Pike, Paul Henderson, Lauren, Kipp, and Maija Heller. Particular thanks goes to Yang Xiang and Phoebe Lam for performing the large particle particulate organic carbon analyses. Mixed layer depths were provided by Mariko Hatta. 


\section{References}

Amiel, D., Cochran, J.K., 2008. Terrestrial and marine POC fluxes derived from and $\delta^{13} \mathrm{C}$ measurements on the Mackenzie Shelf 113, 1-19. doi:10.1029/2007JC004260

Anderson, L.G., Macdonald, R.W., 2015. Observing the Arctic Ocean carbon cycle in a changing environment. Polar Res. 34, 26891. doi:10.3402/polar.v34.26891

Arrigo, K.R., Perovich, D.K., Pickart, R.S., Brown, Z.W., van Dijken, G.L., Lowry, K.E., Mills, M.M., Palmer, M.A., Balch, W.M., Bahr, F., Bates, N.R., Benitez-Nelson, C., Bowler, B., Brownlee, E., Ehn, J.K., Frey, K.E., Garley, R., Laney, S.R., Lubelczyk, L., Mathis, J., Matsuoka, A., Mitchell, B.G., Moore, G.W.K., Ortega-Retuerta, E., Pal, S., Polashenski, C.M., Reynolds, R.A., Schieber, B., Sosik, H.M., Stephens, M., Swift, J.H., 2012. Massive Phytoplankton Blooms Under Arctic Sea Ice. Science 336, 1408-1408. doi:10.1126/science.1215065

Arrigo, K.R., Perovich, D.K., Pickart, R.S., Brown, Z.W., van Dijken, G.L., Lowry, K.E., Mills, M.M., Palmer, M.A., Balch, W.M., Bates, N.R., Benitez-Nelson, C.R., Brownlee, E., Frey, K.E., Laney, S.R., Mathis, J., Matsuoka, A., Greg Mitchell, B., Moore, G.W.K., Reynolds, R.A., Sosik, H.M., Swift, J.H., 2014. Phytoplankton blooms beneath the sea ice in the Chukchi sea. Deep. Res. Part II Top. Stud. Oceanogr. 105, 1-16. doi:10.1016/j.dsr2.2014.03.018

Arrigo, K.R., van Dijken, G.L., 2015. Continued increases in Arctic Ocean primary production. Prog. Oceanogr. 136, 60-70. doi:10.1016/j.pocean.2015.05.002

Arrigo, K.R., Van Dijken, G.L., 2011. Secular trends in Arctic Ocean net primary production. J. Geophys. Res. Ocean. 116, 1-15. doi:10.1029/2011JC007151

Ashjian, C.J., Gallager, S.M., Plourde, S., 2005. Transport of plankton and particles between the Chukchi and Beaufort Seas during summer 2002, described using a Video Plankton Recorder. Deep. Res. Part II Top. Stud. Oceanogr. 52, 3259-3280. doi:10.1016/j.dsr2.2005.10.012

Baskaran, M., Swarzenski, P.W., Porcelli, D., 2003. Role of colloidal material in the removal of 234Th in the Canada basin of the Arctic Ocean. Deep. Res. Part I Oceanogr. Res. Pap. 50, 1353-1373. doi:10.1016/S0967-0637(03)00140-7

Bates, N.R., Cai, W.-J., Mathis, J., 2011. The ocean carbon cycle in the Western Arctic Ocean. Oceanography 24, 186-201. doi:10.5670/oceanog.2011.65

Bates, N.R., Hansell, D.A., Bradley Moran, S., Codispoti, L.A., 2005. Seasonal and spatial distribution of particulate organic matter (POM) in the Chukchi and Beaufort Seas. Deep. Res. Part II Top. Stud. Oceanogr. 52, 3324-3343. doi:10.1016/j.dsr2.2005.10.003

Bishop, J.K.B., Lam, P.J., Wood, T.J., 2012. Getting good particles: Accurate sampling of particles by large volume in-situ filtration. Limnol. Oceanogr. Methods 10, 681-710. doi:10.4319/lom.2012.10.681

Black, E.E., Buesseler, K.O., Pike, S.M., Lam, P.J., 2017. ${ }^{234}$ Th as a tracer of particulate export and remineralization in the southeastern tropical Pacific. Mar. Chem. In Press, Corrected Proof. doi:10.1016/j.marchem.2017.06.009

Boetius, A., Albrecht, S., Bakker, K., Bienhold, C., Felden, J., Fernandez-Mendez, M., Hendricks, S., Katlein, C., Lalande, C., Krumpen, T., Nicolaus, M., Peeken, I., Rabe, B., Rogacheva, A., Rybakova, E., Somavilla, R., Wenzhofer, F., 2013. Export of Algal Biomass from the Melting Arctic Sea Ice. Science 339, 1430-1432. doi:10.1126/science.1231346

Buesseler, K.O., Andrews, J.E., Pike, S., Charette, M.A., 2005. Particle export during the Southern Ocean Iron Experiment (SOFeX). Limnol. Oceanogr. 50, 311-327. doi:10.4319/1o.2005.50.1.0311 
Buesseler, K.O., Barber, R.T., Dickson, M.L., Hiscock, M.R., Moore, J.K., Sambrotto, R., 2003. The effect of marginal ice-edge dynamics on production and export in the Southern Ocean along $170^{\circ}$ W. Deep. Res. Part II Top. Stud. Oceanogr. 50, 579-603. doi:10.1016/S0967-0645(02)00585-4

Buesseler, K.O., Benitez-Nelson, C., Rutgers van der Loeff, M., Andrews, J., Ball, L., Crossin, G., Charette, M.A., 2001. An intercomparison of small- and large-volume techniques for thorium-234 in seawater. Mar. Chem. 74, 15-28.

Buesseler, K.O., Boyd, P.W., 2009. Shedding light on processes that control particle export and flux attenuation in the twilight zone of the open ocean. Limnol. Oceanogr. 54, 1210-1232.

Buitenhuis, E.T., Hashioka, T., Quere, C. Le, 2013. Combined constraints on global ocean primary production using observations and models. Global Biogeochem. Cycles 27, 847-858. doi:10.1002/gbc.20074

Cai, P., Chen, W., Dai, M., Wan, Z., Wang, D., Li, Q., Tang, T., Lv, D., 2008. A high-resolution study of particle export in the southern South China Sea based on ${ }^{234} \mathrm{Th}:{ }^{238} \mathrm{U}$ disequilibrium. J. Geophys. Res. 113, 1-15. doi:10.1029/2007JC004268

Cai, P., Dai, M., Lv, D., Chen, W., 2006. An improvement in the small-volume technique for determining thorium-234 in seawater. Mar. Chem. 100, 282-288. doi:10.1016/j.marchem.2005.10.016

Cai, P., Rutgers Van der Loeff, M., Stimac, I., Nothig, E.M., Lepore, K., Moran, S.B., 2010. Low export flux of particulate organic carbon in the central Arctic Ocean as revealed by ${ }^{234} \mathrm{Th}:{ }^{238} \mathrm{U}$ disequilibrium. J. Geophys. Res. Ocean. 115, 1-21. doi:10.1029/2009JC005595

Carmack, E., Chapman, D.C., 2003. Wind-driven shelf/basin exchange on an Arctic shelf: The joint roles of ice cover extent and shelf-break bathymetry. Geophys. Res. Lett. 30, 3-6. doi:10.1029/2003GL017526

Chen, M., Huang, Y., Cai, P., Guo, L., 2003. Particulate organic carbon export fluxes in the Canada Basin and Bering Sea as derived from ${ }^{234} \mathrm{Th} /{ }^{238} \mathrm{U}$ disequilibria. Arctic 56, 32-44. doi:10.14430/arctic600

Coale, K.H., Bruland, K.W., 1985. ${ }^{234}$ Th: $:{ }^{238} \mathrm{U}$ disequilibria within the California current. Limnol. Oceanogr. 30, 22-33. doi:10.4319/1o.1985.30.1.0022

Cochran, J.K., Barnes, C., Achman, D., Hirschberg, D.J., 1995. Thorium-234/uranium-238 disequilibrium as an indicator of scavenging rates and participate organic carbon fluxes in the Northeast Water Polynya, Greenland. J. Geophys. Res. 100, 4399. doi:10.1029/94JC01954

Comiso, J.C., Parkinson, C.L., Gersten, R., Stock, L., 2008. Accelerated decline in the Arctic sea ice cover. Geophys. Res. Lett. 35, 1-6. doi:10.1029/2007GL031972

Coppola, L., Roy-Barman, M., Mulsow, S., Povinec, P., Jeandel, C., 2005. Low particulate organic carbon export in the frontal zone of the Southern Ocean (Indian sector) revealed by ${ }^{234} \mathrm{Th}$. Deep. Res. Part I Oceanogr. Res. Pap. 52, 51-68. doi:10.1016/j.dsr.2004.07.020

Coupel, P., Ruiz-Pino, D., Sicre, M.A., Chen, J.F., Lee, S.H., Schiffrine, N., Li, H.L., Gascard, J.C., 2015. The impact of freshening on phytoplankton production in the Pacific Arctic Ocean. Prog. Oceanogr. 131, 113-125. doi:10.1016/j.pocean.2014.12.003

Fahl, K., Nöthig, E.M., 2007. Lithogenic and biogenic particle fluxes on the Lomonosov Ridge (central Arctic Ocean) and their relevance for sediment accumulation: Vertical vs. lateral transport. Deep. Res. Part I Oceanogr. Res. Pap. 54, 1256-1272. doi:10.1016/j.dsr.2007.04.014

Fernández-Méndez, M., Wenzhöfer, F., Peeken, I., Sørensen, H.L., Glud, R.N., Boetius, A., 2014. Composition, buoyancy regulation and fate of ice algal aggregates in the Central Arctic Ocean. PLoS One 9. doi:10.1371/journal.pone.0107452 
Forest, A., Sampei, M., Hattori, H., Makabe, R., Sasaki, H., Fukuchi, M., Wassmann, P., Fortier, L., 2007. Particulate organic carbon fluxes on the slope of the Mackenzie Shelf (Beaufort Sea):

Physical and biological forcing of shelf-basin exchanges. J. Mar. Syst. 68, 39-54. doi:10.1016/j.jmarsys.2006.10.008

Gosselin, M., Levasseur, M., Wheeler, P.A., Horner, R.A., Booth, B.C., 1997. New measurements of phytoplankton and ice algal production in the Arctic Ocean. Deep. Res. Part II Top. Stud. Oceanogr. 44, 1623-1644. doi:10.1016/S0967-0645(97)00054-4

Henson, S.A., Sanders, R., Madsen, E., Morris, P.J., Le Moigne, F., Quartly, G.D., 2011. A reduced estimate of the strength of the ocean's biological carbon pump. Geophys. Res. Lett. 38, 1-5. doi:10.1029/2011GL046735

Hill, V., Cota, G., 2005. Spatial patterns of primary production on the shelf, slope and basin of the Western Arctic in 2002. Deep Sea Res. Part II Top. Stud. Oceanogr. 52, 3344-3354. doi:10.1016/j.dsr2.2005.10.001

Hill, V., Cota, G., Stockwell, D., 2005. Spring and summer phytoplankton communities in the Chukchi and Eastern Beaufort Seas. Deep. Res. Part II Top. Stud. Oceanogr. 52, 3369-3385. doi:10.1016/j.dsr2.2005.10.010

Honjo, S., Krishfield, R.A., Eglinton, T.I., Manganini, S.J., Kemp, J.N., Doherty, K., Hwang, J., McKee, T.K., Takizawa, T., 2010. Biological pump processes in the cryopelagic and hemipelagic Arctic Ocean: Canada Basin and Chukchi Rise. Prog. Oceanogr. 85, 137-170. doi:10.1016/j.pocean.2010.02.009

Hwang, J., Eglinton, T.I., Krishfield, R.A., Manganini, S.J., Honjo, S., 2008. Lateral organic carbon supply to the deep Canada Basin. Geophys. Res. Lett. 35, 1-5. doi:10.1029/2008GL034271

Hwang, J., Kim, M., Manganini, S.J., McIntyre, C.P., Haghipour, N., Park, J.J., Krishfield, R.A., Macdonald, R.W., McLaughlin, F.A., Eglinton, T.I., 2015. Temporal and spatial variability of particle transport in the deep Arctic Canada Basin. J. Geophys. Res. Ocean. 120, 2784-2799. doi:10.1002/2014JC010643

Jakobsson, M., 2002. Hypsometry and volume of the Arctic Ocean and its constituent seas. Geochemistry Geophys. Geosystems 3, 1-18.

Klinkhammer, G.P., Palmer, M.R., 1991. Uranium in the oceans: Where it goes and why. Geochim. Cosmochim. Acta 55, 1799-1806. doi:10.1016/0016-7037(91)90024-Y

Lalande, C., Lepore, K., Cooper, L.W., Grebmeier, J.M., Moran, S.B., 2007. Export fluxes of particulate organic carbon in the Chukchi Sea: A comparative study using ${ }^{234} \mathrm{Th} /{ }^{238} \mathrm{U}$ disequilibria and drifting sediment traps. Mar. Chem. 103, 185-196. doi:10.1016/j.marchem.2006.07.004

Lam, P.J., Lee, J.-M., Heller, M.I., Mehic, S., Xiang, Y., Bates, N.R., 2017. Size-fractionated distributions of suspended particle concentration and major phase composition from the U.S. GEOTRACES Eastern Pacific Zonal Transect (GP16). Mar. Chem. Accepted doi:10.1016/j.marchem.2017.08.013

Lam, P.J., Twining, B.S., Jeandel, C., Roychoudhury, A., Resing, J.A., Santschi, P.H., Anderson, R.F., 2015. Methods for analyzing the concentration and speciation of major and trace elements in marine particles. Prog. Oceanogr. 133. doi:10.1016/j.pocean.2015.01.005

Laney, S.R., Krishfield, R.A., Toole, J.M., 2017. The euphotic zone under Arctic Ocean sea ice: Vertical extents and seasonal trends. Limnol. Oceanogr. doi:10.1002/lno.10543 
Lee, Z.P., Weidemann, A., Kindle, J., Arnone, R., Carder, K.L., Davis, C., 2007. Euphotic zone depth: Its derivation and implication to ocean-color remote sensing. J. Geophys. Res. 112, 1-11. doi:10.1029/2006JC003802

Lepore, K., Moran, S.B., 2007. Seasonal changes in thorium scavenging and particle aggregation in the western Arctic Ocean. Deep. Res. Part I Oceanogr. Res. Pap. 54, 919-938. doi:10.1016/j.dsr.2007.03.001

Lepore, K., Moran, S.B., Grebmeier, J.M., Cooper, L.W., Lalande, C., Maslowski, W., Hill, V., Bates, N.R., Hansell, D.A., Mathis, J.T., Kelly, R.P., 2007. Seasonal and interannual changes in particulate organic carbon export and deposition in the Chukchi Sea. J. Geophys. Res. 112, 1-14. doi:10.1029/2006JC003555

Lin, W., Chen, L., Zeng, S., Li, T., Wang, Y., Yu, K., 2016. Residual $\beta$ activity of particulate ${ }^{234}$ Th as a novel proxy for tracking sediment resuspension in the ocean. Nat. Sci. Reports 6, 27069. doi:10.1038/srep27069

Ma, Q., Chen, M., Qiu, Y., Li, Y., 2005. Regional estimates of POC export flux derived from thorium234 in the western Arctic Ocean. Acta Oceanol. Sin. 24, 97-108. doi:10.1080/10643389.2012.728825

Macdonald, R.W., Harner, T., Fyfe, J., 2005. Recent climate change in the Arctic and its impact on contaminant pathways and interpretation of temporal trend data. Sci. Total Environ. 342, 5-86. doi:10.1016/j.scitotenv.2004.12.059

Maiti, K., Buesseler, K.O., Pike, S.M., Benitez-Nelson, C., Cai, P., Chen, W., Cochran, K., Dai, M., Dehairs, F., Gasser, B., 2012. Intercalibration studies of short-lived thorium-234 in the water column and marine particles. Limnol. Oceanogr. Methods 10, 631-644.

doi:10.4319/lom.2012.10.631

Maiti, K., Nelson, C.R.B., Buesseler, K.O., 2010. Insights into particle formation and remineralization using the short-lived radionuclide, Thoruim-234. Geophys. Res. Lett. 37, 1-6. doi:10.1029/2010GL044063

Measures, C.I., 1999. The role of entrained sediments in sea ice in the distribution of aluminium and iron in the surface waters of the Arctic Ocean. Mar. Chem. 68, 59-70. doi:10.1016/S03044203(99)00065-1

Moore, J.K., Doney, S.C., Lindsay, K., 2004. Upper ocean ecosystem dynamics and iron cycling in a global three-dimensional model. Global Biogeochem. Cycles 18, 1-21. doi:10.1029/2004GB002220

Moran, S.B., Ellis, K.M., Smith, J.N., 1997. ${ }^{234} \mathrm{Th} /{ }^{238} \mathrm{U}$ disequilibrium in the central Arctic Ocean: implications for particulate organic carbon export. Deep. Res. Part II 44, 1593-1606.

Moran, S.B., Kelly, R.P., Hagstrom, K., Smith, J.N., Grebmeier, J.M., Cooper, L.W., Cota, G.F., Walsh, J.J., Bates, N.R., Hansell, D.A., Maslowski, W., Nelson, R.P., Mulsow, S., 2005. Seasonal changes in POC export flux in the Chukchi Sea and implications for water column-benthic coupling in Arctic shelves. Deep. Res. Part II 52, 3427-3451. doi:10.1016/j.dsr2.2005.09.011

Moran, S.B., Smith, J.N., 2000. ${ }^{234}$ Th as a tracer of scavenging and particle export in the Beaufort Sea. Cont. Shelf Res. 20, 153-167. doi:10.1016/S0278-4343(99)00065-5

Not, C., Brown, K.A., Ghaleb, B., Hillaire-Marcel, C., 2012. Conservative behavior of uranium vs. salinity in Arctic sea ice and brine. Mar. Chem. 130-131, 33-39. doi:10.1016/j.marchem.2011.12.005 
Ohnemus, D.C., Rauschenberg, S., Cutter, G.A., Fitzsimmons, J.N., Sherrell, R.M., Twining, B.S., 2016. Elevated trace metal content of prokaryotic plankton communities associated with marine oxygen deficient zones. Limnol. Oceanogr. 62, 3-25. doi:10.1002/lno.10363

Owens, S.A., Buesseler, K.O., Sims, K.W.W., 2011. Re-evaluating the ${ }^{238}$ U-salinity relationship in seawater: Implications for the 238U-234Th disequilibrium method. Mar. Chem. 127, 31-39. doi:10.1016/j.marchem.2011.07.005

Owens, S.A., Pike, S., Buesseler, K.O., 2015. Thorium-234 as a tracer of particle dynamics and upper ocean export in the Atlantic Ocean. Deep. Res. II 116, 42-59. doi:10.1016/j.dsr2.2014.11.010

Pates, J.M., Muir, G.K.P., 2007. U-salinity relationships in the Mediterranean: Implications for ${ }^{234} \mathrm{Th}:{ }^{238} \mathrm{U}$ particle flux studies. Mar. Chem. 106, 530-545. doi:10.1016/j.marchem.2007.05.006

Perovich, D.K., Richter-Menge, J.A., 2009. Loss of Sea Ice in the Arctic. Ann. Rev. Mar. Sci. 1, 417441. doi:10.1146/annurev.marine.010908.163805

Rengarajan, R., Sarin, M.M., Krishnaswami, S., 2003. Uranium in the Arabian Sea: Role of denitrification in controlling its distribution. Oceanol. Acta 26, 687-693. doi:10.1016/j.oceact.2003.05.001

Roca-marti, M., Puigcorbe, V., Rutgers van der Loeff, M., Katlein, C., Fernandez-Mendez, M., Peeken, I., Masque, P., 2016. Carbon export fluxes and export efficiency in the central Arctic during the record sea-ice minimum in 2012: a joint ${ }^{234} \mathrm{Th} /{ }^{238} \mathrm{U}$ and ${ }^{210} \mathrm{Po} /{ }^{210} \mathrm{~Pb}$ study. J. Geophys. Res. Ocean. 1-23. doi:10.1002/2016JC011736

Rodriguez y Baena, A.M., Boudjenoun, R., Fowler, S.W., Miquel, J.C., Masqué, P., Sanchez-Cabeza, J.A., Warnau, M., 2008. 234Th-based carbon export during an ice-edge bloom: Sea-ice algae as a likely bias in data interpretation. Earth Planet. Sci. Lett. 269, 595-603. doi:10.1016/j.epsl.2008.03.020

Rutgers van der Loeff, M.M., Buesseler, K., Bathmann, U., Hense, I., Andrews, J., 2002. Comparison of carbon and opal export rates between summer and spring bloom periods in the region of the Antarctic Polar Front, SE Atlantic. Deep. Res. Part II Top. Stud. Oceanogr. 49, 3849-3869. doi:10.1016/S0967-0645(02)00114-5

Rutgers van der Loeff, M.M., Cai, P.H., Stimac, I., Bracher, A., Middag, R., Klunder, M.B., van Heuven, S.M.A.C., 2011. ${ }^{234}$ Th in surface waters: Distribution of particle export flux across the Antarctic Circumpolar Current and in the Weddell Sea during the GEOTRACES expedition ZERO and DRAKE. Deep. Res. Part II Top. Stud. Oceanogr. 58, 2749-2766. doi:10.1016/j.dsr2.2011.02.004

Savoye, N., Benitez-Nelson, C., Burd, A.B., Cochran, J.K., Charette, M., Buesseler, K.O., Jackson, G.A., Roy-Barman, M., Schmidt, S., Elskens, M., 2006. ${ }^{234}$ Th sorption and export models in the water column: A review. Mar. Chem. 100, 234-249. doi:10.1016/j.marchem.2005.10.014

Savoye, N., Buesseler, K.O., Cardinal, D., Dehairs, F., 2004. ${ }^{234}$ Th deficit and excess in the Southern Ocean during spring 2001: Particle export and remineralization. Geophys. Res. Lett. 31, 1-4. doi:10.1029/2004GL019744

Siegel, D.A., Buesseler, K.O., Doney, S.C., Sailley, S.F., Behrenfeld, M.J., Boyd, P.W., 2014. Global assessment of ocean carbon export by combining satellite observations and food-web models. Global Biogeochem. Cycles 28, 181-196. doi:10.1002/2013GB004743

Subha Anand, S., Rengarajan, R., Sarma, V.V.S.S., Sudheer, A.K., Bhushan, R., Singh, S.K., 2017. Spatial variability of upper ocean POC export in the Bay of Bengal and the Indian Ocean determined using particle-reactive ${ }^{234} \mathrm{Th}$. J. Geophys. Res. Ocean. 122, 3753-3770. doi:10.1002/2016JC012639 
Toole, J., Baxter, M.S., Thomson, J., 1987. The behaviour of uranium isotopes with salinity change in three U.K. estuaries. Estuar. Coast. Shelf Sci. 25, 283-297. doi:10.1016/0272-7714(87)90072-2

Trimble, S.M., Baskaran, M., 2005. The role of suspended particulate matter in ${ }^{234} \mathrm{Th}$ scavenging and ${ }^{234}$ Th-derived export fluxes of POC in the Canada Basin of the Arctic Ocean. Mar. Chem. 96, 1-19. doi:10.1016/j.marchem.2004.10.003

Wassmann, P., Bauerfeind, E., Fortier, M., Fukuchi, M., Hargrave, B., Moran, B., Noji, T., Nöthig, E.-M., Olli, K., Peinert, R., Sasaki, H., Shevchenko, V., 2004. Particulate Organic Carbon Flux to the Arctic Ocean Sea Floor, in: Stein, R., Macdonald, R.W. (Eds.), The Organic Carbon Cycle in the Arctic Ocean. Springer, pp. 101-138. doi:10.1007/978-3-642-18912-8_5

Wassmann, P., Reigstad, M., 2011. Future Arctic Ocean Seasonal Ice Zones and Implications for PelagicBenthic Coupling. Oceanography 24, 220-231. doi:10.5670/oceanog.2011.74

Wheeler, P.A., Gosselin, M., Sherr, E., Thibaultc, D., Kirchman, D.L., Benner, R., Whitledge, T.E., 1996. Active cycling of organic carbon in the central Arctic Ocean. Nature. doi:10.1038/380697a0

Woodgate, R.A., Aagaard, K., Weingartner, T.J., 2005. A year in the physical oceanography of the Chukchi Sea: Moored measurements from autumn 1990-1991. Deep. Res. Part II Top. Stud. Oceanogr. 52, 3116-3149. doi:10.1016/j.dsr2.2005.10.016

Woodgate, R.A., Weingartner, T.J., Lindsay, R., 2012. Observed increases in Bering Strait oceanic fluxes from the Pacific to the Arctic from 2001 to 2011 and their impacts on the Arctic Ocean water column. Geophys. Res. Lett. 39, 2-7. doi:10.1029/2012GL054092

Yu, W., He, J., Li, Y., Lin, W., Chen, L., 2012. Particulate organic carbon export fluxes and validation of steady state model of ${ }^{234}$ Th export in the Chukchi Sea. Deep. Res. Part II 84, 63-71. doi:10.1016/j.dsr2.2012.03.003

Zimov, S.A., Schuur, E.A.G., Chapin, F.S.I., 2006. Permafrost and the Global Carbon Budget. Science $312,1612-1613$. 
Table 1: Station information. Total ${ }^{234} \mathrm{Th}$ was sampled at all listed stations and size-fractionated particulate ${ }^{234} \mathrm{Th}$ was measured when noted $(\mathrm{Y})$. Locations where particulates were not sampled (N) included MIZ and ICE stations where only surface samples were taken in the upper $50 \mathrm{~m}$. Start dates for the first ${ }^{234} \mathrm{Th}$ sampling event are listed. Mixed layer depths (MLD) were determined as the depth where the potential density change relative to the surface (at $5 \mathrm{~m}$ ) was $>0.125 \mathrm{~kg} \mathrm{~m}^{-3}$.

\begin{tabular}{|c|c|c|c|c|c|c|}
\hline Station & Region & $\underset{{ }^{\circ} \mathbf{N}}{\text { Latitude }}$ & $\begin{array}{c}\text { Longitude } \\
{ }^{\circ} \mathbf{E}(+),{ }^{\circ} \mathbf{W}(-)\end{array}$ & $\begin{array}{c}\text { Particulates } \\
\text { Sampled }\end{array}$ & Date & $\begin{array}{l}\text { MLD } \\
\text { (m) }\end{array}$ \\
\hline 1 & $\begin{array}{c}\text { Pacific } \\
\text { Endmember }\end{array}$ & 60.22 & -179.05 & $\mathrm{Y}$ & $8 / 13 / 2015$ & 29 \\
\hline 2 & Shelf & 62.26 & -171.64 & $\mathrm{Y}$ & $8 / 14 / 2015$ & 16 \\
\hline 3 & Shelf & 64.01 & -166.65 & $\mathrm{Y}$ & $8 / 15 / 2015$ & 20 \\
\hline 6 & Shelf & 68.00 & -168.11 & $\mathrm{Y}$ & $8 / 17 / 2015$ & 20 \\
\hline 7 & MIZ-Slope & 73.49 & -168.85 & $\mathrm{~N}(\mathrm{MIZ})$ & $8 / 18 / 2015$ & 9 \\
\hline 8 & MIZ-Slope & 73.98 & -168.76 & $\mathrm{~N}(\mathrm{MIZ})$ & $8 / 18 / 2015$ & 7 \\
\hline 9 & MIZ-Slope & 74.50 & -168.88 & N (MIZ) & $8 / 19 / 2015$ & 6 \\
\hline 10 & MIZ-Slope & 74.99 & -170.11 & $\mathrm{Y}$ & $8 / 19 / 2015$ & 10 \\
\hline 12 & MIZ-Basin & 75.78 & -171.29 & $\mathrm{~N}(\mathrm{MIZ})$ & $8 / 20 / 2015$ & 13 \\
\hline 14 & MIZ-Basin & 76.54 & -173.10 & $\mathrm{Y}$ & $8 / 20 / 2015$ & 11 \\
\hline 17 & MIZ-Basin & 78.15 & -176.76 & N (MIZ) & $8 / 22 / 2015$ & 10 \\
\hline 19 & MIZ-Basin & 79.99 & -174.98 & $\mathrm{Y}$ & $8 / 23 / 2015$ & 13 \\
\hline 26 & Basin & 83.76 & -174.82 & $\mathrm{Y}$ & $8 / 28 / 2015$ & 20 \\
\hline 30 & Basin & 87.53 & -180.00 & $\mathrm{Y}$ & $9 / 1 / 2015$ & 22 \\
\hline 31 & Basin & 88.41 & -176.76 & $\mathrm{~N}(\mathrm{ICE})$ & $9 / 4 / 2015$ & -- \\
\hline 32 & Basin & 89.99 & 37.84 & $\mathrm{Y}$ & $9 / 5 / 2015$ & 15 \\
\hline 33 & Basin & 89.99 & 89.25 & $\mathrm{~N}(\mathrm{ICE})$ & 9/7/2015 & -- \\
\hline 34 & Basin & 89.95 & -96.86 & $\mathrm{~N}$ & $9 / 8 / 2015$ & 10 \\
\hline 38 & Basin & 87.83 & -149.50 & $\mathrm{Y}$ & $9 / 10 / 2015$ & 18 \\
\hline 43 & Basin & 85.13 & -150.00 & $\mathrm{Y}$ & $9 / 15 / 2015$ & 31 \\
\hline 46 & MIZ-Basin & 82.49 & -149.83 & $\mathrm{Y}$ & $9 / 15 / 2015$ & 25 \\
\hline 48 & MIZ-Basin & 80.38 & -149.77 & $\mathrm{Y}$ & $9 / 22 / 2015$ & 23 \\
\hline 51 & MIZ-Basin & 77.51 & -148.01 & $\mathrm{~N}(\mathrm{MIZ})$ & $9 / 26 / 2015$ & 25 \\
\hline 52 & MIZ-Basin & 78.18 & -147.83 & $\mathrm{Y}$ & $9 / 27 / 2015$ & 20 \\
\hline 53 & MIZ-Basin & 77.00 & -148.81 & $\mathrm{~N}(\mathrm{MIZ})$ & $9 / 28 / 2015$ & 17 \\
\hline 54 & MIZ-Basin & 76.51 & -149.52 & N (MIZ) & $9 / 28 / 2015$ & 23 \\
\hline 56 & MIZ-Basin & 75.06 & -150.33 & $\mathrm{Y}$ & $9 / 29 / 2015$ & 24 \\
\hline 57 & MIZ-Basin & 73.40 & -156.77 & $\mathrm{Y}$ & $10 / 3 / 2015$ & 18 \\
\hline 60 & Slope & 73.00 & -158.77 & $\mathrm{Y}$ & $10 / 5 / 2015$ & 16 \\
\hline 61 & Shelf & 72.81 & -159.64 & Y & $10 / 5 / 2015$ & 13 \\
\hline 66 & Shelf & 72.00 & -162.50 & $\mathrm{Y}$ & $10 / 7 / 2015$ & 23 \\
\hline
\end{tabular}


Table 2: ${ }^{234}$ Th, POC, and PON fluxes. Values in italics are essentially zero within reported uncertainties or negative. Stations where the potential lateral component was estimated to be $>40 \%$ of the calculated ${ }^{234} \mathrm{Th}$ flux are marked with an asterisk.

\begin{tabular}{|c|c|c|c|c|c|c|c|c|}
\hline \multirow{2}{*}{$\frac{\text { Station }}{1}$} & \multirow{2}{*}{$\begin{array}{c}\text { Depth } \\
50\end{array}$} & \multicolumn{2}{|c|}{$\begin{array}{c}{ }^{234} \text { Th Flux } \\
\left(\text { dpm m m}^{-2} \mathbf{d}^{-1}\right)\end{array}$} & \multicolumn{2}{|c|}{$\begin{array}{c}\begin{array}{c}\text { POC Flux } \\
\left(\mathrm{mmol} \mathrm{m}^{-2} \mathbf{d}^{-1}\right)\end{array} \\
\end{array}$} & \multicolumn{3}{|c|}{ 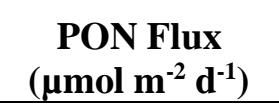 } \\
\hline & & 1170 & \pm 60 & 5 & \pm 1 & 590 & \pm & 40 \\
\hline & 75 & 1590 & \pm 90 & 6 & \pm 1 & 720 & \pm & 50 \\
\hline & 100 & 1600 & \pm 100 & 4.9 & \pm 0.8 & 630 & \pm & 60 \\
\hline & 125 & 1400 & \pm 200 & 3.6 & \pm 0.5 & 500 & \pm & 70 \\
\hline & 150 & 1200 & $\pm \quad 200$ & 2.7 & \pm 0.6 & 380 & \pm & 80 \\
\hline 6 & 50 & 2370 & \pm 40 & 83 & \pm 4 & 13000 & \pm & 1000 \\
\hline \multirow[t]{5}{*}{$10 *$} & 50 & 400 & \pm 90 & 9 & \pm 2 & 1500 & \pm & 300 \\
\hline & 75 & 800 & \pm 100 & 7 & \pm 1 & 1000 & \pm & 200 \\
\hline & 100 & 1300 & \pm 100 & 7.1 & \pm 0.9 & 800 & \pm & 100 \\
\hline & 125 & 1700 & \pm 200 & 7.8 & $\pm \quad 0.9$ & 1000 & \pm & 100 \\
\hline & 150 & 2100 & $\pm \quad 200$ & 9 & \pm 1 & 1200 & \pm & 100 \\
\hline \multirow{5}{*}{14} & 50 & -40 & \pm 80 & -0.1 & \pm 0.3 & -20 & \pm & 30 \\
\hline & 75 & -100 & $\pm \quad 100$ & -0.2 & \pm 0.3 & -20 & \pm & 30 \\
\hline & 100 & -100 & \pm 100 & -0.2 & \pm 0.2 & -20 & \pm & 30 \\
\hline & 125 & -100 & \pm 200 & -0.1 & \pm 0.2 & -10 & \pm & \\
\hline & 150 & -200 & $\pm \quad 200$ & -0.1 & \pm 0.1 & -10 & \pm & 10 \\
\hline \multirow[t]{5}{*}{19} & 50 & -70 & \pm 90 & -0.3 & \pm 0.4 & -40 & \pm & 50 \\
\hline & 75 & -200 & \pm 100 & -0.7 & \pm 0.4 & -80 & \pm & \\
\hline & 100 & -400 & \pm 100 & -0.8 & \pm 0.3 & -80 & \pm & 30 \\
\hline & 125 & -500 & \pm 200 & -0.8 & \pm 0.3 & -90 & \pm & 30 \\
\hline & 150 & -600 & $\pm \quad 200$ & -0.6 & \pm 0.2 & -70 & \pm & 20 \\
\hline \multirow[t]{5}{*}{26} & 50 & 20 & \pm 80 & 0.1 & \pm 0.4 & 10 & \pm & 40 \\
\hline & 75 & -100 & $\pm \quad 100$ & -0.1 & \pm 0.1 & -20 & \pm & 20 \\
\hline & 100 & -300 & \pm 100 & -0.2 & \pm 0.1 & -20 & \pm & \\
\hline & 125 & -400 & \pm 200 & -0.6 & \pm 0.2 & -70 & \pm & 30 \\
\hline & 150 & -600 & $\pm \quad 200$ & -1 & \pm 0.4 & -90 & \pm & 40 \\
\hline \multirow[t]{5}{*}{30} & 50 & -10 & \pm 70 & -0.1 & \pm 0.3 & -10 & \pm & 30 \\
\hline & 75 & 0 & \pm 100 & 0.0 & \pm 0.3 & 0 & \pm & 30 \\
\hline & 100 & -10 & \pm 100 & 0.0 & \pm 0.2 & 0 & \pm & \\
\hline & 125 & 0 & \pm 200 & 0.0 & \pm 0.3 & 0 & \pm & 30 \\
\hline & 150 & -100 & \pm 200 & -0.2 & \pm 0.4 & -10 & \pm & 30 \\
\hline \multirow[t]{5}{*}{32} & 50 & -100 & \pm 90 & -0.5 & \pm 0.4 & -50 & \pm & \\
\hline & 75 & -200 & \pm 100 & -0.7 & \pm 0.5 & -70 & \pm & 60 \\
\hline & 100 & -300 & \pm 100 & -1.2 & \pm 0.6 & -130 & \pm & 70 \\
\hline & 125 & -400 & \pm 200 & -1.4 & \pm 0.7 & -150 & \pm & 70 \\
\hline & 150 & -300 & $\pm \quad 200$ & -1.0 & \pm 0.7 & -110 & \pm & 80 \\
\hline
\end{tabular}




\begin{tabular}{|c|c|c|c|c|c|c|c|}
\hline \multirow{2}{*}{$\begin{array}{c}\text { Station } \\
38\end{array}$} & \multirow{2}{*}{$\frac{\text { Depth }}{50}$} & \multicolumn{2}{|c|}{ 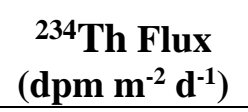 } & \multicolumn{2}{|c|}{$\begin{array}{c}\underset{\left(\mathbf{m m o l} \mathbf{m}^{-2} \mathbf{d}^{-1}\right)}{\text { POC Flux }} \\
\text {. }\end{array}$} & \multicolumn{2}{|c|}{$\underset{\left(\mu \mathrm{mol} \mathbf{m}^{-2} \mathbf{d}^{-1}\right)}{\operatorname{PON} \text { Flux }}$} \\
\hline & & 80 & \pm 80 & 0.3 & \pm 0.3 & 20 & \pm 20 \\
\hline & 75 & 40 & \pm 100 & 0.1 & \pm 0.3 & 10 & \pm 30 \\
\hline & 100 & 40 & \pm 100 & 0.1 & \pm 0.2 & 10 & \pm 20 \\
\hline & 125 & 50 & \pm 200 & 0.1 & \pm 0.3 & 10 & \pm 20 \\
\hline & 150 & 80 & \pm 200 & 0.1 & \pm 0.3 & 10 & \pm 20 \\
\hline \multirow[t]{5}{*}{43} & 50 & 20 & \pm 80 & 0.1 & \pm 0.3 & 10 & \pm 30 \\
\hline & 75 & -30 & \pm 100 & -0.1 & \pm 0.3 & -10 & \pm 30 \\
\hline & 100 & -100 & \pm 200 & -0.2 & \pm 0.2 & -10 & \pm 20 \\
\hline & 125 & -200 & \pm 200 & -0.2 & \pm 0.3 & -20 & \pm 20 \\
\hline & 150 & -30 & \pm 200 & 0.0 & \pm 0.3 & 0 & \pm 20 \\
\hline \multirow[t]{5}{*}{46} & 50 & -150 & \pm 70 & -0.8 & \pm 0.4 & -80 & \pm 40 \\
\hline & 75 & -200 & \pm 100 & -0.5 & \pm 0.2 & -50 & \pm 20 \\
\hline & 100 & -300 & \pm 100 & -0.3 & \pm 0.2 & -30 & \pm 10 \\
\hline & 125 & -300 & \pm 200 & -0.2 & \pm 0.1 & -20 & \pm 10 \\
\hline & 150 & -300 & \pm 200 & -0.2 & \pm 0.1 & -20 & \pm 10 \\
\hline \multirow[t]{5}{*}{48} & 50 & -60 & \pm 80 & -0.3 & \pm 0.4 & -30 & \pm 40 \\
\hline & 75 & -80 & \pm 100 & -0.1 & \pm 0.2 & -20 & \pm 20 \\
\hline & 100 & -20 & \pm 100 & 0.0 & \pm 0.1 & 0 & \pm 10 \\
\hline & 125 & -90 & \pm 100 & -0.1 & \pm 0.1 & -10 & \pm 10 \\
\hline & 150 & -100 & \pm 200 & -0.1 & \pm 0.1 & 0 & \pm 10 \\
\hline \multirow[t]{5}{*}{52} & 50 & -3 & \pm 100 & 0.0 & \pm 0.6 & 0 & \pm 50 \\
\hline & 75 & -90 & \pm 100 & -0.4 & \pm 0.5 & -40 & \pm 50 \\
\hline & 100 & -200 & \pm 100 & -0.2 & \pm 0.2 & -30 & \pm 20 \\
\hline & 125 & -270 & \pm 200 & -0.3 & \pm 0.2 & -30 & \pm 20 \\
\hline & 150 & -300 & \pm 200 & -0.2 & \pm 0.2 & -20 & \pm 10 \\
\hline \multirow[t]{5}{*}{56} & 50 & -200 & \pm 100 & & & & \\
\hline & 75 & -200 & \pm 100 & -0.6 & \pm 0.4 & -60 & \pm 40 \\
\hline & 100 & -200 & \pm 100 & -0.4 & \pm 0.4 & -40 & \pm 40 \\
\hline & 125 & -200 & \pm 200 & -0.2 & \pm 0.2 & -20 & \pm 20 \\
\hline & 150 & -200 & \pm 200 & -0.2 & \pm 0.2 & -20 & \pm 20 \\
\hline \multirow[t]{5}{*}{57} & 50 & 40 & \pm 90 & 0.2 & \pm 0.4 & 20 & \pm 30 \\
\hline & 75 & 100 & \pm 100 & 0.3 & \pm 0.3 & 20 & \pm 20 \\
\hline & 100 & 100 & \pm 100 & 0.2 & \pm 0.2 & 20 & \pm 20 \\
\hline & 125 & 200 & \pm 200 & 0.2 & \pm 0.2 & 20 & \pm 20 \\
\hline & 150 & 100 & \pm 200 & 0.1 & \pm 0.1 & 10 & $\pm \quad 10$ \\
\hline \multirow[t]{5}{*}{$60 *$} & 50 & 560 & \pm 70 & 1.3 & \pm 0.2 & 120 & \pm 20 \\
\hline & 75 & 600 & \pm 100 & 1.2 & \pm 0.2 & 110 & \pm 20 \\
\hline & 100 & 700 & \pm 100 & 1.0 & \pm 0.2 & 110 & \pm 20 \\
\hline & 125 & 900 & \pm 200 & 0.9 & \pm 0.2 & 90 & \pm 20 \\
\hline & 150 & 1400 & $\pm \quad 200$ & 1.2 & $\pm \quad 0.2$ & 110 & $\pm \quad 20$ \\
\hline $61 *$ & 50 & 1180 & $\pm \quad 60$ & 6 & \pm 1 & 640 & $\pm \quad 50$ \\
\hline
\end{tabular}


Table 3: ${ }^{234}$ Th removal rates and residence times for the shelf-slope region. The removal rates of dissolved ${ }^{234} \mathrm{Th}$ with respect to particle scavenging $\left(J_{T h}\right)$ and the particulate removal rates due to particle sinking $\left(P_{T h}\right)$ are reported. The residence times $(\tau)$ of the dissolved and particulate pools with respect to these removal fluxes are included. These parameters were not determined for the Arctic basin because ${ }^{234} \mathrm{Th}$ deficits, if present, were small compared to uncertainties.

\begin{tabular}{|c|c|c|c|c|c|c|c|c|c|c|c|c|c|}
\hline \multirow{2}{*}{$\begin{array}{r}\text { Station } \\
2\end{array}$} & $\begin{array}{c}\text { Depth } \\
\text { Interval }\end{array}$ & \multicolumn{3}{|c|}{ 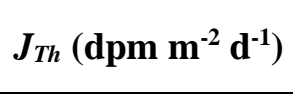 } & \multicolumn{3}{|c|}{$\tau_{D i s s}$} & \multicolumn{3}{|c|}{ 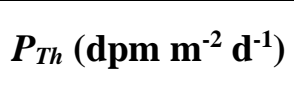 } & \multicolumn{3}{|c|}{$\tau_{\text {Part }}$} \\
\hline & $0-45$ & 1200 & \pm & 100 & 45 & \pm & 5 & 800 & \pm & 100 & 20 & \pm & 3 \\
\hline 3 & $0-25$ & 1190 & \pm & 60 & 12 & \pm & 1 & 1050 & \pm & 60 & 4.6 & \pm & 0.6 \\
\hline \multirow[t]{2}{*}{6} & $0-25$ & 1620 & \pm & 50 & & -- & & 1260 & \pm & 50 & 9.8 & \pm & 0.7 \\
\hline & $0-50$ & 3030 & \pm & 80 & 2.5 & \pm & 0.5 & 2360 & \pm & 80 & 9.9 & \pm & 0.6 \\
\hline \multirow[t]{4}{*}{10} & $0-50$ & 700 & \pm & 100 & 100 & \pm & 20 & 500 & \pm & 100 & 11 & \pm & 3 \\
\hline & $0-100$ & 1900 & \pm & 300 & 80 & \pm & 10 & 1500 & \pm & 300 & 10 & \pm & 2 \\
\hline & $0-150$ & 2900 & \pm & 400 & 80 & \pm & 10 & 2300 & \pm & 400 & 9 & \pm & 2 \\
\hline & $0-200$ & 4000 & \pm & 400 & 77 & \pm & 9 & 3200 & \pm & 400 & 9 & \pm & 1 \\
\hline \multirow[t]{5}{*}{60} & $0-25$ & 1400 & \pm & 100 & 2.7 & \pm & 0.5 & 900 & \pm & 200 & 18 & \pm & 4 \\
\hline & $0-50$ & 1500 & \pm & 100 & 29 & \pm & 5 & 800 & \pm & 200 & 29 & \pm & 6 \\
\hline & $0-100$ & 2500 & \pm & 300 & 48 & \pm & 6 & 900 & \pm & 200 & 60 & \pm & 20 \\
\hline & $0-150$ & 4400 & \pm & 400 & 39 & \pm & 4 & 1600 & \pm & 400 & 60 & \pm & 20 \\
\hline & $0-200$ & 5800 & \pm & 400 & 40 & \pm & 4 & 1800 & \pm & 400 & 80 & \pm & 20 \\
\hline \multirow[t]{2}{*}{61} & $0-25$ & 420 & \pm & 40 & 51 & \pm & 7 & 230 & \pm & 30 & 19 & \pm & 3 \\
\hline & $0-50$ & 1900 & \pm & 200 & 26 & \pm & 3 & 1400 & \pm & 200 & 14 & \pm & 2 \\
\hline 66 & $0-25$ & 660 & \pm & 70 & 46 & \pm & 6 & 330 & \pm & 70 & 33 & \pm & 7 \\
\hline
\end{tabular}


Table 4: Summary of ${ }^{234}$ Th and POC flux statistics. Average fluxes for this study (August to October 2015) and prior studies (1990s to 2010s), split into shelf-slope stations (<1000 m water depth) and basin locations (>1000 $\mathrm{m}$ water depth), are shown for ${ }^{234} \mathrm{Th}$ and POC. The number of data points (n) and minimum-maximum ranges are also included for prior studies.

\begin{tabular}{|c|c|c|c|c|c|c|}
\hline & & $\begin{array}{l}\text { This Study } \\
{ }^{234} \text { Th Flux }\end{array}$ & Prior Stuc & $e^{234}$ & Flux & \\
\hline & Depth Range (m) & $\begin{array}{l}\text { Mean } \pm \text { s.d. } \\
\left(\mathrm{dpm} \mathrm{m}^{-2} \mathrm{~d}^{-1}\right)\end{array}$ & $\begin{array}{l}\text { Mean } \pm \text { s.d. } \\
\left(\mathrm{dpm} \mathrm{m}^{-2} \mathrm{~d}^{-1}\right)\end{array}$ & $\mathrm{n}$ & Min & Max \\
\hline \multirow{2}{*}{ Shelf-Slope } & 26 to 50 & $1136 \pm 774$ & $1002 \pm 691$ & 122 & -1309 & 3918 \\
\hline & 75 to 100 & $1200 \pm 458$ & $1200 \pm 1207$ & 37 & -1166 & 3803 \\
\hline \multirow{3}{*}{$\begin{array}{c}\text { Basin } \\
(>1000 \mathrm{~m})\end{array}$} & 26 to 50 & $-39 \pm 81$ & $626 \pm 528$ & 62 & -160 & 2203 \\
\hline & 75 to 100 & $-149 \pm 158$ & $479 \pm 1027$ & 58 & -2212 & 3984 \\
\hline & 125 to 150 & $-213 \pm 227$ & $726 \pm 653$ & 19 & -220 & 1863 \\
\hline
\end{tabular}

\begin{tabular}{|c|c|c|c|c|c|c|}
\hline & & $\begin{array}{l}\text { This Study } \\
\text { POC Flux }\end{array}$ & Prior Stu & es PO & Flux & \\
\hline & Depth Range (m) & 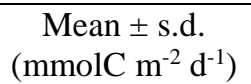 & 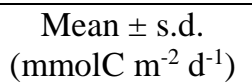 & $\mathrm{n}$ & Min & Max \\
\hline \multirow{2}{*}{ Shelf-Slope } & 26 to 50 & $21 \pm 35$ & $35 \pm 50$ & 104 & -0.4 & 304 \\
\hline & 75 to 100 & $4 \pm 3$ & $26 \pm 40$ & 39 & -0.4 & 164 \\
\hline \multirow{3}{*}{$\begin{array}{c}\text { Basin } \\
(>1000 \mathrm{~m})\end{array}$} & 26 to 50 & $-0.3 \pm 0.3$ & $8 \pm 9$ & 53 & -1 & 46 \\
\hline & 75 to 100 & $-0.3 \pm 0.4$ & $6 \pm 16$ & 47 & -6 & 85 \\
\hline & 125 to 150 & $-0.3 \pm 0.4$ & $5 \pm 5$ & 18 & -3 & 15 \\
\hline
\end{tabular}




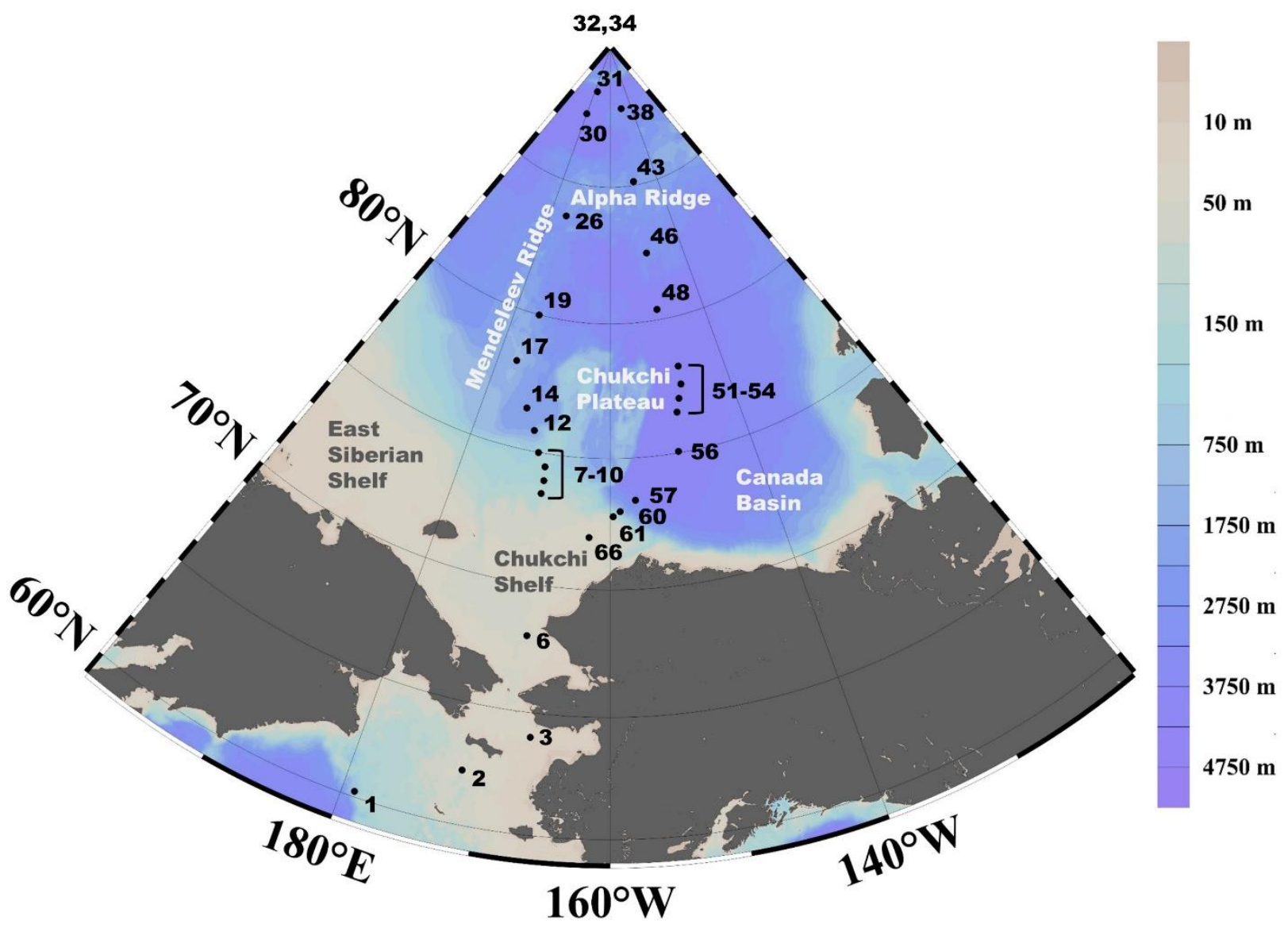

Figure 1: GN01 station locations. Stations where total ${ }^{234} \mathrm{Th}$ samples were taken are shown with the relevant bathymetric features and regions of the Western Artic Ocean. 

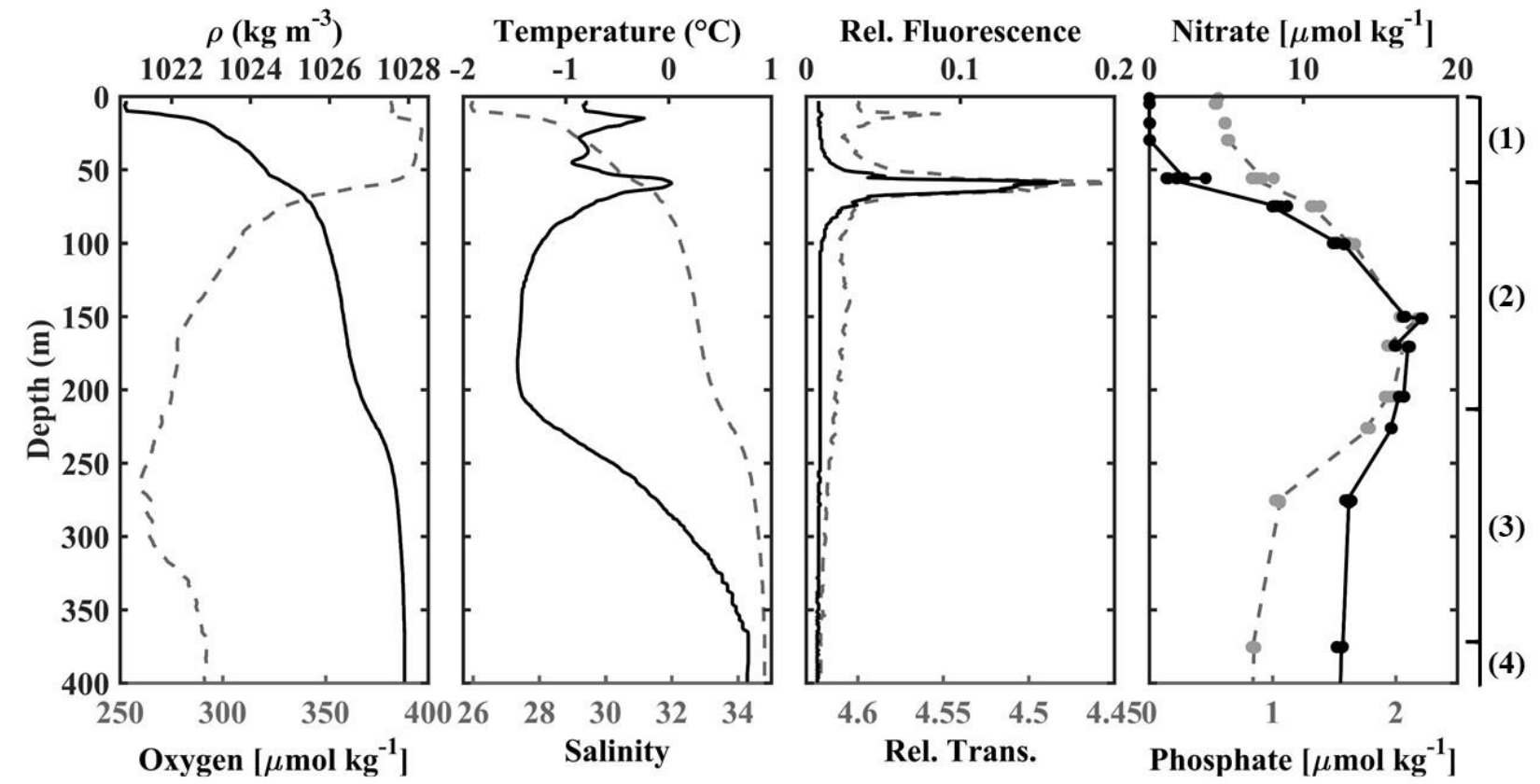

Figure 2: Typical hydrographic and biogeochemical features of the Western Arctic Ocean. The typical upper water column layers of the Western Arctic Ocean (right axis) are shown for NB station $14\left(76.54^{\circ} \mathrm{N}, 173.10^{\circ} \mathrm{W}\right)$, including the polar mixed layer (1), Pacific halocline layer (2), Atlantic halocline layer (3), and Atlantic layer. Transmission and fluorescence have relative units (volts). Black solid lines correspond to the upper axes and dashed grey lines to the lower axes. 

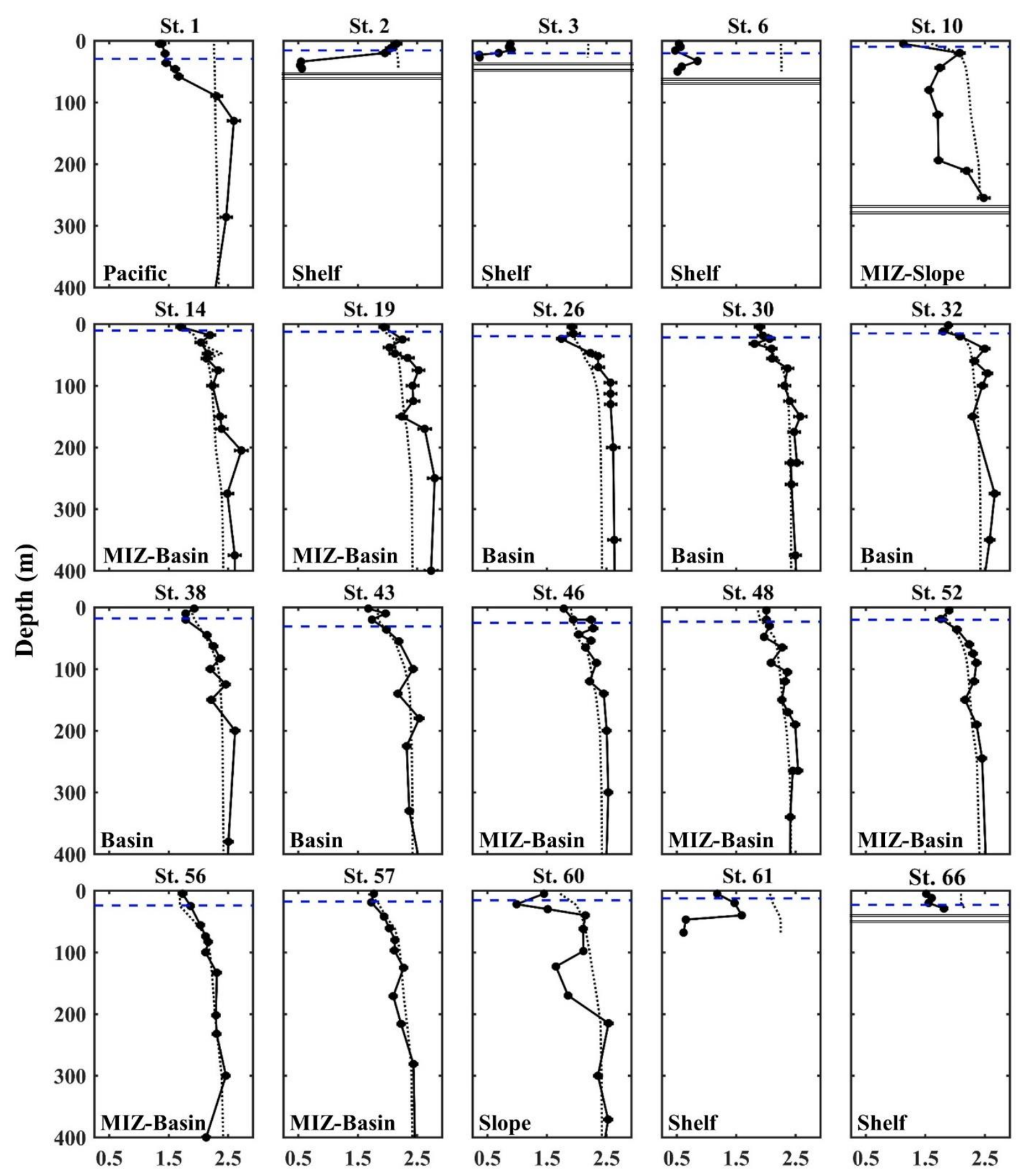

${ }^{234}$ Th and ${ }^{238} \mathrm{U}$ Activities (dpm L $\left.\mathrm{L}^{-1}\right)$

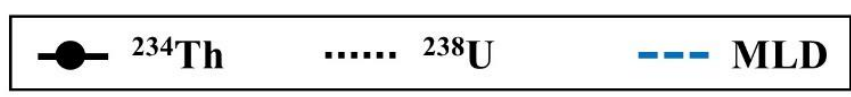

Figure 3: ${ }^{238} \mathbf{U}$ and ${ }^{234} \mathrm{Th}$ activities. Bottom depths are indicated (double lines) if shallower than $400 \mathrm{~m}$. Locations sampled solely as MIZ or ice stations are not shown here (see Figs. 4 and 5). 


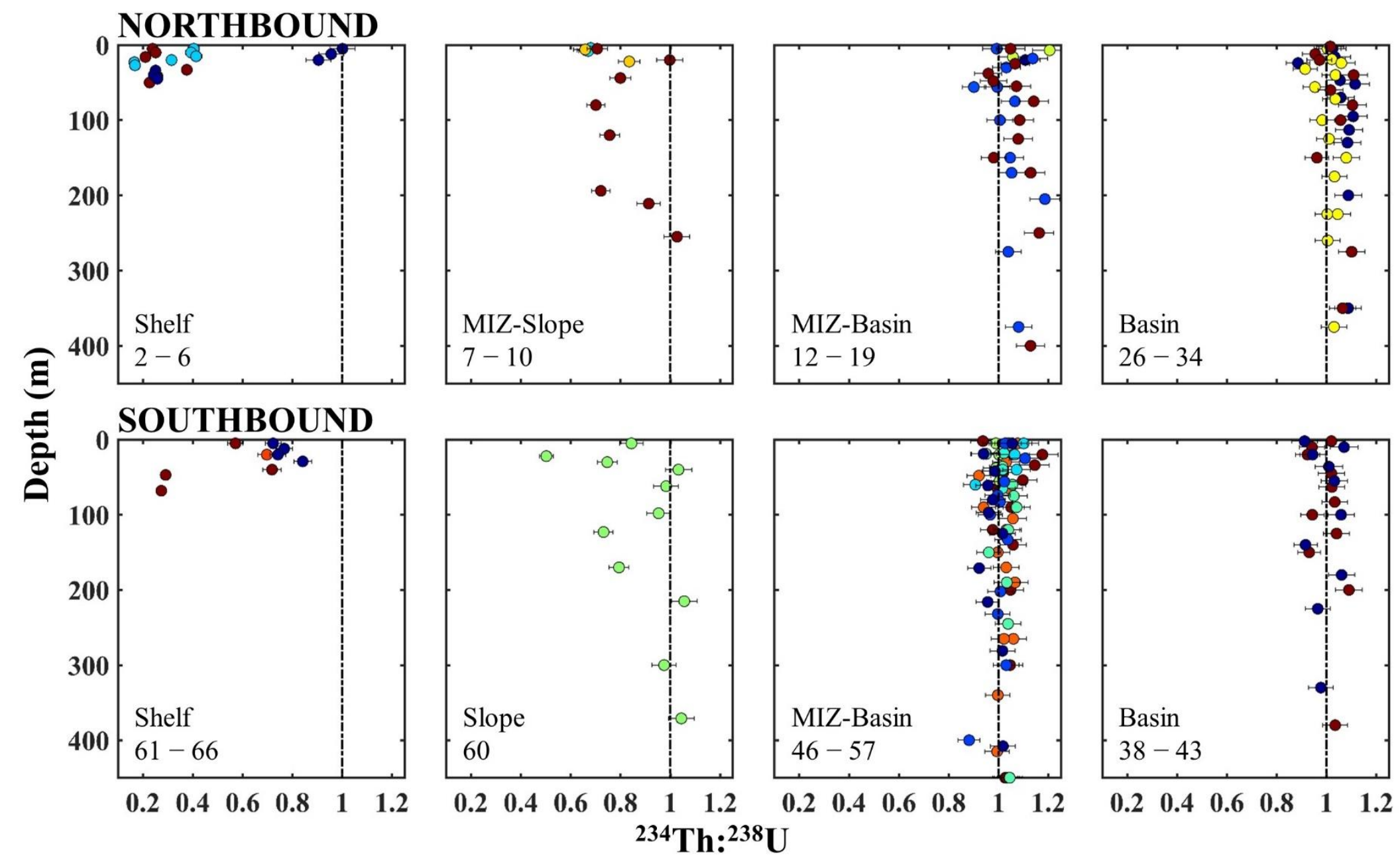

Figure 4: ${ }^{234} \mathbf{T h}:{ }^{238} \mathbf{U}$ by region. The expression of activities as a ratio allows for comparison of disequilibria across the wide range of surface activities observed in the Arctic. As outlined in Table 1, stations were grouped based on similarities in the observed ice concentrations, bathymetry, and depth of $1 \%$ light levels (PAR and fluorescence). Random color assignments are used to distinguish individual stations within a group. ${ }^{234} \mathrm{Th}$ data for MIZ stations that had only a few sampling depths are shown here only and not as separate profiles in Fig. 3. 


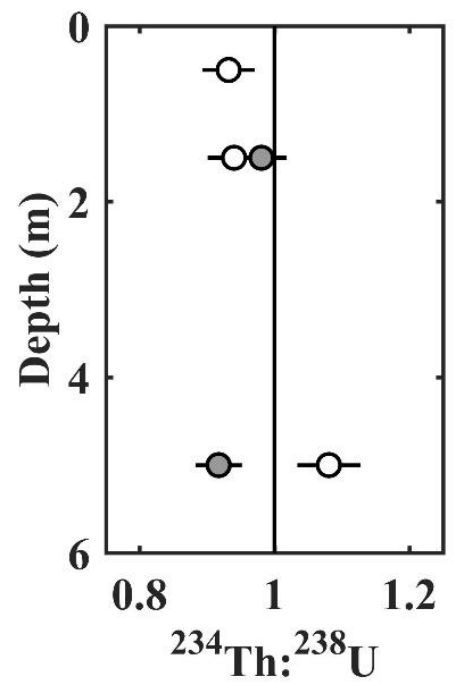

Figure 5: ${ }^{234} \mathbf{T h}:{ }^{238} \mathbf{U}$ at ice stations. Under-ice samples were taken at station 31 (grey; $88.41^{\circ} \mathrm{N}$, $176.76^{\circ} \mathrm{W}$ ) and station 33 (white; $89.99^{\circ} \mathrm{N}, 89.25^{\circ} \mathrm{E}$ ). 


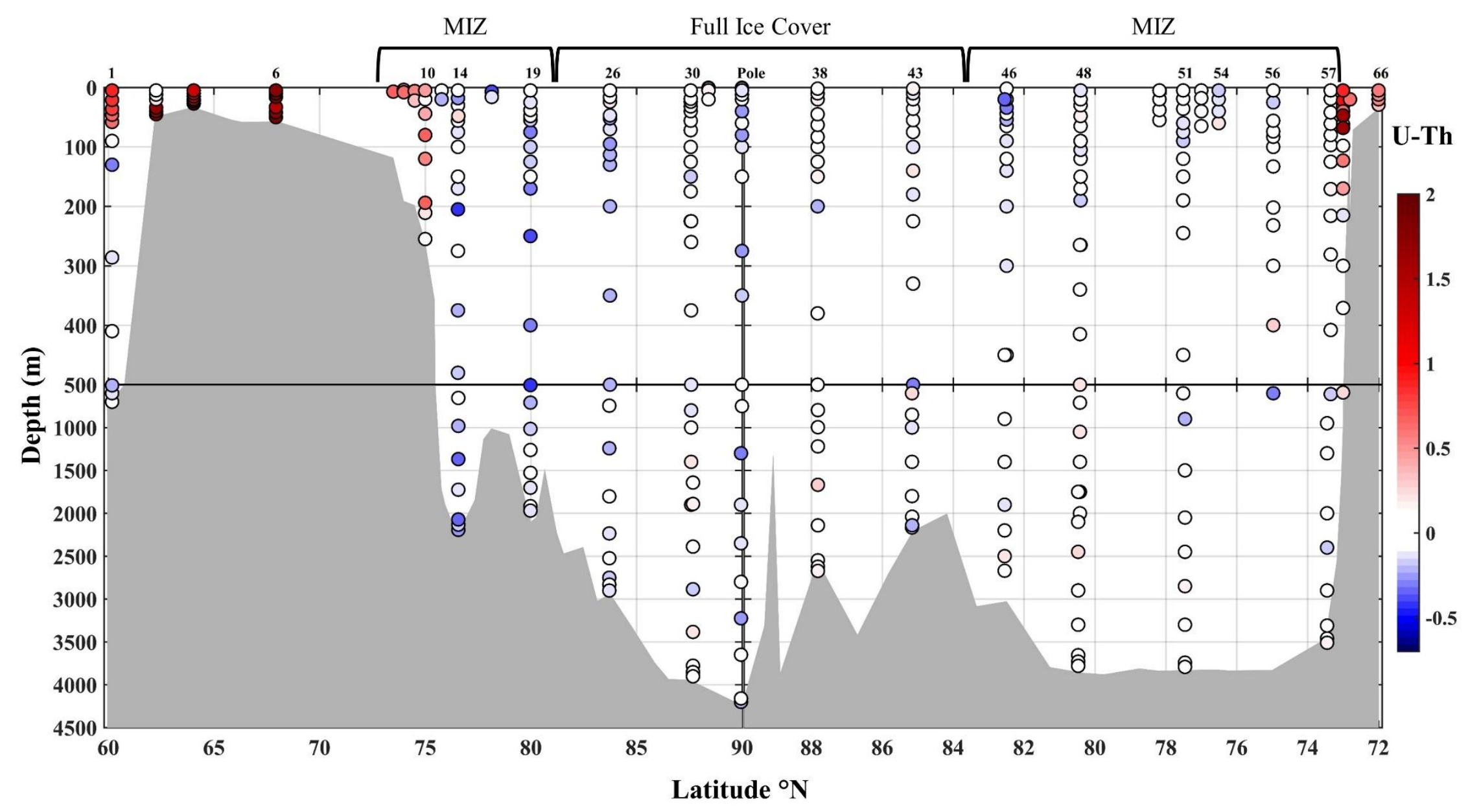

Figure 6: Full water column ${ }^{238} \mathbf{U}$ activity minus ${ }^{234} \mathbf{T h}$ activity $\left(\mathbf{d p m} \mathbf{L}^{-1}\right) .{ }^{234} \mathrm{Th}$ excess over the parent ${ }^{238} \mathrm{U}$ is shown in blue and deficits are shown in red. Some station numbers and the location of the MIZ are shown on the upper axis for reference. 
A

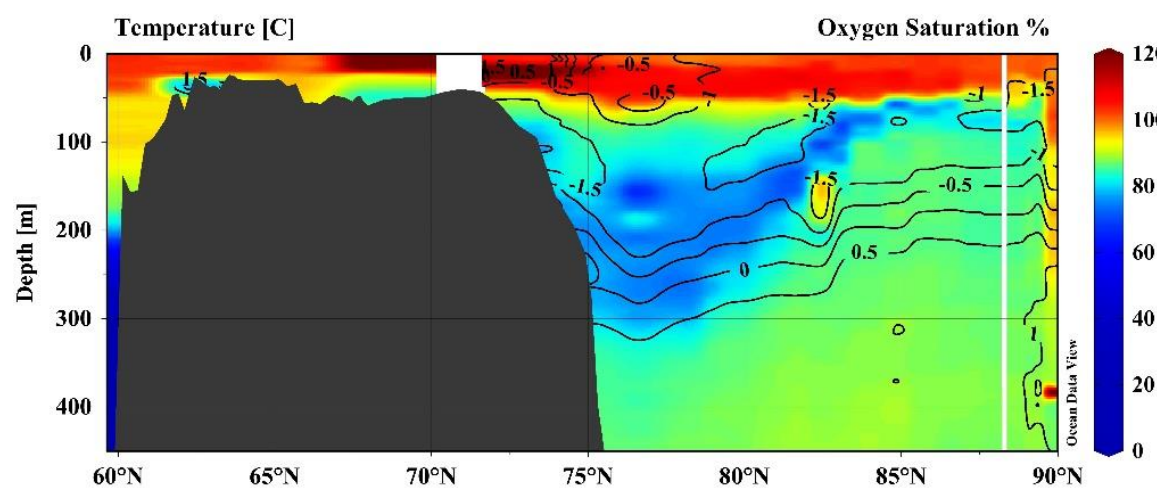

B

Temperature [C]

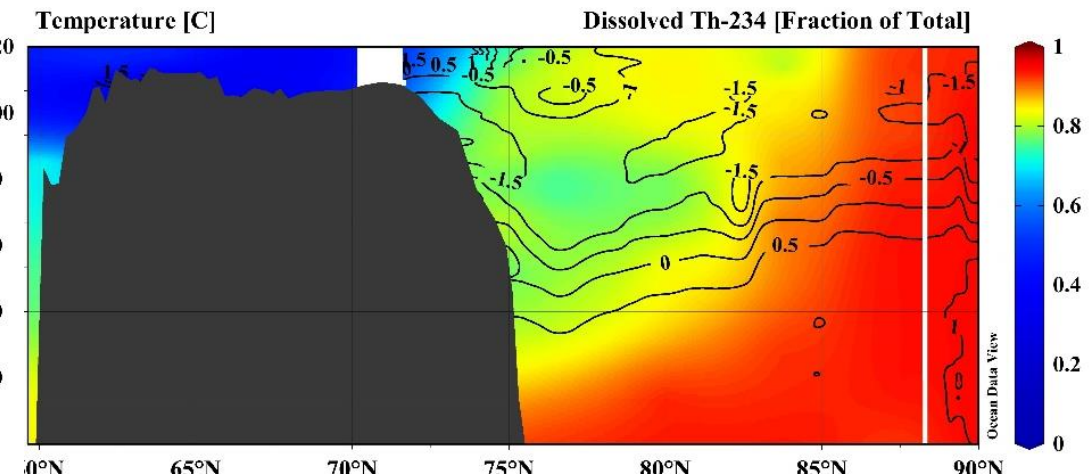

D

Large Particle Th-234 [dpm/L]

Small Particle Th-234 [dpm/L]

Small Particle Th-234 [dpm/L]
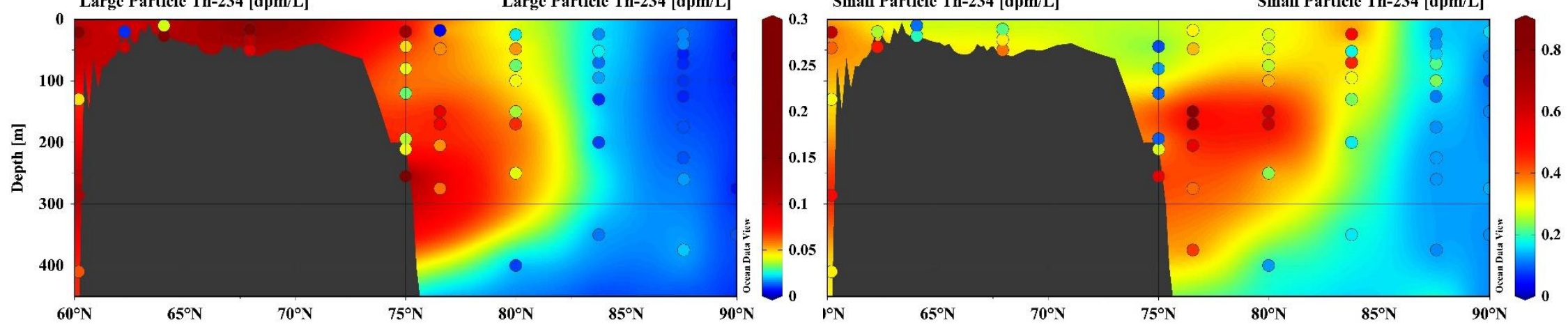

Figure 7: Oxygen saturation, the fraction of dissolved ${ }^{234} \mathrm{Th}$, large particle ${ }^{234} \mathrm{Th}$ activity, and small particle ${ }^{234} \mathrm{Th}$ activity. Oxygen saturation and dissolved fraction data from the NB transect (A, B, C, D) and SB transect (E, F, G, H) are shown with temperature contours. Both large and small fraction ${ }^{234} \mathrm{Th}$ data are show as extrapolated color gradients in the background and individual sampling points in the foreground. 


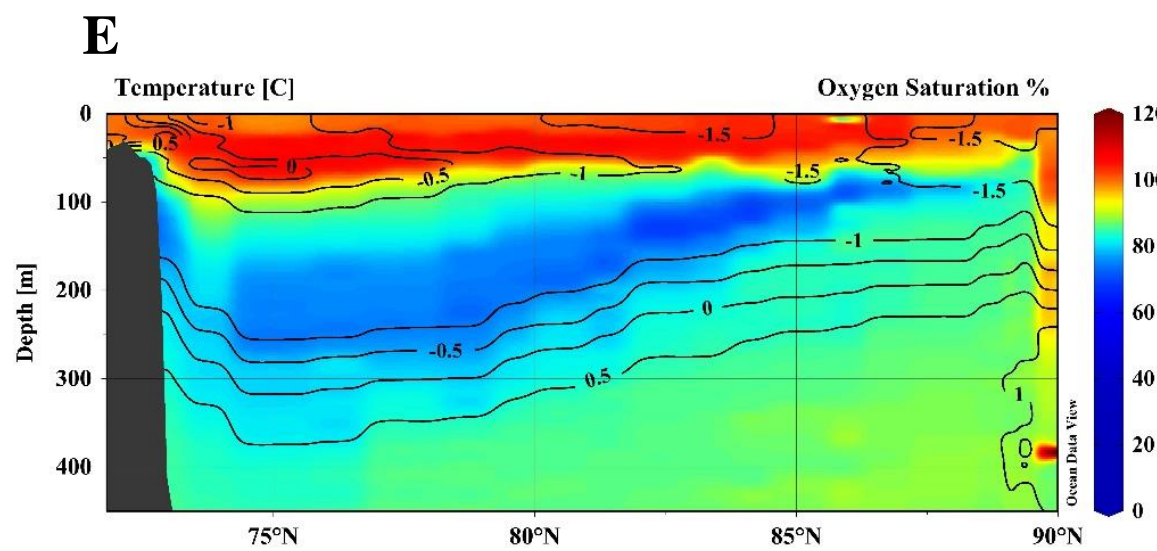

$\mathbf{F}$

Temperature [C]

Dissolved Th-234 [Fraction of Total]

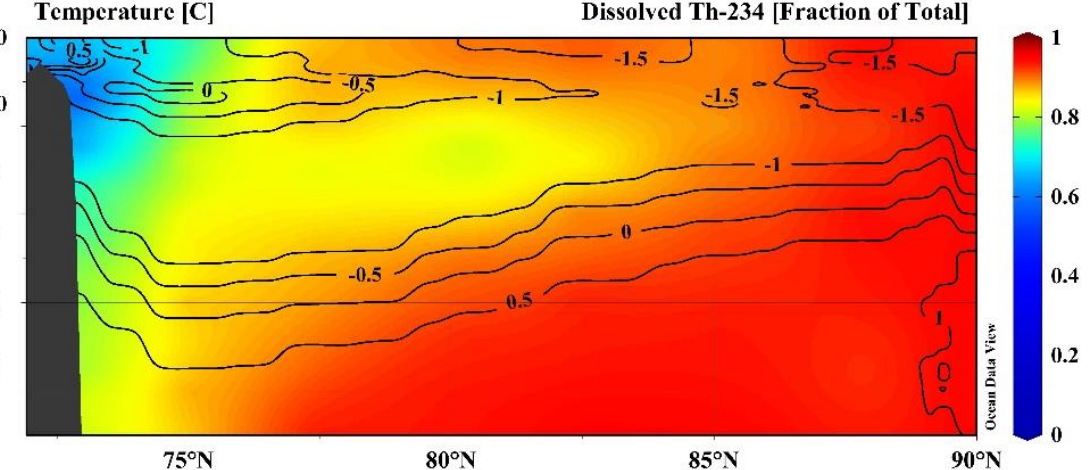

G

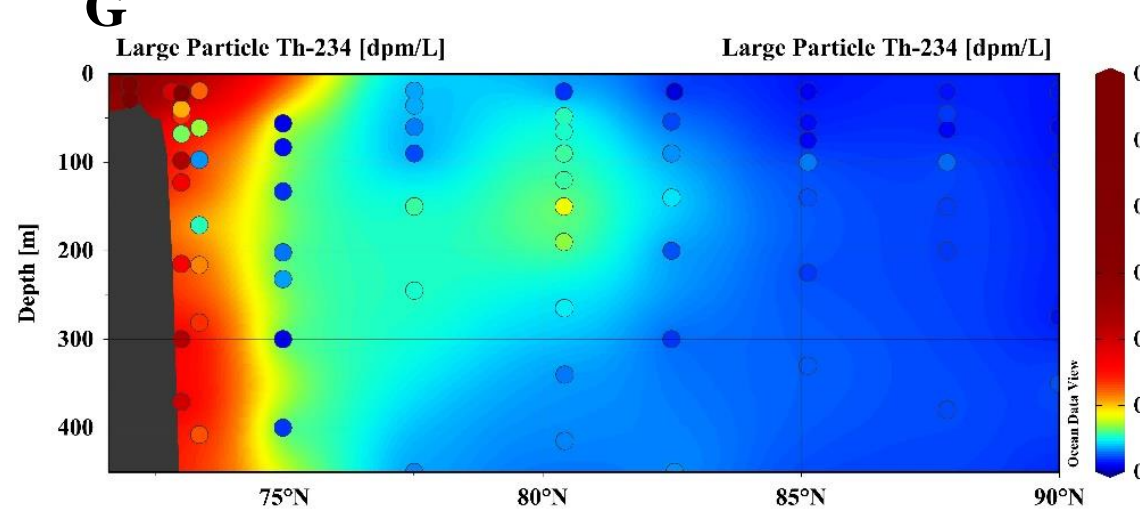

H

Small Particle Th-234 $[\mathrm{dpm} / \mathrm{L}]$

Small Particle Th-234 [dpm/L]

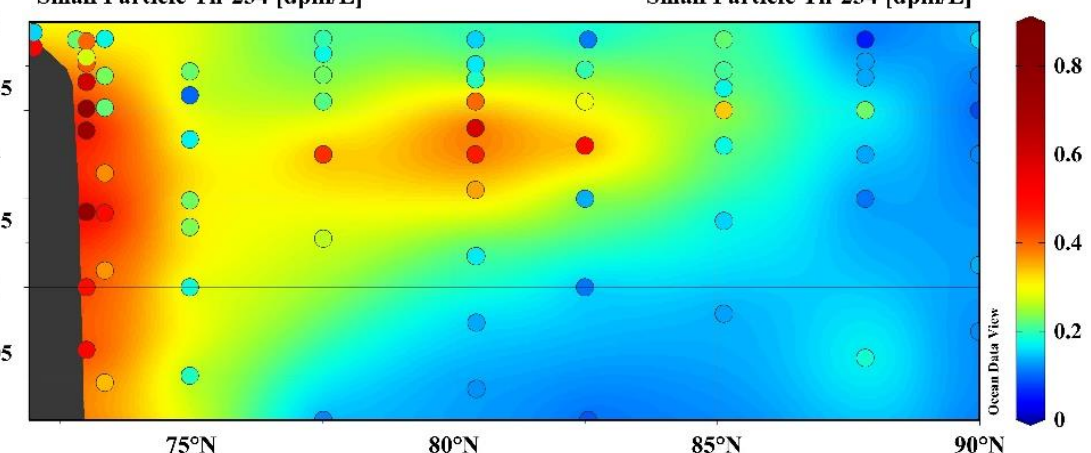

Figure 7: Oxygen saturation, the fraction of dissolved ${ }^{234} \mathrm{Th}$, large particle ${ }^{234} \mathrm{Th}$ activity, and small particle ${ }^{234} \mathrm{Th}$ activity. Oxygen saturation and dissolved fraction data from the NB transect $(\mathrm{A}, \mathrm{B}, \mathrm{C}, \mathrm{D})$ and $\mathrm{SB}$ transect $(\mathrm{E}, \mathrm{F}, \mathrm{G}, \mathrm{H})$ are shown with temperature contours. Both large and small fraction ${ }^{234}$ Th data are show as extrapolated color gradients in the background and individual sampling points in the foreground. 

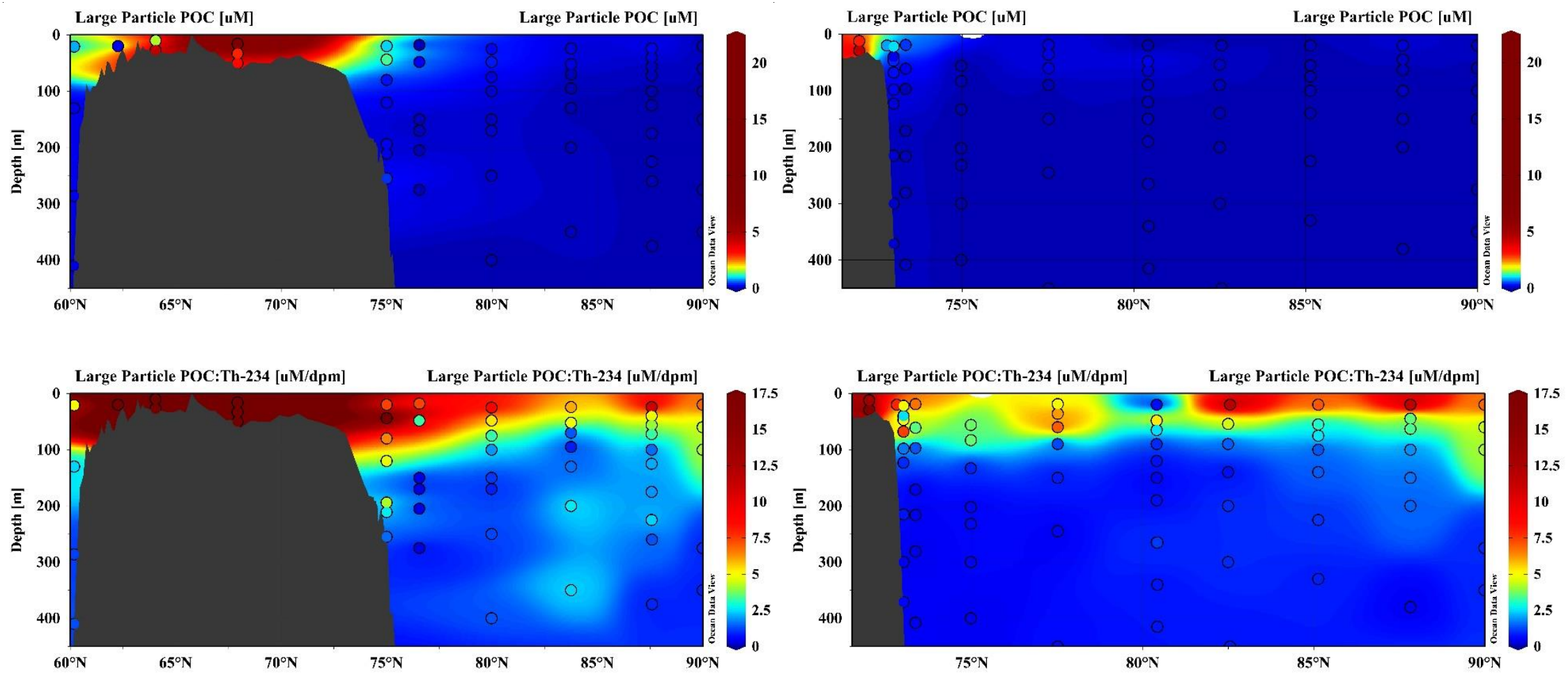

Figure 8: Large particle (>51 $\boldsymbol{\mu m}$ ) POC concentrations and POC: ${ }^{234} \mathbf{T h}$. Individual data points are shown over weighted-average gridding of the same data from the NB (left) and SB (right) transects. 


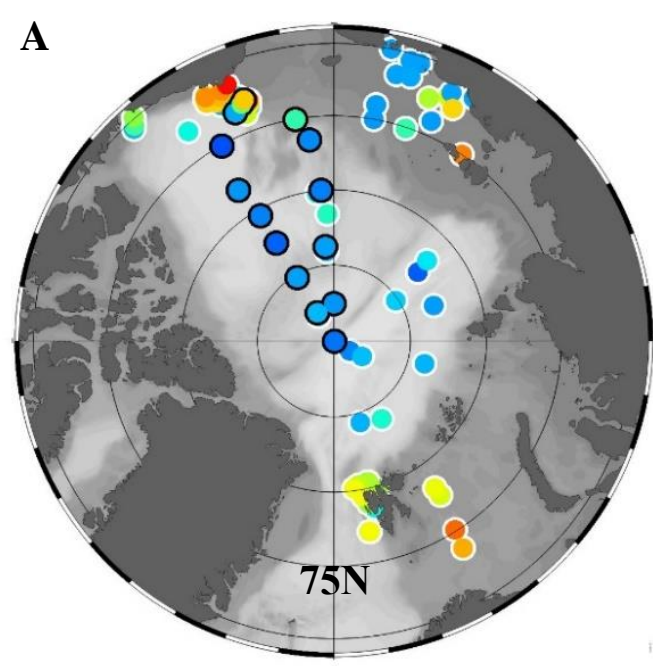

Flux at $\sim 50$ m

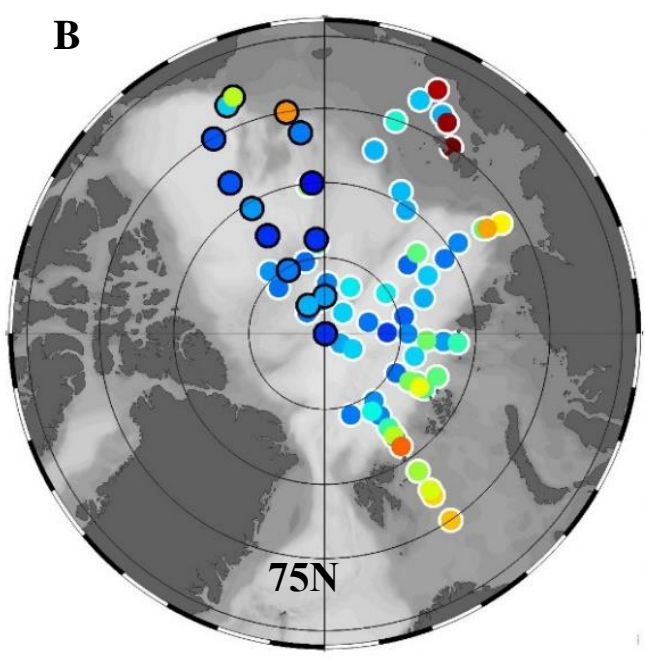

Flux at $\sim 100$ m

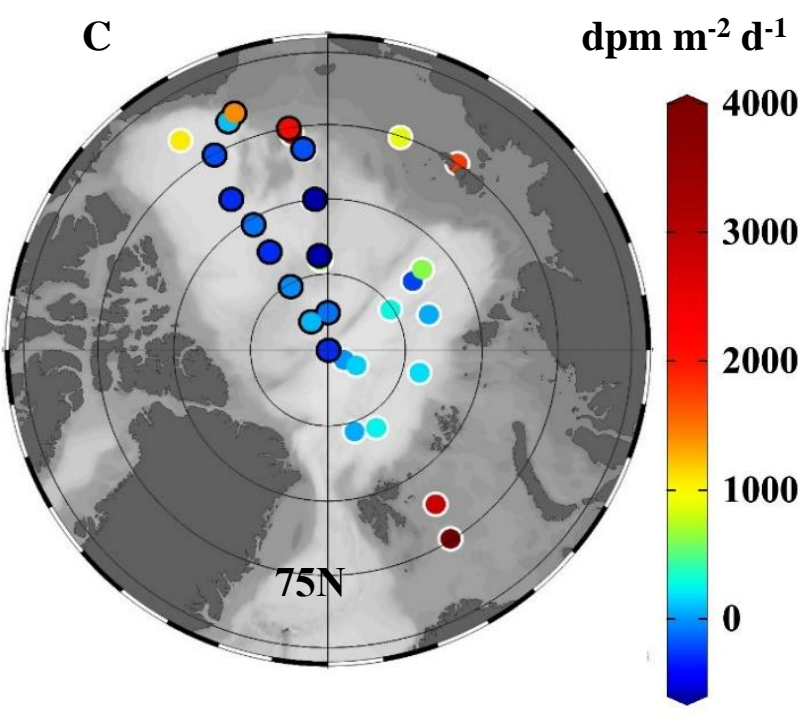

Flux at $\sim 150 \mathrm{~m}$

Figure 9: ${ }^{234}$ Th flux at three depth intervals. Fluxes from this study determined at depths of $50 \mathrm{~m}(\mathrm{~A}), 100 \mathrm{~m}(\mathrm{~B})$, and $150 \mathrm{~m}(\mathrm{C})$ are shown outlined in black. Data from historical studies were included (outlined in white) when the fluxes that were reported fell into three depth zones: $25 \mathrm{~m}$ to $50 \mathrm{~m}, 76 \mathrm{~m}$ to $100 \mathrm{~m}$, and 126 to $150 \mathrm{~m}$ (Amiel et al., 2002; Baskaran et al., 2003; Cai et al., 2010; Chen et al., 2003; Cochran et al., 1995; Coppola et al., 2005; Lalande et al., 2007; Lepore et al., 2007; Ma et al., 2005; Moran et al., 2005, 1997; Moran and Smith, 2000; Roca-marti et al., 2016; Trimble et al., 2004; Trimble and Baskaran, 2005; Yu et al., 2012). Two historical points near the Eurasian shelf and in the Barents Sea had fluxes close to $5000 \mathrm{dpm} \mathrm{m}^{-2} \mathrm{~d}^{-1}$, but the scale was set at $4000 \mathrm{dpm}$ $\mathrm{m}^{-2} \mathrm{~d}^{-1}$ to shown more of the variability in the lower flux range. 


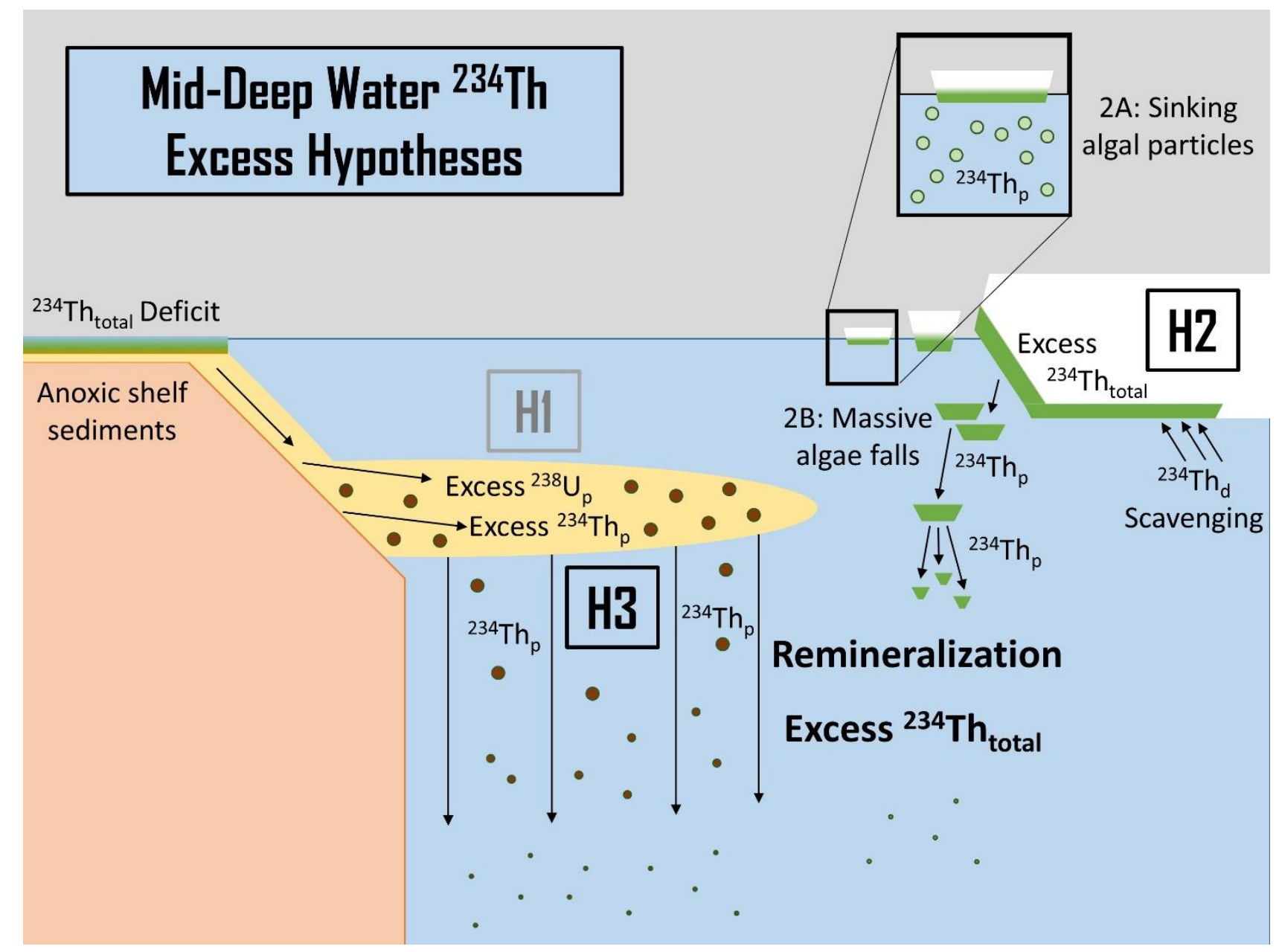

Figure 10: Three potential causes of the mid-deep water ${ }^{234}$ Th activity excesses at NB stations 14, 19, and 26. Details of these hypotheses are discussed in Section 4.4.5. In short, (H1) shows the transport of particulate ${ }^{238} \mathrm{U}$ from shelf-slope waters to the basin in excess of ${ }^{238} \mathrm{U}$ predicted from salinity, (H2) represents under-ice algae scavenging ${ }^{234} \mathrm{Th}$ from the water column that sinks upon ice melt, releasing ${ }^{234} \mathrm{Th}$, and (H3) indicates how particulates with high ${ }^{234} \mathrm{Th}$ could be rapidly transported from a nearby shelf, releasing ${ }^{234} \mathrm{Th}$ to the water column when it sinks. Because (H1) was not supported by the data available for this study, nor by those from prior studies, it has been shown in grey. The dominant means of under-ice algae breakdown and sinking are unknown and therefore, two different mechanisms have been included (2A: individual or aggregated sinking algae material and 2B: massive falls of algal mats). 
A
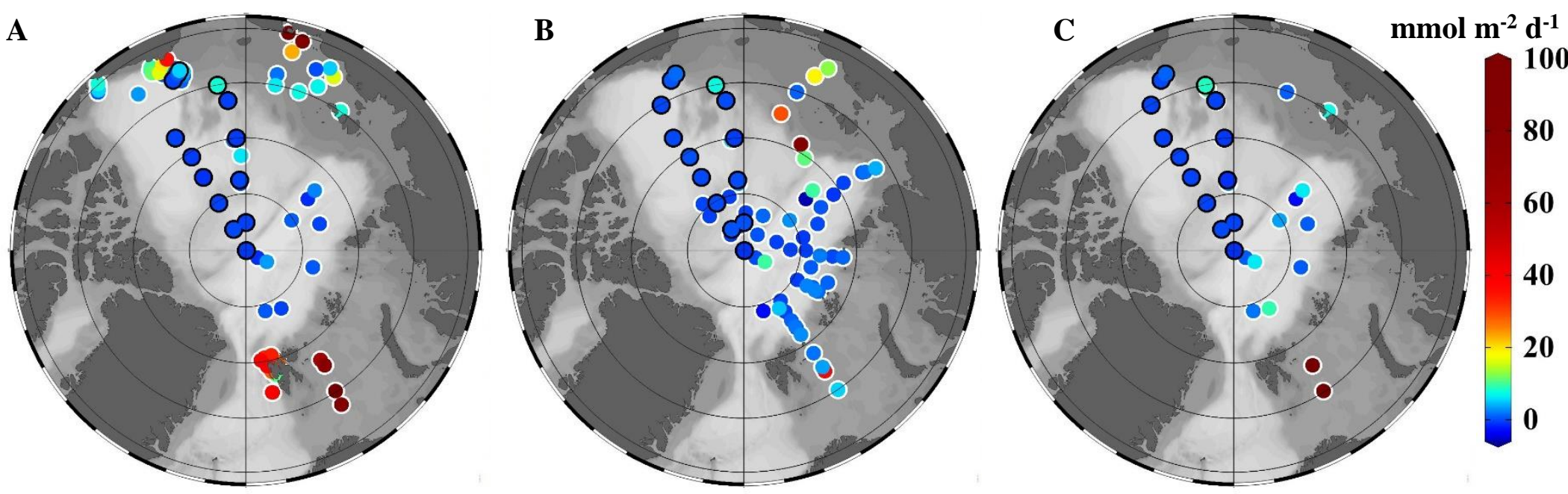

Figure 11: POC flux at three depth intervals. Fluxes from this study determined at depths of $50 \mathrm{~m}(\mathrm{~A}), 100 \mathrm{~m}$ (B), and $150 \mathrm{~m}(\mathrm{C})$ are shown with black circles. Data from historical studies were included when the fluxes that were reported fell into three depth zones: $25 \mathrm{~m}$ to $50 \mathrm{~m}, 76 \mathrm{~m}$ to $100 \mathrm{~m}$, and 126 to $150 \mathrm{~m}$ (Amiel et al., 2002; Amiel and Cochran, 2008; Baskaran et al., 2003; Cai et al., 2010; Chen et al., 2003; Cochran et al., 1995; Coppola et al., 2005; Lalande et al., 2007; Lepore et al., 2007; Ma et al., 2005; Moran et al., 2005, 1997; Moran and Smith, 2000; Roca-marti et al., 2016; Trimble et al., 2004; Trimble and Baskaran, 2005; Yu et al., 2012). Five historical points from shelf regions and in the Barents Sea had fluxes $>100 \mathrm{mmolC} \mathrm{m}^{-2} \mathrm{~d}^{-1}$, but the scale was set at $100 \mathrm{mmol} \mathrm{m}^{-2} \mathrm{~d}^{-1}$ to shown more of the variability in the lower flux range. 


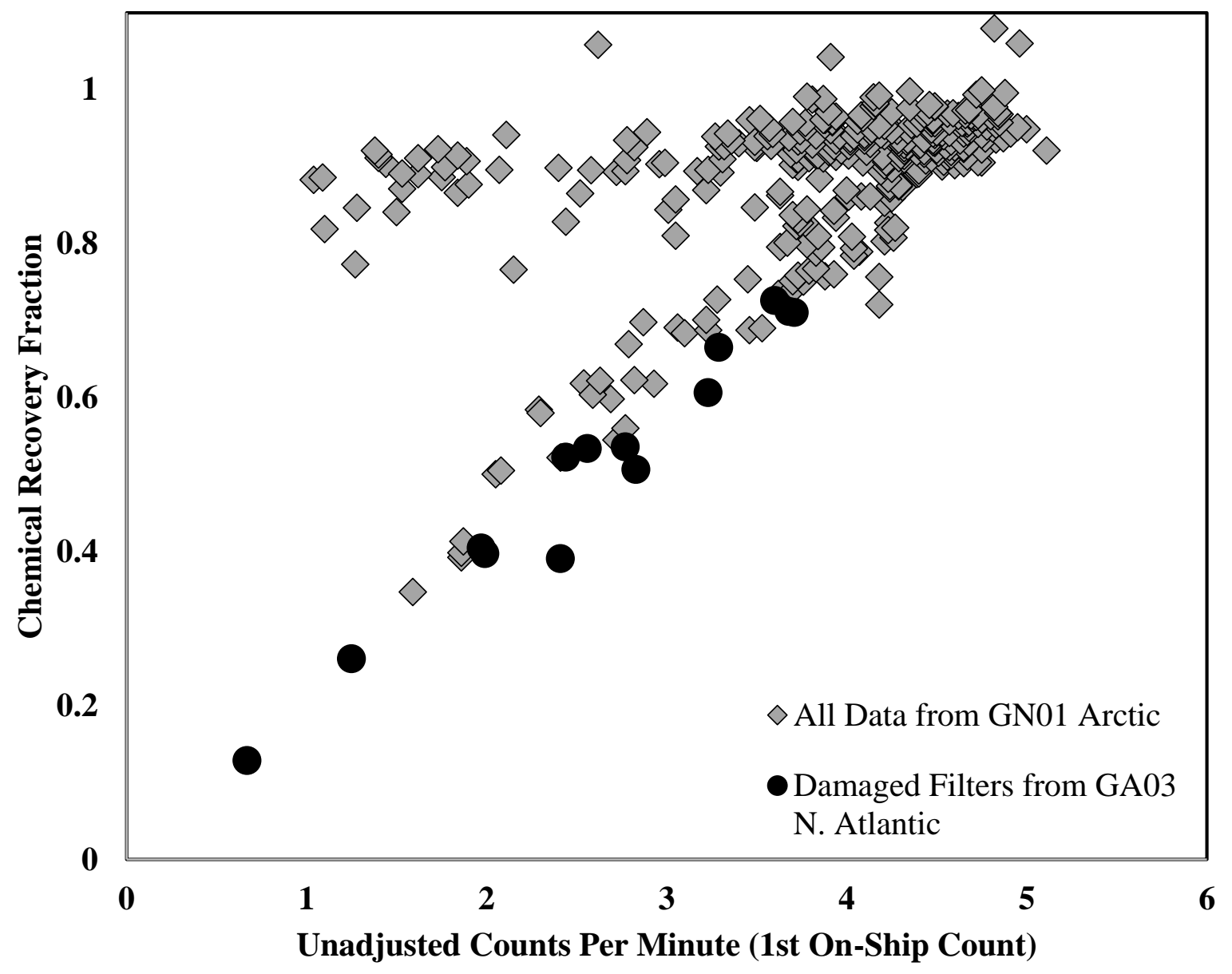

Supplemental Figure 1: CPM for total ${ }^{234} \mathrm{Th}$ samples versus chemical recovery. All total ${ }^{234} \mathrm{Th}$ data are shown with the raw counts per minute from the first of two, on-ship counts. Damaged or broken filters from a prior cruise that utilized the same $4 \mathrm{~L}$ technique and ICP-MS recovery method are shown to illustrate the effect of a known loss of volume from the $4 \mathrm{~L}$ sample. 
Th Recovery Fraction

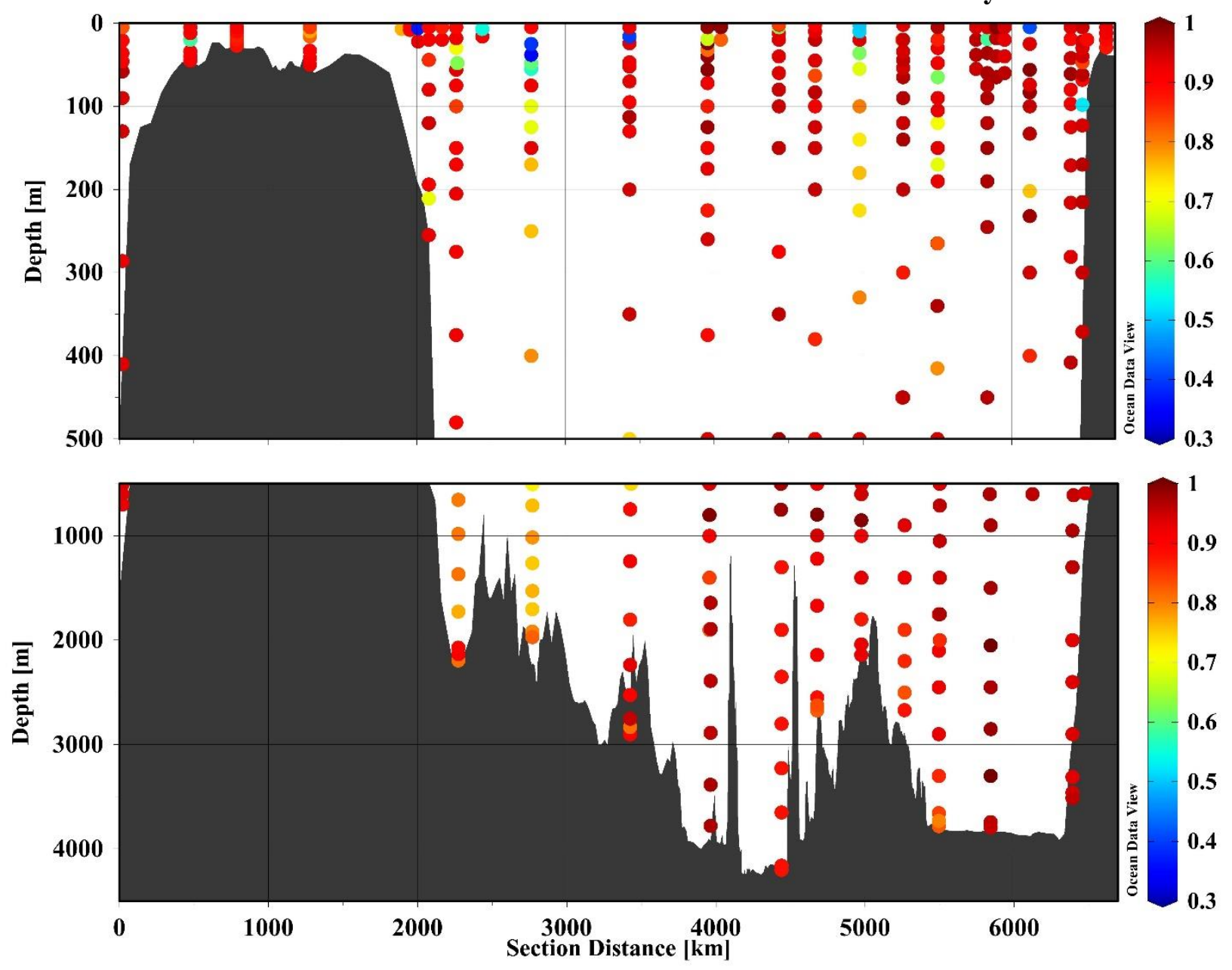

Supplemental Figure 2: Chemical recoveries by location for all total ${ }^{234} \mathbf{T h}$ samples. Reoveries under $40 \%$ were considered to be of poor quality. Distance along the cruise transect is used here from station $1(0 \mathrm{~km})$ to station $66(\sim 6500 \mathrm{~km})$. 


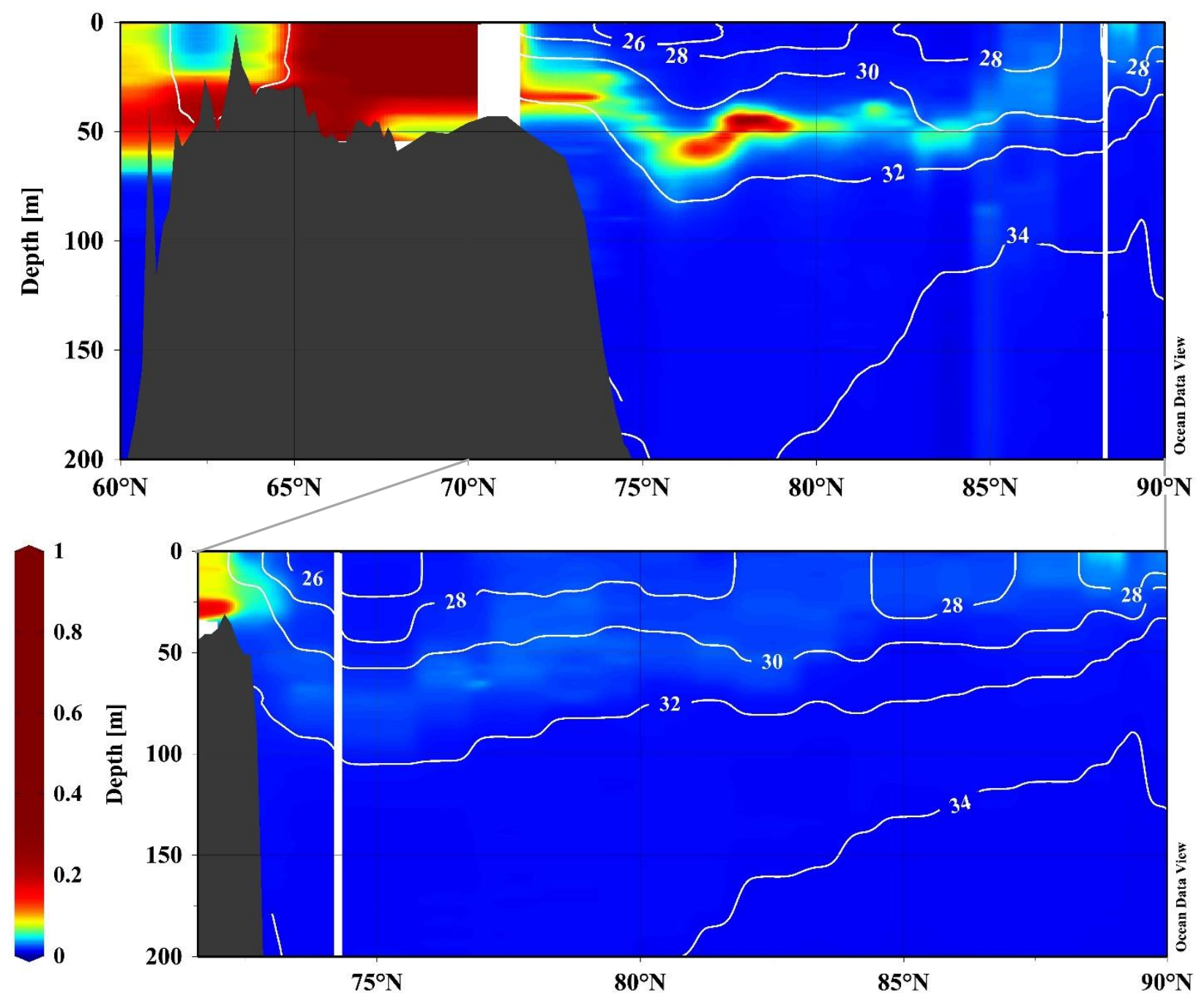

Supplemental Figure 3: Fluorescence data with salinity contours for the NB (upper) and SB (lower) transects. CTD data from all stations were used here, including repeat hydrography stations not sampled for ${ }^{234} \mathrm{Th}$. Regions with insufficient data are masked (white). 


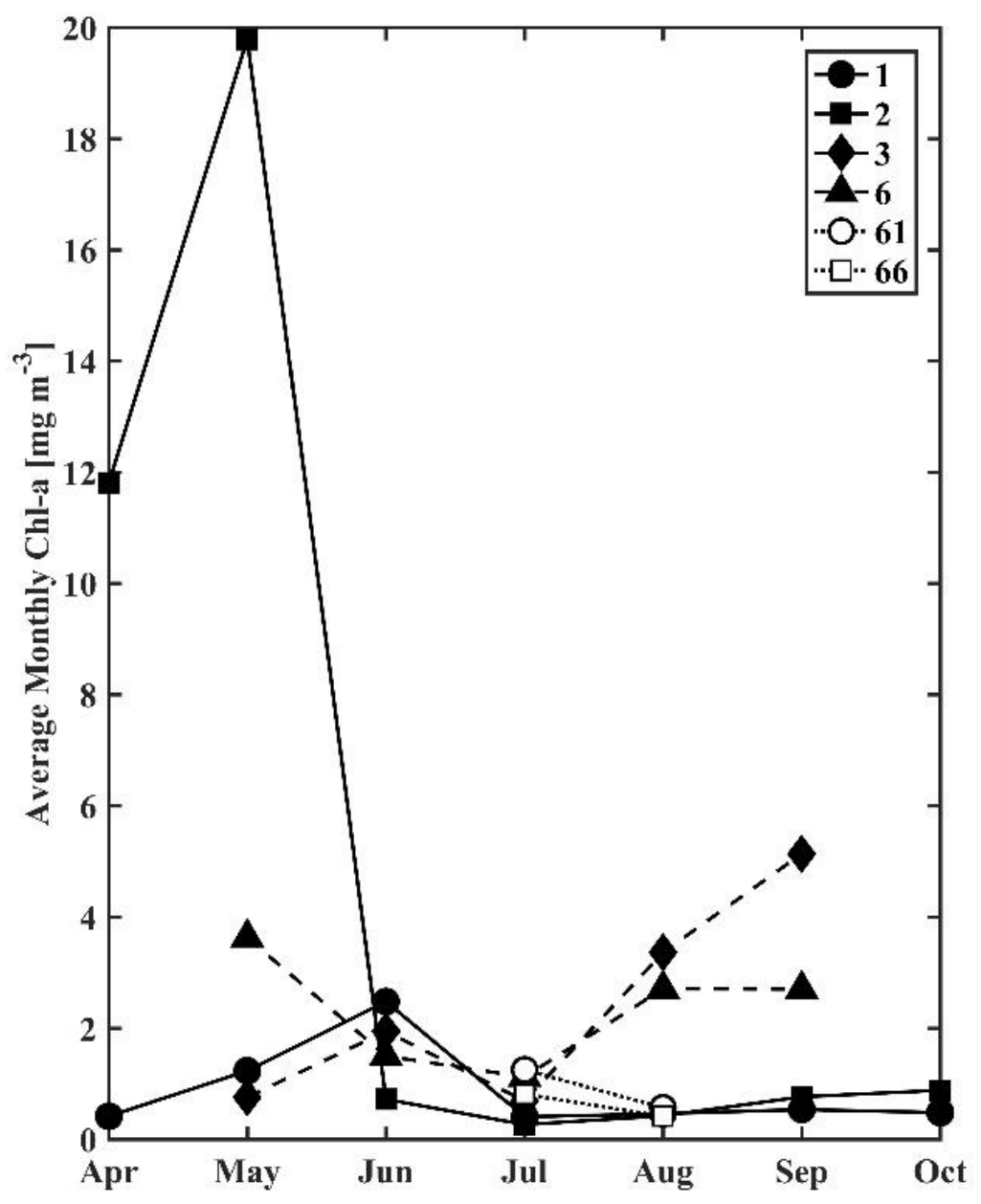

Supplemental Figure 4: Average monthly Chl-a concentrations at station locations. Concentrations are shown at stations which had at least 2 months of data from April to October 2015 to indicate conditions prior to and through station occupation dates from mid-August to midOctober. MODIS-Aqua data was obtained from https://oceandata.sci.gsfc.nasa.gov/MODISAqua/Mapped/Monthly/9km/ (Hu et al., 2012). 

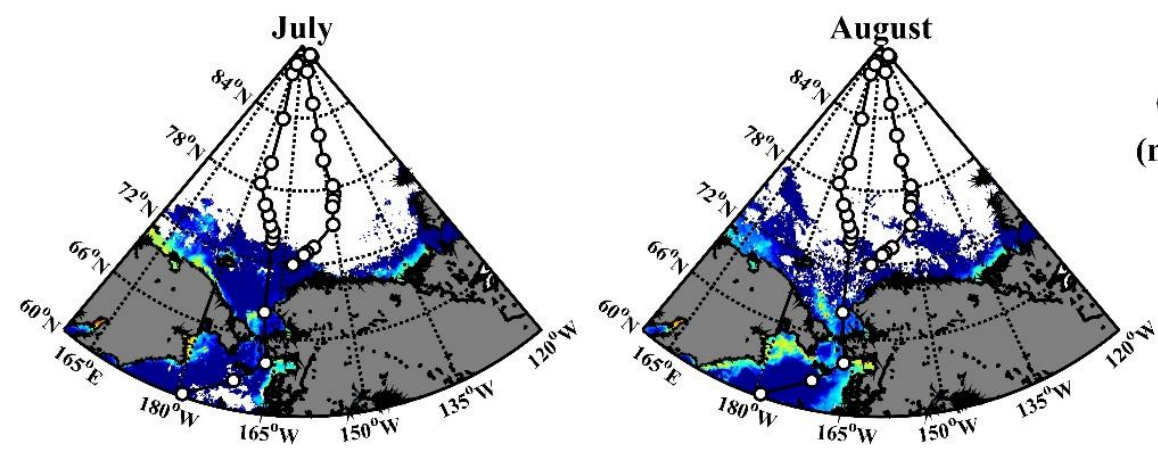

Chl A
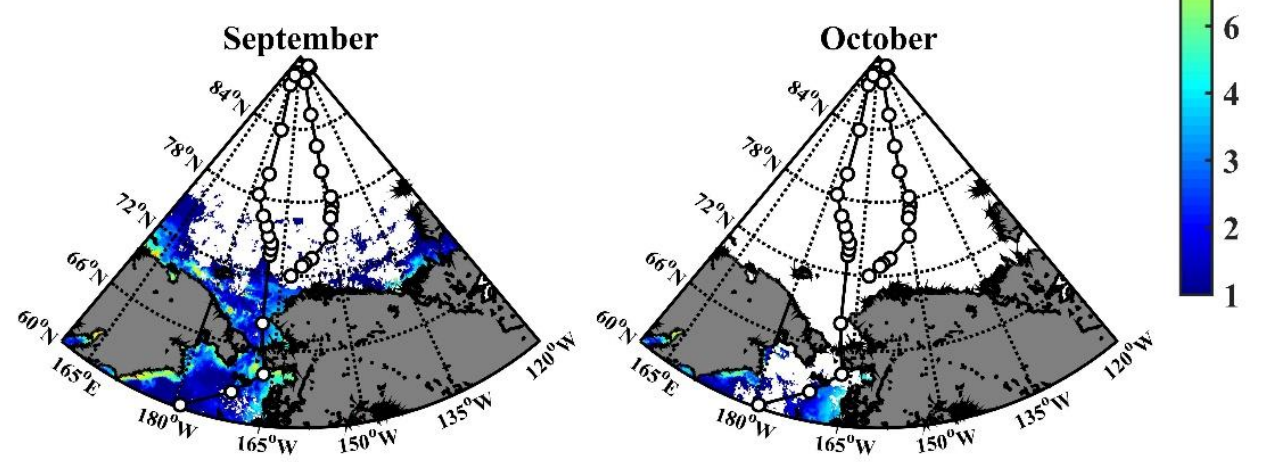

Supplemental Figure 5: Average monthly Chl-a concentrations for the Western Arctic. All available monthly data is shown for the July to October period and white indicates no data. MODIS-Aqua data was obtained from https://oceandata.sci.gsfc.nasa.gov/MODISAqua/Mapped/Monthly/9km/ (Hu et al., 2012). 

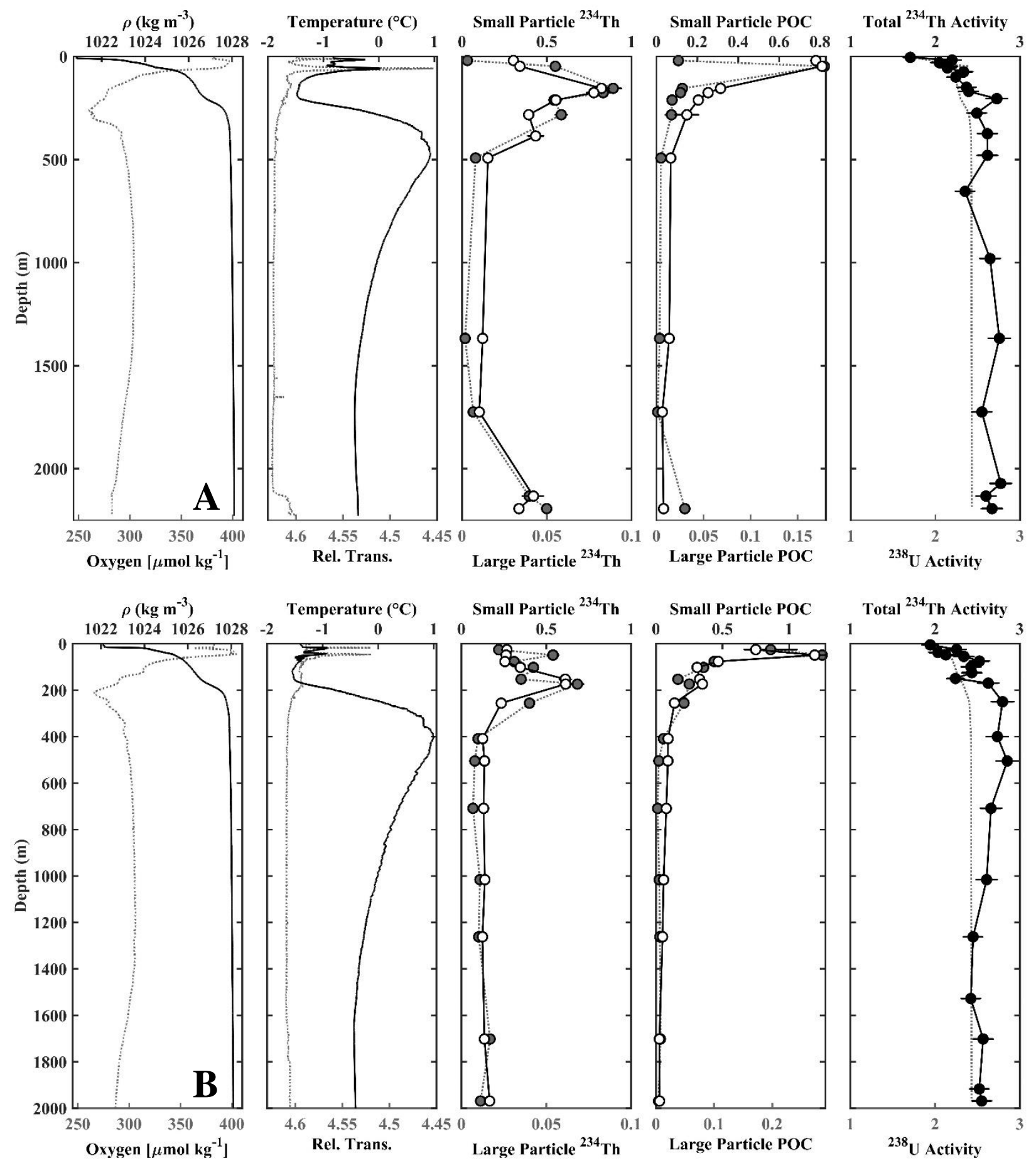

Supplemental Figure 6: Physical and geochemical data from three NB stations with observed ${ }^{234}$ Th excesses. Hydrographic data $\left(1^{\text {st }}\right.$ and $2^{\text {nd }}$ panels $)$, particulate ${ }^{234} \mathrm{Th}$ activities $\left(3^{\text {rd }}, \mathrm{dpm} \mathrm{L}{ }^{-1}\right)$, POC concentrations $\left(4^{\text {th }}, \mu \mathrm{M}\right)$, and total ${ }^{234} \mathrm{Th}$ activities $\left(5^{\text {th }}, \mathrm{dpm} \mathrm{L}^{-1}\right)$ for stations 14 (A), 19 (B), 26 (C), and 46 (D) are shown. Station 46 was included to illustrate the relative magnitude of the ${ }^{234} \mathrm{Th}$ excesses on the SB transect, most of which were within uncertainties of the ${ }^{238} \mathrm{U}$ activities. Black solid lines correspond to the upper axes and dashed grey lines to the lower axes. 

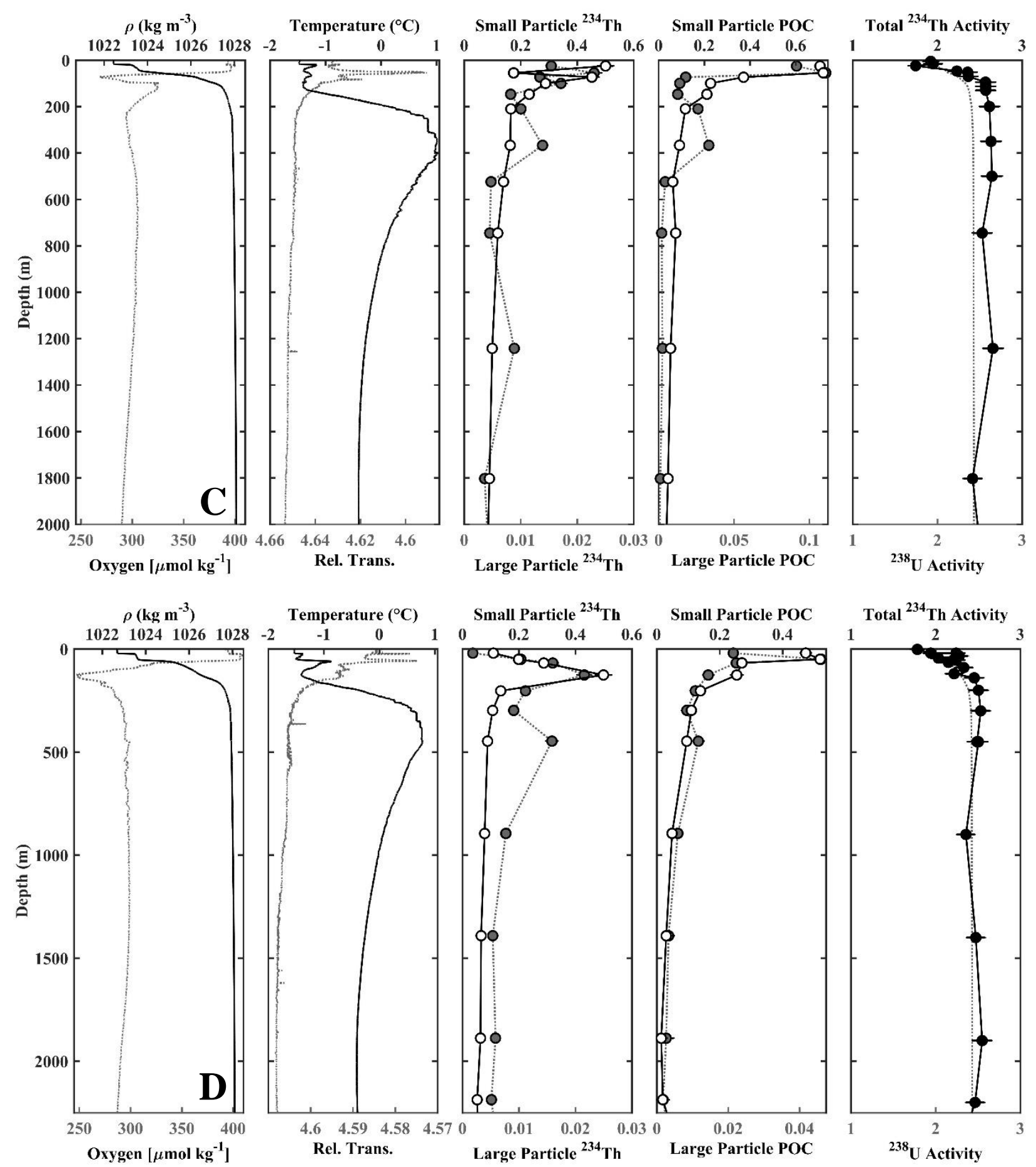

Supplemental Figure 6: Physical and geochemical data from three NB stations with observed ${ }^{234}$ Th excesses. Hydrographic data $\left(1^{\text {st }}\right.$ and $2^{\text {nd }}$ panels $)$, particulate ${ }^{234} \mathrm{Th}$ activities $\left(3^{\text {rd }}, \mathrm{dpm} \mathrm{L}^{-1}\right)$, POC concentrations $\left(4^{\text {th }}, \mu \mathrm{M}\right)$, and total ${ }^{234} \mathrm{Th}$ activities $\left(5^{\text {th }}, \mathrm{dpm} \mathrm{L}^{-1}\right)$ for stations 14 (A), 19 (B), 26 (C), and 46 (D) are shown. Station 46 was included to illustrate the relative magnitude of the ${ }^{234} \mathrm{Th}$ excesses on the SB transect, most of which were within uncertainties of the ${ }^{238} \mathrm{U}$ activities. Black solid lines correspond to the upper axes and dashed grey lines to the lower axes. 


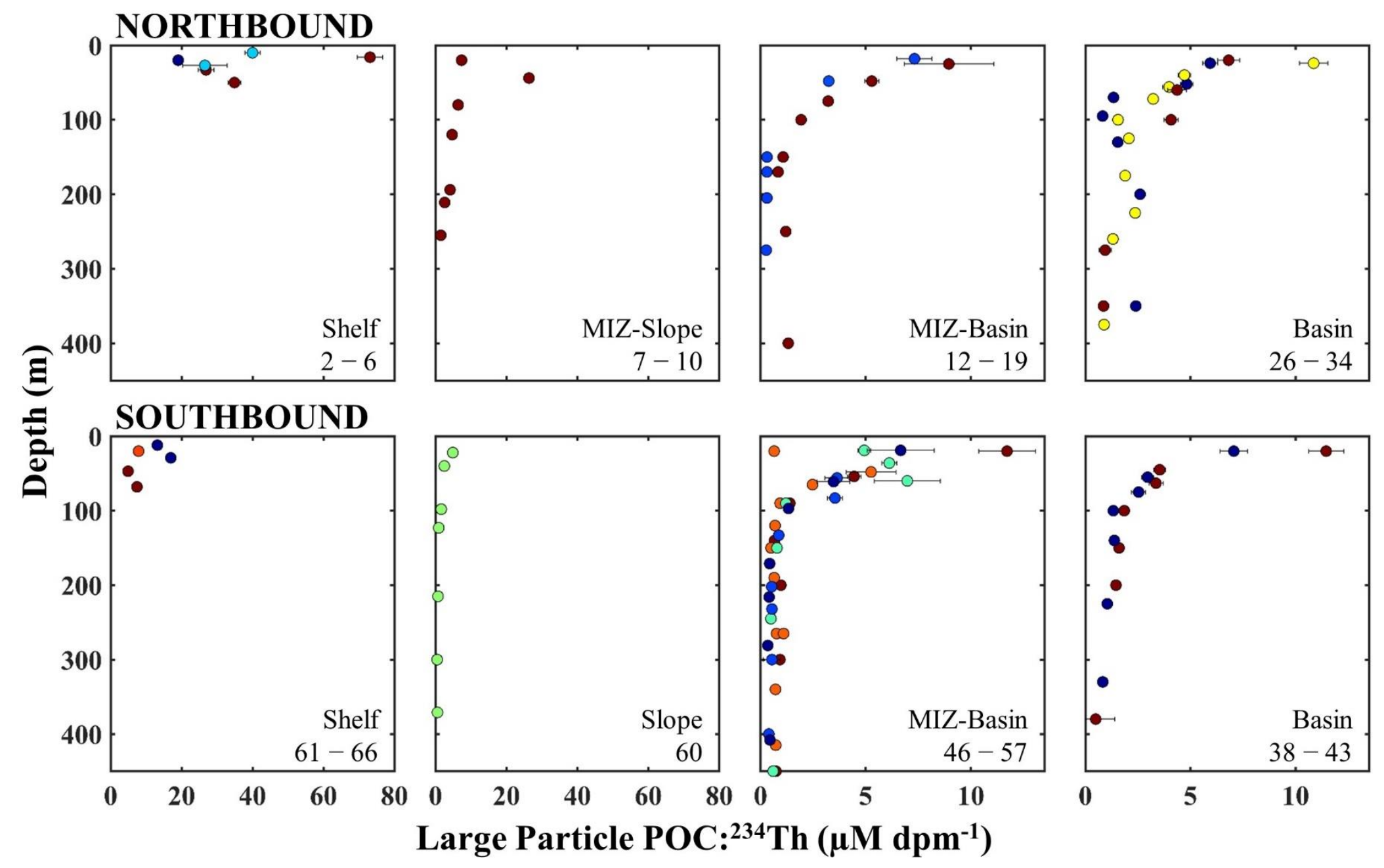

Supplemental Figure 7: POC: ${ }^{234}$ Th by region. Random color assignments are used to distinguish individual stations within a group and a larger scale was used for the shelf and slope panels to include high ratios observed in the upper $100 \mathrm{~m}$ at these locations. 


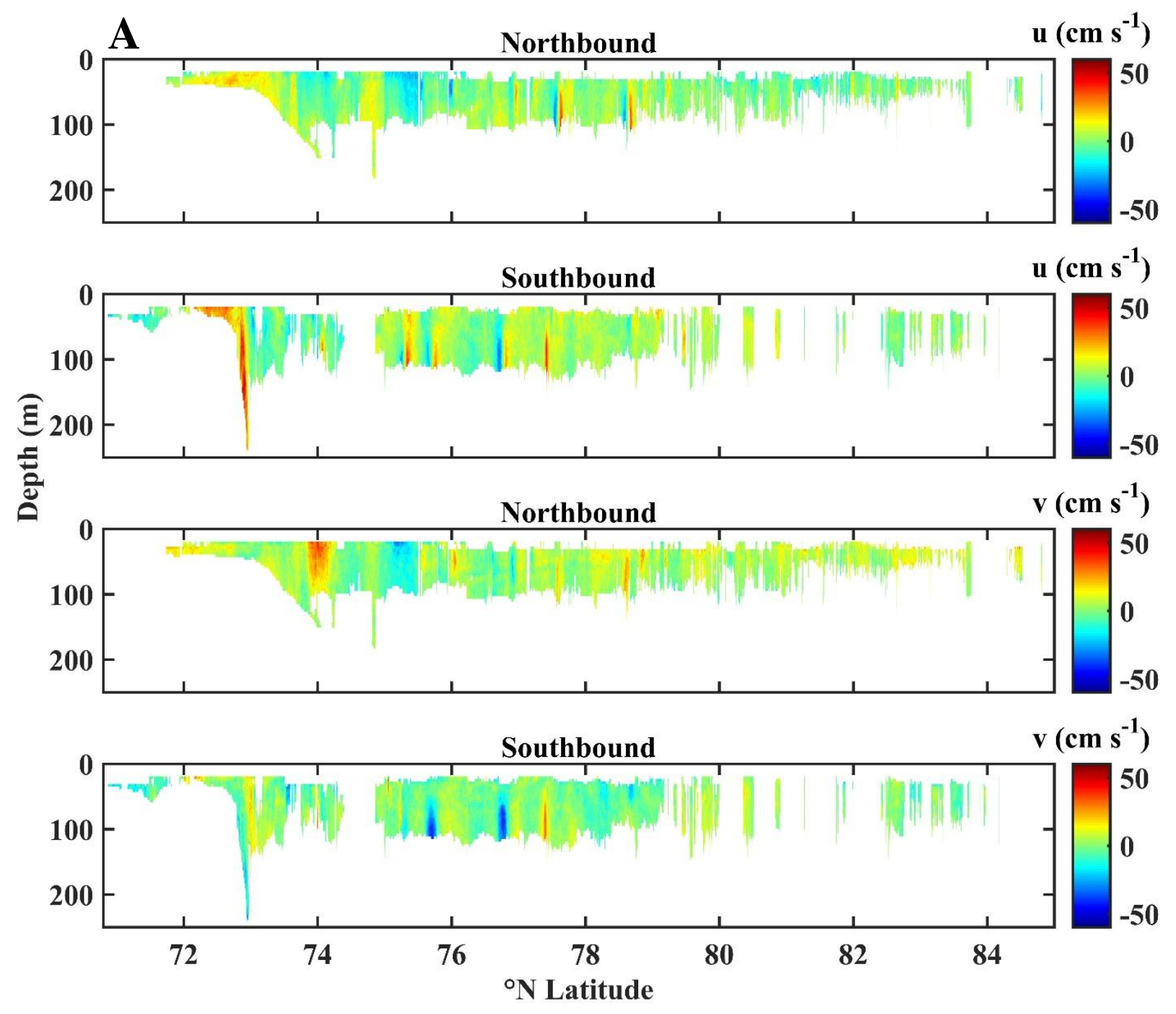

Supplemental Figure 8: ADCP velocity data. De-tided shallow (150 hertz) ADCP velocities in the N-S direction (v) and the E-W direction (u) are shown in 8A, (last downloaded 08/2017 (http://science.whoi.edu/users/seasoar/hly1502/). Positives indicate northward and eastward currents. Raw deep ( 75 hertz) ADCP velocities are shown in $8 \mathrm{~B}$ and $8 \mathrm{C}$ for the latitudes shown and with the same sign conventions (figures directly downloaded from https://currents.soest.hawaii.edu/clivar_co2/hly1502/os75nb/webpy/index.html 10/2017). Note that $8 \mathrm{~A}$ is in units of $\mathrm{cm} \mathrm{s}^{-1}$ and $8 \mathrm{~B}$ and $8 \mathrm{C}$ are in $\mathrm{m} \mathrm{s}^{-1}$. All depths are in $\mathrm{m}$. 


\section{B}

hly1502 os75nb

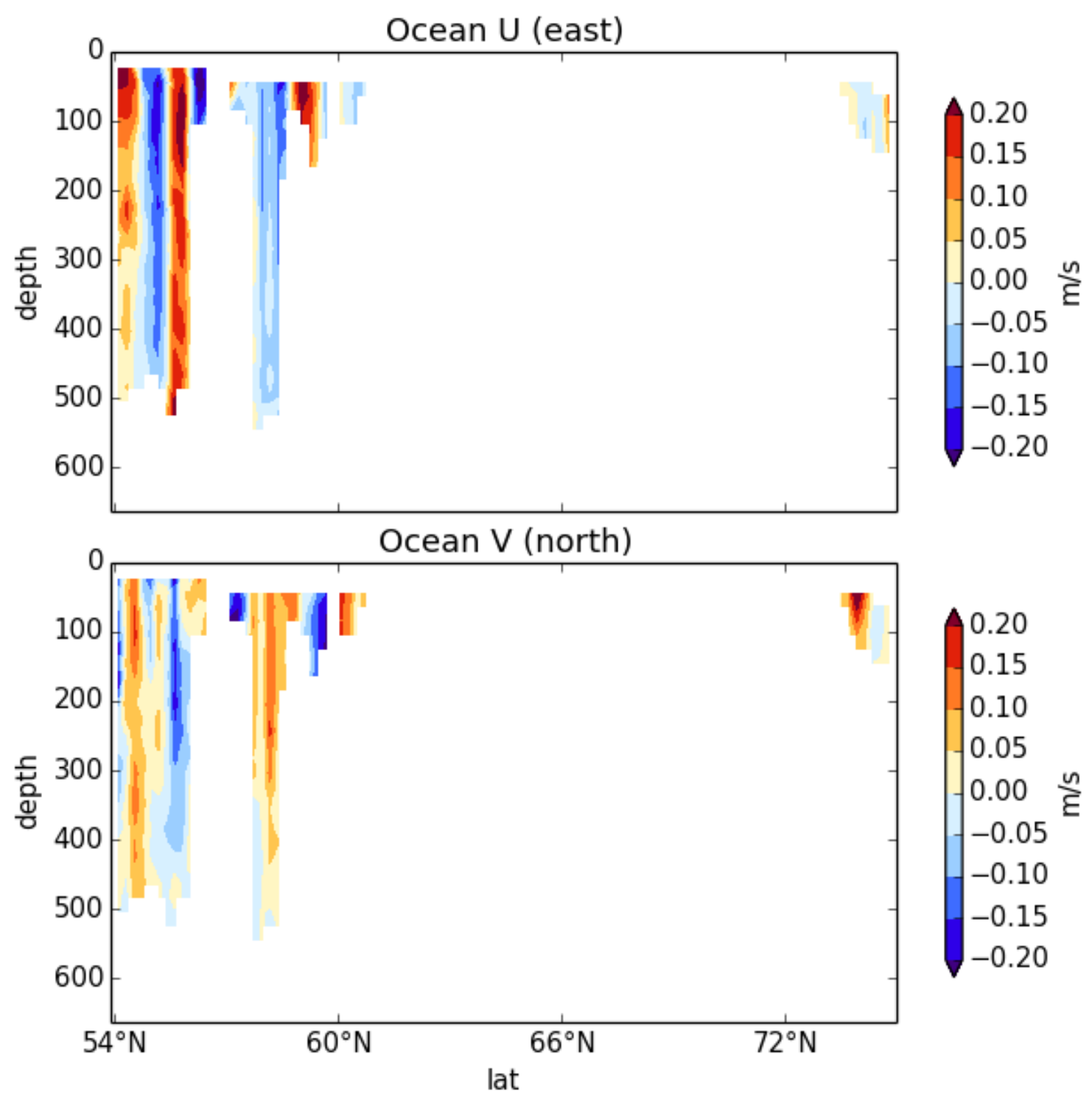

Supplemental Figure 8: ADCP velocity data. De-tided shallow (150 hertz) ADCP velocities in the N-S direction (v) and the E-W direction (u) are shown in 8A, (last downloaded 08/2017 (http://science.whoi.edu/users/seasoar/hly1502/). Positives indicate northward and eastward currents. Raw deep (75 hertz) ADCP velocities are shown in 8B and 8C for the latitudes shown and with the same sign conventions (figures directly downloaded from https://currents.soest.hawaii.edu/clivar_co2/hly1502/os75nb/webpy/index.html 10/2017). Note that $8 \mathrm{~A}$ is in units of $\mathrm{cm} \mathrm{s}^{-1}$ and $8 \mathrm{~B}$ and $8 \mathrm{C}$ are in $\mathrm{m} \mathrm{s}^{-1}$. All depths are in $\mathrm{m}$. 


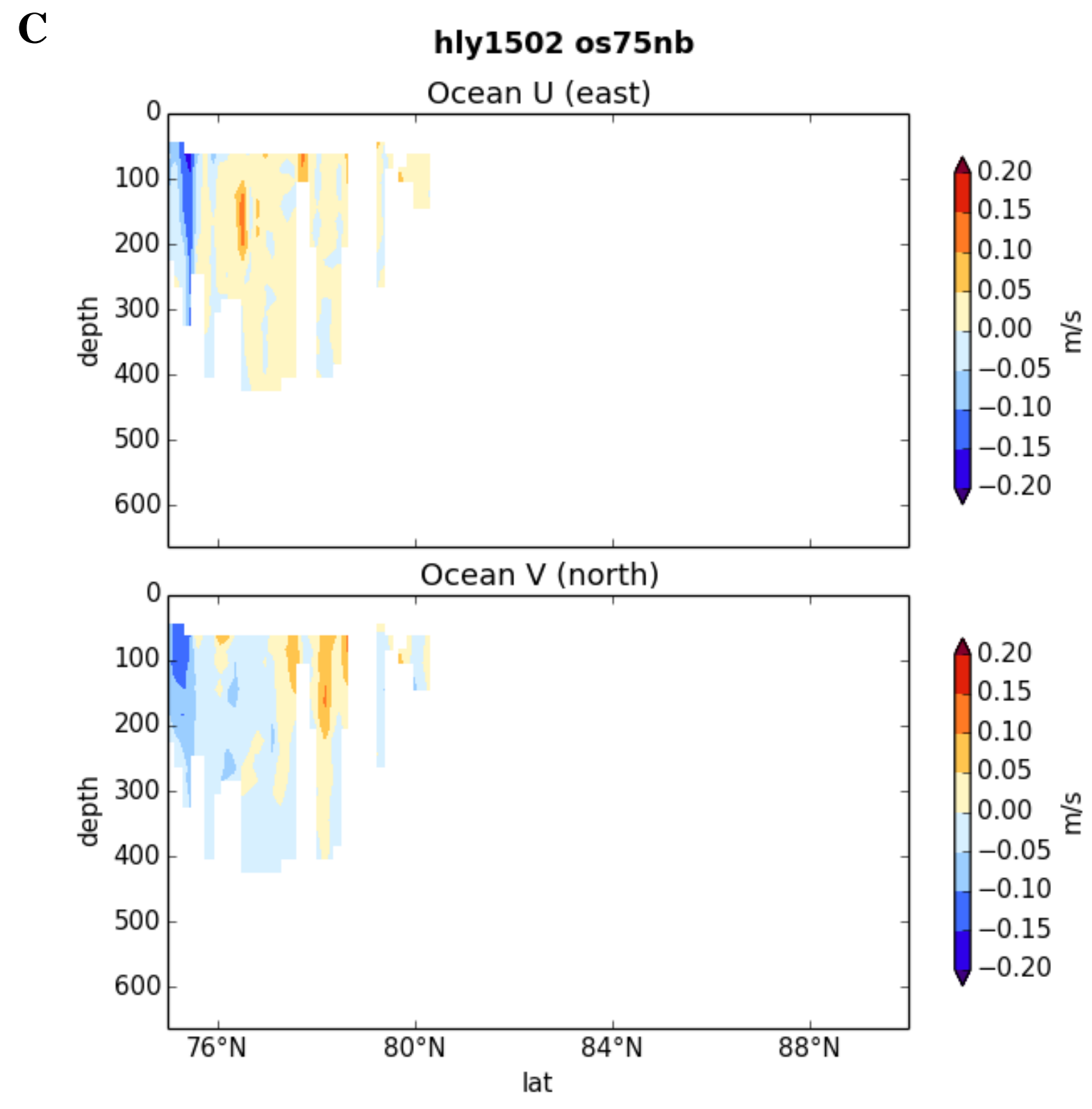

Supplemental Figure 8: ADCP velocity data. De-tided shallow (150 hertz) ADCP velocities in the N-S direction (v) and the E-W direction (u) are shown in 8A, (last downloaded 08/2017 (http://science.whoi.edu/users/seasoar/hly1502/). Positives indicate northward and eastward currents. Raw deep (75 hertz) ADCP velocities are shown in 8B and 8C for the latitudes shown and with the same sign conventions (figures directly downloaded from https://currents.soest.hawaii.edu/clivar_co2/hly1502/os75nb/webpy/index.html 10/2017). Note that $8 \mathrm{~A}$ is in units of $\mathrm{cm} \mathrm{s}^{-1}$ and $8 \mathrm{~B}$ and $8 \mathrm{C}$ are in $\mathrm{m} \mathrm{s}^{-1}$. All depths are in $\mathrm{m}$. 
Chapter 5:

Concluding remarks and future directions 
Since 2010, three major U.S. GEOTRACES campaigns have been completed in three separate ocean basins (Fig. 1), two of which have been utilized in this thesis. Although the focus of these campaigns has been to constrain the budgets of trace metals in the global ocean, they have provided a unique opportunity to utilize the ${ }^{234} \mathrm{Th}^{238} \mathrm{U}$ method to expand our knowledge of the global BCP. This thesis has specifically improved our understanding of the BCP by addressing aspects of a few key questions. First, the questions of 'how much' and 'where' were answered with regard to the processes of export and remineralization in the upper $400 \mathrm{~m}$ along two basin-scale transects in the Southeastern Pacific and the Western Arctic Ocean. Second, factors impacting the BCP and the magnitude of export and remineralization in these regions were examined in an effort to answer 'why'. These factors included oxygen, macro- and micronutrient availability, phytoplankton community dominance, productivity, upwelling, and ice cover. In the case of the Pacific transect, a specific aspect was investigated in more detail: the distribution and cycling of biologicallyrelevant trace metals, $\mathrm{Fe}, \mathrm{Mn}, \mathrm{Cd}$, and Co. Lastly, measurements from prior studies, this thesis, and future work were synthesized in order to address the question of how the BCP may be shifting regionally and globally with climate change. The progress that this thesis has made towards answering these questions in the Southeastern Pacific and the Western Arctic Ocean are summarized below. References to any remaining questions and recommendations for continued work are also outlined.

\subsection{Export and Remineralization of POC across the Peruvian OMZ}

Regarding the improved application of ${ }^{234} \mathrm{Th}$ methodology itself, Chapter 2 illustrated the importance of assessing and incorporating physical parameters in ${ }^{234} \mathrm{Th}$ flux calculations in coastal regions. POC fluxes could have been underestimated by a factor of two to four if the influence of upwelling was not account for in our ${ }^{234} \mathrm{Th}$-based estimates and this directly impacted our interpretation of the strength and efficiency of the BCP for these coastal regions. When possible, in areas with known upwelling or strong lateral currents, efforts should be made to sample along anticipated gradients in ${ }^{234} \mathrm{Th}$. This chapter also illustrated the importance of high resolution sampling through the upper $400 \mathrm{~m}$ to our knowledge of remineralization. From Kwon et al.'s (2009) work, it is clear that small differences in the remineralization length scale can have widereaching implications for atmospheric $\mathrm{CO}_{2}$ and without the high resolution sampling in this study, we would not have been able to accurately assess the amount of carbon that is being effectively sequestered across the $>7500 \mathrm{~km}$ transect. Prior studies of ${ }^{234} \mathrm{Th}$ have often focused on quantifying 
export alone and/or sampling solely in the upper $100 \mathrm{~m}$, but the processes in the water column just below the base of the euphotic zone (Ez) are equally as important when evaluating the efficiency of the BCP.

The range in the strength (0.02 to 0.5$)$ and transfer efficiency (0.1 to 1$)$ of the BCP observed across this transect was greater than has been reported for other regions of the world's ocean. This is perhaps not surprising considering the large gradients in oxygen, productivity, Ez depth, and nutrients across the transect, as well as the fact that few estimates pairing strength and efficiency using Ez as a boundary layer have been published. The amount of POC exported at $100 \mathrm{~m}$ below Ez relative to the amount of surface production was higher in the Shelf (7\% to $13 \%$ POC remaining) compared to the rest of the transect, but consistent with higher NPP, upwelling, higher nutrient concentrations, and a microplankton-dominated surface community. The $\mathrm{BCP}$ at the lowproductivity, picoplankton-dominated gyre stations had relative low strength, but high efficiency (not much attenuation at depth), supporting prior studies which have suggested a decreased attenuation of POC through OMZs (Roullier et al., 2014; Van Mooy et al., 2002). However, within the offshore ODZ region, the percent of POC exported at $100 \mathrm{~m}$ below Ez ranged from $0.4 \%$ to $7 \%$ of surface production and transfer efficiencies ranged from 0.1 to 0.9 . The reason for these large ranges is unknown and reflects our lack of understanding of the impact of ODZs on carbon cycling. Further research is warranted to determine if these growing, functionally anoxic cores could actually lead to greater net attenuation of POC.

One next step would be to confirm whether these remineralization features are permanent (not seasonal) and whether they exist in all low oxygen regions globally. We have concluded here that the expansion of ODZs and OMZs worldwide could have large implications for the global sequestration of carbon if OMZs in fact show lower rates of POC attenuation compared to other oxygenated regions of the global ocean. However, this assumes that these remineralization features are relatively consistent during the year in magnitude and extent, and that the characteristics of the Peruvian OMZ are similar to those of other OMZs globally. Further study of the Peruvian OMZ is needed outside of the austral spring to confirm this (and/or the application of other, longer-lived radioisotopes) and additional work should be performed using the same ${ }^{234}$ Th-based methodologies (and depth resolution) in other OMZs. 
Lastly, it appeared that community structure in the Peruvian OMZ, which was to an extent governed by oxygen levels in the water column, greatly affected particle cycling and the magnitude of remineralization near the upper boundary of the ODZ, and thus, more research is need on what specific factors contribute to the presence of these remineralization features. Without more biological data it is difficult to classify the types of microbes and plankton that are contributing most to the local remineralization. However, the prior studies detailed in Chapter 2 and results from this campaign (Ohnemus et al., 2016) suggest that small plankton (bacteria) are dominant and that both photoautotrophic and chemoautotrophic processes are supporting these communities. We have suggested that shallow remineralization could be largely driven by anaerobic denitrification east of $90^{\circ} \mathrm{W}$ and aerobic respiration west of this point, but further verification is needed to confirm these processes and the associated organisms. As was detailed in both Chapters 2 and 3, particulate trace metal cycling at and below the ODZ boundary is complex and communities here could play an integral role in controlling the availability of elements like Fe, which can be a limiting factor on local productivity (Hutchins et al., 2002).

\subsection{Trace metal cycling across the Peruvian OMZ}

As detailed in Chapter 3, very few upper ocean estimates of trace metal fluxes exist for Fe, $\mathrm{Mn}, \mathrm{Cd}$, and $\mathrm{Co}$, and even fewer have successfully utilized the ${ }^{234} \mathrm{Th}^{238} \mathrm{U}$ method and done so on a scale comparable to this study. There are no published large-scale studies utilizing the ${ }^{234} \mathrm{Th}^{238} \mathrm{U}$ method with co-sampled sinking E: ${ }^{234} \mathrm{Th}$ ratios for Co and Mn fluxes, and only one for Cd flux (Smith et al., 2014). Fe flux has been studied more often with the ${ }^{234} \mathrm{Th}^{238} \mathrm{U}$ method, but efforts of this magnitude have only been performed off the coast of North America and southeast of Africa in the Southern Ocean. Chapter 3 represents a significant addition to this field and shows that the ${ }^{234} \mathrm{Th}^{-238} \mathrm{U}$ method can be successfully applied to trace metals. These estimates are first and foremost of interest to trace metal scientists who are working to reproduce the basic distributions of major trace metals in the full water column. These flux and residence estimates for the upper $200 \mathrm{~m}$ are necessary for understanding both surface food webs and mesopelagic community dynamics, which depend on the rain of materials from above. However, because fluxes reported here will help close the budgets of trace metals in the upper ocean, this in turn will lead to improved estimates from global biogeochemical models that seek to incorporate elements, such as iron, which are of interest to climate modelers. 
Co and Cd flux profiles behaved very similarly to those of POC and P in the upper $200 \mathrm{~m}$, showing high fluxes in the productive coastal shelf region, lower fluxes with increasing distance offshore, and generally lower fluxes with depth. Although there was some indication of particle scavenging of these elements playing a role below $200 \mathrm{~m}$ in the gyre, production and remineralization strongly influenced the fluxes of these two elements in the southeastern tropical Pacific. The results suggest that ${ }^{234} \mathrm{Th}$ could be successfully used for these and other nutrient-like trace metals. It is possible that $\mathrm{E}:{ }^{234}$ Th-depth relationships for sinking particles similar to those used for POC (Black et al., 2017; Owens et al., 2015) could be determined for Cd and Co, which would significantly reduce the amount of large particle measurements required, however further comparison with results from other GEOTRACES efforts are needed to better constrain the range in global variability for the upper $400 \mathrm{~m}$.

Many questions still remain about the particle dynamics of Mn, both within the Peruvian OMZ and throughout the rest of the world's ocean. Because Mn distributions and fluxes were very much controlled by redox conditions unique to the OMZ, additional studies in more oxic ocean basins that look at the possible range in $\mathrm{Mn}:{ }^{234} \mathrm{Th}$ on both large and small particles, Mn flux, and $\mathrm{Mn}$ residence times would be beneficial to our continued understanding of Mn cycling in the upper $400 \mathrm{~m}$. Both particulate (this study) and dissolved (Sanial et al., 2017) flux estimates suggest that lateral transport of Mn from coastal OMZs could be a significant source of $\mathrm{Mn}$ to open ocean communities. We hope that global biogeochemical models that have struggled to incorporate $\mathrm{Mn}$ and reproduce observed $\mathrm{Mn}$ distributions in the Pacific will improve once they incorporate these upper ocean particulate flux estimates and those derived for dissolved $\mathrm{Mn}$.

Although the flux and residence time estimates for Fe are perhaps the most sought-after, the observed trends in these parameters were the most difficult to interpret and constrain for Fe. $\mathrm{Fe}:{ }^{234} \mathrm{Th}$ and Fe flux appeared to be less impacted by the presence of the OMZ than Mn and more influenced by sources (i.e. dust and lateral inputs) and a combination of authigenic, lithogenic and biological processes, whose influences were difficult to separate with the data reported here. The variability in all Fe-related parameters observed across the entire transect suggest that continued high resolution sampling is necessary for ${ }^{234}$ Th-dervied flux estimates and a process study with paired sampling of ${ }^{234} \mathrm{Th}$ and Fe during dust deposition events (i.e. dust collection and sampling of surface waters) could provide valuable insight into some of the Fe: ${ }^{234} \mathrm{Th}$ and Fe flux profile variability. 


\subsection{Export and remineralization of $\mathrm{POC}$ in a changing Arctic}

The surface data ( $0 \mathrm{~m}$ to $200 \mathrm{~m}$ ) included in Chapter 4 support historical results from the Arctic Ocean, which have shown high productivity across the shallow coastal shelves during the spring and summer months and a low productivity, generally inefficient BCP in the central Arctic basin. Continued measurement of export in the central basin is important for monitoring the impacts of climate change on the Arctic BCP and without satellite estimates for NPP, paired measurements of both NPP and export are vital for accurately determining changes in the efficiency of the BCP. ${ }^{234} \mathrm{Th}$ data below $200 \mathrm{~m}$ on the northbound Arctic transect suggest that the BCP may be transporting more POC to depth than previously thought, but we will need other particulate tracers to help confirm the extent of this feature in space and time, as well as to examine the source of the excess ${ }^{234} \mathrm{Th}$. Because of the extraordinary spatial resolution provided by the work detailed in Chapter 4, a myriad of new avenues to investigate have been brought forth and these outstanding questions are summarized below.

Of all the regions of the global ocean, those that are permanently and seasonally ice covered appear to be the least understood with respect to the cycling of carbon and the local processes impacting the BCP. Numerous questions remain to be answered before the Arctic Ocean BCP can be incorporated into current global biogeochemical and predictive climate models with confidence. For example, how prevalent, pervasive, and impactful are under ice blooms? Some in-situ and creative satellite-based estimates (Arrigo et al., 2012; Behrenfeld et al., 2016; Lowry et al., 2014) have identified these events and sought to assess their importance to the annual cycling of carbon in the Arctic Ocean, but more study is needed to refine these broad estimates. It is also unclear, but vital to our Arctic climate models, whether recent increases in NPP and the occurrence of (more) under ice blooms will directly correlate to any increase in export. Concurrent measurements of NPP and export and synoptic sampling campaigns, in particular, would provide valuable data to show whether decreasing ice cover and thickness impacts both NPP and export.

A rather important step in understanding the impact of under-ice blooms is determining the mechanisms and timescales by which sea ice-associated algae (and other communities) are released from the sea ice and potentially transported out of the sunlit upper ocean. Are single cells being released, aggregating, and sinking or do entire algal mats sink in large events as ice retreats? Data on the diversity and mobility of sea-ice associate communities into the water column, as well 
as the delivery of melt pond communities to the underlying waters have been reported (FernándezMéndez et al., 2014; Szymanski and Gradinger, 2016) and massive algae falls over the deep Arctic Ocean basin have been observed (Boetius et al., 2013). However, if large amounts of this organic material is vertically sinking throughout the Arctic Ocean, it has either been missed by the few sediment traps in the Canada Basin or has been degraded before reaching traps at $>3000 \mathrm{~m}$, where the data still support an inefficient Arctic BCP and suggest that most POC originates allochthonously or not from the overlying water column (Honjo et al., 2010; Hwang et al., 2015, 2008). More investigation into these questions could be performed using a series of shallow sediment traps under the ice and by monitoring suspended particulate matter, size-fractionated POC, and community composition during periods of ice retreat and melt.

Many of these unanswered questions about climate change-induced productivity and export changes directly impact how ${ }^{234} \mathrm{Th}$ distributions are controlled and should be interpreted in the Arctic Ocean or other ice-covered regions. These issues have been discussed at length in Chapter 4 , but in summary, the occurrence of shallow ${ }^{234}$ Th excesses appear to be a common feature in the Arctic and Antarctic Oceans and further study is needed to determine how and when these excesses can form. Time series or synoptic analyses of the ${ }^{234} \mathrm{Th}$ activities in sea-ice associated algae and the upper $200 \mathrm{~m}$ of the underlying water column through the spring, summer, and fall in a region that is seasonally covered with ice would provide valuable insight into the evolution of the ${ }^{234} \mathrm{Th}$ activity profiles throughout periods of variable ice cover and bloom cycles. 


\section{References}

Arrigo, K.R., Perovich, D.K., Pickart, R.S., Brown, Z.W., van Dijken, G.L., Lowry, K.E., Mills, M.M., Palmer, M.A., Balch, W.M., Bahr, F., Bates, N.R., Benitez-Nelson, C., Bowler, B., Brownlee, E., Ehn, J.K., Frey, K.E., Garley, R., Laney, S.R., Lubelczyk, L., Mathis, J., Matsuoka, A., Mitchell, B.G., Moore, G.W.K., Ortega-Retuerta, E., Pal, S., Polashenski, C.M., Reynolds, R.A., Schieber, B., Sosik, H.M., Stephens, M., Swift, J.H., 2012. Massive Phytoplankton Blooms Under Arctic Sea Ice. Science 336, 1408-1408. doi:10.1126/science.1215065

Behrenfeld, M.J., Hu, Y., O’Malley, R.T., Boss, E.S., Hostetler, C.A., Siegel, D.A., Sarmiento, J.L., Schulien, J., Hair, J.W., Lu, X., Rodier, S., Scarino, A.J., 2016. Annual boom-bust cycles of polar phytoplankton biomass revealed by space-based lidar. Nat. Geosci. 10, 118-122. doi:10.1038/ngeo2861

Black, E.E., Buesseler, K.O., Pike, S.M., Lam, P.J., 2017. ${ }^{234}$ Th as a tracer of particulate export and remineralization in the southeastern tropical Pacific. Mar. Chem. In Press, Corrected Proof. doi:10.1016/j.marchem.2017.06.009

Boetius, A., Albrecht, S., Bakker, K., Bienhold, C., Felden, J., Fernandez-Mendez, M., Hendricks, S., Katlein, C., Lalande, C., Krumpen, T., Nicolaus, M., Peeken, I., Rabe, B., Rogacheva, A., Rybakova, E., Somavilla, R., Wenzhofer, F., 2013. Export of Algal Biomass from the Melting Arctic Sea Ice. Science 339, 1430-1432. doi:10.1126/science.1231346

Fernández-Méndez, M., Wenzhöfer, F., Peeken, I., Sørensen, H.L., Glud, R.N., Boetius, A., 2014. Composition, buoyancy regulation and fate of ice algal aggregates in the Central Arctic Ocean. PLoS One 9. doi:10.1371/journal.pone.0107452

Honjo, S., Krishfield, R.A., Eglinton, T.I., Manganini, S.J., Kemp, J.N., Doherty, K., Hwang, J., McKee, T.K., Takizawa, T., 2010. Biological pump processes in the cryopelagic and hemipelagic Arctic Ocean: Canada Basin and Chukchi Rise. Prog. Oceanogr. 85, 137-170. doi:10.1016/j.pocean.2010.02.009

Hutchins, D.A., Hare, C.E., Weaver, R.S., Zhang, Y., Firme, G.F., DiTullio, G.R., Alm, M.B., Riseman, S.F., Maucher, J.M., Geesey, M.E., Trick, C.G., Smith, G.J., Rue, E.L., Conn, J., Bruland, K.W., 2002. Phytoplankton iron limitation in the Humboldt Current and Peru Upwelling. Limnol. Oceanogr. 47, 997-1011. doi:10.4319/lo.2002.47.4.0997

Hwang, J., Eglinton, T.I., Krishfield, R.A., Manganini, S.J., Honjo, S., 2008. Lateral organic carbon supply to the deep Canada Basin. Geophys. Res. Lett. 35, 1-5. doi:10.1029/2008GL034271

Hwang, J., Kim, M., Manganini, S.J., McIntyre, C.P., Haghipour, N., Park, J.J., Krishfield, R.A., Macdonald, R.W., McLaughlin, F.A., Eglinton, T.I., 2015. Temporal and spatial variability of particle transport in the deep Arctic Canada Basin. J. Geophys. Res. Ocean. 120, 2784-2799. doi:10.1002/2014JC010643

Lowry, K.E., van Dijken, G.L., Arrigo, K.R., 2014. Evidence of under-ice phytoplankton blooms in the Chukchi Sea from 1998 to 2012. Deep. Res. Part II Top. Stud. Oceanogr. 105, 105-117. doi:10.1016/j.dsr2.2014.03.013

Ohnemus, D.C., Rauschenberg, S., Cutter, G.A., Fitzsimmons, J.N., Sherrell, R.M., Twining, B.S., 2016. Elevated trace metal content of prokaryotic plankton communities associated with marine oxygen deficient zones. Limnol. Oceanogr. 62, 3-25. doi:10.1002/lno.10363

Owens, S.A., Pike, S., Buesseler, K.O., 2015. Thorium-234 as a tracer of particle dynamics and upper ocean export in the Atlantic Ocean. Deep. Res. II 116, 42-59. doi:10.1016/j.dsr2.2014.11.010 
Roca-marti, M., Puigcorbe, V., Rutgers van der Loeff, M., Katlein, C., Fernandez-Mendez, M., Peeken, I., Masque, P., 2016. Carbon export fluxes and export efficiency in the central Arctic during the record sea-ice minimum in 2012: a joint ${ }^{234} \mathrm{Th} /{ }^{238} \mathrm{U}$ and ${ }^{210} \mathrm{Po} /{ }^{210} \mathrm{~Pb}$ study. J. Geophys. Res. Ocean. 1-23. doi:10.1002/2016JC011736

Roullier, F., Berline, L., Guidi, L., Durrieu De Madron, X., Picheral, M., Sciandra, A., Pesant, S., Stemmann, L., 2014. Particle size distribution and estimated carbon flux across the Arabian Sea oxygen minimum zone. Biogeosciences 11, 4541-4557. doi:10.5194/bg-11-4541-2014

Sanial, V., Kipp, L.E., Henderson, P.B., Beek, P. Van, Reyss, J., Hammond, D.E., Hawco, N.J., Saito, M.A., Resing, J.A., Sedwick, P., Moore, W.S., Charette, M.A., 2017. Radium-228 as a tracer of dissolved trace element inputs from the Peruvian continental margin. Mar. Chem. doi:10.1016/j.marchem.2017.05.008

Smith, J.N., Yeats, P.A., Knowlton, S.E., Moran, S.B., 2014. Comparison of ${ }^{234} \mathrm{Th} /{ }^{238} \mathrm{U}$ and mass balance models for estimating metal removal fluxes in the Gulf of Maine and Scotian Shelf. Cont. Shelf Res. 77, 107-117. doi:10.1016/j.csr.2014.01.008

Szymanski, A., Gradinger, R., 2016. The diversity, abundance and fate of ice algae and phytoplankton in the Bering Sea. Polar Biol. 39, 309-325. doi:10.1007/s00300-015-1783-z

Van Mooy, B.A.S., Keil, R.G., Devol, A.H., 2002. Impact of suboxia on sinking particulate organic carbon: Enhanced carbon flux and preferential degradation of amino acids via denitrification. Geochim. Cosmochim. Acta 66, 457-465. 


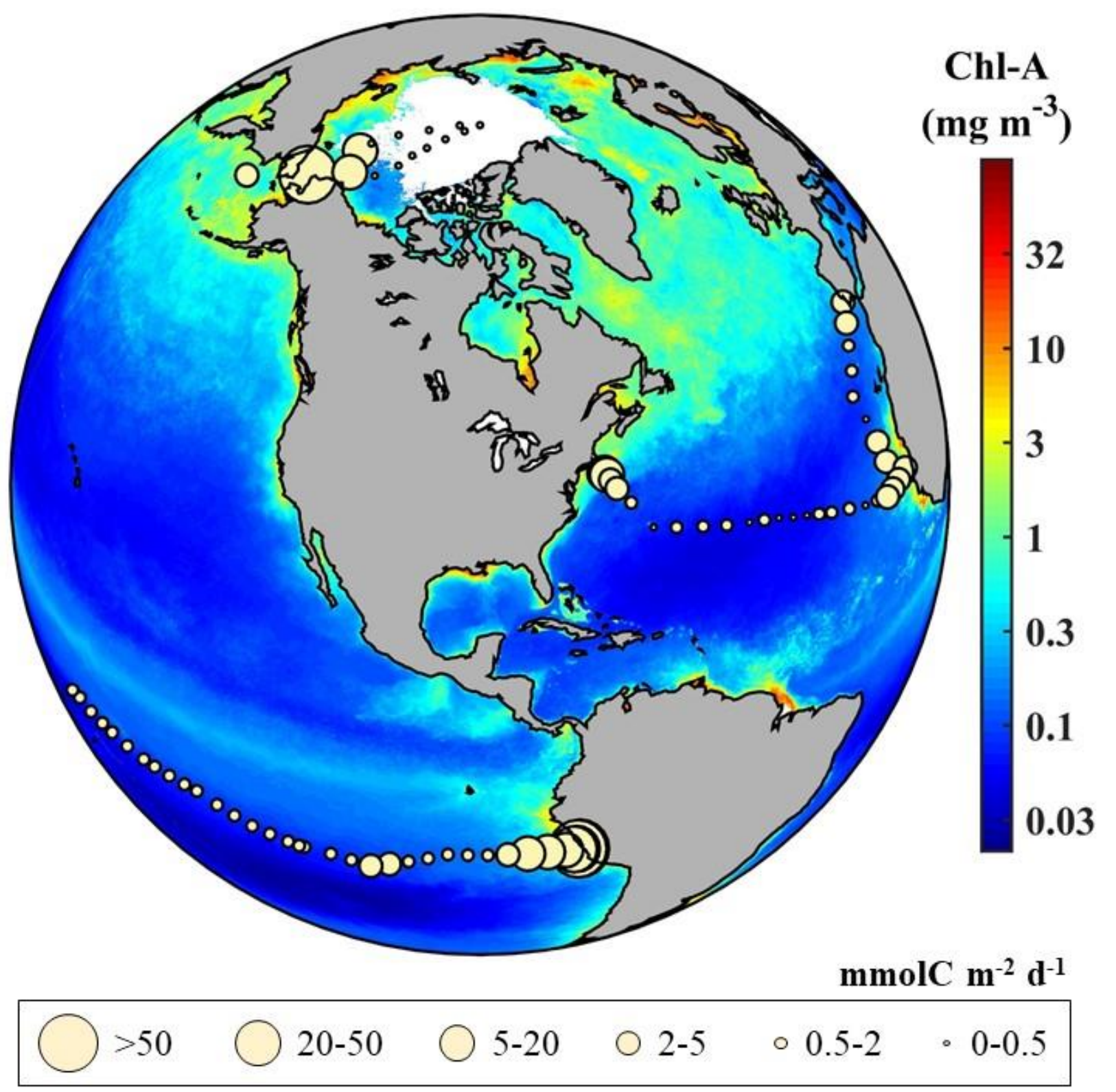

Figure 1: ${ }^{234}$ Th-derived POC export fluxes from the three completed U.S. GEOTRACES campaigns. POC export was calculated at the base of the euphotic zone (Ez) for each individual station on the North Atlantic and Southeastern Pacific transects. Arctic fluxes were reported at the average estimated Ez for a given region (i.e. shelf, open water, under-ice basin). Satellite-derived Chl-A concentrations are shown in the background and areas with no data due to ice and/or cloud cover are indicated in white. 
Appendix:

Method development for the measurement of ${ }^{228} \mathrm{Th}$ on $>51 \mu \mathrm{m}$ particulate samples 


\section{Motivation and project summary}

In principle, the Thorium-228 ( $\mathrm{t} 1 / 2=1.91 \mathrm{y})$ and Radium-228 (t $1 / 2=5.75 \mathrm{y})$ isotope system can be used similarly to the ${ }^{234} \mathrm{Th}-{ }^{238} \mathrm{U}$ method employed in Chapters 2 to 4 of this thesis to determine particulate fluxes of other elements from the surface ocean. However, ${ }^{228} \mathrm{Th}$ has been less commonly used to look at export and remineralization and more often utilized in particle dynamics studies and partitioning models (Cochran et al., 2000; Murnane et al., 1996; Trimble et al., 2004). One of the limiting factors for the measurement of ${ }^{228} \mathrm{Th}$ in many regions of the global ocean, such as the central Pacific and Atlantic, is that total ${ }^{228} \mathrm{Ra}$ activities are $<3 \mathrm{dpm} 100 \mathrm{~L}^{-1}$ in the surface ocean and $<<1 \mathrm{dpm} 100 \mathrm{~L}^{-1}$ throughout the rest of the water column. These low parent activities translate to low total particulate ${ }^{228} \mathrm{Th}$ activities that are difficult to measure without sample volumes $>1000 \mathrm{~L}$. If ${ }^{228} \mathrm{Th}$ activities could be obtained for the large particle size fraction $(>51 \mu \mathrm{m})$ simultaneously with standard dissolved measurements of ${ }^{228} \mathrm{Th}$ and ${ }^{228} \mathrm{Ra}$, the particulate flux of ${ }^{228} \mathrm{Th}$ from the surface ocean could be assessed. Compared to ${ }^{234} \mathrm{Th},{ }^{228} \mathrm{Th}$ has the potential to better capture and reflect the impact of processes occurring well below the euphotic zone, where much of the recycling of sinking particles can occur, due to the longer half-life of ${ }^{228} \mathrm{Th}$. Furthermore, a paired use of high resolution ${ }^{234} \mathrm{Th}$ and ${ }^{228} \mathrm{Th}$ methods could provide new insight into how the biological pump functions at various timescales and remineralization length scales.

The Arctic is a small basin, heavily influenced by its broad coastal shelves (Jakobsson, 2002). This means that ${ }^{228} \mathrm{Ra}$ activities in surface waters, even in the central Arctic, can reach $>10 \mathrm{dpm}$ $100 \mathrm{~L}^{-1}$ (Rutgers Van Der Loeff et al., 2012) and thus, the daughter activities of ${ }^{228} \mathrm{Th}$ are more likely to be measureable without an increase in sample volume. In this appendix, I detail the development of a small chamber method to measure ${ }^{228}$ Th on filters ( $400 \mathrm{~L}$ pumped) also being utilized for large particle ${ }^{234} \mathrm{Th}$ and POC measurements from the U.S. GEOTRACES Western Arctic Ocean campaign. With this method, I was able to produce the most extensive large particle

${ }^{228}$ Th dataset to date (>250 discrete depths sampled at 20 stations from $60^{\circ} \mathrm{N}$ to $90^{\circ} \mathrm{N}$, Figs 1 and 2). This method is non-destructive, unlike traditional alpha spectrometry, and does not require multi-step anion-exchange column processing to purify the samples prior to measurement. This dataset, paired with additional analyses at these same Arctic locations made by myself (small particle ${ }^{228} \mathrm{Th}$ ), Lauren Kipp, Paul Henderson, and Matt Charette (dissolved ${ }^{228} \mathrm{Th}$ and ${ }^{228} \mathrm{Ra}$ ), provides an unparalleled resource for examining the cycling of ${ }^{228} \mathrm{Th}$ in the marine environment. 


\section{Sample collection and ${ }^{228} \mathrm{Th}$ measurements}

Samples were collected on the USCGC Healy from August to October 2015 (See Section 4.2 for additional cruise information). The particulate samples used for initial method development and alpha measurement calibration were collected from the ship's underway system using a custom-built filter head for sampling $>51 \mu \mathrm{m}$ particles with a $142 \mathrm{~mm}$ diameter screen. To capture potential variations in particle loading of the filters and ${ }^{228} \mathrm{Th}$ activities, I collected 18 samples with filter volumes ranging from $16 \mathrm{~L}$ to $1714 \mathrm{~L}$ with filtration times ranging from 1 to 91 hours. These samples were taken between stations 14 and 46 (NB transect: $76^{\circ} \mathrm{N}$ to pole, SB transect: pole to $81^{\circ} \mathrm{N}$ ). The underway system captured surface water (upper $10 \mathrm{~m}$ ) and filtration was generally performed while the ship was moving between stations. Flow rates averaged about $1 \mathrm{~L}$ per minute with a maximum flow rate of $2.4 \mathrm{~L}$ per minute. The high volume McLane pump samples ('water column samples') were taken as detailed in Section 4.2.3.

All of the $142 \mathrm{~mm},>51 \mu \mathrm{m}$ screens were rinsed with pre-filtered seawater directly onto $25 \mathrm{~mm}$

Ag filters, dried, and mounted for ${ }^{234}$ Th shipboard counting. Filters were place on plastic mounts, covered with a layer of mylar, and then covered with 2 layers of aluminum foil. After shipboard beta counting, samples were double-bagged and stored at room temperature. Third counts of the samples began in February 2016. Once the final ${ }^{234}$ Th count was made, the filter was demounted, weighed, and directly placed into the small volume chamber described below. Non-destructive ${ }^{228}$ Th measurements were made using Radium Delayed-Coincidence Counters (RaDeCCs, Maiti et al., 2014). Samples were counted for an average of 23 hours. After measurement, masses were recorded again to ensure than any mass loss could be monitored. A few samples lost mass due to the filtered material cracking and separating from the filter prior to measurement, but we did not have significant mass loss during the 23-hour period of measurement.

\section{Chamber construction}

At first, attempts were made to utilize previously existing, in-line $25 \mathrm{~mm}$ filter holders to measure ${ }^{228} \mathrm{Th}$. Blank filters spiked with high concentrations of ${ }^{228} \mathrm{Th}$ proved to be measureable and cost-effective. However, test filters taken from the eastern coast of Japan, using McLane pumping systems, partially or fully obstructed the flow of the detectors due to the visible particle loading. This method needs to be viable for a wide range in particle loading, especially since the 
parent ${ }^{228} \mathrm{Ra}$ is higher near coastal zones, where particle concentrations generally increase. This in-line method was abandoned for the lateral, flow-over method detailed below.

A miniature version of the chamber described in Maiti et al. (2014) was constructed to hold the $25 \mathrm{~mm} \mathrm{Ag}$ filters (Figs. 3 to 5). The construction was modified to have a single inlet and outlet, an inner diameter of 1 and 1/16 inches. The inner height was set at 11/16 inches with room for 2 removable plastic filter mounts. Samples were run using 2 mounts for easy removal from the chamber. With 2 mounts the filter sits just below the direct flow from the inlet and outlet. It was determined that with this sample setup, particles were not blown from the filters. However, for other filter types, thicker samples, or samples already mounted to another material the option of removing the mounts provides flexibility.

\section{Calibration of RaDeCC systems for small volume chambers}

Of the 18 samples taken from the underway system, 6 were chosen for calibration that had a range in ${ }^{228} \mathrm{Th}$ activities from $0.05 \mathrm{dpm}$ to $1.16 \mathrm{dpm}$ (decays per minute as measured via traditional alpha spectrometry, Fig. 6). The maximum discrete depth sample activity was $0.53 \mathrm{cpm}$. These calibration samples covered the full range in thickness (negligible material on filter to $1.6 \mathrm{~mm}$ of material) and mass $(0.12 \mathrm{~g}$ to $0.31 \mathrm{~g})$ of the discrete depth samples. Blank filters were placed in perforated plastic tupperwares that were attached to the filter head apparatuses during deployment. These filters represent passive dipped blanks (i.e. no volume was pumped through these filters). The average of the dipped blank sample activities was $0.01 \mathrm{cpm}$ and this value was subtracted from all discrete depth samples. The 6 calibration samples were run sequentially through 8 different $\mathrm{RaDeCC}$ systems and then processed for measurement with traditional alpha spectrometry (Table 1). A calibration line was made using this data for each detector, an example of which is shown in Fig. 6, and the RaDeCC efficiency for the small volume chamber method was determined from the slope of the line (Table 1).

Using a larger, but similarly constructed chamber system, Maiti et al. (2014) showed that there was an optimum wetness for QMA filters where the efficiency was greatest. I tested whether wetting 3 different samples would improve the ${ }^{228}$ Th activity (dpm) captured by the detectors (Fig. 7). This volume was determined using the optimum amount ( $5 \mathrm{~mL}$ ) from Maiti et al. (2014) for a $126 \mathrm{~mm}$ 'active' filter diameter and scaling it down for a $25 \mathrm{~mm}$ filter, which amounted to 200 $\mu \mathrm{L}$ of Milli-Q. Two of the filters showed an improved efficiency with sample wetting (Fig. 7) and 
one did not show any difference when uncertainties were considered. I ran all of the discrete depth samples dry because the detector efficiencies were already high enough for the purposes of this Arctic study without wetting (Table 1) and these samples were to be used for POC and PON analyses after ${ }^{228} \mathrm{Th}$ measurements were completed. In the future, further testing could be done to determine whether temporary wetting would change the partitioning of carbon or nitrogen within the samples. If there is no change in partitioning or if POC and PON analyses are not be completed using the samples, wetting could be a means of increasing the efficiency of the system for studies in other basins where ${ }^{228} \mathrm{Th}$ activities might be lower.

Prior to the alpha spectrometry measurements, the calibration filters were placed in a $50 \mathrm{~mL}$ beaker and a known aliquot ( $4.4 \mathrm{mg}$ to $9.8 \mathrm{mg}$ ) of ${ }^{230} \mathrm{Th}$ yield monitor (activity $50.46 \mathrm{dpm} \mathrm{g}^{-1}$ ) was added. Filter were leached with $10 \mathrm{~mL}$ of $8 \mathrm{~N} \mathrm{HNO}_{3}$ and $10 \% \mathrm{H}_{2} \mathrm{O}_{2}$. The beakers were covered with watch glasses and sonicated for 20 minutes. The samples were then left covered overnight. Samples were purified following the procedures in Pike et al. (2005) using a $2 \mathrm{~mL}$ bed-volume anion-exchange column (Bio-Rad Poly-Prep Chromatography column and AG 1-X8, 100-200 mesh chloride form Bio-Rad resin). The columns were first washed with $3 \mathrm{~mL}(\times 3)$ of concentrated $\mathrm{HCl}$, waiting 10 minutes after each addition of acid to dissolve trace impurities. After the last addition the $\mathrm{HCl}$ was allowed to drain completely from the column. The columns were then washed with $4 \mathrm{~mL}(\times 3)$ of $1.2 \mathrm{M} \mathrm{HCl}$, allowing the column to drain between each addition, and then with $4 \mathrm{~mL}(\times 3)$ of Milli-Q water. The columns were then preconditioned by adding $4 \mathrm{~mL}(\times 3)$ of $\mathrm{HNO}_{3}$ (just prior to adding the sample). The sample was added to the column by pouring the contents of the beaker into the column opening (keeping the filter in the beaker). The beaker was rinsed with $4 \mathrm{~mL} 8 \mathrm{M} \mathrm{HNO}_{3}(\times 3)$ and all rinses were added to column. Impurities were removed by washing the column next with $10 \mathrm{~mL}(\times 4) 8 \mathrm{M} \mathrm{HNO}_{3}$ and the wastes were discarded. The thorium was eluted from the column into an acid-cleaned $50 \mathrm{~mL}$ beaker using $5 \mathrm{~mL}(\times 4)$ of $9 \mathrm{M} \mathrm{HCl}$.

Once the elution was complete, the sample liquid was evaporated to dryness. The samples were brought up in $10 \mathrm{~mL}$ of $4 \mathrm{M} \mathrm{HCl}$ and $0.5 \mathrm{~mL}$ concentrated $\mathrm{HCl}$. The samples were then rinsed into plastic beakers with $5 \mathrm{~mL}(\times 2)$ of $4 \mathrm{M} \mathrm{HCl}$ and $200 \mu \mathrm{L}$ of Ce carrier was added. The sample was stirred and $3 \mathrm{~mL}$ of concentrated HF was added. The sample was left covered for 30 minutes. The samples were then filtered using a vacuum flask filtration system. The polypropylene filters (25 mm diameter, $0.1 \mu \mathrm{m}$ ) were dried under a heat lamp and glued sample side up to a cleaned metal planchet for alpha measurement. The alpha spectrometer has been calibrated for this type of flat 
planchet-mounted sample and thus, with the ${ }^{230}$ Th yield monitor I can compare the known dpm from this method to the RaDeCC results to get the efficiency of the RaDeCCs (Table 1). 


\section{References}

Cochran, J.K., Buesseler, K.O., Bacon, M.P., Wang, H.W., Hirschberg, D.J., Ball, L., Andrews, J., Crossin, G., Fleer, A., 2000. Short-lived thorium isotopes $\left({ }^{234} \mathrm{Th},{ }^{228} \mathrm{Th}\right)$ as indicators of POC export and particle cycling in the Ross Sea, Southern Ocean. Deep. Res. Part II Top. Stud. Oceanogr. 47, 3451-3490. doi:10.1016/S0967-0645(00)00075-8

Jakobsson, M., 2002. Hypsometry and volume of the Arctic Ocean and its constituent seas. Geochemistry Geophys. Geosystems 3, 1-18.

Maiti, K., Charette, M.A., Buesseler, K.O., Zhou, K., Henderson, P., Moore, W.S., Morris, P., Kipp, L., 2014. Determination of particulate and dissolved ${ }^{228} \mathrm{Th}$ in seawater using a delayed coincidence counter. Mar. Chem. 177, 196-202. doi:10.1016/j.marchem.2014.12.001

Murnane, R.J., Cochran, J.K., Buesseler, K.O., Bacon, M.P., 1996. Least-squares estimates of thorium, particle, and nutrient cycling rate constants from the JGOFS North Atlantic Bloom Experiment. Deep. Res. Part I Oceanogr. Res. Pap. 43, 239-258. doi:10.1016/0967-0637(96)00004-0

Pike, S.M., Buesseler, K.O., Andrews, J., Savoye, N., 2005. Quantification of ${ }^{234}$ Th recovery in small volume sea water samples by inductively coupled plasma mass spectrometry. J. Radioanal. Nucl. Chem. 263, 355-360.

Rutgers Van der Loeff, M., Cai, P., Stimac, I., Bauch, D., Hanfland, C., Roeske, T., Moran, S.B., 2012. Shelf-basin exchange times of Arctic surface waters estimated from ${ }^{228} \mathrm{Th} /{ }^{228} \mathrm{Ra}$ disequilibrium. J. Geophys. Res. Ocean. 117. doi:10.1029/2011JC007478

Trimble, S.M., Baskaran, M., Porcelli, D., 2004. Scavenging of thorium isotopes in the Canada Basin of the Arctic Ocean. Earth Planet. Sci. Lett. 222, 915-932. doi:10.1016/j.eps1.2004.03.027 
Table 1: Summary of RaDeCC detector efficiency results. The $8 \mathrm{RaDeCC}$ detectors are listed with the line slopes (see Fig. 6) that represent the individual detector efficiencies relative to the alpha spectrometry measurements (i.e. destructive method capturing full ${ }^{228} \mathrm{Th}$ activity present). Alpha spectrometer efficiencies fall between $20 \%$ and $30 \%$, while the results shown here show the RaDeCC small volume chamber system was slightly more efficient.

\begin{tabular}{ccc}
\hline Detector & Slope & R-squared \\
\hline G1 & 0.35 & 0.98 \\
G5 & 0.36 & 0.99 \\
G3 & 0.32 & 0.98 \\
G4 & 0.30 & 0.97 \\
U1 & 0.43 & 0.98 \\
U2 & 0.49 & 0.97 \\
U3 & 0.48 & 0.99 \\
U4 & 0.40 & 0.99 \\
\hline
\end{tabular}



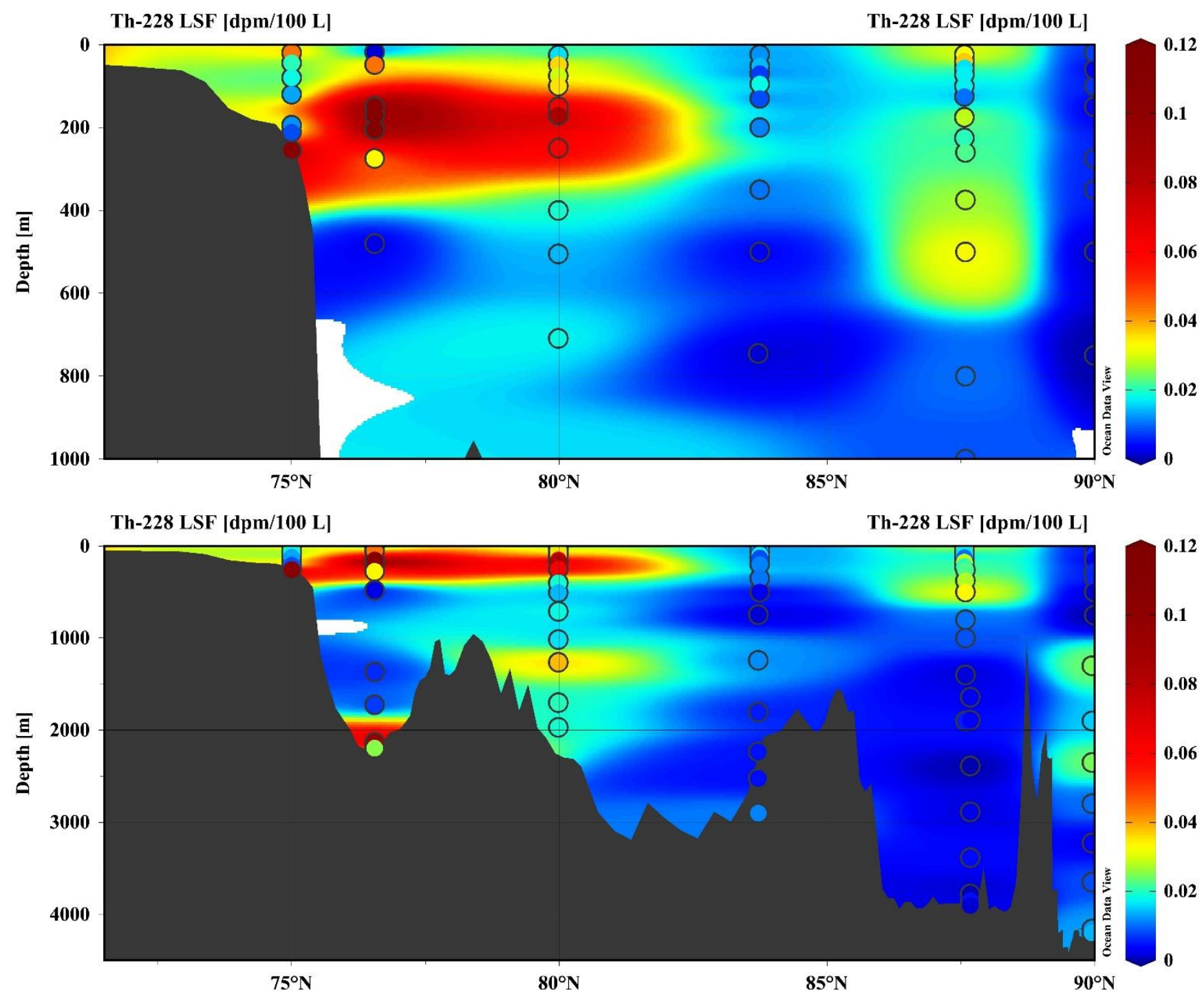

Figure 1: Preliminary ${ }^{228}$ Th activities along the northbound Arctic transect. Activities are shown for the upper $1000 \mathrm{~m}$ (top panel) and the full water column (lower panel). 

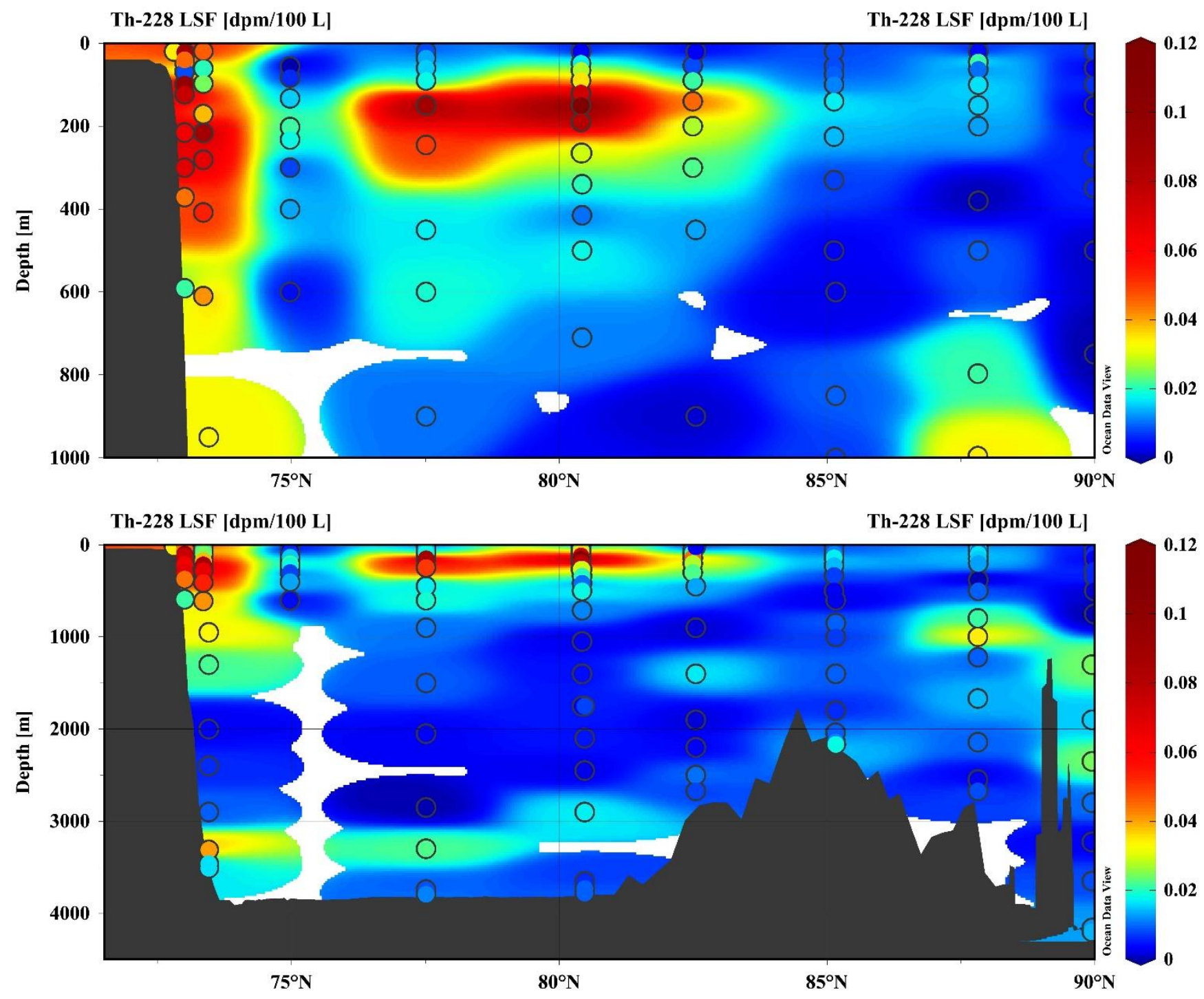

Figure 2: Preliminary ${ }^{228}$ Th activities along the southbound Arctic transect. Activities are shown for the upper $1000 \mathrm{~m}$ (top panel) and the full water column (lower panel). 


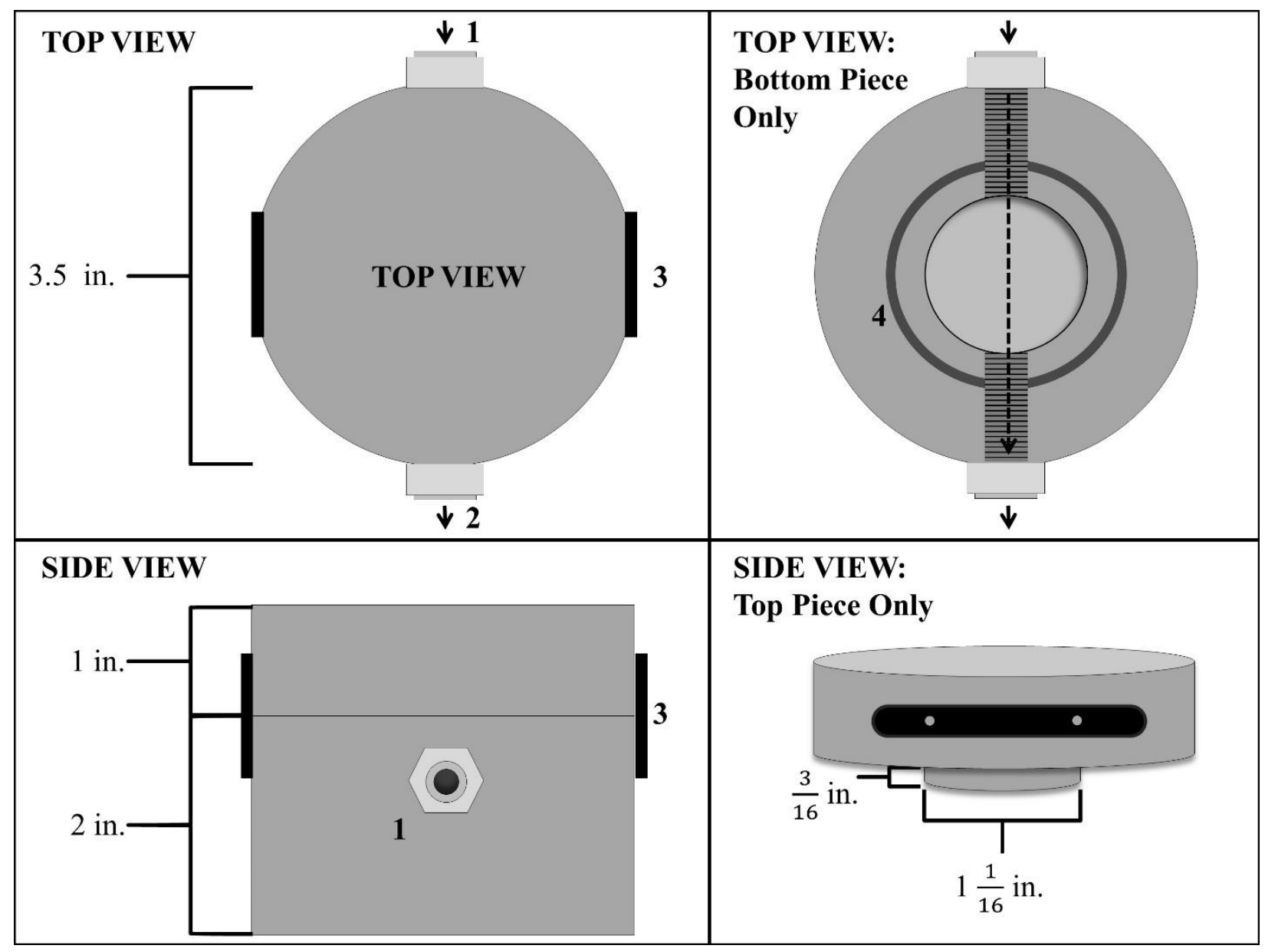

Figure 3: Small volume chamber construction schematics. Approximate size guidelines are shown in inches, but should be adjusted as needed to ensure an air-tight construction. Numbers indicate the following parts: (1) air input location with quick release connector - 1/8 inch tapered pipe threads, (2) air output location with quick release connector - 1/8 inch tapered pipe threads, (3) butterfly clamps on each side of the chamber, and (4) 2-223 $15 / 8$ inch ID o-ring in a groove cut to be just shy of the diameter of o-ring. 


\section{SIDE VIEW: INTERIOR}

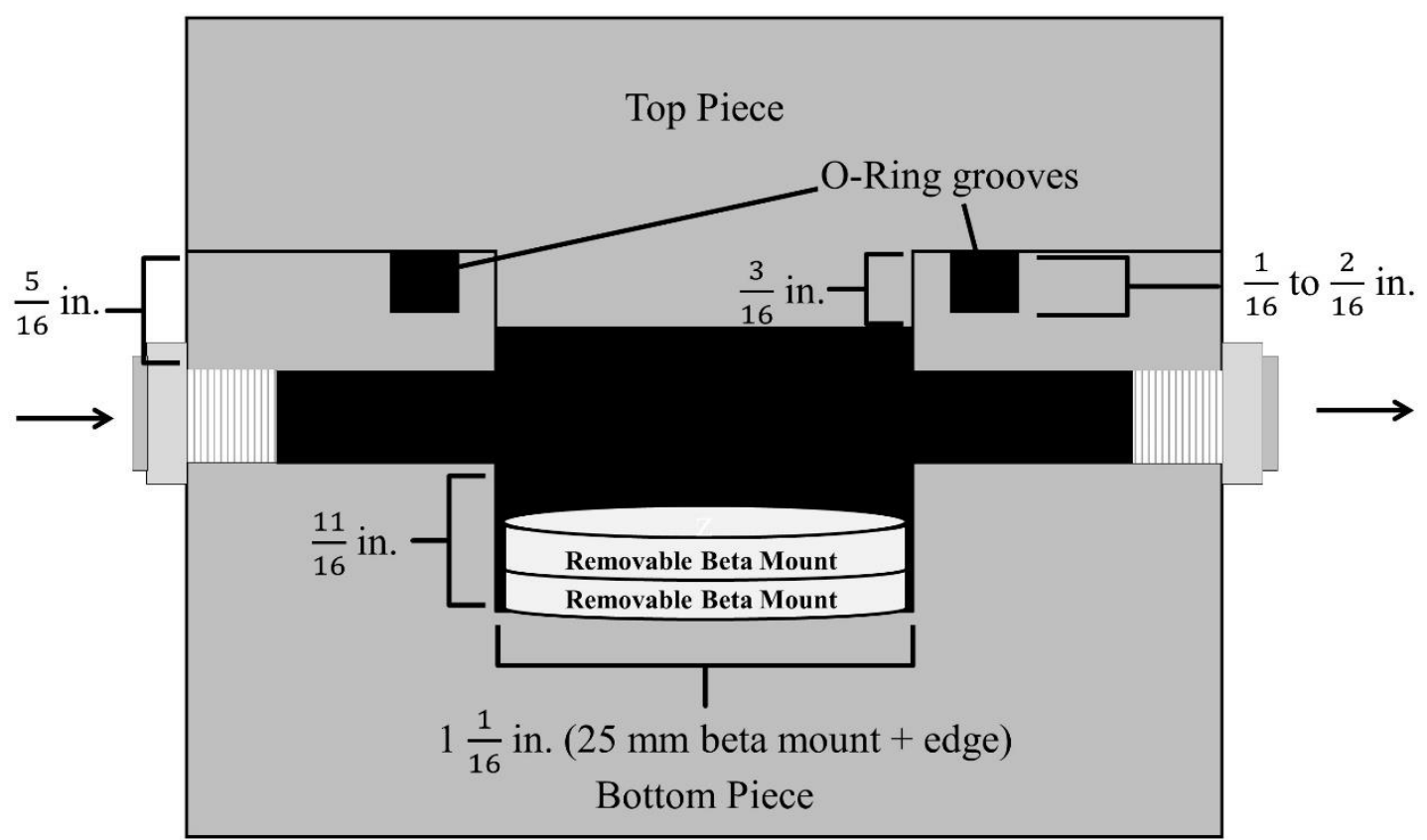

Figure 4: Small volume chamber interior construction schematic. Approximate size guidelines are shown in inches, but should be adjusted as needed to ensure an air-tight construction. The chamber is constructed to fit one or two beta mounts depending on the thickness of the sample. A small space should be left in the central part of the chamber that allows the beta mount to be lifted out of the chamber using standard laboratory forceps. 

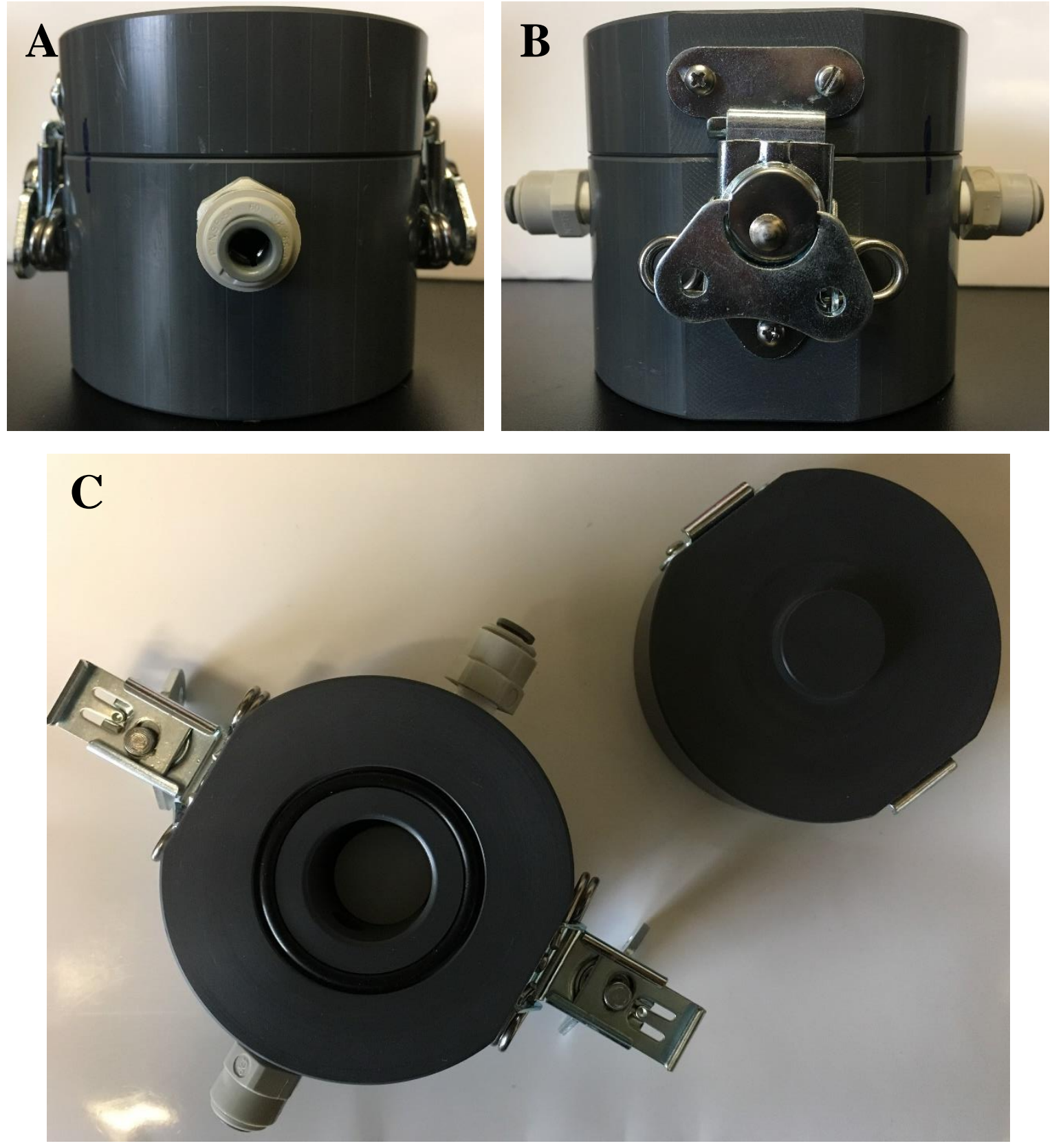

Figure 5: Photographs of small volume chambers. Photos A and B show the side view of the chamber with the input/output and clamp perspectives, respectfully. Photo $\mathrm{C}$ shows a top view of the chamber with the interior visible. The top of the chamber is on the right and the lower piece is on the left. 


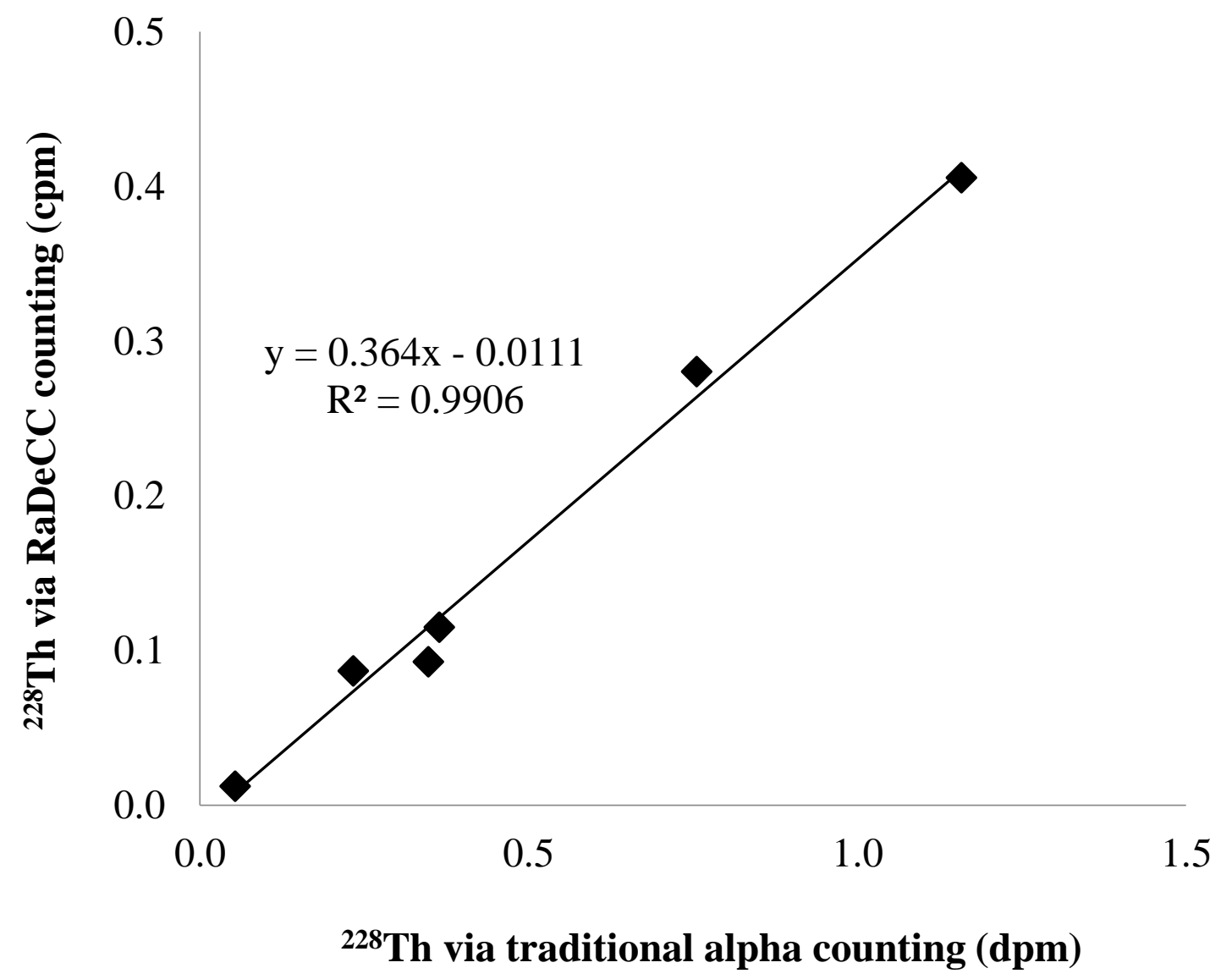

Figure 6: Example calibration for large particle ${ }^{228} \mathrm{Th}$ on RaDeCCs with small volume chambers versus traditional alpha spectrometry. Efficiency calibrations were completed for 11 detectors (data from detector G5 shown). Each individual RaDeCC system efficiency is indicated by the slope of the calibration line, which in this case is $\sim 36.4 \%$. 


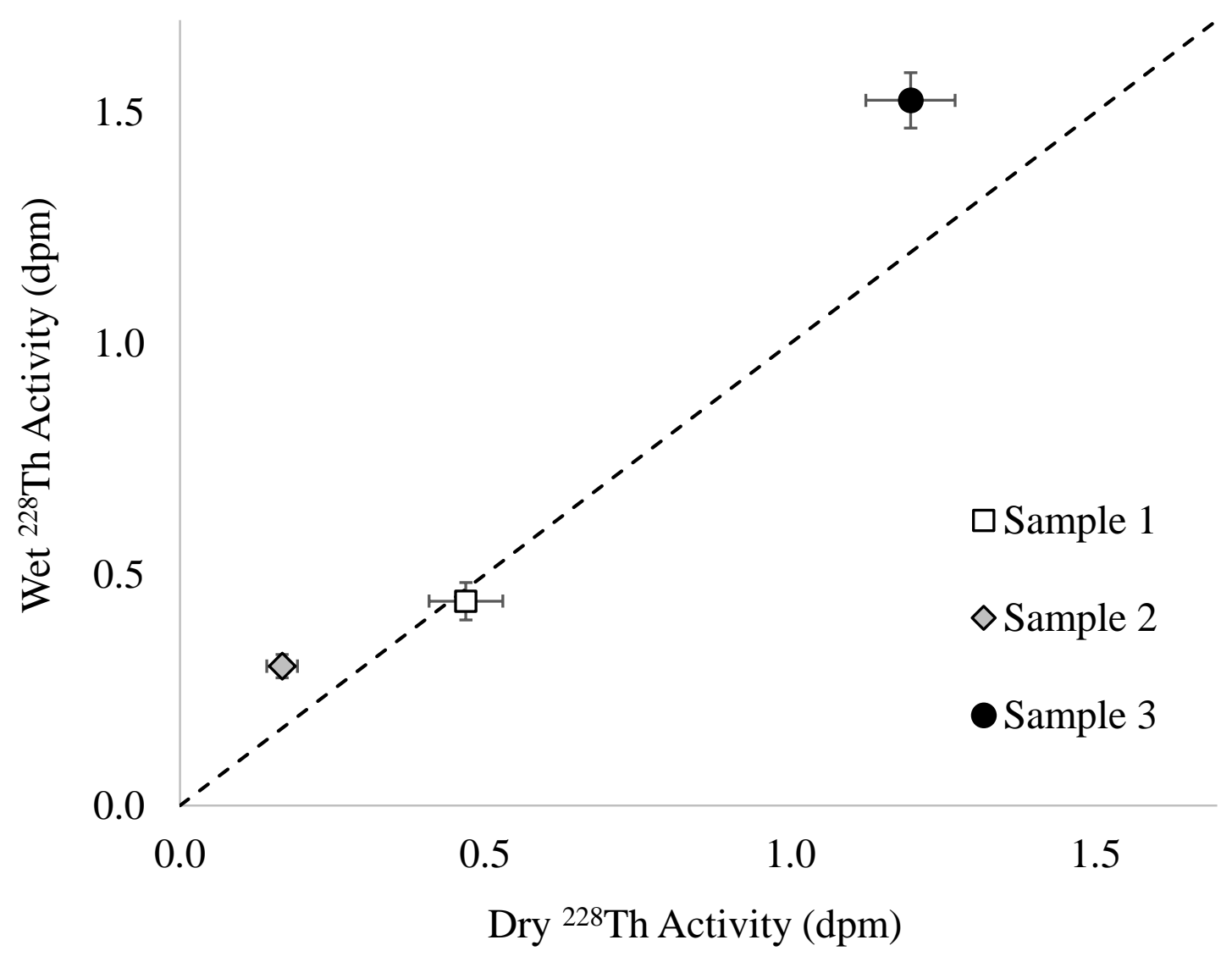

Figure 7: RaDeCC results for wet versus dry measurements. Three samples were measured for $\sim 23$ hours with no water added to the filters and then run a second time for the same duration with $200 \mu \mathrm{L}$ added to the filters just prior. A 1:1 line is shown (dashed) to indicate where there would be no difference in activity between the wet and dry measurements. 